\title{
Extraordinary Acceleration of Cogwheel Helical Self-Organization of Dendronized Perylene Bisimides by the Dendron Sequence Encoding their Tertiary Structure
}

Li Wang, ${ }^{\dagger}, \circ$ Benjamin E. Partridge, ${ }^{\dagger, \square}$ Ning Huang, ${ }^{\dagger}$ James T. Olsen, ${ }^{\dagger}$ Dipankar Sahoo,${ }^{\dagger}$ Xiangbing Zeng, ${ }^{\S}$ Goran Ungar, ${ }^{\S, \#}$ Robert Graf, ${ }^{\diamond}$ Hans W. Spiess ${ }^{\diamond}$ and Virgil Percec ${ }^{\dagger, *}$

$\dagger$ Roy \& Diana Vagelos Laboratories, Department of Chemistry, University of Pennsylvania, Philadelphia, Pennsylvania 19104-6323, United States

${ }^{\circ}$ College of Materials Science and Engineering, Beijing University of Chemical Technology, Beijing 100029, China

$\S$ Department of Materials Science and Engineering, University of Sheffield, Sheffield, S1 3JD, United Kingdom

\# State Key Laboratory for Mechanical Behavior of Materials, Xi'an Jiaotong University, Xi'an 710049, China

${ }^{\diamond}$ Max-Planck Institute for Polymer Research, 55128 Mainz, Germany

*E-mail: percec@sas.upenn.edu

\section{Table of Contents}

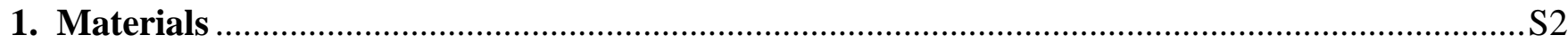

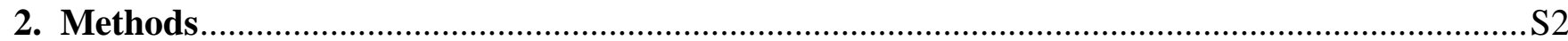

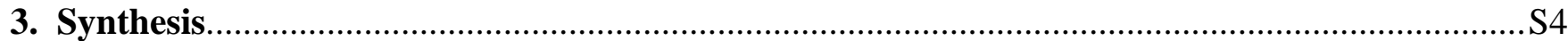

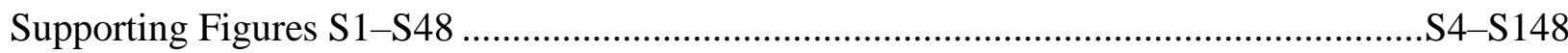

4. Thermal Analysis of Sequence-Defined Hybrid PBIs by DSC ………………....................... 149

Supporting Table ST1 ……………………………………………………………...... S149

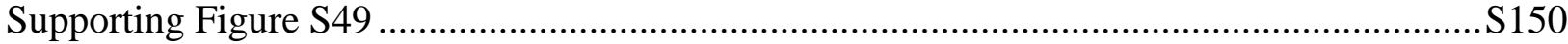

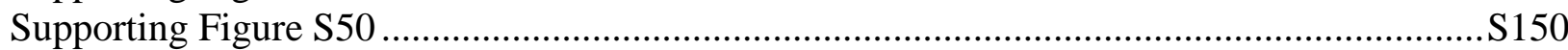

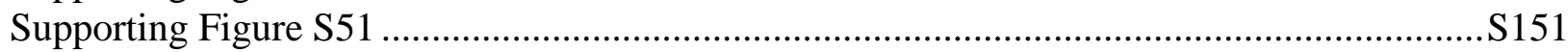

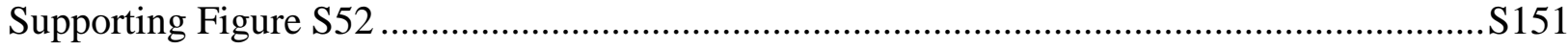

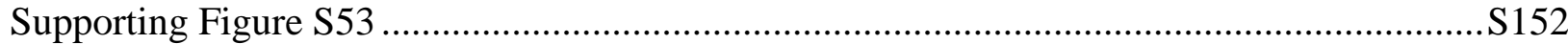

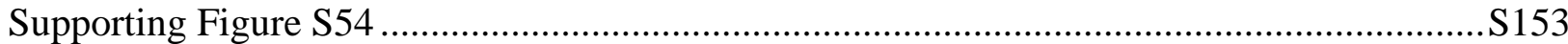

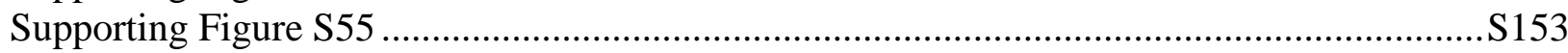

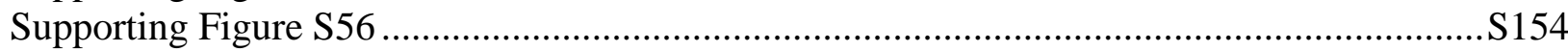

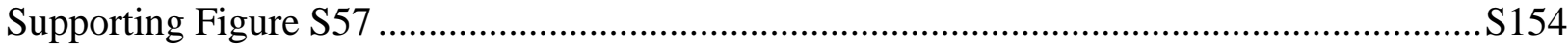

5. Structural Analysis of Sequence-Defined Hybrid PBIs by XRD ………………………........ 155

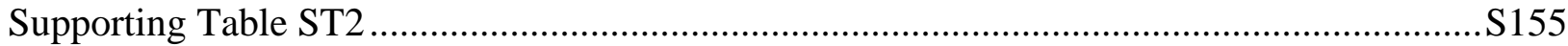

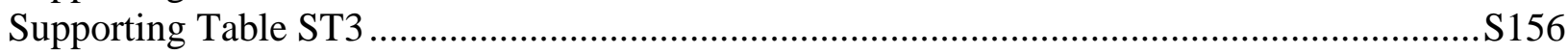

Supporting Table ST4 ……………………………………………………………..... S156

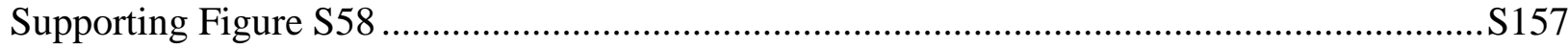

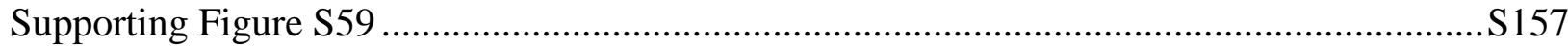

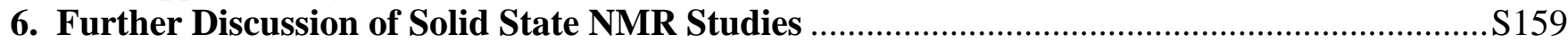

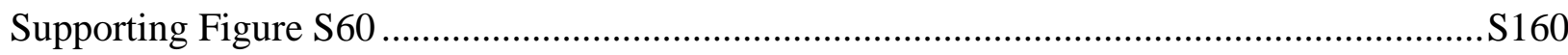

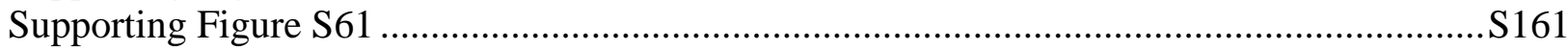

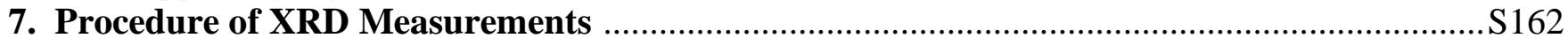

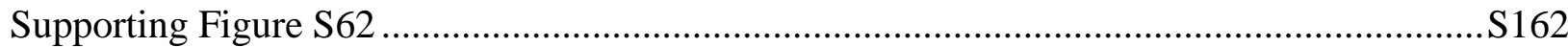

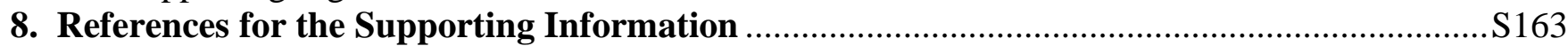




\section{Materials}

Gallic acid (Chem Impex, anhydrous, ACS grade), methanol (Fisher, ACS grade), concentrated sulfuric acid (Fisher, ACS grade), $\mathrm{NaHCO}_{3}$ (Fisher, ACS grade), ethyl acetate (Fisher, ACS grade), anhydrous $\mathrm{MgSO}_{4}$ (Oakwood Chemical, anhydrous), benzyl chloride (Alfa Aesar, 99\%), $\mathrm{K}_{2} \mathrm{CO}_{3}$ (Chem Impex, anhydrous, 99.75\%), KI (Alfa Aesar, 99\%), acetone (Fisher, ACS grade), NaCl (EMD Millipore Corporation, ACS grade), hexanes (Fisher, ACS grade), 1-bromoheptane (Aldrich, 99\%), 1-bromononane (Aldrich, 99\%), ( rac)-1-bromo-3,7-dimethyloctane (Tokyo Chemical Industry Co. Ltd., 93+\%), 1bromohexane (Alfa Aesar 99\%), 1-bromodecane (Alfa Aesar 98\%), palladium on activated carbon catalyst (Aldrich, 10 wt\% loading), hydrogen (Airgas), $\mathrm{LiAlH}_{4}$ (Acros, 95\%), NaOH (VWR Analytical, ACS grade), thionyl chloride (Alfa Aesar, 99.5\%), $\mathrm{NaN}_{3}$ (VWR Life Science), perylenetetracarboxylic acid bisanhydride (PTCDA, Acros, 98\%), $\mathrm{Zn}(\mathrm{OAc})_{2} \cdot 2 \mathrm{H}_{2} \mathrm{O}$ (Acros, 98+\%), imidazle (Aldrich, ACS), concentrated hydrochloric acid (Fisher, ACS grade), triethyl orthoformate (TCI, 98+\%), Amberlyst-15(H)

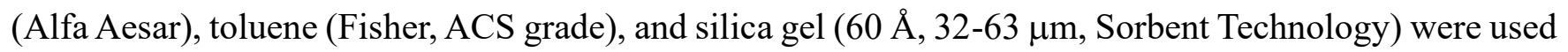
as received. Dichloromethane (DCM, Fisher, ACS grade) was refluxed over $\mathrm{CaH}_{2}$ and freshly distilled before use. THF (Fisher, ACS grade) was refluxed over sodium/benzophenone until the solution turned purple and distilled before use. Dimethylformamide (Fisher, ACS grade) was dried over $4 \AA$ molecular sieves prior to use.

\section{Methods}

NMR. ${ }^{1} \mathrm{H}$ NMR and ${ }^{13} \mathrm{C}$ NMR spectra were recorded on a Bruker Neo 400 instrument at $300 \mathrm{~K}$ using deuterated chloroform $\left(\mathrm{CDCl}_{3}\right)$ as solvent containing trimethylsilane (TMS) as internal standard. All ${ }^{1} \mathrm{H}$ NMR chemical shifts were referenced to TMS at $\delta=0 \mathrm{ppm}$.

HPLC. The purity of the products was determined by a combination of thin-layer chromatography (TLC) on silica gel coated plates and high-performance liquid chromatography (HPLC) using THF as mobile phase at $1 \mathrm{~mL} / \mathrm{min}$, on a Shimadzu LC-10AT high-performance liquid chromatograph equipped with a Perkin Elmer LC-100 oven $\left(40^{\circ} \mathrm{C}\right)$, containing two AM gel columns $\left(5 \times 10^{2}\right.$ and $1 \times 10^{4} \AA$, American Polymer Standards Corp.) in series, a Shimadzu SPD-10A UV detector ( $\lambda=254 \mathrm{~nm})$, a Shimadzu RID10A RI-detector, and a PE Nelson Analytical 900 Series integrator data station.

Differential scanning calorimetry (DSC). Thermal transitions were determined on a TA Instruments Q100 differential scanning calorimeter (DSC) equipped with a refrigerated cooling system at indicated heating and cooling rates. Indium was used as calibration standard. The transition temperatures were calculated as the maxima and minima of their endothermic and exothermic peaks. An Olympus BX51 optical microscope (100× magnification) equipped with a Mettler FP82HT hot stage and a Mettler Toledo FP90 Central Processor was used to verify thermal transitions. Melting points for all solid intermediate compounds were measured using a uni-melt capillary melting point apparatus (Arthur H. Thomas Company) and are uncorrected. Melting points for dendronized PBIs were taken as the peak temperature from DSC upon first heating at $10^{\circ} \mathrm{C} / \mathrm{min}$.

Matrix-assisted laser desorption/ionization time offlight (MALDI-TOF) mass spectrometry. MALDI-TOF mass spectrometry was performed on PerSeptive Biosystems-Voyager-DE (Framingham, MA) mass spectrometer equipped with a nitrogen laser $(337 \mu \mathrm{m})$ and operated in linear mode. Internal calibration was performed using Angiotensin II, and Bombesin as standards. The analytical samples were obtained 
by mixing the THF solutions of samples $(5-10 \mathrm{mg} / \mathrm{mL})$ with the matrix solution $(3,5$-dihydroxybenzoic acid) $(10 \mathrm{mg} / \mathrm{mL})$ in a $1: 1$ to $1: 5 \mathrm{v} / \mathrm{v}$ ratio. The prepared solution $(0.5 \mu \mathrm{L})$ was loaded on a MALDI plate and allowed to dry at $25{ }^{\circ} \mathrm{C}$ before the plate was inserted into the vacuum chamber of the MALDI-TOF instrument. The laser intensity and voltage were adjusted depending on the molecular weight, and the nature of each analyte.

$X$-ray diffraction $(X R D)$. X-ray diffraction (XRD) measurements were performed using $\mathrm{Cu}-\mathrm{K}_{\alpha 1}$ radiation $(\lambda=1.542 \AA)$ from Xeuss 2.0 with the slits of $1.2 \times 1.2 \mathrm{~mm}$ for high flux and $0.7 \times 0.7 \mathrm{~mm}$ for high resolution. To minimize attenuation, and background scattering, an integral vacuum was maintained along the length of the flight tube, and within the sample chamber. Data collected from eight detectors were combined in the first exposure, resulting in black stripe lines in the XRD pattern. The second exposure was conducted with eight detectors moved to different positions. The raw XRD data from the first and the second exposures were combined and integrated to remove the black stripe lines, generating the final XRD raw data. Samples were held in glass capillaries $(1.0 \mathrm{~mm}$ in diameter $)$, mounted in a temperaturecontrolled oven (temperature precision: $\pm 0.1{ }^{\circ} \mathrm{C}$, temperature range: from -10 to $210{ }^{\circ} \mathrm{C}$ ). Aligned samples for fiber XRD experiments were prepared using a custom-made extrusion device. ${ }^{1}$ Powdered sample $(\sim 10 \mathrm{mg})$ was heated inside the extrusion device. After slow cooling, the fiber was extruded in the liquid crystal phase, and cooled to $23{ }^{\circ} \mathrm{C}$. Typically, the aligned samples have a thickness of $0.3-0.7 \mathrm{~mm}$, and a length of 3-7 mm. All XRD measurements were done with the aligned sample axis perpendicular to the beam direction. Sample-to-detector distance was $550 \mathrm{~mm}$. Additional measurements to verify indexed parameters were performed with a sample-to-detector distance of $160 \mathrm{~mm}$. Primary XRD analysis was performed using Datasqueeze (version 3.0.5). ${ }^{2}$

Molecular modeling and XRD simulation. Molecular models of supramolecular assemblies were generated using an iterative methodology described in the main text. Accelrys Materials Studio (version 5.0) was used for model building. Fiber XRD patterns were calculated using Accelrys Cerius2 run on a Silicon Graphics (SGI) Tezro workstation.

Solid state NMR. Variable-temperature ${ }^{13} \mathrm{C}\left\{{ }^{1} \mathrm{H}\right\}$ CP-MAS NMR spectra were recorded with a Bruker Avance III console at $700.23 \mathrm{MHz}{ }^{1} \mathrm{H}\left(176.1 \mathrm{MHz}{ }^{13} \mathrm{C}\right)$ Larmor frequency. A commercial double resonance probe supporting zirconia rotors with $2.5 \mathrm{~mm}$ outer diameter was used. Measurements were performed at $25 \mathrm{kHz}$ MAS spinning frequency using a CP contact time of $1 \mathrm{~ms}$. A RF nutation frequency of $100 \mathrm{kHz}$ was used on both the ${ }^{1} \mathrm{H}$ and ${ }^{13} \mathrm{C}$ channels. The given temperatures were corrected for known deviations due to frictional heating under fast spinning conditions based on the temperature-dependent chemical shift of lead nitrate. 


\section{Synthesis}

The synthesis of the eight dendronized PBI derivatives used in this study is outlined in Figure 1 . The synthetic routes to these PBIs with sequence-defined dendrons are similar to those previously elaborated and reported by our group. ${ }^{3}$ Compounds 3-r, 4-r, 6, 7-r and 8-r were synthesized as described in ref. 3.

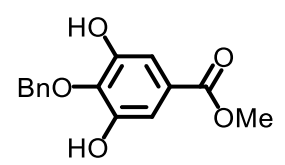

Methyl 4-(benzyloxy)-3,5-dihydroxybenzoate (2). Following a modified literature procedure, ${ }^{4}$ a mixture of 1 (10.00 g, $54.3 \mathrm{mmol}), \mathrm{KHCO}_{3}(10.87 \mathrm{~g}, 108.6 \mathrm{mmol})$ and $\mathrm{KI}$ (53.9 mg, $\left.0.33 \mathrm{mmol}\right)$ was stirred in DMF (300 mL) under $\mathrm{N}_{2}$ protection. Benzyl chloride $(6.25 \mathrm{~mL}, 54.3 \mathrm{mmol})$ was added and the reaction mixture was stirred at $60{ }^{\circ} \mathrm{C}$ for $24 \mathrm{~h}$. Then the mixture was poured into water $(1500 \mathrm{~mL})$ and extracted with EA $(300 \mathrm{~mL} \times 4)$. The combined EA phase was washed with water $(300 \mathrm{~mL} \times 3)$, brine $(300 \mathrm{~mL})$, dried over anhydrous $\mathrm{MgSO}_{4}$, filtered and evaporated in vacuo. After column chromatography $($ EA/Hexanes $=1 / 7$ to $1 / 3), 2$ was obtained as white powder. Product: 10.50 g. Yield: $71 \%$. mp $=131-$ $133{ }^{\circ} \mathrm{C}$. The characterization results of 2 are consistent with those in ref. 3. 


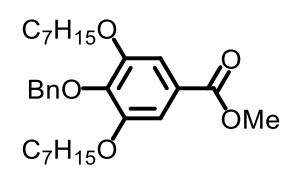

Methyl 4-(benzyloxy)-3,5-bis(octyloxy)benzoate (3-7). Compound 3-7 was synthesized by Williamson etherification under conditions previously reported by our group. ${ }^{5}$ A mixture of $2(1.37 \mathrm{~g}, 5.0 \mathrm{mmol})$ and $\mathrm{K}_{2} \mathrm{CO}_{3}(2.76 \mathrm{~g}, 20.0 \mathrm{mmol})$ was stirred in dry DMF $(20 \mathrm{~mL})$ under $\mathrm{N}_{2}$ protection. 1-Bromoheptane (1.65 $\mathrm{mL}, 10.5 \mathrm{mmol}$ ) was added and the reaction mixture was heated at $120^{\circ} \mathrm{C}$ for $12 \mathrm{~h}$. Then the mixture was cooled, poured into cold water $(100 \mathrm{~mL})$ and extracted with EA $(25 \mathrm{~mL} \times 4)$. The combined EA phase was washed with water $(25 \mathrm{~mL} \times 3)$ and brine $(25 \mathrm{~mL})$, dried over anhydrous $\mathrm{MgSO}_{4}$, filtered and evaporated in vacuo. Column chromatography $(\mathrm{EA} / \mathrm{Hexanes}=1 / 24)$ gave $3-7$ as a colorless oil. Product: $2.21 \mathrm{~g}$. Yield: 94\%. Purity by HPLC: 99+\%. The synthesis procedures for 3-9, 3-6 and 3-10 listed below are similar to that for 3-7.

${ }^{1} \mathrm{H}$ NMR (400 MHz, $\left.\mathrm{CDCl}_{3}, \delta, \mathrm{ppm}\right): 7.50\left(\mathrm{~d}, J=6.8 \mathrm{~Hz}, 2 \mathrm{H}, \mathrm{ArH}\right.$ ortho to $\left.\mathrm{CH}_{2} \mathrm{OAr}\right), 7.38-7.32(\mathrm{~m}, 2 \mathrm{H}$, ArH meta to $\mathrm{CH}_{2} \mathrm{OAr}$ ), 7.32-7.29 (m, 1H, $\mathrm{ArH}$ para to $\mathrm{CH}_{2} \mathrm{OAr}$ ), 7.27 (s, $2 \mathrm{H}, \mathrm{ArH}$ ortho to $\mathrm{CO}_{2} \mathrm{CH}_{3}$ ), $5.09\left(\mathrm{~s}, 2 \mathrm{H},-\mathrm{OCH} H_{2} \mathrm{Ar}\right), 4.02\left(\mathrm{t}, J=6.5 \mathrm{~Hz}, 4 \mathrm{H},-\mathrm{OCH}_{2} \mathrm{CH}_{2}-\right), 3.90\left(\mathrm{~s}, 3 \mathrm{H},-\mathrm{CO}_{2} \mathrm{CH}_{3}\right), 1.87-1.78(\mathrm{~m}, 4 \mathrm{H}$, $\left.-\mathrm{OCH}_{2} \mathrm{CH}_{2-}\right), 1.53-1.43$ (m, 4H, $\left.-\mathrm{O}\left(\mathrm{CH}_{2}\right)_{2} \mathrm{CH}_{2}-\right), 1.43-1.24\left(\mathrm{~m}, 12 \mathrm{H},-\mathrm{O}\left(\mathrm{CH}_{2}\right)_{3}\left(\mathrm{CH}_{2}\right)_{3}-\right), 0.90$ (t, $J=$ $\left.6.8 \mathrm{~Hz}, 6 \mathrm{H},-\mathrm{CH}_{2} \mathrm{CH}_{3}\right) .{ }^{13} \mathrm{C}$ NMR $\left(101 \mathrm{MHz}, \mathrm{CDCl}_{3}, \delta, \mathrm{ppm}\right): 167.01(\mathrm{C}=\mathrm{O}), 152.99$ (ArC-3,5 of benzoate), 141.82 ( $\mathrm{ArC}-4$ of benzoate), 137.94 ( $\mathrm{ArC}-1$ of $\mathrm{Bn}$ ), 128.35 ( $\mathrm{ArC}-3,5$ of Bn), 128.25 ( $\mathrm{ArC}-4$ of $\mathrm{Bn}$ ), 127.94 (ArC-2,6 of $\mathrm{Bn}$ ), 125.21 ( $\mathrm{ArC}-1$ of benzoate), 107.92 (ArC-2,6 of benzoate), 74.95 $\left(\mathrm{ArOCH} \mathrm{H}_{2} \mathrm{Ar}\right), 69.28\left(\mathrm{ArOCH}_{2} \mathrm{CH}_{2}-\right), 52.24\left(-\mathrm{OCH}_{3}\right), \quad[31.92,29.43,29.18,26.17,22.74]$ ($\left.\mathrm{OCH}_{2}\left(\mathrm{CH}_{2}\right)_{5}-\right), 14.21\left(-\mathrm{CH}_{2} \mathrm{CH}_{3}\right)$. MALDI-TOF MS $m / z$ of $[\mathrm{M}+\mathrm{Na}]^{+}$calculated for $\mathrm{C}_{29} \mathrm{H}_{42} \mathrm{O}_{5}: 493.3$; Found: 493.0.
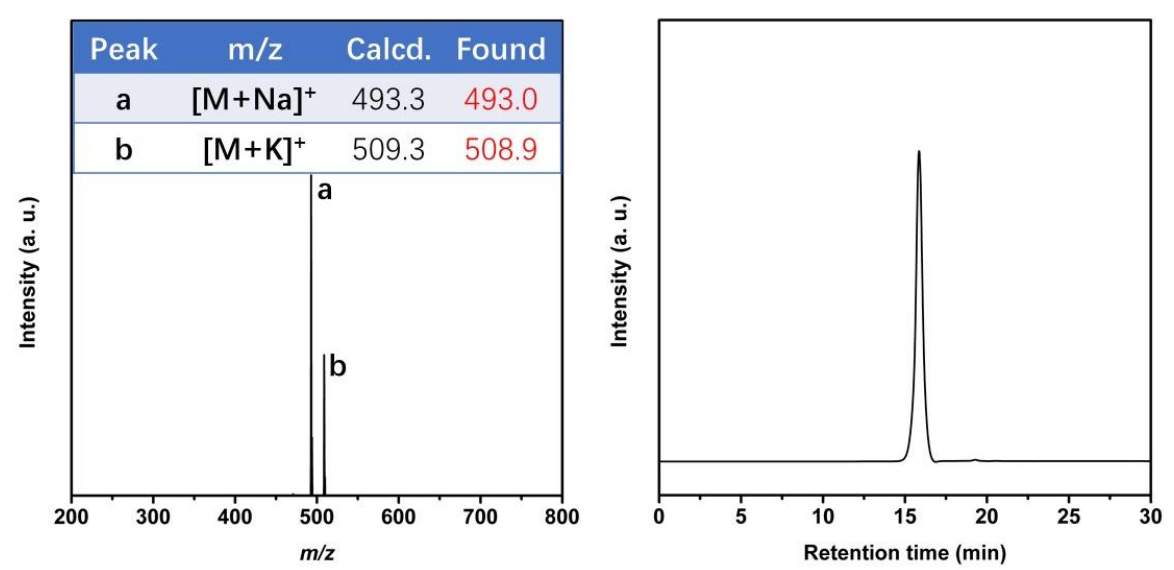

Supporting Figure S1. MALDI-TOF MS spectra (left) and HPLC trace (right) of 3-7. Heptyl groups have been successfully linked to the 3- and 5-positions of 3-7. ${ }^{1} \mathrm{H}$ NMR spectrum is presented on page 6 . 
Supporting Figure S1 (continued). ${ }^{1} \mathrm{H}$ NMR of 3-7.

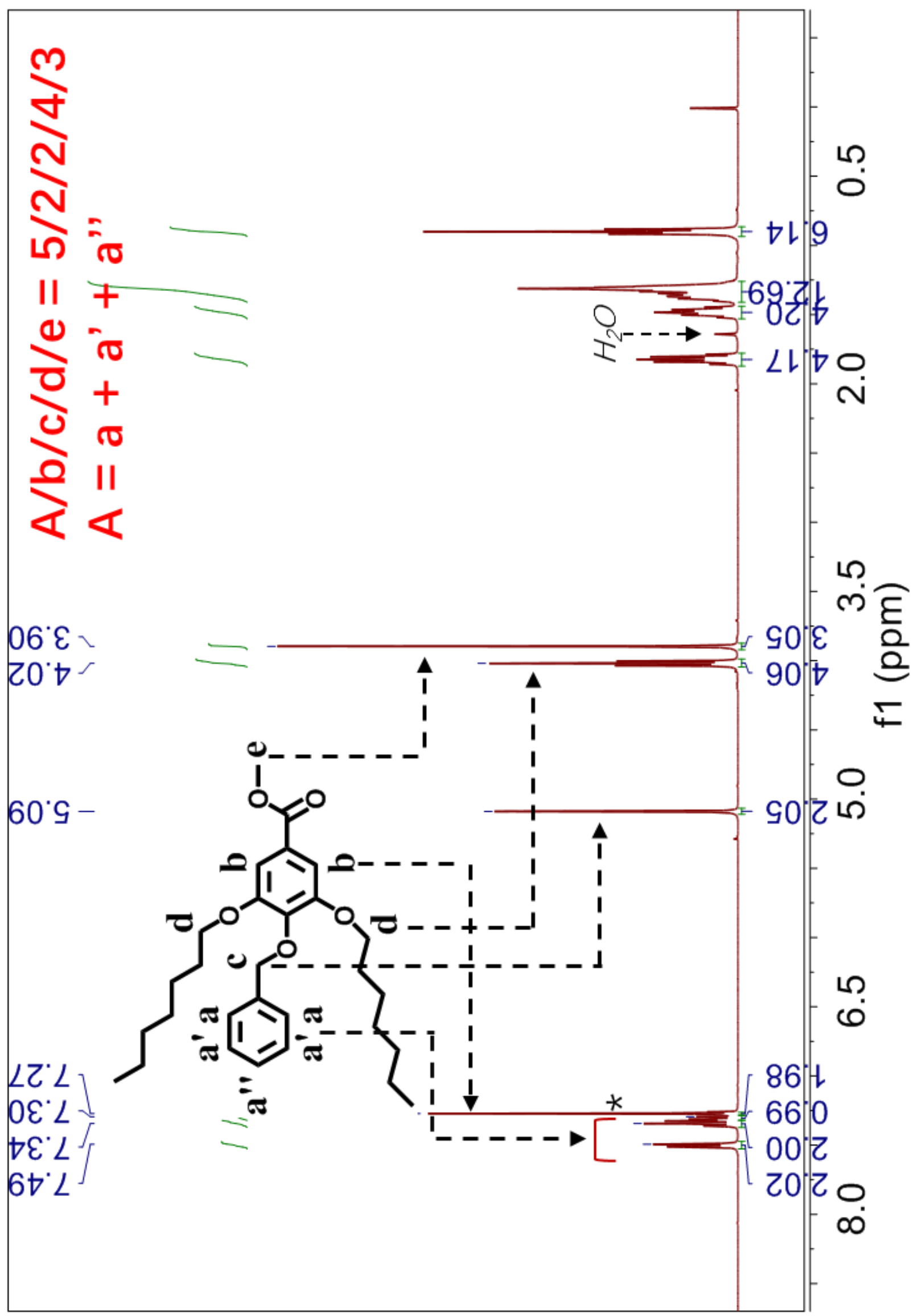




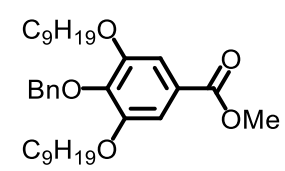

Methyl 4-(benzyloxy)-3,5-bis(octyloxy)benzoate (3-9). Starting from 2 (1.37 g, $5.0 \mathrm{mmol}), \mathrm{K}_{2} \mathrm{CO}_{3}(2.76$ g, $20.0 \mathrm{mmol})$ and 1-bromononane $(2.01 \mathrm{~mL}, 10.5 \mathrm{mmol})$ in dry DMF $(20 \mathrm{~mL})$ at $120{ }^{\circ} \mathrm{C}$ for $12 \mathrm{~h}, \mathbf{3 - 9}$ was obtained as a colorless oil. Product: $2.51 \mathrm{~g}$. Yield: $95 \%$. Purity by HPLC: $99+\%$.

${ }^{1} \mathrm{H}$ NMR $\left(400 \mathrm{MHz}, \mathrm{CDCl}_{3}, \delta, \mathrm{ppm}\right): 7.51$ (d, $J=7.0 \mathrm{~Hz}, 2 \mathrm{H}, \mathrm{ArH}$ ortho to $\left.\mathrm{CH}_{2} \mathrm{OAr}\right), 7.38-7.32(\mathrm{~m}, 2 \mathrm{H}$, $\mathrm{ArH}$ meta to $\mathrm{CH}_{2} \mathrm{OAr}$ ), 7.32-7.29 (m, $1 \mathrm{H}, \mathrm{ArH}$ para to $\mathrm{CH}_{2} \mathrm{OAr}$ ), 7.28 (s, 2H, $\mathrm{ArH}$ ortho to $\mathrm{CO}_{2} \mathrm{CH}_{3}$ ), $5.10\left(\mathrm{~s}, 2 \mathrm{H},-\mathrm{OCH} \mathrm{H}_{2} \mathrm{Ar}\right), 4.02\left(\mathrm{t}, J=6.5 \mathrm{~Hz}, 4 \mathrm{H},-\mathrm{OCH}_{2} \mathrm{CH}_{2}-\right), 3.90\left(\mathrm{~s}, 3 \mathrm{H},-\mathrm{CO}_{2} \mathrm{CH}_{3}\right), 1.88-1.78(\mathrm{~m}, 4 \mathrm{H}$, $\left.-\mathrm{OCH}_{2} \mathrm{CH}_{2}-\right), 1.55-1.43$ (m, 4H, $\left.-\mathrm{O}\left(\mathrm{CH}_{2}\right)_{2} \mathrm{CH}_{2}-\right), 1.43-1.20\left(\mathrm{~m}, 20 \mathrm{H},-\mathrm{O}\left(\mathrm{CH}_{2}\right)_{3}\left(\mathrm{CH}_{2}\right)_{5}-\right), 0.90(\mathrm{t}, J=$ $\left.6.8 \mathrm{~Hz}, 6 \mathrm{H},-\mathrm{CH}_{2} \mathrm{CH}_{3}\right) .{ }^{13} \mathrm{C}$ NMR $\left(101 \mathrm{MHz}, \mathrm{CDCl}_{3}, \delta, \mathrm{ppm}\right): 166.97(\mathrm{C}=\mathrm{O}), 152.97$ (ArC-3,5 of benzoate), 141.82 ( $\mathrm{ArC}-4$ of benzoate), 137.93 ( $\mathrm{ArC}-1$ of $\mathrm{Bn}$ ), 128.33 ( $\mathrm{ArC}-3,5$ of Bn), 128.22 ( $\mathrm{ArC}-4$ of $\mathrm{Bn}$ ), 127.92 ( $\mathrm{ArC}-2,6$ of $\mathrm{Bn}$ ), 125.19 ( $\mathrm{ArC}-1$ of benzoate), 107.90 ( $\mathrm{ArC}-2,6$ of benzoate), 74.94 $(\mathrm{ArOCH} 2 \mathrm{Ar}), 69.26\left(\mathrm{ArOCH}_{2} \mathrm{CH}_{2}-\right), 52.20\left(-\mathrm{OCH}_{3}\right),[32.00,29.67,29.52,29.42,29.39,26.20,22.77]$ ($\left.\mathrm{OCH}_{2}\left(\mathrm{CH}_{2}\right)_{7}-\right), 14.21\left(-\mathrm{CH}_{2} \mathrm{CH}_{3}\right)$. MALDI-TOF MS $m / z$ of $[\mathrm{M}+\mathrm{Na}]^{+}$calculated for $\mathrm{C}_{33} \mathrm{H}_{50} \mathrm{O}_{5}: 549.4$; Found: 549.2 .
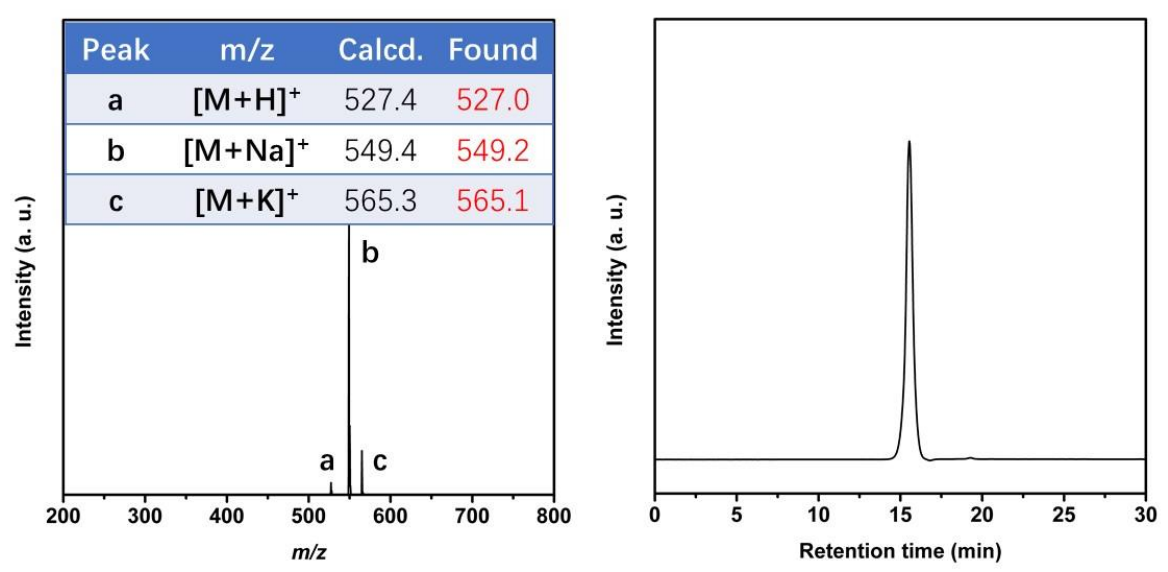

Supporting Figure S2. MALDI-TOF MS spectra (left) and HPLC trace (right) of 3-9. Nonyl groups have been successfully linked to the 3 - and 5-positions of 3-9. ${ }^{1} \mathrm{H}$ NMR spectrum is presented on page 8 . 
Supporting Figure S2 (continued). ${ }^{1} \mathrm{H}$ NMR of 3-9.

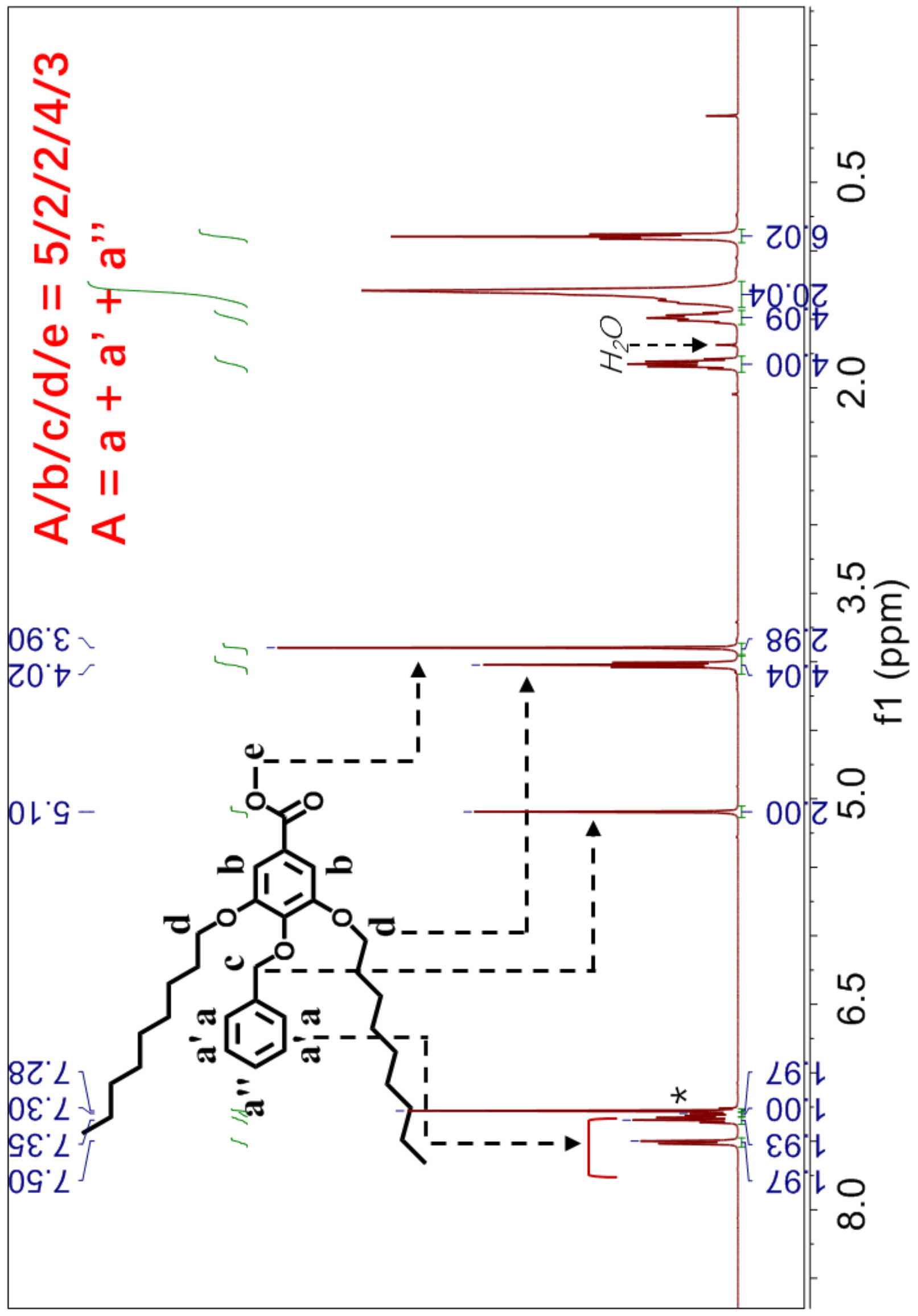




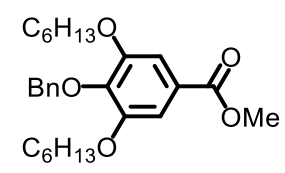

Methyl 4-(benzyloxy)-3,5-bis(hexyloxy)benzoate (3-6). Starting from 2 ( $0.27 \mathrm{~g}, 1.0 \mathrm{mmol}), \mathrm{K}_{2} \mathrm{CO}_{3}(0.83$ g, $6.0 \mathrm{mmol})$ and 1-bromohexane $(0.34 \mathrm{~mL}, 2.4 \mathrm{mmol})$ in dry DMF $(5 \mathrm{~mL})$ at $120^{\circ} \mathrm{C}$ for $3.5 \mathrm{~h}, \mathbf{3 - 6}$ was obtained as a colorless oil. Product: $0.40 \mathrm{~g}$. Yield: $90 \%$. Purity by HPLC: $99+\%$.

${ }^{1} \mathrm{H}$ NMR (500 MHz, $\left.\mathrm{CDCl}_{3}, \delta, \mathrm{ppm}\right): 7.50$ (d, $J=7.2 \mathrm{~Hz}, 2 \mathrm{H}, \mathrm{ArH}$ ortho to $\mathrm{CH}_{2} \mathrm{OAr}$ ), 7.34 (t, $2 \mathrm{H}, \mathrm{ArH}$ meta to $\mathrm{CH}_{2} \mathrm{OAr}$ ), 7.30 (d, $J=6.9 \mathrm{~Hz}, 1 \mathrm{H}, \mathrm{ArH}$ para to $\mathrm{CH}_{2} \mathrm{OAr}$ ), 7.27 (s, $2 \mathrm{H}, \mathrm{ArH}$ ortho to $\mathrm{CO}_{2} \mathrm{CH}_{3}$ ), $5.09\left(\mathrm{~s}, 2 \mathrm{H},-\mathrm{OCH} \mathrm{H}_{2} \mathrm{Ar}\right), 4.02\left(\mathrm{t}, J=6.5 \mathrm{~Hz}, 4 \mathrm{H},-\mathrm{OCH}_{2} \mathrm{CH}_{2}-\right), 3.90\left(\mathrm{~s}, 3 \mathrm{H},-\mathrm{CO}_{2} \mathrm{CH}_{3}\right), 1.86-1.78(\mathrm{~m}, 4 \mathrm{H}$, $\left.-\mathrm{OCH}_{2} \mathrm{CH}_{2}-\right), 1.53-1.44\left(\mathrm{~m}, 4 \mathrm{H},-\mathrm{O}\left(\mathrm{CH}_{2}\right)_{2} \mathrm{CH}_{2}-\right), 1.40-1.29$ (m, 8H, $\left.-\mathrm{O}\left(\mathrm{CH}_{2}\right)_{3}\left(\mathrm{CH}_{2}\right)_{2}-\right), 0.91$ (t, $J=6.7$ $\left.\mathrm{Hz}, 6 \mathrm{H},-\mathrm{CH}_{2} \mathrm{CH}_{3}\right) .{ }^{13} \mathrm{C} \mathrm{NMR}\left(126 \mathrm{MHz}, \mathrm{CDCl}_{3}, \delta, \mathrm{ppm}\right): 167.01(\mathrm{C}=\mathrm{O}), 153.00$ (ArC-3,5 of benzoate), 141.83 ( $\mathrm{ArC}-4$ of benzoate), 137.95 ( $\mathrm{ArC}-1$ of $\mathrm{Bn}$ ), 128.36 ( $\mathrm{ArC}-3,5$ of Bn), 128.25 ( $\mathrm{ArC}-4$ of Bn), 127.95 ( $\mathrm{ArC}-2,6$ of $\mathrm{Bn}$ ), 125.22 ( $\mathrm{ArC}-1$ of benzoate), 107.93 ( $\mathrm{ArC}-2,6$ of benzoate), $74.96\left(\mathrm{ArOCH}_{2} \mathrm{Ar}\right.$ ), 69.29 $\left(\mathrm{ArOCH}_{2} \mathrm{CH}_{2}-\right), 52.25\left(-\mathrm{OCH}_{3}\right),[31.70,29.39,25.89,22.73]\left(-\mathrm{OCH}_{2}\left(\mathrm{CH}_{2}\right)_{4}-\right), 14.16\left(-\mathrm{CH}_{2} \mathrm{CH}_{3}\right)$. MALDI-TOF MS $m / z$ of $[\mathrm{M}+\mathrm{Na}]^{+}$calculated for $\mathrm{C}_{27} \mathrm{H}_{38} \mathrm{O}_{5}$ : 465.3; Found: 464.9.
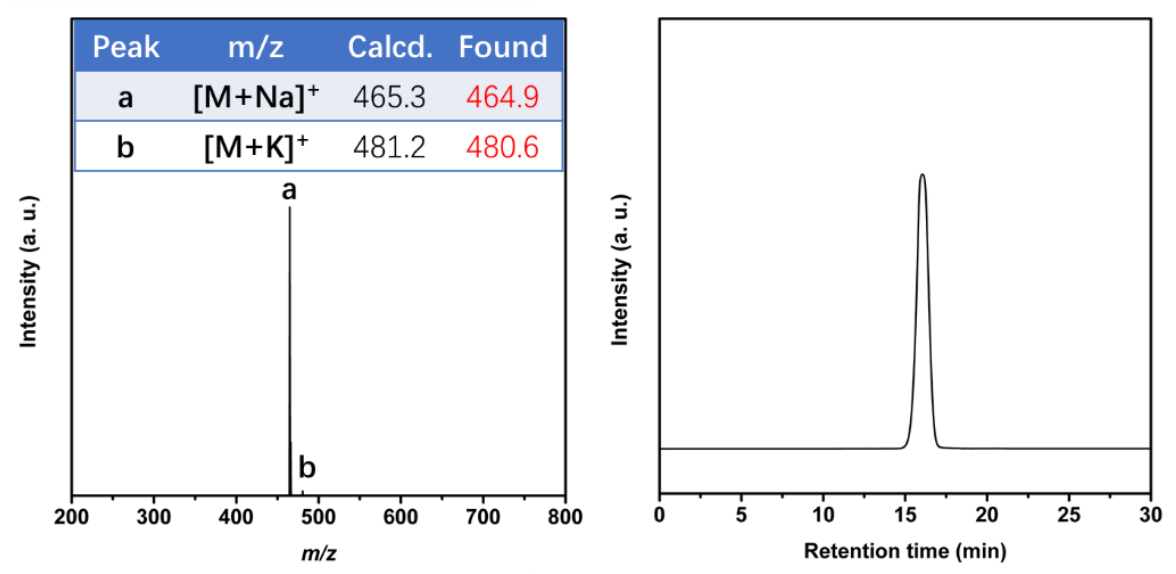

Supporting Figure S3. MALDI-TOF MS spectra (left) and HPLC trace (right) of 3-6. Hexyl groups have been successfully linked to the 3- and 5-positions of 3-6. ${ }^{1} \mathrm{H}$ NMR spectrum is presented on page 10. 
Supporting Figure S3 (continued). ${ }^{1} \mathrm{H}$ NMR of 3-6.

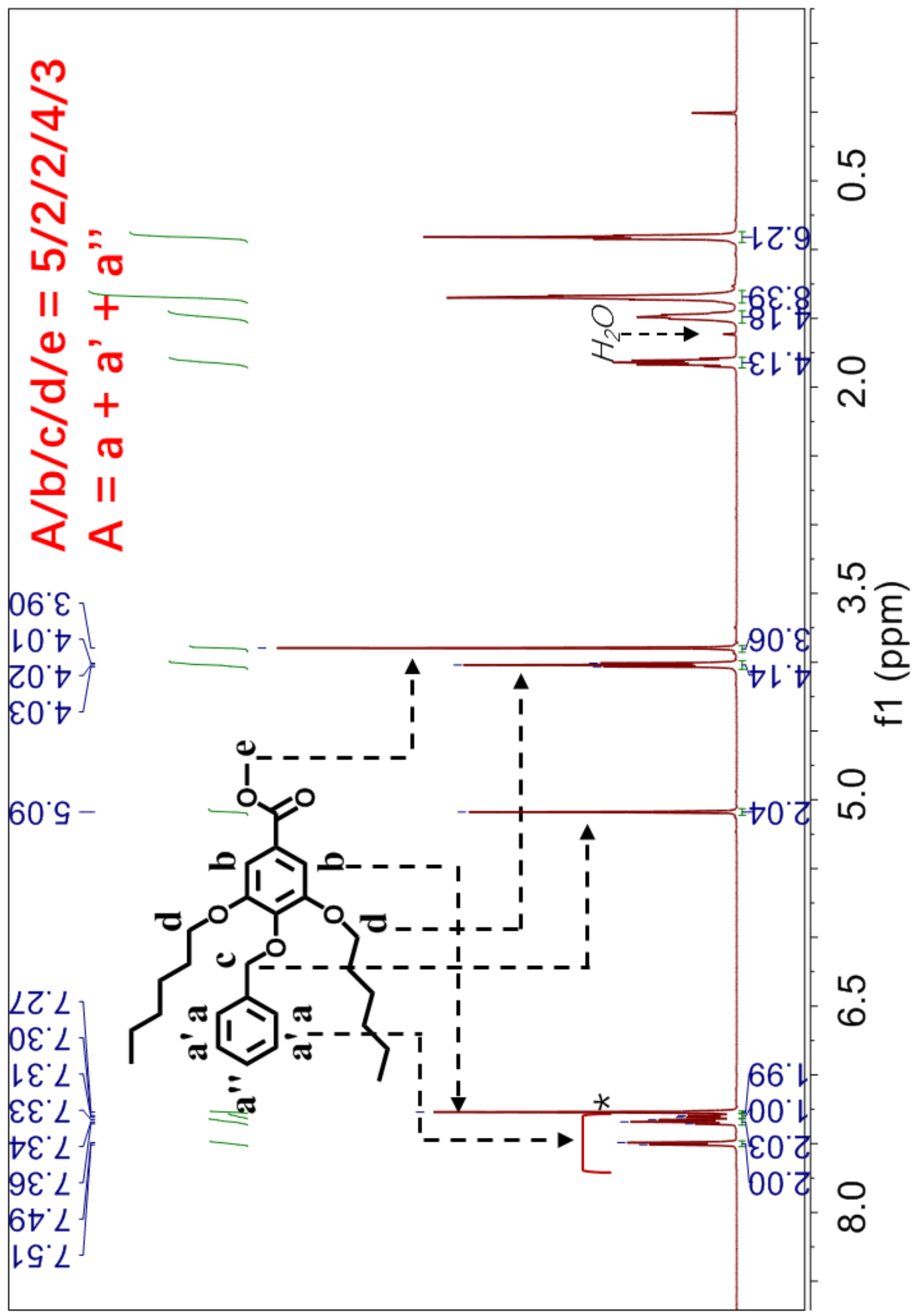




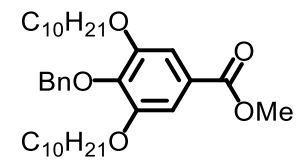

Methyl 4-(benzyloxy)-3,5-bis(hexyloxy)benzoate (3-10). Starting from 2 (0.27 g, $1.0 \mathrm{mmol}), \mathrm{K}_{2} \mathrm{CO}_{3}$ $(0.83 \mathrm{~g}, 6.0 \mathrm{mmol})$ and 1-bromodecane $(0.50 \mathrm{~mL}, 2.4 \mathrm{mmol})$ in dry DMF $(5 \mathrm{~mL})$ at $120{ }^{\circ} \mathrm{C}$ for $3.5 \mathrm{~h}, 3-$ 10 was obtained as a colorless oil. Product: $0.53 \mathrm{~g}$. Yield: $95 \%$. Purity by HPLC: $99+\%$.

${ }^{1} \mathrm{H}$ NMR (500 MHz, $\left.\mathrm{CDCl}_{3}, \delta, \mathrm{ppm}\right): 7.49$ (d, $J=7.2 \mathrm{~Hz}, 2 \mathrm{H}, \mathrm{ArH}$ ortho to $\left.\mathrm{CH}_{2} \mathrm{OAr}\right), 7.37-7.32(\mathrm{~m}, 2 \mathrm{H}$, $\mathrm{ArH}$ meta to $\mathrm{CH}_{2} \mathrm{OAr}$ ), 7.32-7.28 (m, 1H, $\mathrm{ArH}$ para to $\mathrm{CH}_{2} \mathrm{OAr}$ ), 7.26 (s, $2 \mathrm{H}, \mathrm{ArH}$ ortho to $\mathrm{CO}_{2} \mathrm{CH}_{3}$ ), $5.08\left(\mathrm{~s}, 2 \mathrm{H},-\mathrm{OCH} \mathrm{Ar}_{2} \mathrm{Ar}, 4.02\left(\mathrm{t}, J=6.5 \mathrm{~Hz}, 4 \mathrm{H},-\mathrm{OCH}_{2} \mathrm{CH}_{2}-\right), 3.89\left(\mathrm{~s}, 3 \mathrm{H},-\mathrm{CO}_{2} \mathrm{CH}_{3}\right), 1.86-1.77(\mathrm{~m}, 4 \mathrm{H}\right.$, $\left.-\mathrm{OCH}_{2} \mathrm{CH}_{2}-\right), 1.52-1.43$ (m, 4H, $\left.-\mathrm{O}\left(\mathrm{CH}_{2}\right)_{2} \mathrm{CH}_{2}-\right), 1.39-1.20\left(\mathrm{~m}, 24 \mathrm{H},-\mathrm{O}\left(\mathrm{CH}_{2}\right)_{3}\left(\mathrm{CH}_{2}\right)_{6}-\right), 0.88(\mathrm{t}, J=$ $\left.6.9 \mathrm{~Hz}, 6 \mathrm{H},-\mathrm{CH}_{2} \mathrm{CH}_{3}\right) .{ }^{13} \mathrm{C}$ NMR $\left(126 \mathrm{MHz}, \mathrm{CDCl}_{3}, \delta, \mathrm{ppm}\right): 167.05(\mathrm{C}=\mathrm{O}), 153.00(\mathrm{ArC}-3,5$ of benzoate), 140.80 ( $\mathrm{ArC}-4$ of benzoate), 137.95 ( $\mathrm{ArC}-1$ of $\mathrm{Bn}$ ), 128.39 ( $\mathrm{ArC}-3,5$ of $\mathrm{Bn}$ ), 128.27 ( $\mathrm{ArC}-4$ of $\mathrm{Bn}$ ), 127.97 ( $\mathrm{ArC}-2,6$ of $\mathrm{Bn}$ ), 125.22 ( $\mathrm{ArC}-1$ of benzoate), 107.92 ( $\mathrm{ArC}-2,6$ of benzoate), 74.99 $\left(\mathrm{ArOCH} \mathrm{H}_{2} \mathrm{Ar}\right), 69.30\left(\mathrm{ArOCH}_{2} \mathrm{CH}_{2}-\right), 52.29\left(-\mathrm{OCH}_{3}\right)$, [32.04, 29.75, 29.72, 29.55, 29.48, 29.45, 26.24, 22.83] $\left(-\mathrm{OCH}_{2}\left(\mathrm{CH}_{2}\right)_{8}-\right), 14.27\left(-\mathrm{CH}_{2} \mathrm{CH}_{3}\right)$. MALDI-TOF MS $m / z$ of $[\mathrm{M}+\mathrm{Na}]^{+}$calculated for $\mathrm{C}_{35} \mathrm{H}_{54} \mathrm{O}_{5}$ : 577.4; Found: 577.2.
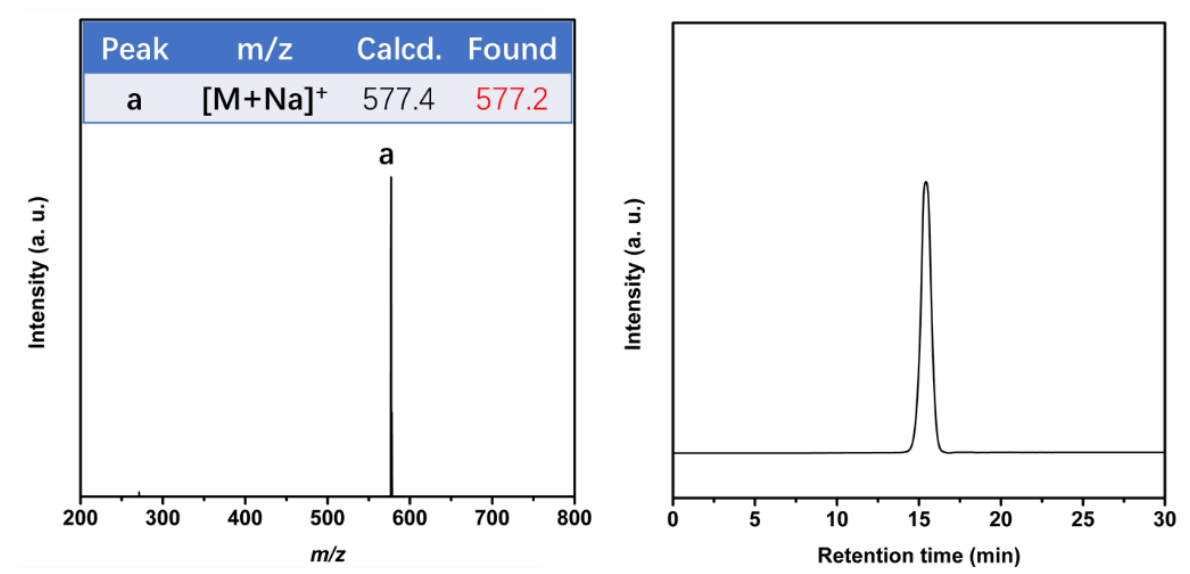

Supporting Figure S4. MALDI-TOF MS spectra (left) and HPLC trace (right) of 3-10. Decyl groups have been successfully linked to the 3- and 5-positions of 3-10. ${ }^{1} \mathrm{H}$ NMR spectrum is presented on page 12. 
Supporting Figure S4 (continued). ${ }^{1} \mathrm{H}$ NMR of 3-10.

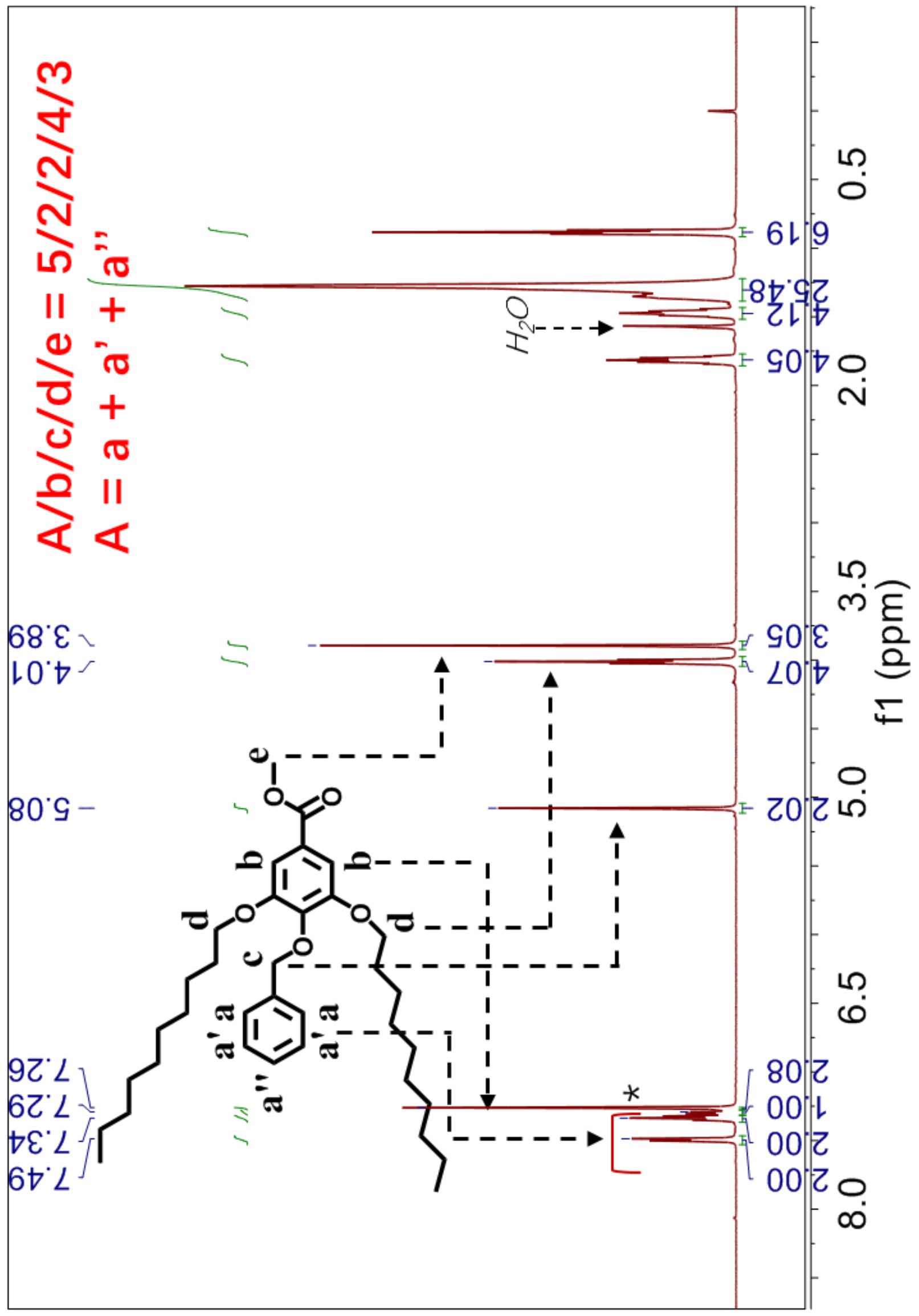




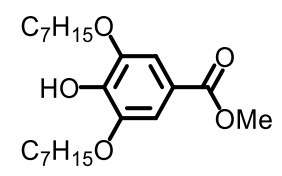

Methyl 3,5-bis(heptyloxy)-4-hydroxybenzoate (4-7). Compound 4-7 was synthesized from 3-7 following a procedure previously reported by our group. ${ }^{6}$ Briefly, 3-7 (2.16 g, $\left.4.6 \mathrm{mmol}\right)$, palladium on activated carbon catalyst $(130 \mathrm{mg}, \mathrm{Pd} 10 \%), \mathrm{CH}_{2} \mathrm{Cl}_{2}(20 \mathrm{~mL})$ and methanol $(20 \mathrm{~mL})$ were added into a $100 \mathrm{~mL}$ round-bottomed flask, which was sealed with a rubber septum under $\mathrm{N}_{2}$ atmosphere. Then the mixture was purged with $\mathrm{H}_{2}$ for 15 min and allowed to react under $\mathrm{H}_{2}$ atmosphere (balloon) at $23{ }^{\circ} \mathrm{C}$. After $24 \mathrm{~h}$, the mixture was filtered through celite and evaporated to dryness. Finally, the product was dried in a vacuum oven until constant weight was reached, giving 4-7 as white crystals. Product: 1.65 g. Yield: 94\%. Purity: $99+\%$. mp: $49-51{ }^{\circ} \mathrm{C}$. The synthesis procedures for 4-9, 4-6 and 4-10 listed below are similar to that for 4-7.

${ }^{1} \mathrm{H}$ NMR (400 MHz, $\mathrm{CDCl}_{3}, \delta$, ppm): 7.29 (s, 2H, $\left.\mathrm{ArH}\right), 5.93$ (s, 1H, $\left.\mathrm{ArOH}\right), 4.07$ (t, $J=6.7 \mathrm{~Hz}, 4 \mathrm{H},-$ $\left.\mathrm{OCH}_{2}-\right), 3.88$ (s, $\left.3 \mathrm{H},-\mathrm{CO}_{2} \mathrm{CH}_{3}\right), 1.88-1.77\left(\mathrm{~m}, 4 \mathrm{H},-\mathrm{OCH}_{2} \mathrm{CH}_{2}-\right), 1.51-1.40\left(\mathrm{~m}, 4 \mathrm{H},-\mathrm{O}\left(\mathrm{CH}_{2}\right)_{2} \mathrm{CH}_{2}-\right.$ ), 1.40-1.21 (m, 12H, $\left.-\mathrm{O}\left(\mathrm{CH}_{2}\right)_{3}\left(\mathrm{CH}_{2}\right)_{3}-\right), 0.88\left(\mathrm{t}, J=6.7 \mathrm{~Hz}, 6 \mathrm{H},-\mathrm{CH}_{2} \mathrm{CH}_{3}\right) .{ }^{13} \mathrm{C} \mathrm{NMR}\left(101 \mathrm{MHz}, \mathrm{CDCl}_{3}\right.$, $\delta$, ppm): $167.09(C=\mathrm{O}), 146.21$ (ArC-3,5), 139.90 (ArC-4), 121.01 (ArC-1), $107.80(\operatorname{ArC}-2,6), 69.63$ $\left(\mathrm{ArOCH}_{2}-\right), 52.14\left(-\mathrm{OCH}_{3}\right),[31.87,29.30,29.14,26.02,22.71]\left(-\mathrm{OCH}_{2}\left(\mathrm{CH}_{2}\right)_{5}-\right), 14.18\left(-\mathrm{CH}_{2} \mathrm{CH}_{3}\right)$. MALDI-TOF MS $m / z$ of $[\mathrm{M}+\mathrm{Na}]^{+}$calculated for $\mathrm{C}_{22} \mathrm{H}_{36} \mathrm{O}_{5}$ : 403.2; Found: 403.5 .
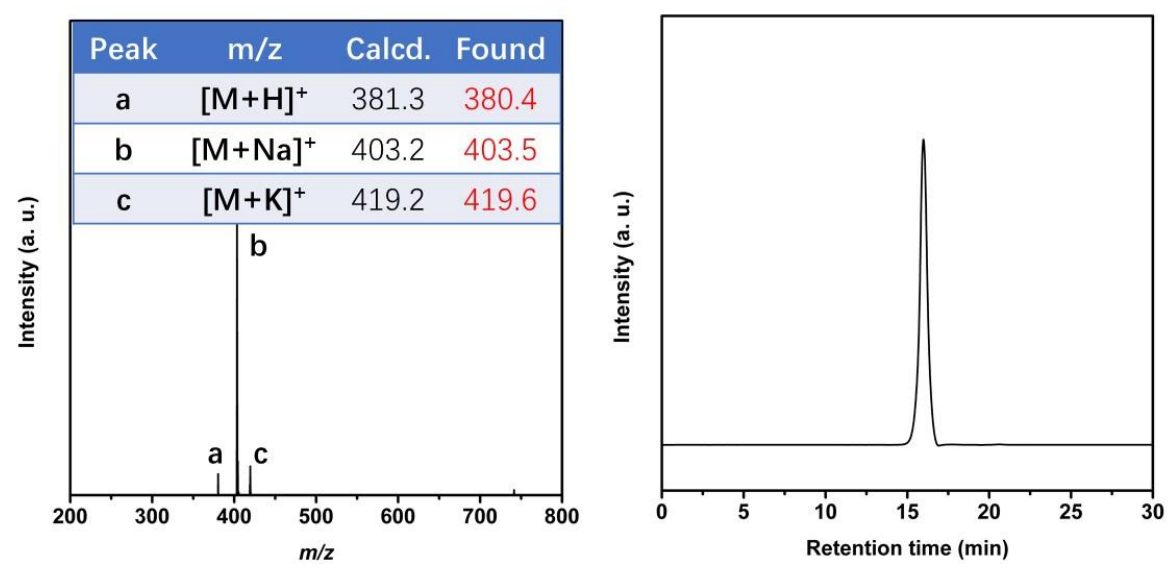

Supporting Figure S5. MALDI-TOF MS spectra (left) and HPLC trace (right) of 4-7. The hydroxyl group in the 4-position has been successfully deprotected in 4-7. ${ }^{1} \mathrm{H}$ NMR spectrum is presented on page 14. 
Supporting Figure S5 (continued). ${ }^{1} \mathrm{H}$ NMR of 4-7.

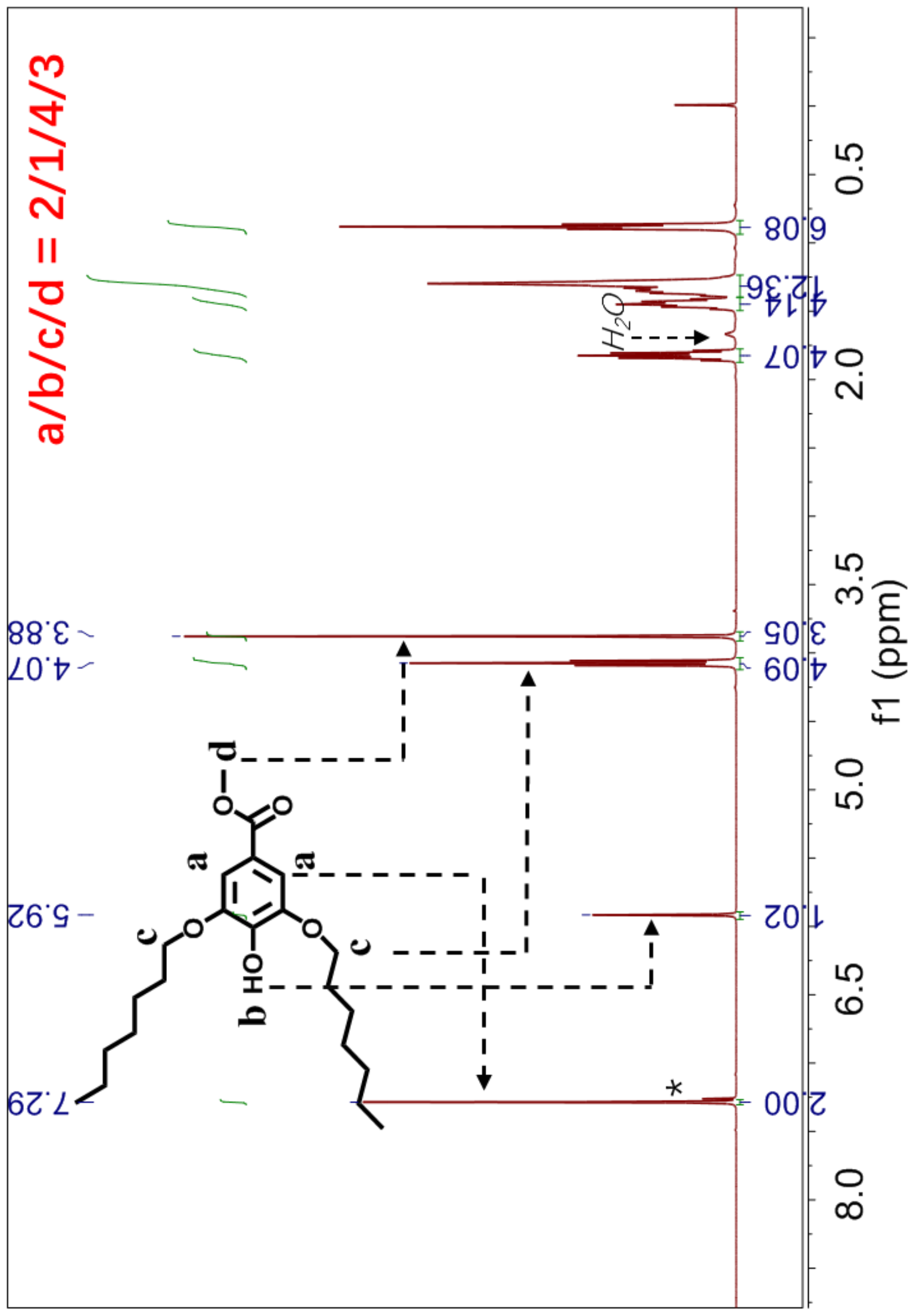




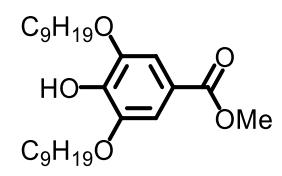

Methyl 3,5-bis(nonyloxy)-4-hydroxybenzoate (4-9). Starting from 3-9 (2.42 g, $4.6 \mathrm{mmol})$ and palladium on activated carbon catalyst $(130 \mathrm{mg}, \mathrm{Pd} 10 \mathrm{wt} \%)$ in $\mathrm{CH}_{2} \mathrm{Cl}_{2}(20 \mathrm{~mL}) /$ methanol $(20 \mathrm{~mL})$ mixture at $23{ }^{\circ} \mathrm{C}$ for $24 \mathrm{~h}, \mathbf{4 - 9}$ was obtained as a white solid. Product: 1.99 g. Yield: $99 \%$. Purity: $99+\%$. mp: $56-57^{\circ} \mathrm{C}$.

${ }^{1} \mathrm{H}$ NMR (400 MHz, $\mathrm{CDCl}_{3}, \delta$, ppm): 7.28 (s, 2H, $\left.\mathrm{ArH}\right), 5.92(\mathrm{~s}, 1 \mathrm{H}, \mathrm{ArOH}), 4.07$ (t, $J=6.6 \mathrm{~Hz}, 4 \mathrm{H},-$ $\left.\mathrm{OCH}_{2}-\right), 3.88$ (s, $\left.3 \mathrm{H},-\mathrm{CO}_{2} \mathrm{CH}_{3}\right), 1.87-1.76\left(\mathrm{~m}, 4 \mathrm{H},-\mathrm{OCH}_{2} \mathrm{CH}_{2}-\right), 1.50-1.39\left(\mathrm{~m}, 4 \mathrm{H},-\mathrm{O}\left(\mathrm{CH}_{2}\right)_{2} \mathrm{CH}_{2}-\right.$ ), 1.39-1.18 (m, 20H, $\left.-\mathrm{O}\left(\mathrm{CH}_{2}\right)_{3}\left(\mathrm{CH}_{2}\right)_{5}-\right), 0.87\left(\mathrm{t}, J=6.7 \mathrm{~Hz}, 6 \mathrm{H},-\mathrm{CH}_{2} \mathrm{CH}_{3}\right) .{ }^{13} \mathrm{C} \mathrm{NMR}\left(101 \mathrm{MHz}, \mathrm{CDCl}_{3}\right.$, $\delta$, ppm): $167.07(C=\mathrm{O}), 146.21(\mathrm{ArC}-3,5), 139.90(\mathrm{ArC}-4), 120.98(\operatorname{ArC}-1), 107.79(\operatorname{ArC}-2,6), 69.62$ $\left(\mathrm{ArOCH}_{2}-\right), 52.12\left(-\mathrm{OCH}_{3}\right)$, [31.97, 29.62, 29.47, 29.35, 29.29, 26.05, 22.77] $\left(-\mathrm{OCH}_{2}\left(\mathrm{CH}_{2}\right){ }_{7}^{-}\right), 14.20(-$ $\left.\mathrm{CH}_{2} \mathrm{CH}_{3}\right)$. MALDI-TOF MS $m / z$ of $[\mathrm{M}+\mathrm{Na}]^{+}$calculated for $\mathrm{C}_{26} \mathrm{H}_{44} \mathrm{O}_{5}: 459.3$; Found: 459.3 .
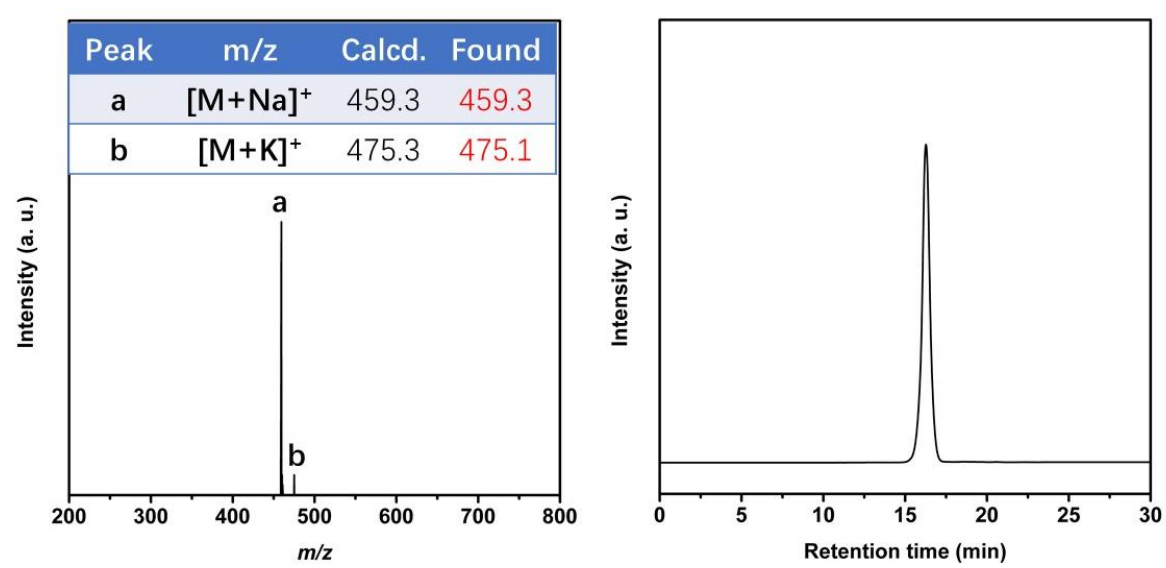

Supporting Figure S6. MALDI-TOF MS spectra (left) and HPLC trace (right) of 4-9. The hydroxyl group in the 4-position has been successfully deprotected in 4-9. ${ }^{1} \mathrm{H}$ NMR spectrum is presented on page 16. 
Supporting Figure S6 (continued). ${ }^{1} \mathrm{H}$ NMR of 4-9.

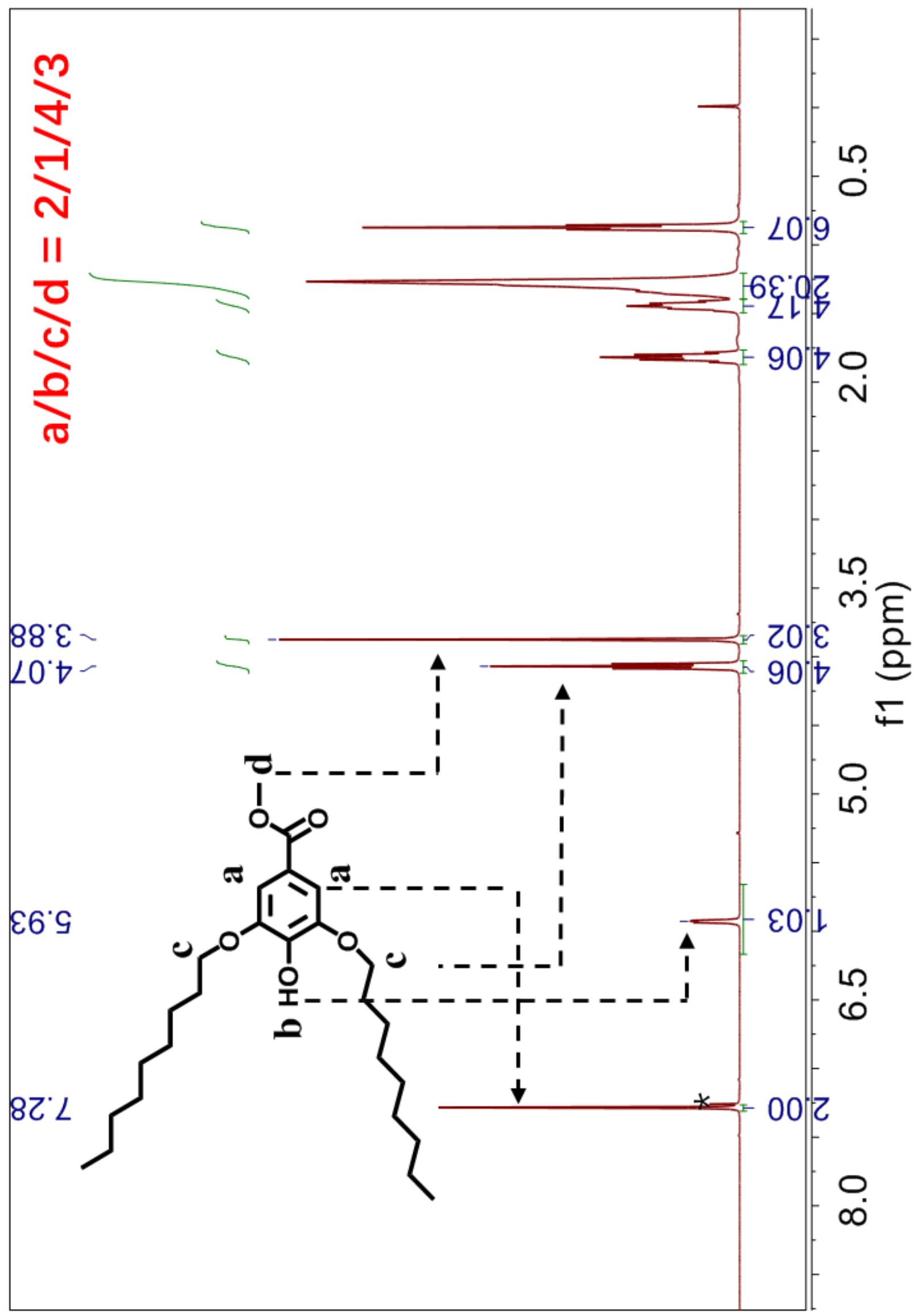




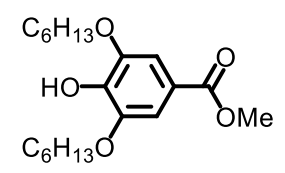

Methyl 3,5-bis(hexyloxy)-4-hydroxybenzoate (4-6). Starting from 3-6 $(0.38 \mathrm{~g}, 0.85 \mathrm{mmol})$ and palladium on activated carbon catalyst $(22 \mathrm{mg}$, $\mathrm{Pd} 10 \mathrm{wt} \%)$ in $\mathrm{CH}_{2} \mathrm{Cl}_{2}(5 \mathrm{~mL}) /$ methanol $(5 \mathrm{~mL})$ mixture at $23{ }^{\circ} \mathrm{C}$ for $24 \mathrm{~h}, \mathbf{4 - 6}$ was obtained as a colorless oil. Product: 0.28 g. Yield: $92 \%$. Purity: $99+\%$.

${ }^{1} \mathrm{H}$ NMR (500 MHz, $\left.\mathrm{CDCl}_{3}, \delta, \mathrm{ppm}\right): 7.29$ (s, 2H, $\mathrm{Ar} H$ ), 5.91 (s, $\left.1 \mathrm{H}, \mathrm{ArOH}\right), 4.07$ (t, $J=6.7 \mathrm{~Hz}, 4 \mathrm{H},-$ $\left.\mathrm{OCH}_{2}-\right), 3.88$ (s, $\left.3 \mathrm{H},-\mathrm{CO}_{2} \mathrm{CH}_{3}\right), 1.86-1.79\left(\mathrm{~m}, 4 \mathrm{H},-\mathrm{OCH}_{2} \mathrm{CH}_{2}-\right), 1.50-1.41\left(\mathrm{~m}, 4 \mathrm{H},-\mathrm{O}\left(\mathrm{CH}_{2}\right)_{2} \mathrm{CH}_{2}-\right.$ ), 1.37-1.28 (m, 8H, $\left.-\mathrm{O}\left(\mathrm{CH}_{2}\right)_{3}\left(\mathrm{CH}_{2}\right)_{2}-\right), 0.90\left(\mathrm{t}, J=7.1 \mathrm{~Hz}, 6 \mathrm{H},-\mathrm{CH}_{2} \mathrm{CH}_{3}\right) .{ }^{13} \mathrm{C}$ NMR $\left(126 \mathrm{MHz}, \mathrm{CDCl}_{3}\right.$, $\delta$, ppm): $167.10(C=\mathrm{O}), 146.20(\mathrm{ArC}-3,5), 139.85$ (ArC-4), 121.01 ( $\mathrm{ArC}-1), 107.76$ (ArC-2,6), 69.62 $\left(\mathrm{ArOCH}_{2} \mathrm{CH}_{2}-\right), 52.17\left(-\mathrm{OCH}_{3}\right),[31.66,29.26,25.75,22.71]\left(-\mathrm{OCH}_{2}\left(\mathrm{CH}_{2}\right)_{4}-\right), 14.14\left(-\mathrm{CH}_{2} \mathrm{CH}_{3}\right)$. MALDI-TOF MS $m / z$ of $[\mathrm{M}+\mathrm{Na}]^{+}$calculated for $\mathrm{C}_{20} \mathrm{H}_{32} \mathrm{O}_{5}: 375.2$; Found: 375.0 .
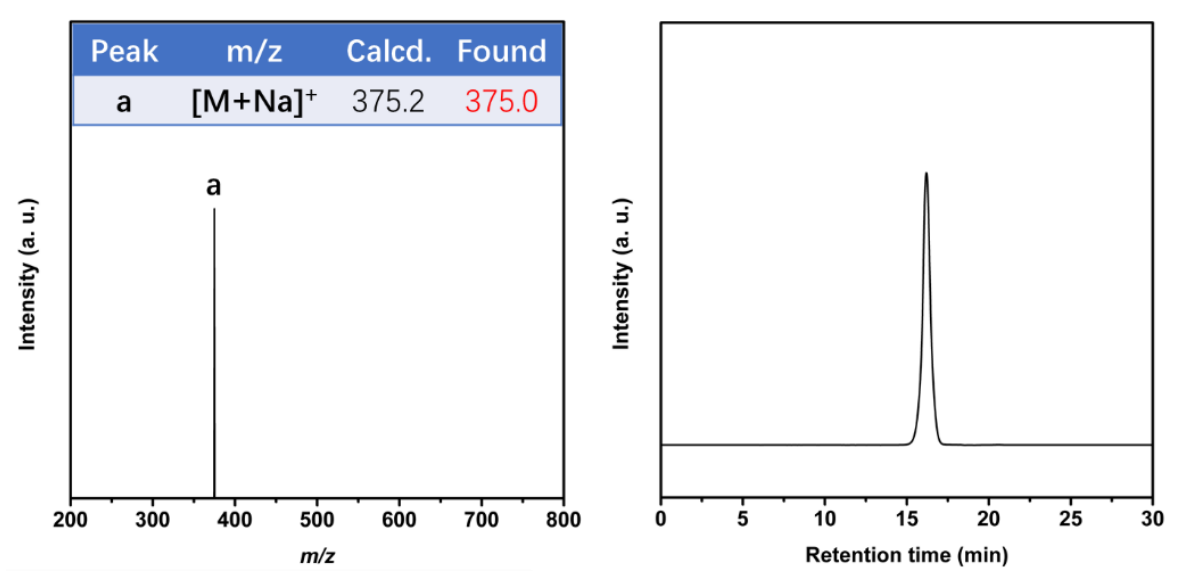

Supporting Figure S7. MALDI-TOF MS spectra (left) and HPLC trace (right) of 4-6. The hydroxyl group in the 4-position has been successfully deprotected in 4-6. ${ }^{1} \mathrm{H}$ NMR spectrum is presented on page 18. 
Supporting Figure S7 (continued). ${ }^{1} \mathrm{H}$ NMR of 4-6.

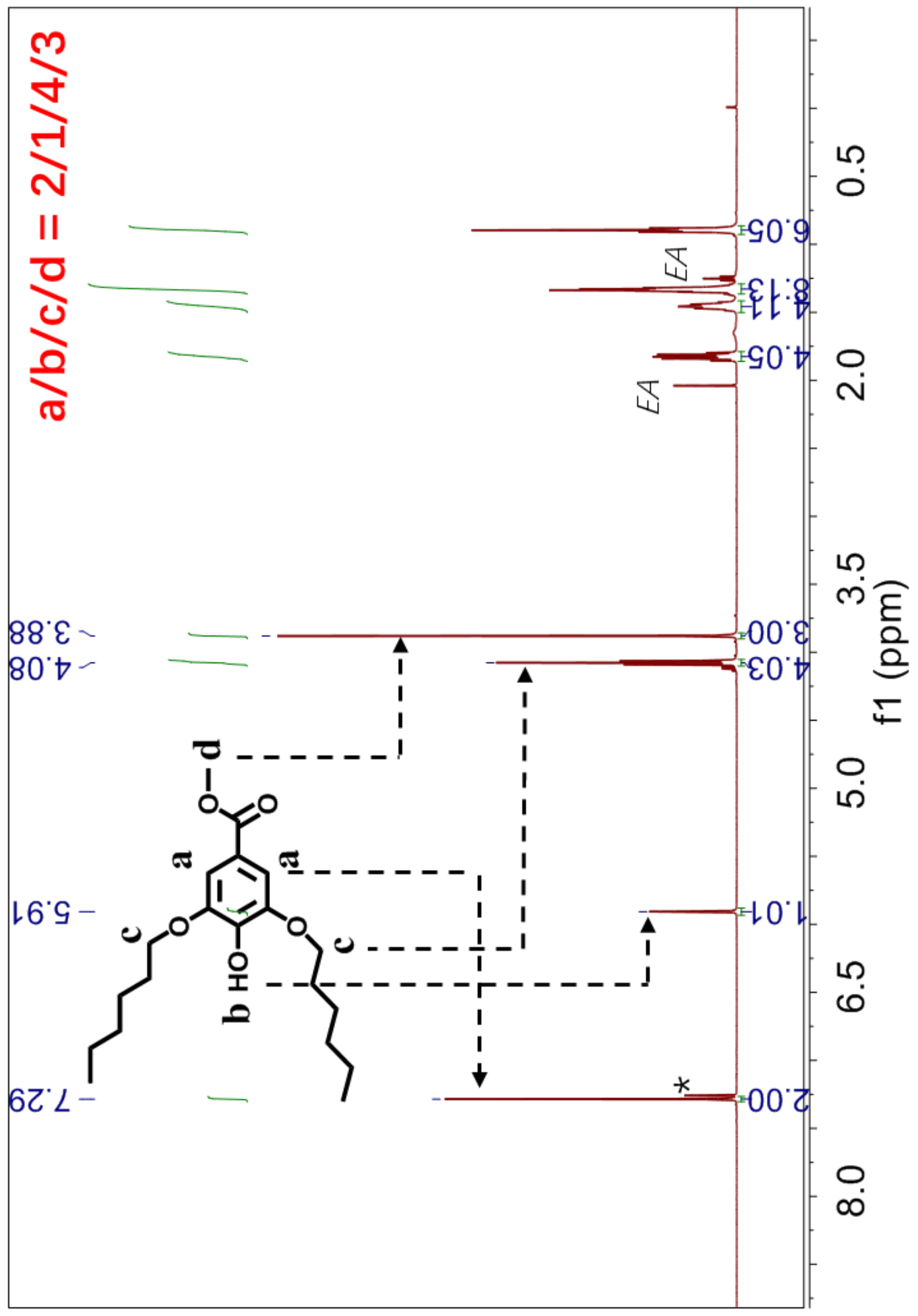




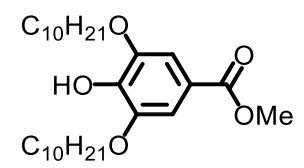

Methyl 3,5-bis(decyloxy)-4-hydroxybenzoate (4-10). Starting from 3-10 (0.44 g, $0.8 \mathrm{mmol})$ and palladium on activated carbon catalyst $(22 \mathrm{mg}$, $\mathrm{Pd} 10 \mathrm{wt} \%)$ in $\mathrm{CH}_{2} \mathrm{Cl}_{2}(5 \mathrm{~mL}) /$ methanol $(5 \mathrm{~mL})$ mixture at $23{ }^{\circ} \mathrm{C}$ for $24 \mathrm{~h}, \mathbf{4 - 1 0}$ was obtained as a white solid. Product: 0.35 g. Yield: $94 \%$. Purity: $99+\%$. mp: $44-$ $45{ }^{\circ} \mathrm{C}$.

${ }^{1} \mathrm{H}$ NMR (500 MHz, $\mathrm{CDCl}_{3}, \delta$, ppm): $7.28(\mathrm{~s}, 2 \mathrm{H}, \mathrm{Ar} H), 5.93$ (s, $\left.1 \mathrm{H}, \mathrm{ArOH}\right), 4.07$ (t, $J=6.7 \mathrm{~Hz}, 4 \mathrm{H},-$ $\left.\mathrm{OCH}_{2}-\right), 3.88$ (s, $\left.3 \mathrm{H},-\mathrm{CO}_{2} \mathrm{CH}_{3}\right), 1.86-1.78\left(\mathrm{~m}, 4 \mathrm{H},-\mathrm{OCH}_{2} \mathrm{CH}_{2}-\right), 1.49-1.40\left(\mathrm{~m}, 4 \mathrm{H},-\mathrm{O}\left(\mathrm{CH}_{2}\right)_{2} \mathrm{CH}_{2}-\right.$ ), 1.38-1.21 (m, 24H, $\left.-\mathrm{O}\left(\mathrm{CH}_{2}\right)_{3}\left(\mathrm{CH}_{2}\right)_{6}-\right), 0.87\left(\mathrm{t}, J=7.0 \mathrm{~Hz}, 6 \mathrm{H},-\mathrm{CH}_{2} \mathrm{CH}_{3}\right) .{ }^{13} \mathrm{C}$ NMR $\left(101 \mathrm{MHz}, \mathrm{CDCl}_{3}\right.$, $\delta$, ppm): $167.10(C=\mathrm{O}), 146.19$ (ArC-3,5), 139.84 (ArC-4), 120.98 (ArC-1), 107.74 (ArC-2,6), 69.60 $\left(\mathrm{ArOCH}_{2}-\right), 52.15\left(-\mathrm{OCH}_{3}\right),[32.00,29.67,29.66,29.48,29.43,29.29,26.06,22.80]\left(-\mathrm{OCH}_{2}\left(\mathrm{CH}_{2}\right)_{8}-\right)$, 14.23 $\left(-\mathrm{CH}_{2} \mathrm{CH}_{3}\right)$. MALDI-TOF MS $m / z$ of $[\mathrm{M}+\mathrm{Na}]^{+}$calculated for $\mathrm{C}_{28} \mathrm{H}_{48} \mathrm{O}_{5}: 487.3$; Found: 487.3.
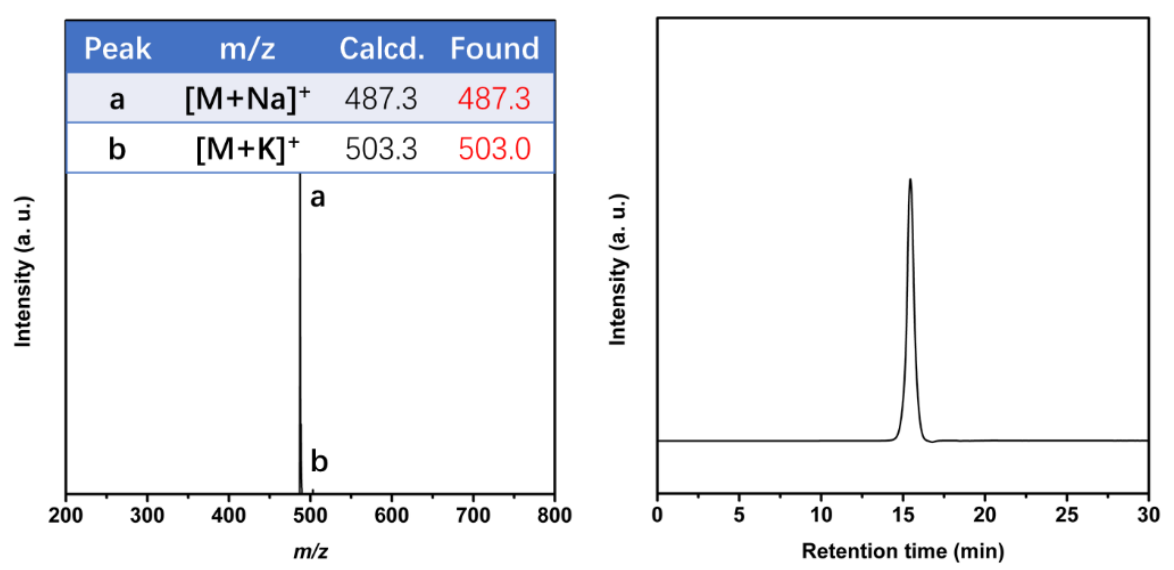

Supporting Figure S8. MALDI-TOF MS spectra (left) and HPLC trace (right) of 4-10. The hydroxyl group in the 4-position has been successfully deprotected in 4-10. ${ }^{1} \mathrm{H}$ NMR spectrum is presented on page 20. 
Supporting Figure S8 (continued). ${ }^{1} \mathrm{H}$ NMR of 4-10.

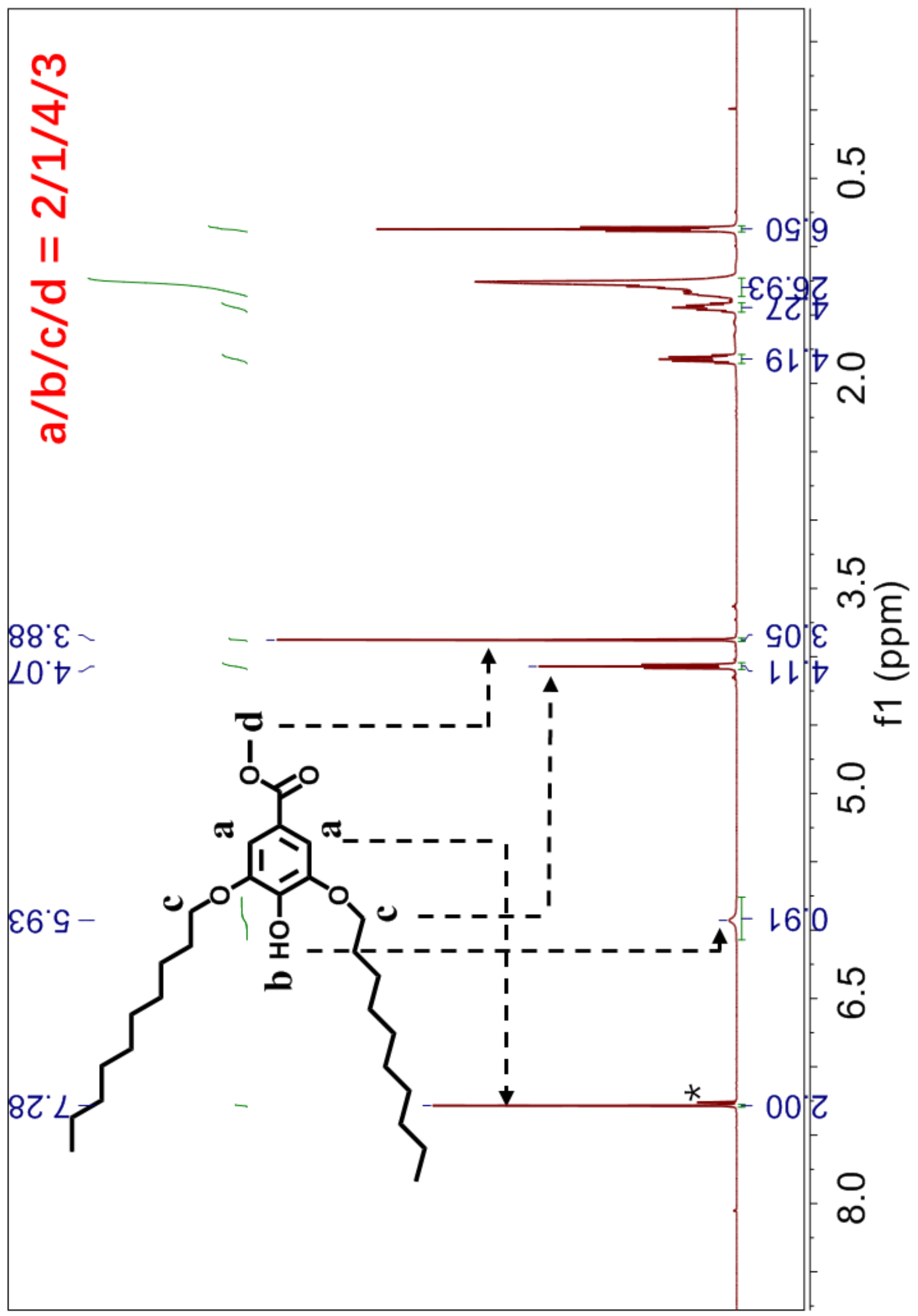




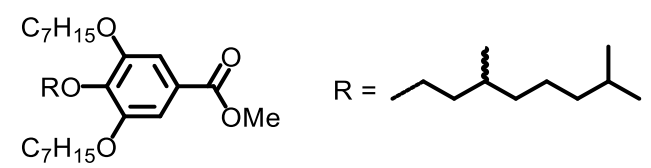

Methyl 4-((3,7-(rac)-dimethyloctyl)oxy)-3,5-bis(heptyloxy)benzoate (5-7r7). Following a procedure similar to that for 3-7, reaction of 4-7 $(1.56 \mathrm{~g}, 4.15 \mathrm{mmol})$ and ( $\mathrm{rac})$-1-bromo-3,7-dimethyloctane $(0.90$ $\mathrm{mL}, 4.4 \mathrm{mmol})$ in the presence of $\mathrm{K}_{2} \mathrm{CO}_{3}(1.15 \mathrm{~g}, 8.3 \mathrm{mmol})$ in dry DMF $(30 \mathrm{~mL})$ at $120{ }^{\circ} \mathrm{C}$ for $6 \mathrm{~h}$ gave 5-7r7 as a colorless oil after column chromatography (EA/hexanes $=1 / 40)$. Product: 2.02 g. Yield: 94\%. Purity by HPLC: $99+\%$. The synthesis procedures for 5-9r9, 5-6r6, 5-10r10, 5-r7r, 5-r9r, 5-r6r and 5r10r listed below are similar to that for 5-7r7.

${ }^{1} \mathrm{H}$ NMR (400 MHz, $\left.\mathrm{CDCl}_{3}, \delta, \mathrm{ppm}\right): 7.25(\mathrm{~s}, 2 \mathrm{H}, \mathrm{Ar} H), 4.12-4.03\left(\mathrm{~m}, 2 \mathrm{H},-\mathrm{OCH}_{2} \mathrm{CH}_{2} \mathrm{CH}\left(\mathrm{CH}_{3}\right)-\right), 4.01$ $\left(\mathrm{t}, J=6.4 \mathrm{~Hz}, 4 \mathrm{H},-\mathrm{OCH}_{2} \mathrm{CH}_{2} \mathrm{CH}_{2}-\right), 3.88\left(\mathrm{~s}, 3 \mathrm{H},-\mathrm{CO}_{2} \mathrm{CH}_{3}\right), 1.88-1.76\left(\mathrm{~m}, 5 \mathrm{H},-\mathrm{CH}_{\left(\mathrm{CH}_{3}\right)_{2} \text { and }-}\right.$

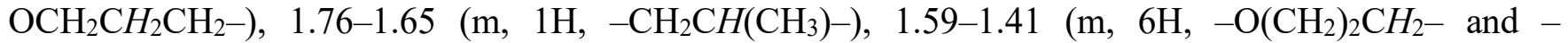
$\left.\mathrm{OCH}_{2} \mathrm{CH}_{2} \mathrm{CH}\left(\mathrm{CH}_{3}\right)^{-}\right)$, 1.41-1.06 (m, $18 \mathrm{H},-\mathrm{O}\left(\mathrm{CH}_{2}\right)_{3}\left(\mathrm{CH}_{2}\right)_{3}-$ and $\left.-\mathrm{CH}\left(\mathrm{CH}_{3}\right)\left(\mathrm{CH}_{2}\right)_{3}-\right), 0.92(\mathrm{~d}, \mathrm{~J}=6.6 \mathrm{~Hz}$, $\left.3 \mathrm{H},-\mathrm{CH}_{2} \mathrm{CH}\left(\mathrm{CH}_{3}\right) \mathrm{CH}_{2}-\right), 0.91-0.82\left(\mathrm{~m}, 12 \mathrm{H},-\mathrm{CH}\left(\mathrm{CH}_{3}\right)_{2}\right.$ and $\left.-\mathrm{CH}_{2} \mathrm{CH}_{3}\right) .{ }^{13} \mathrm{C}^{\mathrm{NMR}}\left(101 \mathrm{MHz}, \mathrm{CDCl}_{3}\right.$, $\delta, \mathrm{ppm}): 167.06(C=\mathrm{O}), 152.97$ (ArC-3,5), 142.48 (ArC-4), 124.80 (ArC-1), 108.09 (ArC-2,6), 71.83 ( $\mathrm{ArOCH} \mathrm{H}_{2}-3,5$ position), 69.27 ( $\mathrm{ArOCH}_{2}-4$ position), $52.21\left(-\mathrm{OCH}_{3}\right)$, [39.48, 37.60, 37.46, 31.95, 29.76, 29.43, 29.18, 28.12, 26.17, 24.85] $\left(-\mathrm{OCH}_{2}\left(\mathrm{CH}_{2}\right)_{4}-\right.$ and $\left.-\mathrm{OCH}_{2} \mathrm{CH}_{2} \mathrm{CH}\left(\mathrm{CH}_{3}\right)\left(\mathrm{CH}_{2}\right)_{3} \mathrm{CH}-\right)$, [22.82, 22.74, $22.72,19.66,14.20]\left(-\mathrm{CH}\left(\mathrm{CH}_{3}\right)_{2},-\mathrm{CH}\left(\mathrm{CH}_{3}\right) \mathrm{CH}_{2}-\right.$ and $\left.-\mathrm{CH}_{2} \mathrm{CH}_{3}\right)$. MALDI-TOF MS $\mathrm{m} / z$ of $[\mathrm{M}+\mathrm{Na}]^{+}$ calculated for $\mathrm{C}_{32} \mathrm{H}_{56} \mathrm{O}_{5}$ : 543.4; Found: 542.9 .
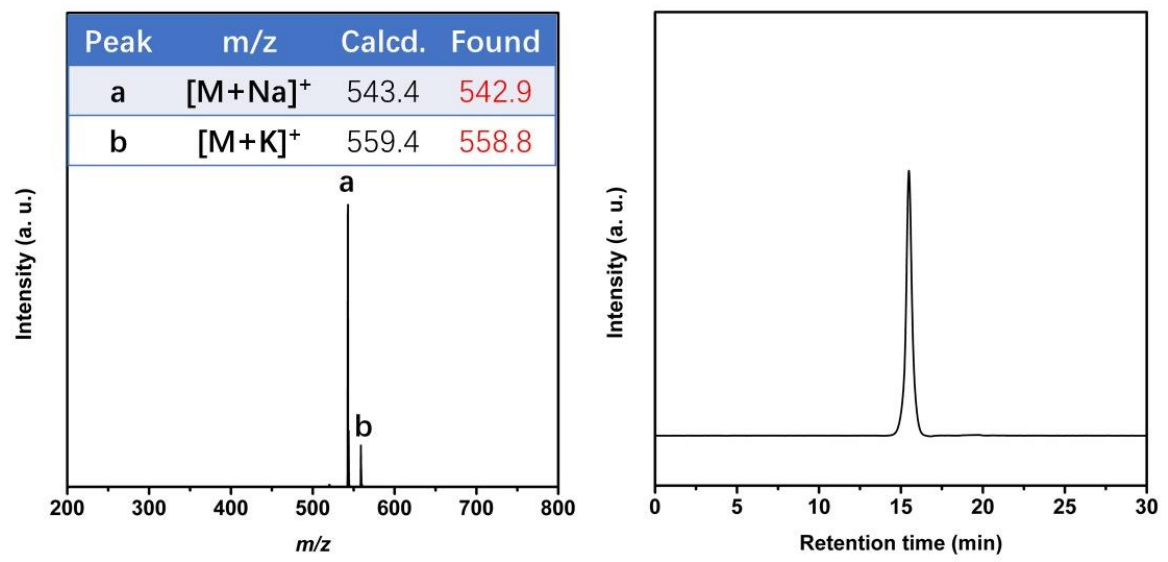

Supporting Figure S9. MALDI-TOF MS spectra (left) and HPLC trace (right) of 5-7r7. Racemic dimethyloctyl group has been successfully linked to the 4-position of 5-7r7. ${ }^{1} \mathrm{H}$ NMR spectrum is presented on page 22 . 
Supporting Figure S9 (continued). ${ }^{1} \mathrm{H}$ NMR of 5-7r7.

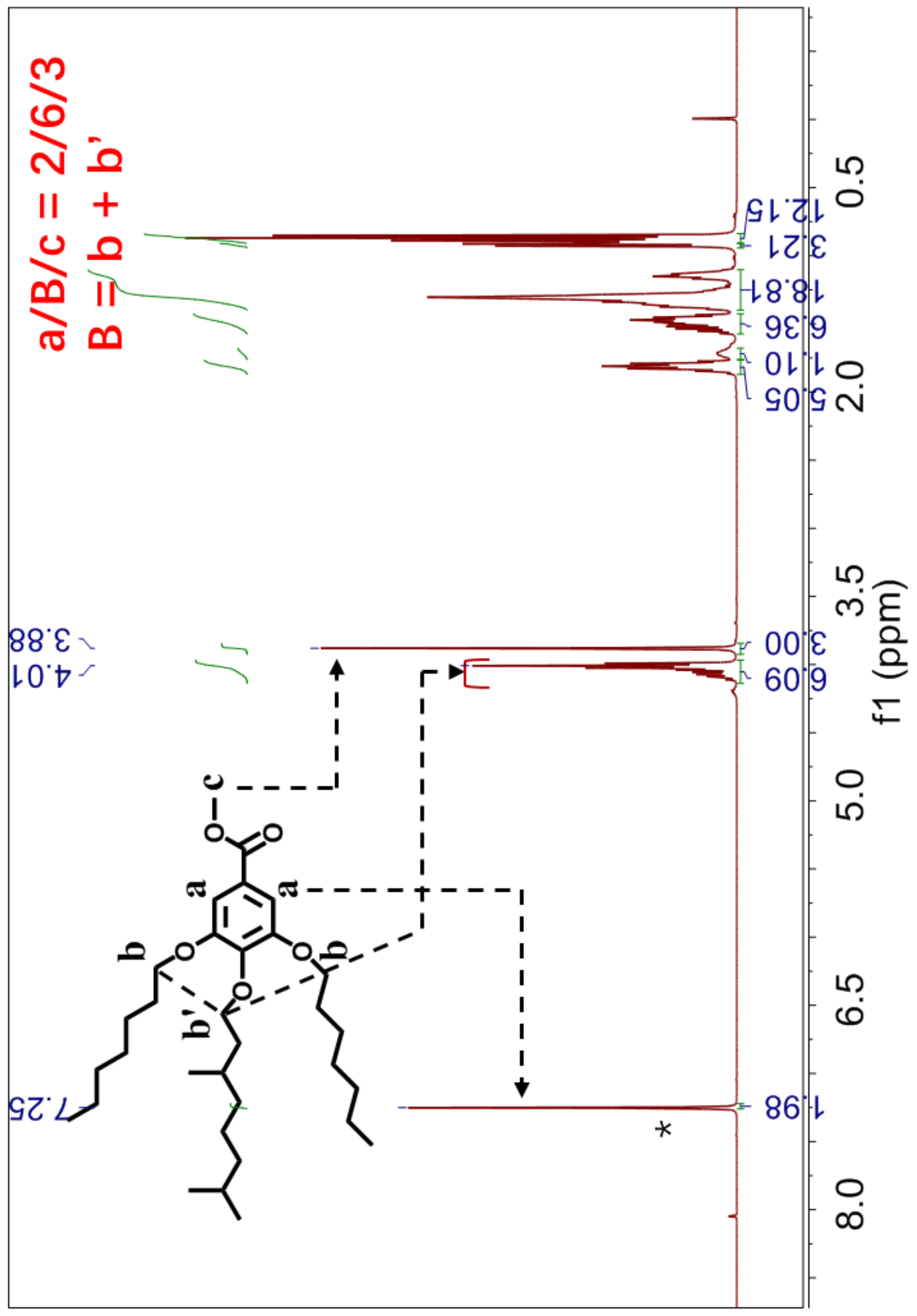




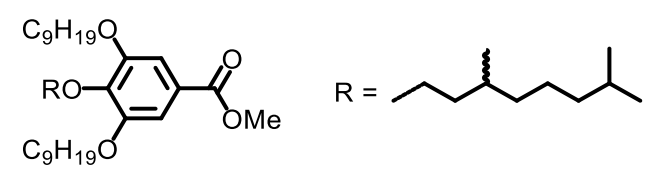

Methyl 4-((3,7-(rac)-dimethyloctyl)oxy)-3,5-bis(nonyloxy)benzoate (5-9r9). Starting from 4-9 (1.92 g, $4.4 \mathrm{mmol}), \mathrm{K}_{2} \mathrm{CO}_{3}(1.22 \mathrm{~g}, 8.8 \mathrm{mmol}$ ) and ( $\mathrm{rac}$ )-1-bromo-3,7-dimethyloctane (0.96 mL, $4.6 \mathrm{mmol})$ in dry DMF (30 mL) at $120{ }^{\circ} \mathrm{C}$ for 6 h, 5-9r9 was obtained as a colorless oil. Product: 2.41 g. Yield: $95 \%$. Purity by HPLC: $99+\%$.

${ }^{1} \mathrm{H}$ NMR (400 MHz, $\left.\mathrm{CDCl}_{3}, \delta, \mathrm{ppm}\right): 7.25$ (s, 2H, $\left.\mathrm{ArH}\right), 4.12-4.03\left(\mathrm{~m}, 2 \mathrm{H},-\mathrm{OCH}_{2} \mathrm{CH}_{2} \mathrm{CH}_{(\mathrm{CH}}\right)-$ ), 4.01 $\left(\mathrm{t}, J=6.5 \mathrm{~Hz}, 4 \mathrm{H},-\mathrm{OCH}_{2} \mathrm{CH}_{2} \mathrm{CH}_{2}-\right), 3.88\left(\mathrm{~s}, 3 \mathrm{H},-\mathrm{CO}_{2} \mathrm{CH}_{3}\right), 1.88-1.76\left(\mathrm{~m}, 5 \mathrm{H},-\mathrm{CH}_{\left(\mathrm{CH}_{3}\right)_{2} \text { and }-}\right.$

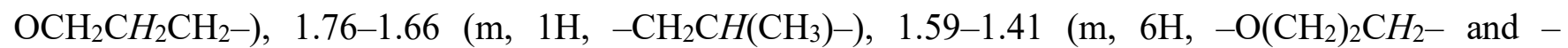
$\left.\mathrm{OCH}_{2} \mathrm{CH}_{2} \mathrm{CH}\left(\mathrm{CH}_{3}\right)-\right), 1.41-1.07\left(\mathrm{~m}, 26 \mathrm{H},-\mathrm{O}\left(\mathrm{CH}_{2}\right)_{3}\left(\mathrm{CH}_{2}\right)_{5}-\right.$ and $\left.-\mathrm{CH}\left(\mathrm{CH}_{3}\right)\left(\mathrm{CH}_{2}\right)_{3}-\right), 0.92(\mathrm{~d}, J=6.6 \mathrm{~Hz}$, $\left.3 \mathrm{H},-\mathrm{CH}_{2} \mathrm{CH}\left(\mathrm{CH}_{3}\right)-\right), 0.91-0.82\left(\mathrm{~m}, 12 \mathrm{H},-\mathrm{CH}\left(\mathrm{CH}_{3}\right)_{2}\right.$ and $\left.-\mathrm{CH}_{2} \mathrm{CH}_{3}\right) .{ }^{13} \mathrm{C} \mathrm{NMR}\left(101 \mathrm{MHz}, \mathrm{CDCl}_{3}, \delta\right.$, ppm): $167.06(C=\mathrm{O}), 152.97$ (ArC-3,5), 142.48 (ArC-4), 124.80 (ArC-1), 108.09 (ArC-2,6), 71.83 ( $\mathrm{ArOCH} \mathrm{H}_{2}-3,5$ position), 69.27 ( $\mathrm{ArOCH}_{2}-4$ position), $52.20\left(-\mathrm{OCH}_{3}\right),[39.48,37.61,37.46,32.03,29.77$, $29.71,29.53,29.44,29.42,28.13,26.21,24.85]\left(-\mathrm{OCH}_{2}\left(\mathrm{CH}_{2}\right)_{6}-\right.$ and $\left.-\mathrm{OCH}_{2} \mathrm{CH}_{2} \mathrm{CH}\left(\mathrm{CH}_{3}\right)\left(\mathrm{CH}_{2}\right)_{3} \mathrm{CH}-\right)$, $[22.82,22.80,22.73,19.66,14.22]\left(-\mathrm{CH}\left(\mathrm{CH}_{3}\right)_{2},-\mathrm{CH}\left(\mathrm{CH}_{3}\right) \mathrm{CH}_{2}-\right.$ and $\left.-\mathrm{CH}_{2} \mathrm{CH}_{3}\right)$. MALDI-TOF MS $m / z$ of $[\mathrm{M}+\mathrm{Na}]^{+}$calculated for $\mathrm{C}_{36} \mathrm{H}_{64} \mathrm{O}_{5}: 599.5$; Found: 598.8 .
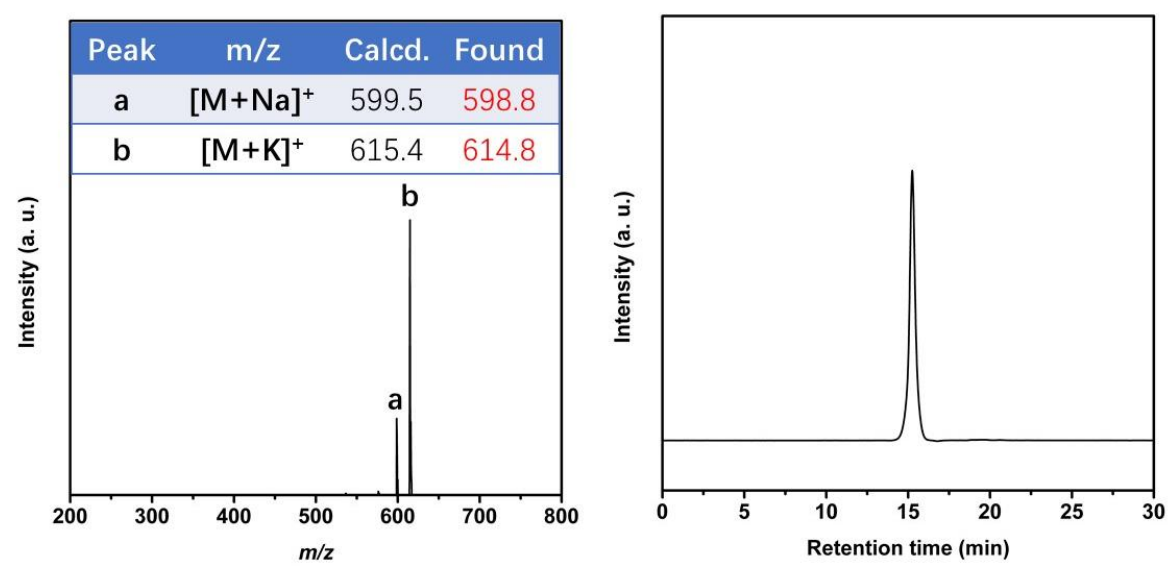

Supporting Figure S10. MALDI-TOF MS spectra (left) and HPLC trace (right) of 5-9r9. Racemic dimethyloctyl group has been successfully linked to the 4-position of 5-9r9. ${ }^{1} \mathrm{H}$ NMR spectrum is presented on page 24 . 
Supporting Figure S10 (continued). ${ }^{1}$ H NMR of 5-9r9.

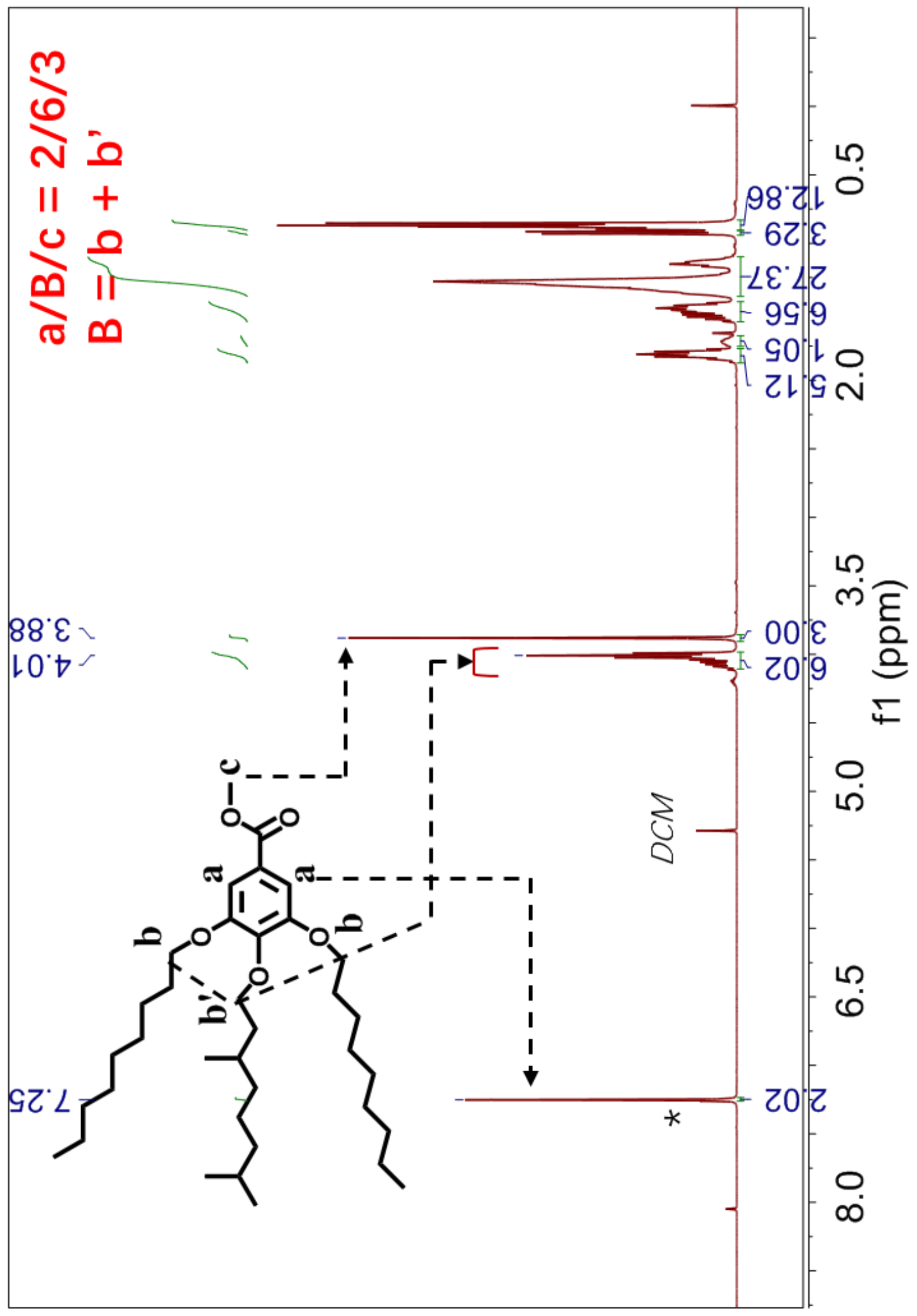




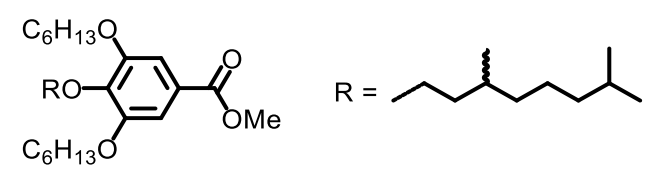

Methyl 4-((3,7-(rac)-dimethyloctyl)oxy)-3,5-bis(hexyloxy)benzoate (5-6r6). Starting from 4-6 (0.21 g, $0.60 \mathrm{mmol}), \mathrm{K}_{2} \mathrm{CO}_{3}(0.25 \mathrm{~g}, 1.8 \mathrm{mmol})$ and ( $\left.\mathrm{rac}\right)$-1-bromo-3,7-dimethyloctane $(0.15 \mathrm{~mL}, 0.70 \mathrm{mmol})$ in dry DMF $(5 \mathrm{~mL})$ at $120^{\circ} \mathrm{C}$ for $3.5 \mathrm{~h}, \mathbf{5 - 6 r 6}$ was obtained as a colorless oil. Product: $0.27 \mathrm{~g}$. Yield: $91 \%$. Purity by HPLC: $99+\%$.

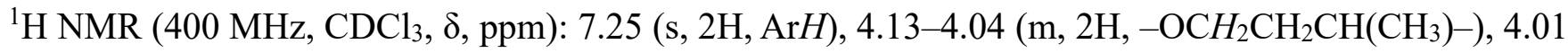
$\left(\mathrm{t}, J=6.5 \mathrm{~Hz}, 4 \mathrm{H},-\mathrm{OCH}_{2} \mathrm{CH}_{2} \mathrm{CH}_{2}-\right), 3.89\left(\mathrm{~s}, 3 \mathrm{H},-\mathrm{CO}_{2} \mathrm{CH}_{3}\right), 1.87-1.76\left(\mathrm{~m}, 5 \mathrm{H},-\mathrm{CH}_{\left(\mathrm{CH}_{3}\right)_{2} \text { and }-}\right.$

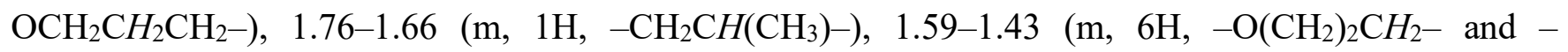
$\left.\mathrm{OCH}_{2} \mathrm{CH}_{2} \mathrm{CH}\left(\mathrm{CH}_{3}\right)-\right), 1.39-1.09\left(\mathrm{~m}, 14 \mathrm{H},-\mathrm{O}\left(\mathrm{CH}_{2}\right)_{3}\left(\mathrm{CH}_{2}\right)_{2}-\right.$ and $\left.-\mathrm{CH}\left(\mathrm{CH}_{3}\right)\left(\mathrm{CH}_{2}\right)_{3}-\right), 0.95-0.83(\mathrm{~m}, 15 \mathrm{H}$, $-\mathrm{CH}_{2} \mathrm{CH}\left(\mathrm{CH}_{3}\right)-,-\mathrm{CH}\left(\mathrm{CH}_{3}\right)_{2}$ and $\left.-\mathrm{CH}_{2} \mathrm{CH}_{3}\right) .{ }^{13} \mathrm{C} \mathrm{NMR}\left(101 \mathrm{MHz}, \mathrm{CDCl}_{3}, \delta, \mathrm{ppm}\right): 167.09(\mathrm{C}=\mathrm{O}), 152.98$ (ArC-3,5), 142.48 (ArC-4), 124.81 (ArC-1), 108.08 (ArC-2,6), 71.85 ( $\mathrm{ArOCH}_{2}-3,5$ position), 69.28 (ArOCH$H_{2}-4$ position), $52.24\left(-\mathrm{OCH}_{3}\right),[39.49,37.61,37.46,31.71,29.77,29.40,28.14,25.89,24.86](-$ $\mathrm{OCH}_{2}\left(\mathrm{CH}_{2}\right)_{3}-$ and $\left.-\mathrm{OCH}_{2} \mathrm{CH}_{2} \mathrm{CH}\left(\mathrm{CH}_{3}\right)\left(\mathrm{CH}_{2}\right)_{3} \mathrm{CH}-\right)$, [22.84, 22.77, 22.74, 19.67, 14.17] $\left(-\mathrm{CH}\left(\mathrm{CH}_{3}\right)_{2},-\right.$ $\mathrm{CH}\left(\mathrm{CH}_{3}\right) \mathrm{CH}_{2}-$ and $\left.-\mathrm{CH}_{2} \mathrm{CH}_{3}\right)$. MALDI-TOF MS $m / z$ of $[\mathrm{M}+\mathrm{Na}]^{+}$calculated for $\mathrm{C}_{30} \mathrm{H}_{52} \mathrm{O}_{5}$ : 515.4; Found: 515.0 .
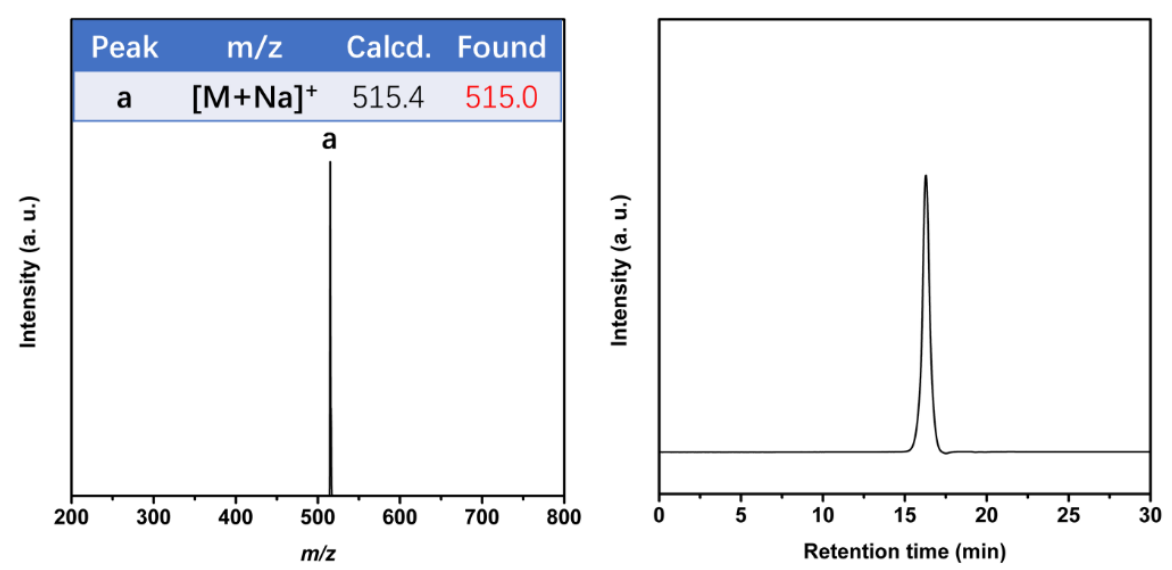

Supporting Figure S11. MALDI-TOF MS spectra (left) and HPLC trace (right) of 5-6r6. Racemic dimethyloctyl group has been successfully linked to the 4-position of 5-6r6. ${ }^{1} \mathrm{H}$ NMR spectrum is presented on page 26 . 
Supporting Figure S11 (continued). ${ }^{1} \mathrm{H}$ NMR of 5-6r6.

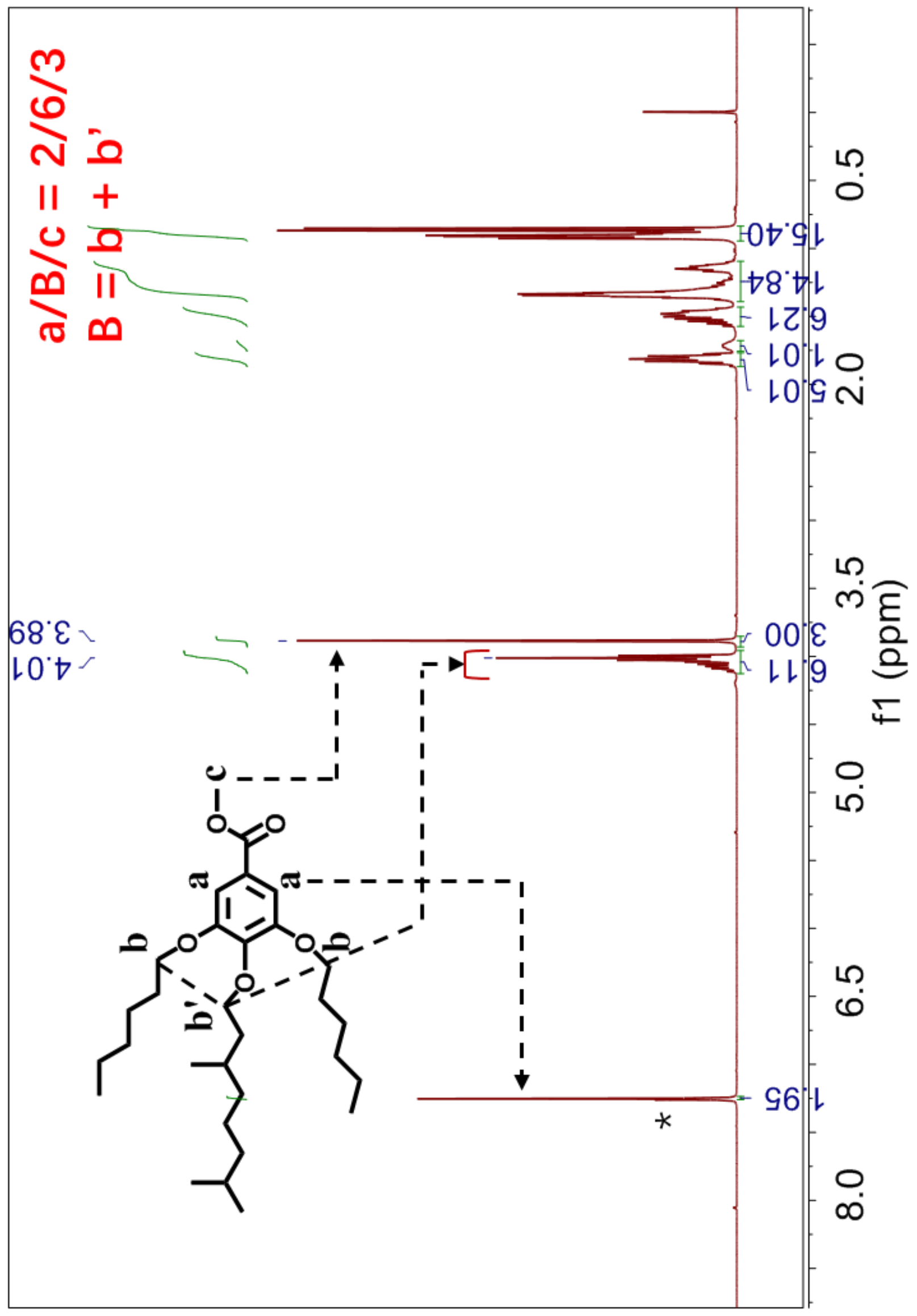




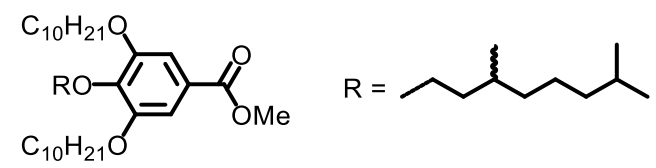

Methyl 3,5-bis(decyloxy)-4-((3,7-(rac)-dimethyloctyl)oxy)benzoate (5-10r10). Starting from 4-10 $(0.28 \mathrm{~g}, 0.60 \mathrm{mmol}), \mathrm{K}_{2} \mathrm{CO}_{3}(0.25 \mathrm{~g}, 1.8 \mathrm{mmol})$ and $(\mathrm{rac})$-1-bromo-3,7-dimethyloctane (0.15 mL, 0.70 mmol) in dry DMF $(5 \mathrm{~mL})$ at $120^{\circ} \mathrm{C}$ for $3.5 \mathrm{~h}, \mathbf{5 - 1 0 r 1 0}$ was obtained as a colorless oil. Product: $0.34 \mathrm{~g}$. Yield: $93 \%$. Purity by HPLC: $99+\%$.

${ }^{1} \mathrm{H}$ NMR (500 MHz, $\left.\mathrm{CDCl}_{3}, \delta, \mathrm{ppm}\right): 7.25(\mathrm{~s}, 2 \mathrm{H}, \mathrm{Ar} H), 4.12-4.03\left(\mathrm{~m}, 2 \mathrm{H},-\mathrm{OCH}_{2} \mathrm{CH}_{2} \mathrm{CH}\left(\mathrm{CH}_{3}\right)-\right), 4.01$ $\left(\mathrm{t}, J=6.5 \mathrm{~Hz}, 4 \mathrm{H},-\mathrm{OCH}_{2} \mathrm{CH}_{2} \mathrm{CH}_{2}-\right), 3.89\left(\mathrm{~s}, 3 \mathrm{H},-\mathrm{CO}_{2} \mathrm{CH}_{3}\right), 1.87-1.76\left(\mathrm{~m}, 5 \mathrm{H},-\mathrm{CH}\left(\mathrm{CH}_{3}\right)_{2}\right.$ and $\left.\mathrm{OCH}_{2} \mathrm{CH}_{2} \mathrm{CH}_{2}-\right)$, $1.76-1.66\left(\mathrm{~m}, 1 \mathrm{H},-\mathrm{CH}_{2} \mathrm{CH}\left(\mathrm{CH}_{3}\right)-\right), 1.58-1.42\left(\mathrm{~m}, 6 \mathrm{H},-\mathrm{O}\left(\mathrm{CH}_{2}\right)_{2} \mathrm{CH}_{2}-\right.$ and $\left.\mathrm{OCH}_{2} \mathrm{CH}_{2} \mathrm{CH}\left(\mathrm{CH}_{3}\right)-\right), 1.39-1.08\left(\mathrm{~m}, 30 \mathrm{H},-\mathrm{O}\left(\mathrm{CH}_{2}\right)_{3}\left(\mathrm{CH}_{2}\right)_{6}-\right.$ and $\left.-\mathrm{CH}\left(\mathrm{CH}_{3}\right)\left(\mathrm{CH}_{2}\right)_{3}-\right), 0.92(\mathrm{~d}, J=6.6 \mathrm{~Hz}$, $\left.3 \mathrm{H},-\mathrm{CH}_{2} \mathrm{CH}\left(\mathrm{CH}_{3}\right)-\right), 0.91-0.83\left(\mathrm{~m}, 12 \mathrm{H},-\mathrm{CH}\left(\mathrm{CH}_{3}\right)_{2}\right.$ and $\left.-\mathrm{CH}_{2} \mathrm{CH}_{3}\right) .{ }^{13} \mathrm{C} \mathrm{NMR}\left(126 \mathrm{MHz}, \mathrm{CDCl}_{3}, \delta\right.$, ppm): $167.10(C=\mathrm{O}), 152.99$ ( $\mathrm{ArC}-3,5), 142.51$ (ArC-4), 124.81 ( $\mathrm{ArC}-1), 108.12(\mathrm{ArC}-2,6), 71.86$ ( $\mathrm{ArOCH}_{2}-3,5$ position), 69.31 ( $\mathrm{ArOCH}_{2}-4$ position), $52.25\left(-\mathrm{OCH}_{3}\right)$, [39.51, 37.63, 37.48, 32.06, 29.78, $29.73,29.55,29.49,29.46,28.15,26.23,24.87]\left(-\mathrm{OCH}_{2}\left(\mathrm{CH}_{2}\right)_{7}\right.$ and $\left.-\mathrm{OCH}_{2} \mathrm{CH}_{2} \mathrm{CH}\left(\mathrm{CH}_{3}\right)\left(\mathrm{CH}_{2}\right)_{3} \mathrm{CH}-\right)$, $[22.85,22.83,22.76,19.69,14.26]\left(-\mathrm{CH}\left(\mathrm{CH}_{3}\right)_{2},-\mathrm{CH}\left(\mathrm{CH}_{3}\right) \mathrm{CH}_{2}-\right.$ and $\left.-\mathrm{CH}_{2} \mathrm{CH}_{3}\right)$. MALDI-TOF MS $\mathrm{m} / \mathrm{z}$ of $[\mathrm{M}+\mathrm{Na}]^{+}$calculated for $\mathrm{C}_{38} \mathrm{H}_{68} \mathrm{O}_{5}: 627.5$; Found: 626.9 .
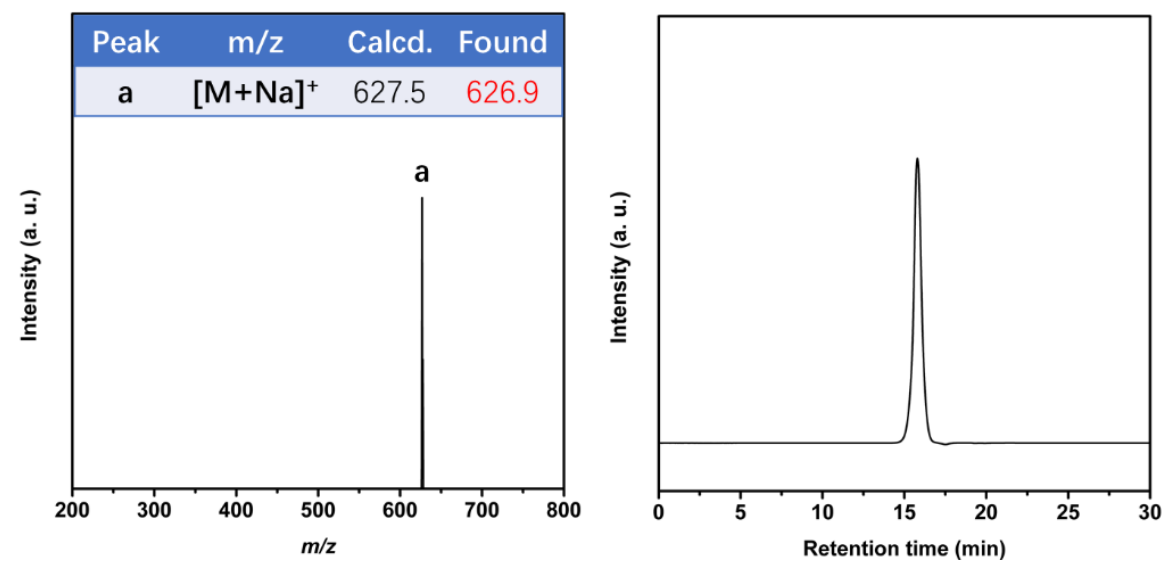

Supporting Figure S12. MALDI-TOF MS spectra (left) and HPLC trace (right) of 5-10r10. Racemic dimethyloctyl group has been successfully linked to the 4-position of $\mathbf{5 - 1 0 r 1 0} .{ }^{1} \mathrm{H}$ NMR spectrum is presented on page 28 . 
Supporting Figure S12 (continued). ${ }^{1} \mathrm{H}$ NMR of 5-10r10.

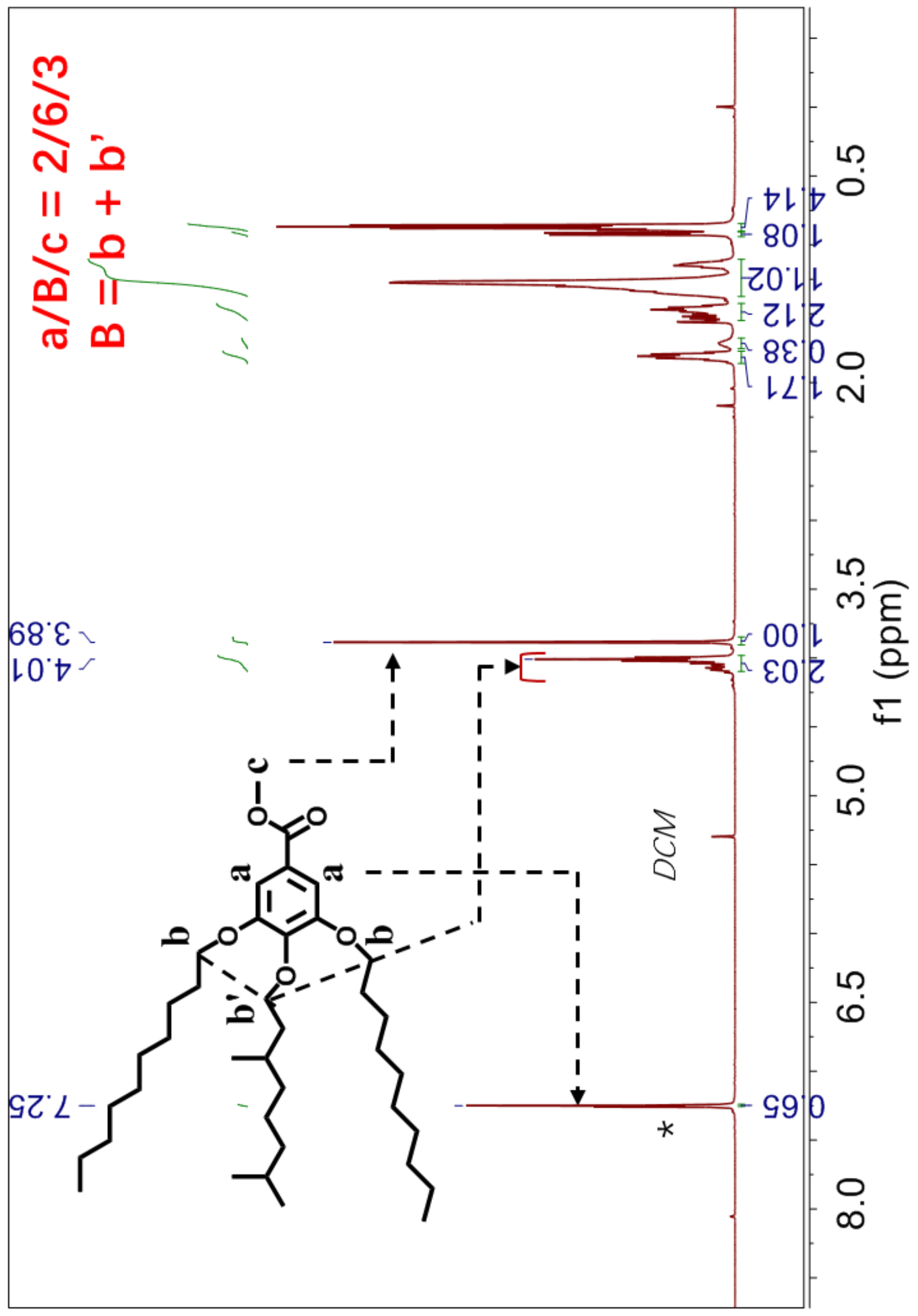


<smiles>[R9]c1cc(C(=O)OC)cc([R9])c1OCC</smiles><smiles>[R]CCC(C)CCCC(C)C</smiles>

Methyl 3,5-bis((3,7-(rac)-dimethyloctyl)oxy)-4-(heptyloxy)benzoate (5-r7r). Starting from 4-r (2.04 g, $4.4 \mathrm{mmol}), \mathrm{K}_{2} \mathrm{CO}_{3}(1.22 \mathrm{~g}, 8.8 \mathrm{mmol})$ and 1-bromo-heptane $(0.73 \mathrm{~mL}, 4.6 \mathrm{mmol})$ in dry DMF (30 mL) at $120^{\circ} \mathrm{C}$ for 4 h, 5-r7r was obtained as a colorless oil. Product: 2.45 g. Yield: 99\%. Purity by HPLC: 99+\%.

${ }^{1} \mathrm{H}$ NMR (400 MHz, $\mathrm{CDCl}_{3}, \delta$ ): ${ }^{1} \mathrm{H}$ NMR (400 MHz, $\left.\mathrm{CDCl}_{3}, \delta, \mathrm{ppm}\right): 7.26$ (s, 2H, ArH), 4.10-3.97 (m, $\left.6 \mathrm{H},-\mathrm{OCH}_{2}-\right), 3.89\left(\mathrm{~s}, 3 \mathrm{H},-\mathrm{CO}_{2} \mathrm{CH}_{3}\right), 1.92-1.81\left(\mathrm{~m}, 2 \mathrm{H},-\mathrm{CH}\left(\mathrm{CH}_{3}\right)_{2}\right), 1.78-1.41(\mathrm{~m}, 10 \mathrm{H},-$ $\mathrm{CH}_{2} \mathrm{CH}\left(\mathrm{CH}_{3}\right)-,-\mathrm{OCH}_{2} \mathrm{CH}_{2} \mathrm{CH}_{2}-,-\mathrm{OCH}_{2} \mathrm{CH}_{2} \mathrm{CH}\left(\mathrm{CH}_{3}\right)-$ and $\left.-\mathrm{OCH}_{2} \mathrm{CH}_{2} \mathrm{CH}_{2}-\right)$, 1.39-1.11 (m, $18 \mathrm{H},-$ $\mathrm{CH}\left(\mathrm{CH}_{3}\right)\left(\mathrm{CH}_{2}\right)_{3}-$ and $\left.-\mathrm{O}\left(\mathrm{CH}_{2}\right)_{3}\left(\mathrm{CH}_{2}\right)_{3}-\right), 0.94\left(\mathrm{~d}, J=6.5 \mathrm{~Hz}, 6 \mathrm{H},-\mathrm{CH}_{2} \mathrm{CH}\left(\mathrm{CH}_{3}\right)-\right), 0.92-0.81(\mathrm{~m}, 15 \mathrm{H}$, $-\mathrm{CH}\left(\mathrm{CH}_{3}\right)_{2}$ and $\left.-\mathrm{CH}_{2} \mathrm{CH}_{3}\right) .{ }^{13} \mathrm{C} \mathrm{NMR}\left(101 \mathrm{MHz}, \mathrm{CDCl}_{3}, \delta, \mathrm{ppm}\right): 167.10(\mathrm{C}=\mathrm{O}), 152.96(\mathrm{ArC}-3,5)$, 142.48 (ArC-4), 124.80 (ArC-1), 108.08 (ArC-2,6), 73.59 ( $\mathrm{ArOCH}_{2}-4$ position), $67.60\left(\mathrm{ArOCH}_{2}-3,5\right.$ position), $52.24\left(-\mathrm{OCH}_{3}\right),[39.39,37.45,36.45,32.04,30.47,29.95,29.36,28.13,26.15,24.87]$ ($\mathrm{OCH}_{2} \mathrm{CH}_{2} \mathrm{CH}\left(\mathrm{CH}_{3}\right)\left(\mathrm{CH}_{2}\right)_{3} \mathrm{CH}-$ and $\left.-\mathrm{OCH}_{2}\left(\mathrm{CH}_{2}\right)_{4}-\right)$, [22.83, 22.73, 19.74, 14.24] $\left(-\mathrm{CH}\left(\mathrm{CH}_{3}\right)_{2}\right.$, $\mathrm{CH}\left(\mathrm{CH}_{3}\right) \mathrm{CH}_{2}-$ and $\left.-\mathrm{CH}_{2} \mathrm{CH}_{3}\right)$. MALDI-TOF MS $m / z$ of $[\mathrm{M}+\mathrm{Na}]^{+}$calculated for $\mathrm{C}_{35} \mathrm{H}_{62} \mathrm{O}_{5}$ : 585.4; Found: 585.1 .
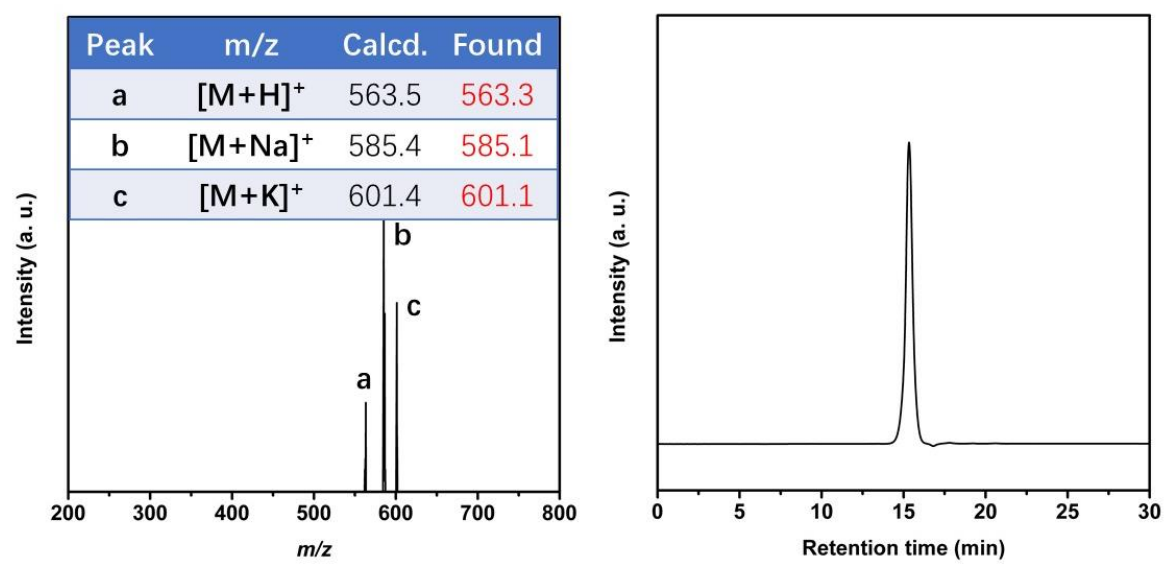

Supporting Figure S13. MALDI-TOF MS spectra (left) and HPLC trace (right) of 5-r7r. Heptyl group has been successfully linked to the 4-position of 5-r7r. ${ }^{1} \mathrm{H}$ NMR spectrum is presented on page 30 . 
Supporting Figure S13 (continued). ${ }^{1} \mathrm{H}$ NMR of 5-r7r.

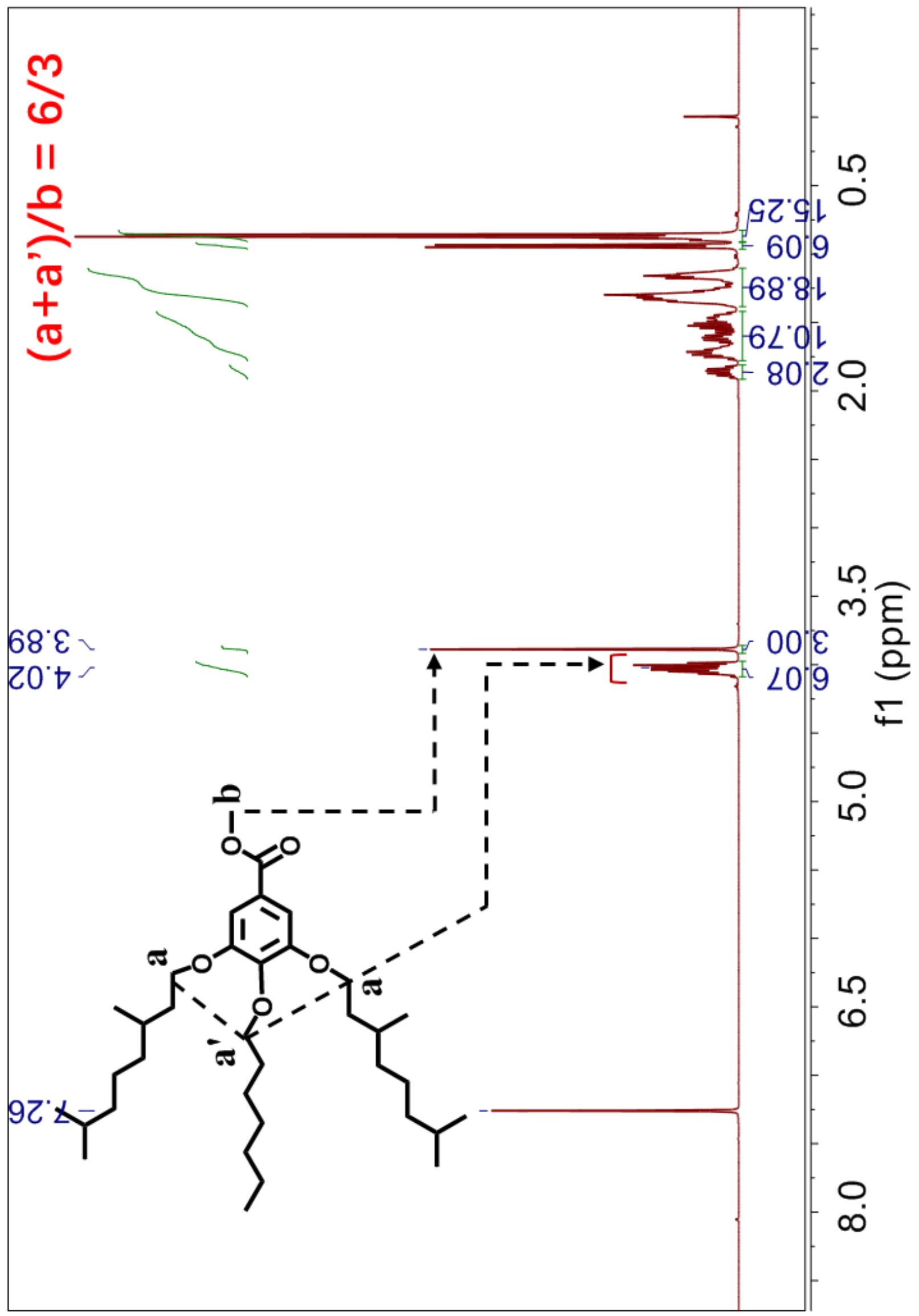


<smiles>[R6]Oc1cc(C(=O)OC)cc([R6])c1O[CH]</smiles><smiles>[R]CCCC(C)CCCC(C)C</smiles>

Methyl 3,5-bis((3,7-(rac)-dimethyloctyl)oxy)-4-(nonyloxy)benzoate (5-r9r). Starting from 4-r (2.04 g, $4.4 \mathrm{mmol}), \mathrm{K}_{2} \mathrm{CO}_{3}(1.22 \mathrm{~g}, 8.8 \mathrm{mmol})$ and 1-bromononane $(0.88 \mathrm{~mL}, 4.6 \mathrm{mmol})$ in dry DMF $(30 \mathrm{~mL})$ at $120^{\circ} \mathrm{C}$ for 4 h, 5-r9r was obtained as a colorless oil. Product: 2.53 g. Yield: 97\%. Purity by HPLC: 99+\%.

${ }^{1} \mathrm{H}$ NMR (400 MHz, $\left.\mathrm{CDCl}_{3}, \delta, \mathrm{ppm}\right): 7.26$ (s, 2H, ArH), 4.10-3.97 (m, 6H, -OCH2-), 3.89 (s, 3H, $\left.\mathrm{CO}_{2} \mathrm{CH}_{3}\right), 1.92-1.80\left(\mathrm{~m}, 2 \mathrm{H},-\mathrm{CH}\left(\mathrm{CH}_{3}\right)_{2}\right), 1.78-1.41\left(\mathrm{~m}, 10 \mathrm{H},-\mathrm{CH}_{2} \mathrm{CH}\left(\mathrm{CH}_{3}\right)-,-\mathrm{OCH}_{2} \mathrm{CH}_{2} \mathrm{CH}_{2}-,-\right.$ $\mathrm{OCH}_{2} \mathrm{CH}_{2} \mathrm{CH}\left(\mathrm{CH}_{3}\right)^{-}$and $\left.-\mathrm{OCH}_{2} \mathrm{CH}_{2} \mathrm{CH}_{2}-\right), \quad 1.41-1.08 \quad\left(\mathrm{~m}, 22 \mathrm{H}, \quad-\mathrm{CH}\left(\mathrm{CH}_{3}\right)\left(\mathrm{CH}_{2}\right)_{3}-\right.$ and $\left.\mathrm{O}\left(\mathrm{CH}_{2}\right)_{3}\left(\mathrm{CH}_{2}\right)_{5}-\right), 0.94\left(\mathrm{~d}, J=6.5 \mathrm{~Hz}, 6 \mathrm{H},-\mathrm{CH}_{2} \mathrm{CH}\left(\mathrm{CH}_{3}\right)-\right), 0.91-0.81\left(\mathrm{~m}, 15 \mathrm{H},-\mathrm{CH}\left(\mathrm{CH}_{3}\right)_{2}\right.$ and $\left.\mathrm{CH}_{2} \mathrm{CH}_{3}\right) .{ }^{13} \mathrm{C} \mathrm{NMR}\left(101 \mathrm{MHz}, \mathrm{CDCl}_{3}, \delta, \mathrm{ppm}\right): 167.09(\mathrm{C}=\mathrm{O}), 152.96$ (ArC-3,5), 142.49 (ArC-4), 124.80 (ArC-1), 108.08 ( $\mathrm{ArC}-2,6), 73.59\left(\mathrm{ArOCH}_{2}-4\right.$ position), $67.60\left(\mathrm{ArOCH}_{2}-3,5\right.$ position), $52.23\left(-\mathrm{OCH}_{3}\right)$, $[39.39, \quad 37.45, \quad 36.45, \quad 32.07,30.47, \quad 29.95,29.80, \quad 29.70,29.50,28.12,26.19,24.87](-$ $\mathrm{OCH}_{2} \mathrm{CH}_{2} \mathrm{CH}\left(\mathrm{CH}_{3}\right)\left(\mathrm{CH}_{2}\right)_{3} \mathrm{CH}-$ and $\left.-\mathrm{OCH}_{2}\left(\mathrm{CH}_{2}\right)_{6}-\right)$, [22.83, 22.73, 19.74, 14.25] $\left(-\mathrm{CH}_{(}\left(\mathrm{CH}_{3}\right)_{2}\right.$, $\mathrm{CH}\left(\mathrm{CH}_{3}\right) \mathrm{CH}_{2}-$ and $\left.-\mathrm{CH}_{2} \mathrm{CH}_{3}\right)$. MALDI-TOF MS $m / z$ of $[\mathrm{M}+\mathrm{Na}]^{+}$calculated for $\mathrm{C}_{37} \mathrm{H}_{66} \mathrm{O}_{5}$ : 613.5; Found: 612.4.
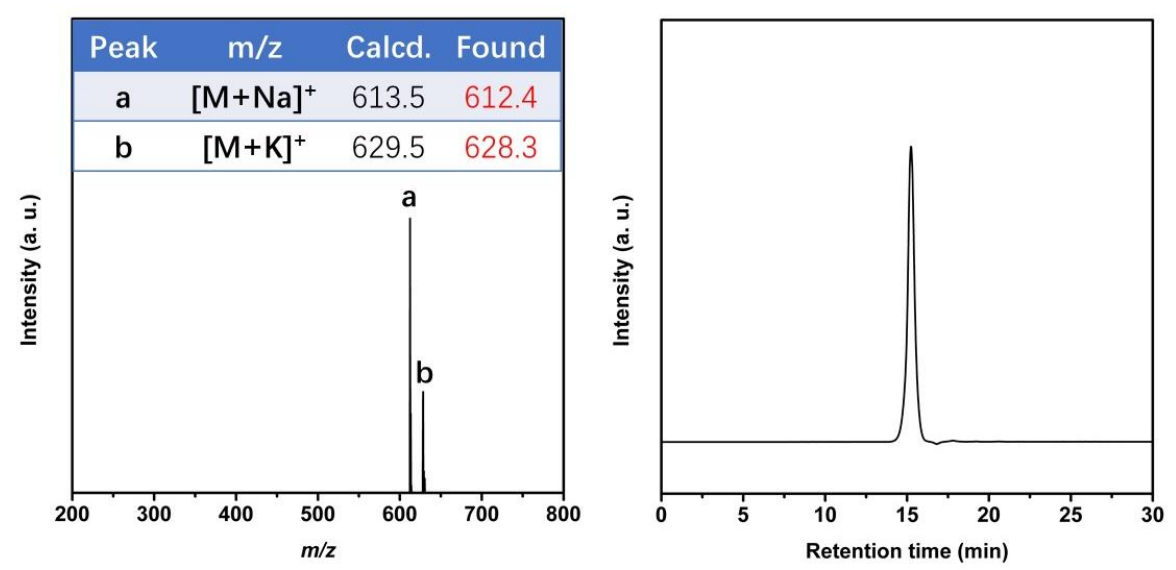

Supporting Figure S14. MALDI-TOF MS spectra (left) and HPLC trace (right) of 5-r9r. Nonyl group has been successfully linked to the 4-position of 5-r9r. ${ }^{1} \mathrm{H}$ NMR spectrum is presented on page 32. 
Supporting Figure S14 (continued). ${ }^{1} \mathrm{H}$ NMR of 5-r9r.

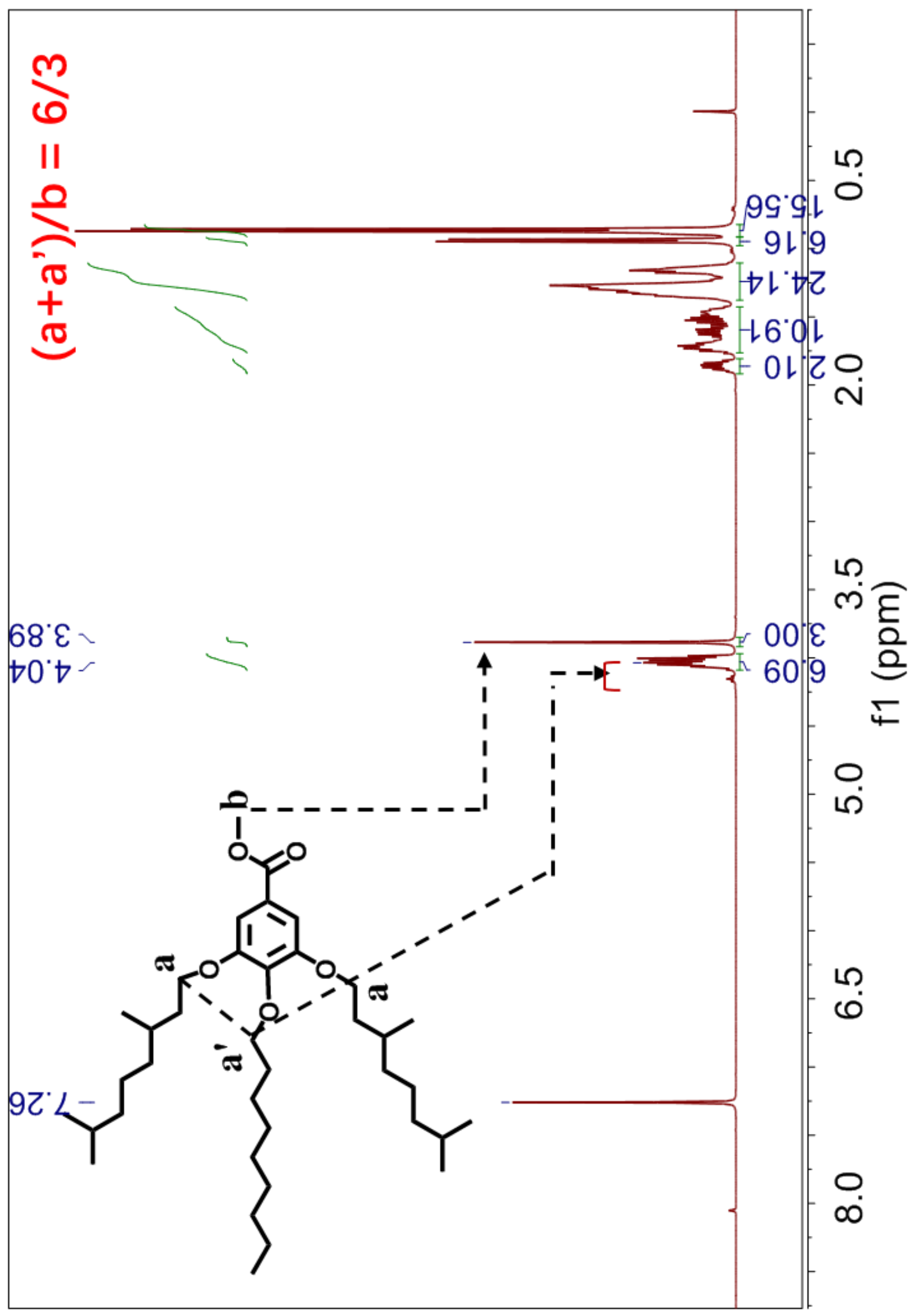


<smiles>[R]CCCC(C)CCCC(C)C</smiles>

Methyl 3,5-bis((3,7-(rac)-dimethyloctyl)oxy)-4-(hexyloxy)benzoate (5-r6r). Starting from 4-r (0.32 g, $0.69 \mathrm{mmol}), \mathrm{K}_{2} \mathrm{CO}_{3}(0.29 \mathrm{~g}, 2.1 \mathrm{mmol})$ and 1-bromo-hexane $(0.11 \mathrm{~mL}, 0.80 \mathrm{mmol})$ in dry DMF $(5 \mathrm{~mL})$ at $120{ }^{\circ} \mathrm{C}$ for 3.5 h, 5-r6r was obtained as a colorless oil. Product: 0.36 g. Yield: $95 \%$. Purity by HPLC: $99+\%$.

${ }^{1} \mathrm{H}$ NMR (500 MHz, $\left.\mathrm{CDCl}_{3}, \delta\right):{ }^{1} \mathrm{H} \mathrm{NMR}\left(400 \mathrm{MHz}, \mathrm{CDCl}_{3}, \delta, \mathrm{ppm}\right): 7.27$ (s, 2H, $\left.\mathrm{ArH}\right), 4.10-3.97$ (m, $\left.6 \mathrm{H},-\mathrm{OCH}_{2}-\right), 3.89\left(\mathrm{~s}, 3 \mathrm{H},-\mathrm{CO}_{2} \mathrm{CH}_{3}\right), 1.92-1.81\left(\mathrm{~m}, 2 \mathrm{H},-\mathrm{CH}\left(\mathrm{CH}_{3}\right)_{2}\right), 1.78-1.67(\mathrm{~m}, 4 \mathrm{H},-$ $\mathrm{CH}_{2} \mathrm{CH}\left(\mathrm{CH}_{3}\right)-$ and $\left.-\mathrm{OCH}_{2} \mathrm{CH}_{2} \mathrm{CH}_{2}-\right), 1.66-1.44\left(\mathrm{~m}, 6 \mathrm{H},-\mathrm{OCH}_{2} \mathrm{CH}_{2} \mathrm{CH}\left(\mathrm{CH}_{3}\right)-\right.$ and $\left.-\mathrm{OCH}_{2} \mathrm{CH}_{2} \mathrm{CH}_{2}-\right)$, 1.39-1.12 (m, 16H, $-\mathrm{CH}\left(\mathrm{CH}_{3}\right)\left(\mathrm{CH}_{2}\right)_{3}-$ and $\left.-\mathrm{O}\left(\mathrm{CH}_{2}\right)_{3}\left(\mathrm{CH}_{2}\right)_{2}-\right), 0.95\left(\mathrm{~d}, J=6.5 \mathrm{~Hz}, 6 \mathrm{H},-\mathrm{CH}_{2} \mathrm{CH}\left(\mathrm{CH}_{3}\right)-\right)$, 0.92-0.85 (m, 15H, $-\mathrm{CH}\left(\mathrm{CH}_{3}\right)_{2}$ and $\left.-\mathrm{CH}_{2} \mathrm{CH}_{3}\right) .{ }^{13} \mathrm{C} \mathrm{NMR}\left(126 \mathrm{MHz}, \mathrm{CDCl}_{3}, \delta, \mathrm{ppm}\right): 167.11(C=\mathrm{O})$, 152.96 (ArC-3,5), 142.46 (ArC-4), 124.80 (ArC-1), 108.05 ( $\mathrm{ArC}-2,6), 73.59$ ( $\mathrm{ArOCH}_{2}-4$ position), 67.59 (ArOCH $2-3,5$ position), $52.26\left(-\mathrm{OCH}_{3}\right),[39.40,37.45,36.44,31.89,30.43,29.95,28.13,25.87,24.87]$ $\left(-\mathrm{OCH}_{2} \mathrm{CH}_{2} \mathrm{CH}\left(\mathrm{CH}_{3}\right)\left(\mathrm{CH}_{2}\right)_{3} \mathrm{CH}-\right.$ and $\left.-\mathrm{OCH}_{2}\left(\mathrm{CH}_{2}\right)_{3}-\right)$, [22.84, 22.74, 19.75, 14.24] $\left(-\mathrm{CH}\left(\mathrm{CH}_{3}\right)_{2}\right.$, $\mathrm{CH}\left(\mathrm{CH}_{3}\right) \mathrm{CH}_{2}$-and $\left.-\mathrm{CH}_{2} \mathrm{CH}_{3}\right)$. MALDI-TOF MS $m / z$ of $[\mathrm{M}+\mathrm{Na}]^{+}$calculated for $\mathrm{C}_{34} \mathrm{H}_{60} \mathrm{O}_{5}$ : 571.4 ; Found: 571.7.
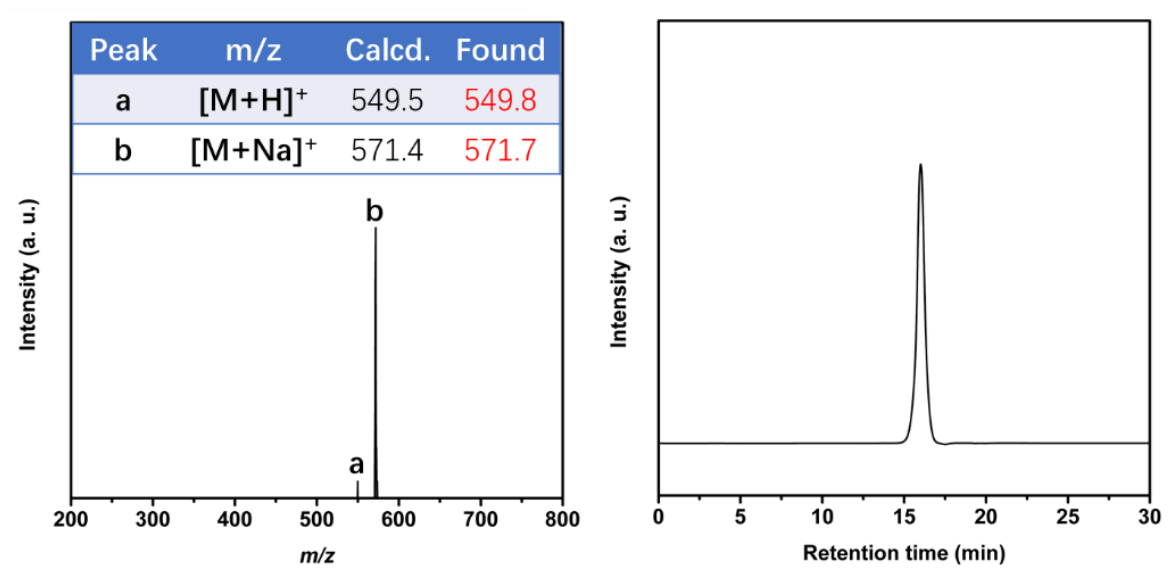

Supporting Figure S15. MALDI-TOF MS spectra (left) and HPLC trace (right) of 5-r6r. Hexyl group has been successfully linked to the 4-position of 5-r6r. ${ }^{1} \mathrm{H}$ NMR spectrum is presented on page 34 . 
Supporting Figure S15 (continued). ${ }^{1} \mathrm{H}$ NMR of 5-r6r.

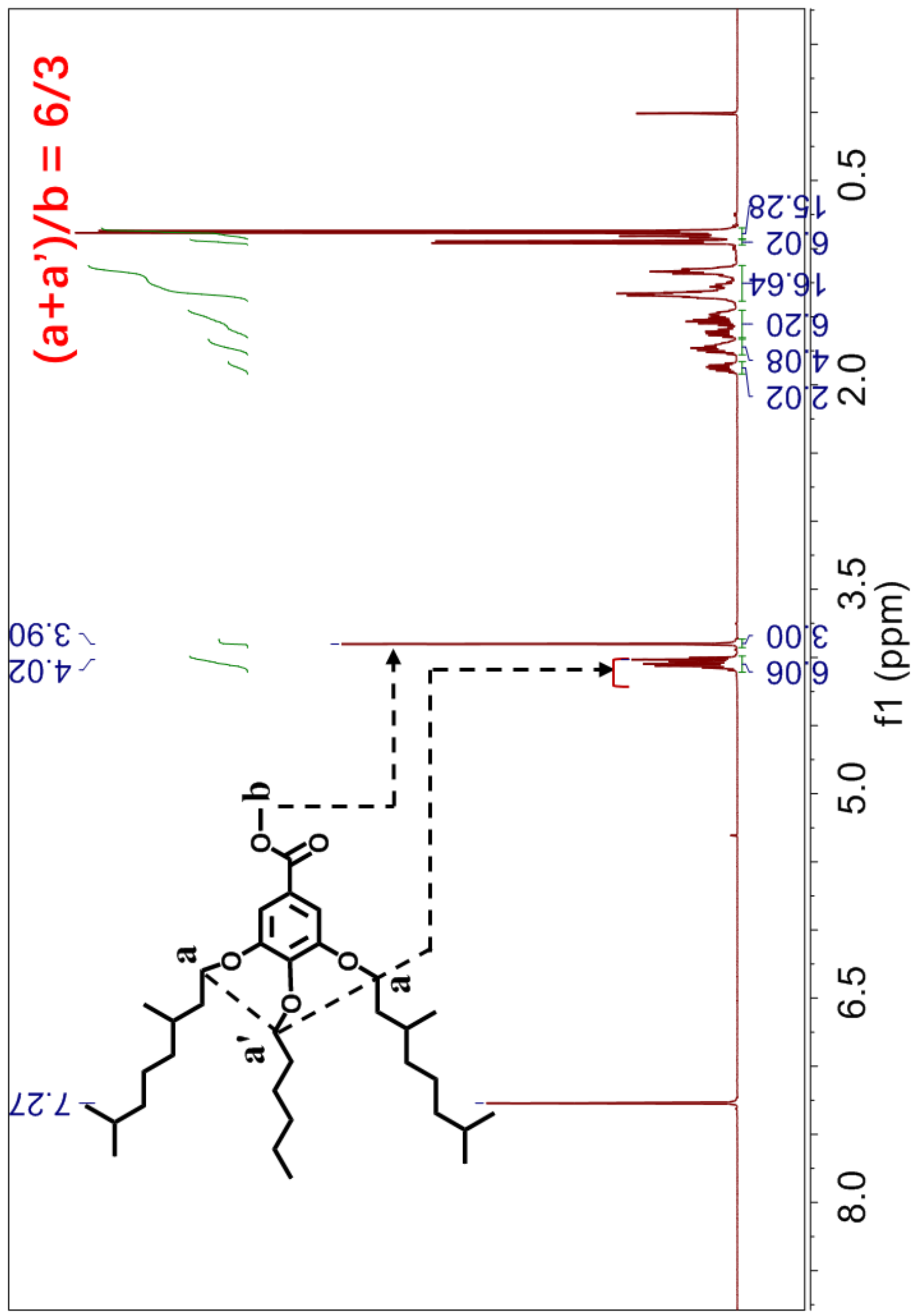


<smiles>[R]CCCC(C)CCCC(C)C</smiles>

Methyl 4-(decyloxy)-3,5-bis((3,7-(rac)-dimethyloctyl)oxy)benzoate (5-r10r). Starting from 4-r (0.23 g, $0.5 \mathrm{mmol}), \mathrm{K}_{2} \mathrm{CO}_{3}(0.25 \mathrm{~g}, 1.8 \mathrm{mmol})$ and 1-bromodecane $(0.12 \mathrm{~mL}, 0.60 \mathrm{mmol})$ in dry DMF $(5 \mathrm{~mL})$ at $120{ }^{\circ} \mathrm{C}$ for 3.5 h, 5-r10r was obtained as a colorless oil. Product: 0.33 g. Yield: 91\%. Purity by HPLC: $99+\%$.

${ }^{1} \mathrm{H}$ NMR (500 MHz, $\left.\mathrm{CDCl}_{3}, \delta, \mathrm{ppm}\right): 7.26$ (s, 2H, $\left.\mathrm{ArH}\right), 4.10-3.97$ (m, 6H, $-\mathrm{OCH}_{2}-$ ), 3.89 (s, 3H, $\left.\mathrm{CO}_{2} \mathrm{CH}_{3}\right), 1.91-1.82\left(\mathrm{~m}, 2 \mathrm{H},-\mathrm{CH}\left(\mathrm{CH}_{3}\right)_{2}\right), 1.77-1.65\left(\mathrm{~m}, 4 \mathrm{H},-\mathrm{CH}_{2} \mathrm{CH}\left(\mathrm{CH}_{3}\right)-\right.$ and $\left.-\mathrm{OCH}_{2} \mathrm{CH}_{2} \mathrm{CH}_{2}-\right)$, 1.65-1.43 (m, 6H, $-\mathrm{OCH}_{2} \mathrm{CH}_{2} \mathrm{CH}\left(\mathrm{CH}_{3}\right)-$ and $\left.-\mathrm{OCH}_{2} \mathrm{CH}_{2} \mathrm{CH}_{2}-\right)$, 1.39-1.11 (m, 24H, $-\mathrm{CH}\left(\mathrm{CH}_{3}\right)\left(\mathrm{CH}_{2}\right)_{3}-$ and $\left.-\mathrm{O}\left(\mathrm{CH}_{2}\right)_{3}\left(\mathrm{CH}_{2}\right)_{6}-\right), 0.94\left(\mathrm{~d}, J=6.6 \mathrm{~Hz}, 6 \mathrm{H},-\mathrm{CH}_{2} \mathrm{CH}\left(\mathrm{CH}_{3}\right)-\right), 0.91-0.83\left(\mathrm{~m}, 15 \mathrm{H},-\mathrm{CH}\left(\mathrm{CH}_{3}\right)_{2}\right.$ and $\left.{ }_{-} \mathrm{CH}_{2} \mathrm{CH}_{3}\right) .{ }^{13} \mathrm{C} \mathrm{NMR}\left(126 \mathrm{MHz}, \mathrm{CDCl}_{3}, \delta, \mathrm{ppm}\right): 167.10(\mathrm{C}=\mathrm{O}), 152.95(\mathrm{ArC}-3,5), 142.44(\mathrm{ArC}-4)$, 124.78 (ArC-1), 108.03 (ArC-2,6), 73.59 ( $\mathrm{ArOCH}_{2}-4$ position), 67.58 ( $\mathrm{ArOCH}_{2}-3,5$ position), 52.25 ($\left.\mathrm{OCH}_{3}\right)$, [39.39, 37.45, 36.44, 32.07, 30.46, 29.95, 29.85, 29.81, 29.71, 29.53, 28.13, 26.19, 24.87] ($\mathrm{OCH}_{2} \mathrm{CH}_{2} \mathrm{CH}\left(\mathrm{CH}_{3}\right)\left(\mathrm{CH}_{2}\right)_{3} \mathrm{CH}-$ and $\left.-\mathrm{OCH}_{2}\left(\mathrm{CH}_{2}\right)_{7}-\right)$, [22.84, 22.74, 19.74, 14.26] $\left(-\mathrm{CH}\left(\mathrm{CH}_{3}\right)_{2}\right.$, $\mathrm{CH}\left(\mathrm{CH}_{3}\right) \mathrm{CH}_{2}-$ and $\left.-\mathrm{CH}_{2} \mathrm{CH}_{3}\right)$. MALDI-TOF MS m/z of $[\mathrm{M}+\mathrm{Na}]^{+}$calculated for $\mathrm{C}_{38} \mathrm{H}_{68} \mathrm{O}_{5}$ : 627.5; Found: 626.9 .
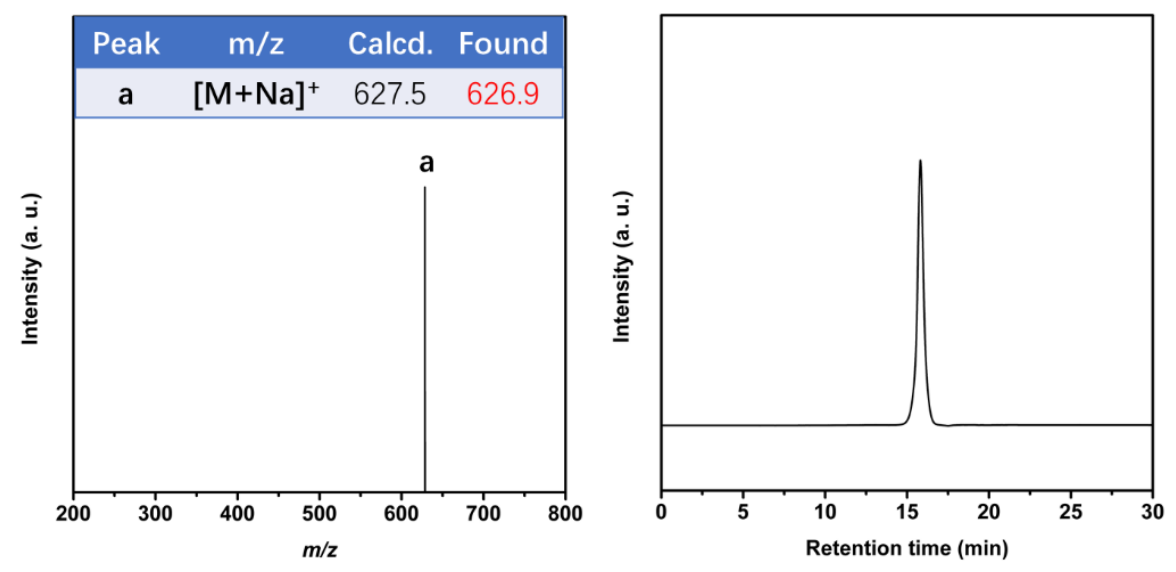

Supporting Figure S16. MALDI-TOF MS spectra (left) and HPLC trace (right) of 5-r10r. Decyl group has been successfully linked to the 4-position of 5-r10r. ${ }^{1} \mathrm{H}$ NMR spectrum is presented on page 36. 
Supporting Figure S16 (continued). ${ }^{1} \mathrm{H}$ NMR of 5-r10r.

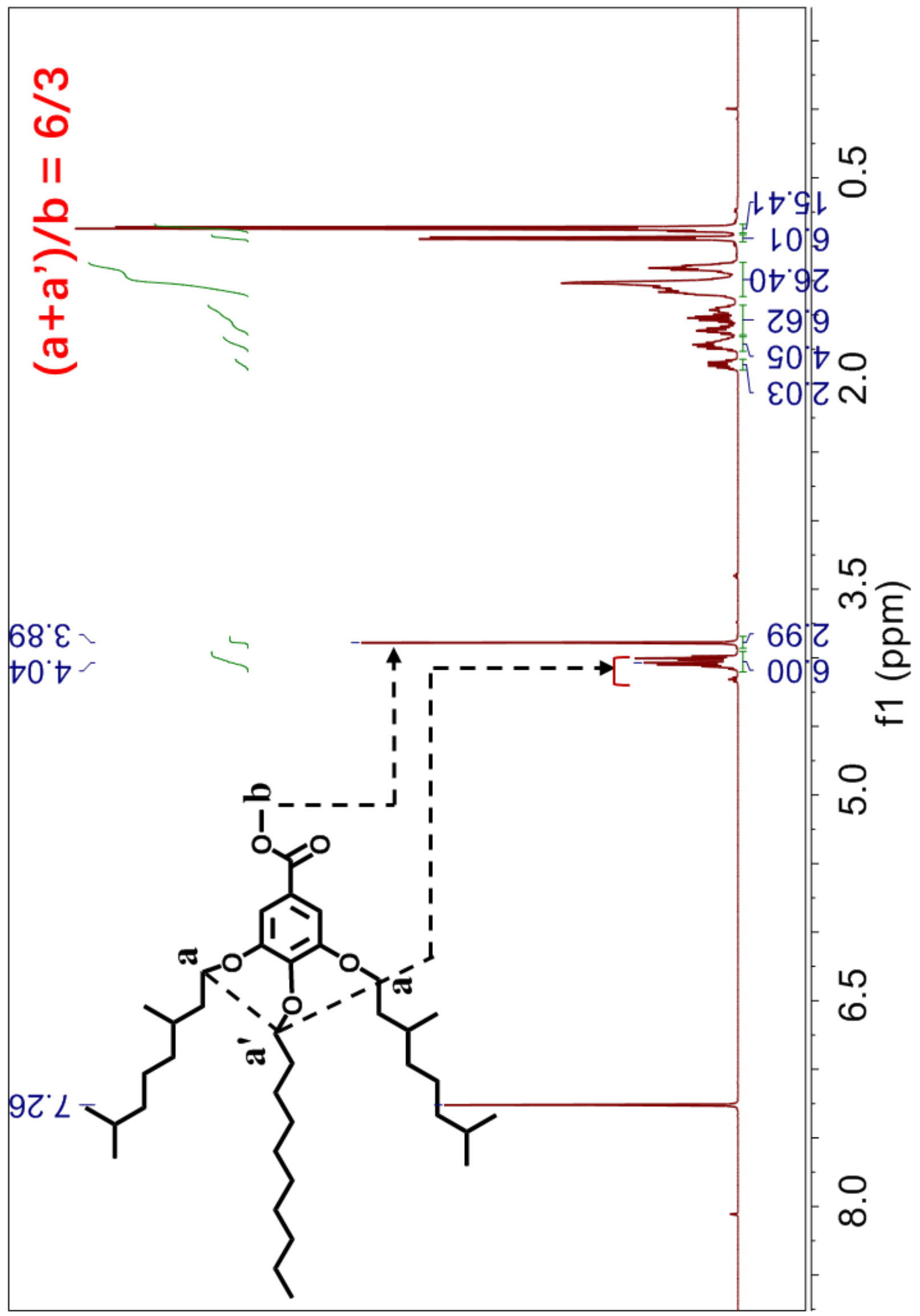




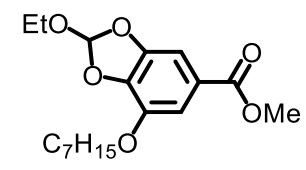

Methyl 2-ethoxy-7-(heptyloxy)benzo[d][1,3]dioxole-5-carboxylate (7-7). Compound 7-7 was synthesized by Williamson etherification under conditions reported by our group. ${ }^{7}$ A mixture of 6 (1.20 g, $5.0 \mathrm{mmol})$ and $\mathrm{K}_{2} \mathrm{CO}_{3}(1.38 \mathrm{~g}, 10.0 \mathrm{mmol})$ was stirred in dry DMF $(20 \mathrm{~mL})$ under $\mathrm{N}_{2}$ protection. 1Bromoheptane $(0.83 \mathrm{~mL}, 5.3 \mathrm{mmol})$ was added and the reaction mixture was heated at $65^{\circ} \mathrm{C}$ for $4 \mathrm{~h}$. Then the mixture was cooled, filtered, poured into cold water $(100 \mathrm{~mL})$ and extracted with EA $(25 \mathrm{~mL} \times 4)$. The combined EA phase was washed with water $(25 \mathrm{~mL} \times 3)$, dried over anhydrous $\mathrm{MgSO}_{4}$, filtered and evaporated in vacuo. The crude product was subjected to column chromatography $(\mathrm{EA} / \mathrm{Hexanes}=1 / 24)$ and 7-7 was obtained as a colorless oil. Product: 1.65 g. Yield: $98 \%$. Purity, HPLC: $99+\%$. The synthesis procedures for 7-9, 7-6 and 7-10 listed below are similar to that for 7-7.

${ }^{1} \mathrm{H}$ NMR $\left(400 \mathrm{MHz}, \mathrm{CDCl}_{3}, \delta, \mathrm{ppm}\right): 7.32\left(\mathrm{~d}, J=1.2 \mathrm{~Hz}, 1 \mathrm{H}, \mathrm{Ar} H\right.$ ortho to $\left.-\mathrm{OC}_{7} \mathrm{H}_{15}\right), 7.21(\mathrm{~d}, J=1.2 \mathrm{~Hz}$, $1 \mathrm{H}, \mathrm{Ar} H$ para to $\left.-\mathrm{OC}_{7} \mathrm{H}_{15}\right), 6.93(\mathrm{~s}, 1 \mathrm{H},-\mathrm{C}(H) \mathrm{OEt}), 4.10\left(\mathrm{t}, J=6.7 \mathrm{~Hz}, 2 \mathrm{H},-\mathrm{OCH}_{2} \mathrm{CH}_{2}-\right), 3.87(\mathrm{~s}, 3 \mathrm{H},-$ $\left.\mathrm{OCH}_{3}\right), 3.74\left(\mathrm{q}, J=7.1 \mathrm{~Hz}, 2 \mathrm{H},-\mathrm{OCH}_{2} \mathrm{CH}_{3}\right), 1.85-1.74\left(\mathrm{~m}, 2 \mathrm{H},-\mathrm{OCH}_{2} \mathrm{CH}_{2}-\right), 1.50-1.39(\mathrm{~m}, 2 \mathrm{H},-$ $\left.\mathrm{O}\left(\mathrm{CH}_{2}\right)_{2} \mathrm{CH}_{2-}\right), 1.39-1.21\left(\mathrm{~m}, 9 \mathrm{H},-\left(\mathrm{CH}_{2}\right)_{3}\left(\mathrm{CH}_{2}\right)_{3}-\right.$ and $\left.-\mathrm{OCH}_{2} \mathrm{CH}_{3}\right), 0.89(\mathrm{t}, J=6.8 \mathrm{~Hz}, 3 \mathrm{H},-$ $\left.\mathrm{CH}_{2} \mathrm{CH}_{2} \mathrm{CH}_{3}\right) .{ }^{13} \mathrm{C} \mathrm{NMR}\left(101 \mathrm{MHz}, \mathrm{CDCl}_{3}, \delta, \mathrm{ppm}\right): 165.56(\mathrm{C}=\mathrm{O}), 147.17\left(\mathrm{ArC}-5\right.$ or $\left.-\mathrm{CH}_{2} \mathrm{OC}\right), 142.29$ ( $\mathrm{ArC}-3$ or $-\mathrm{CHOCCH}$ ), 138.24 ( $\mathrm{ArC}-4$ or $-\mathrm{CHOCC}), 124.31$ ( $\mathrm{ArC}-1$ or $\left.\mathrm{MeO}_{2} \mathrm{CC}\right), 120.09$ (-OCHO-), $111.18\left(\mathrm{ArC}-6\right.$ or $\left.-\mathrm{CH}_{2} \mathrm{OCCH}\right), 103.32(\mathrm{ArC}-2$ or $-\mathrm{CHOCCH}), 69.78\left(\mathrm{ArOCH}_{2}-\right), 59.66\left(-\mathrm{CHOCH}_{2}-\right)$, $52.25\left(-\mathrm{OCH}_{3}\right),[31.85,29.40,29.12,25.94,22.70]\left(-\mathrm{OCH}_{2}\left(\mathrm{CH}_{2}\right)_{5}-\right), 14.88\left(-\mathrm{OCH}_{2} \mathrm{CH}_{3}\right), 14.17(-$ $\mathrm{CH}_{2} \mathrm{CH}_{2} \mathrm{CH}_{3}$ ). MALDI-TOF MS $m / z$ of $[\mathrm{M}+\mathrm{Na}]^{+}$calculated for $\mathrm{C}_{18} \mathrm{H}_{26} \mathrm{O}_{6}$ : 361.2; Found: 361.0.

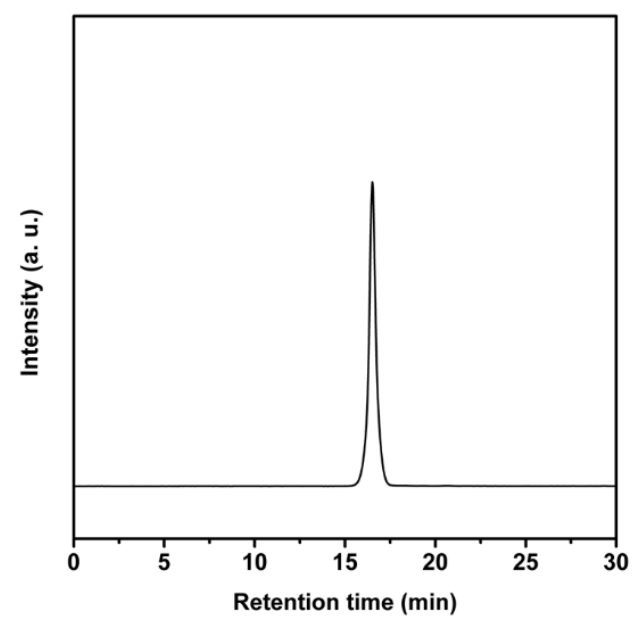

Supporting Figure S17. HPLC trace of 7-7. Heptyl group has been successfully linked to the 7-position of 7-7. ${ }^{1} \mathrm{H}$ NMR spectrum is presented on page 38 . 
Supporting Figure S17 (continued). ${ }^{1} \mathrm{H}$ NMR of 7-7.

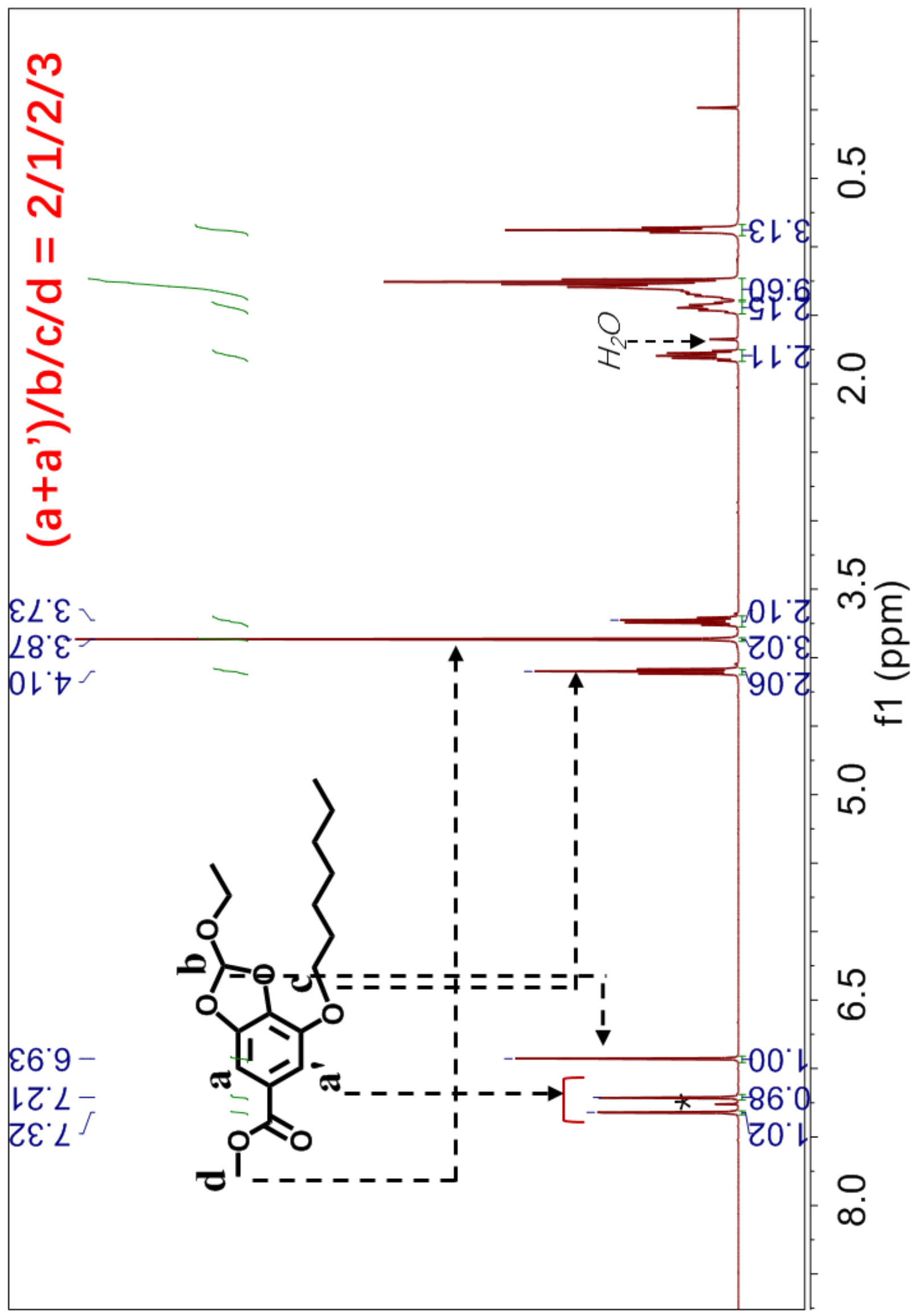




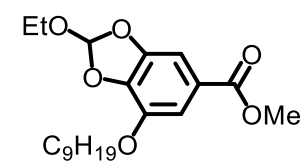

Methyl 2-ethoxy-7-(nonyloxy)benzo[d][1,3]dioxole-5-carboxylate (7-9). Starting from 6 (1.20 g, 5.0 $\mathrm{mmol})$ and 1-bromononane $(1.00 \mathrm{~mL}, 5.25 \mathrm{mmol})$ in the presence of $\mathrm{K}_{2} \mathrm{CO}_{3}(1.38 \mathrm{~g}, 10.0 \mathrm{mmol})$ in dry DMF $(20 \mathrm{~mL})$ at $65^{\circ} \mathrm{C}$ for $4 \mathrm{~h}, \mathbf{7 - 9}$ was obtained as a colorless oil. Product: $1.79 \mathrm{~g}$. Yield: $98 \%$. Purity by HPLC: $99+\%$.

${ }^{1} \mathrm{H}$ NMR (400 MHz, $\mathrm{CDCl}_{3}, \delta$, ppm): $7.32\left(\mathrm{~d}, J=1.3 \mathrm{~Hz}, 1 \mathrm{H}, \mathrm{Ar} H\right.$ ortho to $\left.-\mathrm{OC}_{9} \mathrm{H}_{19}\right), 7.21(\mathrm{~d}, J=1.3 \mathrm{~Hz}$, $1 \mathrm{H}, \mathrm{Ar} H$ para to $\left.-\mathrm{OC}_{9} \mathrm{H}_{19}\right), 6.92(\mathrm{~s}, 1 \mathrm{H},-\mathrm{C}(H) \mathrm{OEt}), 4.10\left(\mathrm{t}, J=6.7 \mathrm{~Hz}, 2 \mathrm{H},-\mathrm{OCH}_{2} \mathrm{CH}_{2}-\right), 3.86(\mathrm{~s}, 3 \mathrm{H},-$ $\left.\mathrm{OCH}_{3}\right), 3.73\left(\mathrm{q}, J=7.1 \mathrm{~Hz}, 2 \mathrm{H},-\mathrm{OCH}_{2} \mathrm{CH}_{3}\right), 1.83-1.75\left(\mathrm{~m}, 2 \mathrm{H},-\mathrm{OCH}_{2} \mathrm{CH}_{2}-\right), 1.50-1.39(\mathrm{~m}, 2 \mathrm{H},-$ $\left.\mathrm{O}\left(\mathrm{CH}_{2}\right)_{2} \mathrm{CH}_{2}-\right), 1.39-1.20\left(\mathrm{~m}, 13 \mathrm{H},-\left(\mathrm{CH}_{2}\right)_{3}\left(\mathrm{CH}_{2}\right)_{5}-\right.$ and $\left.-\mathrm{OCH}_{2} \mathrm{CH}_{3}\right), 0.87$ (t, $J=6.8 \mathrm{~Hz}, 3 \mathrm{H},-$ $\left.\mathrm{CH}_{2} \mathrm{CH}_{2} \mathrm{CH}_{3}\right) .{ }^{13} \mathrm{C}$ NMR (101 MHz, $\left.\mathrm{CDCl}_{3}, \delta, \mathrm{ppm}\right): 166.54(\mathrm{C}=\mathrm{O}), 147.16(\mathrm{ArC}-5), 142.27(\mathrm{ArC}-3)$, 138.23 (ArC-4), 124.29 ( $\mathrm{ArC}-1), 120.08$ (-OCHO-), 111.16 ( $\mathrm{ArC}-6), 103.31$ ( $\mathrm{ArC}-2), 69.77\left(\mathrm{ArOCH}_{2}-\right)$, $59.63\left(-\mathrm{CHOCH}_{2}-\right), 52.23\left(-\mathrm{OCH}_{3}\right),[31.96,29.60,29.45,29.34,29.31,25.96,22.75]\left(-\mathrm{OCH}_{2}\left(\mathrm{CH}_{2}\right)_{7}-\right)$, $14.87\left(-\mathrm{OCH}_{2} \mathrm{CH}_{3}\right), 14.18\left(-\mathrm{CH}_{2} \mathrm{CH}_{2} \mathrm{CH}_{3}\right)$. MALDI-TOF MS $m / z$ of $[\mathrm{M}+\mathrm{Na}]^{+}$calculated for $\mathrm{C}_{20} \mathrm{H}_{30} \mathrm{O}_{6}$ : 389.2; Found: 388.8 .

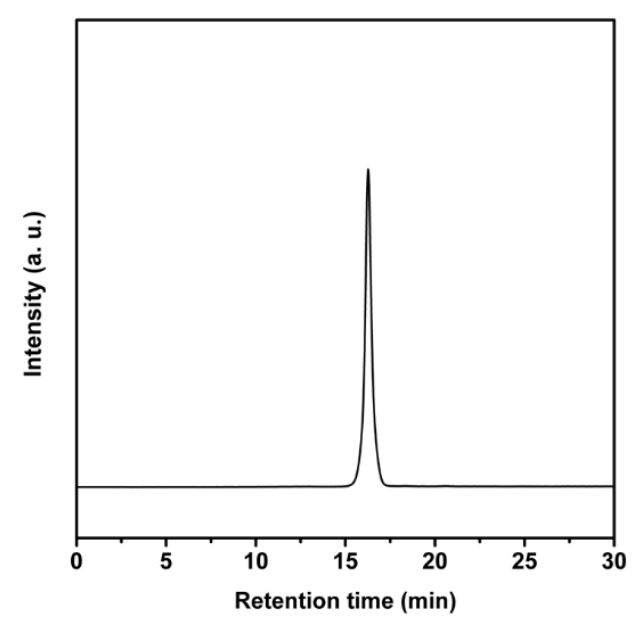

Supporting Figure S18. HPLC trace of 7-9. Nonyl group has been successfully linked to the 7-position of 7-9. ${ }^{1} \mathrm{H}$ NMR spectrum is presented on page 40. 
Supporting Figure S18 (continued). ${ }^{1} \mathrm{H}$ NMR of 7-9.

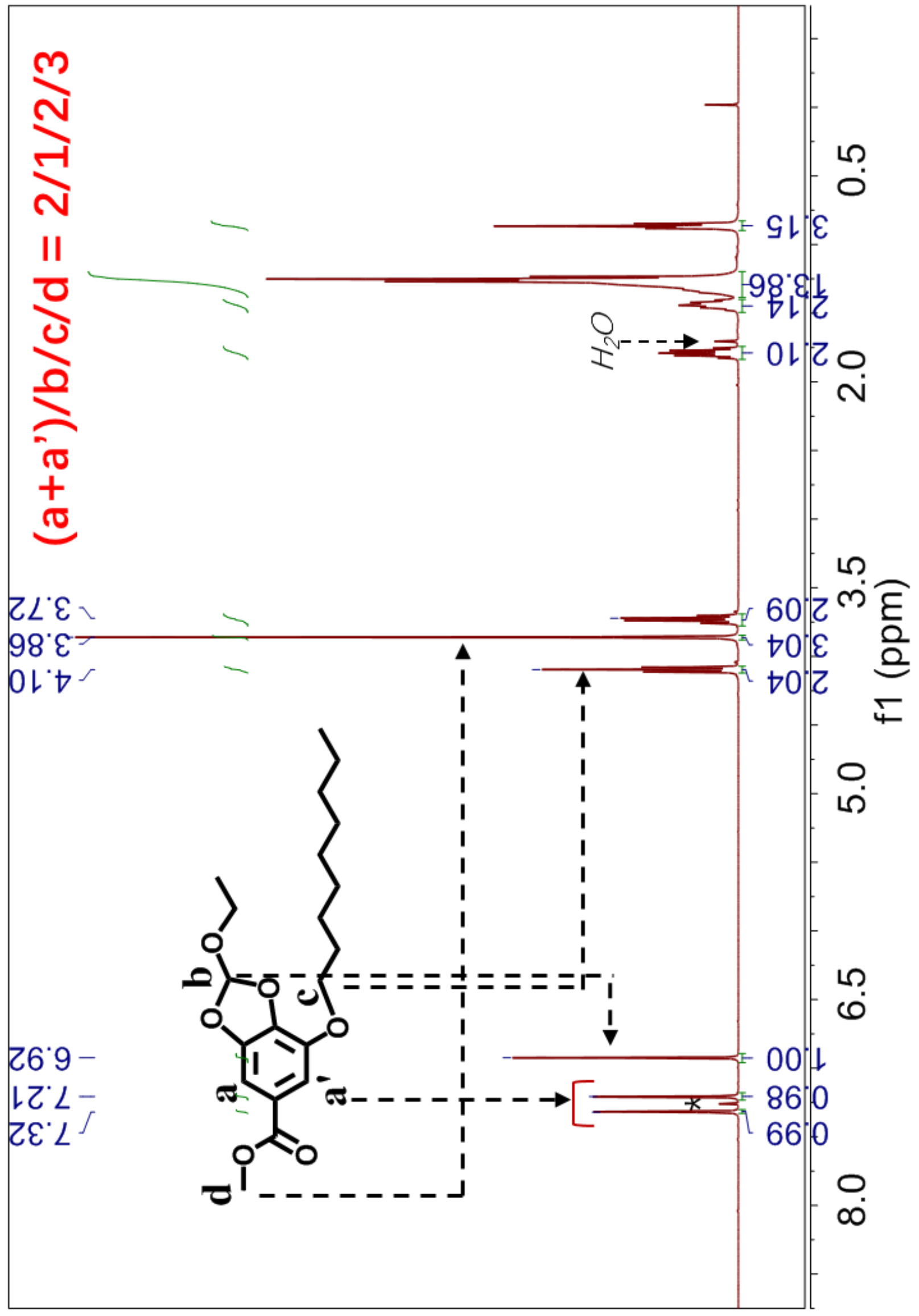




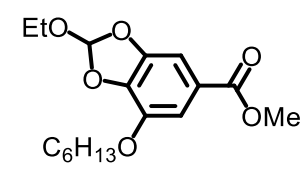

Methyl 2-ethoxy-7-(hexyloxy)benzo[d][1,3]dioxole-5-carboxylate (7-6). Starting from $6(0.48 \mathrm{~g}, 2.0$ $\mathrm{mmol})$ and 1-bromohexane $(0.34 \mathrm{~mL}, 2.4 \mathrm{mmol})$ in the presence of $\mathrm{K}_{2} \mathrm{CO}_{3}(0.83 \mathrm{~g}, 6.0 \mathrm{mmol})$ in dry DMF $(10 \mathrm{~mL})$ at $70{ }^{\circ} \mathrm{C}$ for $4 \mathrm{~h}, 7-6$ was obtained as a colorless oil. Product: $0.64 \mathrm{~g}$. Yield: 98\%. Purity by HPLC: $99+\%$.

${ }^{1} \mathrm{H}$ NMR (400 MHz, $\left.\mathrm{CDCl}_{3}, \delta, \mathrm{ppm}\right): 7.33\left(\mathrm{~d}, J=1.4 \mathrm{~Hz}, 1 \mathrm{H}, \mathrm{ArH}\right.$ ortho to $\left.-\mathrm{OC}_{6} \mathrm{H}_{13}\right), 7.21(\mathrm{~d}, J=1.4 \mathrm{~Hz}$, $1 \mathrm{H}$, ArH para to $\left.-\mathrm{OC}_{6} \mathrm{H}_{13}\right), 6.94(\mathrm{~s}, 1 \mathrm{H},-\mathrm{C}(\mathrm{H}) \mathrm{OEt}), 4.11\left(\mathrm{t}, J=6.7 \mathrm{~Hz}, 2 \mathrm{H},-\mathrm{OCH}_{2} \mathrm{CH}_{2}-\right), 3.88(\mathrm{~s}, 3 \mathrm{H},-$ $\left.\mathrm{OCH}_{3}\right), 3.74\left(\mathrm{q}, J=7.1 \mathrm{~Hz}, 2 \mathrm{H},-\mathrm{OCH}_{2} \mathrm{CH}_{3}\right), 1.85-1.76\left(\mathrm{~m}, 2 \mathrm{H},-\mathrm{OCH}_{2} \mathrm{CH}_{2}-\right), 1.51-1.41(\mathrm{~m}, 2 \mathrm{H},-$ $\left.\mathrm{O}\left(\mathrm{CH}_{2}\right)_{2} \mathrm{CH}_{2}-\right), 1.39-1.30\left(\mathrm{~m}, 4 \mathrm{H},-\left(\mathrm{CH}_{2}\right)_{3}\left(\mathrm{CH}_{2}\right)_{2}-\right), 1.27\left(\mathrm{t}, J=7.1 \mathrm{~Hz}, 3 \mathrm{H},-\mathrm{OCH}_{2} \mathrm{CH}_{3}\right), 0.90(\mathrm{t}, J=7.0$ $\left.\mathrm{Hz}, 3 \mathrm{H},-\mathrm{CH}_{2} \mathrm{CH}_{2} \mathrm{CH}_{3}\right) .{ }^{13} \mathrm{C} \mathrm{NMR}\left(101 \mathrm{MHz}, \mathrm{CDCl}_{3}, \delta, \mathrm{ppm}\right): 166.61(C=\mathrm{O}), 147.20(\operatorname{ArC}-5), 142.32$ (ArC-3), 138.26 (ArC-4), 124.34 (ArC-1), 120.12 (-OCHO-), 111.18 (ArC-6), 103.36 (ArC-2), 69.81 $\left(\mathrm{ArOCH}_{2}-\right), 59.70\left(-\mathrm{CHOCH}_{2}-\right), 52.30\left(-\mathrm{OCH}_{3}\right),[31.66,29.30,25.68,22.71]\left(-\mathrm{OCH}_{2}\left(\mathrm{CH}_{2}\right)_{4}-\right), 14.92(-$ $\left.\mathrm{OCH}_{2} \mathrm{CH}_{3}\right), 14.15\left(-\mathrm{CH}_{2} \mathrm{CH}_{2} \mathrm{CH}_{3}\right)$. MALDI-TOF MS $m / z$ of $[\mathrm{M}+\mathrm{Na}]^{+}$calculated for $\mathrm{C}_{17} \mathrm{H}_{24} \mathrm{O}_{6}: 347.1$; Found: 347.6 .

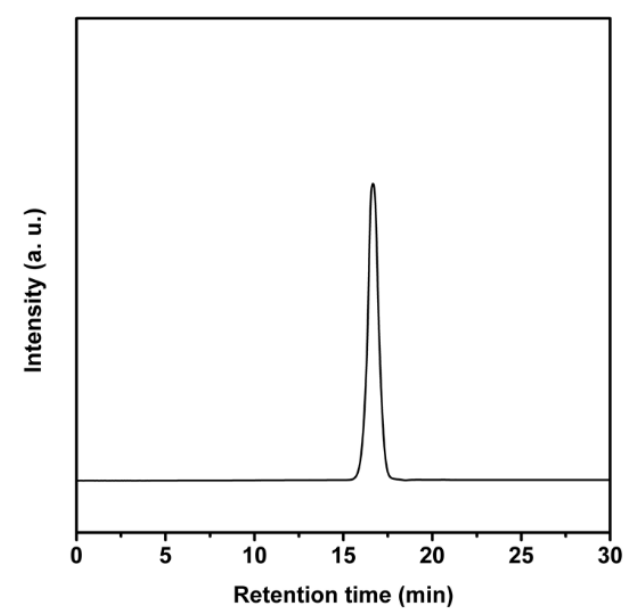

Supporting Figure S19. HPLC trace of 7-6. Hexyl group has been successfully linked to the 7-position of 7-6. ${ }^{1} \mathrm{H}$ NMR spectrum is presented on page 42. 
Supporting Figure S19 (continued). ${ }^{1} \mathrm{H}$ NMR of 7-6.

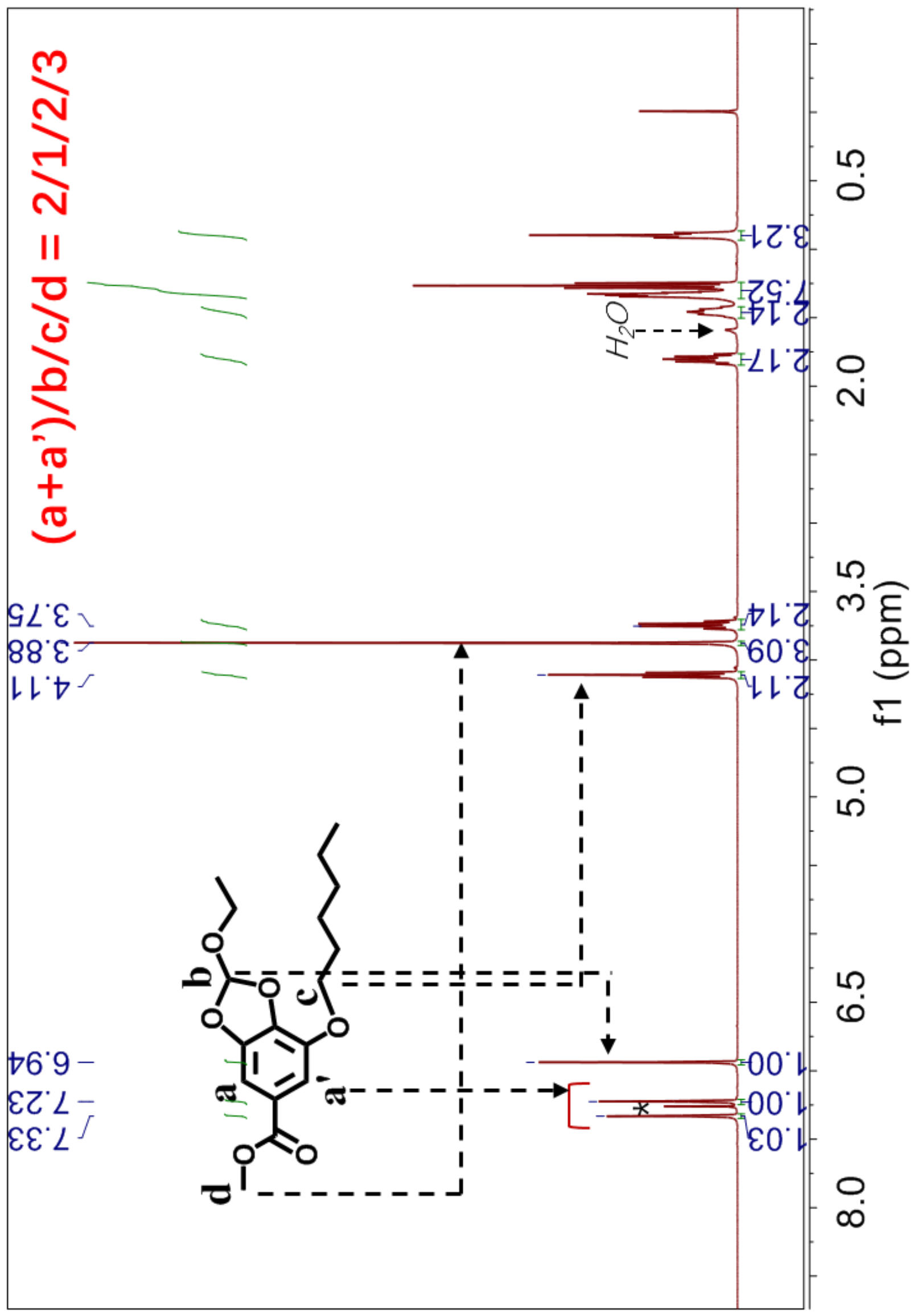




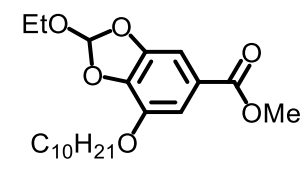

Methyl 2-ethoxy-7-(decyloxy)benzo[d][1,3]dioxole-5-carboxylate (7-10). Starting from 6 (0.48 g, 2.0 $\mathrm{mmol})$ and 1-bromodecane $(0.50 \mathrm{~mL}, 2.4 \mathrm{mmol})$ in the presence of $\mathrm{K}_{2} \mathrm{CO}_{3}(0.83 \mathrm{~g}, 6.0 \mathrm{mmol})$ in dry DMF $(10 \mathrm{~mL})$ at $70{ }^{\circ} \mathrm{C}$ for $4 \mathrm{~h}, \mathbf{7 - 1 0}$ was obtained as a colorless oil. Product: $0.75 \mathrm{~g}$. Yield: $99 \%$. Purity by HPLC: $99+\%$.

${ }^{1} \mathrm{H}$ NMR (400 MHz, $\left.\mathrm{CDCl}_{3}, \delta, \mathrm{ppm}\right): 7.34\left(\mathrm{~d}, J=1.3 \mathrm{~Hz}, 1 \mathrm{H}, \mathrm{Ar} H\right.$ ortho to $\left.-\mathrm{OC}_{10} \mathrm{H}_{21}\right), 7.22(\mathrm{~d}, J=1.4$ $\mathrm{Hz}, 1 \mathrm{H}, \mathrm{Ar} H$ para to $\left.-\mathrm{OC}_{10} \mathrm{H}_{21}\right), 6.94(\mathrm{~s}, 1 \mathrm{H},-\mathrm{C}(H) \mathrm{OEt}), 4.11\left(\mathrm{t}, J=6.7 \mathrm{~Hz}, 2 \mathrm{H},-\mathrm{OCH}_{2} \mathrm{CH}_{2}-\right), 3.88$ (s, $\left.3 \mathrm{H},-\mathrm{OCH}_{3}\right), 3.74\left(\mathrm{q}, J=7.0 \mathrm{~Hz}, 2 \mathrm{H},-\mathrm{OCH}_{2} \mathrm{CH}_{3}\right), 1.85-1.76\left(\mathrm{~m}, 2 \mathrm{H},-\mathrm{OCH}_{2} \mathrm{CH}_{2}-\right), 1.50-1.39(\mathrm{~m}, 2 \mathrm{H},-$ $\left.\mathrm{O}\left(\mathrm{CH}_{2}\right)_{2} \mathrm{CH}_{2}-\right), 1.39-1.20\left(\mathrm{~m}, 15 \mathrm{H},-\left(\mathrm{CH}_{2}\right)_{3}\left(\mathrm{CH}_{2}\right)_{6}-\right.$ and $\left.-\mathrm{OCH}_{2} \mathrm{CH}_{3}\right), 0.87(\mathrm{t}, J=6.8 \mathrm{~Hz}, 3 \mathrm{H},-$ $\left.\mathrm{CH}_{2} \mathrm{CH}_{2} \mathrm{CH}_{3}\right) .{ }^{13} \mathrm{C}$ NMR (101 MHz, $\left.\mathrm{CDCl}_{3}, \delta, \mathrm{ppm}\right): 166.61(\mathrm{C}=\mathrm{O}), 147.20(\mathrm{ArC}-5), 142.32(\mathrm{ArC}-3)$, 138.27 ( $\mathrm{ArC}-4), 124.34$ ( $\mathrm{ArC}-1), 120.12$ (-OCHO-), 111.18 ( $\mathrm{ArC}-6), 103.36(\mathrm{ArC}-2), 69.81\left(\mathrm{ArOCH}_{2}-\right)$, $59.70\left(-\mathrm{CHOCH}_{2}-\right), 52.30\left(-\mathrm{OCH}_{3}\right),[32.03,29.68,29.49,29.45,29.35,26.01,22.81]\left(-\mathrm{OCH}_{2}\left(\mathrm{CH}_{2}\right)_{8}-\right)$, $14.92\left(-\mathrm{OCH}_{2} \mathrm{CH}_{3}\right), 14.25\left(-\mathrm{CH}_{2} \mathrm{CH}_{2} \mathrm{CH}_{3}\right)$. MALDI-TOF MS m/z of $[\mathrm{M}+\mathrm{Na}]^{+}$calculated for $\mathrm{C}_{21} \mathrm{H}_{32} \mathrm{O}_{6}$ : 403.2; Found: 403.5.

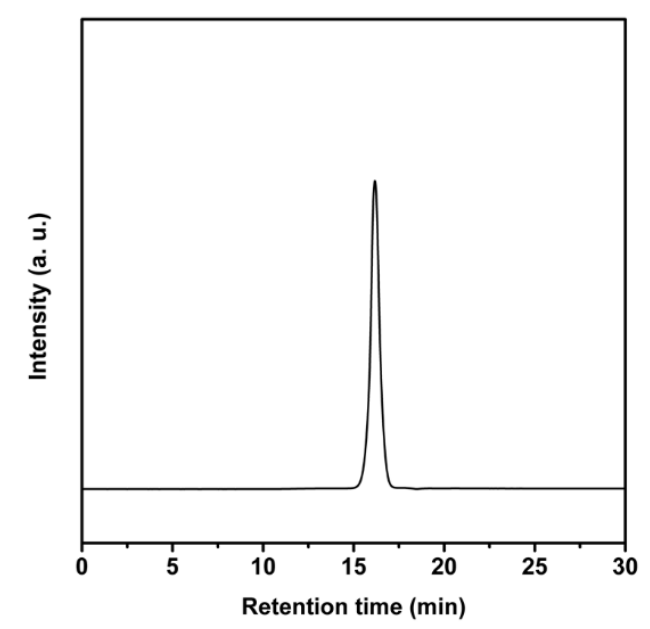

Supporting Figure S20. HPLC trace of 7-10. Decyl group has been successfully linked to the 7-position of 7-10. ${ }^{1} \mathrm{H}$ NMR spectrum is presented on page 44. 
Supporting Figure S20 (continued). ${ }^{1} \mathrm{H}$ NMR of 7-10.

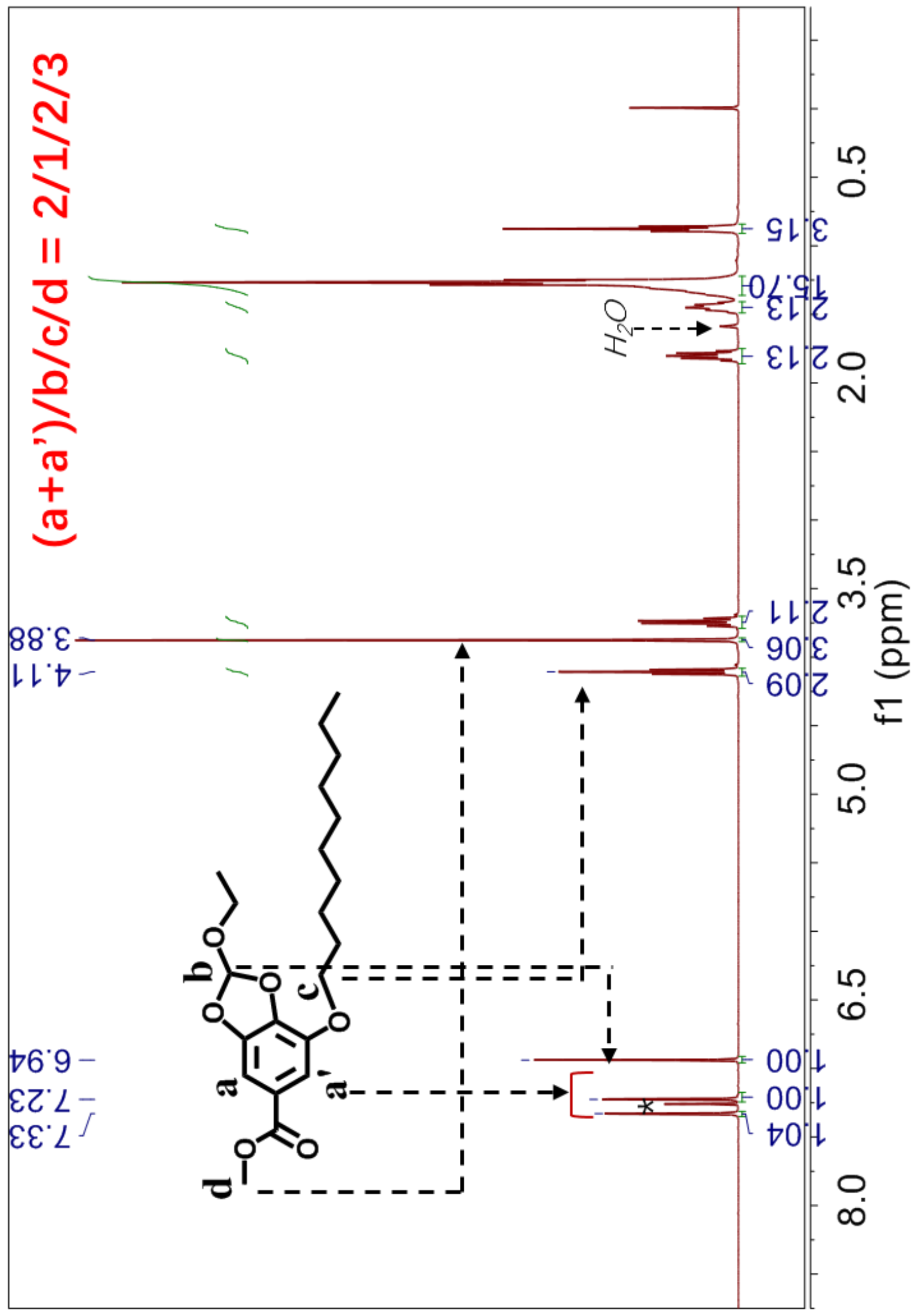


<smiles>CCOc1cc(C(=O)OC)cc(O)c1O</smiles>

Methyl 3,4-dihydroxy-5-(heptyloxy)benzoate (8-7). Compound 8-7 was synthesized by deprotecting the acetal-protected hydroxyl groups of 7-7. ${ }^{7}$ Briefly, $\mathrm{HCl}$ aqueous solution $(2 \mathrm{M}, 15 \mathrm{~mL})$ was added to a methonal solution $(30 \mathrm{~mL})$ of 7-7 (1.56 g, $4.6 \mathrm{mmol})$. The mixture was stirred at $60{ }^{\circ} \mathrm{C}$ for $7 \mathrm{~h}$ and then concentrated in vacuo. After that, it was poured into water $(100 \mathrm{~mL})$ and extracted with $\mathrm{EA}(25 \mathrm{~mL} \times 4)$. The combined EA phase was washed with water $(25 \mathrm{~mL} \times 3)$, brine $(25 \mathrm{~mL})$, dried over anhydrous $\mathrm{MgSO}_{4}$, filtered and evaporated in vacuo. Compound 8-7 was obtained as a white solid. Product: 1.30 g. Yield: 100\%. Purity by HPLC: $99+\%$. mp: $119-121{ }^{\circ} \mathrm{C}$. The synthesis procedures for 8-9, 8-6 and 8-10 listed below are similar to that for 8-7.

${ }^{1} \mathrm{H}$ NMR (400 MHz, $\mathrm{CDCl}_{3}, \delta$, ppm): $7.32\left(\mathrm{~d}, J=1.6 \mathrm{~Hz}, 1 \mathrm{H}\right.$, ArH para to $\left.-\mathrm{OC}_{7} \mathrm{H}_{15}\right), 7.19$ (d, $J=1.6 \mathrm{~Hz}$, 1H, $\mathrm{ArH}$ ortho to $\left.-\mathrm{OC}_{7} \mathrm{H}_{15}\right), 5.82(\mathrm{~s}, 1 \mathrm{H}, \mathrm{ArOH}), 5.37$ (s, $\left.1 \mathrm{H}, \mathrm{ArOH}\right), 4.09$ (t, J=6.6 Hz, 2H, $\left.-\mathrm{OCH}_{2}-\right)$, $3.88\left(\mathrm{~s}, 3 \mathrm{H},-\mathrm{OCH}_{3}\right), 1.87-1.77\left(\mathrm{~m}, 2 \mathrm{H},-\mathrm{OCH}_{2} \mathrm{CH}_{2}-\right), 1.50-1.40\left(\mathrm{~m}, 2 \mathrm{H},-\mathrm{O}\left(\mathrm{CH}_{2}\right)_{2} \mathrm{CH}_{2}-\right), 1.40-1.22(\mathrm{~m}$, $\left.6 \mathrm{H},-\mathrm{O}\left(\mathrm{CH}_{2}\right)_{3}\left(\mathrm{CH}_{2}\right)_{3}-\right), 0.90\left(\mathrm{t}, J=6.8 \mathrm{~Hz}, 3 \mathrm{H},-\mathrm{CH}_{2} \mathrm{CH}_{3}\right) .{ }^{13} \mathrm{C} \mathrm{NMR}\left(101 \mathrm{MHz}, \mathrm{CDCl}_{3}, \delta, \mathrm{ppm}\right): 167.05$ $(C=\mathrm{O}), 145.95$ (ArC-5), 143.50 (ArC-3), 137.02 (ArC-4), 121.95 (ArC-1), [110.90, 105.75] (ArC-2,6), $69.58\left(\mathrm{ArOCH}_{2}-\right), 52.21\left(-\mathrm{OCH}_{3}\right),[31.88,29.29,29.15,26.05,22.74]\left(-\mathrm{OCH}_{2}\left(\mathrm{CH}_{2}\right)_{5}-\right), 14.21(-$ $\left.\mathrm{CH}_{2} \mathrm{CH}_{3}\right)$.

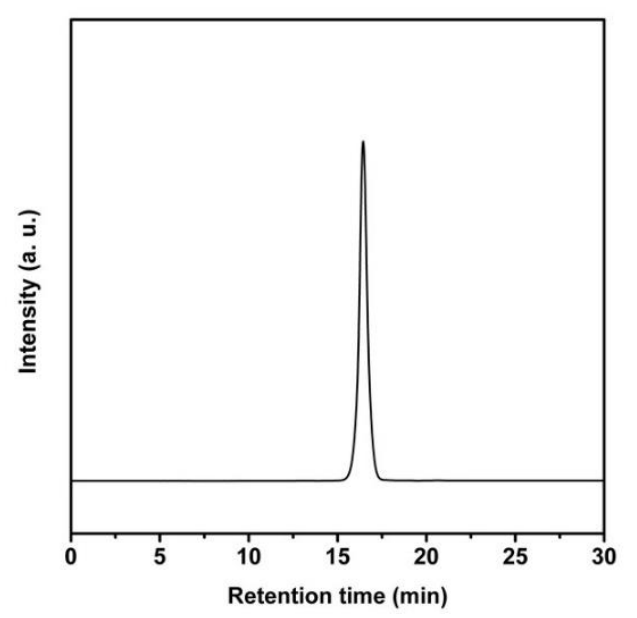

Supporting Figure S21. HPLC trace of 8-7. The hydroxyl groups in the 3- and 4-positions have been successfully deprotected in 8-7. ${ }^{1} \mathrm{H}$ NMR spectrum is presented on page 46. 
Supporting Figure S21 (continued). ${ }^{1} \mathrm{H}$ NMR of 8-7.

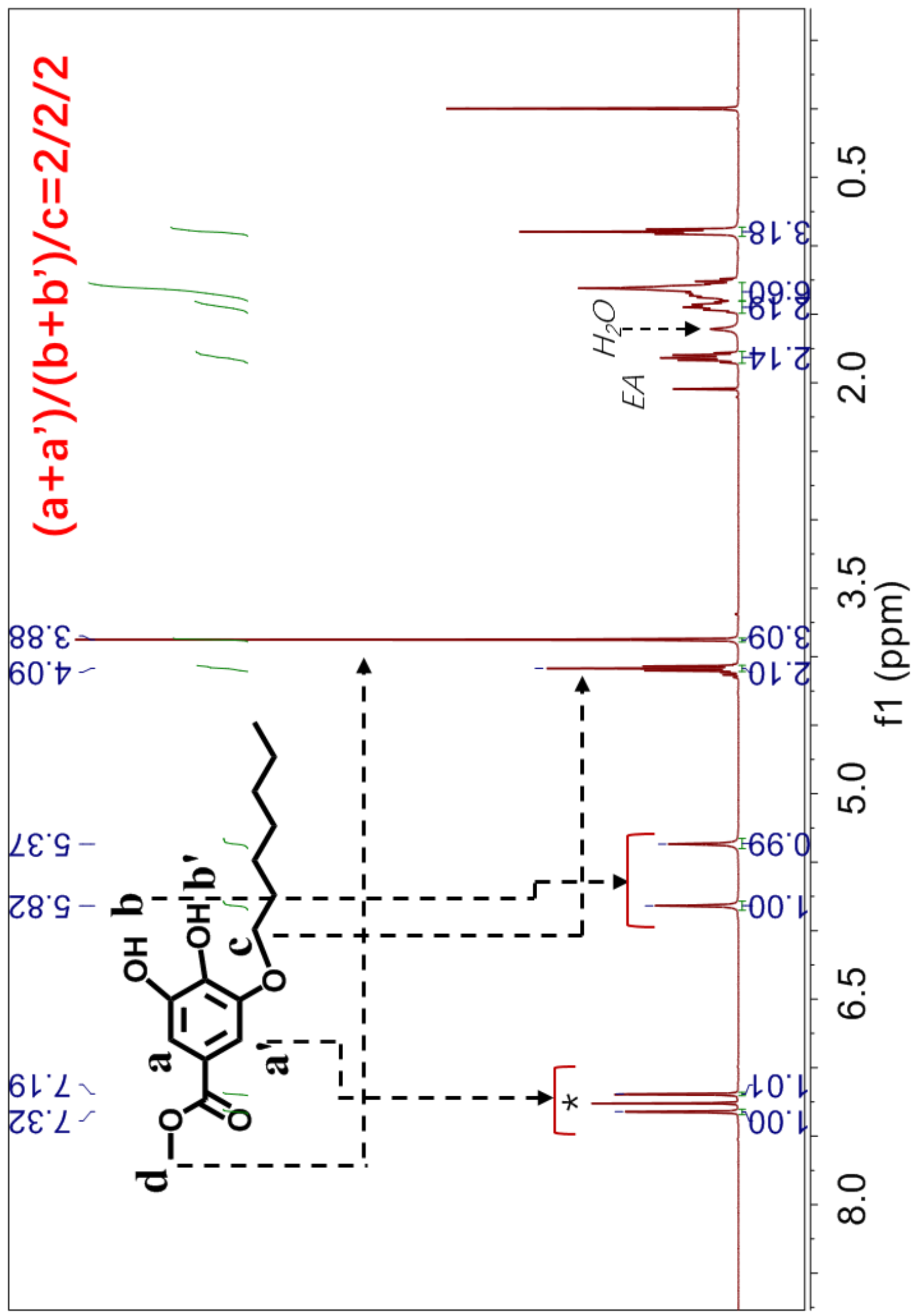




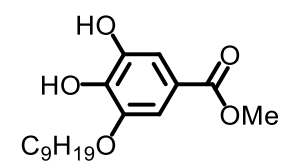

Methyl 3,4-dihydroxy-5-(nonyloxy)benzoate (8-9). Compound 8-9 was obtained as a white solid by deprotecting 7-9 (1.69 g, $4.6 \mathrm{mmol})$ in methanol $(30 \mathrm{~mL})$ using $\mathrm{HCl}$ aqueous solution $(2 \mathrm{M}, 15 \mathrm{~mL})$ at 60 ${ }^{\circ} \mathrm{C}$ for 7 h. Product: 1.57 g. Yield: $100 \%$. Purity by HPLC: $99+\%$. mp: $114-116^{\circ} \mathrm{C}$.

${ }^{1} \mathrm{H} \mathrm{NMR}\left(400 \mathrm{MHz}, \mathrm{CDCl}_{3}, \delta, \mathrm{ppm}\right): 7.32\left(\mathrm{~d}, J=1.5 \mathrm{~Hz}, 1 \mathrm{H}, \mathrm{Ar} H\right.$ para to $\left.-\mathrm{OC}_{9} \mathrm{H}_{19}\right), 7.19$ (d, $J=1.5 \mathrm{~Hz}$, $1 \mathrm{H}, \mathrm{Ar} H$ ortho to $-\mathrm{OC}_{9} \mathrm{H}_{19}$ ), 5.77 (br, s, $\left.1 \mathrm{H}, \mathrm{ArOH}\right), 5.43$ (br, s, $\left.1 \mathrm{H}, \mathrm{ArOH}\right), 4.08$ (t, $J=6.6 \mathrm{~Hz}, 2 \mathrm{H},-$ $\left.\mathrm{OCH}_{2}-\right), 3.88\left(\mathrm{~s}, 3 \mathrm{H},-\mathrm{OCH}_{3}\right), 1.87-1.76\left(\mathrm{~m}, 2 \mathrm{H},-\mathrm{OCH}_{2} \mathrm{CH}_{2}-\right), 1.50-1.40\left(\mathrm{~m}, 2 \mathrm{H},-\mathrm{O}\left(\mathrm{CH}_{2}\right)_{2} \mathrm{CH}_{2}-\right), 1.40-$ $1.21\left(\mathrm{~m}, 10 \mathrm{H},-\mathrm{O}\left(\mathrm{CH}_{2}\right)_{3}\left(\mathrm{CH}_{2}\right)_{5}-\right), 0.89\left(\mathrm{t}, J=6.8 \mathrm{~Hz}, 3 \mathrm{H},-\mathrm{CH}_{2} \mathrm{CH}_{3}\right) .{ }^{13} \mathrm{C} \mathrm{NMR}\left(101 \mathrm{MHz}, \mathrm{CDCl}_{3}, \delta\right.$, ppm): 167.05 ( $C=\mathrm{O}$ ), 145.95 (ArC-5), 143.50 (ArC-3), 137.02 (ArC-4), 121.95 (ArC-1), [110.89, 105.75] $(\mathrm{ArC}-2,6), 69.58\left(\mathrm{ArOCH}_{2}-\right), 52.21\left(-\mathrm{OCH}_{3}\right),[32.00,29.64,29.48,29.38,29.28,26.09,22.80]$ ($\left.\mathrm{OCH}_{2}\left(\mathrm{CH}_{2}\right)_{7}-\right), 14.24\left(-\mathrm{CH}_{2} \mathrm{CH}_{3}\right)$.

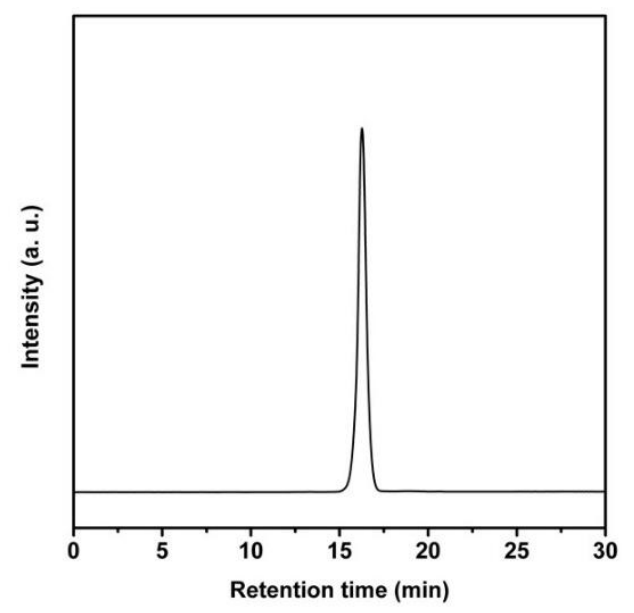

Supporting Figure S22. HPLC trace of 8-9. The hydroxyl groups in the 3- and 4-positions have been successfully deprotected in 8-9. ${ }^{1} \mathrm{H}$ NMR spectrum is presented on page 48 . 
Supporting Figure S22 (continued). ${ }^{1} \mathrm{H}$ NMR of 8-9.

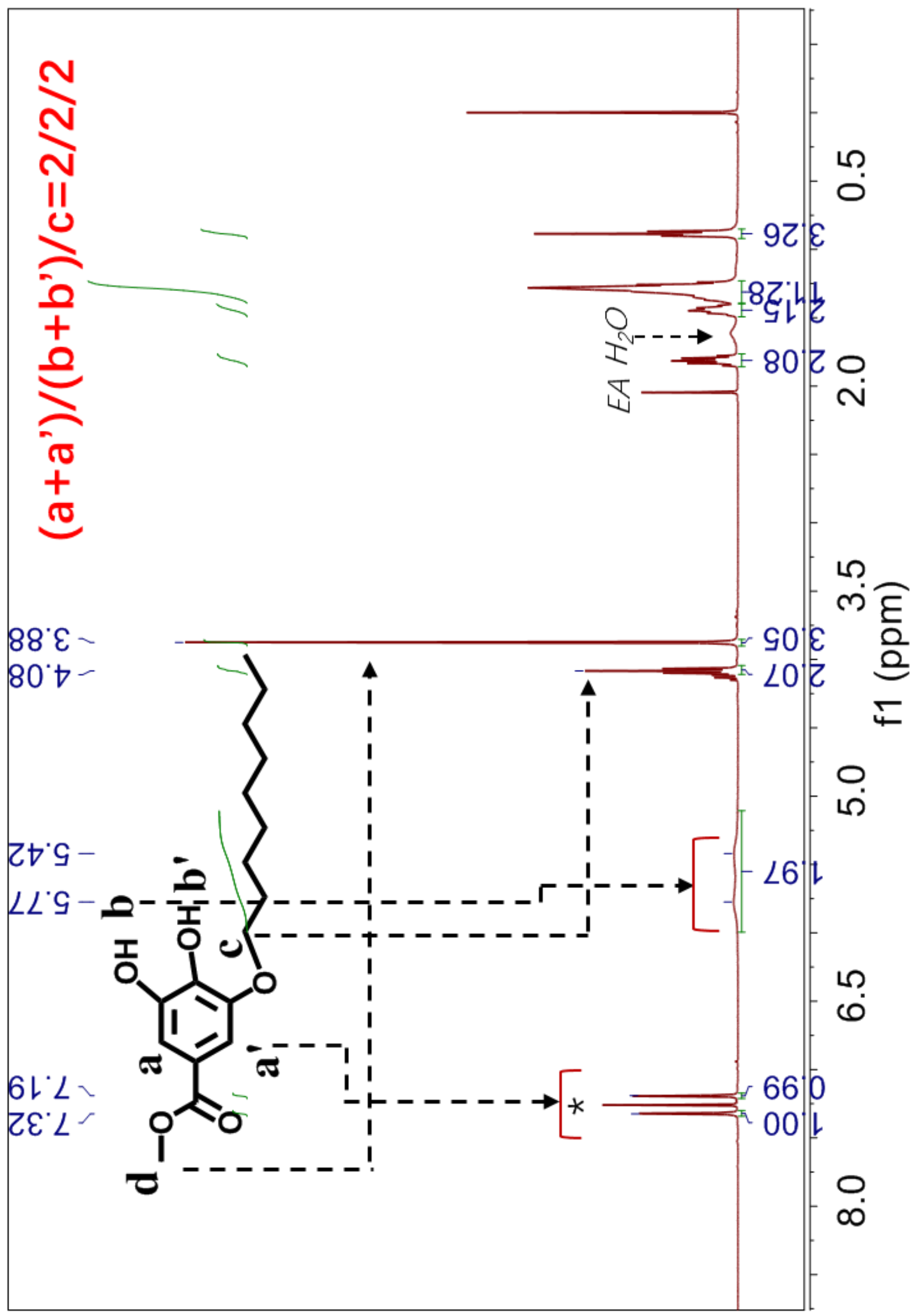




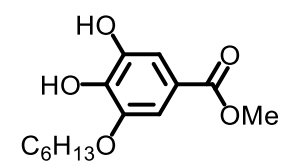

Methyl 3,4-dihydroxy-5-(hexyloxy)benzoate (8-6). Compound 8-6 was obtained as a white solid by deprotecting 7-6 $(0.52 \mathrm{~g}, 1.6 \mathrm{mmol})$ in methanol $(12 \mathrm{~mL})$ using $\mathrm{HCl}$ aqueous solution $(2 \mathrm{M}, 6 \mathrm{~mL})$ at 60 ${ }^{\circ} \mathrm{C}$ for $7 \mathrm{~h}$. Product: 0.42 g. Yield: $99 \%$. Purity by HPLC: $99+\%$. mp: $118-120^{\circ} \mathrm{C}$.

${ }^{1} \mathrm{H}$ NMR (400 MHz, $\left.\mathrm{CDCl}_{3}, \delta, \mathrm{ppm}\right): 7.32$ (d, $J=1.7 \mathrm{~Hz}, 1 \mathrm{H}, \mathrm{ArH}$ para to $\left.-\mathrm{OC}_{6} \mathrm{H}_{13}\right), 7.19$ (d, $J=1.7 \mathrm{~Hz}$, $1 \mathrm{H}, \mathrm{ArH}$ ortho to $\left.-\mathrm{OC}_{6} \mathrm{H}_{13}\right), 5.83(\mathrm{~s}, 1 \mathrm{H}, \mathrm{ArOH}), 5.39(\mathrm{~s}, 1 \mathrm{H}, \mathrm{ArOH}), 4.08\left(\mathrm{t}, J=6.6 \mathrm{~Hz}, 2 \mathrm{H},-\mathrm{OCH}_{2}-\right)$, 3.87 (s, $\left.3 \mathrm{H},-\mathrm{OCH}_{3}\right), 1.87-1.76\left(\mathrm{~m}, 2 \mathrm{H},-\mathrm{OCH}_{2} \mathrm{CH}_{2}-\right), 1.52-1.40\left(\mathrm{~m}, 2 \mathrm{H},-\mathrm{O}\left(\mathrm{CH}_{2}\right)_{2} \mathrm{CH}_{2}-\right), 1.40-1.29$ (m, $\left.4 \mathrm{H},-\mathrm{O}\left(\mathrm{CH}_{2}\right)_{3}\left(\mathrm{CH}_{2}\right)_{2}-\right), 0.91\left(\mathrm{t}, J=7.0 \mathrm{~Hz}, 3 \mathrm{H},-\mathrm{CH}_{2} \mathrm{CH}_{3}\right) .{ }^{13} \mathrm{C} \mathrm{NMR}\left(101 \mathrm{MHz}, \mathrm{CDCl}_{3}, \delta, \mathrm{ppm}\right): 167.05$ $(C=\mathrm{O}), 145.95$ ( $\operatorname{ArC}-5), 143.50(\operatorname{ArC}-3), 137.03$ (ArC-4), 121.96 (ArC-1), [110.90, 105.75] $(\operatorname{ArC}-2,6)$, $69.58\left(\mathrm{ArOCH}_{2}-\right), 52.21\left(-\mathrm{OCH}_{3}\right),[31.65,29.25,25.76,22.71]\left(-\mathrm{OCH}_{2}\left(\mathrm{CH}_{2}\right)_{4}-\right), 14.15\left(-\mathrm{CH}_{2} \mathrm{CH}_{3}\right)$.

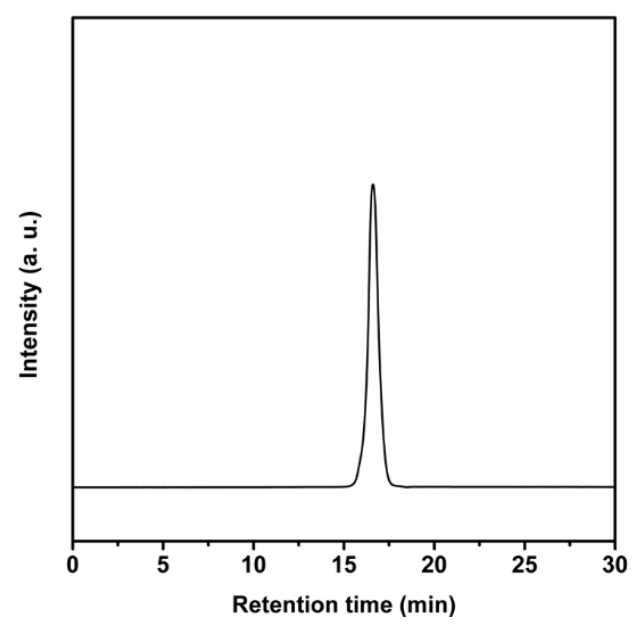

Supporting Figure S23. HPLC trace of 8-6. The hydroxyl groups in the 3- and 4-positions have been successfully deprotected in 8-6. ${ }^{1} \mathrm{H}$ NMR spectrum is presented on page 50 . 
Supporting Figure S23 (continued). ${ }^{1} \mathrm{H}$ NMR of 8-6.

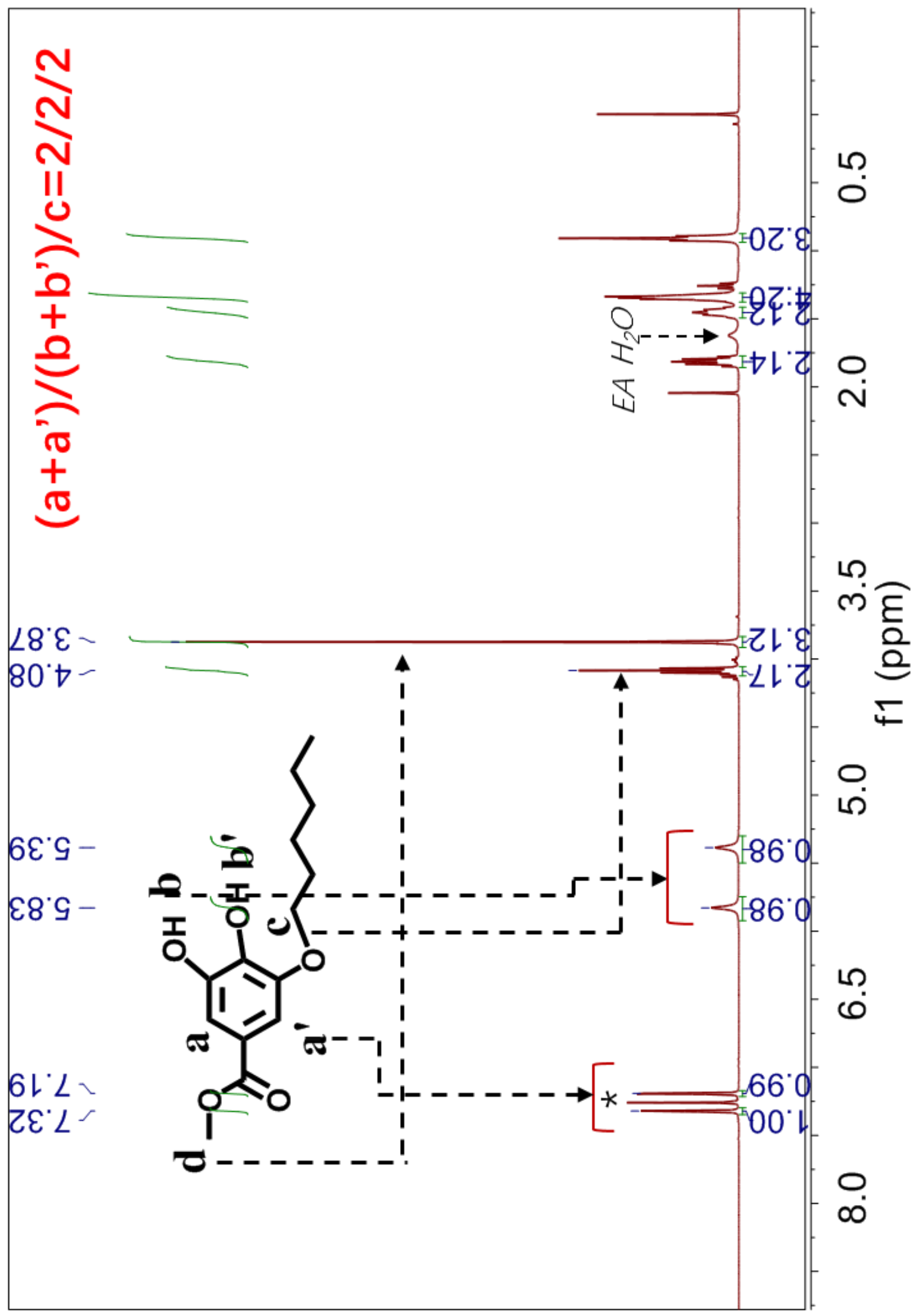




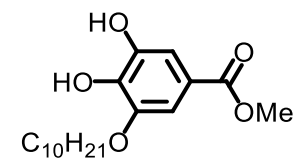

Methyl 3,4-dihydroxy-5-(decyloxy)benzoate (8-10). Compound 8-10 was obtained as a white solid by deprotecting 7-10 $(0.61 \mathrm{~g}, 1.6 \mathrm{mmol})$ in methanol $(12 \mathrm{~mL})$ using $\mathrm{HCl}$ aqueous solution $(2 \mathrm{M}, 6 \mathrm{~mL})$ at 60 ${ }^{\circ} \mathrm{C}$ for $7 \mathrm{~h}$. Product: 0.51 g. Yield: $99 \%$. Purity by HPLC: $99+\%$. mp: $113-114{ }^{\circ} \mathrm{C}$.

${ }^{1} \mathrm{H}$ NMR (400 MHz, $\left.\mathrm{CDCl}_{3}, \delta, \mathrm{ppm}\right): 7.32\left(\mathrm{~d}, J=1.7 \mathrm{~Hz}, 1 \mathrm{H}, \mathrm{Ar} H\right.$ para to $\left.-\mathrm{OC}_{10} \mathrm{H}_{21}\right), 7.19(\mathrm{~d}, J=1.5$ $\mathrm{Hz}, 1 \mathrm{H}, \mathrm{Ar} H$ ortho to $\left.-\mathrm{OC}_{10} \mathrm{H}_{21}\right), 5.82(\mathrm{~s}, 1 \mathrm{H}, \mathrm{ArOH}), 5.37(\mathrm{br}, \mathrm{s}, 1 \mathrm{H}, \mathrm{ArOH}), 4.08(\mathrm{t}, J=6.5 \mathrm{~Hz}, 2 \mathrm{H},-$ $\left.\mathrm{OCH}_{2}-\right), 3.88$ (s, $\left.3 \mathrm{H},-\mathrm{OCH}_{3}\right), 1.86-1.76\left(\mathrm{~m}, 2 \mathrm{H},-\mathrm{OCH}_{2} \mathrm{CH}_{2}-\right), 1.50-1.40\left(\mathrm{~m}, 2 \mathrm{H},-\mathrm{O}\left(\mathrm{CH}_{2}\right)_{2} \mathrm{CH}_{2}-\right), 1.40-$ $1.20\left(\mathrm{~m}, 12 \mathrm{H},-\mathrm{O}\left(\mathrm{CH}_{2}\right)_{3}\left(\mathrm{CH}_{2}\right)_{6}-\right), 0.88\left(\mathrm{t}, J=6.8 \mathrm{~Hz}, 3 \mathrm{H},-\mathrm{CH}_{2} \mathrm{CH}_{3}\right) .{ }^{13} \mathrm{C} \mathrm{NMR}\left(101 \mathrm{MHz}, \mathrm{CDCl}_{3}, \delta\right.$, ppm): 167.05 (C=O), 145.95 (ArC-5), 143.50 (ArC-3), 137.02 (ArC-4), 121.95 (ArC-1), [110.89, 105.75] $(\mathrm{ArC}-2,6), 69.58\left(\mathrm{ArOCH}_{2}-\right), 52.21\left(-\mathrm{OCH}_{3}\right),[32.03,29.68,29.48,29.45,29.28,26.09,22.82](-$ $\left.\mathrm{OCH}_{2}\left(\mathrm{CH}_{2}\right)_{8}-\right), 14.25\left(-\mathrm{CH}_{2} \mathrm{CH}_{3}\right)$.

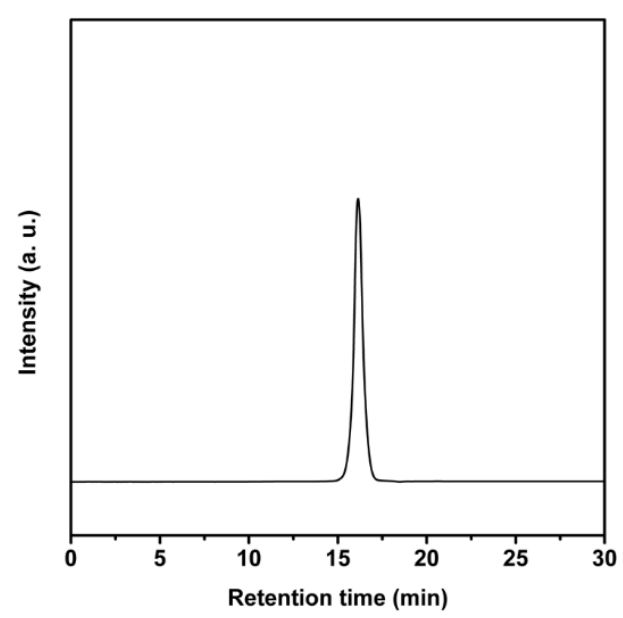

Supporting Figure S24. HPLC trace of 8-10. The hydroxyl groups in the 3- and 4-positions have been successfully deprotected in $\mathbf{8 - 1 0} .{ }^{1} \mathrm{H}$ NMR spectrum is presented on page 52 . 
Supporting Figure S24 (continued). ${ }^{1} \mathrm{H}$ NMR of 8-10.

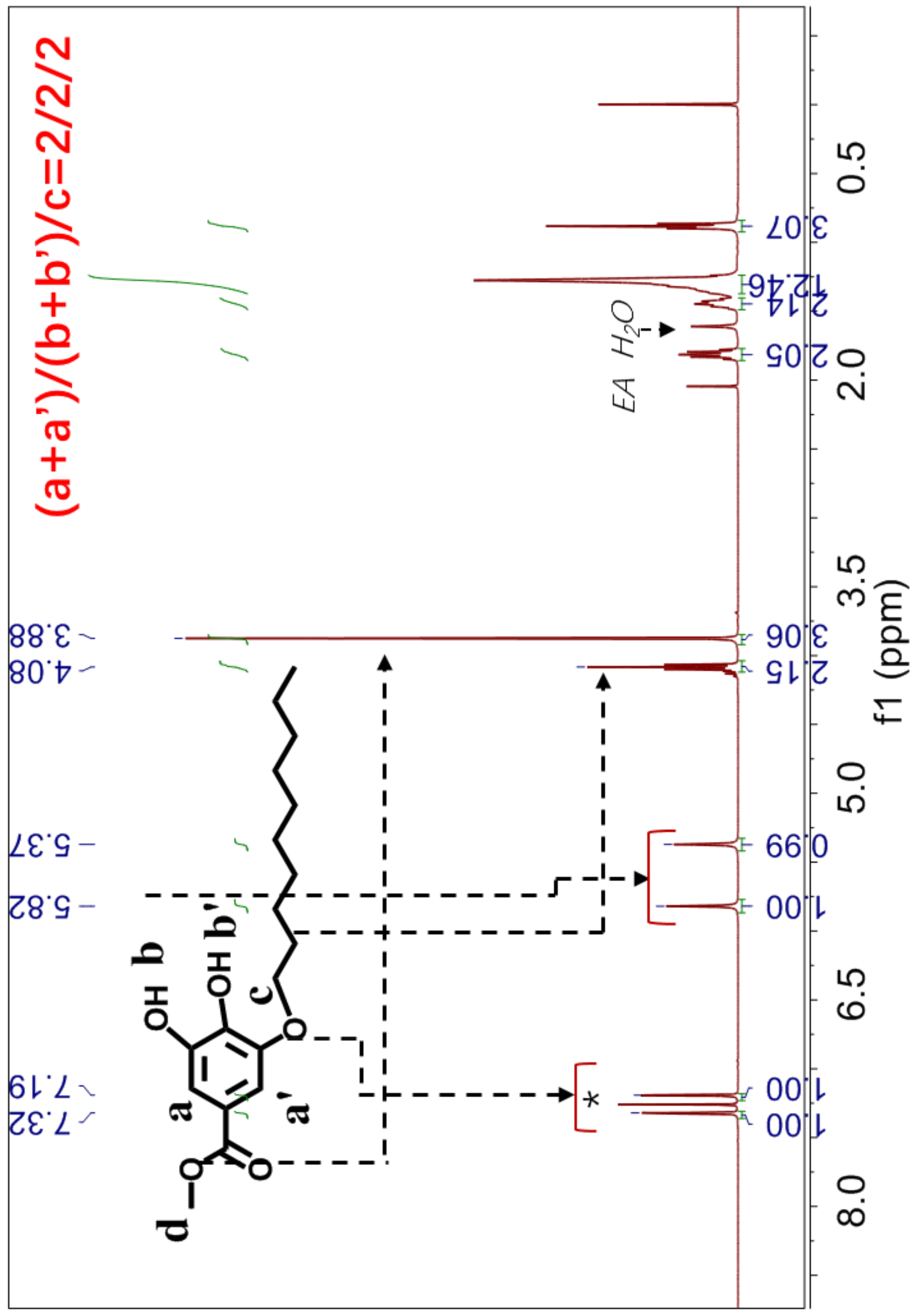




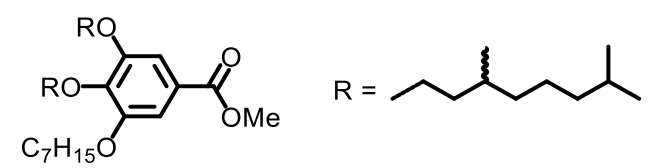

Methyl 3,4-bis(((rac)-3,7-dimethyloctyl)oxy)-5-(heptyloxy)benzoate (5-rr7). Compound 5-rr7 was synthesized according to a procedure reported by our group. ${ }^{7}$ A mixture of 8-7 (1.27 g, $\left.4.5 \mathrm{mmol}\right)$ and $\mathrm{K}_{2} \mathrm{CO}_{3}$ (2.49 g, $18.0 \mathrm{mmol}$ ) was stirred in dry DMF $(20 \mathrm{~mL})$ under $\mathrm{N}_{2}$ protection. (Rac)-1-bromo-3,7dimethyloctane $(1.96 \mathrm{~mL}, 9.5 \mathrm{mmol})$ was added and the reaction mixture was heated at $70{ }^{\circ} \mathrm{C}$ for $4 \mathrm{~h}$. Then the mixture was cooled, poured into cold water $(100 \mathrm{~mL})$ and extracted with EA $(25 \mathrm{~mL} \times 4)$. The combined EA phase was washed with water $(25 \mathrm{~mL} \times 3)$, dried over anhydrous $\mathrm{MgSO}_{4}$, filtered and evaporated in vacuo. Column chromatography $(\mathrm{EA} /$ Hexanes $=1 / 40)$ gave 5-rr7 as a colorless oil. Product: 2.31 g. Yield: 91\%. Purity by HPLC: 99+\%. The synthesis procedures for 5-rr9, 5-rr6, 5-rr10, 5-77r, 599r, 5-66r and 5-1010r listed below are similar to that for 5-rr7.

${ }^{1} \mathrm{H}$ NMR (400 MHz, $\left.\mathrm{CDCl}_{3}, \delta, \mathrm{ppm}\right): 7.26$ (s, 2H, ArH), 4.12-3.97 (m, 6H, -OCH - ), 3.89 (s, 3H, -

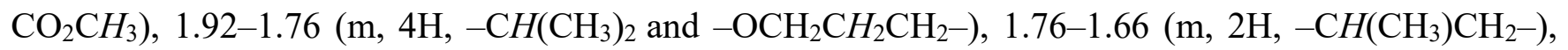
1.66-1.42 (m, 6H, $-\mathrm{OCH}_{2} \mathrm{CH}_{2} \mathrm{CH}\left(\mathrm{CH}_{3}\right)-$ and $\left.-\mathrm{O}\left(\mathrm{CH}_{2}\right)_{2} \mathrm{CH}_{2}-\right), 1.41-1.08\left(\mathrm{~m}, 18 \mathrm{H},-\mathrm{CH}\left(\mathrm{CH}_{3}\right)\left(\mathrm{CH}_{2}\right)_{3}-\right.$ and $\left.-\mathrm{O}\left(\mathrm{CH}_{2}\right)_{3}\left(\mathrm{CH}_{2}\right)_{3}-\right), 0.95\left(\mathrm{~d}, J=6.6 \mathrm{~Hz}, 3 \mathrm{H},-\mathrm{CH}_{2} \mathrm{CH}\left(\mathrm{CH}_{3}\right)_{-}\right), 0.92(\mathrm{~d}, J=6.6 \mathrm{~Hz}, 3 \mathrm{H},-$ $\left.\mathrm{CH}_{2} \mathrm{CH}\left(\mathrm{CH}_{3}\right)^{-}\right)$, $0.90-0.84\left(\mathrm{~m}, 15 \mathrm{H},-\mathrm{CH}\left(\mathrm{CH}_{3}\right)_{2}\right.$ and $\left.-\mathrm{CH}_{2} \mathrm{CH}_{3}\right) .{ }^{13} \mathrm{C} \mathrm{NMR}\left(101 \mathrm{MHz}, \mathrm{CDCl}_{3}, \delta, \mathrm{ppm}\right)$ : $167.09(C=\mathrm{O}), 152.98$ (ArC-3,5), 142.48 (ArC-4), 124.81 (ArC-1), [108.10, 108.06] (ArC-2,6), 71.84 ( $\mathrm{ArOCH} \mathrm{H}_{2}-5$ position), 69.28 ( $\mathrm{ArOCH}_{2}-4$ position), 67.60 ( $\mathrm{ArOCH}_{2}-3$ position), $52.23\left(-\mathrm{OCH}_{3}\right)$, [39.49, $39.40, \quad 37.61, \quad 37.47, \quad 36.44, \quad 31.96,29.97,29.77, \quad 29.45,29.19,28.13,26.18,24.85]$ ($\mathrm{OCH}_{2} \mathrm{CH}_{2} \mathrm{CH}\left(\mathrm{CH}_{3}\right)\left(\mathrm{CH}_{2}\right)_{3} \mathrm{CH}-$ and $\left.-\mathrm{OCH}_{2}\left(\mathrm{CH}_{2}\right)_{4}-\right)$, [22.84, 22.74, 19.76, 19.72, 19.69, 14.22] ($\mathrm{CH}\left(\mathrm{CH}_{3}\right)_{2},-\mathrm{CH}\left(\mathrm{CH}_{3}\right) \mathrm{CH}_{2}-$ and $\left.-\mathrm{CH}_{2} \mathrm{CH}_{3}\right)$. MALDI-TOF MS $m / z$ of $[\mathrm{M}+\mathrm{Na}]^{+}$calculated for $\mathrm{C}_{35} \mathrm{H}_{62} \mathrm{O}_{5}$ : 585.4; Found: 584.9.
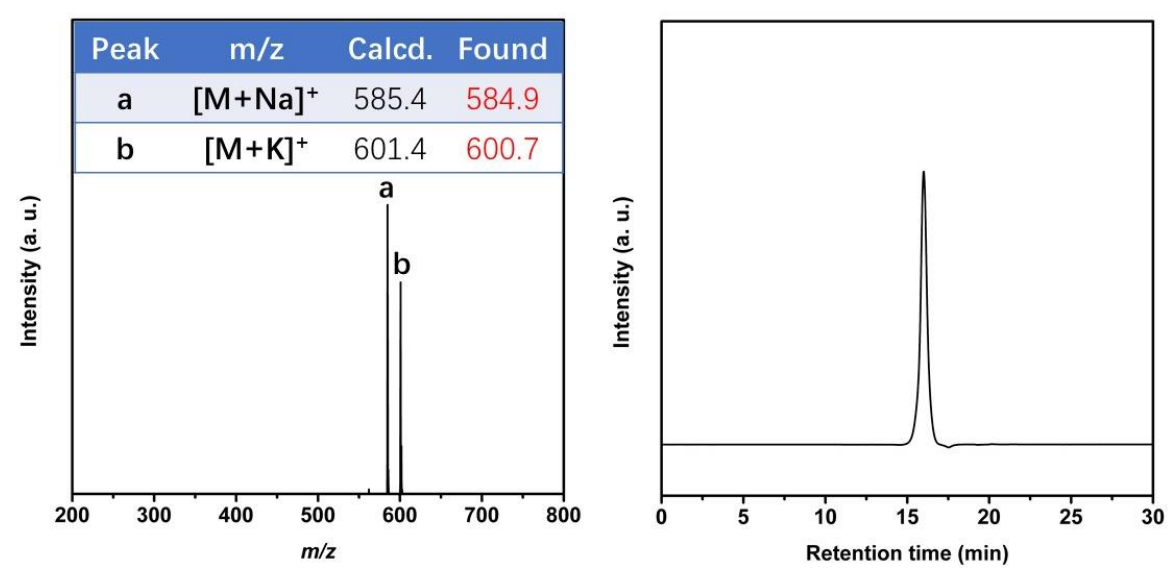

Supporting Figure S25. MALDI-TOF MS spectra (left) and HPLC trace (right) of 5-rr7. Racemic dimethyloctyl groups have been successfully linked to the 3- and 4-positions in 5-rr7. ${ }^{1} \mathrm{H}$ NMR spectrum is presented on page 54 . 
Supporting Figure S25 (continued). ${ }^{1} \mathrm{H}$ NMR of 5-rr7.

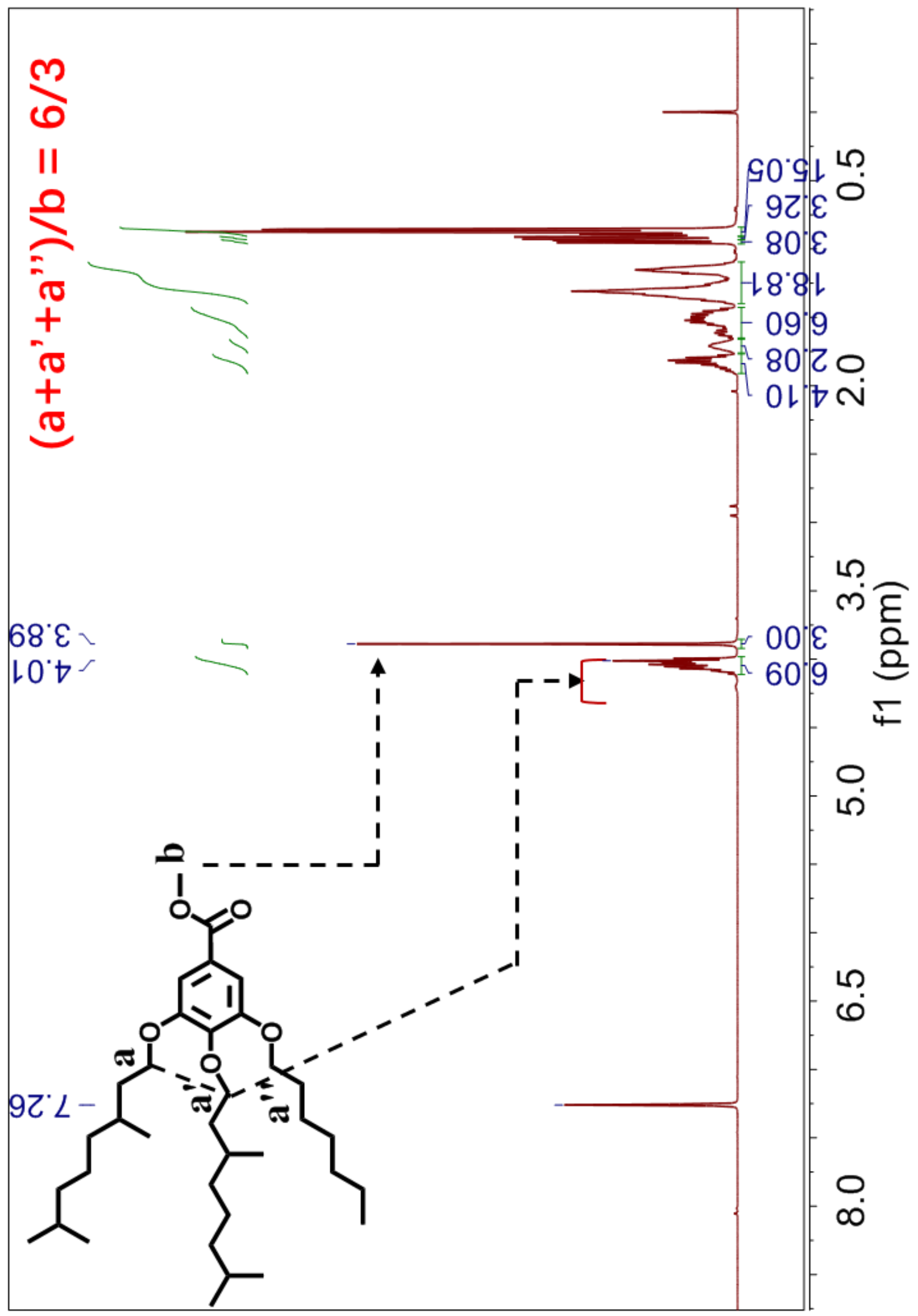


<smiles>[R]CCCC(C)CCCC(C)C</smiles>

Methyl 3,4-bis((3,7-(rac)-dimethyloctyl)oxy)-5-(nonyloxy)benzoate (5-rr9). Starting from 8-9 (1.40 g, $4.5 \mathrm{mmol})$ and $(\mathrm{rac})-1$-bromo-3,7-dimethyloctane $(1.96 \mathrm{~mL}, 9.5 \mathrm{mmol})$ in the presence of $\mathrm{K}_{2} \mathrm{CO}_{3}(2.49 \mathrm{~g}$, $18.0 \mathrm{mmol})$ in dry DMF $(20 \mathrm{~mL})$ at $70{ }^{\circ} \mathrm{C}$ for $4 \mathrm{~h}, \mathbf{5 - r r 9}$ was obtained as a colorless oil. Product: $2.46 \mathrm{~g}$. Yield: 93\%. Purity by HPLC: $99+\%$.

${ }^{1} \mathrm{H}$ NMR (400 MHz, $\left.\mathrm{CDCl}_{3}, \delta, \mathrm{ppm}\right): 7.26$ (s, 2H, $\left.\mathrm{ArH}\right), 4.12-3.97$ (m, 6H, $-\mathrm{OCH}_{2}-$ ), 3.89 (s, 3H, $\left.\mathrm{CO}_{2} \mathrm{CH}_{3}\right), 1.92-1.76\left(\mathrm{~m}, 4 \mathrm{H},-\mathrm{CH}\left(\mathrm{CH}_{3}\right)_{2}\right.$ and $\left.\left.-\mathrm{OCH}_{2} \mathrm{CH}_{2} \mathrm{CH}_{2}-\right), 1.76-1.66\left(\mathrm{~m}, 2 \mathrm{H},-\mathrm{CH}_{(} \mathrm{CH}_{3}\right) \mathrm{CH}_{2}-\right)$, 1.66-1.42 (m, 6H, $-\mathrm{OCH}_{2} \mathrm{CH}_{2} \mathrm{CH}\left(\mathrm{CH}_{3}\right)-$ and $\left.-\mathrm{O}\left(\mathrm{CH}_{2}\right)_{2} \mathrm{CH}_{2}-\right), 1.41-1.08\left(\mathrm{~m}, 22 \mathrm{H},-\mathrm{CH}\left(\mathrm{CH}_{3}\right)\left(\mathrm{CH}_{2}\right)_{3}-\right.$ and $\left.-\mathrm{O}\left(\mathrm{CH}_{2}\right)_{3}\left(\mathrm{CH}_{2}\right)_{5}-\right), 0.95\left(\mathrm{~d}, J=6.3 \mathrm{~Hz}, 3 \mathrm{H},-\mathrm{CH}_{2} \mathrm{CH}\left(\mathrm{CH}_{3}\right)_{-}\right), 0.92(\mathrm{~d}, J=6.6 \mathrm{~Hz}, 3 \mathrm{H},-$ $\left.\mathrm{CH}_{2} \mathrm{CH}\left(\mathrm{CH}_{3}\right)^{-}\right), 0.91-0.84\left(\mathrm{~m}, 15 \mathrm{H},-\mathrm{CH}\left(\mathrm{CH}_{3}\right)_{2}\right.$ and $\left.-\mathrm{CH}_{2} \mathrm{CH}_{3}\right) .{ }^{13} \mathrm{C} \mathrm{NMR}\left(101 \mathrm{MHz}, \mathrm{CDCl}_{3}, \delta, \mathrm{ppm}\right)$ : $167.09(C=\mathrm{O}), 152.98$ (ArC-3,5), 142.48 (ArC-4), 124.81 (ArC-1), [108.10, 108.06] (ArC-2,6), 71.84 ( $\mathrm{ArOCH} \mathrm{H}_{2}-5$ position), 69.28 ( $\mathrm{ArOCH}_{2}-4$ position), 67.59 ( $\mathrm{ArOCH}_{2}-3$ position), $52.23\left(-\mathrm{OCH}_{3}\right),[39.49$, $39.40,37.62,37.47,36.44,32.03,29.96,29.77,29.72,29.54,29.45,29.43,28.13,26.22,24.86]$ ($\mathrm{OCH}_{2} \mathrm{CH}_{2} \mathrm{CH}\left(\mathrm{CH}_{3}\right)\left(\mathrm{CH}_{2}\right)_{3} \mathrm{CH}-$ and $\left.-\mathrm{OCH}_{2}\left(\mathrm{CH}_{2}\right)_{6}-\right)$, [22.84, 22.81, 22.73, 19.76, 19.72, 19.69, 14.24] ($\mathrm{CH}\left(\mathrm{CH}_{3}\right)_{2},-\mathrm{CH}\left(\mathrm{CH}_{3}\right) \mathrm{CH}_{2}-$ and $\left.-\mathrm{CH}_{2} \mathrm{CH}_{3}\right)$. MALDI-TOF MS $m / z$ of $[\mathrm{M}+\mathrm{Na}]^{+}$calculated for $\mathrm{C}_{37} \mathrm{H}_{66} \mathrm{O}_{5}$ : 613.5; Found: 613.1.
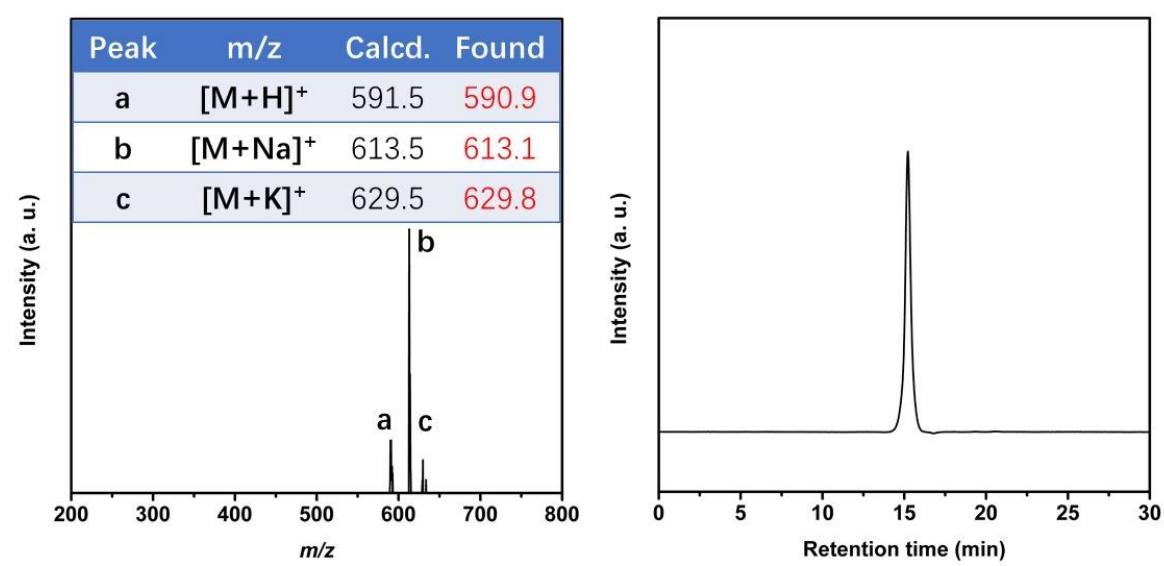

Supporting Figure S26. MALDI-TOF MS spectra (left) and HPLC trace (right) of 5-rr9. Racemic dimethyloctyl groups have been successfully linked to the 3- and 4-positions in 5-rr9. ${ }^{1} \mathrm{H}$ NMR spectrum is presented on page 56. 
Supporting Figure S26 (continued). ${ }^{1} \mathrm{H}$ NMR of 5-rr9.

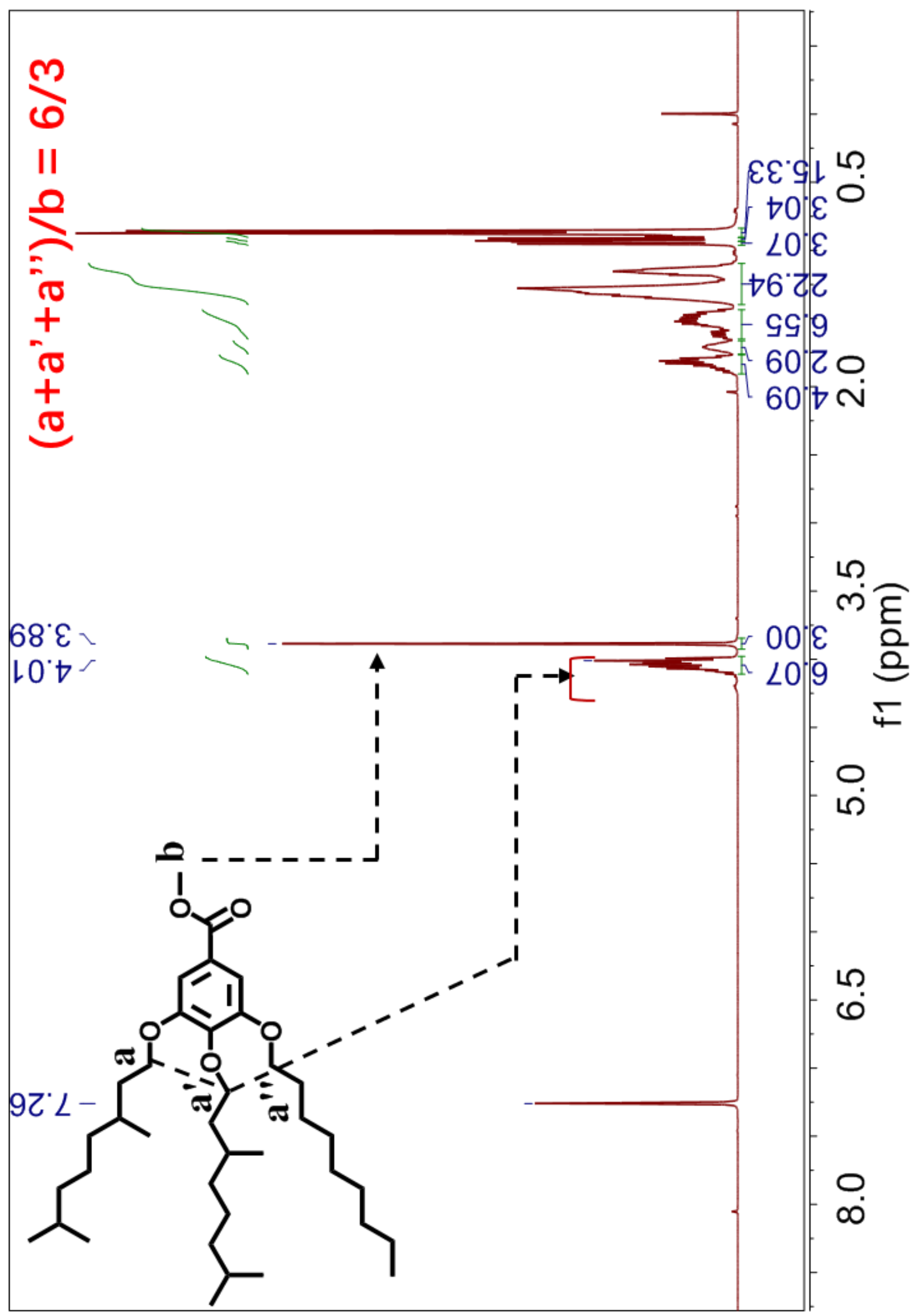


<smiles>[R]CCCC(C)CCCC(C)C</smiles>

Methyl 3,4-bis((3,7-(rac)-dimethyloctyl)oxy)-5-(hexyloxy)benzoate (5-rr6). Starting from 8-6 (0.27 g, $1.0 \mathrm{mmol})$ and $(\mathrm{rac})-1$-bromo-3,7-dimethyloctane $(0.50 \mathrm{~mL}, 2.4 \mathrm{mmol})$ in the presence of $\mathrm{K}_{2} \mathrm{CO}_{3}(0.83 \mathrm{~g}$, $6.0 \mathrm{mmol})$ in dry DMF $(8 \mathrm{~mL})$ at $70{ }^{\circ} \mathrm{C}$ for $4 \mathrm{~h}$, 5-rr6 was obtained as a colorless oil. Product: $0.50 \mathrm{~g}$. Yield: 92\%. Purity by HPLC: $99+\%$.

${ }^{1} \mathrm{H}$ NMR (400 MHz, $\left.\mathrm{CDCl}_{3}, \delta, \mathrm{ppm}\right): 7.26$ (s, 2H, $\left.\mathrm{ArH}\right), 4.11-3.97$ (m, 6H, $\left.-\mathrm{OCH}_{2}-\right), 3.89$ (s, 3H, -

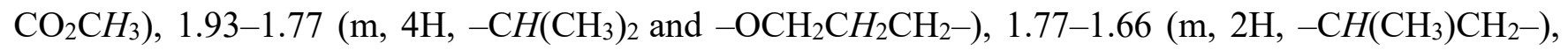
$1.66-1.42\left(\mathrm{~m}, 6 \mathrm{H},-\mathrm{OCH}_{2} \mathrm{CH}_{2} \mathrm{CH}\left(\mathrm{CH}_{3}\right)-\right.$ and $\left.-\mathrm{O}\left(\mathrm{CH}_{2}\right)_{2} \mathrm{CH}_{2}-\right), 1.40-1.09\left(\mathrm{~m}, 16 \mathrm{H},-\mathrm{CH}\left(\mathrm{CH}_{3}\right)\left(\mathrm{CH}_{2}\right)_{3}-\right.$ and $\left.-\mathrm{O}\left(\mathrm{CH}_{2}\right)_{3}\left(\mathrm{CH}_{2}\right)_{2}-\right), 0.97-0.84\left(\mathrm{~m}, 21 \mathrm{H},-\mathrm{CH}_{2} \mathrm{CH}\left(\mathrm{CH}_{3}\right)_{-},-\mathrm{CH}\left(\mathrm{CH}_{3}\right)_{2}\right.$ and $\left.-\mathrm{CH}_{2} \mathrm{CH}_{3}\right) .{ }^{13} \mathrm{C} \mathrm{NMR}(101$ $\left.\mathrm{MHz}, \mathrm{CDCl}_{3}, \delta, \mathrm{ppm}\right): 167.08(C=\mathrm{O}), 152.97$ (ArC-3,5), 142.46 (ArC-4), $124.80(\mathrm{ArC}-1),[108.07,108.03]$ (ArC-2,6), $71.82\left(\mathrm{ArOCH}_{2}-5\right.$ position), $69.26\left(\mathrm{ArOCH}_{2}-4\right.$ position), 67.57 ( $\mathrm{ArOCH}_{2}-3$ position), 52.22 $\left(-\mathrm{OCH}_{3}\right),[39.48,39.39,37.60,37.46,36.43,31.70,29.95,29.76,29.39,28.12,25.89,24.85](-$ $\mathrm{OCH}_{2} \mathrm{CH}_{2} \mathrm{CH}\left(\mathrm{CH}_{3}\right)\left(\mathrm{CH}_{2}\right)_{3} \mathrm{CH}-$ and $\left.-\mathrm{OCH}_{2}\left(\mathrm{CH}_{2}\right)_{3}-\right)$, [22.83, 22.75, 22.73, 19.75, 19.71, 19.68, 14.16] ($\mathrm{CH}\left(\mathrm{CH}_{3}\right)_{2},-\mathrm{CH}\left(\mathrm{CH}_{3}\right) \mathrm{CH}_{2}-$ and $\left.-\mathrm{CH}_{2} \mathrm{CH}_{3}\right)$. MALDI-TOF MS $m / z$ of $[\mathrm{M}+\mathrm{Na}]^{+}$calculated for $\mathrm{C}_{34} \mathrm{H}_{60} \mathrm{O}_{5}$ : 571.4; Found: 571.4.
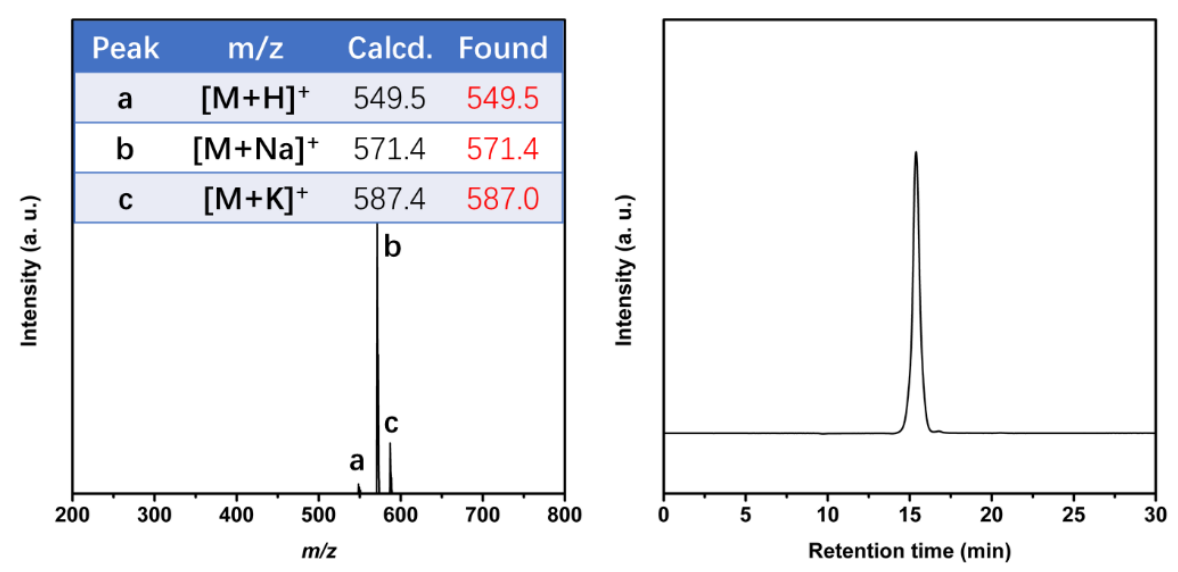

Supporting Figure S27. MALDI-TOF MS spectra (left) and HPLC trace (right) of 5-rr6. Racemic dimethyloctyl groups have been successfully linked to the 3- and 4-positions in 5-rr6. ${ }^{1} \mathrm{H}$ NMR spectrum is presented on page 58 . 
Supporting Figure S27 (continued). ${ }^{1} \mathrm{H}$ NMR of 5-rr6.

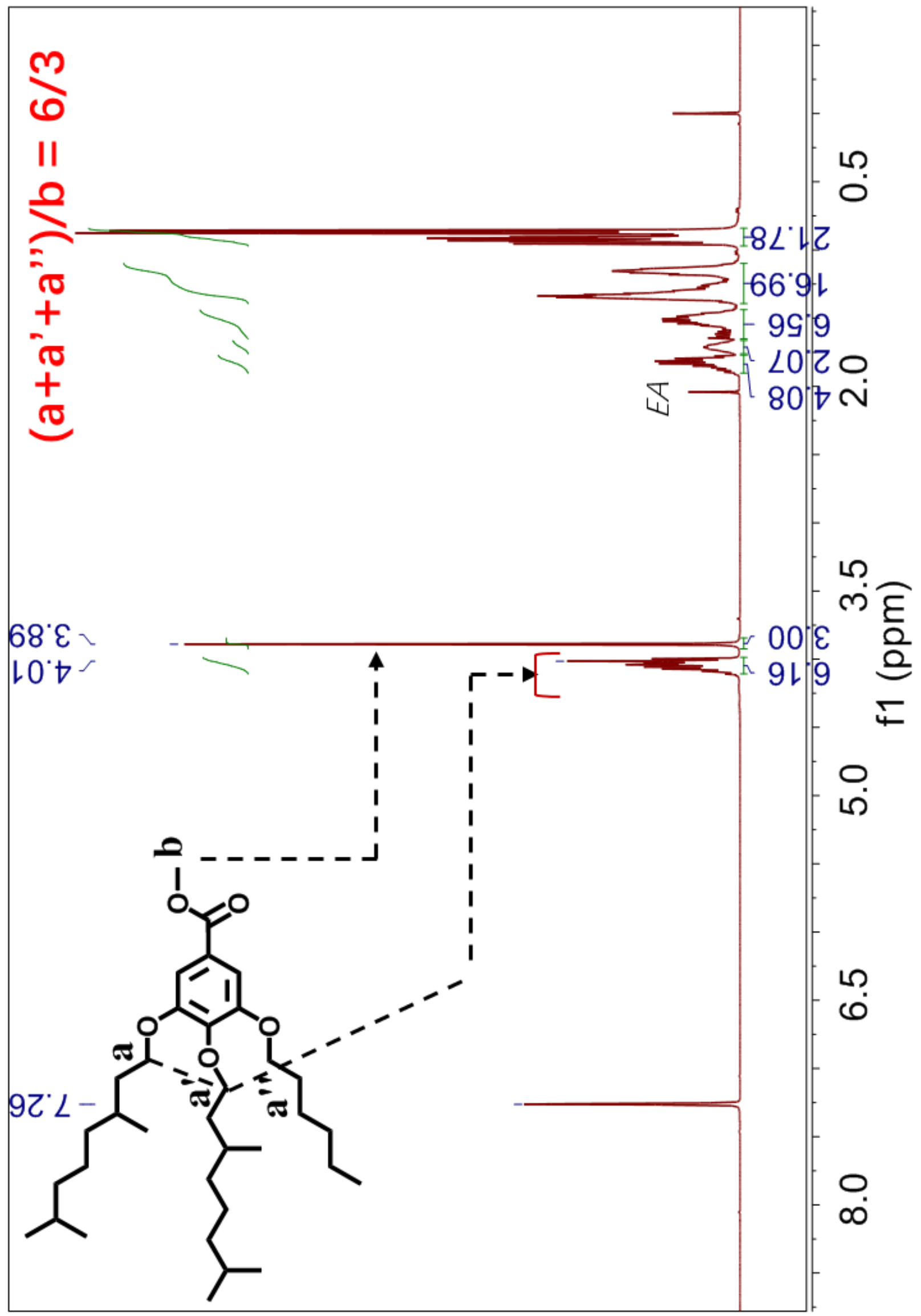


<smiles>[R]CCCC(C)CCCC(C)C</smiles>

Methyl 3-(decyloxy)-4,5-bis((3,7-(rac)-dimethyloctyl)oxy)benzoate (5-rr10). Starting from 8-10 (0.32 $\mathrm{g}, 1.0 \mathrm{mmol})$ and $(\mathrm{rac})-1$-bromo-3,7-dimethyloctane $(0.50 \mathrm{~mL}, 2.4 \mathrm{mmol})$ in the presence of $\mathrm{K}_{2} \mathrm{CO}_{3}(0.83$ g, $6.0 \mathrm{mmol})$ in dry DMF $(8 \mathrm{~mL})$ at $70{ }^{\circ} \mathrm{C}$ for $4 \mathrm{~h}, \mathbf{5 - r r 1 0}$ was obtained as a colorless oil. Product: $0.55 \mathrm{~g}$. Yield: 91\%. Purity by HPLC: 99+\%.

${ }^{1} \mathrm{H}$ NMR (400 MHz, $\left.\mathrm{CDCl}_{3}, \delta, \mathrm{ppm}\right): 7.26$ (s, 2H, ArH), 4.11-3.98 (m, 6H, -OCH2-), 3.89 (s, 3H, -

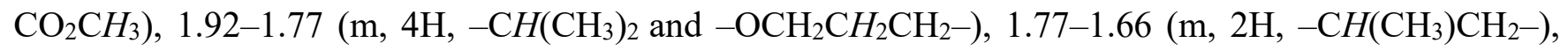
1.65-1.42 (m, 6H, $-\mathrm{OCH}_{2} \mathrm{CH}_{2} \mathrm{CH}\left(\mathrm{CH}_{3}\right)-$ and $\left.-\mathrm{O}\left(\mathrm{CH}_{2}\right)_{2} \mathrm{CH}_{2}-\right), 1.40-1.09\left(\mathrm{~m}, 24 \mathrm{H},-\mathrm{CH}\left(\mathrm{CH}_{3}\right)\left(\mathrm{CH}_{2}\right)_{3}-\right.$ and $\left.-\mathrm{O}\left(\mathrm{CH}_{2}\right)_{3}\left(\mathrm{CH}_{2}\right)_{6}-\right), 0.97-0.83\left(\mathrm{~m}, 21 \mathrm{H},-\mathrm{CH}_{2} \mathrm{CH}\left(\mathrm{CH}_{3}\right)_{-},-\mathrm{CH}\left(\mathrm{CH}_{3}\right)_{2}\right.$ and $\left.-\mathrm{CH}_{2} \mathrm{CH}_{3}\right) .{ }^{13} \mathrm{C}$ NMR $(101$ $\left.\mathrm{MHz}, \mathrm{CDCl}_{3}, \delta, \mathrm{ppm}\right): 167.07(C=\mathrm{O}), 152.97$ (ArC-3,5), 142.46 (ArC-4), 124.80 (ArC-1), [108.07, 108.03] (ArC-2,6), 71.82 ( $\mathrm{ArOCH}_{2}-3$ position), 69.27 ( $\mathrm{ArOCH}_{2}-4$ position), 67.57 ( $\mathrm{ArOCH}_{2}-5$ position), $52.22\left(-\mathrm{OCH}_{3}\right),[39.49,39.39,37.61,37.47,36.43,32.04,29.95,29.76,29.72,29.53,29.48,29.44,28.12$, 26.22, 24.85] $\left(-\mathrm{OCH}_{2} \mathrm{CH}_{2} \mathrm{CH}\left(\mathrm{CH}_{3}\right)\left(\mathrm{CH}_{2}\right)_{3} \mathrm{CH}-\right.$ and $\left.-\mathrm{OCH}_{2}\left(\mathrm{CH}_{2}\right)_{7}-\right)$, [22.83, 22.82, 22.74, 19.75, 19.71, $19.68,14.24]\left(-\mathrm{CH}\left(\mathrm{CH}_{3}\right)_{2},-\mathrm{CH}_{(} \mathrm{CH}_{3}\right) \mathrm{CH}_{2}-$ and $\left.-\mathrm{CH}_{2} \mathrm{CH}_{3}\right)$. MALDI-TOF $\mathrm{MS} \mathrm{m} / z$ of $[\mathrm{M}+\mathrm{Na}]^{+}$ calculated for $\mathrm{C}_{38} \mathrm{H}_{68} \mathrm{O}_{5}$ : 627.5; Found: 628.2 .
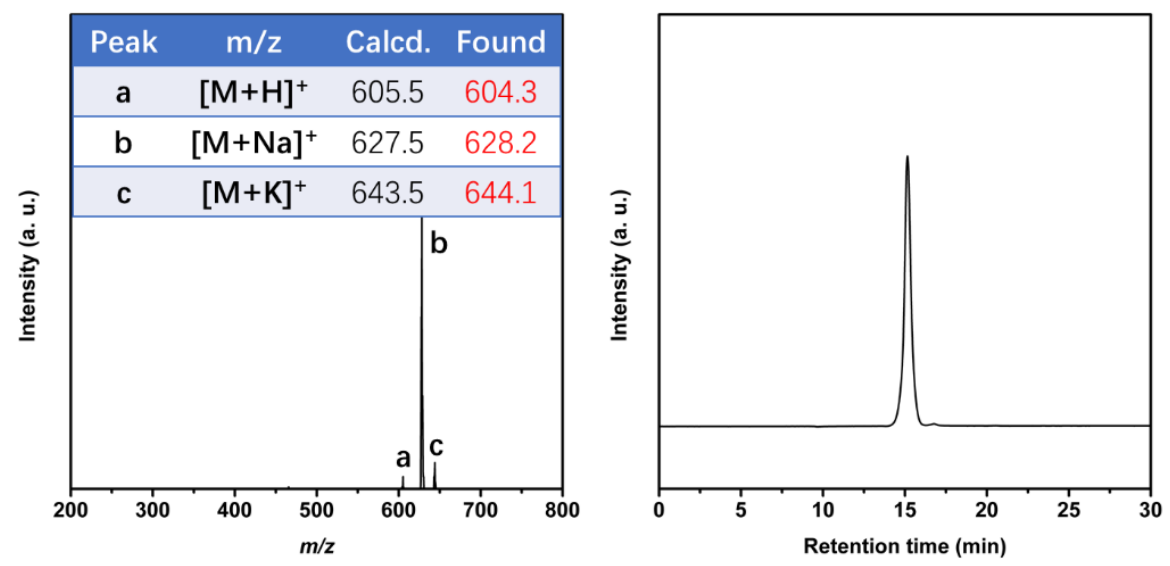

Supporting Figure S28. MALDI-TOF MS spectra (left) and HPLC trace (right) of 5-rr10. Racemic dimethyloctyl groups have been successfully linked to the 3- and 4-positions in 5-rr10. ${ }^{1} \mathrm{H}$ NMR spectrum is presented on page 60 . 
Supporting Figure S28 (continued). ${ }^{1} \mathrm{H}$ NMR of 5-rr10.

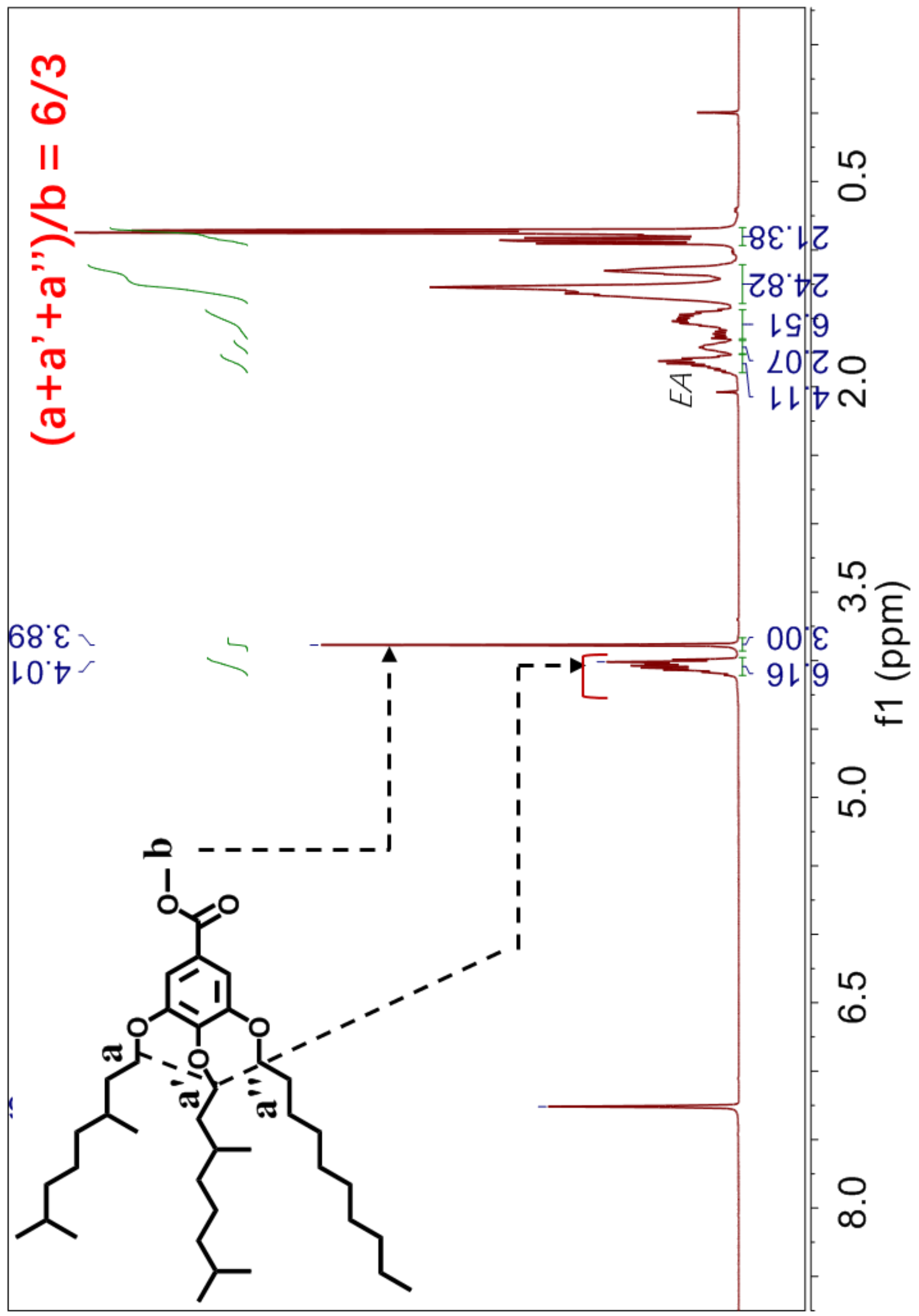


<smiles>[R][CH]CCC(C)CCCC(C)C</smiles>

Methyl 3-((3,7-(rac)-dimethyloctyl)oxy)-4,5-bis(heptyloxy)benzoate (5-77r). Compound 5-77r was obtained as a colorless oil after the reaction of 8-r (1.46 g, $4.5 \mathrm{mmol})$ with 1-bromoheptane $(1.48 \mathrm{~mL}, 9.5$ $\mathrm{mmol})$ in the presence of $\mathrm{K}_{2} \mathrm{CO}_{3}(2.49 \mathrm{~g}, 18.0 \mathrm{mmol})$ in dry DMF $(20 \mathrm{~mL})$ at $70{ }^{\circ} \mathrm{C}$ for $4 \mathrm{~h}$. Product: 2.16 g. Yield: 92\%. Purity by HPLC: $99+\%$.

${ }^{1} \mathrm{H}$ NMR (400 MHz, $\left.\mathrm{CDCl}_{3}, \delta, \mathrm{ppm}\right): 7.26$ (s, 2H, $\left.\mathrm{ArH}\right), 4.10-3.97$ (m, 6H, $-\mathrm{OCH}_{2}-$ ), 3.89 (s, 3H, $\left.\mathrm{CO}_{2} \mathrm{CH}_{3}\right), 1.92-1.66\left(\mathrm{~m}, 6 \mathrm{H},-\mathrm{OCH}_{2} \mathrm{CH}_{2}-\right), 1.66-1.58\left(\mathrm{~m}, 1 \mathrm{H},-\mathrm{CH}\left(\mathrm{CH}_{3}\right)_{2}\right), 1.58-1.41(\mathrm{~m}, 5 \mathrm{H},-$

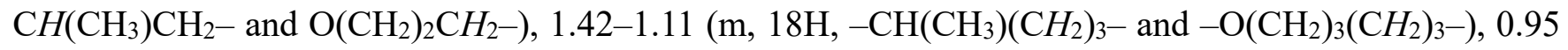
$\left(\mathrm{d}, J=6.5 \mathrm{~Hz}, 3 \mathrm{H},-\mathrm{CH}_{2} \mathrm{CH}\left(\mathrm{CH}_{3}\right)-\right), 0.92-0.82\left(\mathrm{~m}, 12 \mathrm{H},-\mathrm{CH}\left(\mathrm{CH}_{3}\right)_{2}\right.$ and $\left.-\mathrm{CH}_{2} \mathrm{CH}_{3}\right) .{ }^{13} \mathrm{C} \mathrm{NMR}(101 \mathrm{MHz}$, $\left.\mathrm{CDCl}_{3}, \delta, \mathrm{ppm}\right): 167.09(\mathrm{C}=\mathrm{O}), 152.96$ (ArC-3,5), 142.49 (ArC-4), 124.79 (ArC-1), [108.12, 108.07] (ArC-2,6), 73.61 ( $\mathrm{ArOCH}_{2}-4$ position), $69.36\left(\mathrm{ArOCH}_{2}-5\right.$ position), 67.61 ( $\mathrm{ArOCH}_{2}-3$ position), 52.23 $\left(-\mathrm{OCH}_{3}\right),[39.39,37.45,36.45,32.04,31.95,30.47,29.95,29.44,29.35,29.19,28.13,26.17,26.14,24.87]$ $\left(-\mathrm{OCH}_{2} \mathrm{CH}_{2} \mathrm{CH}\left(\mathrm{CH}_{3}\right)\left(\mathrm{CH}_{2}\right)_{3} \mathrm{CH}-\right.$ and $\left.-\mathrm{OCH}_{2}\left(\mathrm{CH}_{2}\right)_{4}-\right)$, [22.83, 22.80, 22.75, 22.73, 19.74, 14.24, 14.22] $\left(-\mathrm{CH}\left(\mathrm{CH}_{3}\right)_{2},-\mathrm{CH}\left(\mathrm{CH}_{3}\right) \mathrm{CH}_{2}-\right.$ and $\left.-\mathrm{CH}_{2} \mathrm{CH}_{3}\right)$. MALDI-TOF MS $m / z$ of $[\mathrm{M}+\mathrm{Na}]^{+}$calculated for $\mathrm{C}_{32} \mathrm{H}_{56} \mathrm{O}_{5}$ : 543.4; Found: 542.8 .
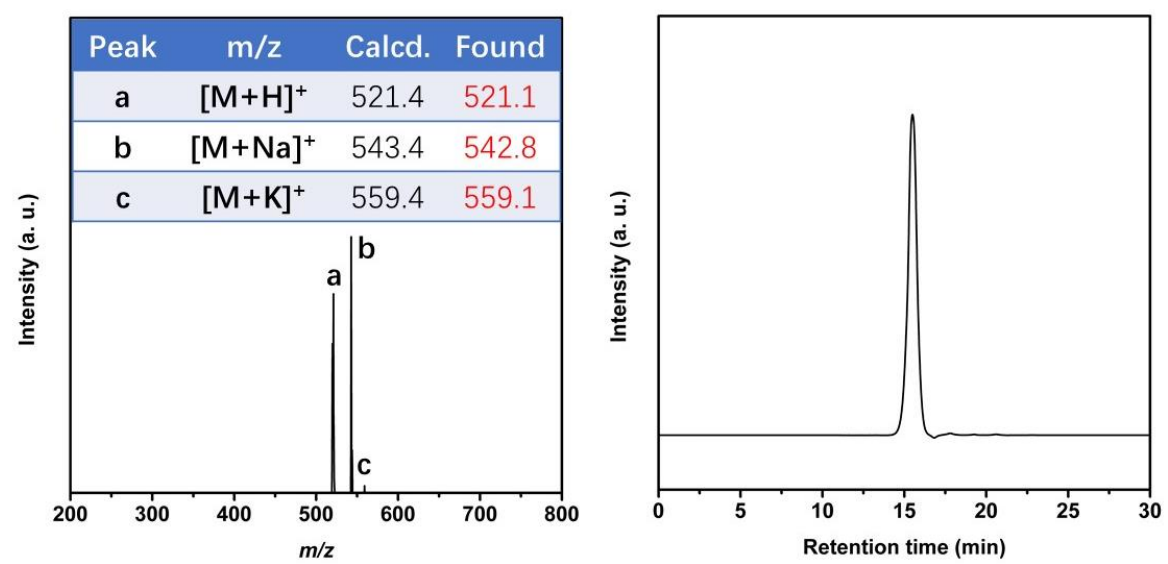

Supporting Figure S29. MALDI-TOF MS spectra (left) and HPLC trace (right) of 5-77r. Heptyl groups have been successfully linked to the 3 - and 4-positions in 5-77r. ${ }^{1} \mathrm{H}$ NMR spectrum is presented on page 62. 
Supporting Figure S29 (continued). ${ }^{1} \mathrm{H}$ NMR of 5-77r.

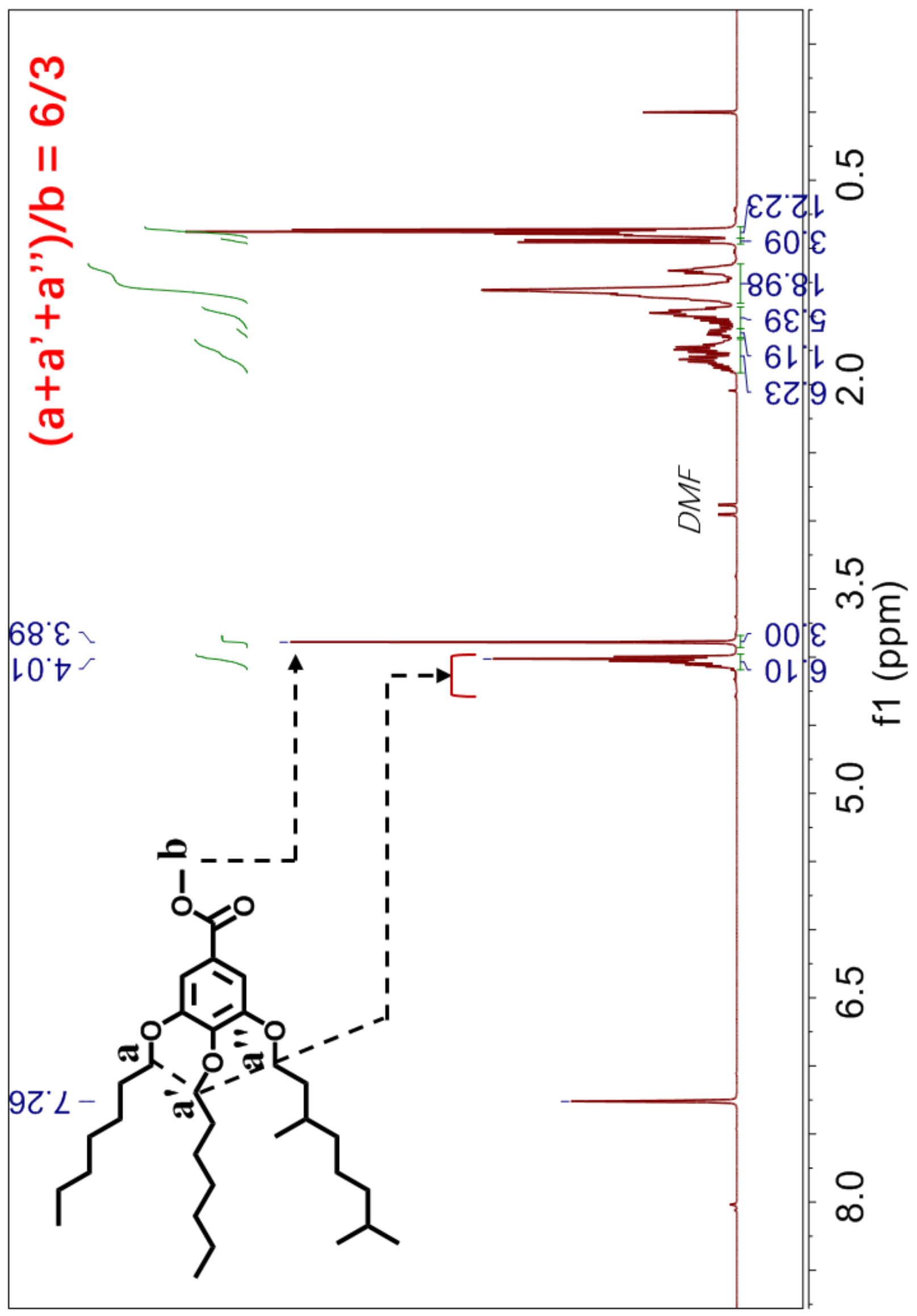


<smiles>[R][CH]CCC(C)CCCC(C)C</smiles>

Methyl 3-((3,7-(rac)-dimethyloctyl)oxy)-4,5-bis(nonyloxy)benzoate (5-99r). Starting from 8-r (1.46 g, $4.5 \mathrm{mmol})$ and 1-bromononane $(1.81 \mathrm{~mL}, 9.5 \mathrm{mmol})$ in the presence of $\mathrm{K}_{2} \mathrm{CO}_{3}(2.49 \mathrm{~g}, 18.0 \mathrm{mmol})$ in dry DMF $(20 \mathrm{~mL})$ at $70{ }^{\circ} \mathrm{C}$ for $4 \mathrm{~h}, \mathbf{5 - 9 9 r}$ was obtained as a light yellow oil. Product: $2.37 \mathrm{~g}$. Yield: $91 \%$. Purity by HPLC: $99+\%$.

${ }^{1} \mathrm{H}$ NMR (400 MHz, $\left.\mathrm{CDCl}_{3}, \delta, \mathrm{ppm}\right): 7.26$ (s, 2H, $\left.\mathrm{ArH}\right), 4.11-3.96$ (m, 6H, $-\mathrm{OCH}_{2}-$ ), 3.89 (s, 3H, $\left.\mathrm{CO}_{2} \mathrm{CH}_{3}\right), 1.93-1.66\left(\mathrm{~m}, 6 \mathrm{H},-\mathrm{OCH}_{2} \mathrm{CH}_{2}-\right), 1.66-1.59\left(\mathrm{~m}, 1 \mathrm{H},-\mathrm{CH}\left(\mathrm{CH}_{3}\right)_{2}\right), 1.59-1.41(\mathrm{~m}, 5 \mathrm{H},-$

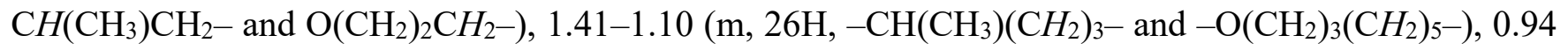
$\left(\mathrm{d}, J=6.5 \mathrm{~Hz}, 3 \mathrm{H},-\mathrm{CH}_{2} \mathrm{CH}\left(\mathrm{CH}_{3}\right)-\right), 0.92-0.82\left(\mathrm{~m}, 12 \mathrm{H},-\mathrm{CH}\left(\mathrm{CH}_{3}\right)_{2}\right.$ and $\left.-\mathrm{CH}_{2} \mathrm{CH}_{3}\right) .{ }^{13} \mathrm{C} \mathrm{NMR}(101 \mathrm{MHz}$, $\left.\mathrm{CDCl}_{3}, \delta, \mathrm{ppm}\right): 167.11(C=\mathrm{O}), 152.97$ (ArC-3,5), 142.50 (ArC-4), 124.80 (ArC-1), [108.13, 108.08] (ArC-2,6), 73.62 ( $\mathrm{ArOCH}_{2}-4$ position), $69.30\left(\mathrm{ArOCH}_{2}-5\right.$ position), $67.62\left(\mathrm{ArOCH}_{2}-3\right.$ position), 52.25 $\left(-\mathrm{OCH}_{3}\right),[39.40,37.46,36.45,32.08,32.05,30.47,29.97,29.82,29.73,29.71,29.54,29.51,29.45,29.43$, 28.13, 26.23, 26.20, 24.88] $\left(-\mathrm{OCH}_{2} \mathrm{CH}_{2} \mathrm{CH}\left(\mathrm{CH}_{3}\right)\left(\mathrm{CH}_{2}\right)_{3} \mathrm{CH}-\right.$ and $\left.-\mathrm{OCH}_{2}\left(\mathrm{CH}_{2}\right)_{6}-\right)$, [22.84, 22.74, 19.75, 14.25] $\left(-\mathrm{CH}\left(\mathrm{CH}_{3}\right)_{2},-\mathrm{CH}\left(\mathrm{CH}_{3}\right) \mathrm{CH}_{2}-\right.$ and $\left.-\mathrm{CH}_{2} \mathrm{CH}_{3}\right)$. MALDI-TOF MS $m / z$ of $[\mathrm{M}+\mathrm{Na}]^{+}$calculated for $\mathrm{C}_{36} \mathrm{H}_{64} \mathrm{O}_{5}$ : 599.5; Found: 599.3.
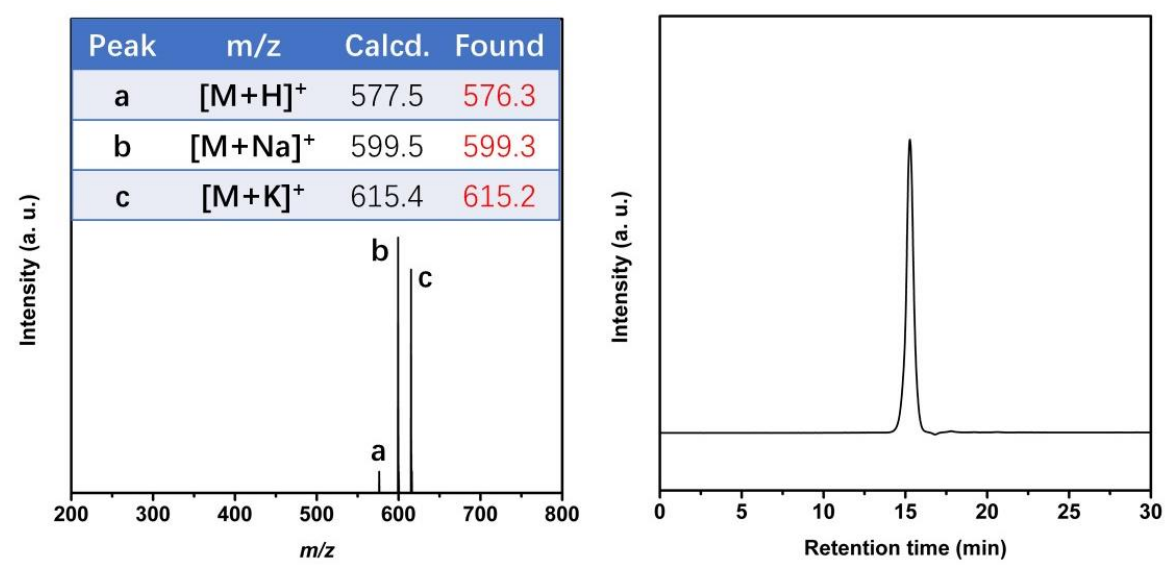

Supporting Figure S30. MALDI-TOF MS spectra (left) and HPLC trace (right) of 5-99r. Nonyl groups have been successfully linked to the 3 - and 4-positions in 5-99r. ${ }^{1} \mathrm{H}$ NMR spectrum is presented on page 64. 
Supporting Figure S30 (continued). ${ }^{1} \mathrm{H}$ NMR of 5-99r.

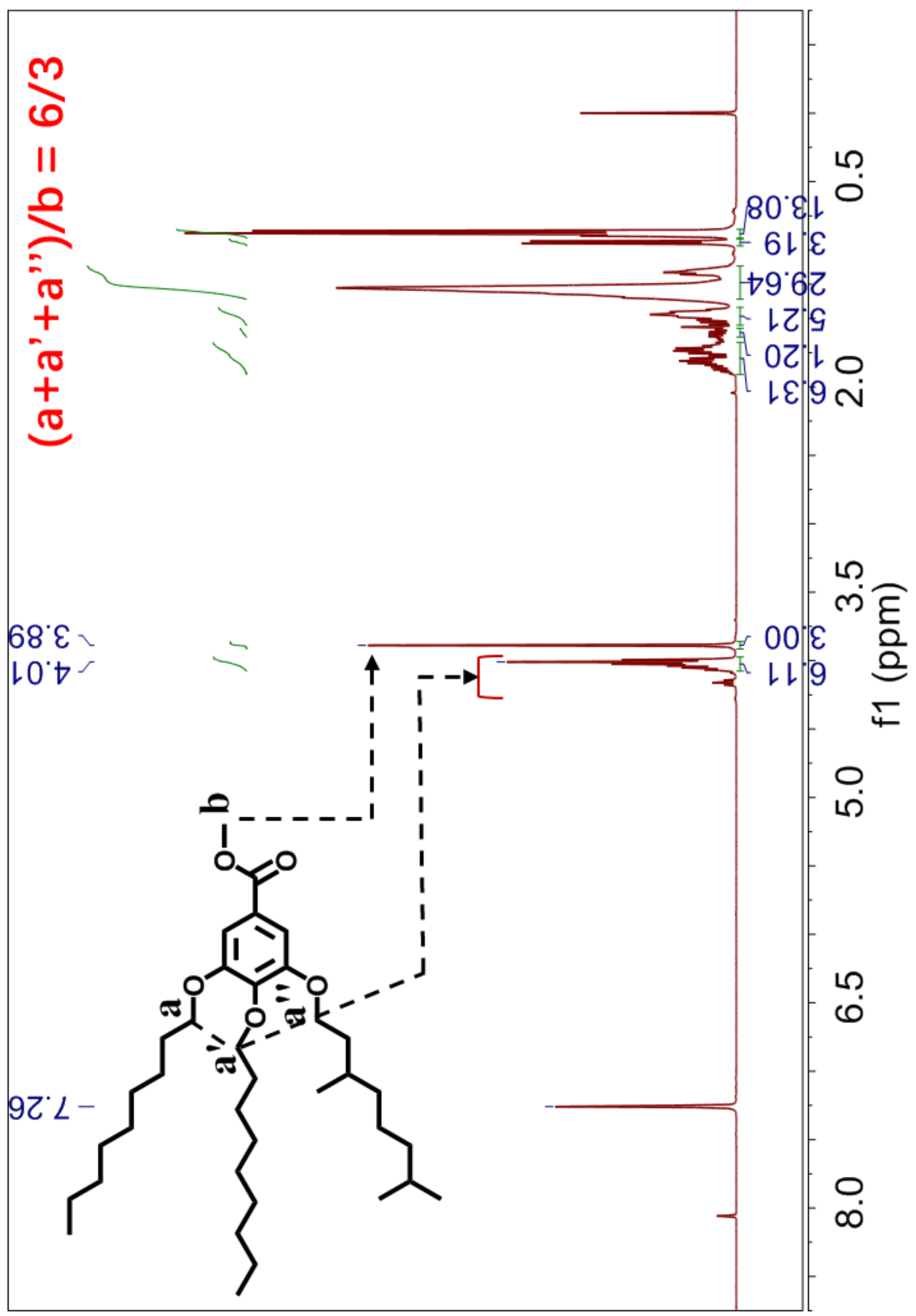


<smiles>[R]CCCC(C)CCCC(C)C</smiles>

Methyl 3-((3,7-(rac)-dimethyloctyl)oxy)-4,5-bis(hexyloxy)benzoate (5-66r). Compound 5-66r was obtained as a colorless oil after the reaction of 8-r $(0.32 \mathrm{~g}, 1.0 \mathrm{mmol})$ with 1-bromohexane $(0.34 \mathrm{~mL}, 2.4$ $\mathrm{mmol})$ in the presence of $\mathrm{K}_{2} \mathrm{CO}_{3}(0.83 \mathrm{~g}, 6.0 \mathrm{mmol})$ in dry DMF $(8 \mathrm{~mL})$ at $70{ }^{\circ} \mathrm{C}$ for $4 \mathrm{~h}$. Product: $0.46 \mathrm{~g}$. Yield: 93\%. Purity by HPLC: $99+\%$.

${ }^{1} \mathrm{H}$ NMR (400 MHz, $\left.\mathrm{CDCl}_{3}, \delta, \mathrm{ppm}\right): 7.26$ (s, 2H, ArH), 4.10-3.97 (m, 6H, -OCH - ), 3.89 (s, 3H, $\left.\mathrm{CO}_{2} \mathrm{CH}_{3}\right), 1.92-1.67\left(\mathrm{~m}, 6 \mathrm{H},-\mathrm{OCH}_{2} \mathrm{CH}_{2}-\right), 1.67-1.57\left(\mathrm{~m}, 1 \mathrm{H},-\mathrm{CH}\left(\mathrm{CH}_{3}\right)_{2}\right), 1.57-1.43(\mathrm{~m}, 5 \mathrm{H},-$

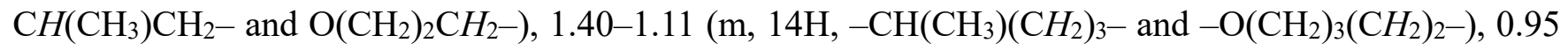
$\left(\mathrm{d}, J=6.6 \mathrm{~Hz}, 3 \mathrm{H},-\mathrm{CH}_{2} \mathrm{CH}\left(\mathrm{CH}_{3}\right)-\right), 0.93-0.83\left(\mathrm{~m}, 12 \mathrm{H},-\mathrm{CH}\left(\mathrm{CH}_{3}\right)_{2}\right.$ and $\left.-\mathrm{CH}_{2} \mathrm{CH}_{3}\right) .{ }^{13} \mathrm{C} \mathrm{NMR}(101 \mathrm{MHz}$, $\left.\mathrm{CDCl}_{3}, \delta, \mathrm{ppm}\right): 167.06(C=\mathrm{O}), 152.95$ (ArC-3,5), 142.46 (ArC-4), 124.78 (ArC-1), [108.09, 108.04] (ArC-2,6), $73.58\left(\mathrm{ArOCH}_{2}-4\right.$ position), $69.27\left(\mathrm{ArOCH}_{2}-5\right.$ position), 67.57 ( $\mathrm{ArOCH}_{2}-3$ position), 52.21 $\left(-\mathrm{OCH}_{3}\right),[39.38,37.43,36.43,31.86,31.67,30.40,29.93,29.38,28.11,25.87,25.84,24.85](-$ $\mathrm{OCH}_{2} \mathrm{CH}_{2} \mathrm{CH}\left(\mathrm{CH}_{3}\right)\left(\mathrm{CH}_{2}\right)_{3} \mathrm{CH}-$ and $\left.-\mathrm{OCH}_{2}\left(\mathrm{CH}_{2}\right)_{3}-\right)$, [22.81, 22.80, 22.74, 22.71, 19.72, 14.20, 14.14] ($\mathrm{CH}\left(\mathrm{CH}_{3}\right)_{2},-\mathrm{CH}\left(\mathrm{CH}_{3}\right) \mathrm{CH}_{2}-$ and $\left.-\mathrm{CH}_{2} \mathrm{CH}_{3}\right)$. MALDI-TOF MS $m / z$ of $[\mathrm{M}+\mathrm{Na}]^{+}$calculated for $\mathrm{C}_{30} \mathrm{H}_{52} \mathrm{O}_{5}$ : 515.4; Found: 514.8 .
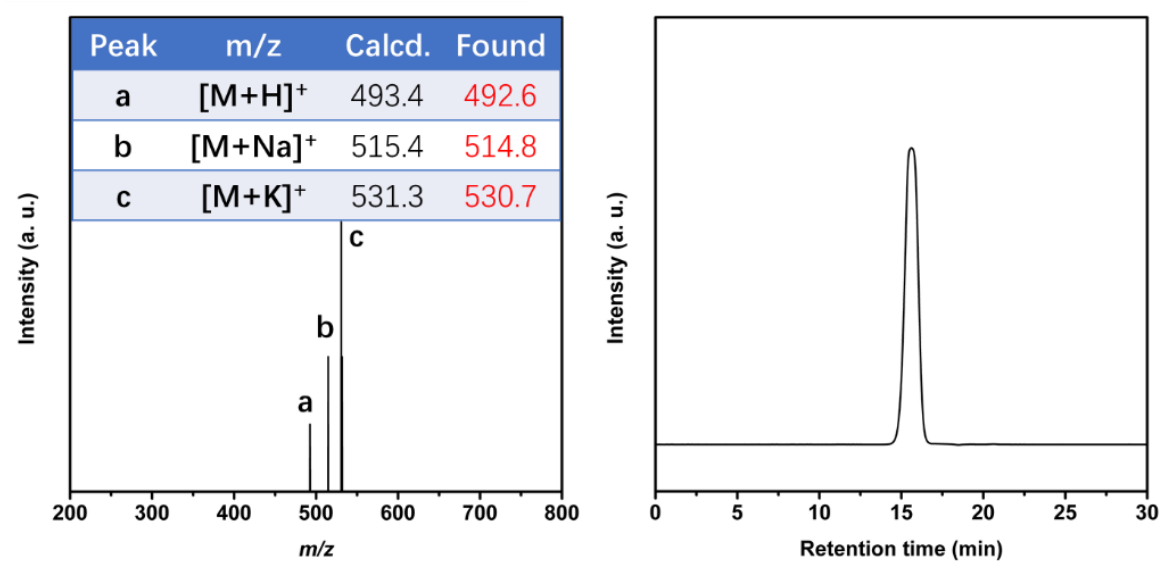

Supporting Figure S31. MALDI-TOF MS spectra (left) and HPLC trace (right) of 5-66r. Hexyl groups have been successfully linked to the 3 - and 4-positions in 5-66r. ${ }^{1} \mathrm{H}$ NMR spectrum is presented on page 66. 
Supporting Figure S31 (continued). ${ }^{1} \mathrm{H}$ NMR of 5-66r.

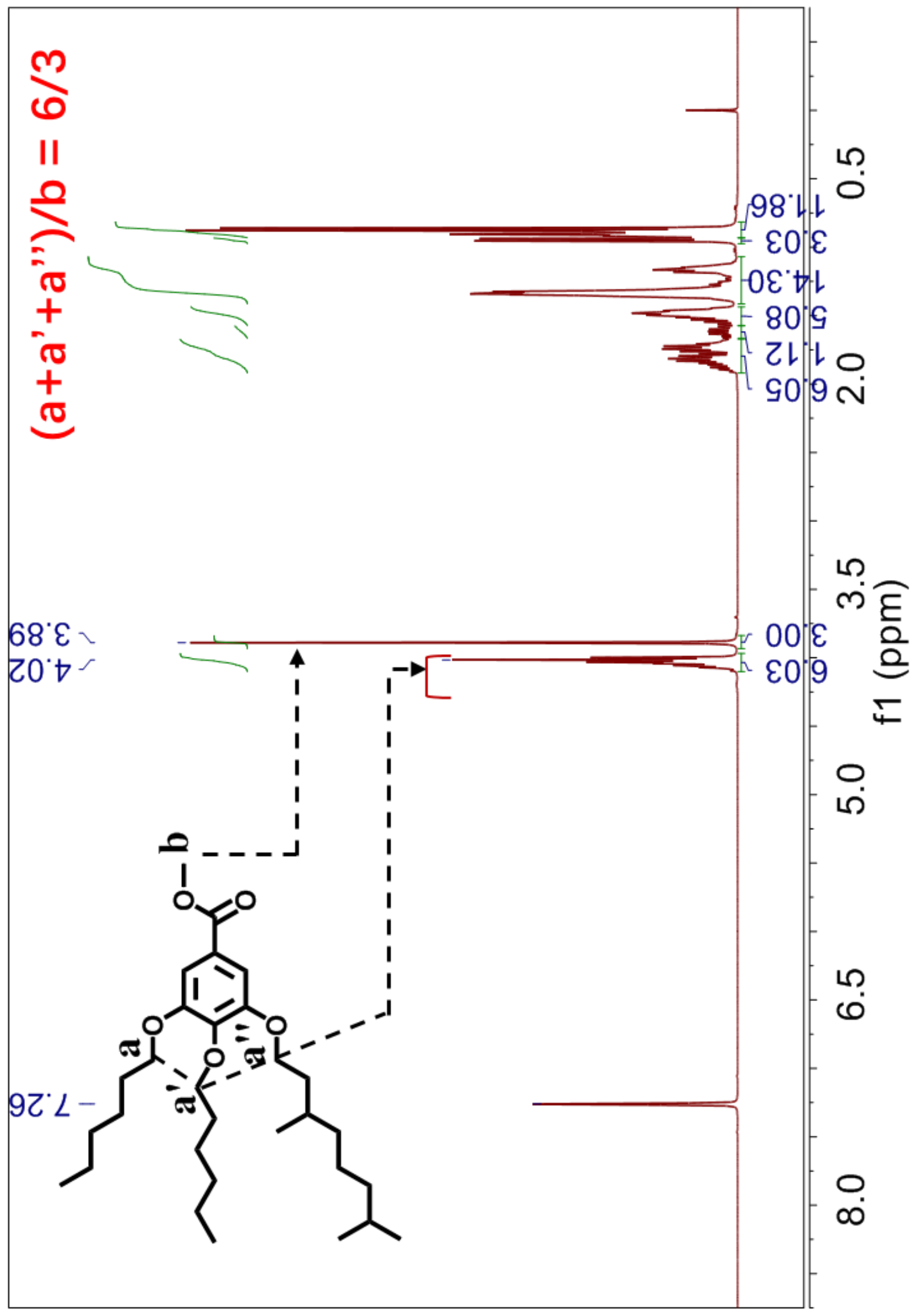


<smiles>[R]CCCC(C)CCCC(C)C</smiles>

Methyl 3,4-bis(decyloxy)-5-((3,7-(rac)-dimethyloctyl)oxy)benzoate (5-1010r). Starting from 8-r (0.32 g, $1.0 \mathrm{mmol})$ and 1-bromodecane $(0.50 \mathrm{~mL}, 2.4 \mathrm{mmol})$ in the presence of $\mathrm{K}_{2} \mathrm{CO}_{3}(0.83 \mathrm{~g}, 6.0 \mathrm{mmol})$ in dry DMF $(8 \mathrm{~mL})$ at $70{ }^{\circ} \mathrm{C}$ for $4 \mathrm{~h}, \mathbf{5 - 1 0 1 0 r}$ was obtained as a colorless oil. Product: 0.56 g. Yield: $92 \%$. Purity by HPLC: $99+\%$.

${ }^{1} \mathrm{H}$ NMR (400 MHz, $\left.\mathrm{CDCl}_{3}, \delta, \mathrm{ppm}\right): 7.26$ (s, 2H, $\left.\mathrm{ArH}\right), 4.10-3.97$ (m, 6H, $-\mathrm{OCH}_{2}-$ ), 3.89 (s, 3H, $\left.\mathrm{CO}_{2} \mathrm{CH}_{3}\right), 1.92-1.68\left(\mathrm{~m}, 6 \mathrm{H},-\mathrm{OCH}_{2} \mathrm{CH}_{2}-\right), 1.67-1.59\left(\mathrm{~m}, 1 \mathrm{H},-\mathrm{CH}\left(\mathrm{CH}_{3}\right)_{2}\right), 1.59-1.42(\mathrm{~m}, 5 \mathrm{H},-$ $\mathrm{CH}\left(\mathrm{CH}_{3}\right) \mathrm{CH}_{2}-$ and $\left.\mathrm{O}\left(\mathrm{CH}_{2}\right)_{2} \mathrm{CH}_{2}-\right), 1.41-1.11\left(\mathrm{~m}, 30 \mathrm{H},-\mathrm{CH}\left(\mathrm{CH}_{3}\right)\left(\mathrm{CH}_{2}\right)_{3}-\right.$ and $\left.-\mathrm{O}\left(\mathrm{CH}_{2}\right)_{3}\left(\mathrm{CH}_{2}\right)_{6}-\right), 0.95$ $\left(\mathrm{d}, J=6.5 \mathrm{~Hz}, 3 \mathrm{H},-\mathrm{CH}_{2} \mathrm{CH}\left(\mathrm{CH}_{3}\right)-\right), 0.92-0.82\left(\mathrm{~m}, 12 \mathrm{H},-\mathrm{CH}\left(\mathrm{CH}_{3}\right)_{2}\right.$ and $\left.-\mathrm{CH}_{2} \mathrm{CH}_{3}\right) .{ }^{13} \mathrm{C} \mathrm{NMR}(101 \mathrm{MHz}$, $\left.\mathrm{CDCl}_{3}, \delta, \mathrm{ppm}\right): 167.06(C=\mathrm{O}), 152.94(\mathrm{ArC}-3,5), 142.47$ (ArC-4), 124.78 (ArC-1), [108.09, 108.04] (ArC-2,6), 73.58 ( $\mathrm{ArOCH}_{2}-4$ position), 69.26 ( $\mathrm{ArOCH}_{2}-3$ position), 67.57 ( $\mathrm{ArOCH}_{2}-5$ position), 52.20 $\left(-\mathrm{OCH}_{3}\right),[39.38,37.43,36.43,32.06,32.04,30.46,29.94,29.85,29.79,29.76,29.71,29.69,29.52,29.48$, 29.43, 28.11, 26.21, 26.18, 24.86] $\left(-\mathrm{OCH}_{2} \mathrm{CH}_{2} \mathrm{CH}\left(\mathrm{CH}_{3}\right)\left(\mathrm{CH}_{2}\right)_{3} \mathrm{CH}-\right.$ and $\left.-\mathrm{OCH}_{2}\left(\mathrm{CH}_{2}\right)_{7}-\right)$, [22.82, 22.71, 19.73, 14.23] $\left(-\mathrm{CH}\left(\mathrm{CH}_{3}\right)_{2},-\mathrm{CH}\left(\mathrm{CH}_{3}\right) \mathrm{CH}_{2}-\right.$ and $\left.-\mathrm{CH}_{2} \mathrm{CH}_{3}\right)$. MALDI-TOF $\mathrm{MS} \mathrm{m} / z$ of $[\mathrm{M}+\mathrm{Na}]^{+}$ calculated for $\mathrm{C}_{38} \mathrm{H}_{68} \mathrm{O}_{5}$ : 627.5; Found: 627.8 .
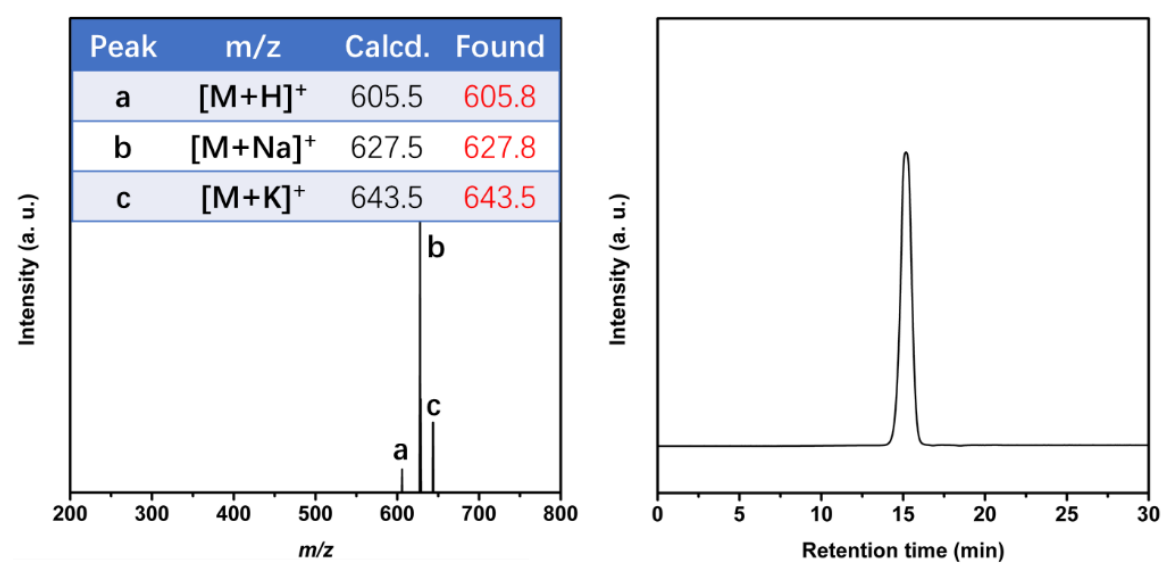

Supporting Figure S32. MALDI-TOF MS spectra (left) and HPLC trace (right) of 5-1010r. Decyl groups have been successfully linked to the 3 - and 4-positions in 5-1010r. ${ }^{1} \mathrm{H}$ NMR spectrum is presented on page 68 . 
Supporting Figure S32 (continued). ${ }^{1} \mathrm{H}$ NMR of 5-1010r.

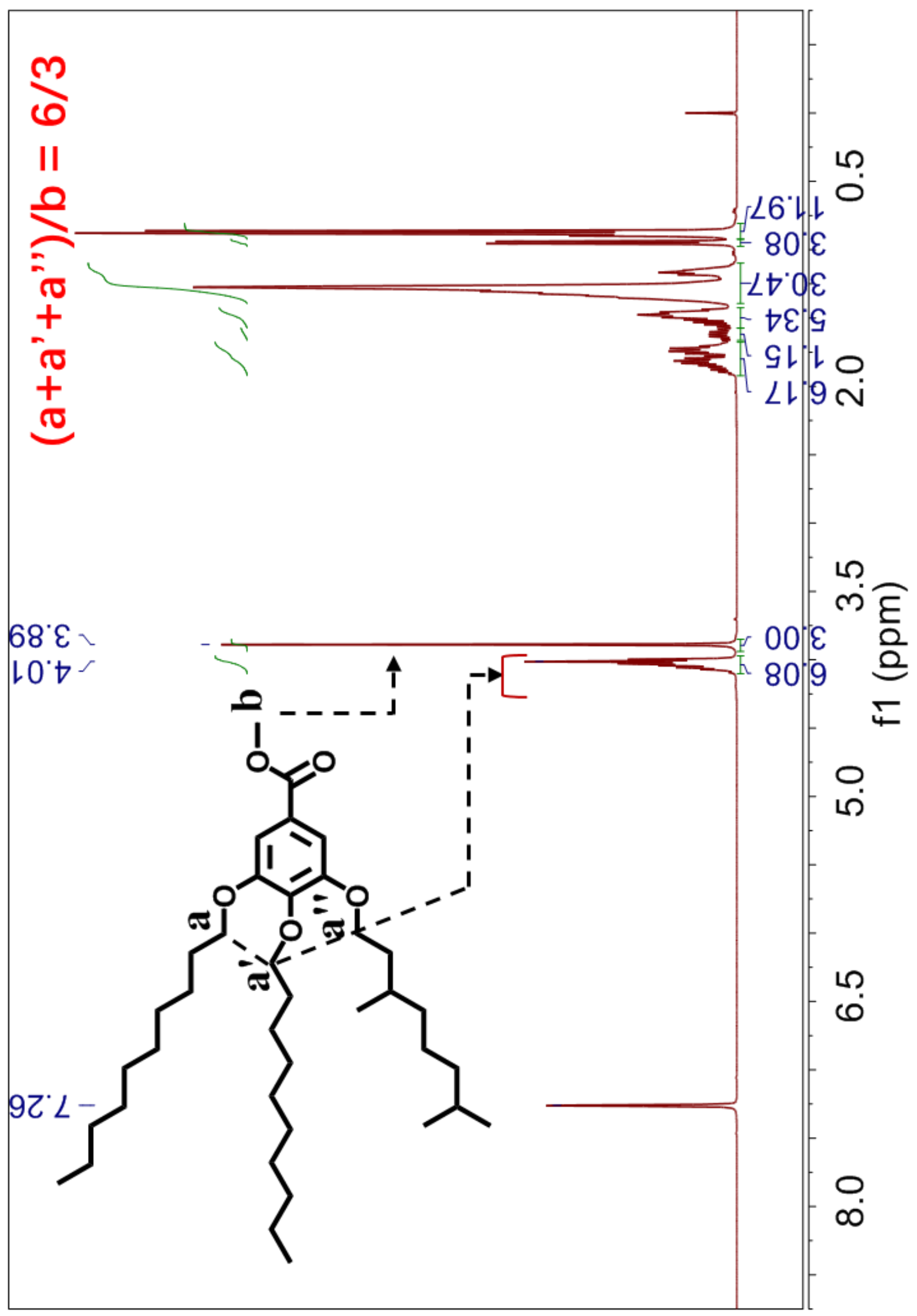


General procedure for preparation of minidendron alcohols 9. The synthesis of minidendron alcohols 9 was conducted according to a procedure reported by our group. ${ }^{8}$ A general example is provided below.
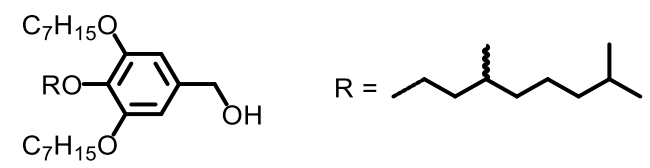

(4-((3,7-(rac)-Dimethyloctyl)oxy)-3,5-bis(heptyloxy)phenyl)methanol (9-7r7). Under $\mathrm{N}_{2}$ protection, 5$7 \mathbf{7} 7(1.04 \mathrm{~g}, 2.0 \mathrm{mmol})$ in dry THF $(4 \mathrm{~mL})$ was added dropwise to a slurry of $\mathrm{LiAlH}_{4}(0.11 \mathrm{~g}, 3.0 \mathrm{mmol})$ in dry THF $(6 \mathrm{~mL})$ at $0{ }^{\circ} \mathrm{C}$. The mixture was stirred at $23{ }^{\circ} \mathrm{C}$ for $1 \mathrm{~h}$. Afterwards, the reaction mixture was cooled to $0{ }^{\circ} \mathrm{C}$ and quenched by the successive addition of $\mathrm{H}_{2} \mathrm{O}(0.1 \mathrm{~mL}), 15 \% \mathrm{NaOH}$ aqueous solution $(0.1 \mathrm{~mL})$ and $\mathrm{H}_{2} \mathrm{O}(0.5 \mathrm{~mL})$. Then, the mixture was filtered through celite, washed with plenty of THF, dried over anhydrous $\mathrm{MgSO}_{4}$, filtered and evaporated in vacuo. Compound 9-7r7 was obtained as a white wax-like oil. Product: 0.97 g. Yield: 98\%. Purity by HPLC: $99+\%$. This was used in the next step without further purification.

${ }^{1} \mathrm{H} \mathrm{NMR}\left(400 \mathrm{MHz}, \mathrm{CDCl}_{3}, \delta, \mathrm{ppm}\right): 6.55$ (s, 2H, $\left.\mathrm{ArH}\right), 4.59\left(\mathrm{~d}, J=4.3 \mathrm{~Hz}, 2 \mathrm{H},-\mathrm{CH}_{2} \mathrm{OH}\right), 4.04-3.90(\mathrm{~m}$, $\left.6 \mathrm{H},-\mathrm{OCH}_{2} \mathrm{CH}_{2}-\right), 1.88-1.66\left(\mathrm{~m}, 6 \mathrm{H},-\mathrm{CH}\left(\mathrm{CH}_{3}\right)_{2},-\mathrm{OCH}_{2} \mathrm{CH}_{2} \mathrm{CH}_{2}-\right.$ and $\left.-\mathrm{CH}\left(\mathrm{CH}_{3}\right) \mathrm{CH}_{2}-\right), 1.59-1.41(\mathrm{~m}$, $6 \mathrm{H},-\mathrm{O}\left(\mathrm{CH}_{2}\right)_{2} \mathrm{CH}_{2}-$ and $\left.-\mathrm{OCH}_{2} \mathrm{CH}_{2} \mathrm{CH}\left(\mathrm{CH}_{3}\right)-\right), 1.41-1.08\left(\mathrm{~m}, 18 \mathrm{H},-\mathrm{CH}\left(\mathrm{CH}_{3}\right)\left(\mathrm{CH}_{2}\right)_{3}-\right.$ and $\left.\mathrm{O}\left(\mathrm{CH}_{2}\right)_{3}\left(\mathrm{CH}_{2}\right)_{3}-\right), 0.92\left(\mathrm{~d}, \mathrm{~J}=6.6 \mathrm{~Hz}, 3 \mathrm{H},-\mathrm{CH}_{2} \mathrm{CH}\left(\mathrm{CH}_{3}\right)-\right), 0.91-0.84\left(\mathrm{~m}, 12 \mathrm{H},-\mathrm{CH}\left(\mathrm{CH}_{3}\right)_{2}\right.$ and $\left.\mathrm{CH}_{2} \mathrm{CH}_{3}\right) .{ }^{13} \mathrm{C} \mathrm{NMR}\left(101 \mathrm{MHz}, \mathrm{CDCl}_{3}, \delta, \mathrm{ppm}\right): 153.41$ (ArC-3,5), 137.68 (ArC-4), $136.23(\mathrm{ArC}-1)$, 105.43 (ArC-2,6), 71.82 ( $\mathrm{ArOCH}_{2}-3,5$ position), $69.22\left(\mathrm{ArOCH}_{2}-4\right.$ position), $65.77\left(-\mathrm{CH} \mathrm{H}_{2} \mathrm{OH}\right),[39.51$, $37.65, \quad 37.47, \quad 31.97,29.85,29.56,29.21,28.13,26.19,24.86] \quad\left(-\mathrm{OCH}_{2}\left(\mathrm{CH}_{2}\right)_{4}-\right.$ and $\left.\mathrm{OCH}_{2} \mathrm{CH}_{2} \mathrm{CH}\left(\mathrm{CH}_{3}\right)\left(\mathrm{CH}_{2}\right)_{3} \mathrm{CH}-\right),[22.84,22.76,22.74,19.69,14.22]\left(-\mathrm{CH}\left(\mathrm{CH}_{3}\right)_{2},-\mathrm{CH}\left(\mathrm{CH}_{3}\right) \mathrm{CH}_{2}-\right.$ and $\mathrm{CH}_{2} \mathrm{CH}_{3}$ ). MALDI-TOF MS $m / z$ of $[\mathrm{M}+\mathrm{Na}]^{+}$calculated for $\mathrm{C}_{31} \mathrm{H}_{56} \mathrm{O}_{4}$ : 515.4; Found: 515.4 .
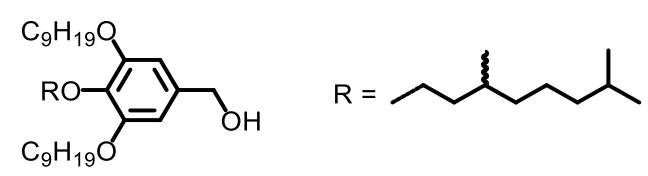

(4-((3,7-(rac)-Dimethyloctyl)oxy)-3,5-bis(nonyloxy)phenyl)methanol (9-9r9). From 5-9r9 (1.15 g, 2.0 mmol $)$ and $\mathrm{LiAlH}_{4}(0.11 \mathrm{~g}, 3.0 \mathrm{mmol})$ in dry THF $(10 \mathrm{~mL})$ at $23{ }^{\circ} \mathrm{C}$ for $1 \mathrm{~h}, 9-9 \mathrm{r} 9$ was obtained as a white wax-like oil. Product: 1.04 g; Yield: 95\%. Purity by HPLC: $99+\%$. This was used in the next step without further purification.

${ }^{1} \mathrm{H}$ NMR (400 MHz, $\left.\mathrm{CDCl}_{3}, \delta, \mathrm{ppm}\right): 6.55$ (s, 2H, $\left.\mathrm{ArH}\right), 4.59\left(\mathrm{~d}, J=5.7 \mathrm{~Hz}, 2 \mathrm{H},-\mathrm{CH}_{2} \mathrm{OH}\right), 4.04-3.90$ (m, $\left.6 \mathrm{H},-\mathrm{OCH}_{2} \mathrm{CH}_{2}-\right), 1.88-1.62\left(\mathrm{~m}, 6 \mathrm{H},-\mathrm{CH}\left(\mathrm{CH}_{3}\right)_{2},-\mathrm{OCH}_{2} \mathrm{CH}_{2} \mathrm{CH}_{2}-\right.$ and $\left.-\mathrm{CH}\left(\mathrm{CH}_{3}\right) \mathrm{CH}_{2}-\right), 1.62-1.41(\mathrm{~m}$, $6 \mathrm{H},-\mathrm{O}\left(\mathrm{CH}_{2}\right)_{2} \mathrm{CH}_{2}-$ and $\left.-\mathrm{OCH}_{2} \mathrm{CH}_{2} \mathrm{CH}\left(\mathrm{CH}_{3}\right)-\right), 1.41-1.06\left(\mathrm{~m}, 26 \mathrm{H},-\mathrm{CH}\left(\mathrm{CH}_{3}\right)\left(\mathrm{CH}_{2}\right)_{3}-\right.$ and $\left.\mathrm{O}\left(\mathrm{CH}_{2}\right)_{3}\left(\mathrm{CH}_{2}\right)_{5}-\right), 0.92\left(\mathrm{~d}, \mathrm{~J}=6.6 \mathrm{~Hz}, 3 \mathrm{H},-\mathrm{CH}_{2} \mathrm{CH}\left(\mathrm{CH}_{3}\right)-\right), 0.91-0.84\left(\mathrm{~m}, 12 \mathrm{H},-\mathrm{CH}\left(\mathrm{CH}_{3}\right)_{2}\right.$ and $\left.\mathrm{CH}_{2} \mathrm{CH}_{3}\right) .{ }^{13} \mathrm{C} \mathrm{NMR}\left(101 \mathrm{MHz}, \mathrm{CDCl}_{3}, \delta, \mathrm{ppm}\right): 153.44$ (ArC-3,5), 137.74 (ArC-4), 136.19 (ArC-1), 105.46 ( $\mathrm{ArC}-2,6), 71.82$ ( $\mathrm{ArOCH}_{2}-3,5$ position), 69.24 ( $\mathrm{ArOCH}_{2}-4$ position), $65.82\left(-\mathrm{CH}_{2} \mathrm{OH}\right),[39.52$, $37.67,37.49,32.04,29.86,29.75,29.57,29.44,28.14,26.25,24.87]\left(-\mathrm{OCH}_{2}\left(\mathrm{CH}_{2}\right)_{6}-\right.$ and $\left.\mathrm{OCH}_{2} \mathrm{CH}_{2} \mathrm{CH}\left(\mathrm{CH}_{3}\right)\left(\mathrm{CH}_{2}\right)_{3} \mathrm{CH}-\right),[22.85,22.82,22.76,19.70,14.25]\left(-\mathrm{CH}\left(\mathrm{CH}_{3}\right)_{2},-\mathrm{CH}\left(\mathrm{CH}_{3}\right) \mathrm{CH}_{2}-\right.$ and $\mathrm{CH}_{2} \mathrm{CH}_{3}$ ). MALDI-TOF MS $m / z$ of $[\mathrm{M}+\mathrm{Na}]^{+}$calculated for $\mathrm{C}_{35} \mathrm{H}_{64} \mathrm{O}_{4}$ : 571.5; Found: 571.5 . 


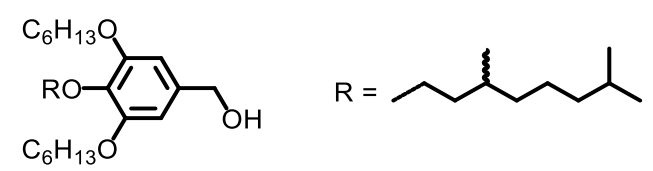

(4-((3,7-(rac)-Dimethyloctyl)oxy)-3,5-bis(hexyloxy)phenyl)methanol (9-6r6). From 5-6r6 (0.24 g, $0.49 \mathrm{mmol})$ and $\mathrm{LiAlH}_{4}(0.028 \mathrm{~g}, 0.74 \mathrm{mmol})$ in dry THF $(4 \mathrm{~mL})$ at $23{ }^{\circ} \mathrm{C}$ for $1 \mathrm{~h}, 9-6 \mathrm{r6}$ was obtained as a white wax-like oil. Product: 0.22 g; Yield: 98\%. Purity by HPLC: 99+\%. This was used in the next step without further purification.

${ }^{1} \mathrm{H}$ NMR (500 MHz, $\left.\mathrm{CDCl}_{3}, \delta, \mathrm{ppm}\right): 6.54$ (s, 2H, $\left.\mathrm{ArH}\right), 4.57$ (s, 2H, $\left.-\mathrm{CH}_{2} \mathrm{OH}\right), 4.05-3.89$ (m, 6H, $\left.\mathrm{OCH}_{2} \mathrm{CH}_{2}-\right)$, 1.91-1.66 (m, $6 \mathrm{H},-\mathrm{CH}\left(\mathrm{CH}_{3}\right)_{2},-\mathrm{OCH}_{2} \mathrm{CH}_{2} \mathrm{CH}_{2}-$ and $\left.-\mathrm{CH}\left(\mathrm{CH}_{3}\right) \mathrm{CH}_{2}-\right), 1.58-1.40(\mathrm{~m}, 6 \mathrm{H}$, $-\mathrm{O}\left(\mathrm{CH}_{2}\right)_{2} \mathrm{CH}_{2}-$ and $\left.-\mathrm{OCH}_{2} \mathrm{CH}_{2} \mathrm{CH}\left(\mathrm{CH}_{3}\right)_{-}\right), \quad 1.40-1.09 \quad\left(\mathrm{~m}, \quad 14 \mathrm{H}, \quad-\mathrm{CH}\left(\mathrm{CH}_{3}\right)\left(\mathrm{CH}_{2}\right)_{3}-\right.$ and $\left.\mathrm{O}\left(\mathrm{CH}_{2}\right)_{3}\left(\mathrm{CH}_{2}\right)_{2}-\right), 0.95-0.84\left(\mathrm{~m}, 15 \mathrm{H},-\mathrm{CH}_{2} \mathrm{CH}\left(\mathrm{CH}_{3}\right)-,-\mathrm{CH}\left(\mathrm{CH}_{3}\right)_{2}\right.$ and $\left.-\mathrm{CH}_{2} \mathrm{CH}_{3}\right) .{ }^{13} \mathrm{C} \mathrm{NMR}(126 \mathrm{MHz}$, $\mathrm{CDCl}_{3}, \delta$, ppm): 153.36 (ArC-3,5), 137.61 (ArC-4), 136.27 (ArC-1), 105.38 (ArC-2,6), $71.80\left(\mathrm{ArOCH}_{2}-\right.$ 3,5 position), 69.19 ( $\mathrm{ArOCH}_{2}-4$ position), $65.70\left(-\mathrm{CH}_{2} \mathrm{OH}\right),[39.49,37.64,37.45,31.72,29.83,29.50$, 28.12, 25.90, 24.84] $\left(-\mathrm{OCH}_{2}\left(\mathrm{CH}_{2}\right)_{3}-\right.$ and $\left.-\mathrm{OCH}_{2} \mathrm{CH}_{2} \mathrm{CH}\left(\mathrm{CH}_{3}\right)\left(\mathrm{CH}_{2}\right)_{3} \mathrm{CH}-\right)$, [22.82, 22.76, 22.72, 19.68, 14.15] $\left(-\mathrm{CH}\left(\mathrm{CH}_{3}\right)_{2},-\mathrm{CH}\left(\mathrm{CH}_{3}\right) \mathrm{CH}_{2}-\right.$ and $\left.-\mathrm{CH}_{2} \mathrm{CH}_{3}\right)$. MALDI-TOF MS $m / z$ of $[\mathrm{M}+\mathrm{Na}]^{+}$calculated for $\mathrm{C}_{29} \mathrm{H}_{52} \mathrm{O}_{4}$ : 487.4; Found: 487.3 .
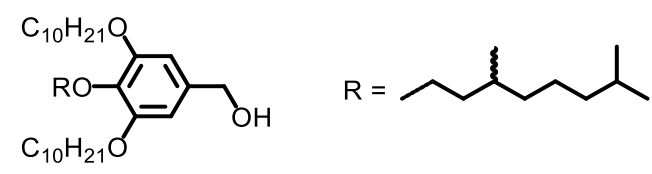

(3,5-Bis(decyloxy)-4-((3,7-(rac)-dimethyloctyl)oxy)phenyl)methanol (9-10r10). From 5-10r10 (0.33 g, $0.55 \mathrm{mmol})$ and $\mathrm{LiAlH}_{4}(0.031 \mathrm{~g}, 0.82 \mathrm{mmol})$ in dry THF $(4 \mathrm{~mL})$ at $23{ }^{\circ} \mathrm{C}$ for $1 \mathrm{~h}, \mathbf{9 - 1 0 r 1 0}$ was obtained as a white wax-like oil. Product: $0.31 \mathrm{~g}$; Yield: 99\%. Purity by HPLC: $99+\%$. This was used in the next step without further purification.

${ }^{1} \mathrm{H}$ NMR (500 MHz, $\left.\mathrm{CDCl}_{3}, \delta, \mathrm{ppm}\right): 6.53$ (s, 2H, $\left.\mathrm{ArH}\right), 4.57$ (d, J=5.7 Hz, 2H, $\left.-\mathrm{CH}_{2} \mathrm{OH}\right), 4.03-3.88(\mathrm{~m}$, $\left.6 \mathrm{H},-\mathrm{OCH}_{2} \mathrm{CH}_{2}-\right), 1.87-1.66\left(\mathrm{~m}, 6 \mathrm{H},-\mathrm{CH}\left(\mathrm{CH}_{3}\right)_{2},-\mathrm{OCH}_{2} \mathrm{CH}_{2} \mathrm{CH}_{2}-\right.$ and $\left.-\mathrm{CH}\left(\mathrm{CH}_{3}\right) \mathrm{CH}_{2}-\right), 1.57-1.41(\mathrm{~m}$, $6 \mathrm{H},-\mathrm{O}\left(\mathrm{CH}_{2}\right)_{2} \mathrm{CH}_{2}-$ and $\left.-\mathrm{OCH}_{2} \mathrm{CH}_{2} \mathrm{CH}\left(\mathrm{CH}_{3}\right)-\right), 1.37-1.08\left(\mathrm{~m}, 30 \mathrm{H},-\mathrm{CH}\left(\mathrm{CH}_{3}\right)\left(\mathrm{CH}_{2}\right)_{3}-\right.$ and $\left.\mathrm{O}\left(\mathrm{CH}_{2}\right)_{3}\left(\mathrm{CH}_{2}\right)_{6}-\right), 0.91\left(\mathrm{~d}, \mathrm{~J}=6.6 \mathrm{~Hz}, 3 \mathrm{H},-\mathrm{CH}_{2} \mathrm{CH}\left(\mathrm{CH}_{3}\right)-\right), 0.89-0.83\left(\mathrm{~m}, 12 \mathrm{H},-\mathrm{CH}\left(\mathrm{CH}_{3}\right)_{2}\right.$ and $\left.\mathrm{CH}_{2} \mathrm{CH}_{3}\right) .{ }^{13} \mathrm{C} \mathrm{NMR}\left(126 \mathrm{MHz}, \mathrm{CDCl}_{3}, \delta, \mathrm{ppm}\right): 153.44$ (ArC-3,5), 137.74 (ArC-4), 136.19 (ArC-1), 105.46 (ArC-2,6), 71.82 ( $\mathrm{ArOCH}_{2}-3,5$ position), 69.24 ( $\mathrm{ArOCH}_{2}-4$ position), $65.82\left(-\mathrm{CH}_{2} \mathrm{OH}\right),[39.52$, $37.67,37.49,32.04,29.86,29.75,29.57,29.44,28.14,26.25,24.87]\left(-\mathrm{OCH}_{2}\left(\mathrm{CH}_{2}\right)_{7}-\right.$ and $\left.\mathrm{OCH}_{2} \mathrm{CH}_{2} \mathrm{CH}\left(\mathrm{CH}_{3}\right)\left(\mathrm{CH}_{2}\right)_{3} \mathrm{CH}-\right)$, [22.85, 22.82, 22.76, 19.70, 14.25] $\left(-\mathrm{CH}\left(\mathrm{CH}_{3}\right)_{2},-\mathrm{CH}\left(\mathrm{CH}_{3}\right) \mathrm{CH}_{2}-\right.$ and $\mathrm{CH}_{2} \mathrm{CH}_{3}$ ). MALDI-TOF MS $m / z$ of $[\mathrm{M}+\mathrm{Na}]^{+}$calculated for $\mathrm{C}_{37} \mathrm{H}_{68} \mathrm{O}_{4}$ : 599.5; Found: 599.0 . 
<smiles>[R]=CCCC(C)CCCC(C)C</smiles>

(3,5-Bis((3,7-(rac)-dimethyloctyl)oxy)-4-(heptyloxy)phenyl)methanol (9-r7r). From 5-r7r (1.13 g, 2.0 mmol) and $\mathrm{LiAlH}_{4}(0.11 \mathrm{~g}, 3.0 \mathrm{mmol})$ in dry THF $(10 \mathrm{~mL})$ at $23{ }^{\circ} \mathrm{C}$ for $1 \mathrm{~h}, \mathbf{9}-\mathbf{r} 7 \mathbf{r}$ was obtained as a colorless oil. Product: 1.06 g; Yield: 99\%. Purity by HPLC: 99+\%. This was used in the next step without further purification.

${ }^{1} \mathrm{H}$ NMR (400 MHz, $\left.\mathrm{CDCl}_{3}, \delta, \mathrm{ppm}\right): 6.56$ (s, 2H, $\mathrm{ArH}$ ), 4.59 (s, 2H, $\left.-\mathrm{CH}_{2} \mathrm{OH}\right), 4.06-3.95$ (m, 4H, -

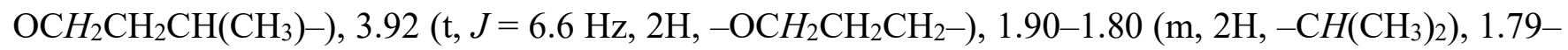
$1.65\left(\mathrm{~m}, 4 \mathrm{H},-\mathrm{CH}\left(\mathrm{CH}_{3}\right) \mathrm{CH}_{2}-\right.$ and $\left.-\mathrm{OCH}_{2} \mathrm{CH}_{2} \mathrm{CH}_{2}-\right)$, $1.65-1.41\left(\mathrm{~m}, 6 \mathrm{H},-\mathrm{OCH}_{2} \mathrm{CH}_{2} \mathrm{CH}_{\left(\mathrm{CH}_{3}\right)-\text { and }-}\right.$ $\left.\mathrm{OCH}_{2} \mathrm{CH}_{2} \mathrm{CH}_{2}-\right), 1.41-1.08\left(\mathrm{~m}, 18 \mathrm{H},-\mathrm{CH}\left(\mathrm{CH}_{3}\right)\left(\mathrm{CH}_{2}\right)_{3}-\right.$ and $\left.-\mathrm{O}\left(\mathrm{CH}_{2}\right)_{3}\left(\mathrm{CH}_{2}\right)_{3}-\right), 0.93(\mathrm{~d}, J=6.6 \mathrm{~Hz}, 6 \mathrm{H}$, $\left.-\mathrm{CH}_{2} \mathrm{CH}\left(\mathrm{CH}_{3}\right)-\right), 0.91-0.84\left(\mathrm{~m}, 15 \mathrm{H},-\mathrm{CH}\left(\mathrm{CH}_{3}\right)_{2}\right.$ and $\left.-\mathrm{CH}_{2} \mathrm{CH}_{3}\right) .{ }^{13} \mathrm{C} \mathrm{NMR}\left(101 \mathrm{MHz}, \mathrm{CDCl}_{3}, \delta, \mathrm{ppm}\right)$ : 153.42 (ArC-3,5), 137.67 (ArC-4), 136.20 (ArC-1), 105.43 (ArC-2,6), 73.55 ( $\mathrm{ArOCH}_{2}-4$ position), 67.54 (ArOCH $\mathrm{H}_{2}-3,5$ position), $65.80\left(-\mathrm{CH}_{2} \mathrm{OH}\right),[39.41,37.47,36.57,32.06,30.47,29.96,29.41,28.13,26.23$, 24.87] $\left(-\mathrm{OCH}_{2} \mathrm{CH}_{2} \mathrm{CH}\left(\mathrm{CH}_{3}\right)\left(\mathrm{CH}_{2}\right)_{3} \mathrm{CH}-\right.$ and $\left.-\mathrm{OCH}_{2}\left(\mathrm{CH}_{2}\right)_{4}-\right)$, [22.84, 22.73, 19.74, 14.25] $\left(-\mathrm{CH}\left(\mathrm{CH}_{3}\right)_{2}\right.$, $-\mathrm{CH}\left(\mathrm{CH}_{3}\right) \mathrm{CH}_{2}-$ and $\left.-\mathrm{CH}_{2} \mathrm{CH}_{3}\right)$. MALDI-TOF MS $m / z$ of $[\mathrm{M}+\mathrm{Na}]^{+}$calculated for $\mathrm{C}_{34} \mathrm{H}_{62} \mathrm{O}_{4}$ : 557.5; Found: 557.1.

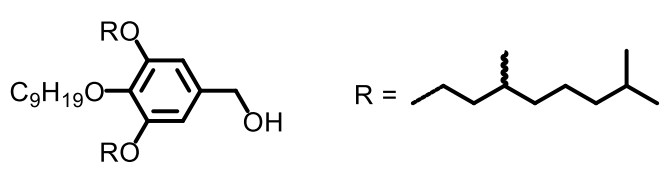

(3,5-Bis((3,7-(rac)-dimethyloctyl)oxy)-4-(nonyloxy)phenyl)methanol (9-r9r). From 5-r9r (1.18 g, 2.0 mmol) and $\mathrm{LiAlH}_{4}(0.11 \mathrm{~g}, 3.0 \mathrm{mmol})$ in dry THF $(10 \mathrm{~mL})$ at $23{ }^{\circ} \mathrm{C}$ for $1 \mathrm{~h}, 9-\mathbf{9} 9 \mathrm{r}$ was obtained as a colorless oil. Product: 1.12 g; Yield: 100\%. Purity by HPLC: 99+\%. This was used in the next step without further purification.

${ }^{1} \mathrm{H}$ NMR (400 MHz, $\left.\mathrm{CDCl}_{3}, \delta, \mathrm{ppm}\right): 6.56$ (s, 2H, $\mathrm{ArH}$ ), 4.59 (s, 2H, $\left.-\mathrm{CH}_{2} \mathrm{OH}\right), 4.06-3.95$ (m, 4H, $\left.\mathrm{OCH}_{2} \mathrm{CH}_{2} \mathrm{CH}\left(\mathrm{CH}_{3}\right)_{-}\right), 3.92\left(\mathrm{t}, J=6.6 \mathrm{~Hz}, 2 \mathrm{H},-\mathrm{OCH}_{2} \mathrm{CH}_{2} \mathrm{CH}_{2}-\right), 1.90-1.80\left(\mathrm{~m}, 2 \mathrm{H},-\mathrm{CH}_{\left.\left(\mathrm{CH}_{3}\right)_{2}\right), 1.78-}\right.$ $1.64\left(\mathrm{~m}, 4 \mathrm{H},-\mathrm{CH}\left(\mathrm{CH}_{3}\right) \mathrm{CH}_{2}-\right.$ and $\left.-\mathrm{OCH}_{2} \mathrm{CH}_{2} \mathrm{CH}_{2}-\right), 1.64-1.41\left(\mathrm{~m}, 6 \mathrm{H},-\mathrm{OCH}_{2} \mathrm{CH}_{2} \mathrm{CH}_{\left(\mathrm{CH}_{3}\right)-\text { and }-}\right.$ $\left.\mathrm{OCH}_{2} \mathrm{CH}_{2} \mathrm{CH}_{2}-\right), 1.41-1.09\left(\mathrm{~m}, 22 \mathrm{H},-\mathrm{CH}\left(\mathrm{CH}_{3}\right)\left(\mathrm{CH}_{2}\right)_{3}-\right.$ and $\left.-\mathrm{O}\left(\mathrm{CH}_{2}\right)_{3}\left(\mathrm{CH}_{2}\right)_{5}-\right), 0.93(\mathrm{~d}, J=6.6 \mathrm{~Hz}, 6 \mathrm{H}$, $\left.-\mathrm{CH}_{2} \mathrm{CH}\left(\mathrm{CH}_{3}\right)-\right), 0.91-0.84\left(\mathrm{~m}, 15 \mathrm{H},-\mathrm{CH}\left(\mathrm{CH}_{3}\right)_{2}\right.$ and $\left.-\mathrm{CH}_{2} \mathrm{CH}_{3}\right) .{ }^{13} \mathrm{C} \mathrm{NMR}\left(101 \mathrm{MHz}, \mathrm{CDCl}_{3}, \delta, \mathrm{ppm}\right)$ : 153.40 (ArC-3,5), 137.66 ( $\mathrm{ArC}-4), 136.21$ (ArC-1), 105.42 ( $\mathrm{ArC}-2,6), 73.54$ ( $\mathrm{ArOCH}_{2}-4$ position), 67.53 (ArOCH$-3,5$ position), $65.77\left(-\mathrm{CH}_{2} \mathrm{OH}\right),[39.41,37.46,36.57,32.08,30.46,29.96,29.82,29.76,29.51$, 28.12, 26.27, 24.87] $\left(-\mathrm{OCH}_{2} \mathrm{CH}_{2} \mathrm{CH}\left(\mathrm{CH}_{3}\right)\left(\mathrm{CH}_{2}\right)_{3} \mathrm{CH}-\right.$ and $\left.-\mathrm{OCH}_{2}\left(\mathrm{CH}_{2}\right)_{6}-\right)$, [22.83, 22.73, 19.73, 14.25] $\left(-\mathrm{CH}\left(\mathrm{CH}_{3}\right)_{2},-\mathrm{CH}\left(\mathrm{CH}_{3}\right) \mathrm{CH}_{2}-\right.$ and $\left.-\mathrm{CH}_{2} \mathrm{CH}_{3}\right)$. MALDI-TOF MS $m / z$ of $[\mathrm{M}+\mathrm{Na}]^{+}$calculated for $\mathrm{C}_{36} \mathrm{H}_{66} \mathrm{O}_{4}$ : 585.5; Found: 585.2. 
<smiles>[R]CCCC(C)CCCC(C)C</smiles>

(3,5-Bis((3,7-(rac)-dimethyloctyl)oxy)-4-(hexyloxy)phenyl)methanol (9-r6r). From 5-r6r (0.30 g, 0.55 mmol) and $\mathrm{LiAlH}_{4}(0.031 \mathrm{~g}, 0.82 \mathrm{mmol})$ in dry THF $(4 \mathrm{~mL})$ at $23{ }^{\circ} \mathrm{C}$ for $1 \mathrm{~h}, \mathbf{9}-\mathbf{r 6 r}$ was obtained as a colorless oil. Product: 0.28 g; Yield: 97\%. Purity by HPLC: $99+\%$. This was used in the next step without further purification.

${ }^{1} \mathrm{H}$ NMR (500 MHz, $\left.\mathrm{CDCl}_{3}, \delta, \mathrm{ppm}\right): 6.56(\mathrm{~s}, 2 \mathrm{H}, \mathrm{ArH}), 4.59\left(\mathrm{~d}, J=6.0 \mathrm{~Hz}, 2 \mathrm{H},-\mathrm{CH}_{2} \mathrm{OH}\right), 4.06-3.95(\mathrm{~m}$, $\left.4 \mathrm{H},-\mathrm{OCH}_{2} \mathrm{CH}_{2} \mathrm{CH}\left(\mathrm{CH}_{3}\right)-\right), 3.93\left(\mathrm{t}, \mathrm{J}=6.6 \mathrm{~Hz}, 2 \mathrm{H},-\mathrm{OCH}_{2} \mathrm{CH}_{2} \mathrm{CH}_{2}-\right), 1.89-1.82\left(\mathrm{~m}, 2 \mathrm{H},-\mathrm{CH}\left(\mathrm{CH}_{3}\right)_{2}\right)$,

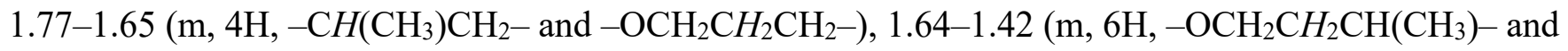
$\left.-\mathrm{OCH}_{2} \mathrm{CH}_{2} \mathrm{CH}_{2}-\right), 1.39-1.10\left(\mathrm{~m}, 16 \mathrm{H},-\mathrm{CH}\left(\mathrm{CH}_{3}\right)\left(\mathrm{CH}_{2}\right)_{3}-\right.$ and $\left.-\mathrm{O}\left(\mathrm{CH}_{2}\right)_{3}\left(\mathrm{CH}_{2}\right)_{2}-\right), 0.93(\mathrm{~d}, J=6.6 \mathrm{~Hz}$, $\left.6 \mathrm{H},-\mathrm{CH}_{2} \mathrm{CH}\left(\mathrm{CH}_{3}\right)-\right), 0.92-0.84\left(\mathrm{~m}, 15 \mathrm{H},-\mathrm{CH}\left(\mathrm{CH}_{3}\right)_{2}\right.$ and $\left.-\mathrm{CH}_{2} \mathrm{CH}_{3}\right) .{ }^{13} \mathrm{C} \mathrm{NMR}\left(126 \mathrm{MHz}, \mathrm{CDCl}_{3}, \delta\right.$, ppm): 153.40 ( $\mathrm{ArC}-3,5), 137.66$ ( $\mathrm{ArC}-4), 136.22$ ( $\mathrm{ArC}-1$ ), 105.42 ( $\mathrm{ArC}-2,6), 73.53$ ( $\mathrm{ArOCH}_{2}-4$ position), $67.52\left(\mathrm{ArOCH}_{2}-3,5\right.$ position), $65.77\left(-\mathrm{CH}_{2} \mathrm{OH}\right),[39.41,37.47,36.57,31.94,30.42,29.95,28.12,25.94$, 24.86] $\left(-\mathrm{OCH}_{2} \mathrm{CH}_{2} \mathrm{CH}\left(\mathrm{CH}_{3}\right)\left(\mathrm{CH}_{2}\right)_{3} \mathrm{CH}-\right.$ and $\left.-\mathrm{OCH}_{2}\left(\mathrm{CH}_{2}\right)_{3}-\right),[22.83,22.72,19.73,14.23]\left(-\mathrm{CH}_{(}\left(\mathrm{CH}_{3}\right)_{2}\right.$, $-\mathrm{CH}\left(\mathrm{CH}_{3}\right) \mathrm{CH}_{2}-$ and $\left.-\mathrm{CH}_{2} \mathrm{CH}_{3}\right)$. MALDI-TOF $\mathrm{MS} m / z$ of $[\mathrm{M}+\mathrm{Na}]^{+}$calculated for $\mathrm{C}_{33} \mathrm{H}_{60} \mathrm{O}_{4}$ : 543.4; Found: 544.2.

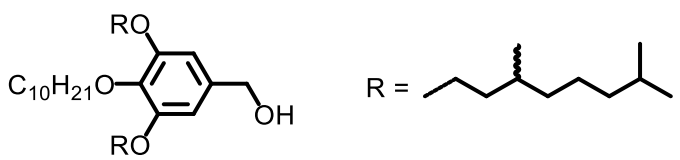

(4-(Decyloxy)-3,5-bis((3,7-(rac)-dimethyloctyl)oxy)phenyl)methanol (9-r10r). From 5-r10r (0.24 g, $0.40 \mathrm{mmol})$ and $\mathrm{LiAlH}_{4}(0.023 \mathrm{~g}, 0.60 \mathrm{mmol})$ in dry THF $(10 \mathrm{~mL})$ at $23{ }^{\circ} \mathrm{C}$ for $1 \mathrm{~h}, 9-\mathbf{9} 10 \mathrm{r}$ was obtained as a colorless oil. Product: $0.21 \mathrm{~g}$; Yield: 90\%. Purity by HPLC: $99+\%$. This was used in the next step without further purification.

${ }^{1} \mathrm{H}$ NMR (500 MHz, $\left.\mathrm{CDCl}_{3}, \delta, \mathrm{ppm}\right): 6.56$ (s, 2H, $\left.\mathrm{ArH}\right), 4.59$ (d, J=6.0 Hz, 2H, $\left.-\mathrm{CH}_{2} \mathrm{OH}\right), 4.06-3.95(\mathrm{~m}$, $\left.4 \mathrm{H},-\mathrm{OCH}_{2} \mathrm{CH}_{2} \mathrm{CH}\left(\mathrm{CH}_{3}\right)-\right), 3.92\left(\mathrm{t}, \mathrm{J}=6.6 \mathrm{~Hz}, 2 \mathrm{H},-\mathrm{OCH}_{2} \mathrm{CH}_{2} \mathrm{CH}_{2}-\right), 1.90-1.79\left(\mathrm{~m}, 2 \mathrm{H},-\mathrm{CH}\left(\mathrm{CH}_{3}\right)_{2}\right)$,

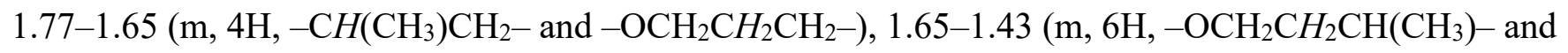
$\left.-\mathrm{OCH}_{2} \mathrm{CH}_{2} \mathrm{CH}_{2}-\right), 1.38-1.10\left(\mathrm{~m}, 24 \mathrm{H},-\mathrm{CH}\left(\mathrm{CH}_{3}\right)\left(\mathrm{CH}_{2}\right)_{3}-\right.$ and $\left.-\mathrm{O}\left(\mathrm{CH}_{2}\right)_{3}\left(\mathrm{CH}_{2}\right)_{6}-\right), 0.93(\mathrm{~d}, J=6.6 \mathrm{~Hz}$, $\left.6 \mathrm{H},-\mathrm{CH}_{2} \mathrm{CH}\left(\mathrm{CH}_{3}\right)-\right), 0.91-0.83\left(\mathrm{~m}, 15 \mathrm{H},-\mathrm{CH}\left(\mathrm{CH}_{3}\right)_{2}\right.$ and $\left.-\mathrm{CH}_{2} \mathrm{CH}_{3}\right) .{ }^{13} \mathrm{C} \mathrm{NMR}\left(126 \mathrm{MHz}, \mathrm{CDCl}_{3}, \delta\right.$, ppm): 153.42 ( $\mathrm{ArC}-3,5), 137.68$ ( $\mathrm{ArC}-4), 136.17$ ( $\mathrm{ArC}-1$ ), 105.42 ( $\mathrm{ArC}-2,6), 73.54$ ( $\mathrm{ArOCH}_{2}-4$ position), $67.52\left(\mathrm{ArOCH}_{2}-3,5\right.$ position), $65.83\left(-\mathrm{CH}_{2} \mathrm{OH}\right),[39.41,37.46,36.57,32.08,30.47,29.96,29.88,29.82$, 29.76, 29.53, 28.13, 26.28, 24.88] $\left(-\mathrm{OCH}_{2} \mathrm{CH}_{2} \mathrm{CH}\left(\mathrm{CH}_{3}\right)\left(\mathrm{CH}_{2}\right)_{3} \mathrm{CH}-\right.$ and $\left.-\mathrm{OCH}_{2}\left(\mathrm{CH}_{2}\right)_{7}-\right)$, [22.85, 22.74, 19.74, 14.26] $\left(-\mathrm{CH}\left(\mathrm{CH}_{3}\right)_{2},-\mathrm{CH}\left(\mathrm{CH}_{3}\right) \mathrm{CH}_{2}-\right.$ and $\left.-\mathrm{CH}_{2} \mathrm{CH}_{3}\right)$. MALDI-TOF MS m/z of $[\mathrm{M}+\mathrm{Na}]^{+}$ calculated for $\mathrm{C}_{37} \mathrm{H}_{68} \mathrm{O}_{4}$ : 599.5; Found: 599.8 . 


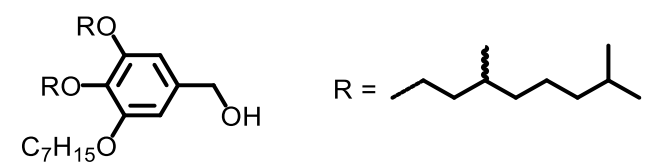

(3,4-Bis((3,7-(rac)-dimethyloctyl)oxy)-5-(heptyloxy)phenyl)methanol (9-rr7). From 5-rr7 (1.13 g, 2.0 mmol) and $\mathrm{LiAlH}_{4}(0.11 \mathrm{~g}, 3.0 \mathrm{mmol})$ in dry THF $(10 \mathrm{~mL})$ at $23{ }^{\circ} \mathrm{C}$ for $1 \mathrm{~h}, 9-\mathrm{rr} 7$ was obtained as a colorless oil. Product: 1.03 g; Yield: 96\%. Purity by HPLC: $99+\%$. This was used in the next step without further purification.

${ }^{1} \mathrm{H}$ NMR (400 MHz, $\left.\mathrm{CDCl}_{3}, \delta, \mathrm{ppm}\right): 6.55$ (s, 2H, $\mathrm{ArH}$ ), 4.58 (s, 2H, $\left.-\mathrm{CH}_{2} \mathrm{OH}\right), 4.07-3.89$ (m, 6H, $\left.\mathrm{OCH}_{2} \mathrm{CH}_{2}-\right), 1.93-1.65\left(\mathrm{~m}, 6 \mathrm{H},-\mathrm{OCH}_{2} \mathrm{CH}_{2} \mathrm{CH}_{2}-,-\mathrm{CH}\left(\mathrm{CH}_{3}\right)_{2}\right.$ and $\left.-\mathrm{CH}\left(\mathrm{CH}_{3}\right) \mathrm{CH}_{2}-\right), 1.65-1.41(\mathrm{~m}, 6 \mathrm{H}$, $-\mathrm{O}\left(\mathrm{CH}_{2}\right)_{2} \mathrm{CH}_{2}-$ and $\left.-\mathrm{OCH}_{2} \mathrm{CH}_{2} \mathrm{CH}\left(\mathrm{CH}_{3}\right)-\right), \quad 1.41-1.08 \quad\left(\mathrm{~m}, \quad 18 \mathrm{H}, \quad-\mathrm{CH}\left(\mathrm{CH}_{3}\right)\left(\mathrm{CH}_{2}\right)_{3}-\right.$ and $\left.\mathrm{O}\left(\mathrm{CH}_{2}\right)_{3}\left(\mathrm{CH}_{2}\right)_{3}-\right), 0.96-0.84\left(\mathrm{~m}, 21 \mathrm{H},-\mathrm{CH}_{3}\right) .{ }^{13} \mathrm{C} \mathrm{NMR}\left(101 \mathrm{MHz}, \mathrm{CDCl}_{3}, \delta, \mathrm{ppm}\right): 153.39(\mathrm{ArC}-3,5)$, 137.63 (ArC-4), 136.24 (ArC-1), [105.41, 105.37] (ArC-2,6), 71.80 ( $\mathrm{ArOCH}_{2}-5$ position), 69.20 (ArOCH $\mathrm{H}_{2}-4$ position), 67.50 ( $\mathrm{ArOCH}_{2}-3$ position), $65.74\left(-\mathrm{CH}_{2} \mathrm{OH}\right),[39.50,39.41,37.65,37.46,36.55$, $31.97,29.96,29.84,29.55,29.21,28.12,26.19,24.85]\left(-\mathrm{OCH}_{2} \mathrm{CH}_{2} \mathrm{CH}\left(\mathrm{CH}_{3}\right)\left(\mathrm{CH}_{2}\right)_{3} \mathrm{CH}-\right.$ and $\left.\mathrm{OCH}_{2}\left(\mathrm{CH}_{2}\right)_{4}-\right)$, [22.83, 22.74, 22.73, 19.74, 19.70, 14.21] $\left(-\mathrm{CH}\left(\mathrm{CH}_{3}\right)_{2},-\mathrm{CH}\left(\mathrm{CH}_{3}\right) \mathrm{CH}_{2}-\right.$ and $\left.-\mathrm{CH}_{2} \mathrm{CH}_{3}\right)$. MALDI-TOF MS $m / z$ of $[\mathrm{M}+\mathrm{Na}]^{+}$calculated for $\mathrm{C}_{34} \mathrm{H}_{62} \mathrm{O}_{4}$ : 557.5; Found: 558.1 .

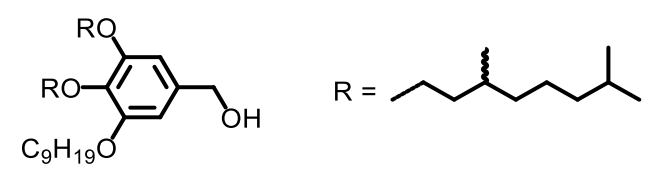

(3,4-Bis((3,7-(rac)-dimethyloctyl)oxy)-5-(nonyloxy)phenyl)methanol (9-rr9). From 5-rr9 (1.18 g, 2.0 mmol) and $\mathrm{LiAlH}_{4}(0.11 \mathrm{~g}, 3.0 \mathrm{mmol})$ in dry THF $(10 \mathrm{~mL})$ at $23{ }^{\circ} \mathrm{C}$ for $1 \mathrm{~h}$, 9-rr9 was obtained as a colorless oil. Product: $1.11 \mathrm{~g}$; Yield: $98 \%$. Purity by HPLC: $99+\%$. This was used in the next step without further purification.

${ }^{1} \mathrm{H}$ NMR (400 MHz, $\left.\mathrm{CDCl}_{3}, \delta, \mathrm{ppm}\right): 6.55$ (s, 2H, $\left.\mathrm{ArH}\right), 4.58\left(\mathrm{~s}, 2 \mathrm{H},-\mathrm{CH}_{2} \mathrm{OH}\right), 4.07-3.90(\mathrm{~m}, 6 \mathrm{H},-$ $\left.\mathrm{OCH}_{2} \mathrm{CH}_{2}-\right), 1.92-1.65\left(\mathrm{~m}, 6 \mathrm{H},-\mathrm{OCH}_{2} \mathrm{CH}_{2} \mathrm{CH}_{2}-,-\mathrm{CH}\left(\mathrm{CH}_{3}\right)_{2}\right.$ and $\left.-\mathrm{CH}\left(\mathrm{CH}_{3}\right) \mathrm{CH}_{2}-\right), 1.65-1.41(\mathrm{~m}, 6 \mathrm{H}$, $-\mathrm{O}\left(\mathrm{CH}_{2}\right)_{2} \mathrm{CH}_{2}-$ and $\left.-\mathrm{OCH}_{2} \mathrm{CH}_{2} \mathrm{CH}\left(\mathrm{CH}_{3}\right)-\right), \quad 1.41-1.08 \quad\left(\mathrm{~m}, \quad 22 \mathrm{H}, \quad-\mathrm{CH}\left(\mathrm{CH}_{3}\right)\left(\mathrm{CH}_{2}\right)_{3}-\right.$ and $\left.\mathrm{O}\left(\mathrm{CH}_{2}\right)_{3}\left(\mathrm{CH}_{2}\right)_{5}-\right), 0.97-0.80\left(\mathrm{~m}, 21 \mathrm{H},-\mathrm{CH}_{3}\right) .{ }^{13} \mathrm{C} \mathrm{NMR}\left(101 \mathrm{MHz}, \mathrm{CDCl}_{3}, \delta, \mathrm{ppm}\right): 153.40(\mathrm{ArC}-3,5)$, 137.65 (ArC-4), 136.24 (ArC-1), [105.42, 105.38] (ArC-2,6), 71.80 ( $\mathrm{ArOCH}_{2}-5$ position), 69.21 (ArOCH $\mathrm{H}_{2}-4$ position), 67.51 ( $\mathrm{ArOCH}_{2}-3$ position), $65.75\left(-\mathrm{CH}_{2} \mathrm{OH}\right),[39.51,39.42,37.66,37.48,36.56$, $32.03,29.97,29.84,29.74,29.56,29.43,28.12,26.24,24.86]\left(-\mathrm{OCH}_{2} \mathrm{CH}_{2} \mathrm{CH}\left(\mathrm{CH}_{3}\right)\left(\mathrm{CH}_{2}\right){ }_{3} \mathrm{CH}-\right.$ and $\left.\mathrm{OCH}_{2}\left(\mathrm{CH}_{2}\right)_{6}-\right)$, [22.84, 22.81, 22.75, 22.73, 19.75, 19.71, 14.24] $\left(-\mathrm{CH}\left(\mathrm{CH}_{3}\right)_{2},-\mathrm{CH}\left(\mathrm{CH}_{3}\right) \mathrm{CH}_{2}-\right.$ and $\mathrm{CH}_{2} \mathrm{CH}_{3}$ ). MALDI-TOF MS m/z of [M+Na] ${ }^{+}$calculated for $\mathrm{C}_{36} \mathrm{H}_{66} \mathrm{O}_{4}$ : 585.5; Found: 585.3. 


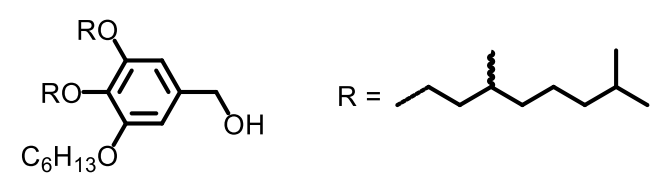

(3,4-Bis((3,7-(rac)-dimethyloctyl)oxy)-5-(hexyloxy)phenyl)methanol (9-rr6). From 5-rr6 (0.44 g, 0.8 mmol) and $\mathrm{LiAlH}_{4}(0.046 \mathrm{~g}, 1.2 \mathrm{mmol})$ in dry THF $(6 \mathrm{~mL})$ at $23{ }^{\circ} \mathrm{C}$ for $1 \mathrm{~h}, 9$-rr6 was obtained as a colorless oil. Product: 0.41 g; Yield: 98\%. Purity by HPLC: $99+\%$. This was used in the next step without further purification.

${ }^{1} \mathrm{H}$ NMR (400 MHz, $\left.\mathrm{CDCl}_{3}, \delta, \mathrm{ppm}\right): 6.56$ (s, 2H, $\left.\mathrm{ArH}\right), 4.59$ (s, 2H, $\left.-\mathrm{CH}_{2} \mathrm{OH}\right), 4.07-3.90$ (m, 6H, $\left.\mathrm{OCH}_{2} \mathrm{CH}_{2}-\right), 1.90-1.65\left(\mathrm{~m}, 6 \mathrm{H},-\mathrm{OCH}_{2} \mathrm{CH}_{2} \mathrm{CH}_{2}-,-\mathrm{CH}\left(\mathrm{CH}_{3}\right)_{2}\right.$ and $\left.-\mathrm{CH}\left(\mathrm{CH}_{3}\right) \mathrm{CH}_{2}-\right), 1.65-1.42(\mathrm{~m}, 6 \mathrm{H}$, $-\mathrm{O}\left(\mathrm{CH}_{2}\right)_{2} \mathrm{CH}_{2}-$ and $\left.-\mathrm{OCH}_{2} \mathrm{CH}_{2} \mathrm{CH}\left(\mathrm{CH}_{3}\right)-\right), \quad 1.39-1.09 \quad\left(\mathrm{~m}, \quad 16 \mathrm{H}, \quad-\mathrm{CH}\left(\mathrm{CH}_{3}\right)\left(\mathrm{CH}_{2}\right)_{3}-\right.$ and $\left.\mathrm{O}\left(\mathrm{CH}_{2}\right)_{3}\left(\mathrm{CH}_{2}\right)_{2}-\right), 0.98-0.83\left(\mathrm{~m}, 21 \mathrm{H},-\mathrm{CH}_{3}\right) .{ }^{13} \mathrm{C} \mathrm{NMR}\left(101 \mathrm{MHz}, \mathrm{CDCl}_{3}, \delta, \mathrm{ppm}\right): 153.42(\mathrm{ArC}-3,5)$, 137.69 (ArC-4), 136.20 (ArC-1), [105.43, 105.39] (ArC-2,6), 71.80 ( $\mathrm{ArOCH}_{2}-5$ position), 69.22 ( $\mathrm{ArOCH} \mathrm{H}_{2}-4$ position), $67.52\left(\mathrm{ArOCH}_{2}-3\right.$ position), $65.81\left(-\mathrm{CH}_{2} \mathrm{OH}\right),[39.51,39.42,37.66,37.48,36.56$, $31.73,29.97,29.84,29.51,28.13,25.91,24.86]\left(-\mathrm{OCH}_{2} \mathrm{CH}_{2} \mathrm{CH}\left(\mathrm{CH}_{3}\right)\left(\mathrm{CH}_{2}\right)_{3} \mathrm{CH}-\right.$ and $\left.-\mathrm{OCH}_{2}\left(\mathrm{CH}_{2}\right)_{3}-\right)$, $[22.85,22.78,22.74,19.75,19.71,14.18]\left(-\mathrm{CH}\left(\mathrm{CH}_{3}\right)_{2},-\mathrm{CH}\left(\mathrm{CH}_{3}\right) \mathrm{CH}_{2}-\right.$ and $\left.-\mathrm{CH}_{2} \mathrm{CH}_{3}\right)$. MALDI-TOF MS $m / z$ of $[\mathrm{M}+\mathrm{Na}]^{+}$calculated for $\mathrm{C}_{33} \mathrm{H}_{60} \mathrm{O}_{4}$ : 543.4; Found: 543.5 .

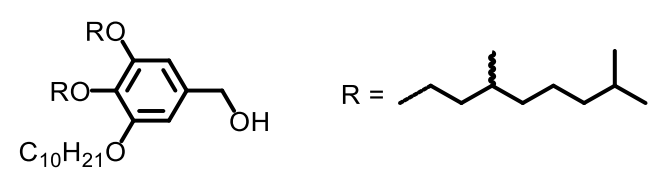

(3-(decyloxy)-4,5-bis((3,7-dimethyloctyl)oxy)phenyl)methanol (9-rr10). From 5-rr10 (0.48 g, 0.8 mmol) and $\mathrm{LiAlH}_{4}(0.046 \mathrm{~g}, 1.2 \mathrm{mmol})$ in dry THF $(6 \mathrm{~mL})$ at $23{ }^{\circ} \mathrm{C}$ for $1 \mathrm{~h}, 9$-rr10 was obtained as a colorless oil. Product: 0.45 g; Yield: 98\%. Purity by HPLC: $99+\%$. This was used in the next step without further purification.

${ }^{1} \mathrm{H}$ NMR (400 MHz, $\mathrm{CDCl}_{3}, \delta$, ppm): 6.56 (overlapping s, 2H, $\mathrm{ArH}$ ), 4.59 (s, J=3.0 Hz, 2H, $-\mathrm{CH} H_{2} \mathrm{OH}$ ), 4.06-3.90 (m, 6H, $\left.-\mathrm{OCH}_{2} \mathrm{CH}_{2}-\right), 1.91-1.40\left(\mathrm{~m}, 12 \mathrm{H},-\mathrm{OCH}_{2} \mathrm{CH}_{2} \mathrm{CH}_{2}-,-\mathrm{CH}\left(\mathrm{CH}_{3}\right)_{2},-\mathrm{CH}_{\left(\mathrm{CH}_{3}\right) \mathrm{CH}_{2}-,-}\right.$ $\mathrm{O}\left(\mathrm{CH}_{2}\right)_{2} \mathrm{CH}_{2}-$ and $\left.-\mathrm{OCH}_{2} \mathrm{CH}_{2} \mathrm{CH}\left(\mathrm{CH}_{3}\right)-\right), 1.40-1.09\left(\mathrm{~m}, 24 \mathrm{H},-\mathrm{CH}\left(\mathrm{CH}_{3}\right)\left(\mathrm{CH}_{2}\right)_{3}-\right.$ and $\left.-\mathrm{O}\left(\mathrm{CH}_{2}\right)_{3}\left(\mathrm{CH}_{2}\right)_{6}-\right)$, 0.97-0.80 (m, 21H, $\left.-\mathrm{CH}_{3}\right) .{ }^{13} \mathrm{C} \mathrm{NMR}\left(101 \mathrm{MHz}, \mathrm{CDCl}_{3}, \delta, \mathrm{ppm}\right): 153.45$ (ArC-3,5), 137.73 (ArC-4), 136.18 (ArC-1), [105.46, 105.41] (ArC-2,6), $71.80\left(\mathrm{ArOCH}_{2}-3\right.$ position), 69.23 ( $\mathrm{ArOCH}_{2}-4$ position), $67.53\left(\mathrm{ArOCH}_{2}-5\right.$ position), $65.84\left(-\mathrm{CH}_{2} \mathrm{OH}\right),[39.52,39.43,37.66,37.49,36.56,32.05,29.98,29.85$, $29.79,29.74,29.56,29.49,28.14,26.25,24.87]\left(-\mathrm{OCH}_{2} \mathrm{CH}_{2} \mathrm{CH}\left(\mathrm{CH}_{3}\right)\left(\mathrm{CH}_{2}\right)_{3} \mathrm{CH}-\right.$ and $\left.-\mathrm{OCH}_{2}\left(\mathrm{CH}_{2}\right)_{7}-\right)$, $[22.86,22.83,22.76,22.75,19.76,19.72,14.26]\left(-\mathrm{CH}\left(\mathrm{CH}_{3}\right)_{2},-\mathrm{CH}\left(\mathrm{CH}_{3}\right) \mathrm{CH}_{2}-\right.$ and $\left.-\mathrm{CH}_{2} \mathrm{CH}_{3}\right)$. MALDITOF MS $m / z$ of $[\mathrm{M}+\mathrm{Na}]^{+}$calculated for $\mathrm{C}_{37} \mathrm{H}_{68} \mathrm{O}_{4}$ : 599.5; Found: 599.5. 
<smiles>[R]=CCCC(C)CCCC(C)C</smiles>

(3-((3,7-(rac)-Dimethyloctyl)oxy)-4,5-bis(heptyloxy)phenyl)methanol (9-77r). From 5-77r (1.04 g, 2.0 mmol) and $\mathrm{LiAlH}_{4}(0.11 \mathrm{~g}, 3.0 \mathrm{mmol})$ in dry THF $(10 \mathrm{~mL})$ at $23{ }^{\circ} \mathrm{C}$ for $1 \mathrm{~h}, \mathbf{9 - 7 7} \mathbf{r}$ was obtained as a colorless oil. Product: 0.96 g; Yield: 98\%. Purity by HPLC: $99+\%$. This was used in the next step without further purification.

${ }^{1} \mathrm{H}$ NMR (400 MHz, $\mathrm{CDCl}_{3}, \delta$, ppm): 6.55 (overlapping s, 2H, $\mathrm{Ar} H$ ), 4.59 (d, J=4.7 Hz, 2H, $-\mathrm{CH}_{2} \mathrm{OH}$ ), 4.07-3.88 (m, 6H, $\left.-\mathrm{OCH}_{2}-\right)$, 1.91-1.40 (m, $12 \mathrm{H},-\mathrm{OCH}_{2} \mathrm{CH}_{2} \mathrm{CH}_{2}-,-\mathrm{CH}\left(\mathrm{CH}_{3}\right)_{2},-\mathrm{CH}_{\left(\mathrm{CH}_{3}\right) \mathrm{CH}_{2}-},-$ $\mathrm{O}\left(\mathrm{CH}_{2}\right)_{2} \mathrm{CH}_{2}-$ and $\left.-\mathrm{OCH}_{2} \mathrm{CH}_{2} \mathrm{CH}\left(\mathrm{CH}_{3}\right)-\right), 1.40-1.08\left(\mathrm{~m}, 18 \mathrm{H},-\mathrm{CH}\left(\mathrm{CH}_{3}\right)\left(\mathrm{CH}_{2}\right)_{3}-\right.$ and $\left.-\mathrm{O}\left(\mathrm{CH}_{2}\right)_{3}\left(\mathrm{CH}_{2}\right)_{3}-\right)$, $0.93\left(\mathrm{~d}, J=6.6 \mathrm{~Hz}, 3 \mathrm{H},-\mathrm{CH}_{2} \mathrm{CH}\left(\mathrm{CH}_{3}\right)-\right), 0.91-0.84\left(\mathrm{~m}, 12 \mathrm{H},-\mathrm{CH}\left(\mathrm{CH}_{3}\right)_{2}\right.$ and $\left.-\mathrm{CH}_{2} \mathrm{CH}_{3}\right) .{ }^{13} \mathrm{C} \mathrm{NMR}(101$ $\mathrm{MHz}, \mathrm{CDCl}_{3}, \delta$, ppm): 153.40 (ArC-3,5), 137.68 (ArC-4), 136.20 (ArC-1), [105.47, 105.42] (ArC-2,6), 73.56 ( $\mathrm{ArOCH}_{2}-4$ position), $69.23\left(\mathrm{ArOCH}_{2}-5\right.$ position), $67.53\left(\mathrm{ArOCH}_{2}-3\right.$ position), $65.78\left(-\mathrm{CH}_{2} \mathrm{OH}\right)$, $[39.41,37.46,36.57,32.06,31.97,30.46,29.96,29.56,29.40,29.21,28.13,26.21,26.19,24.86]$ ($\mathrm{OCH}_{2} \mathrm{CH}_{2} \mathrm{CH}\left(\mathrm{CH}_{3}\right)\left(\mathrm{CH}_{2}\right)_{3} \mathrm{CH}-$ and $\left.-\mathrm{OCH}_{2}\left(\mathrm{CH}_{2}\right)_{4}-\right)$, [22.83, 22.81, 22.75, 22.73, 19.77, 14.24, 14.22] ($\mathrm{CH}\left(\mathrm{CH}_{3}\right)_{2},-\mathrm{CH}\left(\mathrm{CH}_{3}\right) \mathrm{CH}_{2}-$ and $\left.-\mathrm{CH}_{2} \mathrm{CH}_{3}\right)$. MALDI-TOF MS $m / z$ of $[\mathrm{M}+\mathrm{Na}]^{+}$calculated for $\mathrm{C}_{31} \mathrm{H}_{56} \mathrm{O}_{4}$ : 515.4; Found: 515.4.
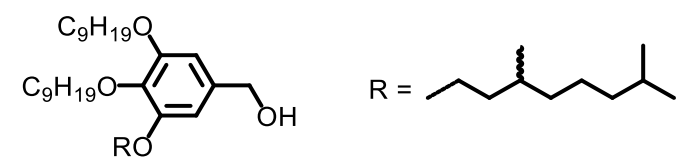

(3-((3,7-(rac)-Dimethyloctyl)oxy)-4,5-bis(nonyloxy)phenyl)methanol (9-99r). From 5-99r (1.15 g, 2.0 mmol) and $\mathrm{LiAlH}_{4}(0.11 \mathrm{~g}, 3.0 \mathrm{mmol})$ in dry THF $(10 \mathrm{~mL})$ at $23{ }^{\circ} \mathrm{C}$ for $1 \mathrm{~h}, \mathbf{9 - 9 9 r}$ was obtained as a colorless oil. Product: 1.06 g; Yield: 97\%. Purity by HPLC: 99+\%. This was used in the next step without further purification.

${ }^{1} \mathrm{H} \mathrm{NMR}\left(400 \mathrm{MHz}, \mathrm{CDCl}_{3}, \delta, \mathrm{ppm}\right): 6.56(\mathrm{~s}, 2 \mathrm{H}, \mathrm{ArH}), 4.59\left(\mathrm{~d}, J=5.3 \mathrm{~Hz}, 2 \mathrm{H},-\mathrm{CH}_{2} \mathrm{OH}\right), 4.07-3.88(\mathrm{~m}$,

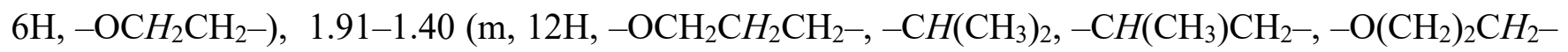
and $\left.-\mathrm{OCH}_{2} \mathrm{CH}_{2} \mathrm{CH}\left(\mathrm{CH}_{3}\right)-\right), 1.40-1.08\left(\mathrm{~m}, 26 \mathrm{H},-\mathrm{CH}\left(\mathrm{CH}_{3}\right)\left(\mathrm{CH}_{2}\right)_{3}-\right.$ and $\left.-\mathrm{O}\left(\mathrm{CH}_{2}\right)_{3}\left(\mathrm{CH}_{2}\right)_{5}-\right), 0.93(\mathrm{~d}, J=$ $\left.6.6 \mathrm{~Hz}, 3 \mathrm{H},-\mathrm{CH}_{2} \mathrm{CH}\left(\mathrm{CH}_{3}\right)-\right), 0.91-0.83\left(\mathrm{~m}, 12 \mathrm{H},-\mathrm{CH}\left(\mathrm{CH}_{3}\right)_{2}\right.$ and $\left.-\mathrm{CH}_{2} \mathrm{CH}_{3}\right) .{ }^{13} \mathrm{C} \mathrm{NMR}(101 \mathrm{MHz}$, $\mathrm{CDCl}_{3}, \delta$, ppm): 153.42 (ArC-3,5), 137.72 (ArC-4), 136.18 (ArC-1), [105.49, 105.44] (ArC-2,6), 73.56 ( $\mathrm{ArOCH} \mathrm{H}_{2}-4$ position), 69.24 ( $\mathrm{ArOCH}_{2}-5$ position), 67.54 ( $\mathrm{ArOCH}_{2}-3$ position), $65.81\left(-\mathrm{CH}_{2} \mathrm{OH}\right)$, [39.41, 37.47, 36.57, 32.09, 32.04, 30.47, 29.97, 29.83, 29.76, 29.74, 29.56, 29.52, 29.44, 28.13, 26.28, 26.24, 24.87] $\left(-\mathrm{OCH}_{2} \mathrm{CH}_{2} \mathrm{CH}\left(\mathrm{CH}_{3}\right)\left(\mathrm{CH}_{2}\right)_{3} \mathrm{CH}-\right.$ and $\left.-\mathrm{OCH}_{2}\left(\mathrm{CH}_{2}\right)_{6}-\right)$, [22.84, 22.73, 19.74, 14.24] $\left(-\mathrm{CH}\left(\mathrm{CH}_{3}\right)_{2}\right.$, $-\mathrm{CH}\left(\mathrm{CH}_{3}\right) \mathrm{CH}_{2}-$ and $\left.-\mathrm{CH}_{2} \mathrm{CH}_{3}\right)$. MALDI-TOF MS $m / z$ of $[\mathrm{M}+\mathrm{Na}]^{+}$calculated for $\mathrm{C}_{35} \mathrm{H}_{64} \mathrm{O}_{4}$ : 571.5; Found: 571.7. 
<smiles>[R]CCCC(C)CCCC(C)C</smiles>

(3-((3,7-(rac)-Dimethyloctyl)oxy)-4,5-bis(hexyloxy)phenyl)methanol (9-66r). From 5-66r (0.39 g, 0.8 mmol) and $\mathrm{LiAlH}_{4}(0.046 \mathrm{~g}, 1.2 \mathrm{mmol})$ in dry THF $(6 \mathrm{~mL})$ at $23{ }^{\circ} \mathrm{C}$ for $1 \mathrm{~h}, \mathbf{9 - 6 6 r}$ was obtained as a colorless oil. Product: 0.37 g; Yield: 99\%. Purity by HPLC: $99+\%$. This was used in the next step without further purification.

${ }^{1} \mathrm{H}$ NMR (400 MHz, $\mathrm{CDCl}_{3}, \delta, \mathrm{ppm}$ ): 6.56 (overlapping s, 2H, $\mathrm{ArH}$ ), 4.59 (d, J=5.7 Hz, 2H, $-\mathrm{CH} \mathrm{H}_{2} \mathrm{OH}$ ),

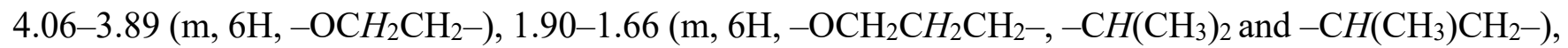
1.66-1.42 (m, 6H, $-\mathrm{O}\left(\mathrm{CH}_{2}\right)_{2} \mathrm{CH}_{2}-$ and $\left.-\mathrm{OCH}_{2} \mathrm{CH}_{2} \mathrm{CH}\left(\mathrm{CH}_{3}\right)-\right), 1.39-1.08\left(\mathrm{~m}, 14 \mathrm{H},-\mathrm{CH}\left(\mathrm{CH}_{3}\right)\left(\mathrm{CH}_{2}\right)_{3}-\right.$ and $\left.-\mathrm{O}\left(\mathrm{CH}_{2}\right)_{3}\left(\mathrm{CH}_{2}\right)_{2}-\right), 0.96-0.83\left(\mathrm{~m}, 15 \mathrm{H},-\mathrm{CH}_{3}\right) .{ }^{13} \mathrm{C} \mathrm{NMR}\left(101 \mathrm{MHz}, \mathrm{CDCl}_{3}, \delta\right.$, ppm): $153.44(\mathrm{ArC}-$ 3,5), 137.73 (ArC-4), 136.16 (ArC-1), [105.49, 105.44] (ArC-2,6), 73.56 ( $\mathrm{ArOCH}_{2}-4$ position), 69.25 (ArOCH $2-5$ position), 67.54 ( $\mathrm{ArOCH}_{2}-3$ position), $65.84\left(-\mathrm{CH}_{2} \mathrm{OH}\right),[39.42,37.47,36.57,31.94,31.72$, $30.42,29.96,29.52,28.14,25.93,25.90,24.87]\left(-\mathrm{OCH}_{2} \mathrm{CH}_{2} \mathrm{CH}\left(\mathrm{CH}_{3}\right)\left(\mathrm{CH}_{2}\right)_{3} \mathrm{CH}-\right.$ and $\left.-\mathrm{OCH}_{2}\left(\mathrm{CH}_{2}\right)_{3}-\right)$, $[22.84,22.77,22.74,19.74,14.25,14.18]\left(-\mathrm{CH}\left(\mathrm{CH}_{3}\right)_{2},-\mathrm{CH}\left(\mathrm{CH}_{3}\right) \mathrm{CH}_{2}-\right.$ and $\left.-\mathrm{CH}_{2} \mathrm{CH}_{3}\right)$. MALDI-TOF MS $m / z$ of $[\mathrm{M}+\mathrm{Na}]^{+}$calculated for $\mathrm{C}_{29} \mathrm{H}_{52} \mathrm{O}_{4}$ : 487.4; Found: 487.1 .

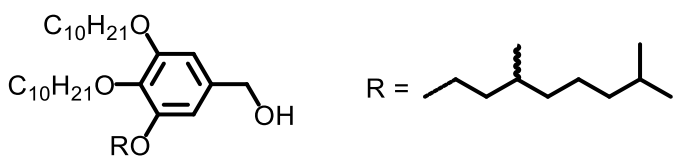

(3,4-bis(decyloxy)-5-((3,7-dimethyloctyl)oxy)phenyl)methanol (9-1010r). From 5-1010r (0.48 g, 0.8 mmol) and $\mathrm{LiAlH}_{4}(0.046 \mathrm{~g}, 1.2 \mathrm{mmol})$ in dry THF $(6 \mathrm{~mL})$ at $23{ }^{\circ} \mathrm{C}$ for $1 \mathrm{~h}, \mathbf{9 - 1 0 1 0 r}$ was obtained as a colorless oil. Product: 0.46 g; Yield: 99\%. Purity by HPLC: 99+\%. This was used in the next step without further purification.

${ }^{1} \mathrm{H}$ NMR (400 MHz, $\left.\mathrm{CDCl}_{3}, \delta, \mathrm{ppm}\right): 6.56$ (s, 2H, $\mathrm{ArH}$ ), 4.59 (s, 2H, $\left.-\mathrm{CH}_{2} \mathrm{OH}\right), 4.06-3.89$ (m, 6H, -

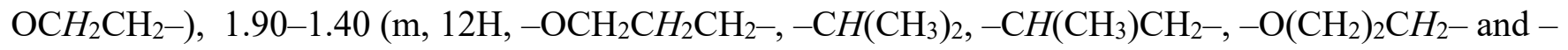
$\left.\mathrm{OCH}_{2} \mathrm{CH}_{2} \mathrm{CH}\left(\mathrm{CH}_{3}\right)-\right), 1.39-1.10\left(\mathrm{~m}, 30 \mathrm{H},-\mathrm{CH}\left(\mathrm{CH}_{3}\right)\left(\mathrm{CH}_{2}\right)_{3}-\right.$ and $\left.-\mathrm{O}\left(\mathrm{CH}_{2}\right)_{3}\left(\mathrm{CH}_{2}\right)_{6}-\right), 0.93(\mathrm{~d}, J=6.6 \mathrm{~Hz}$, $\left.3 \mathrm{H},-\mathrm{CH}_{2} \mathrm{CH}\left(\mathrm{CH}_{3}\right)-\right), 0.91-0.82\left(\mathrm{~m}, 12 \mathrm{H},-\mathrm{CH}\left(\mathrm{CH}_{3}\right)_{2}\right.$ and $\left.-\mathrm{CH}_{2} \mathrm{CH}_{3}\right) .{ }^{13} \mathrm{C} \mathrm{NMR}\left(101 \mathrm{MHz}, \mathrm{CDCl}_{3}, \delta\right.$, ppm): 153.44 (ArC-3,5), 137.73 (ArC-4), 136.16 (ArC-1), [105.49, 105.45] (ArC-2,6), $73.56\left(\mathrm{ArOCH}_{2}-\right.$ 4 position), 69.25 ( $\mathrm{ArOCH}_{2}-3$ position), $67.55\left(\mathrm{ArOCH}_{2}-5\right.$ position), $65.84\left(-\mathrm{CH}_{2} \mathrm{OH}\right),[39.42,37.47$, $36.57,32.09,32.06,30.48,29.97,29.89,29.83,29.80,29.77,29.74,29.56,29.55,29.50,28.14,26.29$, 26.25, 24.88] $\left(-\mathrm{OCH}_{2} \mathrm{CH}_{2} \mathrm{CH}\left(\mathrm{CH}_{3}\right)\left(\mathrm{CH}_{2}\right)_{3} \mathrm{CH}-\right.$ and $\left.-\mathrm{OCH}_{2}\left(\mathrm{CH}_{2}\right)_{7}-\right)$, [22.85, 22.74, 19.75, 14.26] ($\mathrm{CH}\left(\mathrm{CH}_{3}\right)_{2},-\mathrm{CH}\left(\mathrm{CH}_{3}\right) \mathrm{CH}_{2}-$ and $\left.-\mathrm{CH}_{2} \mathrm{CH}_{3}\right)$. MALDI-TOF MS $m / z$ of $[\mathrm{M}+\mathrm{Na}]^{+}$calculated for $\mathrm{C}_{37} \mathrm{H}_{68} \mathrm{O}_{4}$ : 599.5; Found: 599.9. 
General procedure for preparation of minidendron chlorides $\mathbf{1 0}$. The synthesis of minidendron chlorides $\mathbf{1 0}$ was conducted according to a procedure reported by our group. ${ }^{9}$ A general example is provided below.

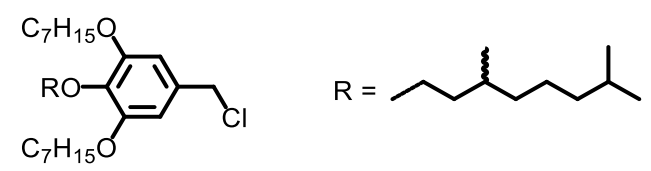

5-(Chloromethyl)-2-((3,7-(rac)-dimethyloctyl)oxy)-1,3-bis(heptyloxy)benzene (10-7r7). To a solution of 9-7r7 $(0.91 \mathrm{~g}, 1.85 \mathrm{mmol})$ in dry DCM $(10 \mathrm{~mL})$ was added a catalytic amount of DMF $(30 \mu \mathrm{L})$, followed by the dropwise addition of $\mathrm{SOCl}_{2}(0.17 \mathrm{~mL}, 2.3 \mathrm{mmol})$ with a syringe. Then the reaction mixture was stirred at $23{ }^{\circ} \mathrm{C}$ for $30 \mathrm{~min}$. After the completion of reaction, the reaction mixture was evaporated in vacuo to remove DCM and excessive $\mathrm{SOCl}_{2}$, giving 10-7r7 as a light yellow oil. Yield: $100 \%$. This was used in the next step without further purification.

${ }^{1} \mathrm{H}$ NMR (400 MHz, $\mathrm{CDCl}_{3}, \delta$, ppm): 6.57 (s, 2H, $\left.\mathrm{ArH}\right), 4.51\left(\mathrm{~s}, 2 \mathrm{H},-\mathrm{CH} \mathrm{Cl}_{2}\right), 4.04-3.92\left(\mathrm{~m}, 6 \mathrm{H},-\mathrm{OCH}_{2}-\right.$ ), 1.88-1.75 (m, 5H, $-\mathrm{CH}\left(\mathrm{CH}_{3}\right)_{2}$ and $\left.-\mathrm{OCH}_{2} \mathrm{CH}_{2} \mathrm{CH}_{2}-\right), 1.75-1.66\left(\mathrm{~m}, 1 \mathrm{H},-\mathrm{CH}\left(\mathrm{CH}_{3}\right) \mathrm{CH}_{2}-\right), 1.58-1.42$ $\left(\mathrm{m}, 6 \mathrm{H},-\mathrm{O}\left(\mathrm{CH}_{2}\right)_{2} \mathrm{CH}_{2}-\right.$ and $\left.-\mathrm{OCH}_{2} \mathrm{CH}_{2} \mathrm{CH}\left(\mathrm{CH}_{3}\right)-\right), 1.42-1.09\left(\mathrm{~m}, 18 \mathrm{H},-\mathrm{CH}\left(\mathrm{CH}_{3}\right)\left(\mathrm{CH}_{2}\right)_{3}-\right.$ and $\left.\left(\mathrm{CH}_{2}\right)_{3}\left(\mathrm{CH}_{2}\right)_{3}-\right), 0.92\left(\mathrm{~d}, J=6.8 \mathrm{~Hz}, 3 \mathrm{H},-\mathrm{CH}_{2} \mathrm{CH}\left(\mathrm{CH}_{3}\right)^{-}\right), 0.91-0.83\left(\mathrm{~m}, 12 \mathrm{H},-\mathrm{CH}\left(\mathrm{CH}_{3}\right)_{2}\right.$ and $\mathrm{CH}_{2} \mathrm{CH}_{3}$ ). ${ }^{13} \mathrm{C}$ NMR (101 MHz, $\mathrm{CDCl}_{3}, \delta$, ppm): 153.36 (ArC-1,3), 138.44 (ArC-2), 132.47 (ArC-5), 107.18 ( $\mathrm{ArC}-4,6), 71.82\left(\mathrm{ArOCH}_{2}-1,3\right.$ position), 69.25 ( $\mathrm{ArOCH}_{2}-2$ position), $47.11\left(-\mathrm{CH}_{2} \mathrm{Cl}\right)$, [39.50, $37.64, \quad 37.47, \quad 31.97,29.83,29.52,29.21,28.13,26.18,24.86] \quad\left(-\mathrm{OCH}_{2}\left(\mathrm{CH}_{2}\right)_{4}-\right.$ and -

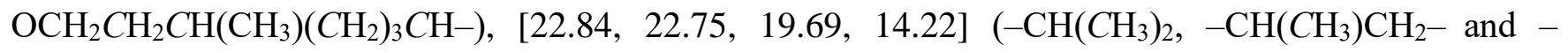
$\mathrm{CH}_{2} \mathrm{CH}_{3}$ ). MALDI-TOF MS m/z of $[\mathrm{M}+\mathrm{Na}]^{+}$calculated for $\mathrm{C}_{31} \mathrm{H}_{55} \mathrm{ClO}_{3}$ : 533.4; Found: 533.2 .

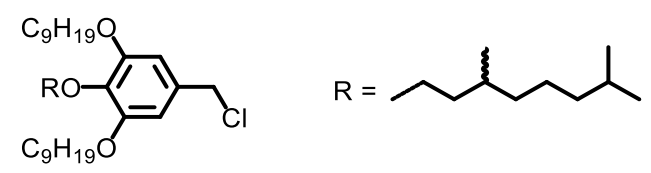

5-(Chloromethyl)-2-((3,7-(rac)-dimethyloctyl)oxy)-1,3-bis(nonyloxy)benzene (10-9r9). From 9-9r9 $(1.02 \mathrm{~g}, 1.85 \mathrm{mmol})$, DMF $(30 \mu \mathrm{L})$ and $\mathrm{SOCl}_{2}(0.17 \mathrm{~mL}, 2.3 \mathrm{mmol})$ in dry DCM $(10 \mathrm{~mL})$ at $23{ }^{\circ} \mathrm{C}$ for 30 min, 10-9r9 was obtained as a light yellow oil in quantitative yield. This was used in the next step without further purification.

${ }^{1} \mathrm{H} \mathrm{NMR}\left(400 \mathrm{MHz}, \mathrm{CDCl}_{3}, \delta, \mathrm{ppm}\right): 6.57$ (s, 2H, $\left.\mathrm{ArH}\right), 4.51\left(\mathrm{~s}, 2 \mathrm{H},-\mathrm{CH}_{2} \mathrm{Cl}\right), 4.06-3.90\left(\mathrm{~m}, 6 \mathrm{H},-\mathrm{OCH}_{2}-\right.$ ), 1.88-1.75 (m, 5H, $-\mathrm{CH}\left(\mathrm{CH}_{3}\right)_{2}$ and $\left.-\mathrm{OCH}_{2} \mathrm{CH}_{2} \mathrm{CH}_{2}-\right)$, 1.75-1.66 (m, 1H, $\left.-\mathrm{CH}\left(\mathrm{CH}_{3}\right) \mathrm{CH}_{2}-\right), 1.58-1.42$ $\left(\mathrm{m}, 6 \mathrm{H},-\mathrm{O}\left(\mathrm{CH}_{2}\right)_{2} \mathrm{CH}_{2}-\right.$ and $\left.-\mathrm{OCH}_{2} \mathrm{CH}_{2} \mathrm{CH}\left(\mathrm{CH}_{3}\right)-\right), 1.41-1.09\left(\mathrm{~m}, 26 \mathrm{H},-\mathrm{CH}\left(\mathrm{CH}_{3}\right)\left(\mathrm{CH}_{2}\right)_{3}-\right.$ and $\left.\left(\mathrm{CH}_{2}\right)_{3}\left(\mathrm{CH}_{2}\right)_{5}-\right), 0.92\left(\mathrm{~d}, J=6.6 \mathrm{~Hz}, 3 \mathrm{H},-\mathrm{CH}_{2} \mathrm{CH}\left(\mathrm{CH}_{3}\right)-\right), 0.91-0.84\left(\mathrm{~m}, 12 \mathrm{H},-\mathrm{CH}\left(\mathrm{CH}_{3}\right)_{2}\right.$ and $\left.\mathrm{CH}_{2} \mathrm{CH}_{3}\right) .{ }^{13} \mathrm{C}$ NMR (101 MHz, $\left.\mathrm{CDCl}_{3}, \delta, \mathrm{ppm}\right): 153.37$ (ArC-1,3), 138.44 (ArC-2), 132.47 (ArC-5), 107.19 (ArC-4,6), 71.82 ( $\mathrm{ArOCH}_{2}-1,3$ position), 69.26 ( $\mathrm{ArOCH}_{2}-2$ position), $47.12\left(-\mathrm{CH}_{2} \mathrm{Cl}\right),[39.51$, $37.65,37.48,32.04,29.84,29.73,29.56,29.52,29.43,28.14,26.23,24.86]\left(-\mathrm{OCH}_{2}\left(\mathrm{CH}_{2}\right)_{6}-\right.$ and $\left.\mathrm{OCH}_{2} \mathrm{CH}_{2} \mathrm{CH}\left(\mathrm{CH}_{3}\right)\left(\mathrm{CH}_{2}\right)_{3} \mathrm{CH}-\right)$, [22.85, 22.82, 22.75, 19.70, 14.24] $\left(-\mathrm{CH}\left(\mathrm{CH}_{3}\right)_{2},-\mathrm{CH}\left(\mathrm{CH}_{3}\right) \mathrm{CH}_{2}-\right.$ and $\mathrm{CH}_{2} \mathrm{CH}_{3}$ ). MALDI-TOF MS m/z of $[\mathrm{M}+\mathrm{Na}]^{+}$calculated for $\mathrm{C}_{35} \mathrm{H}_{63} \mathrm{ClO}_{3}: 589.4$; Found: 589.4 . 


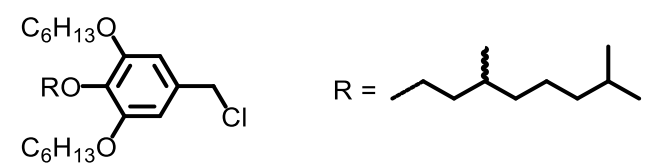

5-(Chloromethyl)-2-((3,7-(rac)-dimethyloctyl)oxy)-1,3-bis(hexyloxy)benzene (10-6r6). From 9-6r6 $(0.21 \mathrm{~g}, 0.45 \mathrm{mmol})$, DMF $(7 \mu \mathrm{L})$ and $\mathrm{SOCl}_{2}(0.041 \mathrm{~mL}, 0.56 \mathrm{mmol})$ in dry DCM $(4 \mathrm{~mL})$ at $23{ }^{\circ} \mathrm{C}$ for 30 min, 10-6r6 was obtained as a light yellow oil in quantitative yield. This was used in the next step without further purification.

${ }^{1} \mathrm{H}$ NMR (500 MHz, $\left.\mathrm{CDCl}_{3}, \delta, \mathrm{ppm}\right): 6.57$ (s, 2H, $\left.\mathrm{ArH}\right), 4.51\left(\mathrm{~s}, 2 \mathrm{H},-\mathrm{CH}_{2} \mathrm{Cl}\right), 4.04-3.91\left(\mathrm{~m}, 6 \mathrm{H},-\mathrm{OCH}_{2}-\right.$ ), 1.87-1.75 (m, 5H, $-\mathrm{CH}\left(\mathrm{CH}_{3}\right)_{2}$ and $\left.-\mathrm{OCH}_{2} \mathrm{CH}_{2} \mathrm{CH}_{2}-\right), 1.75-1.66\left(\mathrm{~m}, 1 \mathrm{H},-\mathrm{CH}\left(\mathrm{CH}_{3}\right) \mathrm{CH}_{2}-\right), 1.57-1.43$ (m, 6H, $-\mathrm{O}\left(\mathrm{CH}_{2}\right)_{2} \mathrm{CH}_{2}-$ and $\left.-\mathrm{OCH}_{2} \mathrm{CH}_{2} \mathrm{CH}\left(\mathrm{CH}_{3}\right)-\right), 1.39-1.09$ (m, $14 \mathrm{H},-\mathrm{CH}\left(\mathrm{CH}_{3}\right)\left(\mathrm{CH}_{2}\right)_{3}-$ and $\left.\left(\mathrm{CH}_{2}\right)_{3}\left(\mathrm{CH}_{2}\right)_{2}-\right), 0.94-0.84\left(\mathrm{~m}, 15 \mathrm{H},-\mathrm{CH}_{2} \mathrm{CH}\left(\mathrm{CH}_{3}\right)-,-\mathrm{CH}\left(\mathrm{CH}_{3}\right)_{2}\right.$ and $\left.-\mathrm{CH}_{2} \mathrm{CH}_{3}\right) .{ }^{13} \mathrm{C} \mathrm{NMR}(126 \mathrm{MHz}$, $\left.\mathrm{CDCl}_{3}, \delta, \mathrm{ppm}\right): 153.38$ ( $\left.\mathrm{ArC}-1,3\right), 138.47$ (ArC-2), 132.47 (ArC-5), 107.21 (ArC-4,6), $71.84\left(\mathrm{ArOCH}_{2}-\right.$ 1,3 position), $69.28\left(\mathrm{ArOCH}_{2}-2\right.$ position), $47.15\left(-\mathrm{CH}_{2} \mathrm{Cl}\right)$, [39.51, 37.66, 37.49, 31.73, 29.85, 29.49, 28.15, 25.91, 24.87] $\left(-\mathrm{OCH}_{2}\left(\mathrm{CH}_{2}\right)_{3}-\right.$ and $\left.-\mathrm{OCH}_{2} \mathrm{CH}_{2} \mathrm{CH}\left(\mathrm{CH}_{3}\right)\left(\mathrm{CH}_{2}\right)_{3} \mathrm{CH}-\right),[22.85,22.78,22.75,19.71$, 14.19] $\left(-\mathrm{CH}\left(\mathrm{CH}_{3}\right)_{2},-\mathrm{CH}\left(\mathrm{CH}_{3}\right) \mathrm{CH}_{2}-\right.$ and $\left.-\mathrm{CH}_{2} \mathrm{CH}_{3}\right)$. MALDI-TOF MS $\mathrm{m} / z$ of $[\mathrm{M}+\mathrm{Na}]^{+}$calculated for $\mathrm{C}_{29} \mathrm{H}_{51} \mathrm{ClO}_{3}$ : 505.3; Found: 505.3.

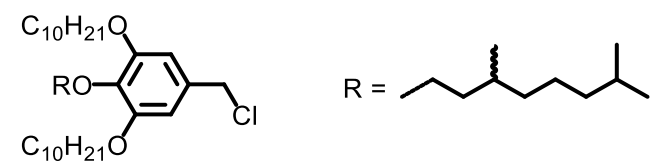

5-(Chloromethyl)-1,3-bis(decyloxy)-2-((3,7-dimethyloctyl)oxy)benzene (10-10r10). From 9-10r10 $(0.29 \mathrm{~g}, 0.50 \mathrm{mmol}), \mathrm{DMF}(7 \mu \mathrm{L})$ and $\mathrm{SOCl}_{2}(0.045 \mathrm{~mL}, 0.62 \mathrm{mmol})$ in dry DCM $(4 \mathrm{~mL})$ at $23{ }^{\circ} \mathrm{C}$ for 30 min, 10-10r10 was obtained as a light yellow oil in quantitative yield. This was used in the next step without further purification.

${ }^{1} \mathrm{H}$ NMR (500 MHz, $\mathrm{CDCl}_{3}, \delta$, ppm): 6.57 (s, 2H, $\left.\mathrm{ArH}\right), 4.51\left(\mathrm{~s}, 2 \mathrm{H},-\mathrm{CH}_{2} \mathrm{Cl}\right), 4.03-3.92\left(\mathrm{~m}, 6 \mathrm{H},-\mathrm{OCH}_{2}-\right.$ ), 1.87-1.75 (m, 5H, $-\mathrm{CH}\left(\mathrm{CH}_{3}\right)_{2}$ and $\left.-\mathrm{OCH}_{2} \mathrm{CH}_{2} \mathrm{CH}_{2}-\right), 1.75-1.67\left(\mathrm{~m}, 1 \mathrm{H},-\mathrm{CH}\left(\mathrm{CH}_{3}\right) \mathrm{CH}_{2}-\right), 1.57-1.42$ (m, $6 \mathrm{H},-\mathrm{O}\left(\mathrm{CH}_{2}\right)_{2} \mathrm{CH}_{2}-$ and $\left.-\mathrm{OCH}_{2} \mathrm{CH}_{2} \mathrm{CH}\left(\mathrm{CH}_{3}\right)-\right), 1.39-1.10\left(\mathrm{~m}, 30 \mathrm{H},-\mathrm{CH}\left(\mathrm{CH}_{3}\right)\left(\mathrm{CH}_{2}\right)_{3}-\right.$ and $\left.\left(\mathrm{CH}_{2}\right)_{3}\left(\mathrm{CH}_{2}\right)_{6}-\right), 0.92\left(\mathrm{~d}, J=6.6 \mathrm{~Hz}, 3 \mathrm{H},-\mathrm{CH}_{2} \mathrm{CH}\left(\mathrm{CH}_{3}\right)-\right), 0.90-0.84\left(\mathrm{~m}, 12 \mathrm{H},-\mathrm{CH}\left(\mathrm{CH}_{3}\right)_{2}\right.$ and $\left.\mathrm{CH}_{2} \mathrm{CH}_{3}\right) .{ }^{13} \mathrm{C}$ NMR $\left(126 \mathrm{MHz}, \mathrm{CDCl}_{3}, \delta, \mathrm{ppm}\right): 153.37$ (ArC-1,3), 138.44 (ArC-2), 132.46 (ArC-5), 107.18 ( $\mathrm{ArC}-4,6), 71.83$ ( $\mathrm{ArOCH}_{2}-1,3$ position), 69.27 ( $\mathrm{ArOCH}_{2}-2$ position), $47.15\left(-\mathrm{CH}_{2} \mathrm{Cl}\right)$, [39.52, $37.66,37.48,32.06,29.84,29.79,29.74,29.56,29.52,29.50,28.15,26.24,24.87]\left(-\mathrm{OCH}_{2}\left(\mathrm{CH}_{2}\right)_{7}-\right.$ and $\left.\mathrm{OCH}_{2} \mathrm{CH}_{2} \mathrm{CH}\left(\mathrm{CH}_{3}\right)\left(\mathrm{CH}_{2}\right)_{3} \mathrm{CH}-\right)$, [22.86, 22.84, 22.77, 19.71, 14.27] $\left(-\mathrm{CH}\left(\mathrm{CH}_{3}\right)_{2},-\mathrm{CH}\left(\mathrm{CH}_{3}\right) \mathrm{CH}_{2}-\right.$ and $\mathrm{CH}_{2} \mathrm{CH}_{3}$ ). MALDI-TOF MS m/z of [M+Na] $]^{+}$calculated for $\mathrm{C}_{37} \mathrm{H}_{67} \mathrm{ClO}_{3}$ : 617.5; Found: 617.4. 


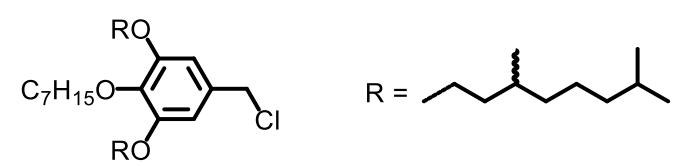

5-(Chloromethyl)-1,3-bis((3,7-(rac)-dimethyloctyl)oxy)-2-(heptyloxy)benzene (10-r7r). From 9-r7r $(0.99 \mathrm{~g}, 1.85 \mathrm{mmol})$, DMF $(30 \mu \mathrm{L})$ and $\mathrm{SOCl}_{2}(0.17 \mathrm{~mL}, 2.3 \mathrm{mmol})$ in dry DCM $(10 \mathrm{~mL})$ at $23{ }^{\circ} \mathrm{C}$ for 30 min, 10-r7r $\mathbf{r}$ was obtained as a light yellow oil in quantitative yield. This was used in the next step without further purification.

${ }^{1} \mathrm{H}$ NMR (400 MHz, $\left.\mathrm{CDCl}_{3}, \delta, \mathrm{ppm}\right): 6.58$ (s, 2H, $\mathrm{ArH}$ ), 4.52 (s, 2H, $\left.-\mathrm{CH}_{2} \mathrm{Cl}\right), 4.08-3.97$ (m, $4 \mathrm{H},-$ $\left.\mathrm{OCH}_{2} \mathrm{CH}_{2} \mathrm{CH}\left(\mathrm{CH}_{3}\right)-\right), 3.94\left(\mathrm{t}, J=6.6 \mathrm{~Hz}, 2 \mathrm{H},-\mathrm{OCH}_{2} \mathrm{CH}_{2} \mathrm{CH}_{2}-\right), 1.91-1.80\left(\mathrm{~m}, 2 \mathrm{H},-\mathrm{CH}\left(\mathrm{CH}_{3}\right)_{2}\right), 1.78-$ $1.66\left(\mathrm{~m}, 4 \mathrm{H},-\mathrm{CH}\left(\mathrm{CH}_{3}\right) \mathrm{CH}_{2}-\right.$ and $\left.-\mathrm{OCH}_{2} \mathrm{CH}_{2} \mathrm{CH}_{2}-\right), 1.65-1.42\left(\mathrm{~m}, 6 \mathrm{H},-\mathrm{OCH}_{2} \mathrm{CH}_{2} \mathrm{CH}\left(\mathrm{CH}_{3}\right)-\right.$ and $\left.\mathrm{OCH}_{2} \mathrm{CH}_{2} \mathrm{CH}_{2}-\right), 1.40-1.10\left(\mathrm{~m}, 18 \mathrm{H},-\mathrm{CH}\left(\mathrm{CH}_{3}\right)\left(\mathrm{CH}_{2}\right)_{3}-\right.$ and $\left.-\left(\mathrm{CH}_{2}\right)_{3}\left(\mathrm{CH}_{2}\right)_{3}-\right), 0.94(\mathrm{~d}, J=6.6 \mathrm{~Hz}, 6 \mathrm{H},-$ $\left.\mathrm{CH}_{2} \mathrm{CH}\left(\mathrm{CH}_{3}\right)-\right), 0.92-0.82\left(\mathrm{~m}, 15 \mathrm{H},-\mathrm{CH}\left(\mathrm{CH}_{3}\right)_{2}\right.$ and $\left.-\mathrm{CH}_{2} \mathrm{CH}_{3}\right) .{ }^{13} \mathrm{C} \mathrm{NMR}\left(101 \mathrm{MHz}, \mathrm{CDCl}_{3}, \delta, \mathrm{ppm}\right)$ : 153.35 (ArC-1,3), 138.43 ( $\mathrm{ArC}-2$ ), 132.45 ( $\mathrm{ArC}-5$ ), 107.18 ( $\mathrm{ArC}-4,6), 73.55$ ( $\mathrm{ArOCH}_{2}-2$ position), 67.57 ( $\mathrm{ArOCH}_{2}-1,3$ position), $47.12\left(-\mathrm{CH}_{2} \mathrm{Cl}\right)$, [39.41, 37.46, 36.52, 32.05, 30.47, 29.95, 29.39, 28.13, 26.20, 24.87] $\left(-\mathrm{OCH}_{2} \mathrm{CH}_{2} \mathrm{CH}\left(\mathrm{CH}_{3}\right)\left(\mathrm{CH}_{2}\right)_{3} \mathrm{CH}-\right.$ and $\left.-\mathrm{OCH}_{2}\left(\mathrm{CH}_{2}\right)_{4}-\right)$, [22.84, 22.82, 22.73, 19.75, 14.25] ($\mathrm{CH}\left(\mathrm{CH}_{3}\right)_{2},-\mathrm{CH}\left(\mathrm{CH}_{3}\right) \mathrm{CH}_{2}-$ and $\left.-\mathrm{CH}_{2} \mathrm{CH}_{3}\right)$. MALDI-TOF $\mathrm{MS} \mathrm{m} / z$ of $[\mathrm{M}+\mathrm{Na}]^{+}$calculated for $\mathrm{C}_{34} \mathrm{H}_{61} \mathrm{ClO}_{3}$ : 575.4; Found: 575.0.

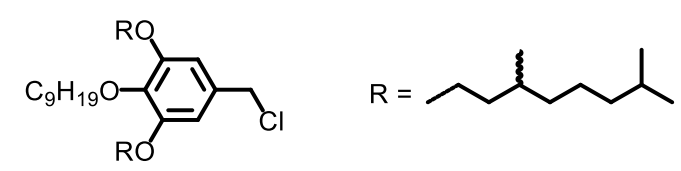

5-(Chloromethyl)-1,3-bis((3,7-(rac)-dimethyloctyl)oxy)-2-(nonyloxy)benzene (10-r9r). From 9-r9r $(1.04 \mathrm{~g}, 1.85 \mathrm{mmol}), \mathrm{DMF}(30 \mu \mathrm{L})$ and $\mathrm{SOCl}_{2}(0.17 \mathrm{~mL}, 2.3 \mathrm{mmol})$ in dry DCM $(10 \mathrm{~mL})$ at $23{ }^{\circ} \mathrm{C}$ for 30 min, 10-r9r was obtained as a light yellow oil in quantitative yield. This was used in the next step without further purification.

${ }^{1} \mathrm{H}$ NMR (400 MHz, $\left.\mathrm{CDCl}_{3}, \delta, \mathrm{ppm}\right): 6.58$ (s, 2H, $\mathrm{ArH}$ ), 4.52 (s, 2H, $\left.-\mathrm{CH}_{2} \mathrm{Cl}\right), 4.08-3.97$ (m, 4H, $\left.\mathrm{OCH}_{2} \mathrm{CH}_{2} \mathrm{CH}\left(\mathrm{CH}_{3}\right)-\right), 3.94\left(\mathrm{t}, J=6.6 \mathrm{~Hz}, 2 \mathrm{H},-\mathrm{OCH}_{2} \mathrm{CH}_{2} \mathrm{CH}_{2}-\right), 1.92-1.80\left(\mathrm{~m}, 2 \mathrm{H},-\mathrm{CH}\left(\mathrm{CH}_{3}\right)_{2}\right), 1.79-$ $1.66\left(\mathrm{~m}, 4 \mathrm{H},-\mathrm{CH}\left(\mathrm{CH}_{3}\right) \mathrm{CH}_{2}-\right.$ and $\left.-\mathrm{OCH}_{2} \mathrm{CH}_{2} \mathrm{CH}_{2}-\right), 1.65-1.42\left(\mathrm{~m}, 6 \mathrm{H},-\mathrm{OCH}_{2} \mathrm{CH}_{2} \mathrm{CH}\left(\mathrm{CH}_{3}\right)-\right.$ and $\left.\mathrm{OCH}_{2} \mathrm{CH}_{2} \mathrm{CH}_{2}-\right), 1.40-1.10\left(\mathrm{~m}, 22 \mathrm{H},-\mathrm{CH}\left(\mathrm{CH}_{3}\right)\left(\mathrm{CH}_{2}\right)_{3}-\right.$ and $\left.-\left(\mathrm{CH}_{2}\right)_{3}\left(\mathrm{CH}_{2}\right)_{5}-\right), 0.94(\mathrm{~d}, J=6.6 \mathrm{~Hz}, 6 \mathrm{H},-$ $\left.\mathrm{CH}_{2} \mathrm{CH}\left(\mathrm{CH}_{3}\right)-\right), 0.92-0.83\left(\mathrm{~m}, 15 \mathrm{H},-\mathrm{CH}\left(\mathrm{CH}_{3}\right)_{2}\right.$ and $\left.-\mathrm{CH}_{2} \mathrm{CH}_{3}\right) .{ }^{13} \mathrm{C} \mathrm{NMR}\left(101 \mathrm{MHz}, \mathrm{CDCl}_{3}, \delta, \mathrm{ppm}\right)$ : 153.35 ( $\mathrm{ArC}-1,3), 138.43$ ( $\mathrm{ArC}-2), 132.45$ ( $\mathrm{ArC}-5), 107.18$ ( $\mathrm{ArC}-4,6), 73.55$ ( $\mathrm{ArOCH}_{2}-2$ position), 67.57 ( $\mathrm{ArOCH}_{2}-1,3$ position), $47.12\left(-\mathrm{CH}_{2} \mathrm{Cl}\right)$, [39.41, 37.46, 36.53, 32.08, 30.47, 29.95, 29.82, 29.74, 29.51, 28.13, 26.26, 24.87] $\left(-\mathrm{OCH}_{2} \mathrm{CH}_{2} \mathrm{CH}\left(\mathrm{CH}_{3}\right)\left(\mathrm{CH}_{2}\right)_{3} \mathrm{CH}-\right.$ and $\left.-\mathrm{OCH}_{2}\left(\mathrm{CH}_{2}\right)_{6}-\right)$, [22.84, 22.74, 19.75, 14.25] $\left(-\mathrm{CH}\left(\mathrm{CH}_{3}\right)_{2},-\mathrm{CH}\left(\mathrm{CH}_{3}\right) \mathrm{CH}_{2}-\right.$ and $\left.-\mathrm{CH}_{2} \mathrm{CH}_{3}\right)$. MALDI-TOF MS m/z of $[\mathrm{M}+\mathrm{Na}]^{+}$calculated for $\mathrm{C}_{36} \mathrm{H}_{65} \mathrm{ClO}_{3}$ : 603.5; Found: 603.4. 


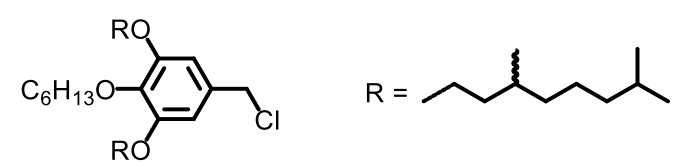

5-(Chloromethyl)-1,3-bis((3,7-(rac)-dimethyloctyl)oxy)-2-(hexyloxy)benzene (10-r6r). From 9-r6r $(0.25 \mathrm{~g}, 0.48 \mathrm{mmol})$, DMF $(7 \mu \mathrm{L})$ and $\mathrm{SOCl}_{2}(0.043 \mathrm{~mL}, 0.60 \mathrm{mmol})$ in dry DCM $(4 \mathrm{~mL})$ at $23{ }^{\circ} \mathrm{C}$ for 30 min, 10-r6r was obtained as a light yellow oil in quantitative yield. This was used in the next step without further purification.

${ }^{1} \mathrm{H}$ NMR $\left(500 \mathrm{MHz}, \mathrm{CDCl}_{3}, \delta, \mathrm{ppm}\right): 6.58$ (s, 2H, $\left.\mathrm{ArH}\right), 4.52$ (s, 2H, $\left.-\mathrm{CH}_{2} \mathrm{Cl}\right), 4.07-3.96(\mathrm{~m}, 4 \mathrm{H},-$ $\left.\mathrm{OCH}_{2} \mathrm{CH}_{2} \mathrm{CH}\left(\mathrm{CH}_{3}\right)-\right), 3.93\left(\mathrm{t}, J=6.6 \mathrm{~Hz}, 2 \mathrm{H},-\mathrm{OCH}_{2} \mathrm{CH}_{2} \mathrm{CH}_{2}-\right), 1.90-1.81\left(\mathrm{~m}, 2 \mathrm{H},-\mathrm{CH}\left(\mathrm{CH}_{3}\right)_{2}\right), 1.77-$ $1.66\left(\mathrm{~m}, 4 \mathrm{H},-\mathrm{CH}\left(\mathrm{CH}_{3}\right) \mathrm{CH}_{2}-\right.$ and $\left.-\mathrm{OCH}_{2} \mathrm{CH}_{2} \mathrm{CH}_{2}-\right), 1.64-1.43\left(\mathrm{~m}, 6 \mathrm{H},-\mathrm{OCH}_{2} \mathrm{CH}_{2} \mathrm{CH}\left(\mathrm{CH}_{3}\right)-\right.$ and $\left.\mathrm{OCH}_{2} \mathrm{CH}_{2} \mathrm{CH}_{2}-\right), 1.39-1.11\left(\mathrm{~m}, 16 \mathrm{H},-\mathrm{CH}\left(\mathrm{CH}_{3}\right)\left(\mathrm{CH}_{2}\right)_{3}-\right.$ and $\left.-\left(\mathrm{CH}_{2}\right)_{3}\left(\mathrm{CH}_{2}\right)_{2}-\right), 0.94(\mathrm{~d}, J=6.6 \mathrm{~Hz}, 6 \mathrm{H},-$ $\left.\mathrm{CH}_{2} \mathrm{CH}\left(\mathrm{CH}_{3}\right)-\right), 0.92-0.84\left(\mathrm{~m}, 15 \mathrm{H},-\mathrm{CH}\left(\mathrm{CH}_{3}\right)_{2}\right.$ and $\left.-\mathrm{CH}_{2} \mathrm{CH}_{3}\right) .{ }^{13} \mathrm{C} \mathrm{NMR}\left(126 \mathrm{MHz}, \mathrm{CDCl}_{3}, \delta, \mathrm{ppm}\right)$ : 153.37 (ArC-3,5), 138.46 (ArC-4), 132.46 ( $\mathrm{ArC}-1), 107.20$ ( $\mathrm{ArC}-2,6), 73.56\left(\mathrm{ArOCH}_{2}-4\right.$ position), 67.59 ( $\mathrm{ArOCH}_{2}-3,5$ position), $47.14\left(-\mathrm{CH}_{2} \mathrm{Cl}\right),[39.42,37.48,36.53,31.94,30.43,29.96,28.14,25.93,24.88]$ $\left(-\mathrm{OCH}_{2} \mathrm{CH}_{2} \mathrm{CH}\left(\mathrm{CH}_{3}\right)\left(\mathrm{CH}_{2}\right)_{3} \mathrm{CH}-\right.$ and $\left.-\mathrm{OCH}_{2}\left(\mathrm{CH}_{2}\right)_{3}-\right)$, [22.85, 22.74, 19.76, 14.25] $\left(-\mathrm{CH}\left(\mathrm{CH}_{3}\right)_{2}\right.$, $\mathrm{CH}\left(\mathrm{CH}_{3}\right) \mathrm{CH}_{2}-$ and $\left.-\mathrm{CH}_{2} \mathrm{CH}_{3}\right)$. MALDI-TOF MS $m / z$ of $[\mathrm{M}+\mathrm{Na}]^{+}$calculated for $\mathrm{C}_{33} \mathrm{H}_{59} \mathrm{ClO}_{3}: 561.4$; Found: 560.9.

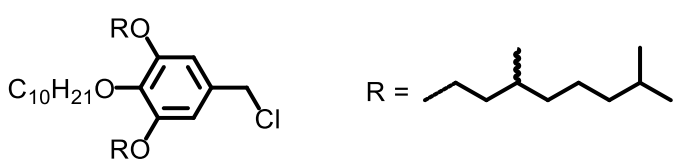

5-(Chloromethyl)-2-(decyloxy)-1,3-bis((3,7-(rac)-dimethyloctyl)oxy)benzene (10-r10r). From 9-r10r $(0.20 \mathrm{~g}, 0.33 \mathrm{mmol}), \mathrm{DMF}(5 \mu \mathrm{L})$ and $\mathrm{SOCl}_{2}(0.030 \mathrm{~mL}, 0.41 \mathrm{mmol})$ in dry DCM $(4 \mathrm{~mL})$ at $23^{\circ} \mathrm{C}$ for 30 min, 10-r10r was obtained as a light yellow oil in quantitative yield. This was used in the next step without further purification.

${ }^{1} \mathrm{H}$ NMR $\left(500 \mathrm{MHz}, \mathrm{CDCl}_{3}, \delta, \mathrm{ppm}\right): 6.58(\mathrm{~s}, 2 \mathrm{H}, \mathrm{ArH}), 4.52(\mathrm{~s}, 2 \mathrm{H},-\mathrm{CH} 2 \mathrm{Cl}), 4.06-3.96(\mathrm{~m}, 4 \mathrm{H},-$ $\left.\mathrm{OCH}_{2} \mathrm{CH}_{2} \mathrm{CH}\left(\mathrm{CH}_{3}\right)-\right), 3.93\left(\mathrm{t}, J=6.6 \mathrm{~Hz}, 2 \mathrm{H},-\mathrm{OCH}_{2} \mathrm{CH}_{2} \mathrm{CH}_{2}-\right), 1.89-1.81\left(\mathrm{~m}, 2 \mathrm{H},-\mathrm{CH}\left(\mathrm{CH}_{3}\right)_{2}\right), 1.76-$ $1.65\left(\mathrm{~m}, 4 \mathrm{H},-\mathrm{CH}\left(\mathrm{CH}_{3}\right) \mathrm{CH}_{2}-\right.$ and $\left.-\mathrm{OCH}_{2} \mathrm{CH}_{2} \mathrm{CH}_{2}-\right), 1.64-1.42\left(\mathrm{~m}, 6 \mathrm{H},-\mathrm{OCH}_{2} \mathrm{CH}_{2} \mathrm{CH}\left(\mathrm{CH}_{3}\right)-\right.$ and $\left.\mathrm{OCH}_{2} \mathrm{CH}_{2} \mathrm{CH}_{2}-\right), 1.38-1.11\left(\mathrm{~m}, 24 \mathrm{H},-\mathrm{CH}\left(\mathrm{CH}_{3}\right)\left(\mathrm{CH}_{2}\right)_{3}-\right.$ and $\left.-\left(\mathrm{CH}_{2}\right)_{3}\left(\mathrm{CH}_{2}\right)_{6}-\right), 0.94(\mathrm{~d}, J=6.6 \mathrm{~Hz}, 6 \mathrm{H},-$ $\left.\mathrm{CH}_{2} \mathrm{CH}\left(\mathrm{CH}_{3}\right)-\right), 0.91-0.83\left(\mathrm{~m}, 15 \mathrm{H},-\mathrm{CH}\left(\mathrm{CH}_{3}\right)_{2}\right.$ and $\left.-\mathrm{CH}_{2} \mathrm{CH}_{3}\right) .{ }^{13} \mathrm{C} \mathrm{NMR}\left(126 \mathrm{MHz}, \mathrm{CDCl}_{3}, \delta, \mathrm{ppm}\right)$ : 153.36 (ArC-1,3), 138.43 ( $\mathrm{ArC}-2$ ), 132.45 ( $\mathrm{ArC}-5), 107.17$ ( $\mathrm{ArC}-4,6), 73.56$ ( $\mathrm{ArOCH}_{2}-2$ position), 67.57 ( $\mathrm{ArOCH}_{2}-1,3$ position), $47.15\left(-\mathrm{CH}_{2} \mathrm{Cl}\right)$, [39.41, 37.47, 36.53, 32.09, 30.48, 29.96, 29.88, 29.82, 29.75, 29.54, 28.14, 26.26, 24.88] $\left(-\mathrm{OCH}_{2} \mathrm{CH}_{2} \mathrm{CH}\left(\mathrm{CH}_{3}\right)\left(\mathrm{CH}_{2}\right)_{3} \mathrm{CH}-\right.$ and $\left.-\mathrm{OCH}_{2}\left(\mathrm{CH}_{2}\right)_{7-}\right)$, [22.85, 22.75, 19.76, 14.27] $\left(-\mathrm{CH}\left(\mathrm{CH}_{3}\right)_{2},-\mathrm{CH}\left(\mathrm{CH}_{3}\right) \mathrm{CH}_{2}-\right.$ and $\left.-\mathrm{CH}_{2} \mathrm{CH}_{3}\right)$. MALDI-TOF MS $m / z$ of $[\mathrm{M}+\mathrm{Na}]^{+}$calculated for $\mathrm{C}_{37} \mathrm{H}_{67} \mathrm{ClO}_{3}$ : 617.5; Found: 617.1. 


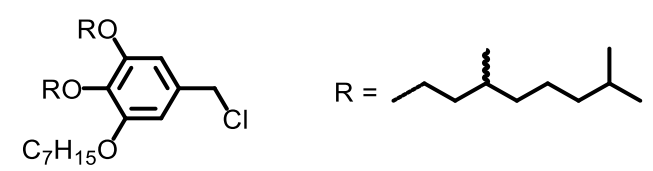

5-(Chloromethyl)-1,2-bis((3,7-(rac)-dimethyloctyl)oxy)-3-(heptyloxy)benzene (10-rr7). From 9-rr7 $(0.99 \mathrm{~g}, 1.85 \mathrm{mmol}), \mathrm{DMF}(30 \mu \mathrm{L})$ and $\mathrm{SOCl}_{2}(0.17 \mathrm{~mL}, 2.3 \mathrm{mmol})$ in dry DCM $(10 \mathrm{~mL})$ at $23{ }^{\circ} \mathrm{C}$ for 30 min, 10-rr7 was obtained as a light yellow oil in quantitative yield. This was used in the next step without further purification.

${ }^{1} \mathrm{H} \mathrm{NMR}\left(400 \mathrm{MHz}, \mathrm{CDCl}_{3}, \delta, \mathrm{ppm}\right): 6.58(\mathrm{~s}, 2 \mathrm{H}, \mathrm{ArH}), 4.52\left(\mathrm{~s}, 2 \mathrm{H},-\mathrm{CH}_{2} \mathrm{Cl}\right), 4.08-3.91\left(\mathrm{~m}, 6 \mathrm{H},-\mathrm{OCH}_{2}-\right)$, 1.93-1.65 (m, 6H, $-\mathrm{OCH}_{2} \mathrm{CH}_{2} \mathrm{CH}_{2}-,-\mathrm{CH}\left(\mathrm{CH}_{3}\right)_{2}$ and $\left.-\mathrm{CH}\left(\mathrm{CH}_{3}\right) \mathrm{CH}_{2}-\right), 1.65-1.42\left(\mathrm{~m}, 6 \mathrm{H},-\mathrm{O}\left(\mathrm{CH}_{2}\right)_{2} \mathrm{CH}_{2}-\right.$ and $\left.-\mathrm{OCH}_{2} \mathrm{CH}_{2} \mathrm{CH}\left(\mathrm{CH}_{3}\right)-\right), 1.42-1.08\left(\mathrm{~m}, 18 \mathrm{H},-\mathrm{CH}\left(\mathrm{CH}_{3}\right)\left(\mathrm{CH}_{2}\right)_{3}-\right.$ and $\left.-\left(\mathrm{CH}_{2}\right)_{3}\left(\mathrm{CH}_{2}\right)_{3}-\right), 0.97-0.83(\mathrm{~m}$, 21H, $\left.-\mathrm{CH}_{3}\right) .{ }^{13} \mathrm{C} \mathrm{NMR}\left(101 \mathrm{MHz}, \mathrm{CDCl}_{3}, \delta, \mathrm{ppm}\right): 153.36$ (ArC-1,3), 138.44 (ArC-2), 132.47 (ArC-5), [107.18, 107.16] (ArC-4,6), $71.81\left(\mathrm{ArOCH}_{2}-3\right.$ position), $69.25\left(\mathrm{ArOCH}_{2}-2\right.$ position), $67.56\left(\mathrm{ArOCH}_{2}-1\right.$ position), $47.11\left(-\mathrm{CH}_{2} \mathrm{Cl}\right)$, [39.51, 39.41, 37.65, 37.48, 36.51, 31.97, 29.96, 29.83, 29.52, 29.21, 28.13, $26.19,24.85]\left(-\mathrm{OCH}_{2} \mathrm{CH}_{2} \mathrm{CH}\left(\mathrm{CH}_{3}\right)\left(\mathrm{CH}_{2}\right)_{3} \mathrm{CH}-\right.$ and $\left.-\mathrm{OCH}_{2}\left(\mathrm{CH}_{2}\right)_{4}-\right)$, [22.85, 22.76, 22.74, 19.76, 19.71, 14.22] $\left(-\mathrm{CH}\left(\mathrm{CH}_{3}\right)_{2},-\mathrm{CH}\left(\mathrm{CH}_{3}\right) \mathrm{CH}_{2}-\right.$ and $\left.-\mathrm{CH}_{2} \mathrm{CH}_{3}\right)$. MALDI-TOF MS $m / z$ of $[\mathrm{M}+\mathrm{Na}]^{+}$calculated for $\mathrm{C}_{34} \mathrm{H}_{61} \mathrm{ClO}_{3}$ : 575.4; Found: 575.3.

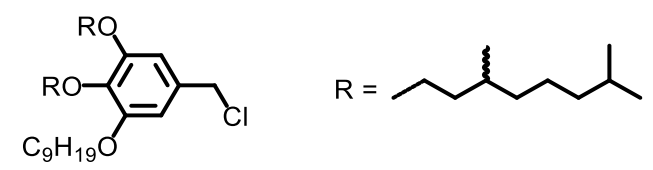

5-(Chloromethyl)-1,2-bis((3,7-(rac)-dimethyloctyl)oxy)-5-(nonyloxy)benzene (10-rr9). From 9-rr9 $(1.04 \mathrm{~g}, 1.85 \mathrm{mmol}), \mathrm{DMF}(30 \mu \mathrm{L})$ and $\mathrm{SOCl}_{2}(0.17 \mathrm{~mL}, 2.3 \mathrm{mmol})$ in dry DCM $(10 \mathrm{~mL})$ at $23{ }^{\circ} \mathrm{C}$ for 30 min, 10-rr9 was obtained as a light yellow oil in quantitative yield. This was used in the next step without further purification.

${ }^{1} \mathrm{H} \mathrm{NMR}\left(400 \mathrm{MHz}, \mathrm{CDCl}_{3}, \delta, \mathrm{ppm}\right): 6.58(\mathrm{~s}, 2 \mathrm{H}, \mathrm{ArH}), 4.52\left(\mathrm{~s}, 2 \mathrm{H},-\mathrm{CH} \mathrm{H}_{2} \mathrm{Cl}\right), 4.07-3.90\left(\mathrm{~m}, 6 \mathrm{H},-\mathrm{OCH}_{2}-\right)$, 1.92-1.65 (m, 6H, $-\mathrm{OCH}_{2} \mathrm{CH}_{2} \mathrm{CH}_{2}-,-\mathrm{CH}\left(\mathrm{CH}_{3}\right)_{2}$ and $\left.-\mathrm{CH}\left(\mathrm{CH}_{3}\right) \mathrm{CH}_{2}-\right), 1.65-1.41\left(\mathrm{~m}, 6 \mathrm{H},-\mathrm{O}\left(\mathrm{CH}_{2}\right)_{2} \mathrm{CH}_{2}-\right.$ and $\left.-\mathrm{OCH}_{2} \mathrm{CH}_{2} \mathrm{CH}\left(\mathrm{CH}_{3}\right)-\right), 1.41-1.07\left(\mathrm{~m}, 22 \mathrm{H},-\mathrm{CH}\left(\mathrm{CH}_{3}\right)\left(\mathrm{CH}_{2}\right)_{3}-\right.$ and $\left.-\left(\mathrm{CH}_{2}\right)_{3}\left(\mathrm{CH}_{2}\right)_{5}-\right), 0.98-0.82(\mathrm{~m}$, 21H, $\left.-\mathrm{CH}_{3}\right) .{ }^{13} \mathrm{C} \mathrm{NMR}\left(101 \mathrm{MHz}, \mathrm{CDCl}_{3}, \delta, \mathrm{ppm}\right): 153.37$ (ArC-1,3), 138.44 (ArC-2), 132.47 (ArC-5), [107.19, 107.17] (ArC-4,6), $71.82\left(\mathrm{ArOCH}_{2}-3\right.$ position), 69.26 ( $\mathrm{ArOCH}_{2}-2$ position), $67.57\left(\mathrm{ArOCH}_{2}-1\right.$ position), $47.12\left(-\mathrm{CH}_{2} \mathrm{Cl}\right),[39.51,39.42,37.65,37.48,36.52,32.04,29.96,29.84,29.73,29.56,29.52$, 29.43, 28.13, 26.24, 24.86] $\left(-\mathrm{OCH}_{2} \mathrm{CH}_{2} \mathrm{CH}\left(\mathrm{CH}_{3}\right)\left(\mathrm{CH}_{2}\right)_{3} \mathrm{CH}-\right.$ and $\left.-\mathrm{OCH}_{2}\left(\mathrm{CH}_{2}\right)_{6}-\right)$, [22.85, 22.82, 22.76, 22.74, 19.77, 19.71, 14.25] $\left(-\mathrm{CH}\left(\mathrm{CH}_{3}\right)_{2},-\mathrm{CH}\left(\mathrm{CH}_{3}\right) \mathrm{CH}_{2}-\right.$ and $\left.-\mathrm{CH}_{2} \mathrm{CH}_{3}\right)$. MALDI-TOF MS $\mathrm{m} / z$ of $[\mathrm{M}+\mathrm{Na}]^{+}$calculated for $\mathrm{C}_{36} \mathrm{H}_{65} \mathrm{ClO}_{3}$ : 603.5; Found: 603.4 . 


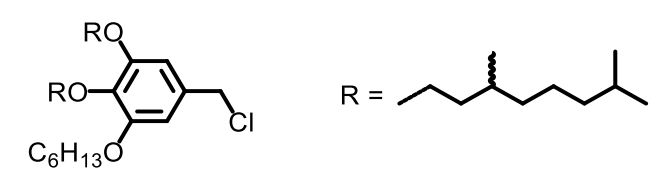

5-(Chloromethyl)-1,2-bis((3,7-(rac)-dimethyloctyl)oxy)-3-(hexyloxy)benzene (10-rr6). From 9-rr6 $(0.33 \mathrm{~g}, 0.64 \mathrm{mmol}), \mathrm{DMF}(10 \mu \mathrm{L})$ and $\mathrm{SOCl}_{2}(60 \mu \mathrm{L}, 0.82 \mathrm{mmol})$ in dry DCM $(6 \mathrm{~mL}), 10$-rr6 was obtained as a light yellow oil in quantitative yield. This was used in the next step without further purification.

${ }^{1} \mathrm{H} \mathrm{NMR}\left(400 \mathrm{MHz}, \mathrm{CDCl}_{3}, \delta, \mathrm{ppm}\right): 6.58(\mathrm{~s}, 2 \mathrm{H}, \mathrm{ArH}), 4.52\left(\mathrm{~s}, 2 \mathrm{H},-\mathrm{CH}_{2} \mathrm{Cl}\right), 4.06-3.91\left(\mathrm{~m}, 6 \mathrm{H},-\mathrm{OCH}_{2}-\right)$, 1.91-1.65 (m, 6H, $-\mathrm{OCH}_{2} \mathrm{CH}_{2} \mathrm{CH}_{2}-,-\mathrm{CH}\left(\mathrm{CH}_{3}\right)_{2}$ and $\left.-\mathrm{CH}\left(\mathrm{CH}_{3}\right) \mathrm{CH}_{2}-\right), 1.65-1.42\left(\mathrm{~m}, 6 \mathrm{H},-\mathrm{O}\left(\mathrm{CH}_{2}\right)_{2} \mathrm{CH}_{2}-\right.$ and $\left.-\mathrm{OCH}_{2} \mathrm{CH}_{2} \mathrm{CH}\left(\mathrm{CH}_{3}\right)-\right), 1.40-1.09\left(\mathrm{~m}, 16 \mathrm{H},-\mathrm{CH}\left(\mathrm{CH}_{3}\right)\left(\mathrm{CH}_{2}\right)_{3}-\right.$ and $\left.-\left(\mathrm{CH}_{2}\right)_{3}\left(\mathrm{CH}_{2}\right)_{2}-\right), 0.97-0.83(\mathrm{~m}$, 21H, $\left.-\mathrm{CH}_{3}\right) .{ }^{13} \mathrm{C} \mathrm{NMR}\left(101 \mathrm{MHz}, \mathrm{CDCl}_{3}, \delta, \mathrm{ppm}\right): 153.37$ (ArC-1,3), 138.44 (ArC-2), 132.47 (ArC-5), 107.18 (ArC-4,6), 71.82 ( $\mathrm{ArOCH}_{2}-3$ position), 69.26 ( $\mathrm{ArOCH}_{2}-2$ position), 67.57 ( $\mathrm{ArOCH}_{2}-1$ position), $47.14\left(-\mathrm{CH}_{2} \mathrm{Cl}\right)$, [39.51, 39.42, 37.64, 37.48, 36.51, 31.73, 29.96, 29.83, 29.48, 28.13, 25.91, 24.86] ($\mathrm{OCH}_{2} \mathrm{CH}_{2} \mathrm{CH}\left(\mathrm{CH}_{3}\right)\left(\mathrm{CH}_{2}\right)_{3} \mathrm{CH}-$ and $\left.-\mathrm{OCH}_{2}\left(\mathrm{CH}_{2}\right)_{3}-\right)$, [22.85, 22.78, 22.75, 19.77, 19.71, 14.18] ($\mathrm{CH}\left(\mathrm{CH}_{3}\right)_{2},-\mathrm{CH}\left(\mathrm{CH}_{3}\right) \mathrm{CH}_{2}-$ and $\left.-\mathrm{CH}_{2} \mathrm{CH}_{3}\right)$. MALDI-TOF MS $m / z$ of $[\mathrm{M}+\mathrm{Na}]^{+}$calculated for $\mathrm{C}_{33} \mathrm{H}_{59} \mathrm{ClO}_{3}$ : 561.4; Found: 561.4.

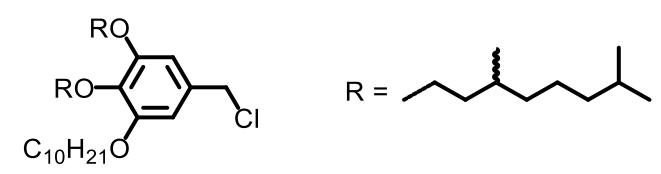

5-(Chloromethyl)-1-(decyloxy)-2,3-bis((3,7-(rac)-dimethyloctyl)oxy)benzene (10-rr10). From 9-rr10 $(0.37 \mathrm{~g}, 0.64 \mathrm{mmol})$, DMF $(10 \mu \mathrm{L})$ and $\mathrm{SOCl}_{2}(60 \mu \mathrm{L}, 0.82 \mathrm{mmol})$ in dry DCM $(6 \mathrm{~mL})$ at $23{ }^{\circ} \mathrm{C}$ for 30 min, 10-rr10 was obtained as a light yellow oil in quantitative yield. This was used in the next step without further purification.

${ }^{1} \mathrm{H} \mathrm{NMR}\left(400 \mathrm{MHz}, \mathrm{CDCl}_{3}, \delta, \mathrm{ppm}\right): 6.58(\mathrm{~s}, 2 \mathrm{H}, \mathrm{ArH}), 4.52\left(\mathrm{~s}, 2 \mathrm{H},-\mathrm{CH} \mathrm{H}_{2} \mathrm{Cl}\right), 4.08-3.89\left(\mathrm{~m}, 6 \mathrm{H},-\mathrm{OCH}_{2}-\right)$, 1.91-1.65 (m, 6H, $-\mathrm{OCH}_{2} \mathrm{CH}_{2} \mathrm{CH}_{2}-,-\mathrm{CH}\left(\mathrm{CH}_{3}\right)_{2}$ and $\left.-\mathrm{CH}\left(\mathrm{CH}_{3}\right) \mathrm{CH}_{2}-\right), 1.64-1.41\left(\mathrm{~m}, 6 \mathrm{H},-\mathrm{O}\left(\mathrm{CH}_{2}\right)_{2} \mathrm{CH}_{2}-\right.$ and $\left.-\mathrm{OCH}_{2} \mathrm{CH}_{2} \mathrm{CH}\left(\mathrm{CH}_{3}\right)-\right), 1.40-1.09\left(\mathrm{~m}, 24 \mathrm{H},-\mathrm{CH}\left(\mathrm{CH}_{3}\right)\left(\mathrm{CH}_{2}\right)_{3}-\right.$ and $\left.-\left(\mathrm{CH}_{2}\right)_{3}\left(\mathrm{CH}_{2}\right)_{6}-\right), 0.98-0.83(\mathrm{~m}$, 21H, $\left.-\mathrm{CH}_{3}\right) .{ }^{13} \mathrm{C} \mathrm{NMR}\left(101 \mathrm{MHz}, \mathrm{CDCl}_{3}, \delta, \mathrm{ppm}\right): 153.37$ (ArC-1,3), 138.44 (ArC-2), 132.47 (ArC-5), [107.18, 107.16] (ArC-4,6), $71.82\left(\mathrm{ArOCH}_{2}-1\right.$ position), $69.26\left(\mathrm{ArOCH}_{2}-2\right.$ position), $67.56\left(\mathrm{ArOCH}_{2}-3\right.$ position), $47.14\left(-\mathrm{CH}_{2} \mathrm{Cl}\right)$, [39.51, 39.42, 37.66, 37.48, 36.51, 32.05, 29.96, 29.84, 29.78, 29.73, 29.56, 29.52, 29.49, 28.14, 26.24, 24.86] $\left(-\mathrm{OCH}_{2} \mathrm{CH}_{2} \mathrm{CH}\left(\mathrm{CH}_{3}\right)\left(\mathrm{CH}_{2}\right)_{3} \mathrm{CH}-\right.$ and $\left.-\mathrm{OCH}_{2}\left(\mathrm{CH}_{2}\right)_{7}-\right)$, [22.85, 22.83, $22.76,22.75,19.77,19.72,14.26]\left(-\mathrm{CH}\left(\mathrm{CH}_{3}\right)_{2},-\mathrm{CH}\left(\mathrm{CH}_{3}\right) \mathrm{CH}_{2}-\right.$ and $\left.-\mathrm{CH}_{2} \mathrm{CH}_{3}\right)$. MALDI-TOF MS $m / z$ of $[\mathrm{M}+\mathrm{Na}]^{+}$calculated for $\mathrm{C}_{37} \mathrm{H}_{67} \mathrm{ClO}_{3}$ : 617.5; Found: 617.7 . 
<smiles>[R]CCCC(C)CCCC(C)C</smiles>

5-(Chloromethyl)-1-((3,7-(rac)-dimethyloctyl)oxy)-2,3-bis(heptyloxy)benzene (10-77r). From 9-77r $(0.91 \mathrm{~g}, 1.85 \mathrm{mmol})$, DMF $(30 \mu \mathrm{L})$ and $\mathrm{SOCl}_{2}(0.17 \mathrm{~mL}, 2.3 \mathrm{mmol})$ in dry DCM $(10 \mathrm{~mL}), \mathbf{1 0 - 7 7 r}$ was obtained as a light yellow oil in quantitative yield. This was used in the next step without further purification.

${ }^{1} \mathrm{H} \mathrm{NMR}\left(400 \mathrm{MHz}, \mathrm{CDCl}_{3}, \delta, \mathrm{ppm}\right): 6.57$ (s, 2H, $\left.\mathrm{ArH}\right), 4.51\left(\mathrm{~s}, 2 \mathrm{H},-\mathrm{CH}_{2} \mathrm{Cl}\right), 4.06-3.89\left(\mathrm{~m}, 6 \mathrm{H},-\mathrm{OCH}_{2}-\right)$, 1.91-1.65 (m, $6 \mathrm{H},-\mathrm{OCH}_{2} \mathrm{CH}_{2} \mathrm{CH}_{2}-,-\mathrm{CH}\left(\mathrm{CH}_{3}\right)_{2}$ and $\left.-\mathrm{CH}\left(\mathrm{CH}_{3}\right) \mathrm{CH}_{2}-\right), 1.65-1.41\left(\mathrm{~m}, 6 \mathrm{H},-\mathrm{O}\left(\mathrm{CH}_{2}\right)_{2} \mathrm{CH}_{2}-\right.$ and $\left.-\mathrm{OCH}_{2} \mathrm{CH}_{2} \mathrm{CH}\left(\mathrm{CH}_{3}\right)-\right), 1.41-1.09\left(\mathrm{~m}, 18 \mathrm{H},-\mathrm{CH}\left(\mathrm{CH}_{3}\right)\left(\mathrm{CH}_{2}\right)_{3}-\right.$ and $\left.-\left(\mathrm{CH}_{2}\right)_{3}\left(\mathrm{CH}_{2}\right)_{3}-\right), 0.94(\mathrm{~d}, J=6.6$ $\left.\mathrm{Hz}, 3 \mathrm{H},-\mathrm{CH}_{2} \mathrm{CH}\left(\mathrm{CH}_{3}\right)-\right), 0.92-0.82\left(\mathrm{~m}, 12 \mathrm{H},-\mathrm{CH}\left(\mathrm{CH}_{3}\right)_{2}\right.$ and $\left.-\mathrm{CH}_{2} \mathrm{CH}_{3}\right) .{ }^{13} \mathrm{C} \mathrm{NMR}\left(101 \mathrm{MHz}, \mathrm{CDCl}_{3}\right.$, $\delta$, ppm): 153.36 (ArC-1,3), 138.43 (ArC-2), 132.46 (ArC-5), [107.21, 107.18] (ArC-4,6), $73.58\left(\mathrm{ArOCH}_{2}-\right.$ 2 position), 69.28 ( $\mathrm{ArOCH}_{2}-3$ position), $67.58\left(\mathrm{ArOCH}_{2}-1\right.$ position), $47.14\left(-\mathrm{CH}_{2} \mathrm{Cl}\right),[39.41,37.47$, $36.52, \quad 32.06, \quad 31.97, \quad 30.47,29.96,29.52,29.39,29.21,28.14,26.20,26.19,24.87](-$ $\mathrm{OCH}_{2} \mathrm{CH}_{2} \mathrm{CH}\left(\mathrm{CH}_{3}\right)\left(\mathrm{CH}_{2}\right)_{3} \mathrm{CH}-$ and $\left.-\mathrm{OCH}_{2}\left(\mathrm{CH}_{2}\right)_{4}-\right)$, [22.84, 22.82, 22.76, 22.74, 19.75, 14.25, 14.23] ($\mathrm{CH}\left(\mathrm{CH}_{3}\right)_{2},-\mathrm{CH}\left(\mathrm{CH}_{3}\right) \mathrm{CH}_{2}-$ and $\left.-\mathrm{CH}_{2} \mathrm{CH}_{3}\right)$. MALDI-TOF $\mathrm{MS} m / z$ of $[\mathrm{M}+\mathrm{Na}]^{+}$calculated for $\mathrm{C}_{31} \mathrm{H}_{55} \mathrm{ClO}_{3}$ : 533.4; Found: 533.0.
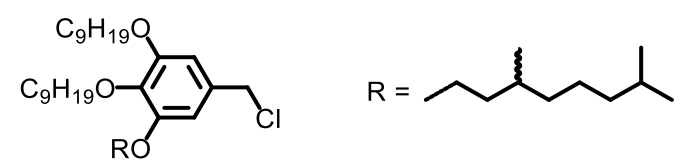

5-(Chloromethyl)-1-((3,7-(rac)-dimethyloctyl)oxy)-2,3-bis(nonyloxy)benzene (10-99r). From 9-99r $(1.02 \mathrm{~g}, 1.85 \mathrm{mmol})$, DMF $(30 \mu \mathrm{L})$ and $\mathrm{SOCl}_{2}(0.17 \mathrm{~mL}, 2.3 \mathrm{mmol})$ in dry DCM $(10 \mathrm{~mL}), \mathbf{1 0 - 9 9 r}$ was obtained as a light yellow oil in quantitative yield. This was used in the next step without further purification.

${ }^{1} \mathrm{H} \mathrm{NMR}\left(400 \mathrm{MHz}, \mathrm{CDCl}_{3}, \delta, \mathrm{ppm}\right): 6.58(\mathrm{~s}, 2 \mathrm{H}, \mathrm{ArH}), 4.51\left(\mathrm{~s}, 2 \mathrm{H},-\mathrm{CH}_{2} \mathrm{Cl}\right), 4.06-3.90\left(\mathrm{~m}, 6 \mathrm{H},-\mathrm{OCH}_{2}-\right)$,

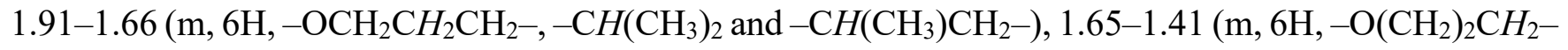
and $\left.-\mathrm{OCH}_{2} \mathrm{CH}_{2} \mathrm{CH}\left(\mathrm{CH}_{3}\right)-\right), 1.41-1.09\left(\mathrm{~m}, 26 \mathrm{H},-\mathrm{CH}\left(\mathrm{CH}_{3}\right)\left(\mathrm{CH}_{2}\right)_{3}-\right.$ and $\left.-\left(\mathrm{CH}_{2}\right)_{3}\left(\mathrm{CH}_{2}\right)_{5}-\right), 0.94(\mathrm{~d}, J=6.6$ $\left.\mathrm{Hz}, 3 \mathrm{H},-\mathrm{CH}_{2} \mathrm{CH}\left(\mathrm{CH}_{3}\right)-\right), 0.92-0.83\left(\mathrm{~m}, 12 \mathrm{H},-\mathrm{CH}\left(\mathrm{CH}_{3}\right)_{2}\right.$ and $\left.-\mathrm{CH}_{2} \mathrm{CH}_{3}\right) .{ }^{13} \mathrm{C} \mathrm{NMR}\left(101 \mathrm{MHz}, \mathrm{CDCl}_{3}\right.$, $\delta$, ppm): 153.35 (ArC-1,3), 138.44 (ArC-2), 132.45 (ArC-5), [107.21, 107.18] (ArC-4,6), $73.57\left(\mathrm{ArOCH}_{2}-\right.$ 2 position), 69.27 ( $\mathrm{ArOCH}_{2}-3$ position), $67.58\left(\mathrm{ArOCH}_{2}-1\right.$ position), $47.12\left(-\mathrm{CH}_{2} \mathrm{Cl}\right),[39.41,37.46$, $36.52,32.08,32.04,30.47,29.95,29.82,29.74,29.55,29.52,29.43,28.13,26.25,26.23,24.87]$ ($\mathrm{OCH}_{2} \mathrm{CH}_{2} \mathrm{CH}\left(\mathrm{CH}_{3}\right)\left(\mathrm{CH}_{2}\right)_{3} \mathrm{CH}-$ and $\left.-\mathrm{OCH}_{2}\left(\mathrm{CH}_{2}\right)_{6}-\right)$, [22.84, 22.82, 22.73, 19.75, 14.24$]\left(-\mathrm{CH}\left(\mathrm{CH}_{3}\right)_{2},-\right.$ $\mathrm{CH}\left(\mathrm{CH}_{3}\right) \mathrm{CH}_{2}-$ and $\left.-\mathrm{CH}_{2} \mathrm{CH}_{3}\right)$. MALDI-TOF MS $m / z$ of $[\mathrm{M}+\mathrm{Na}]^{+}$calculated for $\mathrm{C}_{35} \mathrm{H}_{63} \mathrm{ClO}_{3}: 589.4$; Found: 589.4. 
<smiles>[R]CCCC(C)CCCC(C)C</smiles>

5-(Chloromethyl)-1-((3,7-(rac)-dimethyloctyl)oxy)-2,3-bis(hexyloxy)benzene (10-66r). From 9-66r $(0.30 \mathrm{~g}, 0.64 \mathrm{mmol}), \mathrm{DMF}(10 \mu \mathrm{L})$ and $\mathrm{SOCl}_{2}(60 \mu \mathrm{L}, 0.82 \mathrm{mmol})$ in dry DCM $(6 \mathrm{~mL}), \mathbf{1 0 - 6 6 r}$ was obtained as a light yellow oil in quantitative yield. This was used in the next step without further purification.

${ }^{1} \mathrm{H} \mathrm{NMR}\left(400 \mathrm{MHz}, \mathrm{CDCl}_{3}, \delta, \mathrm{ppm}\right): 6.58(\mathrm{~s}, 2 \mathrm{H}, \mathrm{ArH}), 4.52\left(\mathrm{~s}, 2 \mathrm{H},-\mathrm{CH}_{2} \mathrm{Cl}\right), 4.07-3.89\left(\mathrm{~m}, 6 \mathrm{H},-\mathrm{OCH}_{2}-\right)$,

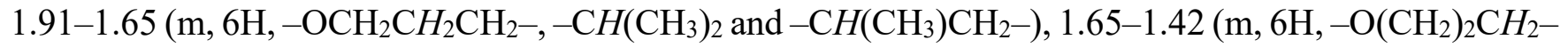
and $\left.-\mathrm{OCH}_{2} \mathrm{CH}_{2} \mathrm{CH}\left(\mathrm{CH}_{3}\right)-\right), 1.40-1.09\left(\mathrm{~m}, 14 \mathrm{H},-\mathrm{CH}\left(\mathrm{CH}_{3}\right)\left(\mathrm{CH}_{2}\right)_{3}-\right.$ and $\left.-\left(\mathrm{CH}_{2}\right)_{3}\left(\mathrm{CH}_{2}\right)_{2}-\right), 0.94(\mathrm{~d}, J=6.6$ $\left.\mathrm{Hz}, 3 \mathrm{H},-\mathrm{CH}_{2} \mathrm{CH}\left(\mathrm{CH}_{3}\right)-\right), 0.92-0.82\left(\mathrm{~m}, 12 \mathrm{H},-\mathrm{CH}\left(\mathrm{CH}_{3}\right)_{2}\right.$ and $\left.-\mathrm{CH}_{2} \mathrm{CH}_{3}\right) .{ }^{13} \mathrm{C} \mathrm{NMR}\left(101 \mathrm{MHz}, \mathrm{CDCl}_{3}\right.$,

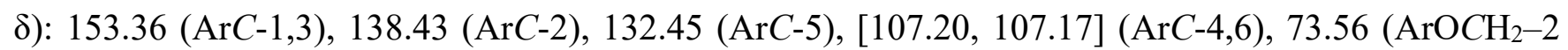
position), 69.26 ( $\mathrm{ArOCH}_{2}-3$ position), 67.57 ( $\mathrm{ArOCH}_{2}-1$ position), $47.13\left(-\mathrm{CH}_{2} \mathrm{Cl}\right),[39.41,37.47,36.52$, $31.92,31.71,30.42,29.95,29.47,28.14,25.90,25.89,24.87]\left(-\mathrm{OCH}_{2} \mathrm{CH}_{2} \mathrm{CH}\left(\mathrm{CH}_{3}\right)\left(\mathrm{CH}_{2}\right)_{3} \mathrm{CH}-\right.$ and $\left.\mathrm{OCH}_{2}\left(\mathrm{CH}_{2}\right)_{3}-\right)$, [22.83, 22.76, 22.74, 19.75, 14.24, 14.16] $\left(-\mathrm{CH}\left(\mathrm{CH}_{3}\right)_{2},-\mathrm{CH}\left(\mathrm{CH}_{3}\right) \mathrm{CH}_{2}-\right.$ and $\left.-\mathrm{CH}_{2} \mathrm{CH}_{3}\right)$. MALDI-TOF MS $m / z$ of $[\mathrm{M}+\mathrm{Na}]^{+}$calculated for $\mathrm{C}_{29} \mathrm{H}_{51} \mathrm{ClO}_{3}$ : 505.3; Found: 505.5.

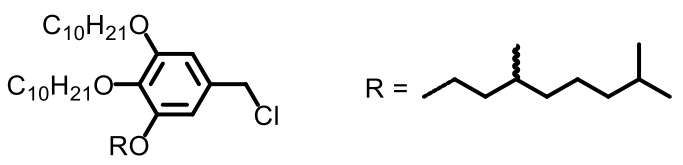

5-(Chloromethyl)-1,2-bis(decyloxy)-3-((3,7-dimethyloctyl)oxy)benzene (10-1010r). From 9-1010r $(0.37 \mathrm{~g}, 0.64 \mathrm{mmol}), \mathrm{DMF}(10 \mu \mathrm{L})$ and $\mathrm{SOCl}_{2}(60 \mu \mathrm{L}, 0.82 \mathrm{mmol})$ in dry DCM $(6 \mathrm{~mL}), \mathbf{1 0 - 1 0 1 0 r}$ was obtained as a light yellow oil in quantitative yield. This was used in the next step without further purification.

${ }^{1} \mathrm{H}$ NMR (400 MHz, $\left.\mathrm{CDCl}_{3}, \delta\right): 6.57$ (s, 2H, $\left.\mathrm{ArH}\right), 4.51$ (s, 2H, $\left.-\mathrm{CH}_{2} \mathrm{Cl}\right), 4.07-3.89$ (m, 6H, $\left.-\mathrm{OCH}_{2}-\right)$, 1.90-1.65 (m, 6H, $-\mathrm{OCH}_{2} \mathrm{CH}_{2} \mathrm{CH}_{2}-,-\mathrm{CH}\left(\mathrm{CH}_{3}\right)_{2}$ and $\left.-\mathrm{CH}\left(\mathrm{CH}_{3}\right) \mathrm{CH}_{2}-\right), 1.64-1.41\left(\mathrm{~m}, 6 \mathrm{H},-\mathrm{O}\left(\mathrm{CH}_{2}\right)_{2} \mathrm{CH}_{2}-\right.$ and $\left.-\mathrm{OCH}_{2} \mathrm{CH}_{2} \mathrm{CH}\left(\mathrm{CH}_{3}\right)-\right), 1.40-1.11\left(\mathrm{~m}, 30 \mathrm{H},-\mathrm{CH}\left(\mathrm{CH}_{3}\right)\left(\mathrm{CH}_{2}\right)_{3}-\right.$ and $\left.-\left(\mathrm{CH}_{2}\right)_{3}\left(\mathrm{CH}_{2}\right)_{6}-\right), 0.94(\mathrm{~d}, J=6.6$ $\left.\mathrm{Hz}, 3 \mathrm{H},-\mathrm{CH}_{2} \mathrm{CH}\left(\mathrm{CH}_{3}\right)-\right), 0.92-0.83\left(\mathrm{~m}, 12 \mathrm{H},-\mathrm{CH}\left(\mathrm{CH}_{3}\right)_{2}\right.$ and $\left.-\mathrm{CH}_{2} \mathrm{CH}_{3}\right) .{ }^{13} \mathrm{C} \mathrm{NMR}\left(101 \mathrm{MHz}, \mathrm{CDCl}_{3}\right.$, $\delta$, ppm): 153.36 (ArC-1,3), 138.44 (ArC-2), 132.45 (ArC-5), [107.21, 107.18] (ArC-4,6), $73.57\left(\mathrm{ArOCH}_{2}-\right.$ 2 position), 69.27 ( $\mathrm{ArOCH} \mathrm{H}_{2}-1$ position), $67.58\left(\mathrm{ArOCH}_{2}-3\right.$ position), $47.14\left(-\mathrm{CH}_{2} \mathrm{Cl}\right),[39.41,37.47$, 36.53 , 32.09, 32.06, 30.47, 29.96, 29.88, 29.82, 29.79, 29.75, 29.54, 29.50, 28.14, 26.26, 26.24, 24.88] ($\mathrm{OCH}_{2} \mathrm{CH}_{2} \mathrm{CH}\left(\mathrm{CH}_{3}\right)\left(\mathrm{CH}_{2}\right)_{3} \mathrm{CH}-$ and $\left.-\mathrm{OCH}_{2}\left(\mathrm{CH}_{2}\right)_{7}-\right)$, [22.85, 22.75, 19.76, 14.26$]\left(-\mathrm{CH}\left(\mathrm{CH}_{3}\right)_{2}\right.$, $\mathrm{CH}\left(\mathrm{CH}_{3}\right) \mathrm{CH}_{2}-$ and $\left.-\mathrm{CH}_{2} \mathrm{CH}_{3}\right)$. MALDI-TOF MS $m / z$ of $[\mathrm{M}+\mathrm{Na}]^{+}$calculated for $\mathrm{C}_{37} \mathrm{H}_{67} \mathrm{ClO}_{3}$ : 617.5; Found: 617.7. 
General procedure for preparation of minidendron azides 11. The synthesis of minidendron azides 11 was conducted according to a procedure reported by our group. ${ }^{10}$ A general example is provided below.
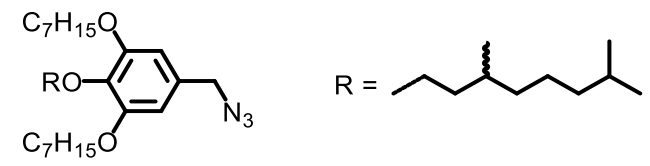

5-(Azidomethyl)-2-((3,7-(rac)-dimethyloctyl)oxy)-1,3-bis(heptyloxy)benzene (11-7r7). $\mathrm{NaN}_{3}(0.14 \mathrm{~g}$, $2.2 \mathrm{mmol})$ was added to a solution of 10-7r7 $(0.89 \mathrm{~g}, 1.75 \mathrm{mmol})$ in DMF $(10 \mathrm{~mL})$ and the reaction mixture was stirred at $23{ }^{\circ} \mathrm{C}$ for $9 \mathrm{~h}$. Then, the reaction mixture was poured into $50 \mathrm{~mL}$ cold water and extracted with EA $(25 \mathrm{~mL} \times 4)$. The combined EA phase was washed with water $(25 \mathrm{~mL} \times 3)$, dried over anhydrous $\mathrm{MgSO}_{4}$, filtered and evaporated in vacuo. Compound 11-7r7 was obtained as a light yellow oil. Product: 0.88 g. Yield: 97\%. Purity by HPLC: 99+\%. This was used in the next step without further purification.

${ }^{1} \mathrm{H}$ NMR (400 MHz, $\left.\mathrm{CDCl}_{3}, \delta, \mathrm{ppm}\right): 6.49$ (s, 2H, $\mathrm{ArH}$ ), 4.24 (s, 2H, $-\mathrm{CH}_{2} \mathrm{~N}_{3}$ ), 4.05-3.92 (m, 6H, -

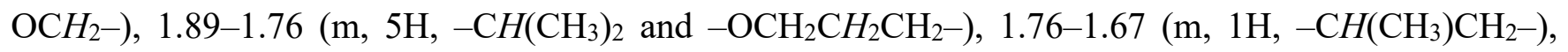
1.59-1.42 (m, 6H, $-\mathrm{O}\left(\mathrm{CH}_{2}\right)_{2} \mathrm{CH}_{2}-$ and $\left.-\mathrm{OCH}_{2} \mathrm{CH}_{2} \mathrm{CH}\left(\mathrm{CH}_{3}\right)-\right), 1.41-1.10\left(\mathrm{~m}, 18 \mathrm{H},-\mathrm{CH}\left(\mathrm{CH}_{3}\right)\left(\mathrm{CH}_{2}\right)_{3}-\right.$ and $\left.-\left(\mathrm{CH}_{2}\right)_{3}\left(\mathrm{CH}_{2}\right)_{3}-\right), 0.95-0.84\left(\mathrm{~m}, 15 \mathrm{H},-\mathrm{CH}_{3}\right) .{ }^{13} \mathrm{C} \mathrm{NMR}\left(101 \mathrm{MHz}, \mathrm{CDCl}_{3}, \delta\right.$, ppm): $153.51(\mathrm{ArC}-$ 1,3), 138.29 (ArC-2), 130.50 (ArC-5), 106.75 ( $\mathrm{ArC}-4,6), 71.80\left(\mathrm{ArOCH}_{2}-1,3\right.$ position), $69.29\left(\mathrm{ArOCH}_{2}-\right.$ 2 position), $55.32\left(-\mathrm{CH}_{2} \mathrm{~N}_{3}\right),[39.50,37.64,37.48,31.96,29.84,29.53,29.21,28.13,26.18,24.86](-$ $\mathrm{OCH}_{2}\left(\mathrm{CH}_{2}\right)_{4}-$ and $\left.-\mathrm{OCH}_{2} \mathrm{CH}_{2} \mathrm{CH}\left(\mathrm{CH}_{3}\right)\left(\mathrm{CH}_{2}\right)_{3} \mathrm{CH}-\right)$, [22.84, 22.75, 19.69, 14.22$]\left(-\mathrm{CH}\left(\mathrm{CH}_{3}\right)_{2}\right.$, $\mathrm{CH}\left(\mathrm{CH}_{3}\right) \mathrm{CH}_{2}-$ and $\left.-\mathrm{CH}_{2} \mathrm{CH}_{3}\right)$. MALDI-TOF MS $m / z$ of $[\mathrm{M}+\mathrm{Na}]^{+}$calculated for $\mathrm{C}_{31} \mathrm{H}_{55} \mathrm{~N}_{3} \mathrm{O}_{3}: 540.4$; Found: 540.5 .

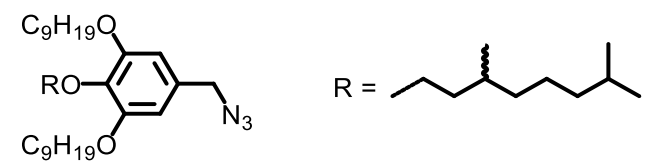

5-(Azidomethyl)-2-((3,7-(rac)-dimethyloctyl)oxy)-1,3-bis(nonyloxy)benzene (11-9r9). From 10-9r9 $(0.99 \mathrm{~g}, 1.75 \mathrm{mmol})$ and $\mathrm{NaN}_{3}(0.14 \mathrm{~g}, 2.2 \mathrm{mmol})$ in DMF $(10 \mathrm{~mL})$ at $23{ }^{\circ} \mathrm{C}$ for $9 \mathrm{~h}, \mathbf{1 1 - 9 r 9}$ was obtained as a light yellow oil. Product: 0.92 g. Yield: $92 \%$. Purity by HPLC: $99+\%$. This was used in the next step without further purification.

${ }^{1} \mathrm{H}$ NMR (400 MHz, $\left.\mathrm{CDCl}_{3}, \delta, \mathrm{ppm}\right): 6.49$ (s, 2H, $\left.\mathrm{ArH}\right), 4.24\left(\mathrm{~s}, 2 \mathrm{H},-\mathrm{CH}_{2} \mathrm{~N}_{3}\right), 4.05-3.90\left(\mathrm{~m}, 6 \mathrm{H},-\mathrm{OCH}_{2}-\right.$ ), 1.89-1.75 (m, 5H, $-\mathrm{CH}\left(\mathrm{CH}_{3}\right)_{2}$ and $\left.-\mathrm{OCH}_{2} \mathrm{CH}_{2} \mathrm{CH}_{2}-\right), 1.75-1.66\left(\mathrm{~m}, 1 \mathrm{H},-\mathrm{CH}\left(\mathrm{CH}_{3}\right) \mathrm{CH}_{2}-\right), 1.57-1.41$ $\left(\mathrm{m}, 6 \mathrm{H},-\mathrm{O}\left(\mathrm{CH}_{2}\right)_{2} \mathrm{CH}_{2}-\right.$ and $\left.-\mathrm{OCH}_{2} \mathrm{CH}_{2} \mathrm{CH}\left(\mathrm{CH}_{3}\right)-\right)$, $1.41-1.09\left(\mathrm{~m}, 26 \mathrm{H},-\mathrm{CH}\left(\mathrm{CH}_{3}\right)\left(\mathrm{CH}_{2}\right)_{3}-\right.$ and $\left.\left(\mathrm{CH}_{2}\right)_{3}\left(\mathrm{CH}_{2}\right)_{5}-\right), 0.96-0.82\left(\mathrm{~m}, 15 \mathrm{H},-\mathrm{CH}_{3}\right) .{ }^{13} \mathrm{C} \mathrm{NMR}\left(101 \mathrm{MHz}, \mathrm{CDCl}_{3}, \delta, \mathrm{ppm}\right): 153.52(\mathrm{ArC}-1,3)$, 138.30 (ArC-2), 130.51 (ArC-5), 106.75 (ArC-4,6), $71.81\left(\mathrm{ArOCH}_{2}-1,3\right.$ position), $69.31\left(\mathrm{ArOCH}_{2}-2\right.$ position), $55.33\left(-\mathrm{CH}_{2} \mathrm{~N}_{3}\right)$, [39.52, 37.66, 37.49, 32.04, 29.85, 29.74, 29.56, 29.53, 29.43, 28.14, 26.24, 24.87] $\left(-\mathrm{OCH}_{2}\left(\mathrm{CH}_{2}\right)_{6}-\right.$ and $\left.-\mathrm{OCH}_{2} \mathrm{CH}_{2} \mathrm{CH}\left(\mathrm{CH}_{3}\right)\left(\mathrm{CH}_{2}\right)_{3} \mathrm{CH}-\right)$, [22.85, 22.82, 22.75, 19.71, 14.25] ($\mathrm{CH}\left(\mathrm{CH}_{3}\right)_{2},-\mathrm{CH}\left(\mathrm{CH}_{3}\right) \mathrm{CH}_{2}-$ and $\left.-\mathrm{CH}_{2} \mathrm{CH}_{3}\right)$. MALDI-TOF $\mathrm{MS} m / z$ of $[\mathrm{M}+\mathrm{Na}]^{+}$calculated for $\mathrm{C}_{35} \mathrm{H}_{63} \mathrm{~N}_{3} \mathrm{O}_{3}$ : 596.5; Found: 596.5. 


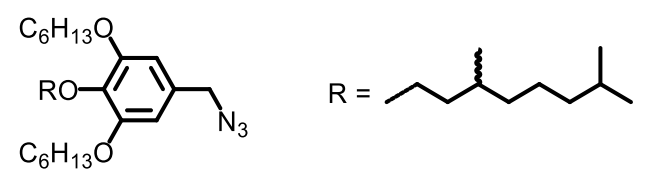

5-(Azidomethyl)-2-((3,7-(rac)-dimethyloctyl)oxy)-1,3-bis(hexyloxy)benzene (11-6r6). From 10-6r6 $(0.19 \mathrm{~g}, 0.39 \mathrm{mmol})$ and $\mathrm{NaN}_{3}(0.032 \mathrm{~g}, 0.49 \mathrm{mmol})$ in $\mathrm{DMF}(6 \mathrm{~mL})$ at $23^{\circ} \mathrm{C}$ for $9 \mathrm{~h}, \mathbf{1 1 - 6 r 6}$ was obtained as a light yellow oil. Product: 0.18 g. Yield: $91 \%$. Purity by HPLC: $99+\%$. This was used in the next step without further purification.

${ }^{1} \mathrm{H}$ NMR $\left(500 \mathrm{MHz}, \mathrm{CDCl}_{3}, \delta, \mathrm{ppm}\right): 6.49$ (s, 2H, $\left.\mathrm{Ar} H\right), 4.24$ (s, 2H, $\left.-\mathrm{CH}_{2} \mathrm{~N}_{3}\right), 4.05-3.89(\mathrm{~m}, 6 \mathrm{H},-$ $\left.\mathrm{OCH}_{2}-\right), 1.87-1.75\left(\mathrm{~m}, 5 \mathrm{H},-\mathrm{CH}\left(\mathrm{CH}_{3}\right)_{2}\right.$ and $\left.-\mathrm{OCH}_{2} \mathrm{CH}_{2} \mathrm{CH}_{2}-\right), 1.75-1.66\left(\mathrm{~m}, 1 \mathrm{H},-\mathrm{CH}\left(\mathrm{CH}_{3}\right) \mathrm{CH}_{2}-\right)$, 1.57-1.42 (m, 6H, $-\mathrm{O}\left(\mathrm{CH}_{2}\right)_{2} \mathrm{CH}_{2}-$ and $\left.-\mathrm{OCH}_{2} \mathrm{CH}_{2} \mathrm{CH}\left(\mathrm{CH}_{3}\right)-\right), 1.39-1.08\left(\mathrm{~m}, 14 \mathrm{H},-\mathrm{CH}\left(\mathrm{CH}_{3}\right)\left(\mathrm{CH}_{2}\right)_{3}-\right.$ and $\left.-\left(\mathrm{CH}_{2}\right)_{3}\left(\mathrm{CH}_{2}\right)_{2}-\right), 0.95-0.81\left(\mathrm{~m}, 15 \mathrm{H},-\mathrm{CH}_{3}\right) .{ }^{13} \mathrm{C} \mathrm{NMR}\left(126 \mathrm{MHz}, \mathrm{CDCl}_{3}, \delta, \mathrm{ppm}\right): 153.50(\mathrm{ArC}-$ 1,3), 138.25 (ArC-2), 130.50 ( $\mathrm{ArC}-5), 106.71$ ( $\mathrm{ArC}-4,6), 71.79\left(\mathrm{ArOCH}_{2}-1,3\right.$ position), $69.28\left(\mathrm{ArOCH}_{2}-\right.$ 2 position), $55.33\left(-\mathrm{CH}_{2} \mathrm{~N}_{3}\right),[39.50,37.64,37.47,31.73,29.83,29.48,28.14,25.90,24.87]$ ($\mathrm{OCH}_{2}\left(\mathrm{CH}_{2}\right)_{3}-$ and $\left.-\mathrm{OCH}_{2} \mathrm{CH}_{2} \mathrm{CH}\left(\mathrm{CH}_{3}\right)\left(\mathrm{CH}_{2}\right)_{3} \mathrm{CH}-\right)$, [22.84, 22.77, 22.74, 19.69, 14.18] $\left(-\mathrm{CH}_{2}\left(\mathrm{CH}_{3}\right)_{2},-\right.$ $\mathrm{CH}\left(\mathrm{CH}_{3}\right) \mathrm{CH}_{2}-$ and $\left.-\mathrm{CH}_{2} \mathrm{CH}_{3}\right)$. MALDI-TOF MS $m / z$ of $[\mathrm{M}+\mathrm{Na}]^{+}$calculated for $\mathrm{C}_{29} \mathrm{H}_{51} \mathrm{~N}_{3} \mathrm{O}_{3}: 512.4$; Found: 511.9 .
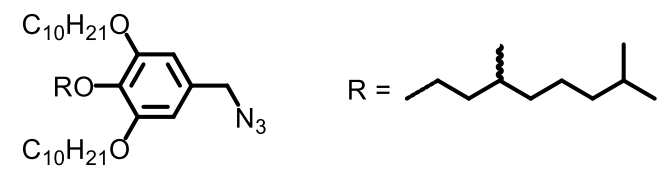

5-(Azidomethyl)-1,3-bis(decyloxy)-2-((3,7-(rac)-dimethyloctyl)oxy)benzene (11-10r10). From 10$10 \mathrm{r} 10(0.22 \mathrm{~g}, 0.36 \mathrm{mmol})$ and $\mathrm{NaN}_{3}(0.029 \mathrm{~g}, 0.45 \mathrm{mmol})$ in DMF $(6 \mathrm{~mL})$ at $23{ }^{\circ} \mathrm{C}$ for $9 \mathrm{~h}, \mathbf{1 1 - 1 0 r 1 0}$ was obtained as a light yellow oil. Product: $0.21 \mathrm{~g}$. Yield: $94 \%$. Purity by HPLC: $99+\%$. This was used in the next step without further purification.

${ }^{1} \mathrm{H}$ NMR $\left(500 \mathrm{MHz}, \mathrm{CDCl}_{3}, \delta, \mathrm{ppm}\right): 6.49$ (s, $\left.2 \mathrm{H}, \mathrm{ArH}\right), 4.24\left(\mathrm{~s}, 2 \mathrm{H},-\mathrm{CH}_{2} \mathrm{~N}_{3}\right), 4.05-3.88\left(\mathrm{~m}, 6 \mathrm{H},-\mathrm{OCH}_{2}-\right.$ ), 1.87-1.75 (m, 5H, $-\mathrm{CH}\left(\mathrm{CH}_{3}\right)_{2}$ and $\left.-\mathrm{OCH}_{2} \mathrm{CH}_{2} \mathrm{CH}_{2}-\right)$, 1.75-1.66 (m, $\left.1 \mathrm{H},-\mathrm{CH}\left(\mathrm{CH}_{3}\right) \mathrm{CH}_{2}-\right), 1.57-1.41$ (m, $6 \mathrm{H},-\mathrm{O}\left(\mathrm{CH}_{2}\right)_{2} \mathrm{CH}_{2}-$ and $\left.-\mathrm{OCH}_{2} \mathrm{CH}_{2} \mathrm{CH}\left(\mathrm{CH}_{3}\right)^{-}\right), 1.39-1.08\left(\mathrm{~m}, 30 \mathrm{H},-\mathrm{CH}\left(\mathrm{CH}_{3}\right)\left(\mathrm{CH}_{2}\right)_{3}-\right.$ and $\left.\left(\mathrm{CH}_{2}\right)_{3}\left(\mathrm{CH}_{2}\right)_{6}-\right), 0.95-0.80\left(\mathrm{~m}, 15 \mathrm{H},-\mathrm{CH}_{3}\right) .{ }^{13} \mathrm{C}$ NMR $\left(101 \mathrm{MHz}, \mathrm{CDCl}_{3}, \delta, \mathrm{ppm}\right): 153.53(\mathrm{ArC}-1,3)$, 138.32 (ArC-2), 130.50 ( $\mathrm{ArC}-5), 106.77$ ( $\mathrm{ArC}-4,6), 71.81\left(\mathrm{ArOCH}_{2}-1,3\right.$ position), $69.32\left(\mathrm{ArOCH}_{2}-2\right.$ position), $55.34\left(-\mathrm{CH}_{2} \mathrm{~N}_{3}\right)$, [39.52, 37.66, 37.50, 32.06, 29.86, 29.79, 29.74, 29.57, 29.54, 29.49, 28.15, 26.24, 24.88] $\left(-\mathrm{OCH}_{2}\left(\mathrm{CH}_{2}\right)_{7}-\right.$ and $\left.-\mathrm{OCH}_{2} \mathrm{CH}_{2} \mathrm{CH}\left(\mathrm{CH}_{3}\right)\left(\mathrm{CH}_{2}\right)_{3} \mathrm{CH}-\right)$, [22.86, 22.83, 22.76, 19.72, 14.26] $\left(-\mathrm{CH}\left(\mathrm{CH}_{3}\right)_{2},-\mathrm{CH}\left(\mathrm{CH}_{3}\right) \mathrm{CH}_{2}-\right.$ and $\left.-\mathrm{CH}_{2} \mathrm{CH}_{3}\right)$. MALDI-TOF MS $\mathrm{m} / z$ of $[\mathrm{M}+\mathrm{Na}]^{+}$calculated for $\mathrm{C}_{37} \mathrm{H}_{67} \mathrm{~N}_{3} \mathrm{O}_{3}$ : 624.5; Found: 624.4. 

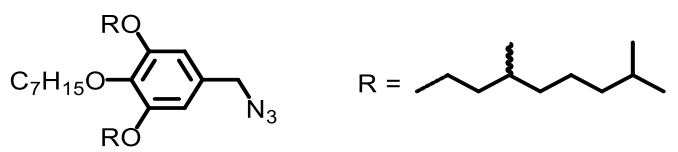

5-(Azidomethyl)-1,3-bis((3,7-(rac)-dimethyloctyl)oxy)-2-(heptyloxy)benzene (11-r7r). From 10-r7r $(0.97 \mathrm{~g}, 1.75 \mathrm{mmol})$ and $\mathrm{NaN}_{3}(0.14 \mathrm{~g}, 2.2 \mathrm{mmol})$ in $\mathrm{DMF}(10 \mathrm{~mL})$ at $23{ }^{\circ} \mathrm{C}$ for $9 \mathrm{~h}, \mathbf{1 1 - r 7 r}$ was obtained as a light yellow oil. Product: 0.93 g. Yield: $95 \%$. Purity by HPLC: $99+\%$. This was used in the next step without further purification.

${ }^{1} \mathrm{H}$ NMR (400 MHz, $\left.\mathrm{CDCl}_{3}, \delta, \mathrm{ppm}\right): 6.50$ (s, 2H, $\left.\mathrm{ArH}\right), 4.25$ (s, 2H, $-\mathrm{CH}_{2} \mathrm{~N}_{3}$ ), 4.08-3.97 (m, 4H, $\left.\mathrm{OCH}_{2} \mathrm{CH}_{2} \mathrm{CH}\left(\mathrm{CH}_{3}\right)_{-}\right), 3.94\left(\mathrm{t}, J=6.6 \mathrm{~Hz}, 2 \mathrm{H},-\mathrm{OCH}_{2} \mathrm{CH}_{2} \mathrm{CH}_{2}-\right), 1.91-1.80\left(\mathrm{~m}, 2 \mathrm{H},-\mathrm{CH}_{\left.\left(\mathrm{CH}_{3}\right)_{2}\right), 1.78-}\right.$ $1.65\left(\mathrm{~m}, 4 \mathrm{H},-\mathrm{CH}\left(\mathrm{CH}_{3}\right) \mathrm{CH}_{2}-\right.$ and $\left.-\mathrm{OCH}_{2} \mathrm{CH}_{2} \mathrm{CH}_{2}-\right), 1.65-1.42\left(\mathrm{~m}, 6 \mathrm{H},-\mathrm{OCH}_{2} \mathrm{CH}_{2} \mathrm{CH}_{\left(\mathrm{CH}_{3}\right)-\text { and }-}\right.$ $\left.\mathrm{OCH}_{2} \mathrm{CH}_{2} \mathrm{CH}_{2}-\right), 1.40-1.10\left(\mathrm{~m}, 18 \mathrm{H},-\mathrm{CH}\left(\mathrm{CH}_{3}\right)\left(\mathrm{CH}_{2}\right)_{3}-\right.$ and $\left.-\left(\mathrm{CH}_{2}\right)_{3}\left(\mathrm{CH}_{2}\right)_{3}-\right), 0.94(\mathrm{~d}, J=6.6 \mathrm{~Hz}, 6 \mathrm{H},-$ $\left.\mathrm{CH}_{2} \mathrm{CH}\left(\mathrm{CH}_{3}\right)_{-}\right), 0.92-0.83\left(\mathrm{~m}, 15 \mathrm{H},-\mathrm{CH}\left(\mathrm{CH}_{3}\right)_{2}\right.$ and $\left.-\mathrm{CH}_{2} \mathrm{CH}_{3}\right) .{ }^{13} \mathrm{C} \mathrm{NMR}\left(101 \mathrm{MHz}, \mathrm{CDCl}_{3}, \delta, \mathrm{ppm}\right)$ : 153.50 (ArC-1,3), 138.28 (ArC-2), 130.51 (ArC-5), 106.74 (ArC-4,6), 73.53 ( $\mathrm{ArOCH}_{2}-2$ position), 67.61 (ArOCH$H_{2}-1,3$ position), $55.34\left(-\mathrm{CH}_{2} \mathrm{~N}_{3}\right)$, [39.40, 37.47, 36.53, 32.06, 30.47, 29.95, 29.40, 28.13, 26.21, 24.87] $\left(-\mathrm{OCH}_{2} \mathrm{CH}_{2} \mathrm{CH}\left(\mathrm{CH}_{3}\right)\left(\mathrm{CH}_{2}\right)_{3} \mathrm{CH}-\right.$ and $\left.-\mathrm{OCH}_{2}\left(\mathrm{CH}_{2}\right)_{4}-\right)$, [22.84, 22.73, 19.75, 14.25] $\left(-\mathrm{CH}_{(}\left(\mathrm{CH}_{3}\right)_{2}\right.$, $-\mathrm{CH}\left(\mathrm{CH}_{3}\right) \mathrm{CH}_{2}-$ and $\left.-\mathrm{CH}_{2} \mathrm{CH}_{3}\right)$. MALDI-TOF MS $m / z$ of $[\mathrm{M}+\mathrm{Na}]^{+}$calculated for $\mathrm{C}_{34} \mathrm{H}_{61} \mathrm{~N}_{3} \mathrm{O}_{3}$ : 582.5; Found: 582.2 .
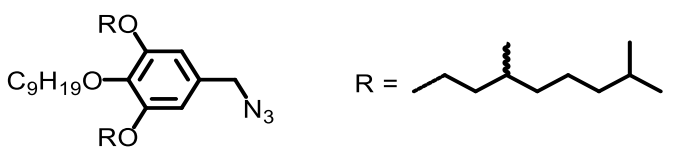

5-(Azidomethyl)-1,3-bis((3,7-(rac)-dimethyloctyl)oxy)-2-(nonyloxy)benzene (11-r9r). From 10-r9r $(1.02 \mathrm{~g}, 1.75 \mathrm{mmol})$ and $\mathrm{NaN}_{3}(0.14 \mathrm{~g}, 2.2 \mathrm{mmol})$ in DMF $(10 \mathrm{~mL})$ at $23{ }^{\circ} \mathrm{C}$ for $9 \mathrm{~h}, \mathbf{1 1 - r 9 r}$ was obtained as a light yellow oil. Product: $1.00 \mathrm{~g}$. Yield: $97 \%$. Purity by HPLC: $99+\%$. This was used in the next step without further purification.

${ }^{1} \mathrm{H}$ NMR (400 MHz, $\left.\mathrm{CDCl}_{3}, \delta, \mathrm{ppm}\right): 6.50$ (s, 2H, $\left.\mathrm{ArH}\right), 4.25$ (s, 2H, $-\mathrm{CH}_{2} \mathrm{~N}_{3}$ ), 4.08-3.98 (m, 4H, -

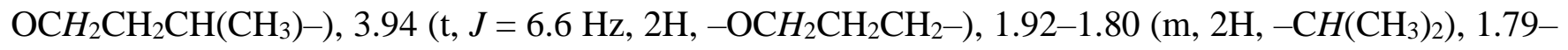

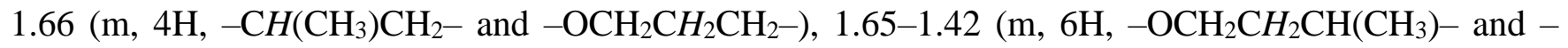
$\left.\mathrm{OCH}_{2} \mathrm{CH}_{2} \mathrm{CH}_{2}-\right), 1.40-1.10\left(\mathrm{~m}, 22 \mathrm{H},-\mathrm{CH}\left(\mathrm{CH}_{3}\right)\left(\mathrm{CH}_{2}\right)_{3}-\right.$ and $\left.-\left(\mathrm{CH}_{2}\right)_{3}\left(\mathrm{CH}_{2}\right)_{5}-\right), 0.94(\mathrm{~d}, J=6.6 \mathrm{~Hz}, 6 \mathrm{H},-$ $\left.\mathrm{CH}_{2} \mathrm{CH}\left(\mathrm{CH}_{3}\right)_{-}\right), 0.92-0.82\left(\mathrm{~m}, 15 \mathrm{H},-\mathrm{CH}\left(\mathrm{CH}_{3}\right)_{2}\right.$ and $\left.-\mathrm{CH}_{2} \mathrm{CH}_{3}\right) .{ }^{13} \mathrm{C} \mathrm{NMR}\left(101 \mathrm{MHz}, \mathrm{CDCl}_{3}, \delta, \mathrm{ppm}\right)$ : 153.50 (ArC-1,3), 138.28 (ArC-2), 130.50 (ArC-5), 106.73 (ArC-4,6), 73.53 ( $\mathrm{ArOCH}_{2}-2$ position), 67.61 (ArOCH $\mathrm{H}_{2}-1,3$ position), $55.33\left(-\mathrm{CH}_{2} \mathrm{~N}_{3}\right)$, [39.40, 37.47, 36.53, 32.08, 30.47, 29.95, 29.82, 29.74, 29.51, 28.13, 26.26, 24.87] $\left(-\mathrm{OCH}_{2} \mathrm{CH}_{2} \mathrm{CH}\left(\mathrm{CH}_{3}\right)\left(\mathrm{CH}_{2}\right)_{3} \mathrm{CH}-\right.$ and $\left.-\mathrm{OCH}_{2}\left(\mathrm{CH}_{2}\right)_{6}-\right)$, [22.83, 22.73, 19.74, 14.25] $\left(-\mathrm{CH}\left(\mathrm{CH}_{3}\right)_{2},-\mathrm{CH}\left(\mathrm{CH}_{3}\right) \mathrm{CH}_{2}-\right.$ and $\left.-\mathrm{CH}_{2} \mathrm{CH}_{3}\right)$. MALDI-TOF MS $m / z$ of $[\mathrm{M}+\mathrm{Na}]^{+}$calculated for $\mathrm{C}_{36} \mathrm{H}_{65} \mathrm{~N}_{3} \mathrm{O}_{3}$ : 610.5; Found: 610.1 . 

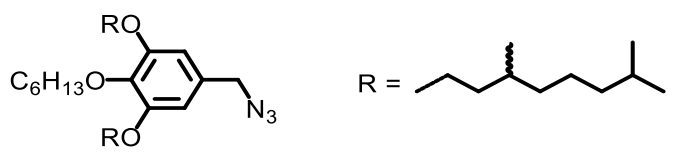

5-(Azidomethyl)-1,3-bis((3,7-(rac)-dimethyloctyl)oxy)-2-(hexyloxy)benzene (11-r6r). From 10-r6r

$(0.24 \mathrm{~g}, 0.44 \mathrm{mmol})$ and $\mathrm{NaN}_{3}(0.036 \mathrm{~g}, 0.55 \mathrm{mmol})$ in $\mathrm{DMF}(6 \mathrm{~mL})$ at $23{ }^{\circ} \mathrm{C}$ for $9 \mathrm{~h}, \mathbf{1 1 - r 6 r}$ was obtained as a light yellow oil. Product: 0.22 g. Yield: $91 \%$. Purity by HPLC: $99+\%$. This was used in the next step without further purification.

${ }^{1} \mathrm{H}$ NMR (500 MHz, $\left.\mathrm{CDCl}_{3}, \delta, \mathrm{ppm}\right): 6.50$ (s, 2H, $\left.\mathrm{ArH}\right), 4.25$ (s, 2H, $\left.-\mathrm{CH}_{2} \mathrm{~N}_{3}\right), 4.07-3.96(\mathrm{~m}, 4 \mathrm{H},-$ $\left.\mathrm{OCH}_{2} \mathrm{CH}_{2} \mathrm{CH}\left(\mathrm{CH}_{3}\right)_{-}\right), 3.94\left(\mathrm{t}, J=6.6 \mathrm{~Hz}, 2 \mathrm{H},-\mathrm{OCH}_{2} \mathrm{CH}_{2} \mathrm{CH}_{2}-\right), 1.90-1.81\left(\mathrm{~m}, 2 \mathrm{H},-\mathrm{CH}_{\left.\left(\mathrm{CH}_{3}\right)_{2}\right), 1.78-}\right.$

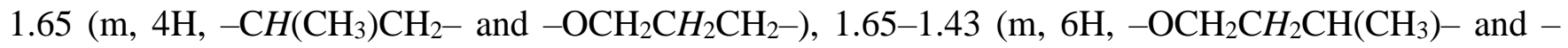
$\left.\mathrm{OCH}_{2} \mathrm{CH}_{2} \mathrm{CH}_{2}-\right), 1.38-1.10\left(\mathrm{~m}, 16 \mathrm{H},-\mathrm{CH}\left(\mathrm{CH}_{3}\right)\left(\mathrm{CH}_{2}\right)_{3}-\right.$ and $\left.-\left(\mathrm{CH}_{2}\right)_{3}\left(\mathrm{CH}_{2}\right)_{2}-\right), 0.94(\mathrm{~d}, J=6.6 \mathrm{~Hz}, 6 \mathrm{H},-$ $\left.\mathrm{CH}_{2} \mathrm{CH}\left(\mathrm{CH}_{3}\right)_{-}\right), 0.92-0.83\left(\mathrm{~m}, 15 \mathrm{H},-\mathrm{CH}\left(\mathrm{CH}_{3}\right)_{2}\right.$ and $\left.-\mathrm{CH}_{2} \mathrm{CH}_{3}\right) .{ }^{13} \mathrm{C} \mathrm{NMR}\left(126 \mathrm{MHz}, \mathrm{CDCl}_{3}, \delta, \mathrm{ppm}\right)$ : 153.49 (ArC-1,3), 138.25 (ArC-2), 130.50 (ArC-5), 106.70 ( $\mathrm{ArC}-4,6), 73.52$ ( $\mathrm{ArOCH}_{2}-2$ position), 67.58 (ArOCH $\mathrm{H}_{2}-1,3$ position), $55.33\left(-\mathrm{CH}_{2} \mathrm{~N}_{3}\right)$, [39.40, 37.47, 36.52, 31.93, 30.42, 29.94, 28.13, 25.93, 24.87] $\left(-\mathrm{OCH}_{2} \mathrm{CH}_{2} \mathrm{CH}\left(\mathrm{CH}_{3}\right)\left(\mathrm{CH}_{2}\right)_{3} \mathrm{CH}-\right.$ and $\left.-\mathrm{OCH}_{2}\left(\mathrm{CH}_{2}\right)_{3}-\right)$, [22.84, 22.73, 19.74, 14.25] $\left(-\mathrm{CH}\left(\mathrm{CH}_{3}\right)_{2}\right.$, $\mathrm{CH}\left(\mathrm{CH}_{3}\right) \mathrm{CH}_{2}-$ and $\left.-\mathrm{CH}_{2} \mathrm{CH}_{3}\right)$. MALDI-TOF MS $m / z$ of $[\mathrm{M}+\mathrm{Na}]^{+}$calculated for $\mathrm{C}_{33} \mathrm{H}_{59} \mathrm{~N}_{3} \mathrm{O}_{3}$ : 568.4; Found: 568.1.

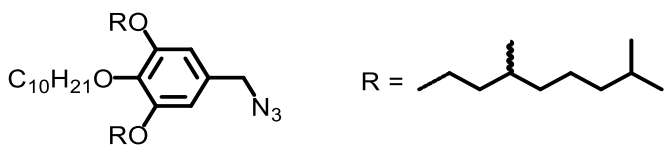

5-(Azidomethyl)-2-(decyloxy)-1,3-bis((3,7-(rac)-dimethyloctyl)oxy)benzene (11-r10r). From 10-r10r $(0.16 \mathrm{~g}, 0.27 \mathrm{mmol})$ and $\mathrm{NaN}_{3}(0.022 \mathrm{~g}, 0.34 \mathrm{mmol})$ in DMF $(6 \mathrm{~mL})$ at $23{ }^{\circ} \mathrm{C}$ for $9 \mathrm{~h}, \mathbf{1 1}-\mathbf{r 1 0 r}$ was obtained as a light yellow oil. Product: 0.15 g. Yield: $97 \%$. Purity by HPLC: $99+\%$. This was used in the next step without further purification.

${ }^{1} \mathrm{H}$ NMR (500 MHz, $\left.\mathrm{CDCl}_{3}, \delta, \mathrm{ppm}\right): 6.50$ (s, 2H, $\left.\mathrm{ArH}\right), 4.25$ (s, 2H, $-\mathrm{CH}_{2} \mathrm{~N}_{3}$ ), 4.06-3.96 (m, 4H, $\left.\left.\mathrm{OCH}_{2} \mathrm{CH}_{2} \mathrm{CH}\left(\mathrm{CH}_{3}\right)-\right), 3.94\left(\mathrm{t}, J=6.6 \mathrm{~Hz}, 2 \mathrm{H},-\mathrm{OCH}_{2} \mathrm{CH}_{2} \mathrm{CH}_{2}-\right), 1.89-1.80\left(\mathrm{~m}, 2 \mathrm{H},-\mathrm{CH}_{(\mathrm{CH}}\right)_{2}\right), 1.77-$

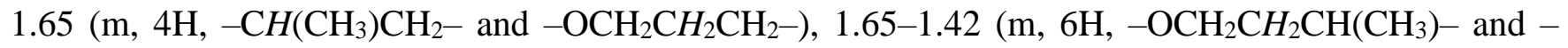
$\left.\mathrm{OCH}_{2} \mathrm{CH}_{2} \mathrm{CH}_{2}-\right), 1.39-1.09\left(\mathrm{~m}, 24 \mathrm{H},-\mathrm{CH}\left(\mathrm{CH}_{3}\right)\left(\mathrm{CH}_{2}\right)_{3}-\right.$ and $\left.-\left(\mathrm{CH}_{2}\right)_{3}\left(\mathrm{CH}_{2}\right)_{6}-\right), 0.94(\mathrm{~d}, J=6.5 \mathrm{~Hz}, 6 \mathrm{H},-$ $\left.\mathrm{CH}_{2} \mathrm{CH}\left(\mathrm{CH}_{3}\right)-\right)$, 0.91-0.79 (m, 15H, $-\mathrm{CH}\left(\mathrm{CH}_{3}\right)_{2}$ and $\left.-\mathrm{CH}_{2} \mathrm{CH}_{3}\right) .{ }^{13} \mathrm{C} \mathrm{NMR}\left(126 \mathrm{MHz}, \mathrm{CDCl}_{3}, \delta, \mathrm{ppm}\right)$ : 153.52 (ArC-1,3), 138.31 (ArC-2), 130.51 (ArC-5), 106.76 ( $\mathrm{ArC}-4,6), 73.55$ ( $\mathrm{ArOCH}_{2}-2$ position), 67.64 (ArOCH$H_{2}-1,3$ position), $55.35\left(-\mathrm{CH}_{2} \mathrm{~N}_{3}\right)$, [39.42, 37.48, 36.55, 32.09, 30.49, 29.97, 29.88, 29.83, 29.76, 29.54, 28.14, 26.28, 24.88] $\left(-\mathrm{OCH}_{2} \mathrm{CH}_{2} \mathrm{CH}\left(\mathrm{CH}_{3}\right)\left(\mathrm{CH}_{2}\right)_{3} \mathrm{CH}-\right.$ and $\left.-\mathrm{OCH}_{2}\left(\mathrm{CH}_{2}\right)_{7}-\right)$, [22.85, 22.75, 19.76, 14.26] $\left(-\mathrm{CH}\left(\mathrm{CH}_{3}\right)_{2},-\mathrm{CH}\left(\mathrm{CH}_{3}\right) \mathrm{CH}_{2}-\right.$ and $\left.-\mathrm{CH}_{2} \mathrm{CH}_{3}\right)$. MALDI-TOF MS $m / z$ of $[\mathrm{M}+\mathrm{Na}]^{+}$calculated for $\mathrm{C}_{37} \mathrm{H}_{67} \mathrm{~N}_{3} \mathrm{O}_{3}$ : 624.5; Found: 623.7 . 


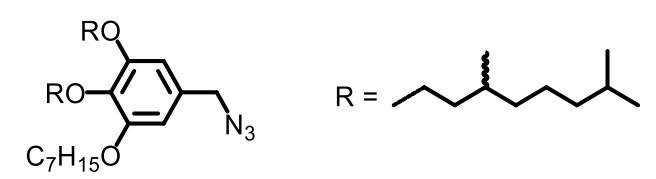

5-(Azidomethyl)-1,2-bis((3,7-(rac)-dimethyloctyl)oxy)-3-(heptyloxy)benzene (11-rr7). From 10-rr7 $(0.97 \mathrm{~g}, 1.75 \mathrm{mmol})$ and $\mathrm{NaN}_{3}(0.14 \mathrm{~g}, 2.2 \mathrm{mmol})$ in $\mathrm{DMF}(10 \mathrm{~mL})$ at $23{ }^{\circ} \mathrm{C}$ for $9 \mathrm{~h}, \mathbf{1 1}-\mathrm{rr} 7$ was obtained as a light yellow oil. Product: $0.91 \mathrm{~g}$. Yield: $93 \%$. Purity by HPLC: $99+\%$. This was used in the next step without further purification.

${ }^{1} \mathrm{H}$ NMR (400 MHz, $\left.\mathrm{CDCl}_{3}, \delta, \mathrm{ppm}\right): 6.50$ (s, 2H, $\left.\mathrm{ArH}\right), 4.24$ (s, 2H, $-\mathrm{CH}_{2} \mathrm{~N}_{3}$ ), 4.07-3.91 (m, 6H, -

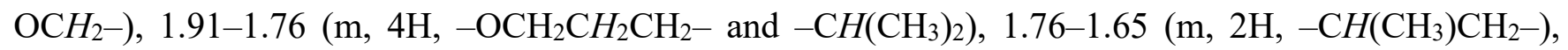
1.64-1.42 (m, 6H, $-\mathrm{O}\left(\mathrm{CH}_{2}\right)_{2} \mathrm{CH}_{2}-$ and $\left.-\mathrm{OCH}_{2} \mathrm{CH}_{2} \mathrm{CH}\left(\mathrm{CH}_{3}\right)-\right), 1.41-1.09\left(\mathrm{~m}, 18 \mathrm{H},-\mathrm{CH}\left(\mathrm{CH}_{3}\right)\left(\mathrm{CH}_{2}\right)_{3}-\right.$ and $\left.-\left(\mathrm{CH}_{2}\right)_{3}\left(\mathrm{CH}_{2}\right)_{3}-\right), 0.98-0.83\left(\mathrm{~m}, 21 \mathrm{H},-\mathrm{CH}_{3}\right) .{ }^{13} \mathrm{C} \mathrm{NMR}\left(101 \mathrm{MHz}, \mathrm{CDCl}_{3}, \delta\right.$, ppm): $153.52(\mathrm{ArC}-$ 1,3), 138.29 (ArC-2), 130.51 (ArC-5), 106.74 (ArC-4,6), $71.80\left(\mathrm{ArOCH}_{2}-3\right.$ position), $69.30\left(\mathrm{ArOCH}_{2}-2\right.$ position), 67.61 ( $\mathrm{ArOCH}_{2}-1$ position), $55.34\left(-\mathrm{CH}_{2} \mathrm{~N}_{3}\right)$, [39.51, 39.41, 37.66, 37.49, 36.52, 31.97, 29.96, $29.85,29.53,29.22,28.13,26.19,24.87]\left(-\mathrm{OCH}_{2} \mathrm{CH}_{2} \mathrm{CH}\left(\mathrm{CH}_{3}\right)\left(\mathrm{CH}_{2}\right)_{3} \mathrm{CH}-\right.$ and $\left.-\mathrm{OCH}_{2}\left(\mathrm{CH}_{2}\right)_{4}-\right)$, [22.85, $22.76,22.74,19.76,19.74,14.23]\left(-\mathrm{CH}\left(\mathrm{CH}_{3}\right)_{2},-\mathrm{CH}\left(\mathrm{CH}_{3}\right) \mathrm{CH}_{2}-\right.$ and $\left.-\mathrm{CH}_{2} \mathrm{CH}_{3}\right)$. MALDI-TOF MS $m / z$ of $[\mathrm{M}+\mathrm{Na}]^{+}$calculated for $\mathrm{C}_{34} \mathrm{H}_{61} \mathrm{~N}_{3} \mathrm{O}_{3}$ : 582.5; Found: 582.5 .

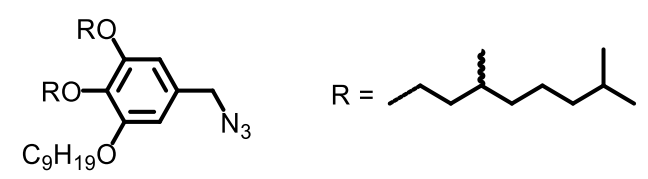

5-(Azidomethyl)-1,2-bis((3,7-(rac)-dimethyloctyl)oxy)-5-(nonyloxy)benzene (11-rr9). From 10-rr9 (1.02 g, $1.75 \mathrm{mmol})$ and $\mathrm{NaN}_{3}(0.14 \mathrm{~g}, 2.2 \mathrm{mmol})$ in DMF $(10 \mathrm{~mL})$ at $23{ }^{\circ} \mathrm{C}$ for $9 \mathrm{~h}, \mathbf{1 1 - r r 9}$ was obtained as a light yellow oil. Product: $1.03 \mathrm{~g}$. Yield: $100 \%$. Purity by HPLC: $99+\%$. This was used in the next step without further purification.

${ }^{1} \mathrm{H} \mathrm{NMR}\left(400 \mathrm{MHz}, \mathrm{CDCl}_{3}, \delta, \mathrm{ppm}\right): 6.50$ (s, 2H, $\left.\mathrm{ArH}\right), 4.24\left(\mathrm{~s}, 2 \mathrm{H},-\mathrm{CH}_{2} \mathrm{~N}_{3}\right), 4.08-3.91\left(\mathrm{~m}, 6 \mathrm{H},-\mathrm{OCH}_{2}-\right.$ ), 1.92-1.76 (m, 4H, $-\mathrm{OCH}_{2} \mathrm{CH}_{2} \mathrm{CH}_{2}-$ and $\left.-\mathrm{CH}\left(\mathrm{CH}_{3}\right)_{2}\right), 1.76-1.65\left(\mathrm{~m}, 2 \mathrm{H},-\mathrm{CH}\left(\mathrm{CH}_{3}\right) \mathrm{CH}_{2}-\right), 1.65-1.42$ $\left(\mathrm{m}, 6 \mathrm{H},-\mathrm{O}\left(\mathrm{CH}_{2}\right)_{2} \mathrm{CH}_{2}-\right.$ and $\left.-\mathrm{OCH}_{2} \mathrm{CH}_{2} \mathrm{CH}\left(\mathrm{CH}_{3}\right)-\right), 1.41-1.09\left(\mathrm{~m}, 22 \mathrm{H},-\mathrm{CH}\left(\mathrm{CH}_{3}\right)\left(\mathrm{CH}_{2}\right)_{3}-\right.$ and $\left.\left(\mathrm{CH}_{2}\right)_{3}\left(\mathrm{CH}_{2}\right)_{5}-\right), 0.97-0.84\left(\mathrm{~m}, 21 \mathrm{H},-\mathrm{CH}_{3}\right) .{ }^{13} \mathrm{C} \mathrm{NMR}\left(101 \mathrm{MHz}, \mathrm{CDCl}_{3}, \delta, \mathrm{ppm}\right): 153.50(\mathrm{ArC}-1,3)$, 138.28 (ArC-2), 130.51 (ArC-5), 106.73 (ArC-4,6), $71.78\left(\mathrm{ArOCH}_{2}-3\right.$ position), $69.28\left(\mathrm{ArOCH}_{2}-2\right.$ position), $67.59\left(\mathrm{ArOCH}_{2}-1\right.$ position), $55.32\left(-\mathrm{CH}_{2} \mathrm{~N}_{3}\right)$, [39.50, 39.40, 37.64, 37.48, 36.51, 32.03, 29.95, $29.83,29.72,29.55,29.53,29.42,28.12,26.23,24.86]\left(-\mathrm{OCH}_{2} \mathrm{CH}_{2} \mathrm{CH}\left(\mathrm{CH}_{3}\right)\left(\mathrm{CH}_{2}\right)_{3} \mathrm{CH}-\right.$ and $\left.\mathrm{OCH}_{2}\left(\mathrm{CH}_{2}\right)_{6}-\right)$, [22.84, 22.81, 22.74, 22.73, 19.75, 19.71, 14.23] $\left(-\mathrm{CH}\left(\mathrm{CH}_{3}\right)_{2},-\mathrm{CH}\left(\mathrm{CH}_{3}\right) \mathrm{CH}_{2}-\right.$ and $\mathrm{CH}_{2} \mathrm{CH}_{3}$ ). MALDI-TOF MS $m / z$ of $[\mathrm{M}+\mathrm{Na}]^{+}$calculated for $\mathrm{C}_{36} \mathrm{H}_{65} \mathrm{~N}_{3} \mathrm{O}_{3}: 610.5$; Found: 610.5 . 


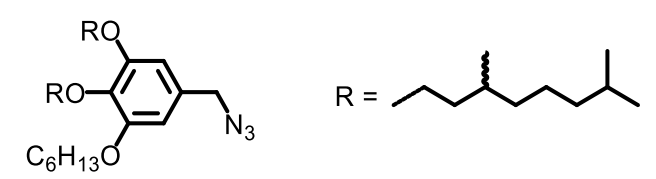

5-(Azidomethyl)-1,2-bis((3,7-(rac)-dimethyloctyl)oxy)-5-(hexyloxy)benzene (11-rr6). From 10-rr6 $(0.29 \mathrm{~g}, 0.54 \mathrm{mmol})$ and $\mathrm{NaN}_{3}(0.044 \mathrm{~g}, 0.68 \mathrm{mmol})$ in $\mathrm{DMF}(6 \mathrm{~mL})$ at $23{ }^{\circ} \mathrm{C}$ for $9 \mathrm{~h}, \mathbf{1 1 - r r 6}$ was obtained as a light yellow oil. Product: 0.29 g. Yield: $97 \%$. Purity by HPLC: $99+\%$. This was used in the next step without further purification.

${ }^{1} \mathrm{H}$ NMR (400 MHz, $\left.\mathrm{CDCl}_{3}, \delta, \mathrm{ppm}\right): 6.49$ (s, 2H, $\left.\mathrm{ArH}\right), 4.25\left(\mathrm{~s}, 2 \mathrm{H},-\mathrm{CH}_{2} \mathrm{~N}_{3}\right), 4.07-3.91(\mathrm{~m}, 6 \mathrm{H},-$

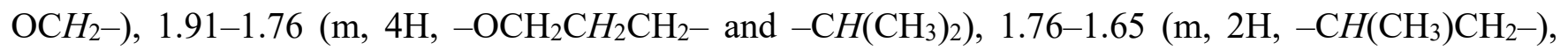
1.64-1.43 (m, 6H, $-\mathrm{O}\left(\mathrm{CH}_{2}\right)_{2} \mathrm{CH}_{2}-$ and $\left.-\mathrm{OCH}_{2} \mathrm{CH}_{2} \mathrm{CH}\left(\mathrm{CH}_{3}\right)-\right), 1.40-1.09\left(\mathrm{~m}, 16 \mathrm{H},-\mathrm{CH}\left(\mathrm{CH}_{3}\right)\left(\mathrm{CH}_{2}\right)_{3}-\right.$ and $\left.-\left(\mathrm{CH}_{2}\right)_{3}\left(\mathrm{CH}_{2}\right)_{2}-\right), 0.98-0.83\left(\mathrm{~m}, 21 \mathrm{H},-\mathrm{CH}_{3}\right) .{ }^{13} \mathrm{C} \mathrm{NMR}\left(101 \mathrm{MHz}, \mathrm{CDCl}_{3}, \delta\right.$, ppm): $153.52(\mathrm{ArC}-$ 1,3), 138.29 (ArC-2), 130.51 (ArC-5), 106.73 ( $\mathrm{ArC}-4,6), 71.79\left(\mathrm{ArOCH}_{2}-3\right.$ position), $69.29\left(\mathrm{ArOCH}_{2}-2\right.$ position), 67.60 ( $\mathrm{ArOCH}_{2}-1$ position), $55.34\left(-\mathrm{CH}_{2} \mathrm{Cl}\right)$, [39.51, 39.41, 37.66, 37.48, 36.52, 31.74, 29.96, 29.84, 29.49, 28.13, 25.91, 24.87] $\left(-\mathrm{OCH}_{2} \mathrm{CH}_{2} \mathrm{CH}\left(\mathrm{CH}_{3}\right)\left(\mathrm{CH}_{2}\right)_{3} \mathrm{CH}-\right.$ and $\left.-\mathrm{OCH}_{2}\left(\mathrm{CH}_{2}\right)_{3}-\right)$, [22.85, 22.77, 22.75, 19.77, 19.72, 14.18] $\left(-\mathrm{CH}\left(\mathrm{CH}_{3}\right)_{2},-\mathrm{CH}\left(\mathrm{CH}_{3}\right) \mathrm{CH}_{2}-\right.$ and $\left.-\mathrm{CH}_{2} \mathrm{CH}_{3}\right)$. MALDI-TOF MS $\mathrm{m} / z$ of $[\mathrm{M}+\mathrm{Na}]^{+}$calculated for $\mathrm{C}_{33} \mathrm{H}_{59} \mathrm{~N}_{3} \mathrm{O}_{3}$ : 568.4; Found: 568.7 .

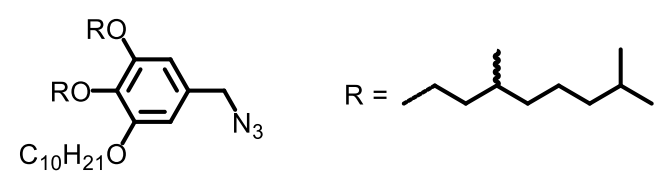

5-(Azidomethyl)-1-(decyloxy)-2,3-bis((3,7-(rac)-dimethyloctyl)oxy)benzene (11-rr10). From 10 -rr10 $(0.32 \mathrm{~g}, 0.54 \mathrm{mmol})$ and $\mathrm{NaN}_{3}(0.044 \mathrm{~g}, 0.68 \mathrm{mmol})$ in $\mathrm{DMF}(6 \mathrm{~mL})$ at $23{ }^{\circ} \mathrm{C}$ for $9 \mathrm{~h}, \mathbf{1 1 - r r 1 0}$ was obtained as a light yellow oil. Product: $0.31 \mathrm{~g}$. Yield: $95 \%$. Purity by HPLC: $99+\%$. This was used in the next step without further purification.

${ }^{1} \mathrm{H}$ NMR (400 MHz, $\left.\mathrm{CDCl}_{3}, \delta, \mathrm{ppm}\right): 6.49$ (s, 2H, $\left.\mathrm{ArH}\right), 4.24\left(\mathrm{~s}, 2 \mathrm{H},-\mathrm{CH}_{2} \mathrm{~N}_{3}\right), 4.07-3.91\left(\mathrm{~m}, 6 \mathrm{H},-\mathrm{OCH}_{2}-\right.$ ), 1.91-1.75 (m, 4H, $-\mathrm{OCH}_{2} \mathrm{CH}_{2} \mathrm{CH}_{2}-$ and $\left.-\mathrm{CH}\left(\mathrm{CH}_{3}\right)_{2}\right), 1.75-1.65\left(\mathrm{~m}, 2 \mathrm{H},-\mathrm{CH}\left(\mathrm{CH}_{3}\right) \mathrm{CH}_{2}-\right), 1.65-1.42$ $\left(\mathrm{m}, 6 \mathrm{H},-\mathrm{O}\left(\mathrm{CH}_{2}\right)_{2} \mathrm{CH}_{2}-\right.$ and $\left.-\mathrm{OCH}_{2} \mathrm{CH}_{2} \mathrm{CH}\left(\mathrm{CH}_{3}\right)-\right)$, $1.39-1.10\left(\mathrm{~m}, 24 \mathrm{H},-\mathrm{CH}\left(\mathrm{CH}_{3}\right)\left(\mathrm{CH}_{2}\right)_{3}-\right.$ and $\left.\left(\mathrm{CH}_{2}\right)_{3}\left(\mathrm{CH}_{2}\right)_{6}-\right), 0.97-0.83\left(\mathrm{~m}, 21 \mathrm{H},-\mathrm{CH}_{3}\right) .{ }^{13} \mathrm{C} \mathrm{NMR}\left(101 \mathrm{MHz}, \mathrm{CDCl}_{3}, \delta, \mathrm{ppm}\right): 153.52(\mathrm{ArC}-1,3)$, 138.29 (ArC-2), 130.51 (ArC-5), 106.74 (ArC-4,6), $71.79\left(\mathrm{ArOCH}_{2}-1\right.$ position), $69.30\left(\mathrm{ArOCH}_{2}-2\right.$ position), $67.60\left(\mathrm{ArOCH}_{2}-3\right.$ position), $55.34\left(-\mathrm{CH}_{2} \mathrm{~N}_{3}\right),[39.52,39.42,37.65,37.49,36.52,32.06,29.96$, $29.85,29.78,29.73,29.56,29.53,29.49,28.14,26.24,24.87]\left(-\mathrm{OCH}_{2} \mathrm{CH}_{2} \mathrm{CH}\left(\mathrm{CH}_{3}\right)\left(\mathrm{CH}_{2}\right){ }_{3} \mathrm{CH}-\right.$ and $\left.\mathrm{OCH}_{2}\left(\mathrm{CH}_{2}\right)_{7}-\right)$, [22.85, 22.83, 22.76, 22.74, 19.77, 19.72, 14.26] $\left(-\mathrm{CH}\left(\mathrm{CH}_{3}\right)_{2},-\mathrm{CH}\left(\mathrm{CH}_{3}\right) \mathrm{CH}_{2}-\right.$ and $\mathrm{CH}_{2} \mathrm{CH}_{3}$ ). MALDI-TOF MS $m / z$ of $[\mathrm{M}+\mathrm{Na}]^{+}$calculated for $\mathrm{C}_{37} \mathrm{H}_{67} \mathrm{~N}_{3} \mathrm{O}_{3}$ : 624.5; Found: 625.0. 
<smiles>[O]c1cc(C[N+](=O)[O-])cc(OCCOCCO)c1O</smiles><smiles>CC(C)CCCC(C)CC[PH3]</smiles>

5-(Azidomethyl)-1-((3,7-(rac)-dimethyloctyl)oxy)-2,3-bis(heptyloxy)benzene (11-77r). From 10-77r $(0.89 \mathrm{~g}, 1.75 \mathrm{mmol})$ and $\mathrm{NaN}_{3}(0.14 \mathrm{~g}, 2.2 \mathrm{mmol})$ in DMF $(10 \mathrm{~mL})$ at $23{ }^{\circ} \mathrm{C}$ for $9 \mathrm{~h}, \mathbf{1 1 - 7 7 r}$ was obtained as a light yellow oil. Product: $0.91 \mathrm{~g}$. Yield: $100 \%$. Purity by HPLC: $99+\%$. This was used in the next step without further purification.

${ }^{1} \mathrm{H}$ NMR (400 MHz, $\left.\mathrm{CDCl}_{3}, \delta, \mathrm{ppm}\right): 6.50(\mathrm{~s}, 2 \mathrm{H}, \mathrm{ArH}), 4.24\left(\mathrm{~s}, 2 \mathrm{H},-\mathrm{CH}_{2} \mathrm{~N}_{3}\right), 4.09-3.89\left(\mathrm{~m}, 6 \mathrm{H},-\mathrm{OCH}_{2}-\right.$ ), 1.93-1.67 (m, 6H, $-\mathrm{OCH}_{2} \mathrm{CH}_{2} \mathrm{CH}_{2-},-\mathrm{CH}\left(\mathrm{CH}_{3}\right)_{2}$ and $\left.-\mathrm{CH}\left(\mathrm{CH}_{3}\right) \mathrm{CH}_{2}-\right), 1.66-1.42\left(\mathrm{~m}, 6 \mathrm{H},-\mathrm{O}\left(\mathrm{CH}_{2}\right)-\right.$ ${ }_{2} \mathrm{CH}_{2}-$ and $\left.-\mathrm{OCH}_{2} \mathrm{CH}_{2} \mathrm{CH}\left(\mathrm{CH}_{3}\right)-\right), 1.42-1.10\left(\mathrm{~m}, 18 \mathrm{H},-\mathrm{CH}\left(\mathrm{CH}_{3}\right)\left(\mathrm{CH}_{2}\right)_{3}-\right.$ and $\left.-\left(\mathrm{CH}_{2}\right)_{3}\left(\mathrm{CH}_{2}\right)_{3}-\right), 0.95$ (d, $\left.J=6.6 \mathrm{~Hz}, 3 \mathrm{H},-\mathrm{CH}_{2} \mathrm{CH}\left(\mathrm{CH}_{3}\right)-\right), 0.93-0.84\left(\mathrm{~m}, 12 \mathrm{H},-\mathrm{CH}\left(\mathrm{CH}_{3}\right)_{2}\right.$ and $\left.-\mathrm{CH}_{2} \mathrm{CH}_{3}\right) .{ }^{13} \mathrm{C} \mathrm{NMR}(101 \mathrm{MHz}$, $\left.\mathrm{CDCl}_{3}, \delta, \mathrm{ppm}\right): 153.48(\mathrm{ArC}-1,3), 138.27(\mathrm{ArC}-2), 130.48(\mathrm{ArC}-5),[106.74,106.71](\mathrm{ArC}-4,6), 73.51$ ( $\mathrm{ArOCH}_{2}-2$ position), 69.27 ( $\mathrm{ArOCH} \mathrm{H}_{2}-3$ position), 67.58 ( $\mathrm{ArOCH} \mathrm{H}_{2}-1$ position), $55.30\left(-\mathrm{CH}_{2} \mathrm{~N}_{3}\right)$, [39.38, $37.45, \quad 36.51, \quad 32.04, \quad 31.94, \quad 29.93,29.51,29.37, \quad 29.19,28.11, \quad 26.19, \quad 26.16, \quad 24.85] \quad(-$ $\mathrm{OCH}_{2} \mathrm{CH}_{2} \mathrm{CH}\left(\mathrm{CH}_{3}\right)\left(\mathrm{CH}_{2}\right)_{3} \mathrm{CH}-$ and $\left.-\mathrm{OCH}_{2}\left(\mathrm{CH}_{2}\right)_{4}\right)$, [22.80, 22.73, 22.71, 19.72, 14.22, 14.19] ($\mathrm{CH}\left(\mathrm{CH}_{3}\right)_{2},-\mathrm{CH}\left(\mathrm{CH}_{3}\right) \mathrm{CH}_{2}-$ and $\left.-\mathrm{CH}_{2} \mathrm{CH}_{3}\right)$. MALDI-TOF MS $\mathrm{m} / z$ of $[\mathrm{M}+\mathrm{Na}]^{+}$calculated for $\mathrm{C}_{31} \mathrm{H}_{55} \mathrm{~N}_{3} \mathrm{O}_{3}$ : 540.4; Found: 540.6.

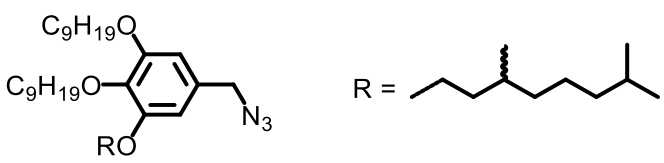

5-(Azidomethyl)-1-((3,7-(rac)-dimethyloctyl)oxy)-2,3-bis(nonyloxy)benzene (11-99r). From 10-99r $(0.99 \mathrm{~g}, 1.75 \mathrm{mmol})$ and $\mathrm{NaN}_{3}(0.14 \mathrm{~g}, 2.2 \mathrm{mmol})$ in DMF $(10 \mathrm{~mL})$ at $23{ }^{\circ} \mathrm{C}$ for $9 \mathrm{~h}, \mathbf{1 1 - 9 9 r}$ was obtained as a light yellow oil. Product: 0.98 g. Yield: $98 \%$. Purity by HPLC: $99+\%$. This was used in the next step without further purification.

${ }^{1} \mathrm{H}$ NMR (400 MHz, $\left.\mathrm{CDCl}_{3}, \delta, \mathrm{ppm}\right): 6.49$ (s, 2H, $\left.\mathrm{ArH}\right), 4.24\left(\mathrm{~s}, 2 \mathrm{H},-\mathrm{CH}_{2} \mathrm{~N}_{3}\right), 4.08-3.90\left(\mathrm{~m}, 6 \mathrm{H},-\mathrm{OCH}_{2}-\right.$ ), 1.92-1.66 (m, 6H, $-\mathrm{OCH}_{2} \mathrm{CH}_{2} \mathrm{CH}_{2}-,-\mathrm{CH}\left(\mathrm{CH}_{3}\right)_{2}$ and $\left.-\mathrm{CH}\left(\mathrm{CH}_{3}\right) \mathrm{CH}_{2}-\right), 1.65-1.42\left(\mathrm{~m}, 6 \mathrm{H},-\mathrm{O}\left(\mathrm{CH}_{2}\right)-\right.$ ${ }_{2} \mathrm{CH}_{2}-$ and $\left.-\mathrm{OCH}_{2} \mathrm{CH}_{2} \mathrm{CH}\left(\mathrm{CH}_{3}\right)-\right), 1.42-1.10\left(\mathrm{~m}, 26 \mathrm{H},-\mathrm{CH}\left(\mathrm{CH}_{3}\right)\left(\mathrm{CH}_{2}\right)_{3}-\right.$ and $\left.-\left(\mathrm{CH}_{2}\right)_{3}\left(\mathrm{CH}_{2}\right)_{5}-\right), 0.94(\mathrm{~d}$, $\left.J=6.6 \mathrm{~Hz}, 3 \mathrm{H},-\mathrm{CH}_{2} \mathrm{CH}\left(\mathrm{CH}_{3}\right)-\right), 0.92-0.81\left(\mathrm{~m}, 12 \mathrm{H},-\mathrm{CH}\left(\mathrm{CH}_{3}\right)_{2}\right.$ and $\left.-\mathrm{CH}_{2} \mathrm{CH}_{3}\right) .{ }^{13} \mathrm{C} \mathrm{NMR}(101 \mathrm{MHz}$, $\mathrm{CDCl}_{3}, \delta$, ppm): 153.49 (ArC-1,3), 138.28 (ArC-2), 130.49 (ArC-5), [106.75, 106.73] (ArC-4,6), 73.53 (ArOCH $\mathrm{H}_{2}-2$ position), 69.29 ( $\mathrm{ArOCH}_{2}-3$ position), 67.60 ( $\mathrm{ArOCH}_{2}-1$ position), $55.31\left(-\mathrm{CH}_{2} \mathrm{~N}_{3}\right)$, [39.40, $37.46,36.52,32.08,32.03,30.46,29.94,29.82$, 29.73, 29.55, 29.52, 29.51, 29.42, 28.12, 26.25, 26.22, 24.86] $\left(-\mathrm{OCH}_{2} \mathrm{CH}_{2} \mathrm{CH}\left(\mathrm{CH}_{3}\right)\left(\mathrm{CH}_{2}\right)_{3} \mathrm{CH}-\right.$ and $\left.-\mathrm{OCH}_{2}\left(\mathrm{CH}_{2}\right)_{6}-\right)$, [22.80, 22.72, 19.73, 14.23] $\left(-\mathrm{CH}_{(}\left(\mathrm{CH}_{3}\right)_{2}\right.$, $-\mathrm{CH}\left(\mathrm{CH}_{3}\right) \mathrm{CH}_{2}-$ and $\left.-\mathrm{CH}_{2} \mathrm{CH}_{3}\right)$. MALDI-TOF MS $m / z$ of $[\mathrm{M}+\mathrm{Na}]^{+}$calculated for $\mathrm{C}_{35} \mathrm{H}_{63} \mathrm{~N}_{3} \mathrm{O}_{3}$ : 596.5; Found: 596.6. 
<smiles></smiles><smiles>CC(C)CCCC(C)CC[PH3-]</smiles>

5-(Azidomethyl)-1-((3,7-(rac)-dimethyloctyl)oxy)-2,3-bis(hexyloxy)benzene (11-66r). From 10-66r $(0.26 \mathrm{~g}, 0.54 \mathrm{mmol})$ and $\mathrm{NaN}_{3}(0.044 \mathrm{~g}, 0.68 \mathrm{mmol})$ in $\mathrm{DMF}(6 \mathrm{~mL})$ at $23{ }^{\circ} \mathrm{C}$ for $9 \mathrm{~h}, \mathbf{1 1 - 6 6 r}$ was obtained as a light yellow oil. Product: 0.25 g. Yield: $96 \%$. Purity by HPLC: $99+\%$. This was used in the next step without further purification.

${ }^{1} \mathrm{H} \mathrm{NMR}\left(400 \mathrm{MHz}, \mathrm{CDCl}_{3}, \delta, \mathrm{ppm}\right): 6.49$ (s, 2H, $\left.\mathrm{ArH}\right), 4.24\left(\mathrm{~s}, 2 \mathrm{H},-\mathrm{CH}_{2} \mathrm{~N}_{3}\right), 4.09-3.88\left(\mathrm{~m}, 6 \mathrm{H},-\mathrm{OCH}_{2}-\right.$ ), 1.91-1.66 (m, 6H, $-\mathrm{OCH}_{2} \mathrm{CH}_{2} \mathrm{CH}_{2}-,-\mathrm{CH}\left(\mathrm{CH}_{3}\right)_{2}$ and $\left.-\mathrm{CH}\left(\mathrm{CH}_{3}\right) \mathrm{CH}_{2}-\right), 1.65-1.42\left(\mathrm{~m}, 6 \mathrm{H},-\mathrm{O}\left(\mathrm{CH}_{2}\right)-\right.$ ${ }_{2} \mathrm{CH}_{2}-$ and $\left.-\mathrm{OCH}_{2} \mathrm{CH}_{2} \mathrm{CH}\left(\mathrm{CH}_{3}\right)-\right), 1.40-1.10\left(\mathrm{~m}, 14 \mathrm{H},-\mathrm{CH}\left(\mathrm{CH}_{3}\right)\left(\mathrm{CH}_{2}\right)_{3}-\right.$ and $\left.-\left(\mathrm{CH}_{2}\right)_{3}\left(\mathrm{CH}_{2}\right)_{2}-\right), 0.94(\mathrm{~d}$, $\left.J=6.6 \mathrm{~Hz}, 3 \mathrm{H},-\mathrm{CH}_{2} \mathrm{CH}\left(\mathrm{CH}_{3}\right)-\right), 0.93-0.84\left(\mathrm{~m}, 12 \mathrm{H},-\mathrm{CH}\left(\mathrm{CH}_{3}\right)_{2}\right.$ and $\left.-\mathrm{CH}_{2} \mathrm{CH}_{3}\right) .{ }^{13} \mathrm{C} \mathrm{NMR}(101 \mathrm{MHz}$, $\mathrm{CDCl}_{3}, \delta, \mathrm{ppm}$ ): 153.50 (ArC-1,3), 138.28 (ArC-2), 130.50 (ArC-5), [106.75, 106.73] (ArC-4,6), 73.53 ( $\mathrm{ArOCH} \mathrm{H}_{2}-2$ position), $69.30\left(\mathrm{ArOCH}_{2}-3\right.$ position), $67.60\left(\mathrm{ArOCH} \mathrm{H}_{2}-1\right.$ position), $55.33\left(-\mathrm{CH}_{2} \mathrm{~N}_{3}\right),[39.40$, $37.47, \quad 36.52, \quad 31.92, \quad 31.71, \quad 30.42, \quad 29.94, \quad 29.48, \quad 28.13, \quad 25.91, \quad 25.89, \quad 24.87] \quad(-$ $\mathrm{OCH}_{2} \mathrm{CH}_{2} \mathrm{CH}\left(\mathrm{CH}_{3}\right)\left(\mathrm{CH}_{2}\right)_{3} \mathrm{CH}-$ and $\left.-\mathrm{OCH}_{2}\left(\mathrm{CH}_{2}\right)_{3}-\right)$, [22.83, 22.76, 22.73, 19.74, 14.23, 14.16] ($\mathrm{CH}\left(\mathrm{CH}_{3}\right)_{2},-\mathrm{CH}\left(\mathrm{CH}_{3}\right) \mathrm{CH}_{2}-$ and $\left.-\mathrm{CH}_{2} \mathrm{CH}_{3}\right)$. MALDI-TOF MS $m / z$ of $[\mathrm{M}+\mathrm{Na}]^{+}$calculated for $\mathrm{C}_{29} \mathrm{H}_{51} \mathrm{~N}_{3} \mathrm{O}_{3}$ : 512.4; Found: 512.4.
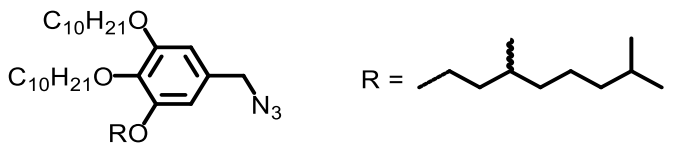

5-(Azidomethyl)-1,2-bis(decyloxy)-3-((3,7-dimethyloctyl)oxy)benzene (11-1010r). From 10-1010r $(0.32 \mathrm{~g}, 0.54 \mathrm{mmol})$ and $\mathrm{NaN}_{3}(0.044 \mathrm{~g}, 0.68 \mathrm{mmol})$ in $\mathrm{DMF}(6 \mathrm{~mL})$ at $23{ }^{\circ} \mathrm{C}$ for $9 \mathrm{~h}, \mathbf{1 1 - 1 0 1 0 r}$ was obtained as a light yellow oil. Product: 0.31 g. Yield: $95 \%$. Purity by HPLC: $99+\%$. This was used in the next step without further purification.

${ }^{1} \mathrm{H}$ NMR (400 MHz, $\left.\mathrm{CDCl}_{3}, \delta, \mathrm{ppm}\right): 6.49$ (s, 2H, $\left.\mathrm{ArH}\right), 4.24\left(\mathrm{~s}, 2 \mathrm{H},-\mathrm{CH}_{2} \mathrm{~N}_{3}\right), 4.07-3.89\left(\mathrm{~m}, 6 \mathrm{H},-\mathrm{OCH}_{2}-\right.$ ), 1.91-1.65 (m, 6H, $-\mathrm{OCH}_{2} \mathrm{CH}_{2} \mathrm{CH}_{2}-,-\mathrm{CH}\left(\mathrm{CH}_{3}\right)_{2}$ and $\left.-\mathrm{CH}\left(\mathrm{CH}_{3}\right) \mathrm{CH}_{2}-\right), 1.65-1.42\left(\mathrm{~m}, 6 \mathrm{H},-\mathrm{O}\left(\mathrm{CH}_{2}\right)-\right.$ ${ }_{2} \mathrm{CH}_{2}-$ and $\left.-\mathrm{OCH}_{2} \mathrm{CH}_{2} \mathrm{CH}\left(\mathrm{CH}_{3}\right)-\right), 1.40-1.10\left(\mathrm{~m}, 30 \mathrm{H},-\mathrm{CH}\left(\mathrm{CH}_{3}\right)\left(\mathrm{CH}_{2}\right)_{3}-\right.$ and $\left.-\left(\mathrm{CH}_{2}\right)_{3}\left(\mathrm{CH}_{2}\right)_{6}-\right), 0.94(\mathrm{~d}$, $\left.J=6.5 \mathrm{~Hz}, 3 \mathrm{H},-\mathrm{CH}_{2} \mathrm{CH}\left(\mathrm{CH}_{3}\right)-\right), 0.92-0.82\left(\mathrm{~m}, 12 \mathrm{H},-\mathrm{CH}\left(\mathrm{CH}_{3}\right)_{2}\right.$ and $\left.-\mathrm{CH}_{2} \mathrm{CH}_{3}\right) .{ }^{13} \mathrm{C} \mathrm{NMR}(101 \mathrm{MHz}$, $\left.\mathrm{CDCl}_{3}, \delta, \mathrm{ppm}\right): 153.50$ (ArC-1,3), 138.29 (ArC-2), 130.49 (ArC-5), [106.76, 106.74] (ArC-4,6), 73.55 ( $\mathrm{ArOCH} \mathrm{H}_{2}-2$ position), 69.30 ( $\mathrm{ArOCH}_{2}-1$ position), 67.61 ( $\mathrm{ArOCH}_{2}-3$ position), $55.33\left(-\mathrm{CH}_{2} \mathrm{~N}_{3}\right)$, [39.41, $37.47,36.53,32.09,32.06,30.48,29.95,29.88,29.82,29.79,29.75,29.73,29.54,29.50,28.13,26.27$, 26.23, 24.87] $\left(-\mathrm{OCH}_{2} \mathrm{CH}_{2} \mathrm{CH}\left(\mathrm{CH}_{3}\right)\left(\mathrm{CH}_{2}\right)_{3} \mathrm{CH}-\right.$ and $\left.-\mathrm{OCH}_{2}\left(\mathrm{CH}_{2}\right)_{7}-\right)$, [22.84, 22.74, 19.75, 14.25] ($\mathrm{CH}\left(\mathrm{CH}_{3}\right)_{2},-\mathrm{CH}\left(\mathrm{CH}_{3}\right) \mathrm{CH}_{2}-$ and $\left.-\mathrm{CH}_{2} \mathrm{CH}_{3}\right)$. MALDI-TOF MS $m / z$ of $[\mathrm{M}+\mathrm{Na}]^{+}$calculated for $\mathrm{C}_{37} \mathrm{H}_{67} \mathrm{~N}_{3} \mathrm{O}_{3}$ : 624.5; Found: 625.1 . 
General procedure for preparation of minidendron amines 12. The synthesis of minidendron amines 12 was conducted according to a procedure reported by our group. ${ }^{10}$ A general example is provided below.
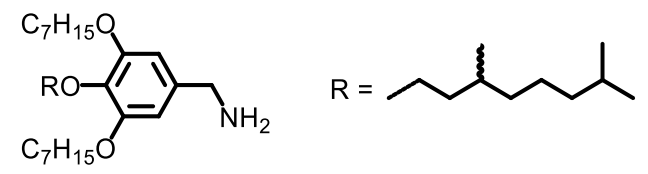

(4-((3,7-(rac)-Dimethyloctyl)oxy)-3,5-bis(heptyloxy)phenyl)methanamine (12-7r7). Under $\quad \mathrm{N}_{2}$ protection, 11-7r7 $(0.80 \mathrm{~g}, 1.55 \mathrm{mmol})$ in dry THF $(4 \mathrm{~mL})$ was added dropwise to a slurry of $\mathrm{LiAlH}_{4}$ $(0.087 \mathrm{~g}, 2.3 \mathrm{mmol})$ in dry THF $(6 \mathrm{~mL})$ at $0{ }^{\circ} \mathrm{C}$. The mixture was stirred at $23{ }^{\circ} \mathrm{C}$ for $1 \mathrm{~h}$. Afterwards, the reaction mixture was cooled to $0{ }^{\circ} \mathrm{C}$ and quenched by the successive addition of $\mathrm{H}_{2} \mathrm{O}(0.1 \mathrm{~mL}), 15 \% \mathrm{NaOH}$ aqueous solution $(0.1 \mathrm{~mL})$ and $\mathrm{H}_{2} \mathrm{O}(0.5 \mathrm{~mL})$. Then, the mixture was filtered through celite, washed with plenty of THF, dried over anhydrous $\mathrm{MgSO}_{4}$, filtered and evaporated in vacuo. Compound 12-7r7 was obtained as a white wax-like oil. Product: 0.76 g. Yield: $100 \%$. This was used in the next step without further purification.

${ }^{1} \mathrm{H}$ NMR (400 MHz, $\left.\mathrm{CDCl}_{3}, \delta, \mathrm{ppm}\right): 6.51$ (s, 2H, $\left.\mathrm{ArH}\right), 4.03-3.89$ (m, 6H, $-\mathrm{OCH}_{2}-$ ), 3.78 (s, 2H, $\left.\mathrm{CH}_{2} \mathrm{NH}_{2}\right), 1.87-1.74\left(\mathrm{~m}, 5 \mathrm{H},-\mathrm{CH}\left(\mathrm{CH}_{3}\right)_{2}\right.$ and $\left.-\mathrm{OCH}_{2} \mathrm{CH}_{2} \mathrm{CH}_{2}-\right), 1.74-1.64\left(\mathrm{~m}, 1 \mathrm{H},-\mathrm{CH}_{(}\left(\mathrm{CH}_{3}\right) \mathrm{CH}_{2}-\right)$, 1.58-1.41 (m, 6H, $-\mathrm{O}\left(\mathrm{CH}_{2}\right)_{2} \mathrm{CH}_{2}-$ and $\left.-\mathrm{OCH}_{2} \mathrm{CH}_{2} \mathrm{CH}\left(\mathrm{CH}_{3}\right)-\right), 1.40-1.08\left(\mathrm{~m}, 18 \mathrm{H},-\mathrm{CH}\left(\mathrm{CH}_{3}\right)\left(\mathrm{CH}_{2}\right)_{3}-\right.$ and $\left.-\left(\mathrm{CH}_{2}\right)_{3}\left(\mathrm{CH}_{2}\right)_{3}-\right), 0.95-0.82\left(\mathrm{~m}, 15 \mathrm{H},-\mathrm{CH}_{3}\right) .{ }^{13} \mathrm{C} \mathrm{NMR}\left(101 \mathrm{MHz}, \mathrm{CDCl}_{3}, \delta, \mathrm{ppm}\right): 153.41(\mathrm{ArC}-$ 3,5), 137.34 ( $\mathrm{ArC}-4), 137.27$ ( $\mathrm{ArC}-1), 105.79$ ( $\mathrm{ArC}-2,6), 71.81\left(\mathrm{ArOCH}_{2}-3,5\right.$ position), $69.26\left(\mathrm{ArOCH}_{2}-\right.$ 4 position), $46.42\left(-\mathrm{CH}_{2} \mathrm{NH}_{2}\right)$, [39.50, 37.66, 37.48, 31.97, 29.86, 29.58, 29.22, 28.13, 26.20, 24.86] ($\mathrm{OCH}_{2}\left(\mathrm{CH}_{2}\right)_{4}-$ and $\left.-\mathrm{OCH}_{2} \mathrm{CH}_{2} \mathrm{CH}\left(\mathrm{CH}_{3}\right)\left(\mathrm{CH}_{2}\right)_{3} \mathrm{CH}-\right)$, [22.84, 22.76, 19.69, 14.22] $\left(-\mathrm{CH}_{(} \mathrm{CH}_{3}\right)_{2}$, $\mathrm{CH}\left(\mathrm{CH}_{3}\right) \mathrm{CH}_{2}-$ and $\left.-\mathrm{CH}_{2} \mathrm{CH}_{3}\right)$. MALDI-TOF MS m/z of $[\mathrm{M}+\mathrm{Na}]^{+}$calculated for $\mathrm{C}_{31} \mathrm{H}_{57} \mathrm{NO}_{3}$ : 514.4; Found: 514.5 .

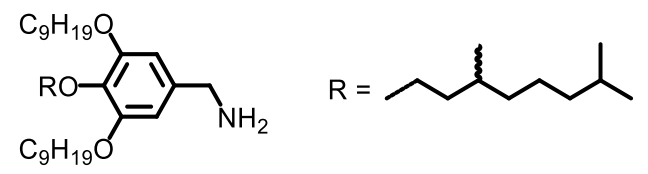

(4-((3,7-(rac)-Dimethyloctyl)oxy)-3,5-bis(nonyloxy)phenyl)methanamine (12-9r9). From 11-9r9 $(0.89 \mathrm{~g}, 1.55 \mathrm{mmol})$ and $\mathrm{LiAlH}_{4}(0.087 \mathrm{~g}, 2.3 \mathrm{mmol})$ in dry THF $(10 \mathrm{~mL})$ at $23{ }^{\circ} \mathrm{C}$ for $1 \mathrm{~h}, \mathbf{1 2 - 9 r 9}$ was obtained as a white wax-like oil. Product: $0.85 \mathrm{~g}$; Yield: $100 \%$. This was used in the next step without further purification.

${ }^{1} \mathrm{H}$ NMR (400 MHz, $\left.\mathrm{CDCl}_{3}, \delta, \mathrm{ppm}\right): 6.51$ (s, 2H, ArH), 4.02-3.90 (m, 6H, -OCH - ), 3.78 (s, 2H, -

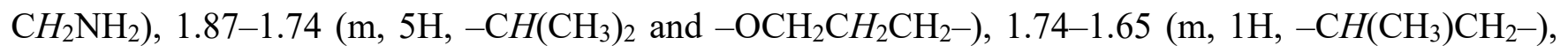
1.59-1.40 (m, 6H, $-\mathrm{O}\left(\mathrm{CH}_{2}\right)_{2} \mathrm{CH}_{2}-$ and $\left.-\mathrm{OCH}_{2} \mathrm{CH}_{2} \mathrm{CH}\left(\mathrm{CH}_{3}\right)-\right), 1.40-1.08\left(\mathrm{~m}, 26 \mathrm{H},-\mathrm{CH}\left(\mathrm{CH}_{3}\right)\left(\mathrm{CH}_{2}\right)_{3}-\right.$ and $\left.-\left(\mathrm{CH}_{2}\right)_{3}\left(\mathrm{CH}_{2}\right)_{5}-\right), 0.96-0.81\left(\mathrm{~m}, 15 \mathrm{H},-\mathrm{CH}_{3}\right) .{ }^{13} \mathrm{C} \mathrm{NMR}\left(101 \mathrm{MHz}, \mathrm{CDCl}_{3}, \delta, \mathrm{ppm}\right): 153.42(\mathrm{ArC}-$ 3,5), 137.33 (ArC-4), 137.27 ( $\mathrm{ArC}-1), 105.79$ ( $\mathrm{ArC}-2,6), 71.81\left(\mathrm{ArOCH}_{2}-3,5\right.$ position), $69.27\left(\mathrm{ArOCH}_{2}-\right.$ 4 position), $46.42\left(-\mathrm{CH}_{2} \mathrm{NH}_{2}\right)$, [39.51, 37.66, 37.48, 32.03, 29.86, 29.74, 29.57, 29.43, 28.13, 26.25, 24.86] $\left(-\mathrm{OCH}_{2}\left(\mathrm{CH}_{2}\right)_{6}-\right.$ and $\left.-\mathrm{OCH}_{2} \mathrm{CH}_{2} \mathrm{CH}\left(\mathrm{CH}_{3}\right)\left(\mathrm{CH}_{2}\right)_{3} \mathrm{CH}-\right)$, [22.84, 22.81, 22.74, 19.69, 14.24] $\left(-\mathrm{CH}\left(\mathrm{CH}_{3}\right)_{2}\right.$, $-\mathrm{CH}\left(\mathrm{CH}_{3}\right) \mathrm{CH}_{2}-$ and $\left.-\mathrm{CH}_{2} \mathrm{CH}_{3}\right)$. MALDI-TOF MS $m / z$ of $[\mathrm{M}+\mathrm{Na}]^{+}$calculated for $\mathrm{C}_{35} \mathrm{H}_{65} \mathrm{NO}_{3}$ : 570.5; Found: 570.8 . 


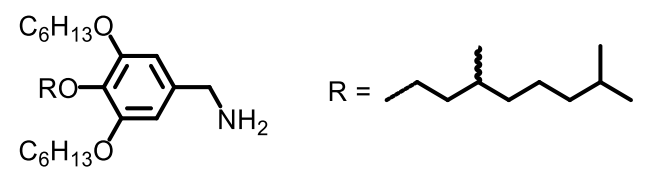

(3,5-bis(hexyloxy)-4-((3,7-(rac)-dimethyloctyl)oxy)phenyl)methanamine (12-6r6). From 11-6r6 $(0.084 \mathrm{~g}, 0.17 \mathrm{mmol})$ and $\mathrm{LiAlH}_{4}(0.010 \mathrm{~g}, 0.26 \mathrm{mmol})$ in dry THF $(4 \mathrm{~mL})$ at $23{ }^{\circ} \mathrm{C}$ for $1 \mathrm{~h}, \mathbf{1 2 - 6 r 6}$ was obtained as a light yellow oil. Product: $0.075 \mathrm{~g}$; Yield: 93\%. This was used in the next step without further purification.

${ }^{1} \mathrm{H}$ NMR (500 MHz, $\left.\mathrm{CDCl}_{3}, \delta, \mathrm{ppm}\right): 6.51$ (s, 2H, $\left.\mathrm{ArH}\right), 4.02-3.91\left(\mathrm{~m}, 6 \mathrm{H},-\mathrm{OCH}_{2}-\right), 3.78(\mathrm{~s}, 2 \mathrm{H},-$

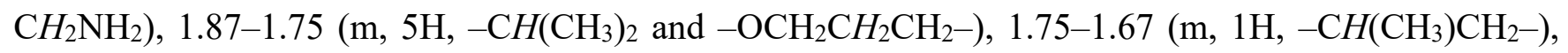
1.58-1.41 (m, 6H, $-\mathrm{O}\left(\mathrm{CH}_{2}\right)_{2} \mathrm{CH}_{2}-$ and $\left.-\mathrm{OCH}_{2} \mathrm{CH}_{2} \mathrm{CH}\left(\mathrm{CH}_{3}\right)-\right), 1.39-1.09\left(\mathrm{~m}, 14 \mathrm{H},-\mathrm{CH}\left(\mathrm{CH}_{3}\right)\left(\mathrm{CH}_{2}\right)_{3}-\right.$ and $\left.-\left(\mathrm{CH}_{2}\right)_{3}\left(\mathrm{CH}_{2}\right)_{2}-\right), 0.95-0.83\left(\mathrm{~m}, 15 \mathrm{H},-\mathrm{CH}_{3}\right) .{ }^{13} \mathrm{C} \mathrm{NMR}\left(126 \mathrm{MHz}, \mathrm{CDCl}_{3}, \delta, \mathrm{ppm}\right): 153.40(\mathrm{ArC}-$ 3,5), 138.65 (ArC-4), 137.20 ( $\mathrm{ArC}-1)$, 105.62 ( $\mathrm{ArC}-2,6), 71.82\left(\mathrm{ArOCH}_{2}-3,5\right.$ position), $69.29\left(\mathrm{ArOCH}_{2}-\right.$ 4 position), $46.92\left(-\mathrm{CH}_{2} \mathrm{NH}_{2}\right),[39.52,37.67,37.50,31.75,29.87,29.57,28.15,25.93,24.87]$ ($\mathrm{OCH}_{2}\left(\mathrm{CH}_{2}\right)_{3}-$ and $\left.-\mathrm{OCH}_{2} \mathrm{CH}_{2} \mathrm{CH}\left(\mathrm{CH}_{3}\right)\left(\mathrm{CH}_{2}\right)_{3} \mathrm{CH}-\right)$, [22.85, 22.78, 22.75, 19.72, 14.18] $\left(-\mathrm{CH}\left(\mathrm{CH}_{3}\right)_{2},-\right.$ $\mathrm{CH}\left(\mathrm{CH}_{3}\right) \mathrm{CH}_{2}-$ and $\left.-\mathrm{CH}_{2} \mathrm{CH}_{3}\right)$. MALDI-TOF MS m/z of $[\mathrm{M}+\mathrm{Na}]^{+}$calculated for $\mathrm{C}_{29} \mathrm{H}_{53} \mathrm{NO}_{3}$ : 486.4; Found: 486.4.

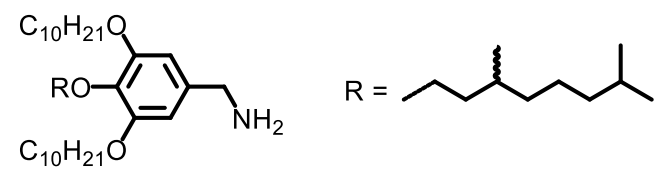

(3,5-Bis(decyloxy)-4-((3,7-(rac)-dimethyloctyl)oxy)phenyl)methanamine (12-10r10). From 11-10r10 $(0.055 \mathrm{~g}, 0.091 \mathrm{mmol})$ and $\mathrm{LiAlH}_{4}(0.005 \mathrm{~g}, 0.14 \mathrm{mmol})$ in dry $\mathrm{THF}(4 \mathrm{~mL})$ at $23{ }^{\circ} \mathrm{C}$ for $1 \mathrm{~h}, \mathbf{1 2 - 1 0 r 1 0}$ was obtained as a white wax-like oil. Product: $0.047 \mathrm{~g}$; Yield: $89 \%$. This was used in the next step without further purification.

${ }^{1} \mathrm{H}$ NMR (500 MHz, $\left.\mathrm{CDCl}_{3}, \delta, \mathrm{ppm}\right): 6.50$ (s, 2H, ArH), 4.03-3.90 (m, 6H, -OCH $\mathrm{O}_{2}-$ ), 3.77 (s, 2H, -

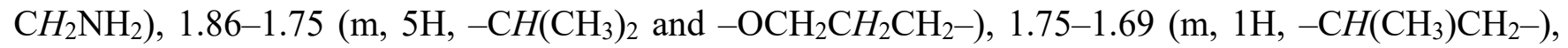
1.57-1.41 (m, 6H, $-\mathrm{O}\left(\mathrm{CH}_{2}\right)_{2} \mathrm{CH}_{2}-$ and $\left.-\mathrm{OCH}_{2} \mathrm{CH}_{2} \mathrm{CH}\left(\mathrm{CH}_{3}\right)-\right), 1.38-1.09\left(\mathrm{~m}, 30 \mathrm{H},-\mathrm{CH}\left(\mathrm{CH}_{3}\right)\left(\mathrm{CH}_{2}\right)_{3}-\right.$ and $\left.-\left(\mathrm{CH}_{2}\right)_{3}\left(\mathrm{CH}_{2}\right)_{6}-\right), 0.95-0.83\left(\mathrm{~m}, 15 \mathrm{H},-\mathrm{CH}_{3}\right) .{ }^{13} \mathrm{C} \mathrm{NMR}\left(126 \mathrm{MHz}, \mathrm{CDCl}_{3}, \delta\right.$, ppm): $153.38(\mathrm{ArC}-$ 3,5), 138.60 (ArC-4), 137.19 ( $\mathrm{ArC}-1), 105.62$ ( $\mathrm{ArC}-2,6), 71.81\left(\mathrm{ArOCH}_{2}-3,5\right.$ position), $69.27\left(\mathrm{ArOCH}_{2}-\right.$ 4 position $), 46.88\left(-\mathrm{CH}_{2} \mathrm{NH}_{2}\right)$, [39.52, 37.67, 37.50, 32.04, 29.87, 29.78, 29.73, 29.61, 29.56, 29.48, 28.13, $26.25,24.86]\left(-\mathrm{OCH}_{2}\left(\mathrm{CH}_{2}\right)_{7}-\right.$ and $\left.-\mathrm{OCH}_{2} \mathrm{CH}_{2} \mathrm{CH}\left(\mathrm{CH}_{3}\right)\left(\mathrm{CH}_{2}\right)_{3} \mathrm{CH}-\right),[22.84,22.82,22.74,19.72,14.23]$ $\left(-\mathrm{CH}\left(\mathrm{CH}_{3}\right)_{2},-\mathrm{CH}\left(\mathrm{CH}_{3}\right) \mathrm{CH}_{2}-\right.$ and $\left.-\mathrm{CH}_{2} \mathrm{CH}_{3}\right)$. MALDI-TOF MS $m / z$ of $[\mathrm{M}+\mathrm{Na}]^{+}$calculated for $\mathrm{C}_{37} \mathrm{H}_{69} \mathrm{NO}_{3}$ : 598.5; Found: 598.0. 
(N)

(3,5-Bis((3,7-(rac)-dimethyloctyl)oxy)-4-(heptyloxy)phenyl)methanamine (12-r7r). From 11-r7r $(0.87 \mathrm{~g}, 1.55 \mathrm{mmol})$ and $\mathrm{LiAlH}_{4}(0.087 \mathrm{~g}, 2.3 \mathrm{mmol})$ in dry THF $(10 \mathrm{~mL})$ at $23{ }^{\circ} \mathrm{C}$ for $1 \mathrm{~h}, 12-\mathbf{r} 7 \mathbf{r}$ was obtained as a light yellow oil. Product: $0.81 \mathrm{~g}$; Yield: $98 \%$. This was used in the next step without further purification.

${ }^{1} \mathrm{H}$ NMR (400 MHz, $\mathrm{CDCl}_{3}, \delta$, ppm): 6.51 (s, 2H, $\left.\mathrm{ArH}\right), 4.05-3.95\left(\mathrm{~m}, 4 \mathrm{H},-\mathrm{OCH}_{2} \mathrm{CH}_{2} \mathrm{CH}_{\left(\mathrm{CH}_{3}\right)-}\right), 3.92$ $\left(\mathrm{t}, J=6.6 \mathrm{~Hz}, 2 \mathrm{H},-\mathrm{OCH}_{2} \mathrm{CH}_{2} \mathrm{CH}_{2}-\right), 3.78\left(\mathrm{~s}, 2 \mathrm{H},-\mathrm{CH}_{2} \mathrm{NH}_{2}\right), 1.90-1.78\left(\mathrm{~m}, 2 \mathrm{H},-\mathrm{CH}\left(\mathrm{CH}_{3}\right)_{2}\right), 1.78-1.64$

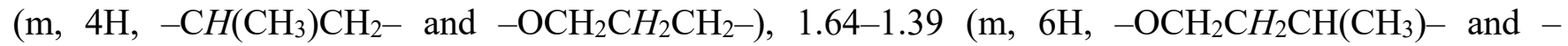
$\left.\mathrm{OCH}_{2} \mathrm{CH}_{2} \mathrm{CH}_{2}-\right), 1.39-1.10\left(\mathrm{~m}, 18 \mathrm{H},-\mathrm{CH}\left(\mathrm{CH}_{3}\right)\left(\mathrm{CH}_{2}\right)_{3}-\right.$ and $\left.-\left(\mathrm{CH}_{2}\right)_{3}\left(\mathrm{CH}_{2}\right)_{3}{ }^{-}\right), 0.94(\mathrm{~d}, J=6.5 \mathrm{~Hz}, 6 \mathrm{H},-$ $\left.\mathrm{CH}_{2} \mathrm{CH}\left(\mathrm{CH}_{3}\right)^{-}\right), 0.91-0.82\left(\mathrm{~m}, 15 \mathrm{H},-\mathrm{CH}\left(\mathrm{CH}_{3}\right)_{2}\right.$ and $\left.-\mathrm{CH}_{2} \mathrm{CH}_{3}\right) .{ }^{13} \mathrm{C} \mathrm{NMR}\left(101 \mathrm{MHz}, \mathrm{CDCl}_{3}, \delta, \mathrm{ppm}\right)$ : 153.39 (ArC-3,5), 137.70 (ArC-4), 137.24 (ArC-1), 105.70 ( $\mathrm{ArC}-2,6), 73.53$ ( $\mathrm{ArOCH}_{2}-4$ position), 67.55 (ArOCH $\mathrm{H}_{2}-3,5$ position), $46.57\left(-\mathrm{CH}_{2} \mathrm{NH}_{2}\right)$, [39.40, 37.47, 36.60, 32.05, 30.47, 29.94, 29.40, 28.12, 26.22, 24.86] $\left(-\mathrm{OCH}_{2} \mathrm{CH}_{2} \mathrm{CH}\left(\mathrm{CH}_{3}\right)\left(\mathrm{CH}_{2}\right)_{3} \mathrm{CH}-\right.$ and $\left.-\mathrm{OCH}_{2}\left(\mathrm{CH}_{2}\right)_{4}-\right)$, [22.82, 22.72, 19.73, 14.24] $\left(-\mathrm{CH}_{(}\left(\mathrm{CH}_{3}\right)_{2}\right.$, $-\mathrm{CH}\left(\mathrm{CH}_{3}\right) \mathrm{CH}_{2}-$ and $\left.-\mathrm{CH}_{2} \mathrm{CH}_{3}\right)$. MALDI-TOF MS $m / z$ of $[\mathrm{M}+\mathrm{Na}]^{+}$calculated for $\mathrm{C}_{34} \mathrm{H}_{63} \mathrm{NO}_{3}$ : 556.5; Found: 556.6.

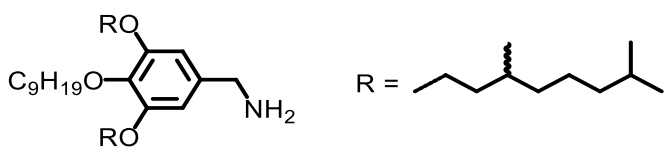

(3,5-Bis((3,7-(rac)-dimethyloctyl)oxy)-4-(nonyloxy)phenyl)methanamine (12-r9r). From 11-r9r (0.91 g, $1.55 \mathrm{mmol})$ and $\mathrm{LiAlH}_{4}(0.087 \mathrm{~g}, 2.3 \mathrm{mmol})$ in dry THF $(10 \mathrm{~mL})$ at $23{ }^{\circ} \mathrm{C}$ for $1 \mathrm{~h}, \mathbf{1 2}-\mathbf{r 9 r}$ was obtained as a light yellow oil. Product: $0.86 \mathrm{~g}$; Yield: 99\%. This was used in the next step without further purification.

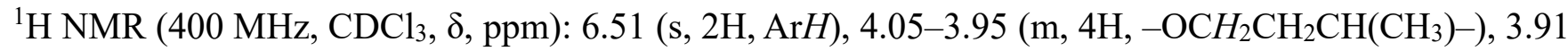
(t, $\left.J=6.6 \mathrm{~Hz}, 2 \mathrm{H},-\mathrm{OCH}_{2} \mathrm{CH}_{2} \mathrm{CH}_{2}-\right), 3.78\left(\mathrm{~s}, 2 \mathrm{H},-\mathrm{CH}_{2} \mathrm{NH}_{2}\right), 1.90-1.79\left(\mathrm{~m}, 2 \mathrm{H},-\mathrm{CH}\left(\mathrm{CH}_{3}\right)_{2}\right), 1.77-1.64$

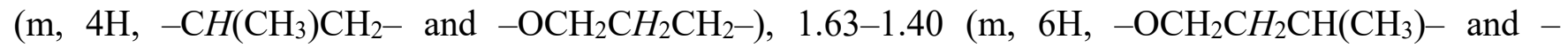
$\left.\mathrm{OCH}_{2} \mathrm{CH}_{2} \mathrm{CH}_{2}-\right), 1.40-1.09\left(\mathrm{~m}, 22 \mathrm{H},-\mathrm{CH}\left(\mathrm{CH}_{3}\right)\left(\mathrm{CH}_{2}\right)_{3}-\right.$ and $\left.-\left(\mathrm{CH}_{2}\right)_{3}\left(\mathrm{CH}_{2}\right)_{5}-\right), 0.91(\mathrm{~d}, J=6.6 \mathrm{~Hz}, 6 \mathrm{H},-$ $\left.\mathrm{CH}_{2} \mathrm{CH}\left(\mathrm{CH}_{3}\right)^{-}\right)$, 0.90-0.83 (m, 15H, $-\mathrm{CH}\left(\mathrm{CH}_{3}\right)_{2}$ and $\left.-\mathrm{CH}_{2} \mathrm{CH}_{3}\right) .{ }^{13} \mathrm{C} \mathrm{NMR}\left(101 \mathrm{MHz}, \mathrm{CDCl}_{3}, \delta, \mathrm{ppm}\right)$ : 153.39 (ArC-3,5), 137.63 (ArC-4), 137.25 (ArC-1), 105.71 ( $\mathrm{ArC}-2,6), 73.53$ ( $\mathrm{ArOCH}_{2}-4$ position), 67.55 (ArOCH $2-3,5$ position), 46.55 $\left(-\mathrm{CH}_{2} \mathrm{NH}_{2}\right)$, [39.40, 37.47, 36.60, 32.07, 30.47, 29.94, 29.81, 29.75, 29.50, $28.11,26.27,24.86]\left(-\mathrm{OCH}_{2} \mathrm{CH}_{2} \mathrm{CH}\left(\mathrm{CH}_{3}\right)\left(\mathrm{CH}_{2}\right)_{3} \mathrm{CH}-\right.$ and $\left.-\mathrm{OCH}_{2}\left(\mathrm{CH}_{2}\right)_{6-}\right),[22.82,22.72,19.72,14.24]$ $\left(-\mathrm{CH}\left(\mathrm{CH}_{3}\right)_{2},-\mathrm{CH}\left(\mathrm{CH}_{3}\right) \mathrm{CH}_{2}-\right.$ and $\left.-\mathrm{CH}_{2} \mathrm{CH}_{3}\right)$. MALDI-TOF MS $m / z$ of $\left[\mathrm{M}+\mathrm{Na}^{+}\right.$calculated for $\mathrm{C}_{36} \mathrm{H}_{67} \mathrm{NO}_{3}$ : 584.5; Found: 584.5. 


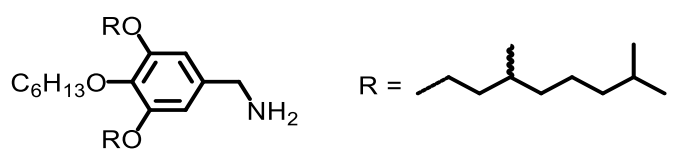

(3,5-Bis((3,7-(rac)-dimethyloctyl)oxy)-4-(hexyloxy)phenyl)methanamine (12-r6r). From 11-r6r $(0.070 \mathrm{~g}, 0.13 \mathrm{mmol})$ and $\mathrm{LiAlH}_{4}(0.007 \mathrm{~g}, 0.19 \mathrm{mmol})$ in dry THF $(4 \mathrm{~mL})$ at $23{ }^{\circ} \mathrm{C}$ for $1 \mathrm{~h}, \mathbf{1 2}-\mathbf{r 6 r}$ was obtained as a light yellow oil. Product: $0.063 \mathrm{~g}$; Yield: $95 \%$. This was used in the next step without further purification.

${ }^{1} \mathrm{H}$ NMR (500 MHz, $\mathrm{CDCl}_{3}, \delta$, ppm): 6.52 (s, 2H, $\left.\mathrm{ArH}\right), 4.07-3.96\left(\mathrm{~m}, 4 \mathrm{H},-\mathrm{OCH}_{2} \mathrm{CH}_{2} \mathrm{CH}_{\left(\mathrm{CH}_{3}\right)-}\right), 3.92$ $\left(\mathrm{t}, J=6.7 \mathrm{~Hz}, 2 \mathrm{H},-\mathrm{OCH}_{2} \mathrm{CH}_{2} \mathrm{CH}_{2}-\right), 3.79\left(\mathrm{~s}, 2 \mathrm{H},-\mathrm{CH}_{2} \mathrm{NH}_{2}\right), 1.89-1.81\left(\mathrm{~m}, 2 \mathrm{H},-\mathrm{CH}\left(\mathrm{CH}_{3}\right)_{2}\right), 1.77-1.66$

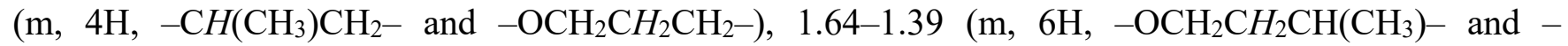
$\left.\mathrm{OCH}_{2} \mathrm{CH}_{2} \mathrm{CH}_{2}-\right), 1.38-1.11\left(\mathrm{~m}, 16 \mathrm{H},-\mathrm{CH}\left(\mathrm{CH}_{3}\right)\left(\mathrm{CH}_{2}\right)_{3}-\right.$ and $\left.-\left(\mathrm{CH}_{2}\right)_{3}\left(\mathrm{CH}_{2}\right)_{2}-\right), 0.94(\mathrm{~d}, J=6.6 \mathrm{~Hz}, 6 \mathrm{H},-$ $\left.\mathrm{CH}_{2} \mathrm{CH}\left(\mathrm{CH}_{3}\right)^{-}\right), 0.92-0.83\left(\mathrm{~m}, 15 \mathrm{H},-\mathrm{CH}\left(\mathrm{CH}_{3}\right)_{2}\right.$ and $\left.-\mathrm{CH}_{2} \mathrm{CH}_{3}\right) .{ }^{13} \mathrm{C} \mathrm{NMR}\left(126 \mathrm{MHz}, \mathrm{CDCl}_{3}, \delta, \mathrm{ppm}\right)$ : 153.40 (ArC-3,5), 138.67 (ArC-4), 137.20 (ArC-1), 105.64 (ArC-2,6), 73.55 ( $\mathrm{ArOCH}_{2}-4$ position), 67.60 (ArOCH$H_{2}-3,5$ position), $46.94\left(-\mathrm{CH}_{2} \mathrm{NH}_{2}\right)$, [39.44, 37.50, 36.65, 31.96, 30.45, 29.98, 28.14, 25.97, 24.88] $\left(-\mathrm{OCH}_{2} \mathrm{CH}_{2} \mathrm{CH}\left(\mathrm{CH}_{3}\right)\left(\mathrm{CH}_{2}\right)_{3} \mathrm{CH}-\right.$ and $\left.-\mathrm{OCH}_{2}\left(\mathrm{CH}_{2}\right)_{3}-\right)$, [22.84, 22.74, 19.77, 14.25$]\left(-\mathrm{CH}\left(\mathrm{CH}_{3}\right)_{2}\right.$, $\mathrm{CH}\left(\mathrm{CH}_{3}\right) \mathrm{CH}_{2}-$ and $\left.-\mathrm{CH}_{2} \mathrm{CH}_{3}\right)$. MALDI-TOF MS m/z of $[\mathrm{M}+\mathrm{Na}]^{+}$calculated for $\mathrm{C}_{33} \mathrm{H}_{61} \mathrm{NO}_{3}$ : 542.5; Found: 542.1.

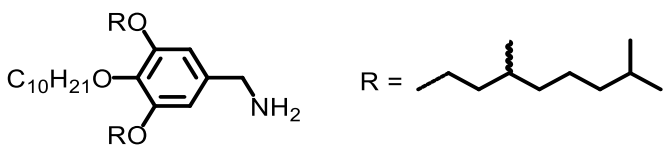

(4-(Decyloxy)-3,5-Bis((3,7-(rac)-dimethyloctyl)oxy)phenyl)methanamine (12-r10r). From 11-r10r $(0.070 \mathrm{~g}, 0.12 \mathrm{mmol})$ and $\mathrm{LiAlH}_{4}(0.007 \mathrm{~g}, 0.18 \mathrm{mmol})$ in dry THF $(4 \mathrm{~mL})$ at $23{ }^{\circ} \mathrm{C}$ for $1 \mathrm{~h}, 12-\mathbf{1 0}$ was obtained as a light yellow oil. Product: $0.059 \mathrm{~g}$; Yield: $88 \%$. This was used in the next step without further purification.

${ }^{1} \mathrm{H}$ NMR (500 MHz, $\mathrm{CDCl}_{3}, \delta$, ppm): 6.52 (s, 2H, $\left.\mathrm{ArH}\right), 4.06-3.96\left(\mathrm{~m}, 4 \mathrm{H},-\mathrm{OCH}_{2} \mathrm{CH}_{2} \mathrm{CH}_{\left(\mathrm{CH}_{3}\right)-}\right), 3.92$ (t, $\left.J=6.6 \mathrm{~Hz}, 2 \mathrm{H},-\mathrm{OCH}_{2} \mathrm{CH}_{2} \mathrm{CH}_{2}-\right), 3.79\left(\mathrm{~s}, 2 \mathrm{H},-\mathrm{CH}_{2} \mathrm{NH}_{2}\right), 1.89-1.81\left(\mathrm{~m}, 2 \mathrm{H},-\mathrm{CH}\left(\mathrm{CH}_{3}\right)_{2}\right), 1.76-1.66$ $\left(\mathrm{m}, 4 \mathrm{H},-\mathrm{CH}\left(\mathrm{CH}_{3}\right) \mathrm{CH}_{2}-\right.$ and $\left.-\mathrm{OCH}_{2} \mathrm{CH}_{2} \mathrm{CH}_{2}-\right), 1.63-1.42\left(\mathrm{~m}, 6 \mathrm{H},-\mathrm{OCH}_{2} \mathrm{CH}_{2} \mathrm{CH}\left(\mathrm{CH}_{3}\right)-\right.$ and $\left.\mathrm{OCH}_{2} \mathrm{CH}_{2} \mathrm{CH}_{2}-\right), 1.37-1.10\left(\mathrm{~m}, 24 \mathrm{H},-\mathrm{CH}\left(\mathrm{CH}_{3}\right)\left(\mathrm{CH}_{2}\right)_{3}-\right.$ and $\left.-\left(\mathrm{CH}_{2}\right)_{3}\left(\mathrm{CH}_{2}\right)_{6}-\right), 0.94(\mathrm{~d}, J=6.6 \mathrm{~Hz}, 6 \mathrm{H},-$ $\left.\mathrm{CH}_{2} \mathrm{CH}\left(\mathrm{CH}_{3}\right)^{-}\right)$, 0.90-0.83 (m, 15H, $-\mathrm{CH}\left(\mathrm{CH}_{3}\right)_{2}$ and $\left.-\mathrm{CH}_{2} \mathrm{CH}_{3}\right) .{ }^{13} \mathrm{C} \mathrm{NMR}\left(126 \mathrm{MHz}, \mathrm{CDCl}_{3}, \delta, \mathrm{ppm}\right)$ : 153.40 (ArC-3,5), 138.67 (ArC-4), 137.23 (ArC-1), 105.66 ( $\mathrm{ArC}-2,6), 73.55$ ( $\mathrm{ArOCH}_{2}-4$ position), 67.62 (ArOCH $2-3,5$ position), $46.94\left(-\mathrm{CH}_{2} \mathrm{NH}_{2}\right)$, [39.44, 37.50, 36.66, 32.08, 30.50, 29.99, 29.88, 29.82, 29.78, 29.53, 28.14, 26.31, 24.88] $\left(-\mathrm{OCH}_{2} \mathrm{CH}_{2} \mathrm{CH}\left(\mathrm{CH}_{3}\right)\left(\mathrm{CH}_{2}\right)_{3} \mathrm{CH}-\right.$ and $\left.-\mathrm{OCH}_{2}\left(\mathrm{CH}_{2}\right)_{7-}\right)$, [22.84 22.74, 19.77, 14.25] $\left(-\mathrm{CH}\left(\mathrm{CH}_{3}\right)_{2},-\mathrm{CH}\left(\mathrm{CH}_{3}\right) \mathrm{CH}_{2}-\right.$ and $\left.-\mathrm{CH}_{2} \mathrm{CH}_{3}\right)$. MALDI-TOF MS $m / z$ of $[\mathrm{M}+\mathrm{Na}]^{+}$calculated for $\mathrm{C}_{37} \mathrm{H}_{69} \mathrm{NO}_{3}$ : 598.5; Found: 598.7. 
(n)

(3,4-Bis((3,7-(rac)-dimethyloctyl)oxy)-5-(heptyloxy)phenyl)methanamine (12-rr7). From 11-rr7 (0.87 g, $1.55 \mathrm{mmol})$ and $\mathrm{LiAlH}_{4}(0.087 \mathrm{~g}, 2.3 \mathrm{mmol})$ in dry THF $(10 \mathrm{~mL})$ at $23{ }^{\circ} \mathrm{C}$ for $1 \mathrm{~h}, \mathbf{1 2}-\mathbf{r r} 7$ was obtained as a light yellow oil. Product: $0.82 \mathrm{~g}$; Yield: 99\%. This was used in the next step without further purification.

${ }^{1} \mathrm{H}$ NMR (400 MHz, $\left.\mathrm{CDCl}_{3}, \delta, \mathrm{ppm}\right): 6.51(\mathrm{~s}, 2 \mathrm{H}, \mathrm{ArH}), 4.06-3.89$ (m, 6H, $\left.-\mathrm{OCH}_{2}-\right), 3.78(\mathrm{~s}, 2 \mathrm{H},-$

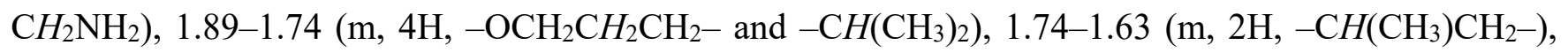
1.63-1.41 (m, 6H, $-\mathrm{O}\left(\mathrm{CH}_{2}\right)_{2} \mathrm{CH}_{2}-$ and $\left.-\mathrm{OCH}_{2} \mathrm{CH}_{2} \mathrm{CH}\left(\mathrm{CH}_{3}\right)-\right), 1.40-1.07\left(\mathrm{~m}, 18 \mathrm{H},-\mathrm{CH}\left(\mathrm{CH}_{3}\right)\left(\mathrm{CH}_{2}\right)_{3}-\right.$ and $\left.-\left(\mathrm{CH}_{2}\right)_{3}\left(\mathrm{CH}_{2}\right)_{3}-\right), 0.96-0.81\left(\mathrm{~m}, 21 \mathrm{H},-\mathrm{CH}_{3}\right) .{ }^{13} \mathrm{C} \mathrm{NMR}\left(101 \mathrm{MHz}, \mathrm{CDCl}_{3}, \delta, \mathrm{ppm}\right): 153.40(\mathrm{ArC}-$ 3,5), 137.54 (ArC-4), 137.28 (ArC-1), [105.74, 105.71] (ArC-2,6), $71.29\left(\mathrm{ArOCH}_{2}-5\right.$ position), 69.25 (ArOCH $\mathrm{H}_{2}-4$ position), $67.54\left(\mathrm{ArOCH}_{2}-3\right.$ position), $46.51\left(-\mathrm{CH}_{2} \mathrm{NH}_{2}\right)$, [39.50, 39.41, 37.64, 37.48, 36.59, $31.97,29.96,29.85,29.58,29.22,28.12,26.20,24.85]\left(-\mathrm{OCH}_{2} \mathrm{CH}_{2} \mathrm{CH}\left(\mathrm{CH}_{3}\right)\left(\mathrm{CH}_{2}\right)_{3} \mathrm{CH}-\right.$ and $\left.\mathrm{OCH}_{2}\left(\mathrm{CH}_{2}\right)_{4}-\right)$, [22.83, 22.74, 19.74, 19.70, 14.22] $\left(-\mathrm{CH}\left(\mathrm{CH}_{3}\right)_{2},-\mathrm{CH}\left(\mathrm{CH}_{3}\right) \mathrm{CH}_{2}-\right.$ and $\left.-\mathrm{CH}_{2} \mathrm{CH}_{3}\right)$. MALDI-TOF MS $m / z$ of $[\mathrm{M}+\mathrm{Na}]^{+}$calculated for $\mathrm{C}_{34} \mathrm{H}_{63} \mathrm{NO}_{3}$ : 556.5; Found: 556.6.

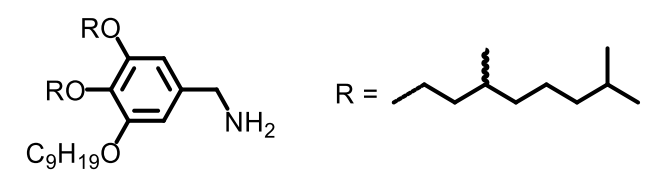

(3,4-Bis((3,7-(rac)-dimethyloctyl)oxy)-5-(nonyloxy)phenyl)methanamine (12-rr9). From 11-rr9 (0.91 g, $1.55 \mathrm{mmol})$ and $\mathrm{LiAlH}_{4}(0.087 \mathrm{~g}, 2.3 \mathrm{mmol})$ in dry THF $(10 \mathrm{~mL})$ at $23{ }^{\circ} \mathrm{C}$ for $1 \mathrm{~h}, \mathbf{1 2}-\mathbf{r r} 9$ was obtained as a light yellow oil. Product: $0.87 \mathrm{~g}$; Yield: $100 \%$. This was used in the next step without further purification.

${ }^{1} \mathrm{H}$ NMR (400 MHz, $\left.\mathrm{CDCl}_{3}, \delta, \mathrm{ppm}\right): 6.50$ (s, 2H, $\left.\mathrm{ArH}\right), 4.05-3.89$ (m, 6H, $-\mathrm{OCH}_{2}-$ ), 3.77 (s, 2H, -

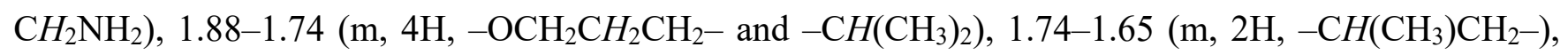
$1.65-1.40\left(\mathrm{~m}, 6 \mathrm{H},-\mathrm{O}\left(\mathrm{CH}_{2}\right)_{2} \mathrm{CH}_{2}-\right.$ and $\left.-\mathrm{OCH}_{2} \mathrm{CH}_{2} \mathrm{CH}\left(\mathrm{CH}_{3}\right)-\right), 1.40-1.07\left(\mathrm{~m}, 22 \mathrm{H},-\mathrm{CH}\left(\mathrm{CH}_{3}\right)\left(\mathrm{CH}_{2}\right)_{3}-\right.$ and $\left.-\left(\mathrm{CH}_{2}\right)_{3}\left(\mathrm{CH}_{2}\right)_{5}-\right), 0.95-0.81\left(\mathrm{~m}, 21 \mathrm{H},-\mathrm{CH}_{3}\right) .{ }^{13} \mathrm{C} \mathrm{NMR}\left(101 \mathrm{MHz}, \mathrm{CDCl}_{3}, \delta\right.$, ppm): $153.35(\mathrm{ArC}-$ 3,5), 138.07 (ArC-4), 137.16 (ArC-1), [105.51, 105.58] (ArC-2,6), $71.74\left(\mathrm{ArOCH}_{2}-5\right.$ position), 69.20 (ArOCH $2-4$ position), 67.49 ( $\mathrm{ArOCH}_{2}-3$ position), $46.66\left(-\mathrm{CH}_{2} \mathrm{NH}_{2}\right),[39.47,39.38,37.62,37.61,37.45$, $36.56, \quad 31.99, \quad 29.91, \quad 29.81, \quad 29.70, \quad 29.56, \quad 29.53, \quad 29.39, \quad 28.08, \quad 26.21, \quad 24.82] \quad(-$ $\mathrm{OCH}_{2} \mathrm{CH}_{2} \mathrm{CH}\left(\mathrm{CH}_{3}\right)\left(\mathrm{CH}_{2}\right)_{3} \mathrm{CH}-$ and $\left.-\mathrm{OCH}_{2}\left(\mathrm{CH}_{2}\right)_{6}-\right)$, [22.80, 22.77, 22.70, 22.69, 19.71, 19.67, 14.19] ($\mathrm{CH}\left(\mathrm{CH}_{3}\right)_{2},-\mathrm{CH}\left(\mathrm{CH}_{3}\right) \mathrm{CH}_{2}-$ and $\left.-\mathrm{CH}_{2} \mathrm{CH}_{3}\right)$. MALDI-TOF MS $m / z$ of $[\mathrm{M}+\mathrm{Na}]^{+}$calculated for $\mathrm{C}_{36} \mathrm{H}_{67} \mathrm{NO}_{3}$ : 584.5; Found: 584.7. 


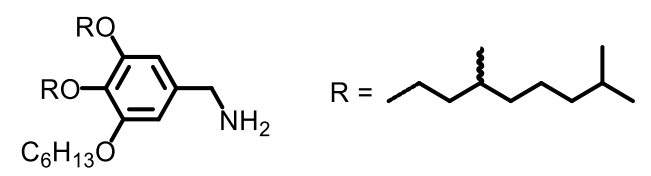

(3,4-Bis((3,7-(rac)-dimethyloctyl)oxy)-5-(hexyloxy)phenyl)methanamine (12-rr6). From 11-rr6 (0.24 g, $0.44 \mathrm{mmol})$ and $\mathrm{LiAlH}_{4}(0.025 \mathrm{~g}, 0.66 \mathrm{mmol})$ in dry THF $(4 \mathrm{~mL})$ at $23{ }^{\circ} \mathrm{C}$ for $1 \mathrm{~h}, \mathbf{1 2}-\mathbf{r r 6}$ was obtained as a light yellow oil. Product: $0.22 \mathrm{~g}$; Yield: $98 \%$. This was used in the next step without further purification.

${ }^{1} \mathrm{H}$ NMR (400 MHz, $\left.\mathrm{CDCl}_{3}, \delta, \mathrm{ppm}\right): 6.51$ (s, 2H, ArH), 4.07-3.89 (m, 6H, -OCH - ), 3.78 (s, 2H, -

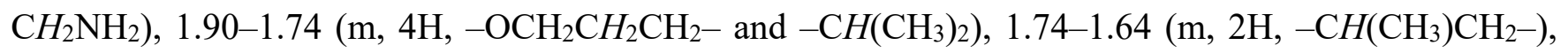
1.64-1.42 (m, 6H, $-\mathrm{O}\left(\mathrm{CH}_{2}\right)_{2} \mathrm{CH}_{2}-$ and $\left.-\mathrm{OCH}_{2} \mathrm{CH}_{2} \mathrm{CH}\left(\mathrm{CH}_{3}\right)-\right), 1.38-1.07\left(\mathrm{~m}, 16 \mathrm{H},-\mathrm{CH}\left(\mathrm{CH}_{3}\right)\left(\mathrm{CH}_{2}\right)_{3}-\right.$ and $\left.-\left(\mathrm{CH}_{2}\right)_{3}\left(\mathrm{CH}_{2}\right)_{2}-\right), 0.96-0.82\left(\mathrm{~m}, 21 \mathrm{H},-\mathrm{CH}_{3}\right) .{ }^{13} \mathrm{C} \mathrm{NMR}\left(101 \mathrm{MHz}, \mathrm{CDCl}_{3}, \delta, \mathrm{ppm}\right): 153.36(\mathrm{ArC}-$ 3,5), 138.62 (ArC-4), 137.12 (ArC-1), [105.54, 105.52] (ArC-2,6), 71.77 ( $\mathrm{ArOCH}_{2}-5$ position), 69.23 (ArOCH $\mathrm{H}_{2}-4$ position), 67.51 ( $\mathrm{ArOCH}_{2}-3$ position), $46.90\left(-\mathrm{CH}_{2} \mathrm{NH}_{2}\right)$, [39.49, 39.41, 37.65, 37.64, 37.48, $36.59,31.72,29.94,29.83,29.54,28.11,25.90,24.84]\left(-\mathrm{OCH}_{2} \mathrm{CH}_{2} \mathrm{CH}\left(\mathrm{CH}_{3}\right)\left(\mathrm{CH}_{2}\right)_{3} \mathrm{CH}-\right.$ and $\left.\mathrm{OCH}_{2}\left(\mathrm{CH}_{2}\right)_{3}-\right)$, [22.83, 22.76, 22.72, 19.75, 19.71, 14.17] $\left(-\mathrm{CH}\left(\mathrm{CH}_{3}\right)_{2},-\mathrm{CH}\left(\mathrm{CH}_{3}\right) \mathrm{CH}_{2}-\right.$ and $\left.-\mathrm{CH}_{2} \mathrm{CH}_{3}\right)$. MALDI-TOF MS $m / z$ of $[\mathrm{M}+\mathrm{Na}]^{+}$calculated for $\mathrm{C}_{33} \mathrm{H}_{61} \mathrm{NO}_{3}$ : 542.5; Found: 542.4.

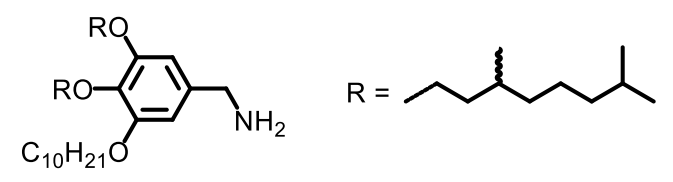

(3-(decyloxy)-4,5-bis((3,7-(rac)-dimethyloctyl)oxy)phenyl)methanamine (12-rr10). From $11-r r 10$ $(0.26 \mathrm{~g}, 0.44 \mathrm{mmol})$ and $\mathrm{LiAlH}_{4}(0.025 \mathrm{~g}, 0.66 \mathrm{mmol})$ in dry THF $(4 \mathrm{~mL})$ at $23{ }^{\circ} \mathrm{C}$ for $1 \mathrm{~h}, 12-r r 10$ was obtained as a light yellow oil. Product: $0.25 \mathrm{~g}$; Yield: $98 \%$. This was used in the next step without further purification.

${ }^{1} \mathrm{H}$ NMR (400 MHz, $\left.\mathrm{CDCl}_{3}, \delta, \mathrm{ppm}\right): 6.51$ (s, 2H, ArH), 4.06-3.88 (m, 6H, -OCH - ), 3.78 (s, 2H, -

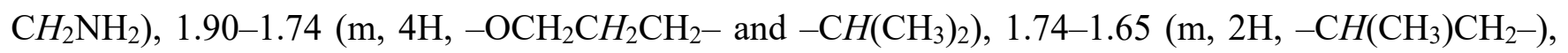
$1.65-1.41\left(\mathrm{~m}, 6 \mathrm{H},-\mathrm{O}\left(\mathrm{CH}_{2}\right)_{2} \mathrm{CH}_{2}-\right.$ and $\left.-\mathrm{OCH}_{2} \mathrm{CH}_{2} \mathrm{CH}\left(\mathrm{CH}_{3}\right)-\right), 1.40-1.09\left(\mathrm{~m}, 24 \mathrm{H},-\mathrm{CH}\left(\mathrm{CH}_{3}\right)\left(\mathrm{CH}_{2}\right)_{3}-\right.$ and $\left.-\left(\mathrm{CH}_{2}\right)_{3}\left(\mathrm{CH}_{2}\right)_{6}-\right), 0.96-0.82\left(\mathrm{~m}, 21 \mathrm{H},-\mathrm{CH}_{3}\right) .{ }^{13} \mathrm{C} \mathrm{NMR}\left(101 \mathrm{MHz}, \mathrm{CDCl}_{3}, \delta\right.$, ppm): $153.37(\mathrm{ArC}-$ 3,5), 138.62 (ArC-4), 137.13 (ArC-1), [105.56, 105.52] (ArC-2,6), $71.78\left(\mathrm{ArOCH}_{2}-3\right.$ position), 69.24 (ArOCH $\mathrm{H}_{2}-4$ position), 67.52 ( $\mathrm{ArOCH}_{2}-5$ position), $46.90\left(-\mathrm{CH}_{2} \mathrm{NH}_{2}\right),[39.51,39.41,37.65,37.49,36.60$, $32.04, \quad 29.95, \quad 29.85, \quad 29.78, \quad 29.72, \quad 29.59, \quad 29.56, \quad 29.48, \quad 28.12, \quad 26.24, \quad 24.85] \quad(-$ $\mathrm{OCH}_{2} \mathrm{CH}_{2} \mathrm{CH}\left(\mathrm{CH}_{3}\right)\left(\mathrm{CH}_{2}\right)_{3} \mathrm{CH}-$ and $\left.-\mathrm{OCH}_{2}\left(\mathrm{CH}_{2}\right)_{7}-\right)$, [22.84, 22.81, 22.75, 22.73, 19.76, 19.72, 14.24] ($\mathrm{CH}\left(\mathrm{CH}_{3}\right)_{2},-\mathrm{CH}\left(\mathrm{CH}_{3}\right) \mathrm{CH}_{2}-$ and $\left.-\mathrm{CH}_{2} \mathrm{CH}_{3}\right)$. MALDI-TOF MS $m / z$ of $[\mathrm{M}+\mathrm{Na}]^{+}$calculated for $\mathrm{C}_{37} \mathrm{H}_{69} \mathrm{NO}_{3}$ : 598.5; Found: 598.7. 
(1)

(3-((3,7-(rac)-Dimethyloctyl)oxy)-4,5-bis(heptyloxy)phenyl)methanamine (12-77r). From 11-77r $(0.80 \mathrm{~g}, 1.55 \mathrm{mmol})$ and $\mathrm{LiAlH}_{4}(0.087 \mathrm{~g}, 2.3 \mathrm{mmol})$ in dry THF $(10 \mathrm{~mL})$ at $23{ }^{\circ} \mathrm{C}$ for $1 \mathrm{~h}, \mathbf{1 2 - 7 7 r}$ was obtained as a light yellow oil. Product: $0.76 \mathrm{~g}$; Yield: $100 \%$. This was used in the next step without further purification.

${ }^{1} \mathrm{H}$ NMR (400 MHz, $\left.\mathrm{CDCl}_{3}, \delta, \mathrm{ppm}\right): 6.50$ (s, 2H, $\left.\mathrm{ArH}\right), 4.05-3.88$ (m, 6H, $-\mathrm{OCH}_{2}-$ ), 3.77 (s, 2H, $\left.\mathrm{CH}_{2} \mathrm{NH}_{2}\right), 1.89-1.64\left(\mathrm{~m}, 6 \mathrm{H},-\mathrm{OCH}_{2} \mathrm{CH}_{2} \mathrm{CH}_{2}-,-\mathrm{CH}\left(\mathrm{CH}_{3}\right)_{2}\right.$ and $\left.-\mathrm{CH}\left(\mathrm{CH}_{3}\right) \mathrm{CH}_{2}-\right)$, 1.62-1.40 (m, 6H, $\mathrm{O}\left(\mathrm{CH}_{2}\right)_{2} \mathrm{CH}_{2}-$ and $\left.-\mathrm{OCH}_{2} \mathrm{CH}_{2} \mathrm{CH}\left(\mathrm{CH}_{3}\right)-\right), 1.40-1.09\left(\mathrm{~m}, 18 \mathrm{H},-\mathrm{CH}\left(\mathrm{CH}_{3}\right)\left(\mathrm{CH}_{2}\right)_{3}-\right.$ and $\left.-\left(\mathrm{CH}_{2}\right)_{3}\left(\mathrm{CH}_{2}\right)_{3}-\right)$, 0.96-0.81 (m, 15H, $\left.-\mathrm{CH}_{3}\right) .{ }^{13} \mathrm{C}$ NMR (101 MHz, $\left.\mathrm{CDCl}_{3}, \delta, \mathrm{ppm}\right): 153.33$ (ArC-3,5), $138.03(\mathrm{ArC}-4)$, 137.16 (ArC-1), [105.65, 105.61] (ArC-2,6), 73.50 ( $\mathrm{ArOCH}_{2}-4$ position), 69.21 ( $\mathrm{ArOCH}_{2}-5$ position), 67.50 ( $\mathrm{ArOCH}_{2}-3$ position), $46.65\left(-\mathrm{CH}_{2} \mathrm{NH}_{2}\right)$, [39.37, 37.43, 36.57, 32.02, 31.93, 30.43, 29.91, 29.56, 29.37, 29.18, 28.08, 26.19, 26.16, 24.83] $\left(-\mathrm{OCH}_{2} \mathrm{CH}_{2} \mathrm{CH}\left(\mathrm{CH}_{3}\right)\left(\mathrm{CH}_{2}\right)_{3} \mathrm{CH}-\right.$ and $\left.-\mathrm{OCH}_{2}\left(\mathrm{CH}_{2}\right)_{4}-\right)$, [22.79, 22.77, 22.71, 22.68, 19.70, 14.20, 14.18] $\left(-\mathrm{CH}\left(\mathrm{CH}_{3}\right)_{2},-\mathrm{CH}\left(\mathrm{CH}_{3}\right) \mathrm{CH}_{2}-\right.$ and $\left.-\mathrm{CH}_{2} \mathrm{CH}_{3}\right)$. MALDI-TOF MS $m / z$ of $[\mathrm{M}+\mathrm{Na}]^{+}$calculated for $\mathrm{C}_{31} \mathrm{H}_{57} \mathrm{NO}_{3}$ : 514.4; Found: 514.5 .

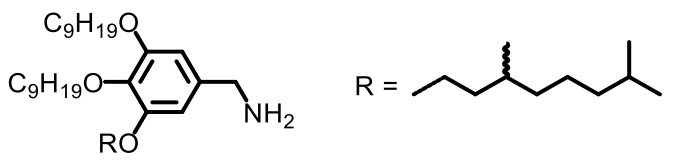

(3-((3,7-(rac)-Dimethyloctyl)oxy)-4,5-bis(nonyloxy)phenyl)methanamine (12-99r). From 11-99r $(0.89 \mathrm{~g}, 1.55 \mathrm{mmol})$ and $\mathrm{LiAlH}_{4}(0.087 \mathrm{~g}, 2.3 \mathrm{mmol})$ in dry THF $(10 \mathrm{~mL})$ at $23{ }^{\circ} \mathrm{C}$ for $1 \mathrm{~h}, \mathbf{1 2 - 9 9 r}$ was obtained as a light yellow oil. Product: $0.84 \mathrm{~g}$; Yield: 99\%. This was used in the next step without further purification.

${ }^{1} \mathrm{H}$ NMR (400 MHz, $\left.\mathrm{CDCl}_{3}, \delta, \mathrm{ppm}\right): 6.50$ (s, 2H, ArH), 4.05-3.87 (m, 6H, -OCH2-), 3.77 (s, 2H, $\left.\mathrm{CH}_{2} \mathrm{NH}_{2}\right), 1.89-1.63\left(\mathrm{~m}, 6 \mathrm{H},-\mathrm{OCH}_{2} \mathrm{CH}_{2} \mathrm{CH}_{2}-,-\mathrm{CH}\left(\mathrm{CH}_{3}\right)_{2}\right.$ and $\left.-\mathrm{CH}\left(\mathrm{CH}_{3}\right) \mathrm{CH}_{2}-\right), 1.63-1.40(\mathrm{~m}, 6 \mathrm{H},-$ $\mathrm{O}\left(\mathrm{CH}_{2}\right)_{2} \mathrm{CH}_{2}-$ and $\left.-\mathrm{OCH}_{2} \mathrm{CH}_{2} \mathrm{CH}\left(\mathrm{CH}_{3}\right)-\right), 1.40-1.07\left(\mathrm{~m}, 26 \mathrm{H},-\mathrm{CH}\left(\mathrm{CH}_{3}\right)\left(\mathrm{CH}_{2}\right)_{3}-\right.$ and $\left.-\left(\mathrm{CH}_{2}\right)_{3}\left(\mathrm{CH}_{2}\right)_{5}-\right)$, 0.96-0.80 (m, 15H, $\left.-\mathrm{CH}_{3}\right) .{ }^{13} \mathrm{C}$ NMR (101 MHz, $\left.\mathrm{CDCl}_{3}, \delta, \mathrm{ppm}\right): 153.36$ (ArC-3,5), 137.89 (ArC-4), 137.20 (ArC-1), [105.69, 105.65] (ArC-2,6), 73.52 ( $\mathrm{ArOCH}_{2}-4$ position), 69.24 ( $\mathrm{ArOCH}_{2}-5$ position), $67.53\left(\mathrm{ArOCH}_{2}-3\right.$ position), $46.61\left(-\mathrm{CH}_{2} \mathrm{NH}_{2}\right),[39.39,37.45,36.58,32.05,32.01,30.45,29.93,29.80$, $29.73,29.72,29.57,29.54,29.49,29.41,28.10,26.25,26.22,24.85]\left(-\mathrm{OCH}_{2} \mathrm{CH}_{2} \mathrm{CH}\left(\mathrm{CH}_{3}\right)\left(\mathrm{CH}_{2}\right)_{3} C \mathrm{CH}-\right.$ and $\left.-\mathrm{OCH}_{2}\left(\mathrm{CH}_{2}\right)_{6}-\right)$, [22.80, 22.70, 19.71, 14.21] $\left(-\mathrm{CH}\left(\mathrm{CH}_{3}\right)_{2},-\mathrm{CH}\left(\mathrm{CH}_{3}\right) \mathrm{CH}_{2}-\right.$ and $\left.-\mathrm{CH}_{2} \mathrm{CH}_{3}\right)$. MALDITOF MS $m / z$ of $[\mathrm{M}+\mathrm{Na}]^{+}$calculated for $\mathrm{C}_{35} \mathrm{H}_{65} \mathrm{NO}_{3}: 570.5$; Found: 570.4 . 


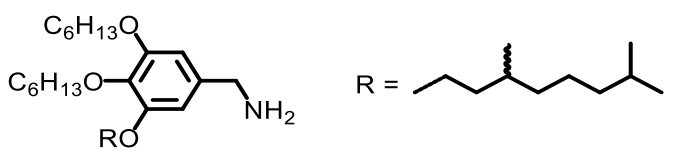

(3-((3,7-(rac)-Dimethyloctyl)oxy)-4,5-bis(hexyloxy)phenyl)methanamine (12-66r). From 11-66r (0.22 g, $0.44 \mathrm{mmol})$ and $\mathrm{LiAlH}_{4}(0.025 \mathrm{~g}, 0.66 \mathrm{mmol})$ in dry THF $(4 \mathrm{~mL})$ at $23{ }^{\circ} \mathrm{C}$ for $1 \mathrm{~h}, \mathbf{1 2 - 6 6 r}$ was obtained as a light yellow oil. Product: $0.20 \mathrm{~g}$; Yield: $97 \%$. This was used in the next step without further purification.

${ }^{1} \mathrm{H}$ NMR (400 MHz, $\left.\mathrm{CDCl}_{3}, \delta, \mathrm{ppm}\right): 6.51$ (s, 2H, ArH), 4.07-3.88 (m, 6H, -OCH - ), 3.78 (s, 2H, $\left.\mathrm{CH}_{2} \mathrm{NH}_{2}\right), 1.90-1.65\left(\mathrm{~m}, 6 \mathrm{H},-\mathrm{OCH}_{2} \mathrm{CH}_{2} \mathrm{CH}_{2}-,-\mathrm{CH}\left(\mathrm{CH}_{3}\right)_{2}\right.$ and $\left.-\mathrm{CH}\left(\mathrm{CH}_{3}\right) \mathrm{CH}_{2}-\right)$, 1.64-1.42 (m, 6H, $\mathrm{O}\left(\mathrm{CH}_{2}\right)_{2} \mathrm{CH}_{2}-$ and $\left.-\mathrm{OCH}_{2} \mathrm{CH}_{2} \mathrm{CH}\left(\mathrm{CH}_{3}\right)-\right), 1.39-1.10\left(\mathrm{~m}, 14 \mathrm{H},-\mathrm{CH}\left(\mathrm{CH}_{3}\right)\left(\mathrm{CH}_{2}\right)_{3}-\right.$ and $\left.-\left(\mathrm{CH}_{2}\right)_{3}\left(\mathrm{CH}_{2}\right)_{2}-\right)$, 0.96-0.83 (m, 15H, $\left.-\mathrm{CH}_{3}\right) .{ }^{13} \mathrm{C}$ NMR (101 MHz, $\left.\mathrm{CDCl}_{3}, \delta, \mathrm{ppm}\right): 153.36$ (ArC-3,5), $138.62(\mathrm{ArC}-4)$, 137.13 (ArC-1), [105.59, 105.55] (ArC-2,6), 73.53 ( $\mathrm{ArOCH}_{2}-4$ position), 69.25 ( $\mathrm{ArOCH}_{2}-5$ position), 67.54 ( $\mathrm{ArOCH}_{2}-3$ position), $46.90\left(-\mathrm{CH}_{2} \mathrm{NH}_{2}\right)$, [39.41, 37.47, 36.60, 31.93, 31.71, 30.42, 29.94, 29.55, 28.13, 25.93, 25.90, 24.86] $\left(-\mathrm{OCH}_{2} \mathrm{CH}_{2} \mathrm{CH}\left(\mathrm{CH}_{3}\right)\left(\mathrm{CH}_{2}\right)_{3} \mathrm{CH}-\right.$ and $\left.-\mathrm{OCH}_{2}\left(\mathrm{CH}_{2}\right)_{3}-\right)$, [22.83, 22.76, 22.72, $19.74,14.23,14.16]\left(-\mathrm{CH}\left(\mathrm{CH}_{3}\right)_{2},-\mathrm{CH}\left(\mathrm{CH}_{3}\right) \mathrm{CH}_{2}-\right.$ and $\left.-\mathrm{CH}_{2} \mathrm{CH}_{3}\right)$. MALDI-TOF MS $m / z$ of $[\mathrm{M}+\mathrm{Na}]^{+}$ calculated for $\mathrm{C}_{29} \mathrm{H}_{53} \mathrm{NO}_{3}$ : 486.4; Found: 486.2 .

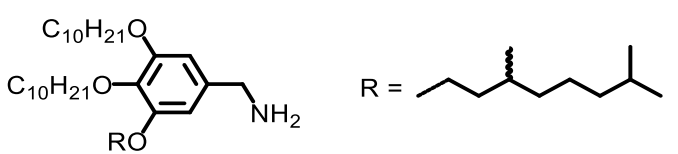

(3,4-Bis(decyloxy)-5-((3,7-(rac)-dimethyloctyl)oxy)phenyl)methanamine (12-1010r). From 11-1010r $(0.26 \mathrm{~g}, 0.44 \mathrm{mmol})$ and $\mathrm{LiAlH}_{4}(0.025 \mathrm{~g}, 0.66 \mathrm{mmol})$ in dry THF $(4 \mathrm{~mL})$ at $23{ }^{\circ} \mathrm{C}$ for $1 \mathrm{~h}, 12-1010 r$ was obtained as a light yellow oil. Product: $0.25 \mathrm{~g}$; Yield: $99 \%$. This was used in the next step without further purification.

${ }^{1} \mathrm{H}$ NMR (400 MHz, $\left.\mathrm{CDCl}_{3}, \delta, \mathrm{ppm}\right): 6.51$ (s, 2H, $\left.\mathrm{ArH}\right), 4.07-3.87$ (m, 6H, $-\mathrm{OCH}_{2}-$ ), 3.78 (s, 2H, $\left.\mathrm{CH}_{2} \mathrm{NH}_{2}\right), 1.09-1.65\left(\mathrm{~m}, 6 \mathrm{H},-\mathrm{OCH}_{2} \mathrm{CH}_{2} \mathrm{CH}_{2-}-,-\mathrm{CH}\left(\mathrm{CH}_{3}\right)_{2}\right.$ and $\left.-\mathrm{CH}\left(\mathrm{CH}_{3}\right) \mathrm{CH}_{2}-\right), 1.65-1.40(\mathrm{~m}, 6 \mathrm{H}$, $\mathrm{O}\left(\mathrm{CH}_{2}\right)_{2} \mathrm{CH}_{2}-$ and $\left.-\mathrm{OCH}_{2} \mathrm{CH}_{2} \mathrm{CH}\left(\mathrm{CH}_{3}\right)-\right), 1.39-1.10\left(\mathrm{~m}, 30 \mathrm{H},-\mathrm{CH}\left(\mathrm{CH}_{3}\right)\left(\mathrm{CH}_{2}\right)_{3}-\right.$ and $\left.-\left(\mathrm{CH}_{2}\right)_{3}\left(\mathrm{CH}_{2}\right)_{6}-\right)$, $0.93\left(\mathrm{~d}, J=6.6 \mathrm{~Hz}, 3 \mathrm{H},-\mathrm{CH}_{2} \mathrm{CH}\left(\mathrm{CH}_{3}\right)-\right), 0.91-0.82\left(\mathrm{~m}, 12 \mathrm{H},-\mathrm{CH}\left(\mathrm{CH}_{3}\right)_{2}\right.$ and $\left.-\mathrm{CH}_{2} \mathrm{CH}_{3}\right) .{ }^{13} \mathrm{C} \mathrm{NMR}(101$ $\mathrm{MHz}, \mathrm{CDCl}_{3}, \delta$, ppm): 153.36 (ArC-3,5), 138.60 (ArC-4), 137.14 (ArC-1), [105.60, 105.56] (ArC-2,6), 73.54 ( $\mathrm{ArOCH}_{2}-4$ position), $69.26\left(\mathrm{ArOCH}_{2}-3\right.$ position), 67.55 ( $\mathrm{ArOCH} \mathrm{H}_{2}-5$ position), $46.90\left(-\mathrm{CH}_{2} \mathrm{NH}_{2}\right)$, $[39.41,37.47,36.61,32.07,32.05,30.48,29.95,29.88,29.81,29.79,29.76,29.73,29.60,29.55,29.53$, 29.49, 28.12, 26.28, 26.24, 24.87] $\left(-\mathrm{OCH}_{2} \mathrm{CH}_{2} \mathrm{CH}\left(\mathrm{CH}_{3}\right)\left(\mathrm{CH}_{2}\right)_{3} \mathrm{CH}-\right.$ and $\left.-\mathrm{OCH}_{2}\left(\mathrm{CH}_{2}\right)_{7}-\right)$, [22.83, 22.73, $19.75,14.24]\left(-\mathrm{CH}\left(\mathrm{CH}_{3}\right)_{2},-\mathrm{CH}\left(\mathrm{CH}_{3}\right) \mathrm{CH}_{2}-\right.$ and $\left.-\mathrm{CH}_{2} \mathrm{CH}_{3}\right)$. MALDI-TOF $\mathrm{MS} \mathrm{m} / z$ of $[\mathrm{M}+\mathrm{Na}]^{+}$ calculated for $\mathrm{C}_{37} \mathrm{H}_{69} \mathrm{NO}_{3}$ : 598.5; Found: 598.1 . 
General procedure for preparation of dendronized PBIs. The synthesis of dendronized PBIs was conducted according to a procedure reported by our group. ${ }^{10} \mathrm{~A}$ general example is provided below.
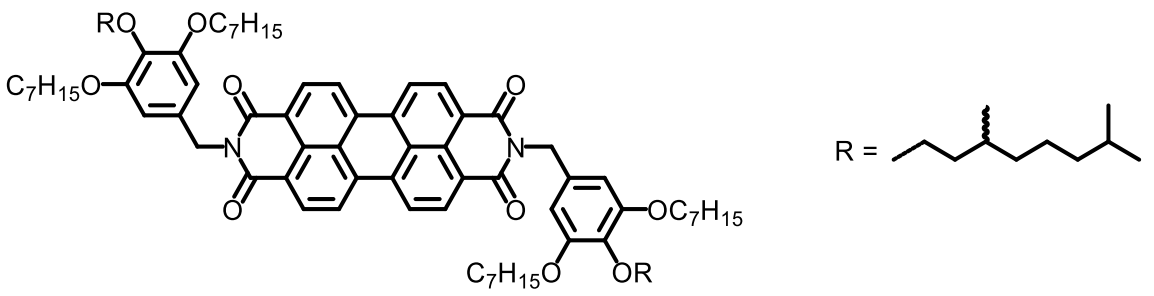

7r7-PBI. A mixture of 12-7r7 $(0.71 \mathrm{~g}, 1.45 \mathrm{mmol})$, perylenetetracarboxylic acid bisanhydride $(0.26 \mathrm{~g}$, $0.66 \mathrm{mmol}), \mathrm{Zn}(\mathrm{OAc})_{2} \cdot 2 \mathrm{H}_{2} \mathrm{O}(0.145 \mathrm{~g}, 0.66 \mathrm{mmol})$ and imidazole $(3 \mathrm{~g})$ was stirred at $180{ }^{\circ} \mathrm{C}$ for $6 \mathrm{~h}$ under $\mathrm{N}_{2}$ protection. Then the reaction mixture was cooled to $23{ }^{\circ} \mathrm{C}$, dissolved in a minimum amount of THF and poured into a stirring mixture of $2 \mathrm{M} \mathrm{HCl}(180 \mathrm{~mL})$ and methanol $(60 \mathrm{~mL})$. The precipitate was filtered, washed with water $(40 \mathrm{~mL})$ and methanol $(40 \mathrm{~mL})$ and dried. The crude product was subjected to column chromatography using DCM as eluent. The product was purified further by being dissolved in $\mathrm{CHCl}_{3}$ and precipitated in methanol. 7r7-PBI was obtained as a red solid. Product: 0.72 g. Yield: $81 \%$. Purity by HPLC: $99+\%$. mp: $209^{\circ} \mathrm{C}$.

${ }^{1} \mathrm{H}$ NMR (400 MHz, $\mathrm{CDCl}_{3}, \delta$, ppm): 8.55 (d, $J=7.9 \mathrm{~Hz}, 4 \mathrm{H}, \mathrm{PBI}, 1,6,7,12$-positions), 8.38 (d, $J=8.0$ $\mathrm{Hz}, 4 \mathrm{H}, \mathrm{PBI}, 2,5,8,11$-positions), 6.85 (s, 4H, ArH of dendrons), 5.29 (s, 4H, -NCH$H_{2}-$ ), 4.01 (t, $J=6.6$ $\left.\mathrm{Hz}, 8 \mathrm{H},-\mathrm{OCH}_{2}\left(\mathrm{CH}_{2}\right)_{3}-\right)$, 3.98-3.87 (m, 4H, $\left.-\mathrm{OCH}_{2} \mathrm{CH}_{2} \mathrm{CH}\left(\mathrm{CH}_{3}\right)_{-}\right)$, 1.85-1.60 (m, $12 \mathrm{H},-\mathrm{CH}\left(\mathrm{CH}_{3}\right)_{2},-$ $\mathrm{OCH}_{2} \mathrm{CH}_{2} \mathrm{CH}_{2}-$ and $\left.-\mathrm{CH}\left(\mathrm{CH}_{3}\right) \mathrm{CH}_{2}-\right), 1.54-1.41\left(\mathrm{~m}, 12 \mathrm{H},-\mathrm{O}\left(\mathrm{CH}_{2}\right)_{2} \mathrm{CH}_{2}-\right.$ and $\left.-\mathrm{OCH}_{2} \mathrm{CH}_{2} \mathrm{CH}\left(\mathrm{CH}_{3}\right)-\right)$, 1.40-1.05 (m, 36H, $-\mathrm{CH}\left(\mathrm{CH}_{3}\right)\left(\mathrm{CH}_{2}\right)_{3}-$ and $\left.-\left(\mathrm{CH}_{2}\right)_{3}\left(\mathrm{CH}_{2}\right)_{3}-\right), 0.91-0.80\left(\mathrm{~m}, 30 \mathrm{H},-\mathrm{CH}_{3}\right) .{ }^{13} \mathrm{C}$ NMR $(101$ $\left.\mathrm{MHz}, \mathrm{CDCl}_{3}, \delta, \mathrm{ppm}\right): 162.60(C=\mathrm{O}), 153.19$ (ArC-3,5), 137.96 (ArC-4), 133.53 (ArC-1), [132.36, 130.90, 128.11, 125.07, 122.60, 122.46] (perylene C), 108.66 (ArC-2,6), $71.85\left(\mathrm{ArOCH}_{2}-3,5\right.$ position), 69.34 ( $\mathrm{ArOCH}_{2}-4$ position), $43.75\left(-\mathrm{CH}_{2} \mathrm{~N}\right),[39.45,37.63,37.53,32.02,29.90,29.66,29.30,28.07,26.29$, 24.83] $\left(-\mathrm{OCH}_{2}\left(\mathrm{CH}_{2}\right)_{4}-\right.$ and $\left.-\mathrm{OCH}_{2} \mathrm{CH}_{2} \mathrm{CH}\left(\mathrm{CH}_{3}\right)\left(\mathrm{CH}_{2}\right)_{3} \mathrm{CH}-\right),[22.79,22.68,19.68,14.25]\left(-\mathrm{CH}_{(}\left(\mathrm{CH}_{3}\right)_{2}\right.$, $-\mathrm{CH}\left(\mathrm{CH}_{3}\right) \mathrm{CH}_{2}-$ and $\left.-\mathrm{CH}_{2} \mathrm{CH}_{3}\right)$. MALDI-TOF MS $m / z$ of $[\mathrm{M}+\mathrm{Na}]^{+}$calculated for $\mathrm{C}_{86} \mathrm{H}_{118} \mathrm{~N}_{2} \mathrm{O}_{10}:$ 1361.9; Found: 1362.5 .
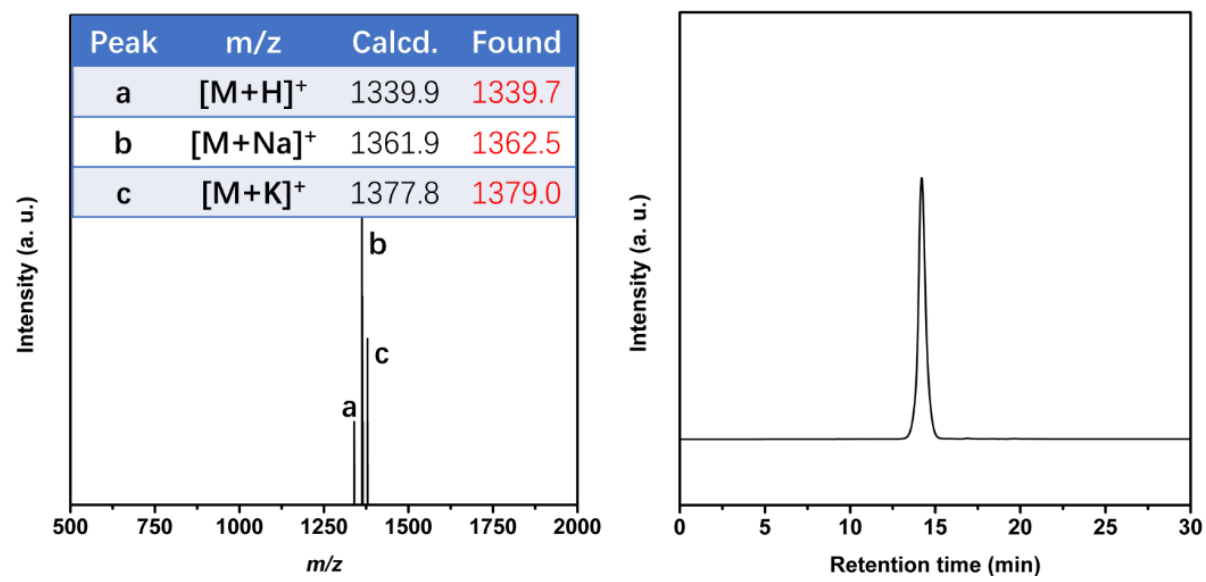

Supporting Figure S33. MALDI-TOF MS spectra (left) and HPLC trace (right) of 7r7-PBI. Target molecule 7r7-PBI has been successfully synthesized. ${ }^{1} \mathrm{H}$ and ${ }^{13} \mathrm{C}$ NMR spectra are presented on pages 102 and 103 , respectively. 
Supporting Figure S33 (continued). ${ }^{1} \mathrm{H}$ NMR of 7r7-PBI.

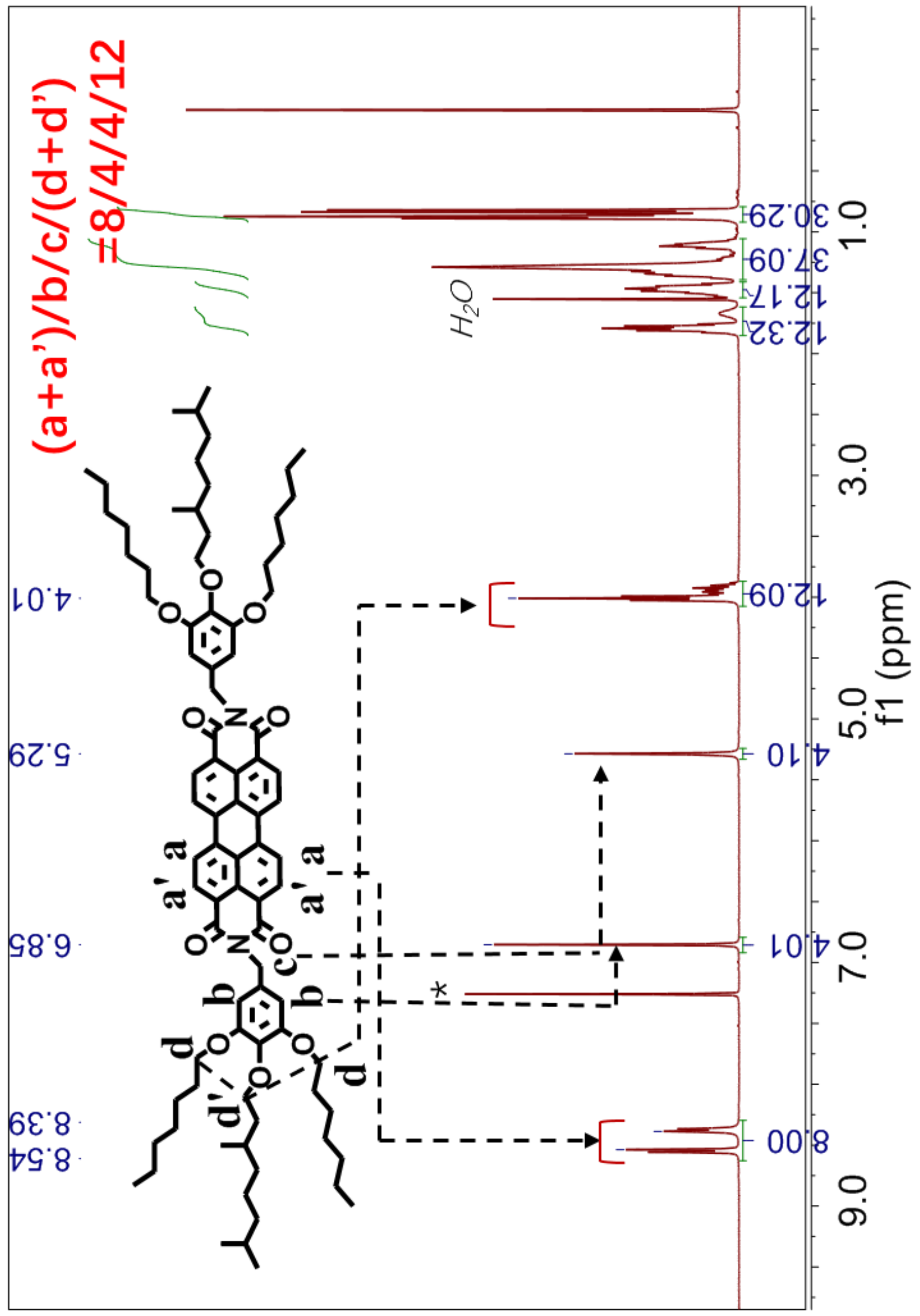


Supporting Figure S33 (continued). ${ }^{13} \mathrm{C}$ NMR of 7r7-PBI.

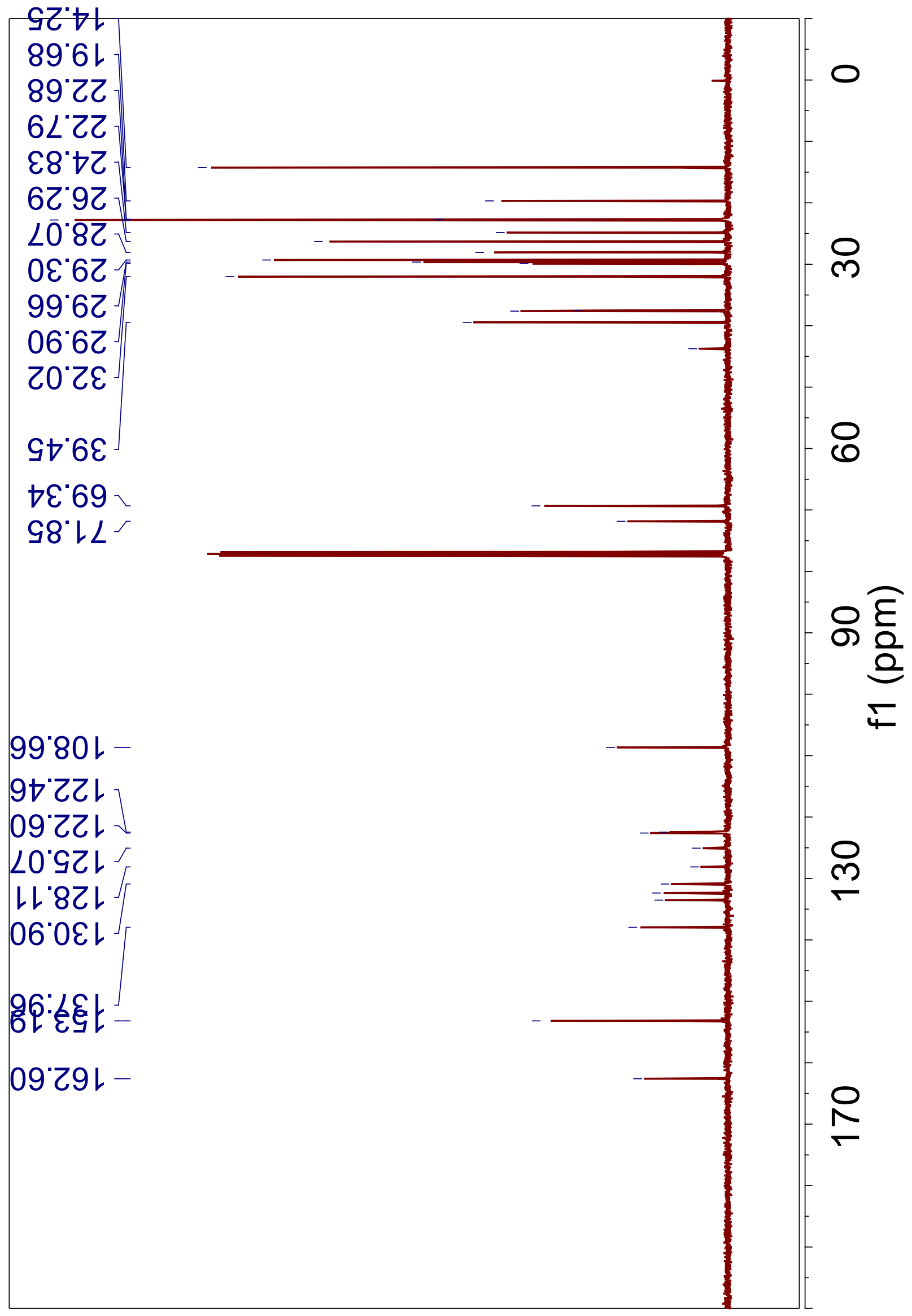




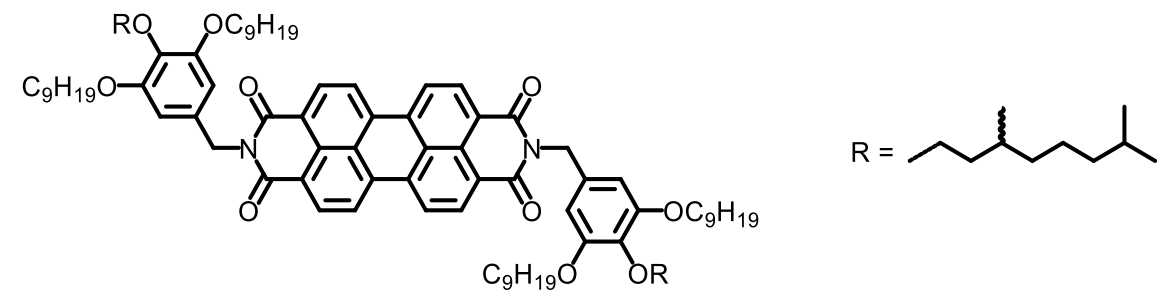

9r9-PBI. From 12-9r9 (0.79 g, $1.45 \mathrm{mmol})$, PTCDA (0.26 g, $0.66 \mathrm{mmol})$ and $\mathrm{Zn}(\mathrm{OAc})_{2} \cdot 2 \mathrm{H}_{2} \mathrm{O}(0.145 \mathrm{~g}$, $0.66 \mathrm{mmol})$ in imidazole $(3 \mathrm{~g})$ at $180{ }^{\circ} \mathrm{C}$ for $6 \mathrm{~h}, 9 \mathrm{r} 9-\mathrm{PBI}$ was obtained as a red solid. Product: $0.70 \mathrm{~g}$. Yield: $73 \%$. Purity by HPLC: $99+\%$. mp: $207^{\circ} \mathrm{C}$.

${ }^{1} \mathrm{H}$ NMR (400 MHz, $\mathrm{CDCl}_{3}, \delta, \mathrm{ppm}$ ): 8.54 (d, $J=7.9 \mathrm{~Hz}, 4 \mathrm{H}, \mathrm{PBI}, 1,6,7,12$-positions), 8.36 (d, $J=8.0$ $\mathrm{Hz}, 4 \mathrm{H}, \mathrm{PBI}, 2,5,8,11$-positions), 6.85 (s, 4H, $\mathrm{Ar} H$ of dendrons), 5.29 (s, 4H, $-\mathrm{NCH}_{2}-$ ), 4.01 (t, $J=6.4$ $\left.\mathrm{Hz}, 8 \mathrm{H},-\mathrm{OCH}_{2}\left(\mathrm{CH}_{2}\right)_{3}-\right)$, 3.99-3.87 (m, $\left.4 \mathrm{H},-\mathrm{OCH}_{2} \mathrm{CH}_{2} \mathrm{CH}\left(\mathrm{CH}_{3}\right)-\right), 1.86-1.61\left(\mathrm{~m}, 12 \mathrm{H},-\mathrm{CH}_{2}\left(\mathrm{CH}_{3}\right)_{2},-\right.$ $\mathrm{OCH}_{2} \mathrm{CH}_{2} \mathrm{CH}_{2}-$ and $\left.-\mathrm{CH}\left(\mathrm{CH}_{3}\right) \mathrm{CH}_{2}-\right), 1.54-1.41\left(\mathrm{~m}, 12 \mathrm{H},-\mathrm{O}\left(\mathrm{CH}_{2}\right)_{2} \mathrm{CH}_{2}-\right.$ and $\left.-\mathrm{OCH}_{2} \mathrm{CH}_{2} \mathrm{CH}\left(\mathrm{CH}_{3}\right)-\right)$, 1.39-1.04 (m, 52H, $-\mathrm{CH}\left(\mathrm{CH}_{3}\right)\left(\mathrm{CH}_{2}\right)_{3}-$ and $\left.-\left(\mathrm{CH}_{2}\right)_{3}\left(\mathrm{CH}_{2}\right)_{5}-\right), 0.91-0.80\left(\mathrm{~m}, 30 \mathrm{H},-\mathrm{CH}_{3}\right) .{ }^{13} \mathrm{C}$ NMR $(101$ $\left.\mathrm{MHz}, \mathrm{CDCl}_{3}, \delta, \mathrm{ppm}\right): 162.54(C=\mathrm{O}), 153.19$ (ArC-3,5), 137.94 ( $\left.\mathrm{ArC}-4\right), 133.46$ ( $\left.\mathrm{ArC}-1\right)$, [132.36, 130.86, 128.04, 124.99, 122.55, 122.41] (perylene $C$ ), 108.65 ( $\mathrm{ArC}-2,6), 71.84$ ( $\mathrm{ArOCH}_{2}-3,5$ position), 69.33 ( $\mathrm{ArOCH}_{2}-4$ position), $43.71\left(-\mathrm{CH}_{2} \mathrm{~N}\right)$, [39.45, 37.64, 37.53, 32.06, 29.90, 29.80, 29.67, 29.48, 28.07, 26.36, 24.84] $\left(-\mathrm{OCH}_{2}\left(\mathrm{CH}_{2}\right)_{6}-\right.$ and $\left.-\mathrm{OCH}_{2} \mathrm{CH}_{2} \mathrm{CH}\left(\mathrm{CH}_{3}\right)\left(\mathrm{CH}_{2}\right)_{3} \mathrm{CH}-\right)$, [22.82, 22.78, 22.68, 19.68, 14.23] $\left(-\mathrm{CH}\left(\mathrm{CH}_{3}\right)_{2},-\mathrm{CH}\left(\mathrm{CH}_{3}\right) \mathrm{CH}_{2}-\right.$ and $\left.-\mathrm{CH}_{2} \mathrm{CH}_{3}\right)$. MALDI-TOF MS m/z of $[\mathrm{M}+\mathrm{Na}]^{+}$calculated for $\mathrm{C}_{94} \mathrm{H}_{134} \mathrm{~N}_{2} \mathrm{O}_{10}$ : 1474.0; Found: 1474.7 .
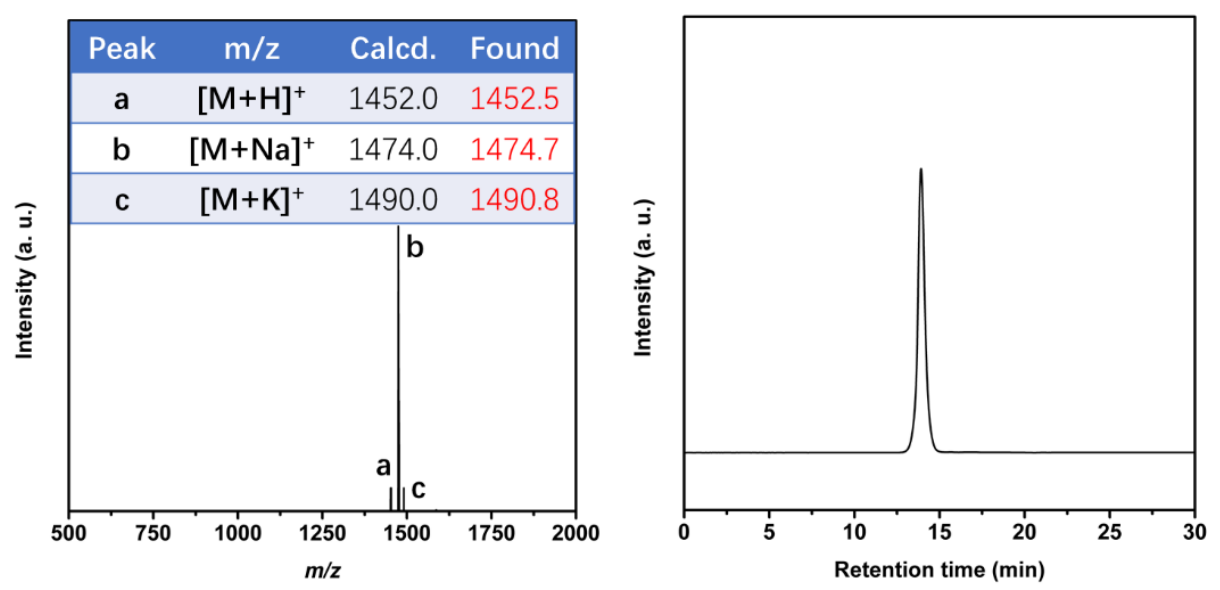

Supporting Figure S34. MALDI-TOF MS spectra (left) and HPLC trace (right) of 9r9-PBI. Target molecule 9r9-PBI has been successfully synthesized. ${ }^{1} \mathrm{H}$ and ${ }^{13} \mathrm{C}$ NMR spectra are presented on pages 105 and 106 , respectively. 
Supporting Figure S34 (continued). ${ }^{1} \mathrm{H}$ NMR of 9r9-PBI.

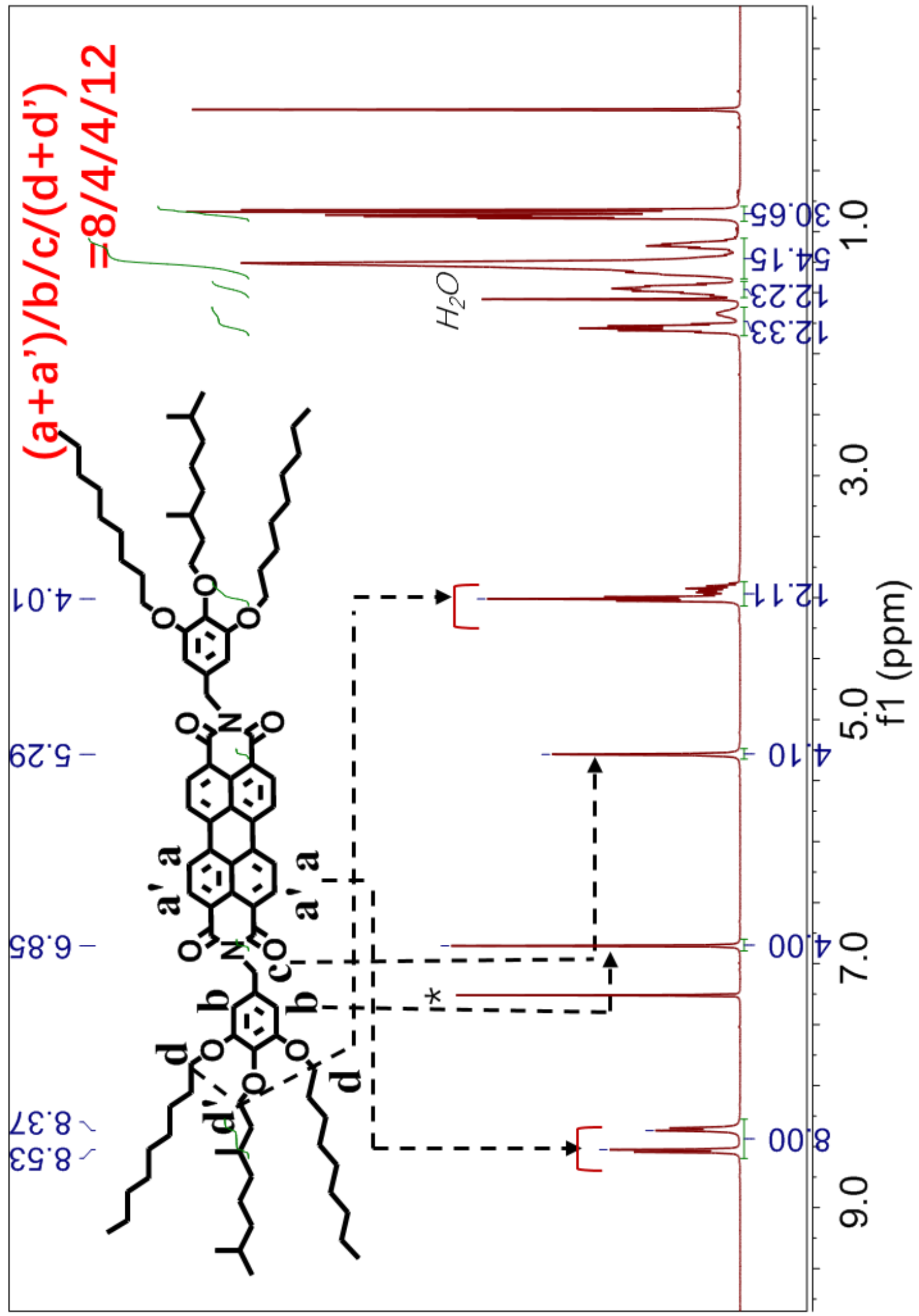


Supporting Figure S34 (continued). ${ }^{13} \mathrm{C}$ NMR of 9r9-PBI.

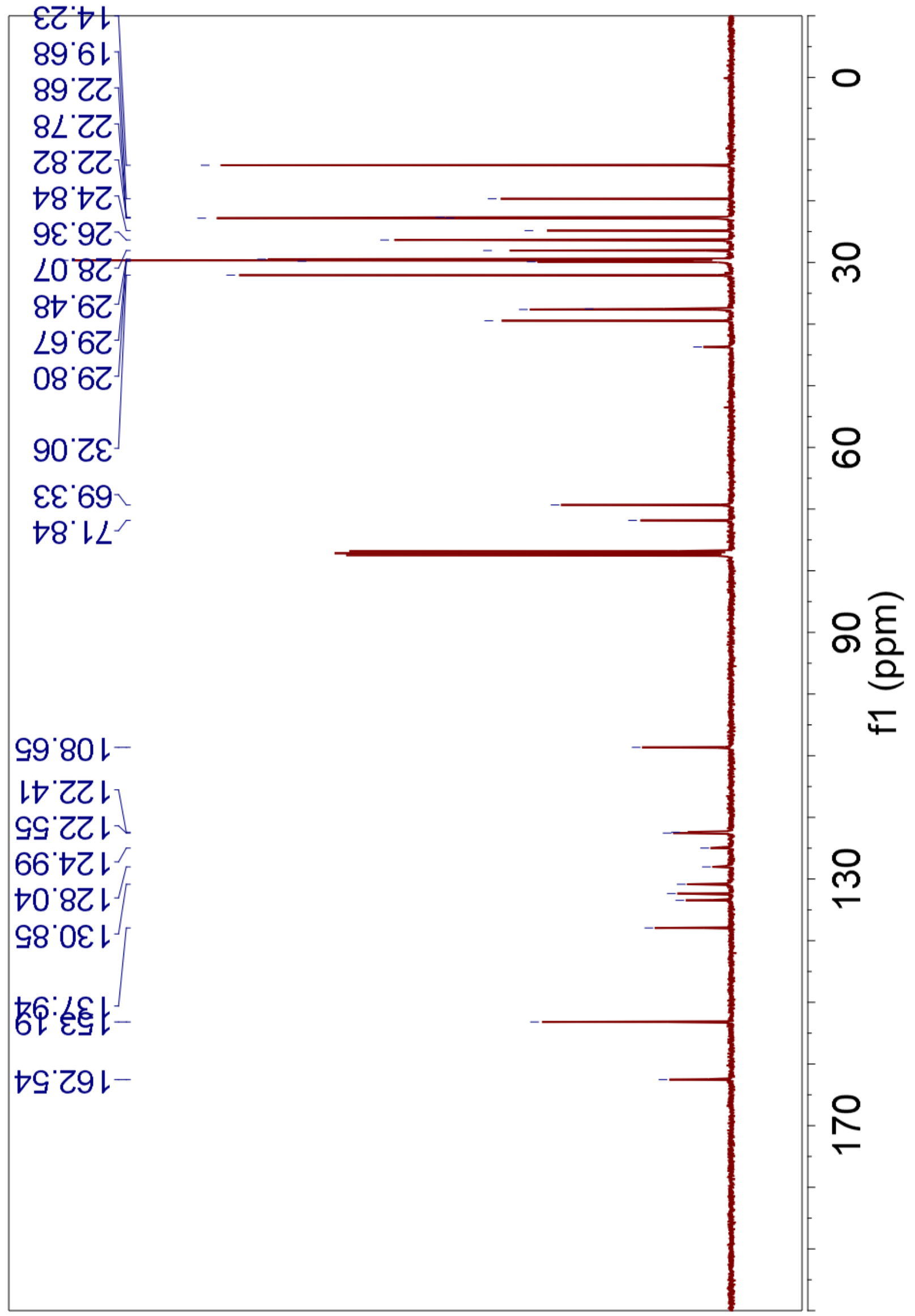



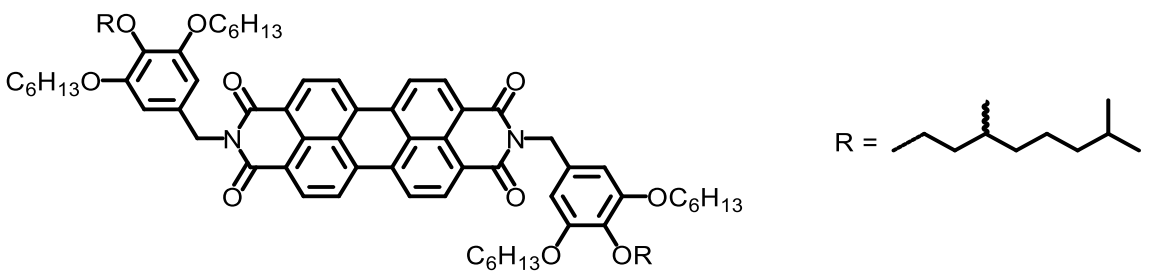

6r6-PBI. From 12-6r6 (1.02 g, $2.2 \mathrm{mmol})$, PTCDA (0.39 g, $1.0 \mathrm{mmol})$ and $\mathrm{Zn}(\mathrm{OAc})_{2} \cdot 2 \mathrm{H}_{2} \mathrm{O}(0.22 \mathrm{~g}, 1.0$ $\mathrm{mmol})$ in imidazole $(3 \mathrm{~g})$ at $160^{\circ} \mathrm{C}$ for $16 \mathrm{~h}, \mathbf{6 r 6}-\mathrm{PBI}$ was obtained as a red solid. Product: $0.78 \mathrm{~g}$. Yield: $61 \%$. Purity by HPLC: $99+\%$. mp: $216^{\circ} \mathrm{C}$.

${ }^{1} \mathrm{H}$ NMR (500 MHz, $\mathrm{CDCl}_{3}, \delta, \mathrm{ppm}$ ): 8.49 (d, $J=7.9 \mathrm{~Hz}, 4 \mathrm{H}, \mathrm{PBI}, 1,6,7,12$-positions), 8.29 (d, $J=7.7$ $\mathrm{Hz}, 4 \mathrm{H}, \mathrm{PBI}, 2,5,8,11$-positions), 6.86 (s, 4H, $\mathrm{Ar} H$ of dendrons), 5.28 (s, 4H, $-\mathrm{NCH}_{2}-$ ), 4.02 (t, $J=6.5$ $\left.\mathrm{Hz}, 8 \mathrm{H},-\mathrm{OCH}_{2}\left(\mathrm{CH}_{2}\right)_{3}-\right), 4.00-3.89\left(\mathrm{~m}, 4 \mathrm{H},-\mathrm{OCH}_{2} \mathrm{CH}_{2} \mathrm{CH}\left(\mathrm{CH}_{3}\right)-\right), 1.85-1.62\left(\mathrm{~m}, 12 \mathrm{H},-\mathrm{CH}_{2}\left(\mathrm{CH}_{3}\right)_{2},-\right.$ $\mathrm{OCH}_{2} \mathrm{CH}_{2} \mathrm{CH}_{2}-$ and $\left.-\mathrm{CH}\left(\mathrm{CH}_{3}\right) \mathrm{CH}_{2}-\right), 1.55-1.43\left(\mathrm{~m}, 12 \mathrm{H},-\mathrm{O}\left(\mathrm{CH}_{2}\right)_{2} \mathrm{CH}_{2}-\right.$ and $\left.-\mathrm{OCH}_{2} \mathrm{CH}_{2} \mathrm{CH}\left(\mathrm{CH}_{3}\right)-\right)$, 1.38-1.06 (m, 28H, $-\mathrm{CH}\left(\mathrm{CH}_{3}\right)\left(\mathrm{CH}_{2}\right)_{3}-$ and $\left.-\left(\mathrm{CH}_{2}\right)_{3}\left(\mathrm{CH}_{2}\right)_{2}-\right), 0.93-0.86\left(\mathrm{~m}, 18 \mathrm{H},-\mathrm{CH}_{2} \mathrm{CH}_{3}\right.$ and $\left.\mathrm{CH}_{2} \mathrm{CH}\left(\mathrm{CH}_{3}\right)-\right), 0.83$ (d, $\left.J=6.5 \mathrm{~Hz}, 12 \mathrm{H},-\mathrm{CH}\left(\mathrm{CH}_{3}\right)_{2}\right) \cdot{ }^{13} \mathrm{C} \mathrm{NMR}\left(101 \mathrm{MHz}, \mathrm{CDCl}_{3}, \delta, \mathrm{ppm}\right): 162.59$ $(C=\mathrm{O}), 153.19$ (ArC-3,5), 137.95 (ArC-4), 133.53 (ArC-1), [132.36, 130.91, 128.10, 125.06, 122.60, 122.46] (perylene $C$ ), 108.66 ( $\mathrm{ArC}-2,6), 71.84$ ( $\mathrm{ArOCH}_{2}-3,5$ position), 69.33 ( $\mathrm{ArOCH}_{2}-4$ position), 43.75 $\left(-\mathrm{CH}_{2} \mathrm{~N}\right),[39.45,37.63,37.52,31.81,29.89,29.60,28.08,26.00,24.83]\left(-\mathrm{OCH}_{2}\left(\mathrm{CH}_{2}\right)_{3}-\right.$ and $\left.\mathrm{OCH}_{2} \mathrm{CH}_{2} \mathrm{CH}\left(\mathrm{CH}_{3}\right)\left(\mathrm{CH}_{2}\right)_{3} \mathrm{CH}-\right)$, [22.82, 22.78, 22.68, 19.68, 14.23] $\left(-\mathrm{CH}\left(\mathrm{CH}_{3}\right)_{2},-\mathrm{CH}\left(\mathrm{CH}_{3}\right) \mathrm{CH}_{2}-\right.$ and $\mathrm{CH}_{2} \mathrm{CH}_{3}$ ). MALDI-TOF MS $m / z$ of $[\mathrm{M}+\mathrm{Na}]^{+}$calculated for $\mathrm{C}_{82} \mathrm{H}_{110} \mathrm{~N}_{2} \mathrm{O}_{10}$ : 1305.8; Found: 1305.6.
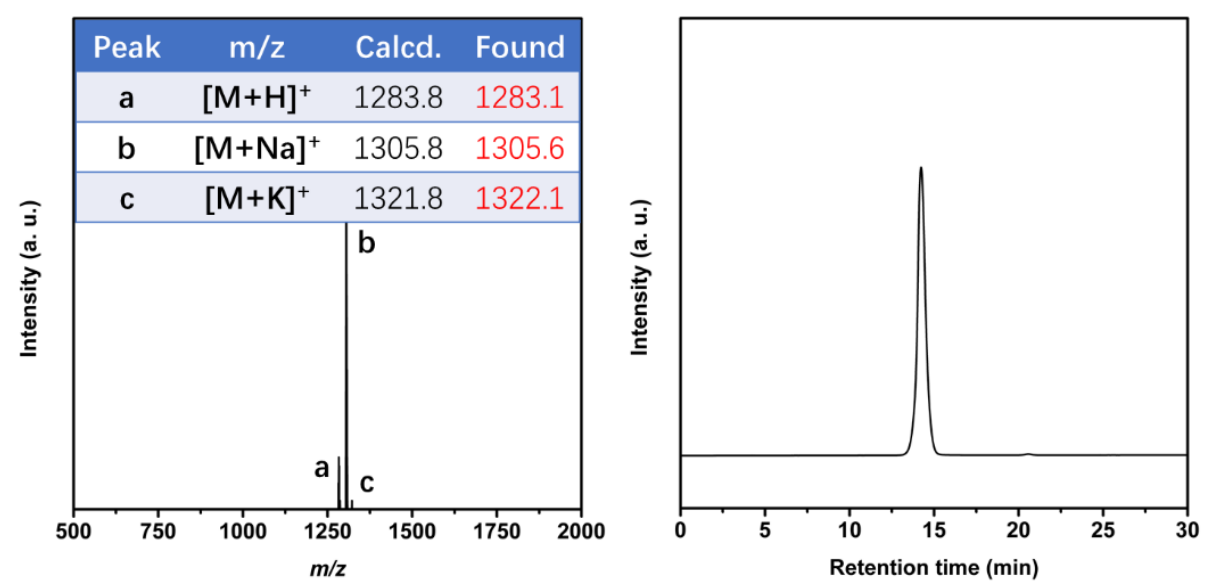

Supporting Figure S35. MALDI-TOF MS spectra (left) and HPLC trace (right) of 6r6-PBI. Target molecule 6r6-PBI has been successfully synthesized. ${ }^{1} \mathrm{H}$ and ${ }^{13} \mathrm{C}$ NMR spectra are presented on pages 108 and 109 , respectively. 
Supporting Figure S35 (continued). ${ }^{1} \mathrm{H}$ NMR of 6r6-PBI.

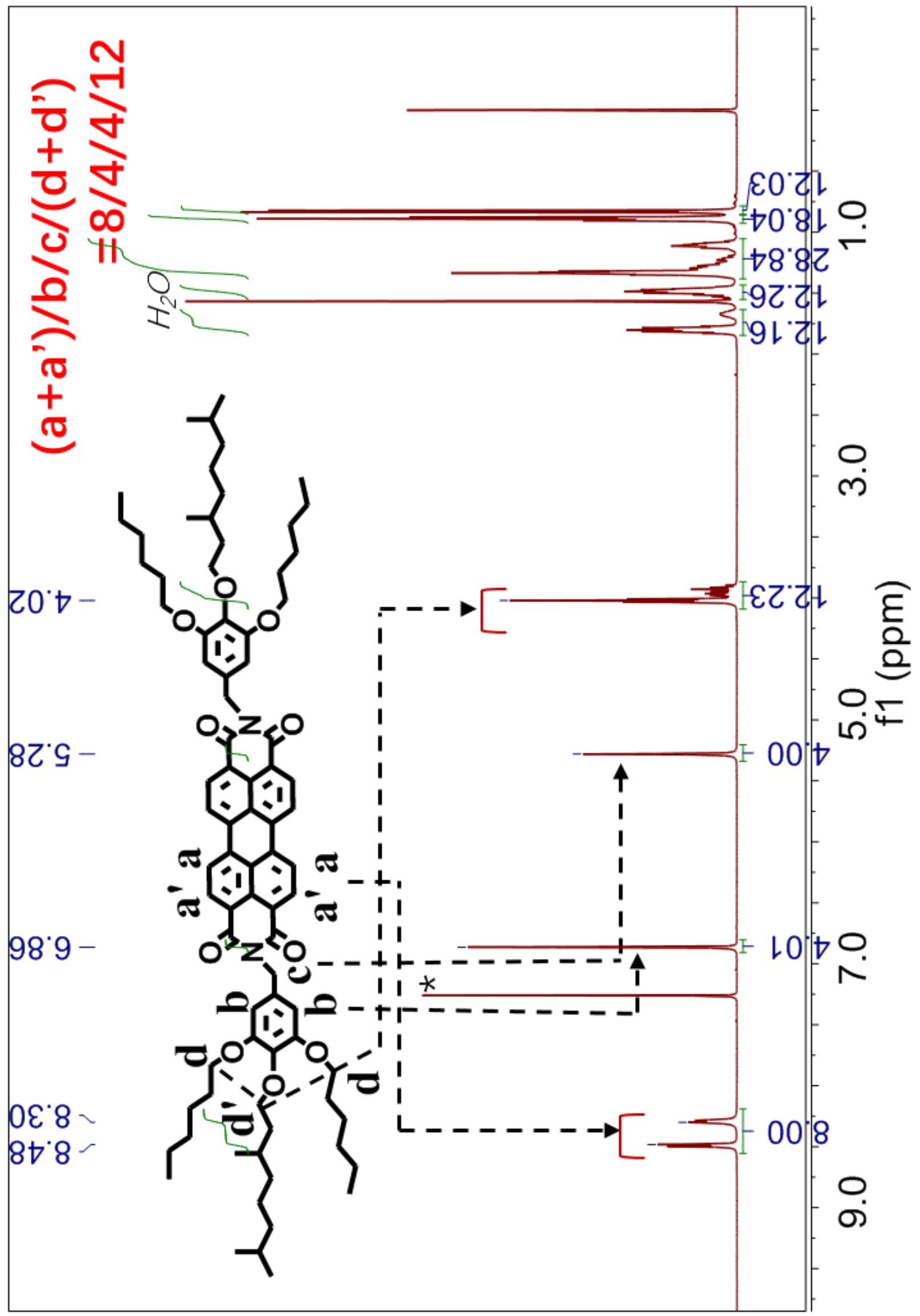


Supporting Figure S35 (continued). ${ }^{13} \mathrm{C}$ NMR of 6r6-PBI.

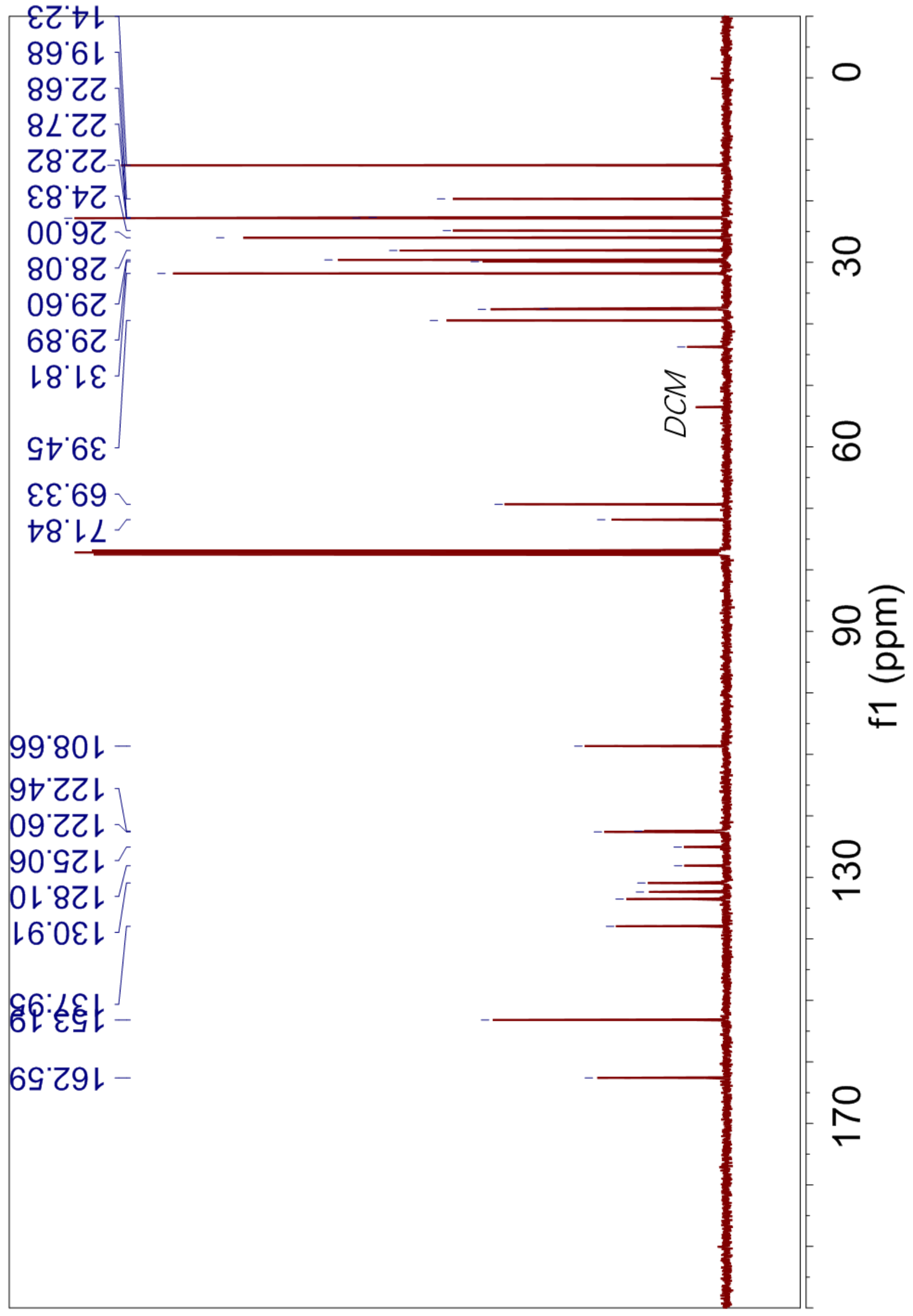




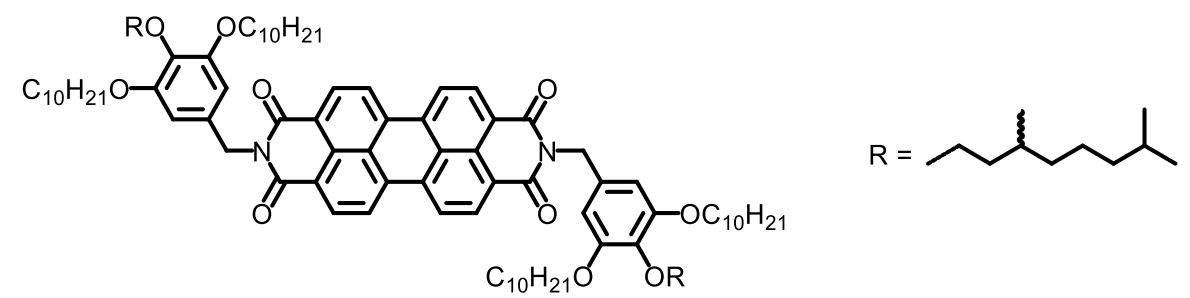

10r10-PBI. From 12-10r10 (1.27 g, $2.2 \mathrm{mmol})$, PTCDA $(0.39 \mathrm{~g}, 1.0 \mathrm{mmol})$ and $\mathrm{Zn}(\mathrm{OAc})_{2} \cdot 2 \mathrm{H}_{2} \mathrm{O}(0.22 \mathrm{~g}$, $1.0 \mathrm{mmol})$ in imidazole $(3 \mathrm{~g})$ at $160{ }^{\circ} \mathrm{C}$ for $16 \mathrm{~h}, \mathbf{1 0}$ r10-PBI was obtained as a red solid. Product: $0.88 \mathrm{~g}$. Yield: $58 \%$. Purity by HPLC: $99+\%$. mp: $209^{\circ} \mathrm{C}$.

${ }^{1} \mathrm{H}$ NMR (500 MHz, $\left.\mathrm{CDCl}_{3}, \delta, \mathrm{ppm}\right): 8.54$ (d, $J=7.9 \mathrm{~Hz}, 4 \mathrm{H}, \mathrm{PBI}, 1,6,7,12$-positions), 8.36 (d, $J=7.9$ $\mathrm{Hz}, 4 \mathrm{H}, \mathrm{PBI}, 2,5,8,11$-positions), 6.85 (s, 4H, $\mathrm{Ar} H$ of dendrons), 5.28 (s, 4H, $\left.-\mathrm{NCH}_{2}-\right), 4.01$ (t, $J=6.5$ $\left.\mathrm{Hz}, 8 \mathrm{H},-\mathrm{OCH}_{2}\left(\mathrm{CH}_{2}\right)_{3}-\right)$, 3.98-3.86 (m, 4H, $\left.-\mathrm{OCH}_{2} \mathrm{CH}_{2} \mathrm{CH}\left(\mathrm{CH}_{3}\right)-\right), 1.84-1.62\left(\mathrm{~m}, 12 \mathrm{H},-\mathrm{CH}_{\left(\mathrm{CH}_{3}\right)_{2},-}\right.$ $\mathrm{OCH}_{2} \mathrm{CH}_{2} \mathrm{CH}_{2}$ and $\left.-\mathrm{CH}\left(\mathrm{CH}_{3}\right) \mathrm{CH}_{2}-\right), 1.54-1.41\left(\mathrm{~m}, 12 \mathrm{H},-\mathrm{O}\left(\mathrm{CH}_{2}\right)_{2} \mathrm{CH}_{2}-\right.$ and $\left.-\mathrm{OCH}_{2} \mathrm{CH}_{2} \mathrm{CH}\left(\mathrm{CH}_{3}\right)^{-}\right)$, 1.37-1.07 (m, 60H, $-\mathrm{CH}\left(\mathrm{CH}_{3}\right)\left(\mathrm{CH}_{2}\right)_{3}-$ and $\left.-\left(\mathrm{CH}_{2}\right)_{3}\left(\mathrm{CH}_{2}\right)_{6}-\right), 0.91-0.80\left(\mathrm{~m}, 30 \mathrm{H},-\mathrm{CH}_{3}\right) .{ }^{13} \mathrm{C} \mathrm{NMR}(101$ $\left.\mathrm{MHz}, \mathrm{CDCl}_{3}, \delta, \mathrm{ppm}\right): 162.68(\mathrm{C}=\mathrm{O}), 153.20$ (ArC-3,5), 137.97 (ArC-4), 133.64 (ArC-1), [132.34, 130.98, 128.24, 125.20, 122.69, 122.51] (perylene $C$ ), 108.62 ( $\mathrm{ArC}-2,6), 71.84$ ( $\mathrm{ArOCH}_{2}-3,5$ position), 69.33 ( $\mathrm{ArOCH}_{2}-4$ position), $43.79\left(-\mathrm{CH}_{2} \mathrm{~N}\right),[39.46,37.65,37.53,32.07,29.89,29.85,29.78,29.67,29.52$, 28.09, 26.36, 24.84] $\left(-\mathrm{OCH}_{2}\left(\mathrm{CH}_{2}\right)_{7}-\right.$ and $\left.-\mathrm{OCH}_{2} \mathrm{CH}_{2} \mathrm{CH}\left(\mathrm{CH}_{3}\right)\left(\mathrm{CH}_{2}\right)_{3} \mathrm{CH}-\right)$, [22.82, 22.80, 22.70, 19.69, 14.25] $\left(-\mathrm{CH}\left(\mathrm{CH}_{3}\right)_{2},-\mathrm{CH}\left(\mathrm{CH}_{3}\right) \mathrm{CH}_{2}-\right.$ and $\left.-\mathrm{CH}_{2} \mathrm{CH}_{3}\right)$. MALDI-TOF MS $m / z$ of $[\mathrm{M}+\mathrm{Na}]^{+}$calculated for $\mathrm{C}_{98} \mathrm{H}_{142} \mathrm{~N}_{2} \mathrm{O}_{10}$ : 1530.1; Found: 1530.4 .
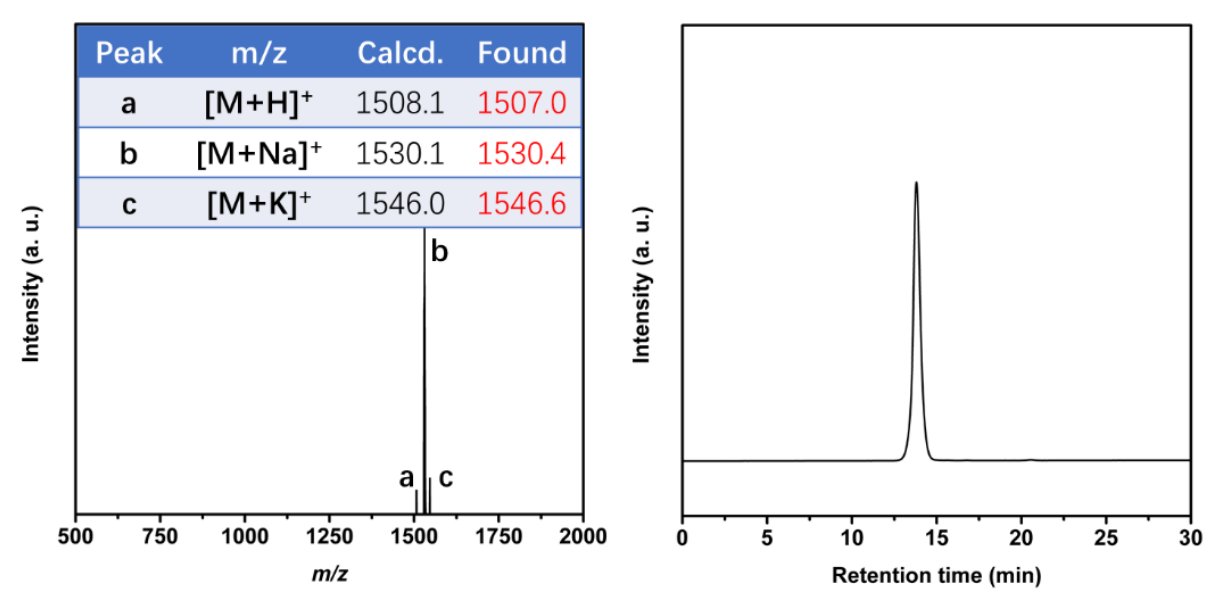

Supporting Figure S36. MALDI-TOF MS spectra (left) and HPLC trace (right) of 10r10-PBI. Target molecule 10r10-PBI has been successfully synthesized. ${ }^{1} \mathrm{H}$ and ${ }^{13} \mathrm{C}$ NMR spectra are presented on pages 111 and 112 , respectively. 
Supporting Figure S36 (continued). ${ }^{1} \mathrm{H}$ NMR of 10r10-PBI.

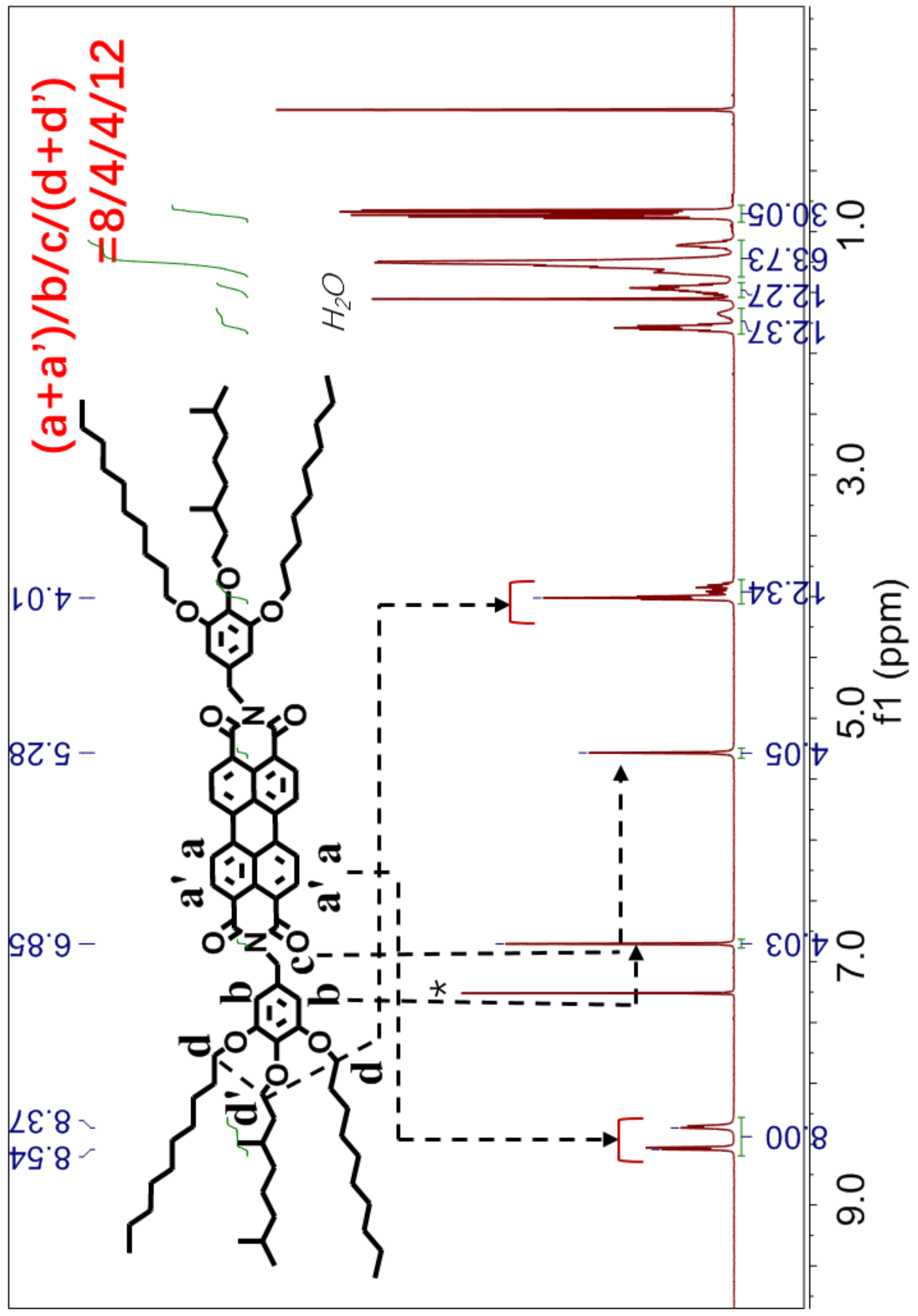


Supporting Figure S36 (continued). ${ }^{13} \mathrm{C}$ NMR of 10r10-PBI.

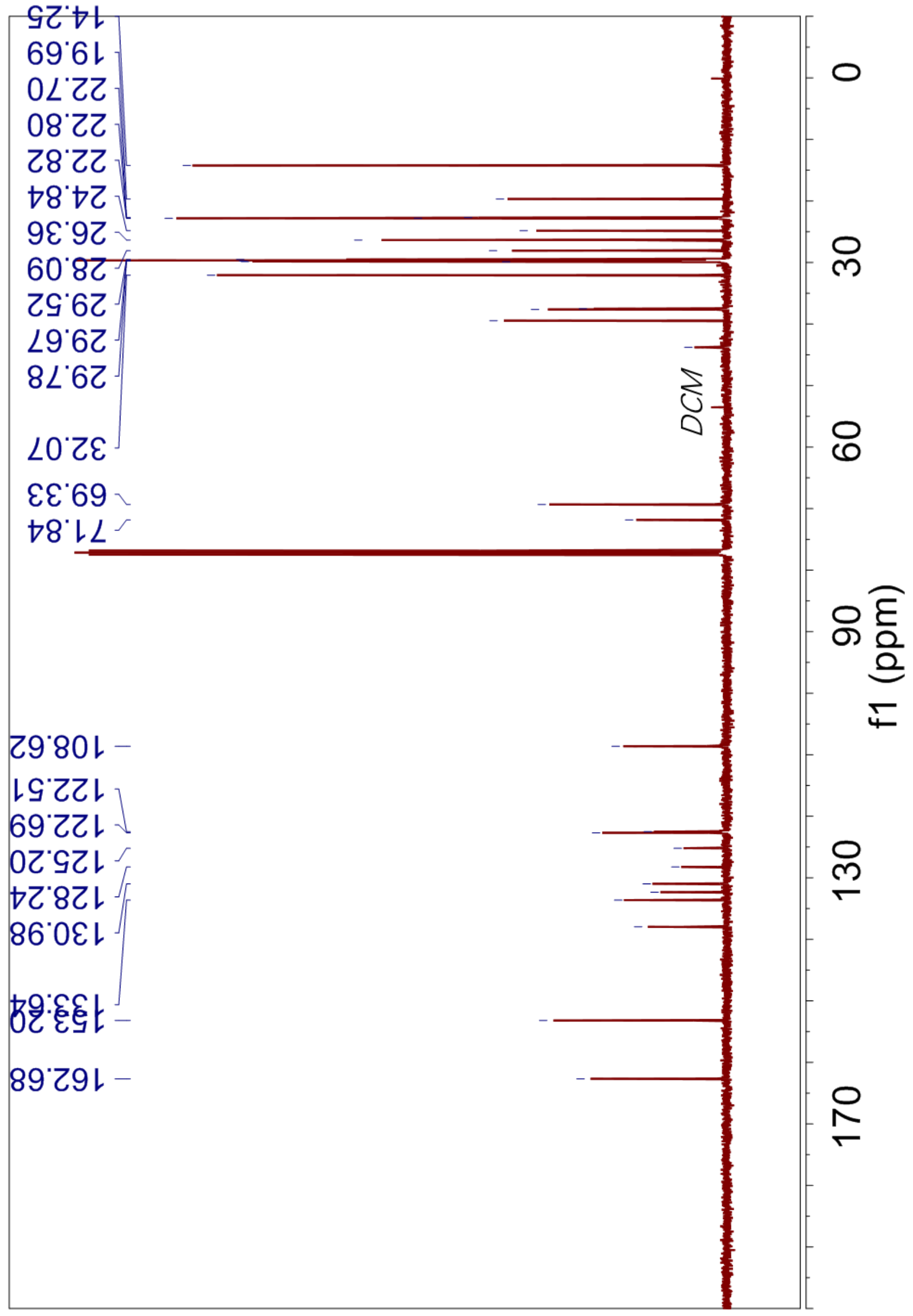




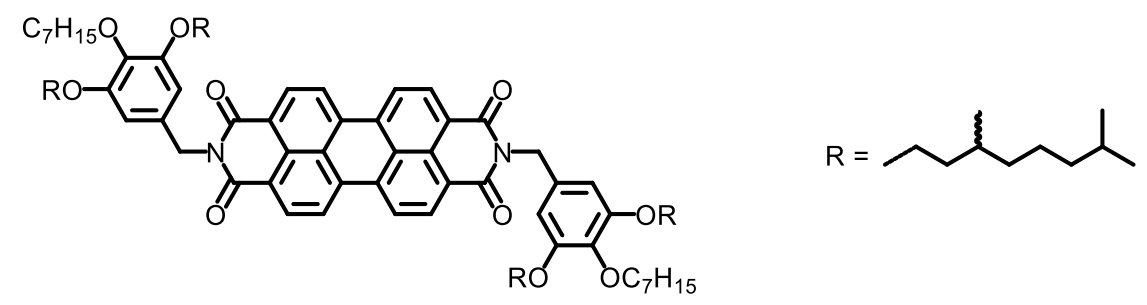

r7r-PBI. From 12-r7r (0.77 g, $1.45 \mathrm{mmol})$, PTCDA $(0.26 \mathrm{~g}, 0.66 \mathrm{mmol})$ and $\mathrm{Zn}(\mathrm{OAc})_{2} \cdot 2 \mathrm{H}_{2} \mathrm{O}(0.145 \mathrm{~g}$, $0.66 \mathrm{mmol})$ in imidazole $(3 \mathrm{~g})$ at $180{ }^{\circ} \mathrm{C}$ for $6 \mathrm{~h}$, r7r-PBI was obtained as a red solid. Product: $0.70 \mathrm{~g}$. Yield: $75 \%$. Purity by HPLC: $99+\%$. mp: $225^{\circ} \mathrm{C}$.

${ }^{1} \mathrm{H}$ NMR (400 MHz, $\left.\mathrm{CDCl}_{3}, \delta, \mathrm{ppm}\right): 8.67$ (d, $J=8.0 \mathrm{~Hz}, 4 \mathrm{H}, \mathrm{PBI}, 1,6,7,12$-positions), 8.55 (d, $J=8,1$ $\mathrm{Hz}, 4 \mathrm{H}, \mathrm{PBI}, 2,5,8,11$-positions), 6.84 (s, 4H, ArH of dendrons), 5.30 (s, 4H, - $\mathrm{NCH}_{2}-$ ), 4.08-3.97 (m, 8H, $\left.-\mathrm{OCH}_{2} \mathrm{CH}_{2} \mathrm{CH}\left(\mathrm{CH}_{3}\right)^{-}\right)$), $3.88\left(\mathrm{t}, J=6.7 \mathrm{~Hz}, 4 \mathrm{H},-\mathrm{OCH}_{2} \mathrm{CH}_{2} \mathrm{CH}_{2}-\right), 1.89-1.77\left(\mathrm{~m}, 4 \mathrm{H},-\mathrm{CH}\left(\mathrm{CH}_{3}\right)_{2}\right), 1.74-$ $1.64\left(\mathrm{~m}, 8 \mathrm{H},-\mathrm{CH}\left(\mathrm{CH}_{3}\right) \mathrm{CH}_{2}-\right.$ and $\left.-\mathrm{OCH}_{2} \mathrm{CH}_{2} \mathrm{CH}_{2}-\right), 1.62-1.37\left(\mathrm{~m}, 12 \mathrm{H},-\mathrm{OCH}_{2} \mathrm{CH}_{2} \mathrm{CH}\left(\mathrm{CH}_{3}\right)-\right.$ and $\left.\mathrm{OCH}_{2} \mathrm{CH}_{2} \mathrm{CH}_{2}-\right), 1.36-1.08\left(\mathrm{~m}, 36 \mathrm{H},-\mathrm{CH}\left(\mathrm{CH}_{3}\right)\left(\mathrm{CH}_{2}\right)_{3}-\right.$ and $\left.-\left(\mathrm{CH}_{2}\right)_{3}\left(\mathrm{CH}_{2}\right)_{3}-\right), 0.92(\mathrm{~d}, \mathrm{~J}=6.5 \mathrm{~Hz}, 12 \mathrm{H}$, $\left.-\mathrm{CH}_{2} \mathrm{CH}\left(\mathrm{CH}_{3}\right)-\right), 0.89-0.80\left(\mathrm{~m}, 30 \mathrm{H},-\mathrm{CH}\left(\mathrm{CH}_{3}\right)_{2}\right.$ and $\left.-\mathrm{CH}_{2} \mathrm{CH}_{3}\right) .{ }^{13} \mathrm{C} \mathrm{NMR}\left(101 \mathrm{MHz}, \mathrm{CDCl}_{3}, \delta, \mathrm{ppm}\right)$ : $162.80(C=\mathrm{O}), 153.17$ (ArC-3,5), 138.00 (ArC-4), 133.81 (ArC-1), [132.33, 131.08, 128.44, 125.42, 122.84, 122.61] (perylene C), 108.62 (ArC-2,6), $73.55\left(\mathrm{ArOCH}_{2}-4\right.$ position), $67.66\left(\mathrm{ArOCH}_{2}-3,5\right.$ position), $43.84\left(-\mathrm{CH}_{2} \mathrm{~N}\right),[39.44,37.57,36.67,32.03,30.50,30.00,29.38,28.14,26.23,24.91]$ ($\mathrm{OCH}_{2} \mathrm{CH}_{2} \mathrm{CH}\left(\mathrm{CH}_{3}\right)\left(\mathrm{CH}_{2}\right)_{3} \mathrm{CH}-$ and $\left.-\mathrm{OCH}_{2}\left(\mathrm{CH}_{2}\right)_{4}-\right)$, [22.86, 22.79, 22.74, 19.78, 14.21] $\left(-\mathrm{CH}\left(\mathrm{CH}_{3}\right)_{2},-\right.$ $\mathrm{CH}\left(\mathrm{CH}_{3}\right) \mathrm{CH}_{2}-$ and $\left.-\mathrm{CH}_{2} \mathrm{CH}_{3}\right)$. MALDI-TOF MS $m / z$ of $[\mathrm{M}+\mathrm{Na}]^{+}$calculated for $\mathrm{C}_{92} \mathrm{H}_{130} \mathrm{~N}_{2} \mathrm{O}_{10}$ : 1446.0; Found: 1446.2 .
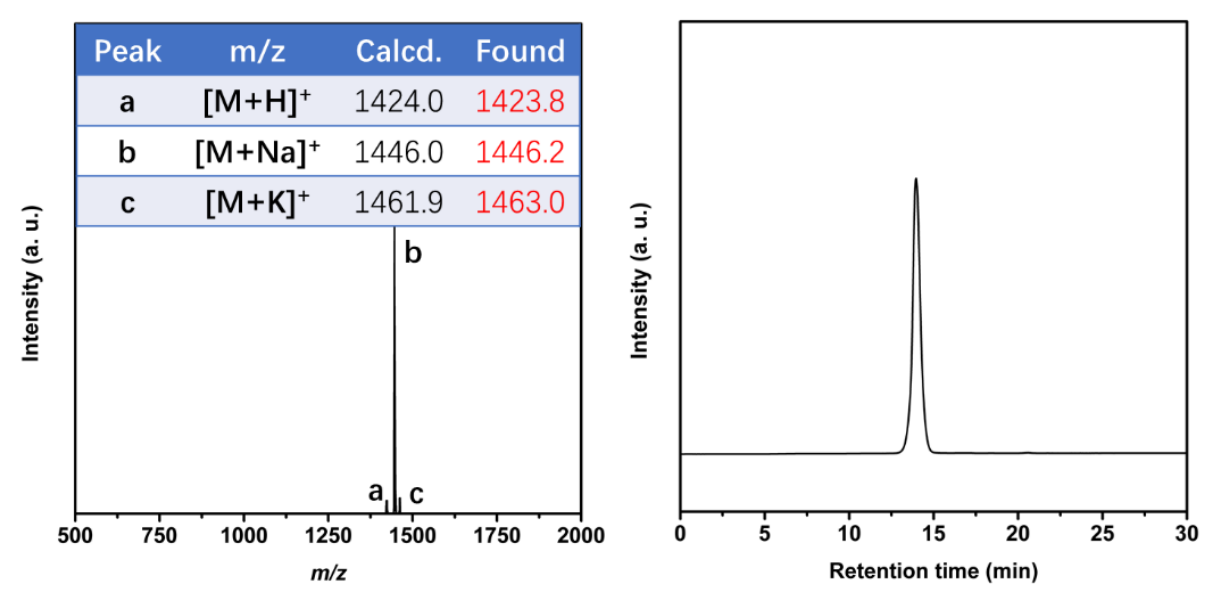

Supporting Figure S37. MALDI-TOF MS spectra (left) and HPLC trace (right) of r7r-PBI. Target molecule r7r-PBI has been successfully synthesized. ${ }^{1} \mathrm{H}$ and ${ }^{13} \mathrm{C}$ NMR spectra are presented on pages 114 and 115 , respectively. 
Supporting Figure S37 (continued). ${ }^{1} \mathrm{H}$ NMR of r r r-PBI.

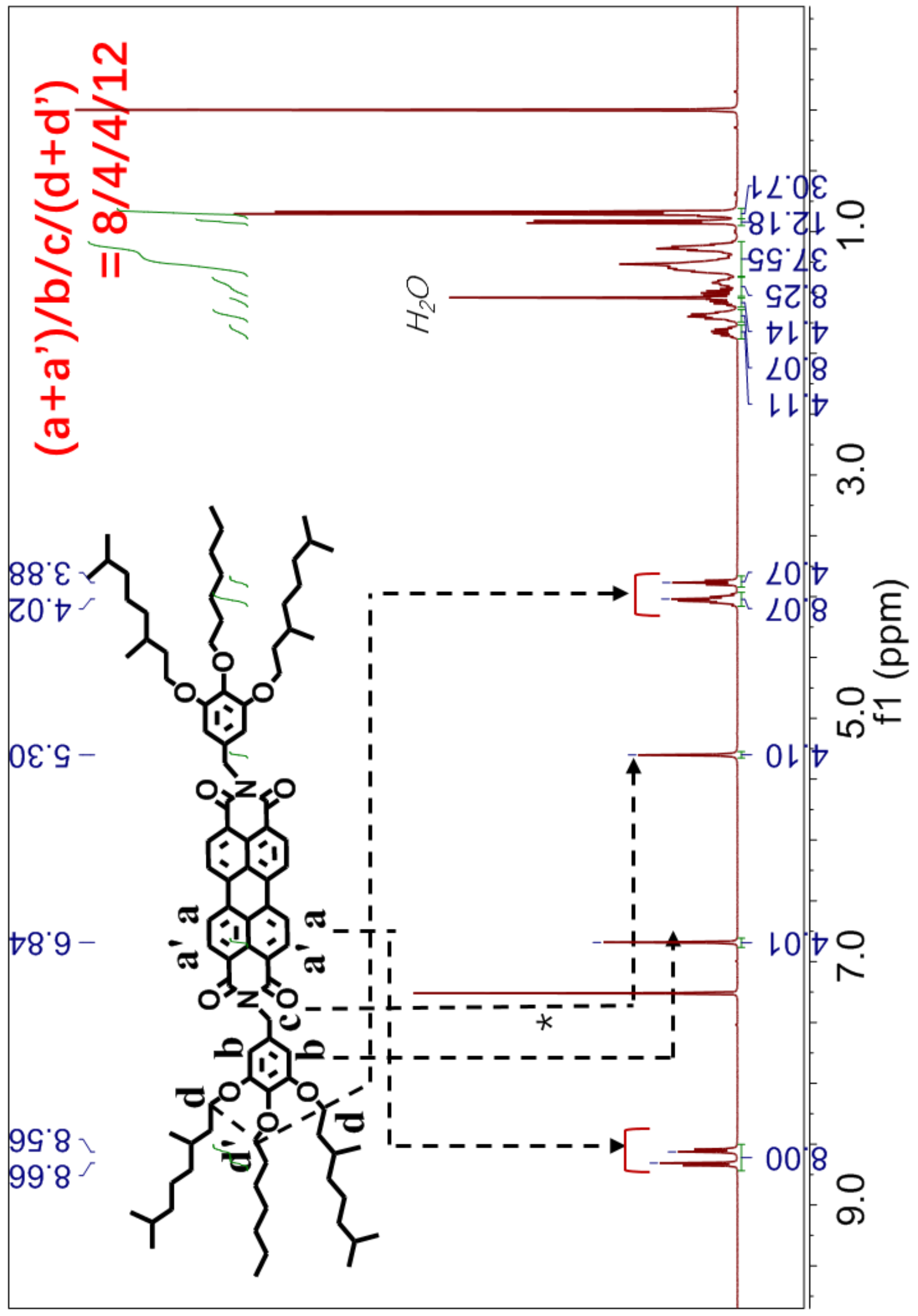


Supporting Figure S37 (continued). ${ }^{13} \mathrm{C}$ NMR of r7r-PBI.

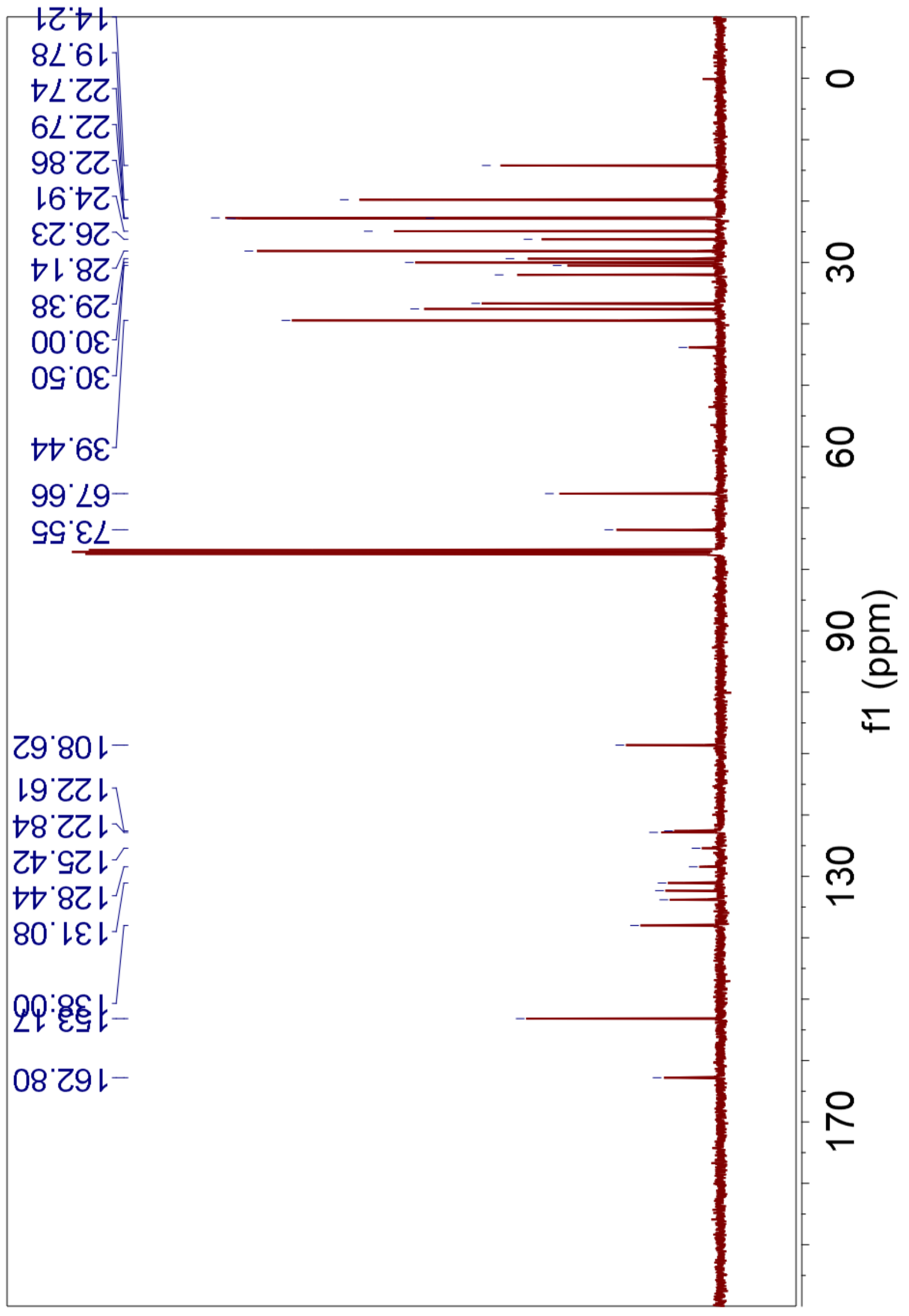




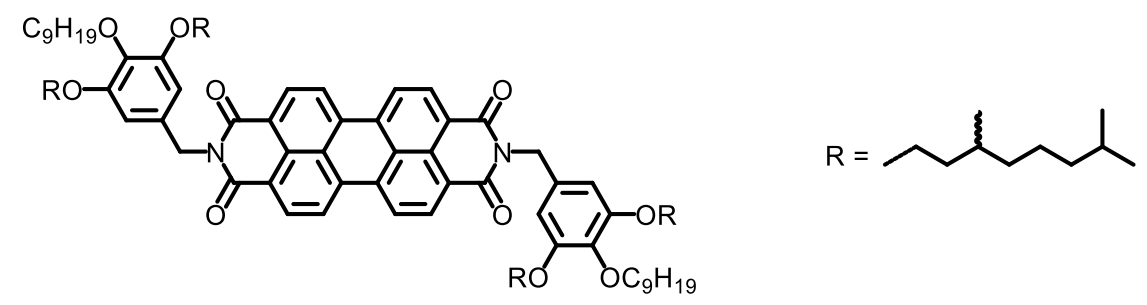

r9r-PBI. From 12-r9r (0.81 g, $1.45 \mathrm{mmol})$, PTCDA $(0.26 \mathrm{~g}, 0.66 \mathrm{mmol})$ and $\mathrm{Zn}(\mathrm{OAc})_{2} \cdot 2 \mathrm{H}_{2} \mathrm{O}(0.145 \mathrm{~g}$, $0.66 \mathrm{mmol})$ in imidazole $(3 \mathrm{~g})$ at $180{ }^{\circ} \mathrm{C}$ for $6 \mathrm{~h}$, r9r-PBI was obtained as a red solid. Product: $0.73 \mathrm{~g}$. Yield: $75 \%$. Purity by HPLC: $99+\%$. mp: $222{ }^{\circ} \mathrm{C}$.

${ }^{1} \mathrm{H}$ NMR (400 MHz, $\left.\mathrm{CDCl}_{3}, \delta, \mathrm{ppm}\right): 8.71$ (d, $J=8.0 \mathrm{~Hz}, 4 \mathrm{H}, \mathrm{PBI}, 1,6,7,12$-positions), 8.62 (d, $J=8.1$ $\mathrm{Hz}, 4 \mathrm{H}, \mathrm{PBI}, 2,5,8,11$-positions), 6.83 (s, 4H, ArH of dendrons), 5.30 (s, 4H, -NCH2-), 4.08-3.96 (m, 8H, $\left.-\mathrm{OCH}_{2} \mathrm{CH}_{2} \mathrm{CH}\left(\mathrm{CH}_{3}\right)^{-}\right)$), $3.88\left(\mathrm{t}, J=6.6 \mathrm{~Hz}, 4 \mathrm{H},-\mathrm{OCH}_{2} \mathrm{CH}_{2} \mathrm{CH}_{2}-\right), 1.87-1.77\left(\mathrm{~m}, 4 \mathrm{H},-\mathrm{CH}\left(\mathrm{CH}_{3}\right)_{2}\right), 1.74-$ $1.64\left(\mathrm{~m}, 8 \mathrm{H},-\mathrm{CH}\left(\mathrm{CH}_{3}\right) \mathrm{CH}_{2}-\right.$ and $\left.-\mathrm{OCH}_{2} \mathrm{CH}_{2} \mathrm{CH}_{2}-\right), 1.62-1.37\left(\mathrm{~m}, 12 \mathrm{H},-\mathrm{OCH}_{2} \mathrm{CH}_{2} \mathrm{CH}\left(\mathrm{CH}_{3}\right)-\right.$ and $\left.\mathrm{OCH}_{2} \mathrm{CH}_{2} \mathrm{CH}_{2}-\right), 1.36-1.08\left(\mathrm{~m}, 44 \mathrm{H},-\mathrm{CH}\left(\mathrm{CH}_{3}\right)\left(\mathrm{CH}_{2}\right)_{3}-\right.$ and $\left.-\left(\mathrm{CH}_{2}\right)_{3}\left(\mathrm{CH}_{2}\right)_{5}-\right), 0.92(\mathrm{~d}, \mathrm{~J}=6.5 \mathrm{~Hz}, 12 \mathrm{H}$, $\left.-\mathrm{CH}_{2} \mathrm{CH}\left(\mathrm{CH}_{3}\right)_{-}\right), 0.89-0.81\left(\mathrm{~m}, 30 \mathrm{H},-\mathrm{CH}\left(\mathrm{CH}_{3}\right)_{2}\right.$ and $\left.-\mathrm{CH}_{2} \mathrm{CH}_{3}\right) .{ }^{13} \mathrm{C} \mathrm{NMR}\left(101 \mathrm{MHz}, \mathrm{CDCl}_{3}, \delta, \mathrm{ppm}\right)$ : $163.09(C=\mathrm{O}), 153.17$ (ArC-3,5), 138.04 (ArC-4), 134.20 (ArC-1), [132.27, 131.34, 128.87, 125.88, 123.11, 122.86] (perylene C), 108.54 (ArC-2,6), $73.55\left(\mathrm{ArOCH}_{2}-4\right.$ position), $67.65\left(\mathrm{ArOCH}_{2}-3,5\right.$ position), $43.96\left(-\mathrm{CH}_{2} \mathrm{~N}\right),[39.44,37.56,36.66,32.05,30.49,29.98,29.80,29.75,29.49,28.13,26.27$, 24.90] $\left(-\mathrm{OCH}_{2} \mathrm{CH}_{2} \mathrm{CH}\left(\mathrm{CH}_{3}\right)\left(\mathrm{CH}_{2}\right)_{3} \mathrm{CH}-\right.$ and $\left.-\mathrm{OCH}_{2}\left(\mathrm{CH}_{2}\right)_{6}-\right)$, [22.86, 22.81, 22.75, 19.77, 14.23] ($\mathrm{CH}\left(\mathrm{CH}_{3}\right)_{2},-\mathrm{CH}\left(\mathrm{CH}_{3}\right) \mathrm{CH}_{2}-$ and $\left.-\mathrm{CH}_{2} \mathrm{CH}_{3}\right)$. MALDI-TOF MS $m / z$ of $[\mathrm{M}+\mathrm{Na}]^{+}$calculated for $\mathrm{C}_{96} \mathrm{H}_{138} \mathrm{~N}_{2} \mathrm{O}_{10}$ : 1502.0; Found: 1503.4.
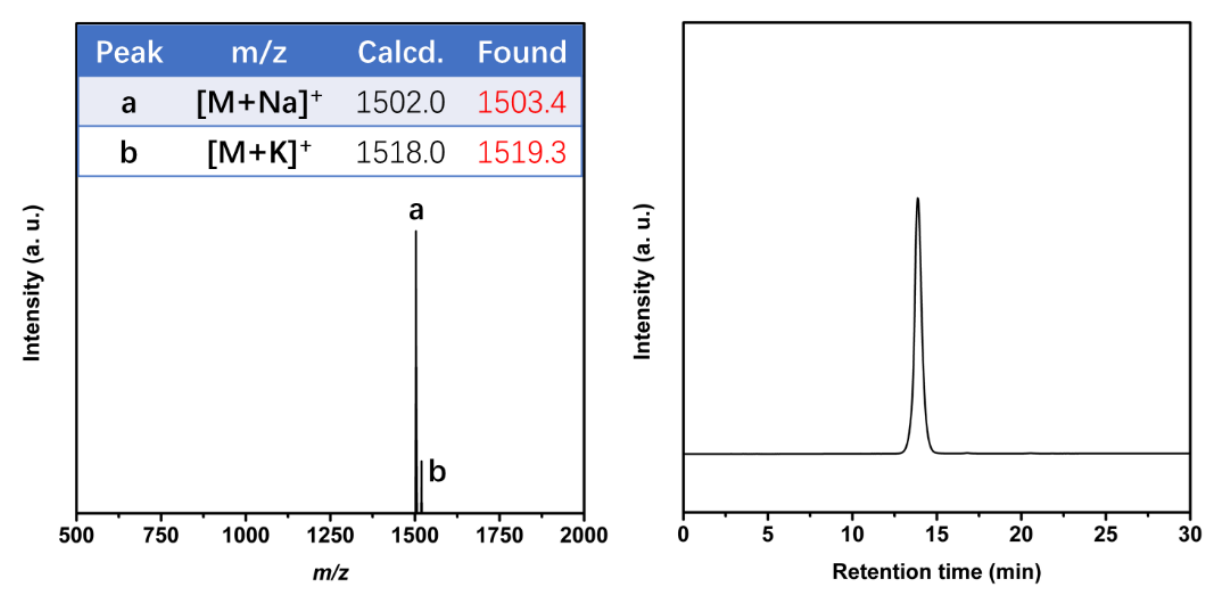

Supporting Figure S38. MALDI-TOF MS spectra (left) and HPLC trace (right) of r9r-PBI. Target molecule r9r-PBI has been successfully synthesized. ${ }^{1} \mathrm{H}$ and ${ }^{13} \mathrm{C}$ NMR spectra are presented on pages 117 and 118 , respectively. 
Supporting Figure S38 (continued). ${ }^{1} \mathrm{H}$ NMR of r9r-PBI.

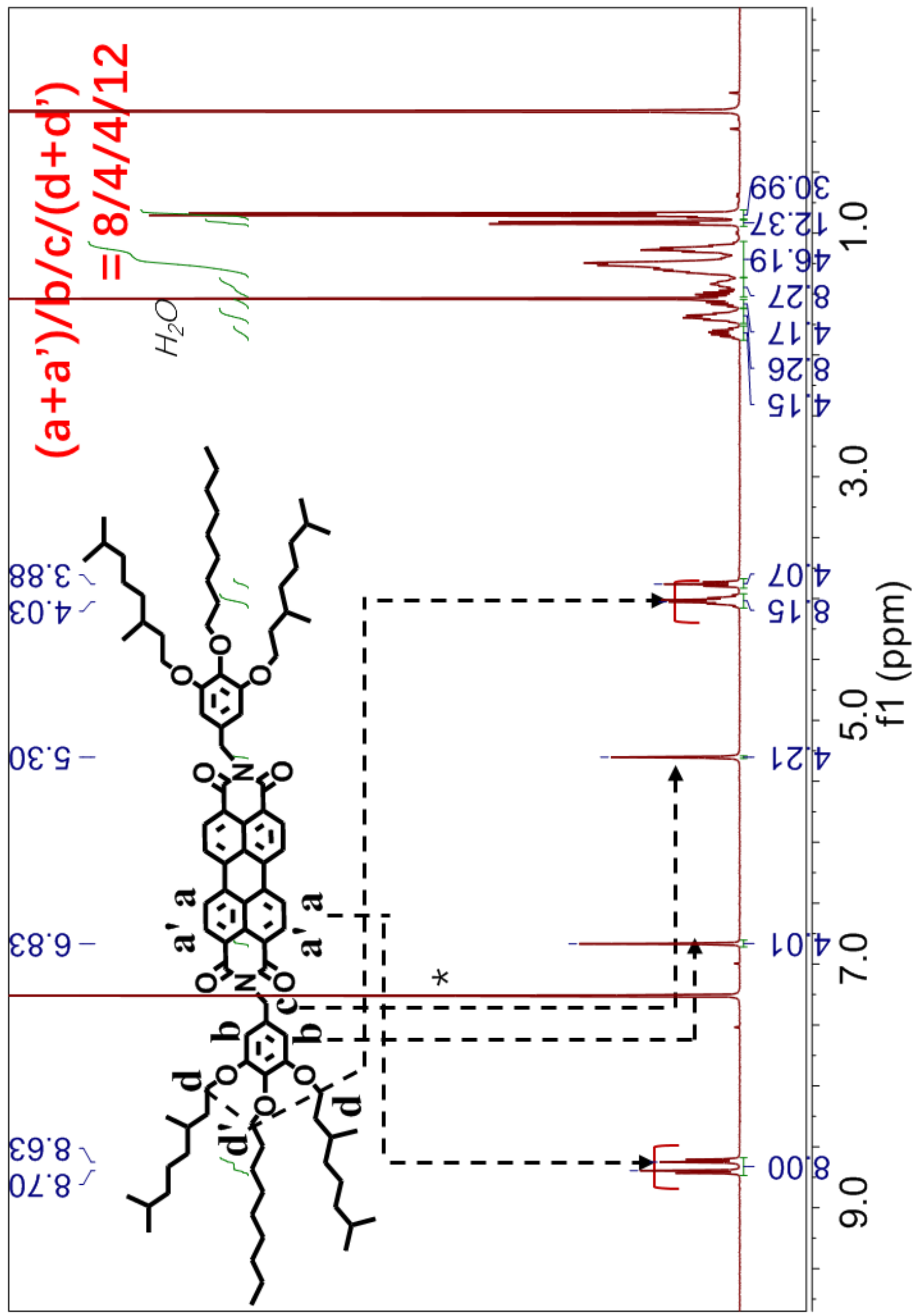


Supporting Figure S38 (continued). ${ }^{13} \mathrm{C}$ NMR of r9r-PBI.

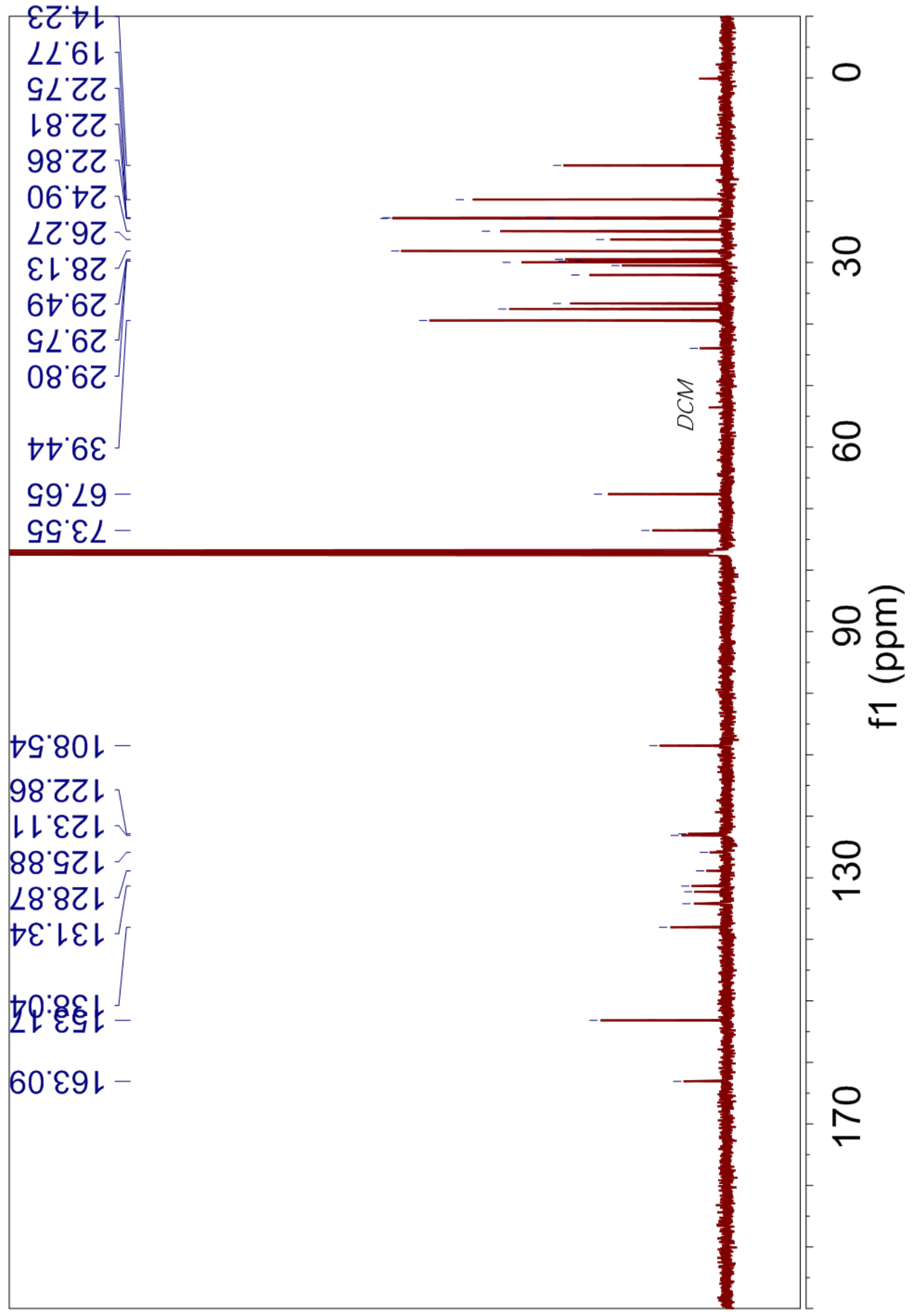



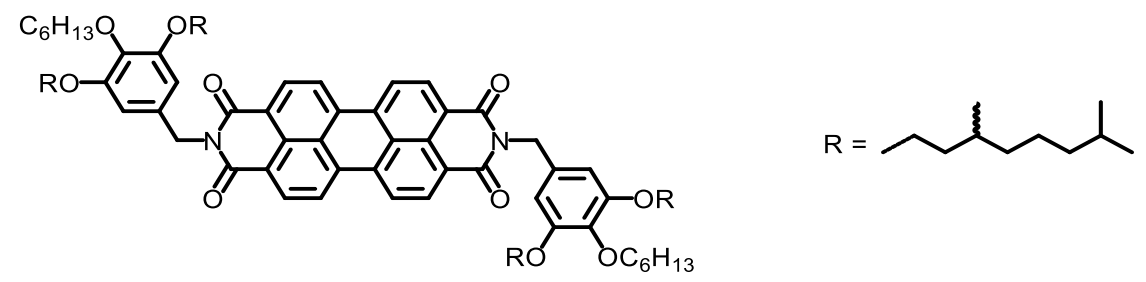

r6r-PBI. From 12-r6r (1.14 g, $2.2 \mathrm{mmol})$, PTCDA (0.39 g, $1.0 \mathrm{mmol})$ and $\mathrm{Zn}(\mathrm{OAc})_{2} \cdot 2 \mathrm{H}_{2} \mathrm{O}(0.22 \mathrm{~g}, 1.0$ mmol) in imidazole $(3 \mathrm{~g})$ at $160{ }^{\circ} \mathrm{C}$ for $14 \mathrm{~h}, \mathbf{r 6 r}-\mathbf{P B I}$ was obtained as a red solid. Product: $0.87 \mathrm{~g}$. Yield: $67 \%$. Purity by HPLC: $99+\%$. mp: $229^{\circ} \mathrm{C}$.

${ }^{1} \mathrm{H}$ NMR (500 MHz, $\mathrm{CDCl}_{3}, \delta$, ppm): 8.42 (d, $J=7.8 \mathrm{~Hz}, 4 \mathrm{H}, \mathrm{PBI}, 1,6,7,12$-positions), 8.18 (d, $J=7.8$ $\mathrm{Hz}, 4 \mathrm{H}, \mathrm{PBI}, 2,5,8,11$-positions), 6.88 (s, 4H, $\mathrm{Ar} H$ of dendrons), 5.28 (s, 4H, $-\mathrm{NCH}_{2}-$ ), 4.13-4.01 (m, 8H, $\left.-\mathrm{OCH}_{2} \mathrm{CH}_{2} \mathrm{CH}\left(\mathrm{CH}_{3}\right)-\right), 3.91\left(\mathrm{t}, J=6.7 \mathrm{~Hz}, 4 \mathrm{H},-\mathrm{OCH}_{2} \mathrm{CH}_{2} \mathrm{CH}_{2}-\right), 1.91-1.82\left(\mathrm{~m}, 4 \mathrm{H},-\mathrm{CH}\left(\mathrm{CH}_{3}\right)_{2}\right), 1.77-$ $1.66\left(\mathrm{~m}, 8 \mathrm{H},-\mathrm{CH}\left(\mathrm{CH}_{3}\right) \mathrm{CH}_{2}-\right.$ and $\left.-\mathrm{OCH}_{2} \mathrm{CH}_{2} \mathrm{CH}_{2}-\right), 1.66-1.40\left(\mathrm{~m}, 12 \mathrm{H},-\mathrm{OCH}_{2} \mathrm{CH}_{2} \mathrm{CH}\left(\mathrm{CH}_{3}\right)-\right.$ and $\left.\mathrm{OCH}_{2} \mathrm{CH}_{2} \mathrm{CH}_{2}-\right), 1.39-1.09$ (m, 32H, $-\mathrm{CH}\left(\mathrm{CH}_{3}\right)\left(\mathrm{CH}_{2}\right)_{3}-$ and $\left.-\left(\mathrm{CH}_{2}\right)_{3}\left(\mathrm{CH}_{2}\right)_{2}-\right), 0.95$ (d, $J=6.6 \mathrm{~Hz}, 12 \mathrm{H}$, $\left.-\mathrm{CH}_{2} \mathrm{CH}\left(\mathrm{CH}_{3}\right)-\right), 0.90-0.81\left(\mathrm{~m}, 30 \mathrm{H},-\mathrm{CH}\left(\mathrm{CH}_{3}\right)_{2}\right.$ and $\left.-\mathrm{CH}_{2} \mathrm{CH}_{3}\right) .{ }^{13} \mathrm{C} \mathrm{NMR}\left(101 \mathrm{MHz}, \mathrm{CDCl}_{3}, \delta, \mathrm{ppm}\right)$ : $162.71(C=\mathrm{O}), 153.17(\mathrm{ArC}-3,5), 137.97(\operatorname{ArC}-4), 133.70(\operatorname{ArC}-1),[132.34,131.01,128.30,125.28$, 122.75, 122.55] (perylene $C$ ), 108.63 ( $\mathrm{ArC}-2,6), 73.54$ ( $\mathrm{ArOCH}_{2}-4$ position), 67.65 ( $\mathrm{ArOCH}_{2}-3,5$ position), $43.81\left(-\mathrm{CH}_{2} \mathrm{~N}\right), \quad[39.44,37.57,36.67,31.91,30.45,30.00,28.14,25.94,24.92]$ ($\mathrm{OCH}_{2} \mathrm{CH}_{2} \mathrm{CH}\left(\mathrm{CH}_{3}\right)\left(\mathrm{CH}_{2}\right)_{3} \mathrm{CH}-$ and $\left.-\mathrm{OCH}_{2}\left(\mathrm{CH}_{2}\right)_{3}-\right)$, [22.86, 22.80, 22.74, 19.78, 14.21] $\left(-\mathrm{CH}_{\left(-\mathrm{CH}_{3}\right)_{2},-}\right.$ $\mathrm{CH}\left(\mathrm{CH}_{3}\right) \mathrm{CH}_{2}$-and $\left.-\mathrm{CH}_{2} \mathrm{CH}_{3}\right)$. MALDI-TOF MS $m / z$ of $[\mathrm{M}+\mathrm{Na}]^{+}$calculated for $\mathrm{C}_{90} \mathrm{H}_{126} \mathrm{~N}_{2} \mathrm{O}_{10}$ : 1417.9; Found: 1418.4 .
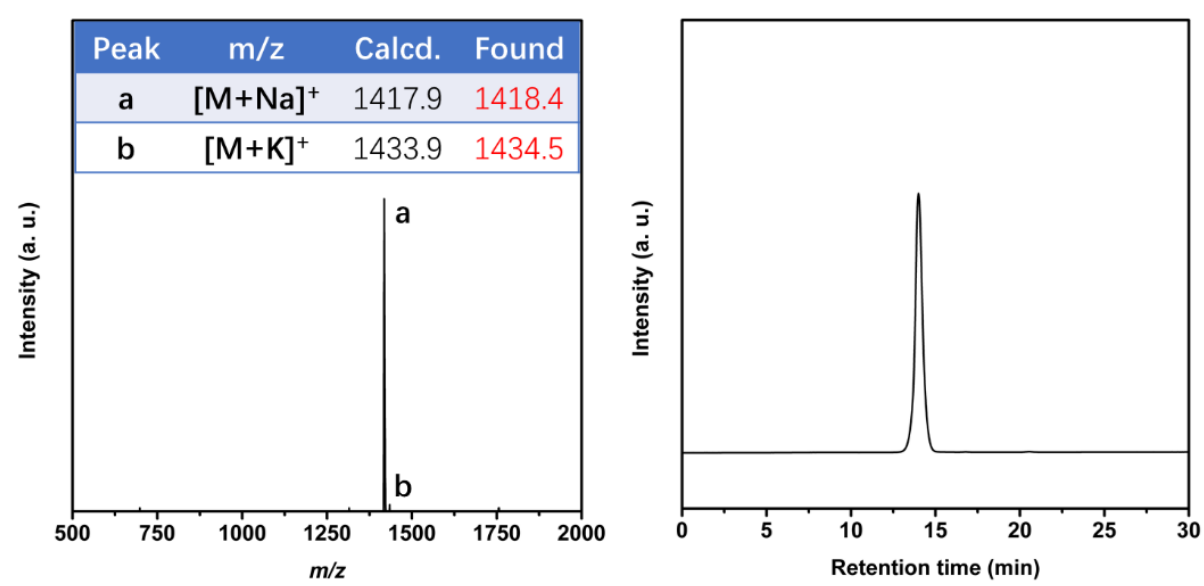

Supporting Figure S39. MALDI-TOF MS spectra (left) and HPLC trace (right) of r6r-PBI. Target molecule r6r-PBI has been successfully synthesized. ${ }^{1} \mathrm{H}$ and ${ }^{13} \mathrm{C}$ NMR spectra are presented on pages 120 and 121 , respectively. 
Supporting Figure S39 (continued). ${ }^{1} \mathrm{H}$ NMR of r6r-PBI.

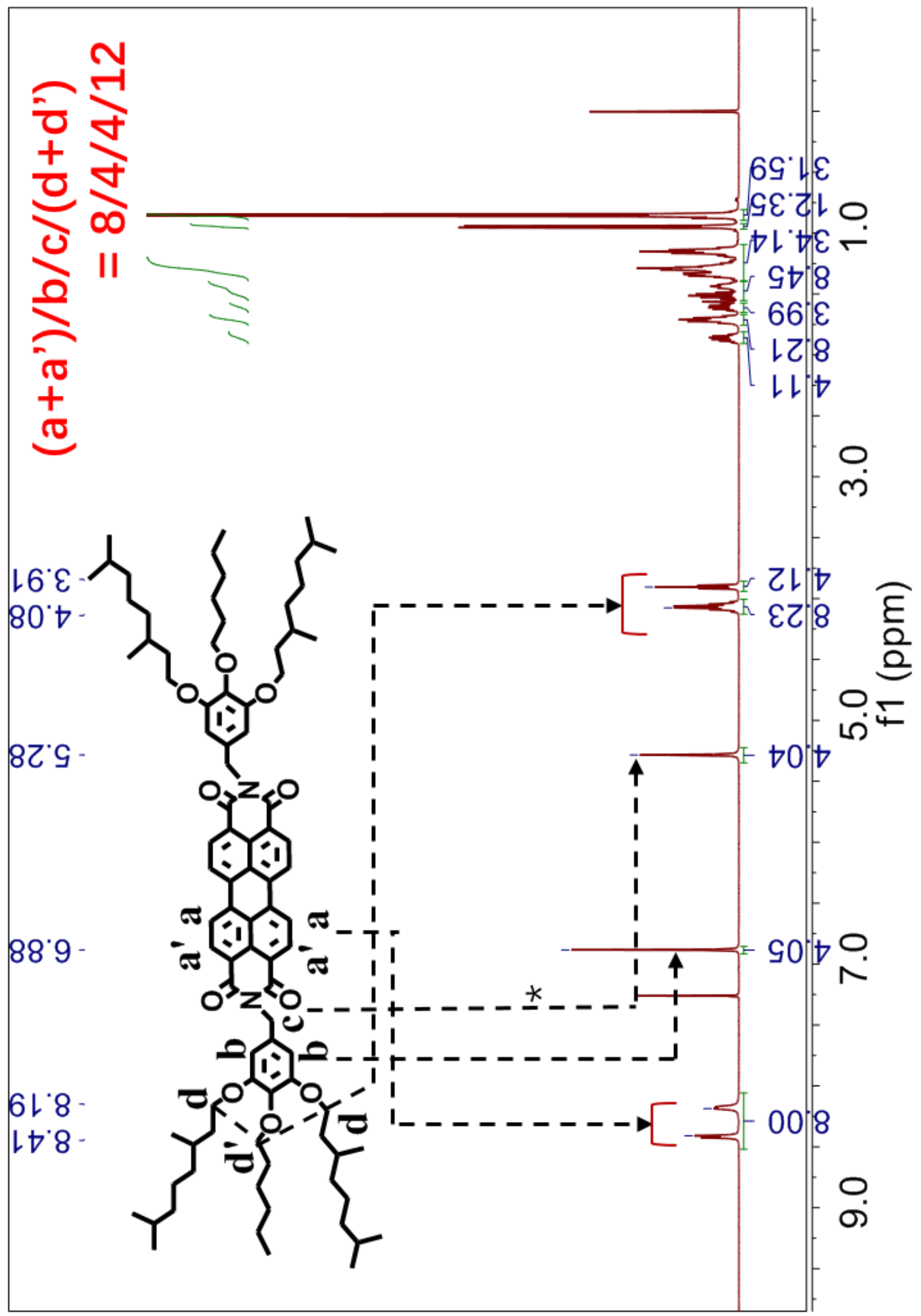


Supporting Figure S39 (continued). ${ }^{13} \mathrm{C}$ NMR of r6r-PBI.

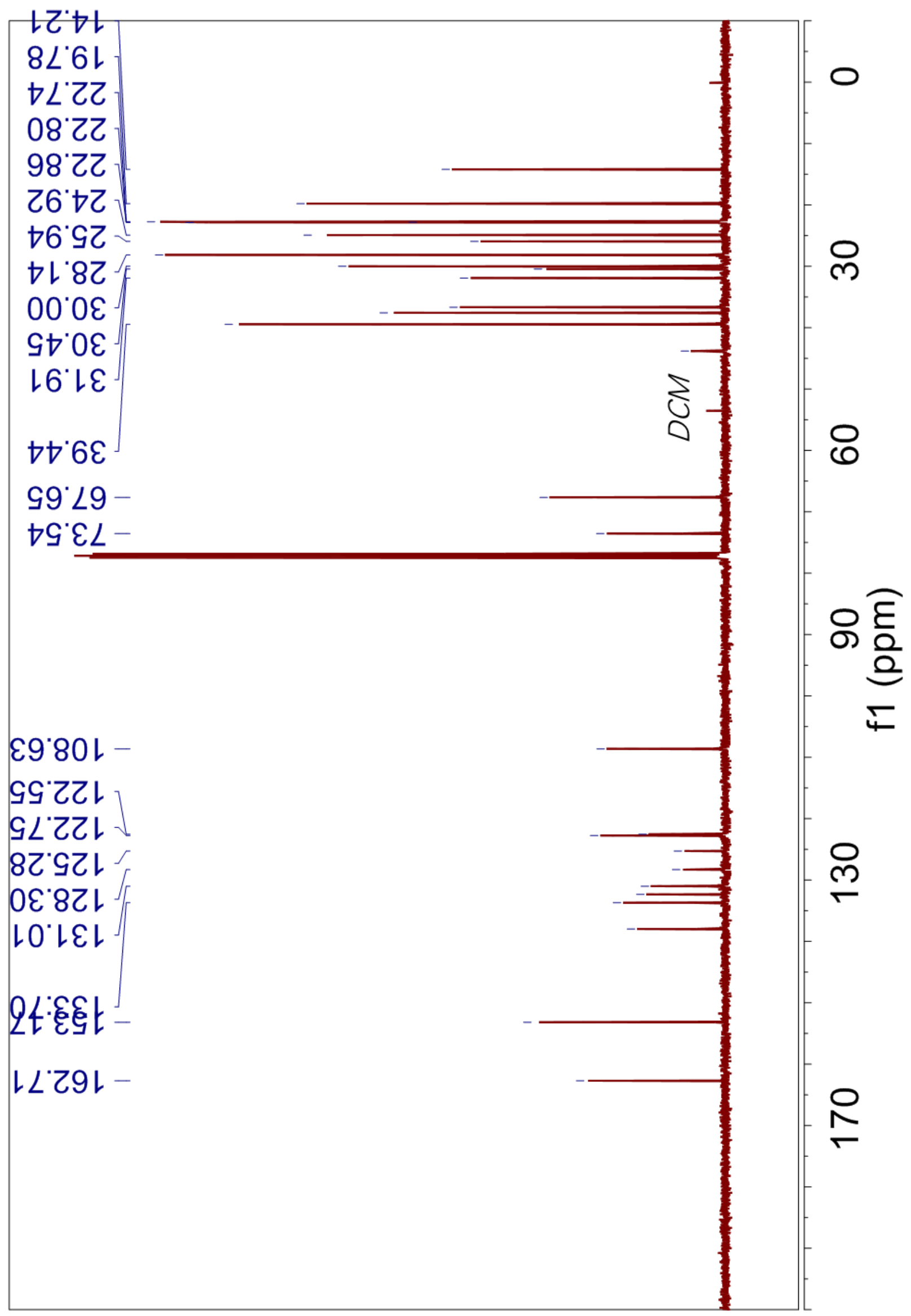




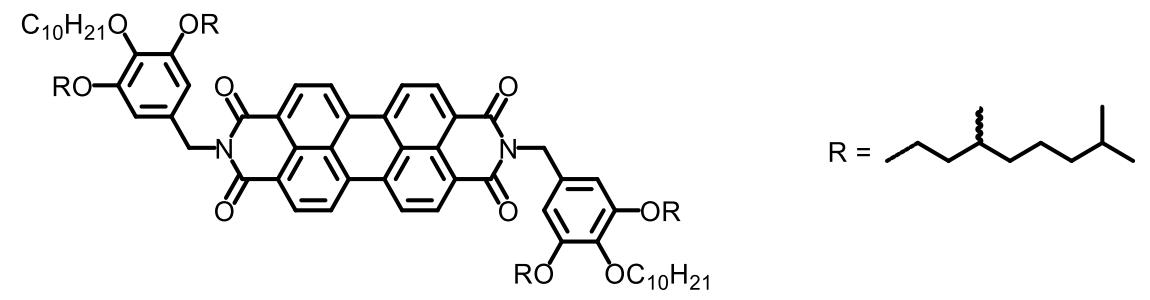

r10r-PBI. From 12-r10r (1.27 g, $2.2 \mathrm{mmol})$, PTCDA $(0.39 \mathrm{~g}, 1.0 \mathrm{mmol})$ and $\mathrm{Zn}(\mathrm{OAc})_{2} \cdot 2 \mathrm{H}_{2} \mathrm{O}(0.22 \mathrm{~g}$, $1.0 \mathrm{mmol})$ in imidazole $(3 \mathrm{~g})$ at $160{ }^{\circ} \mathrm{C}$ for $18 \mathrm{~h}$, r10r-PBI was obtained as a red solid. Product: $1.06 \mathrm{~g}$. Yield: $70 \%$. Purity by HPLC: $99+\%$. mp: $222{ }^{\circ} \mathrm{C}$.

${ }^{1} \mathrm{H}$ NMR (500 MHz, $\left.\mathrm{CDCl}_{3}, \delta, \mathrm{ppm}\right): 8.55$ (d, $J=8.0 \mathrm{~Hz}, 4 \mathrm{H}, \mathrm{PBI}, 1,6,7,12$-positions), 8.37 (d, $J=8.1$ $\mathrm{Hz}, 4 \mathrm{H}, \mathrm{PBI}, 2,5,8,11$-positions), 6.86 (s, 4H, ArH of dendrons), 5.29 (s, 4H, -NCH $\mathrm{N}_{2}$ ), 4.11-3.99 (m, 8H, $\left.-\mathrm{OCH}_{2} \mathrm{CH}_{2} \mathrm{CH}\left(\mathrm{CH}_{3}\right)-\right), 3.89\left(\mathrm{t}, J=6.7 \mathrm{~Hz}, 4 \mathrm{H},-\mathrm{OCH}_{2} \mathrm{CH}_{2} \mathrm{CH}_{2}-\right), 1.89-1.80\left(\mathrm{~m}, 4 \mathrm{H},-\mathrm{CH}_{\left.\left(\mathrm{CH}_{3}\right)_{2}\right), 1.75-}\right.$ $1.65\left(\mathrm{~m}, 8 \mathrm{H},-\mathrm{CH}\left(\mathrm{CH}_{3}\right) \mathrm{CH}_{2}-\right.$ and $\left.-\mathrm{OCH}_{2} \mathrm{CH}_{2} \mathrm{CH}_{2}-\right), 1.63-1.38\left(\mathrm{~m}, 12 \mathrm{H},-\mathrm{OCH}_{2} \mathrm{CH}_{2} \mathrm{CH}_{\left(\mathrm{CH}_{3}\right)-\text { and }-}\right.$ $\left.\mathrm{OCH}_{2} \mathrm{CH}_{2} \mathrm{CH}_{2}-\right), 1.37-1.09\left(\mathrm{~m}, 48 \mathrm{H},-\mathrm{CH}\left(\mathrm{CH}_{3}\right)\left(\mathrm{CH}_{2}\right)_{3}-\right.$ and $\left.-\left(\mathrm{CH}_{2}\right)_{3}\left(\mathrm{CH}_{2}\right)_{6}-\right), 0.93(\mathrm{~d}, J=6.6 \mathrm{~Hz}, 12 \mathrm{H}$, $\left.-\mathrm{CH}_{2} \mathrm{CH}\left(\mathrm{CH}_{3}\right)-\right), 0.89-0.82\left(\mathrm{~m}, 30 \mathrm{H},-\mathrm{CH}\left(\mathrm{CH}_{3}\right)_{2}\right.$ and $\left.-\mathrm{CH}_{2} \mathrm{CH}_{3}\right) .{ }^{13} \mathrm{C} \mathrm{NMR}\left(101 \mathrm{MHz}, \mathrm{CDCl}_{3}, \delta, \mathrm{ppm}\right)$ : $162.67(C=\mathrm{O}), 153.17$ (ArC-3,5), 137.98 (ArC-4), 133.65 (ArC-1), [132.34, 130.97, 128.25, 125.22, 122.71, 122.51] (perylene C), 108.65 (ArC-2,6), 73.56 ( $\mathrm{ArOCH}_{2}-4$ position), $67.65\left(\mathrm{ArOCH}_{2}-3,5\right.$ position), $43.79\left(-\mathrm{CH}_{2} \mathrm{~N}\right),[39.44,37.57,36.68,32.03,30.50,30.01,29.84,29.78,29.73,29.48,28.14$, $26.28,24.92]\left(-\mathrm{OCH}_{2} \mathrm{CH}_{2} \mathrm{CH}\left(\mathrm{CH}_{3}\right)\left(\mathrm{CH}_{2}\right)_{3} \mathrm{CH}-\right.$ and $\left.-\mathrm{OCH}_{2}\left(\mathrm{CH}_{2}\right)_{7}-\right),[22.86,22.79,22.75,19.78,14.21]$ $\left(-\mathrm{CH}\left(\mathrm{CH}_{3}\right)_{2},-\mathrm{CH}\left(\mathrm{CH}_{3}\right) \mathrm{CH}_{2}-\right.$ and $\left.-\mathrm{CH}_{2} \mathrm{CH}_{3}\right)$. MALDI-TOF MS $m / z$ of $\left[\mathrm{M}+\mathrm{Na}^{+}\right.$calculated for $\mathrm{C}_{98} \mathrm{H}_{142} \mathrm{~N}_{2} \mathrm{O}_{10}$ : 1530.1; Found: 1530.8 .
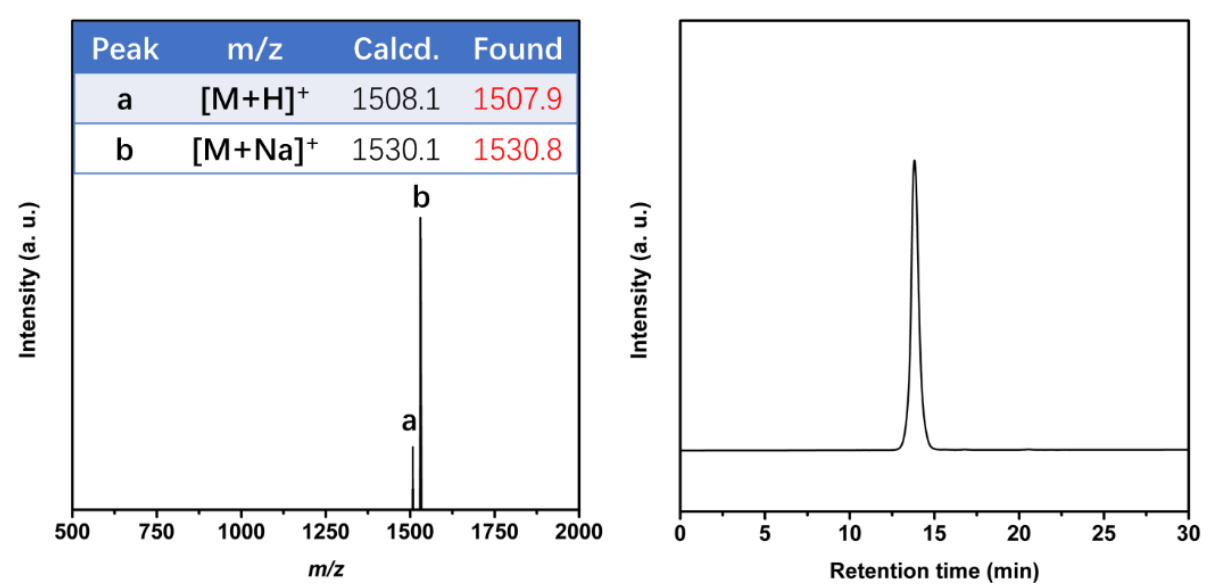

Supporting Figure S40. MALDI-TOF MS spectra (left) and HPLC trace (right) of r10r-PBI. Target molecule r10r-PBI has been successfully synthesized. ${ }^{1} \mathrm{H}$ and ${ }^{13} \mathrm{C}$ NMR spectra are presented on pages 123 and 124 , respectively. 
Supporting Figure S40 (continued). ${ }^{1} \mathrm{H}$ NMR of r10r-PBI.

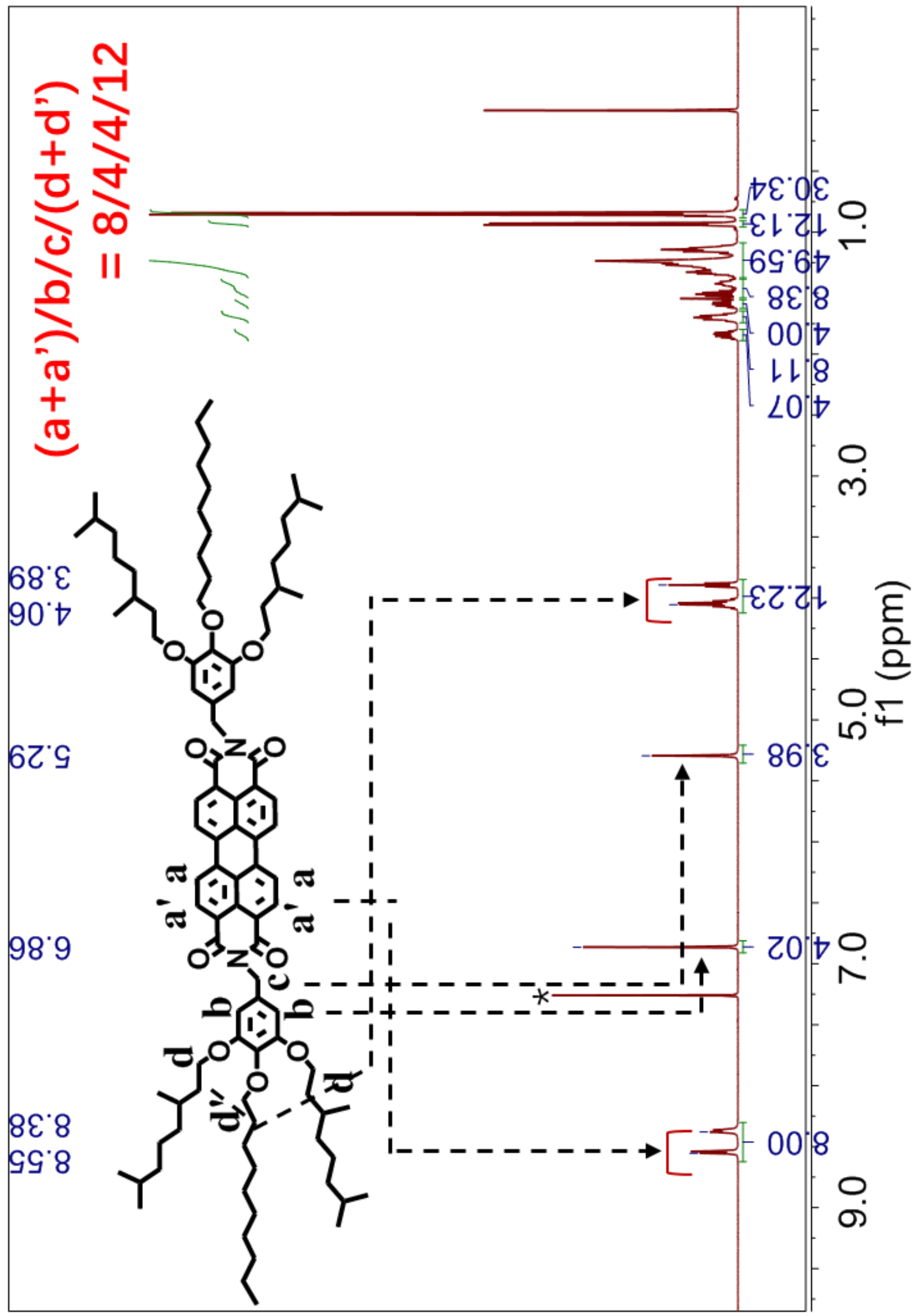


Supporting Figure $\mathbf{S 4 0}$ (continued). ${ }^{13} \mathrm{C}$ NMR of r10r-PBI.

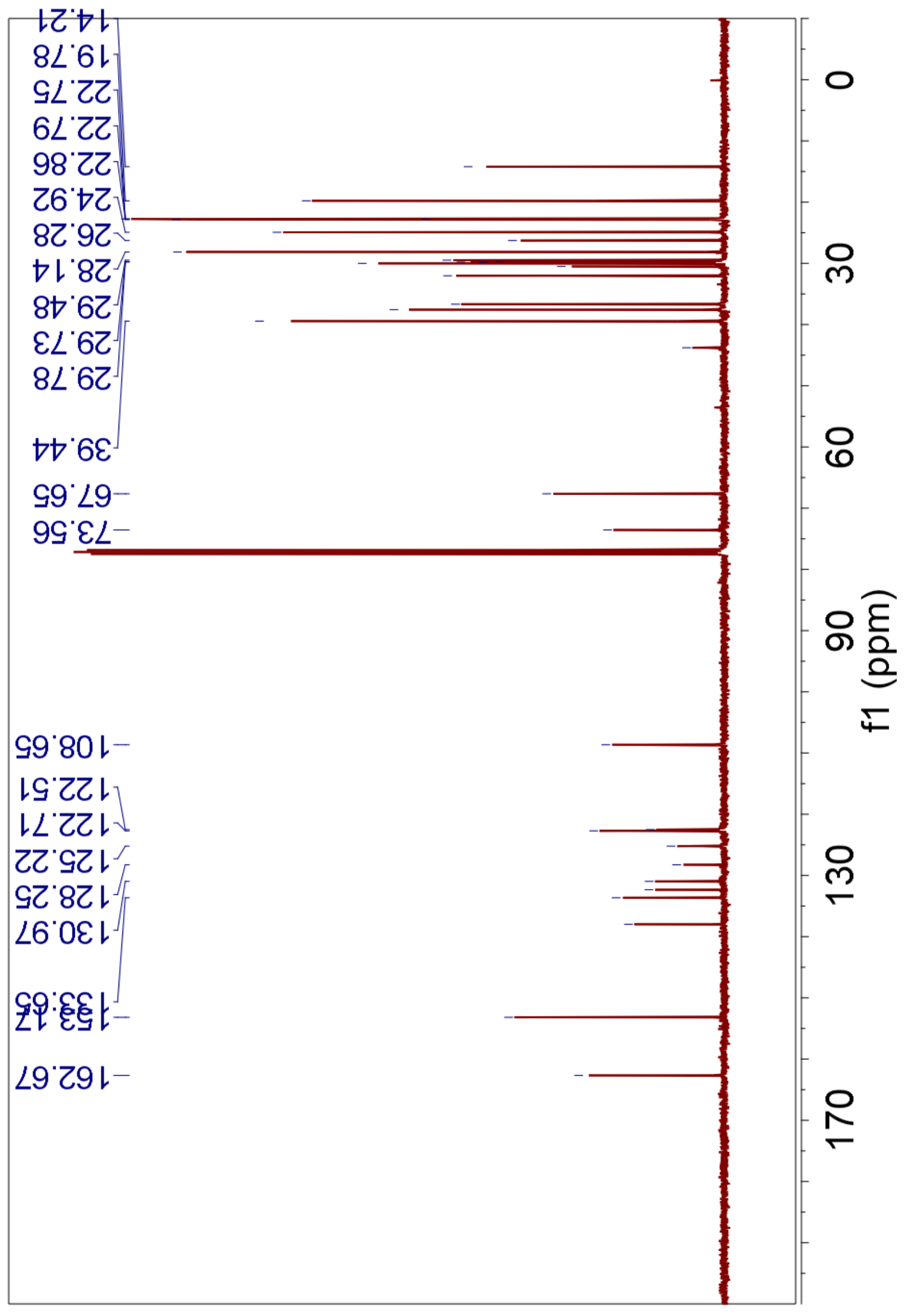




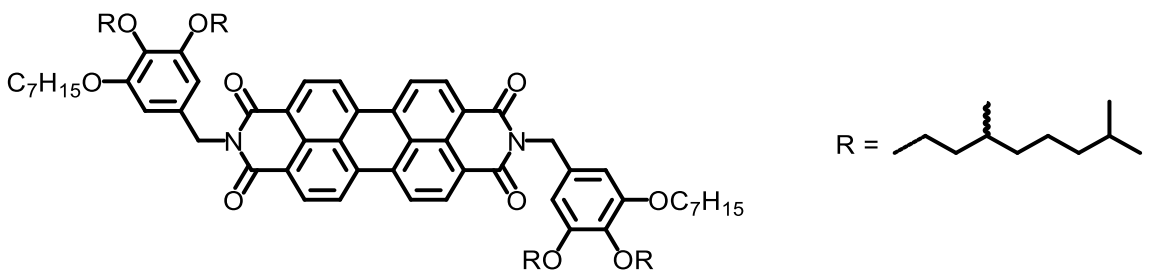

rr7-PBI. From 12-rr7 (0.77 g, $1.45 \mathrm{mmol})$, PTCDA (0.26 g, $0.66 \mathrm{mmol})$ and $\mathrm{Zn}(\mathrm{OAc})_{2} \cdot 2 \mathrm{H}_{2} \mathrm{O}(0.145 \mathrm{~g}$, $0.66 \mathrm{mmol})$ in imidazole $(3 \mathrm{~g})$ at $180{ }^{\circ} \mathrm{C}$ for $6 \mathrm{~h}$, rr7-PBI was obtained as a red solid. Product: $0.44 \mathrm{~g}$. Yield: 47\%. Purity by HPLC: $99+\%$. mp: $205{ }^{\circ} \mathrm{C}$.

${ }^{1} \mathrm{H}$ NMR (400 MHz, $\left.\mathrm{CDCl}_{3}, \delta, \mathrm{ppm}\right): 8.56$ (d, $J=7.9 \mathrm{~Hz}, 4 \mathrm{H}, \mathrm{PBI}, 1,6,7,12$-positions), 8.39 (d, $J=8.0$ $\mathrm{Hz}, 4 \mathrm{H}, \mathrm{PBI}, 2,5,8,11$-positions), 6.86 (d, $J=3.5 \mathrm{~Hz}, 4 \mathrm{H}, \mathrm{ArH}$ of dendrons), 5.29 (s, 4H, $-\mathrm{NCH}_{2}-$ ), 4.12$3.85\left(\mathrm{~m}, 12 \mathrm{H},-\mathrm{OCH}_{2}-\right), 1.91-1.74\left(\mathrm{~m}, 8 \mathrm{H},-\mathrm{OCH}_{2} \mathrm{CH}_{2} \mathrm{CH}_{2}-\right.$ and $\left.-\mathrm{CH}\left(\mathrm{CH}_{3}\right)_{2}\right), 1.74-1.63(\mathrm{~m}, 4 \mathrm{H},-$ $\left.\mathrm{CH}\left(\mathrm{CH}_{3}\right) \mathrm{CH}_{2}-\right)$, 1.63-1.41 (m, $12 \mathrm{H},-\mathrm{O}\left(\mathrm{CH}_{2}\right)_{2} \mathrm{CH}_{2}-$ and $\left.-\mathrm{OCH}_{2} \mathrm{CH}_{2} \mathrm{CH}\left(\mathrm{CH}_{3}\right)-\right), 1.40-1.04(\mathrm{~m}, 36 \mathrm{H},-$ $\mathrm{CH}\left(\mathrm{CH}_{3}\right)\left(\mathrm{CH}_{2}\right)_{3}-$ and $\left.-\left(\mathrm{CH}_{2}\right)_{3}\left(\mathrm{CH}_{2}\right)_{3}-\right), 0.93\left(\mathrm{~d}, \mathrm{~J}=6.5 \mathrm{~Hz}, 6 \mathrm{H},-\mathrm{CH}_{2} \mathrm{CH}\left(\mathrm{CH}_{3}\right)-\right), 0.91-0.79(\mathrm{~m}, 36 \mathrm{H},-$ $\mathrm{CH}_{2} \mathrm{CH}\left(\mathrm{CH}_{3}\right)_{-},-\mathrm{CH}\left(\mathrm{CH}_{3}\right)_{2}$ and $\left.-\mathrm{CH}_{2} \mathrm{CH}_{3}\right) .{ }^{13} \mathrm{C} \mathrm{NMR}\left(101 \mathrm{MHz}, \mathrm{CDCl}_{3}, \delta, \mathrm{ppm}\right): 162.81(C=\mathrm{O}), 153.20$ $(\mathrm{ArC}-3,5), 138.01$ (ArC-4), 133.82 (ArC-1), [132.34, 131.09, 128.44, 125.42, 122.83, 122.62] (perylene C), 108.61 (ArC-2,6), $71.85\left(\mathrm{ArOCH}_{2}-5\right.$ position), $69.34\left(\mathrm{ArOCH}_{2}-4\right.$ position), $67.66\left(\mathrm{ArOCH}_{2}-3\right.$ position), $43.85\left(-\mathrm{CH}_{2} \mathrm{~N}\right),[39.46,39.45,37.64,37.60,37.57,37.52,36.66,32.01,30.02,29.89,29.65$, 29.30, 28.13, 28.08, 26.29, 24.92, 24.83] $\left(-\mathrm{OCH}_{2} \mathrm{CH}_{2} \mathrm{CH}\left(\mathrm{CH}_{3}\right)\left(\mathrm{CH}_{2}\right)_{3} \mathrm{CH}-\right.$ and $\left.-\mathrm{OCH}_{2}\left(\mathrm{CH}_{2}\right)_{4}-\right)$, [22.86, 22.80, 22.79, 22.75, 22.70, 19.80, 19.76, 19.70, 14.26] $\left(-\mathrm{CH}\left(\mathrm{CH}_{3}\right)_{2},-\mathrm{CH}\left(\mathrm{CH}_{3}\right) \mathrm{CH}_{2}-\right.$ and $\left.-\mathrm{CH}_{2} \mathrm{CH}_{3}\right)$. MALDI-TOF MS $m / z$ of $[\mathrm{M}+\mathrm{Na}]^{+}$calculated for $\mathrm{C}_{92} \mathrm{H}_{130} \mathrm{~N}_{2} \mathrm{O}_{10}$ : 1446.0; Found: 1447.5 .
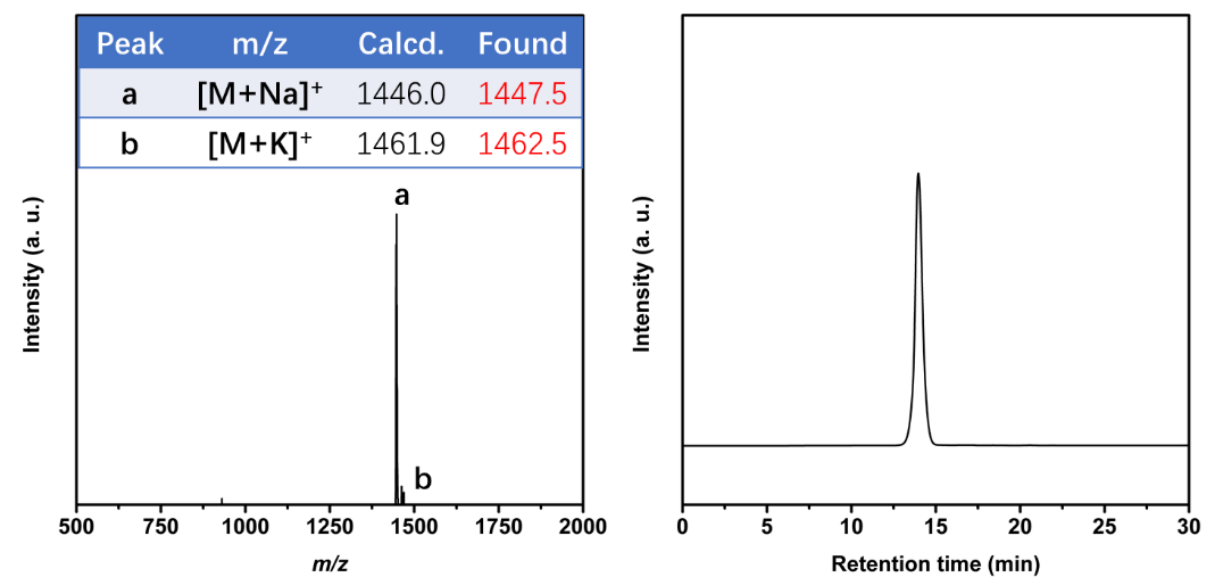

Supporting Figure S41. MALDI-TOF MS spectra (left) and HPLC trace (right) of rr7-PBI. Target molecule rr7-PBI has been successfully synthesized. ${ }^{1} \mathrm{H}$ and ${ }^{13} \mathrm{C}$ NMR spectra are presented on pages 126 and 127 , respectively. 
Supporting Figure S41 (continued). ${ }^{1} \mathrm{H}$ NMR of rr7-PBI.

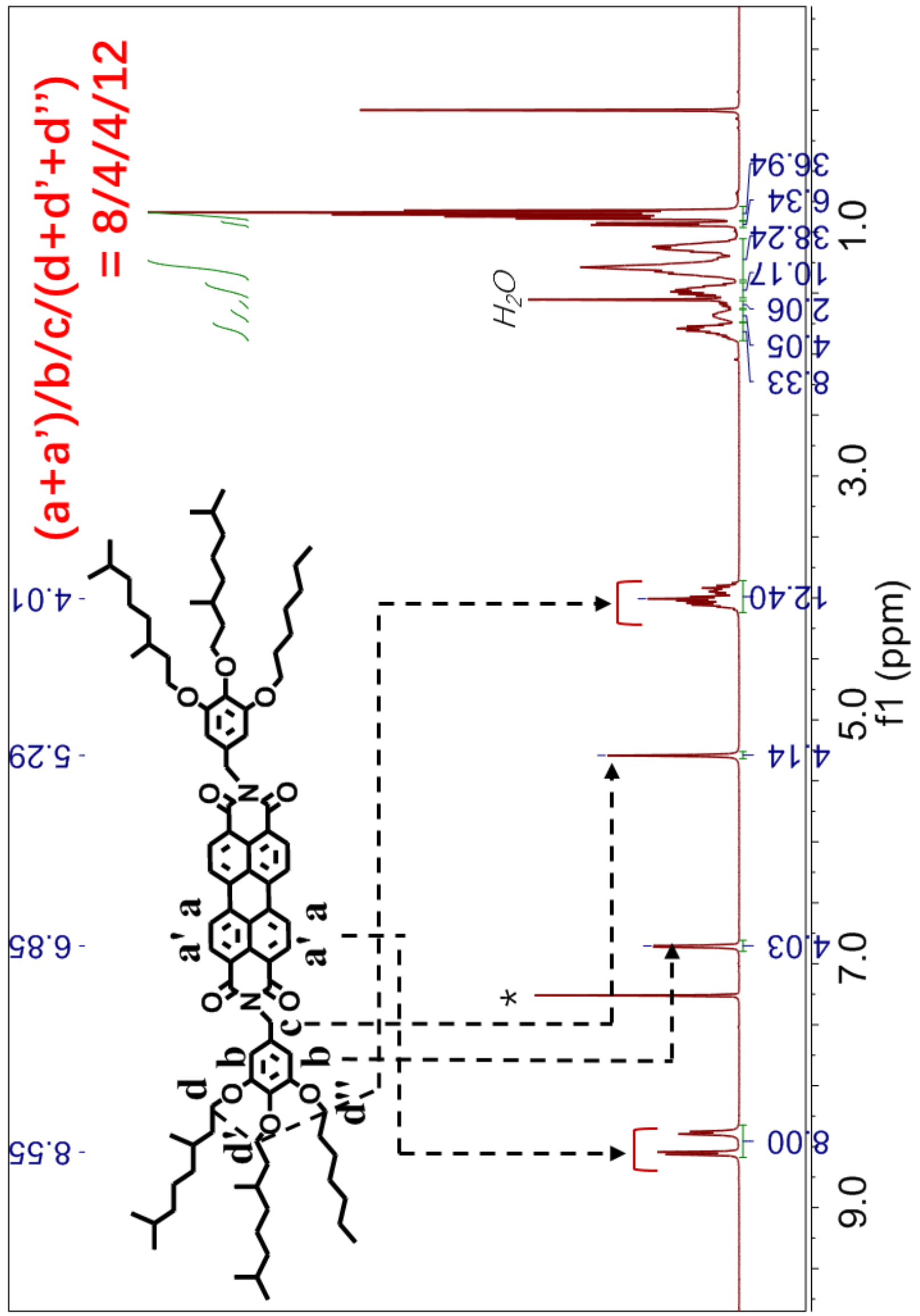


Supporting Figure $\mathbf{S 4 1}$ (continued). ${ }^{13} \mathrm{C}$ NMR of rr7-PBI.

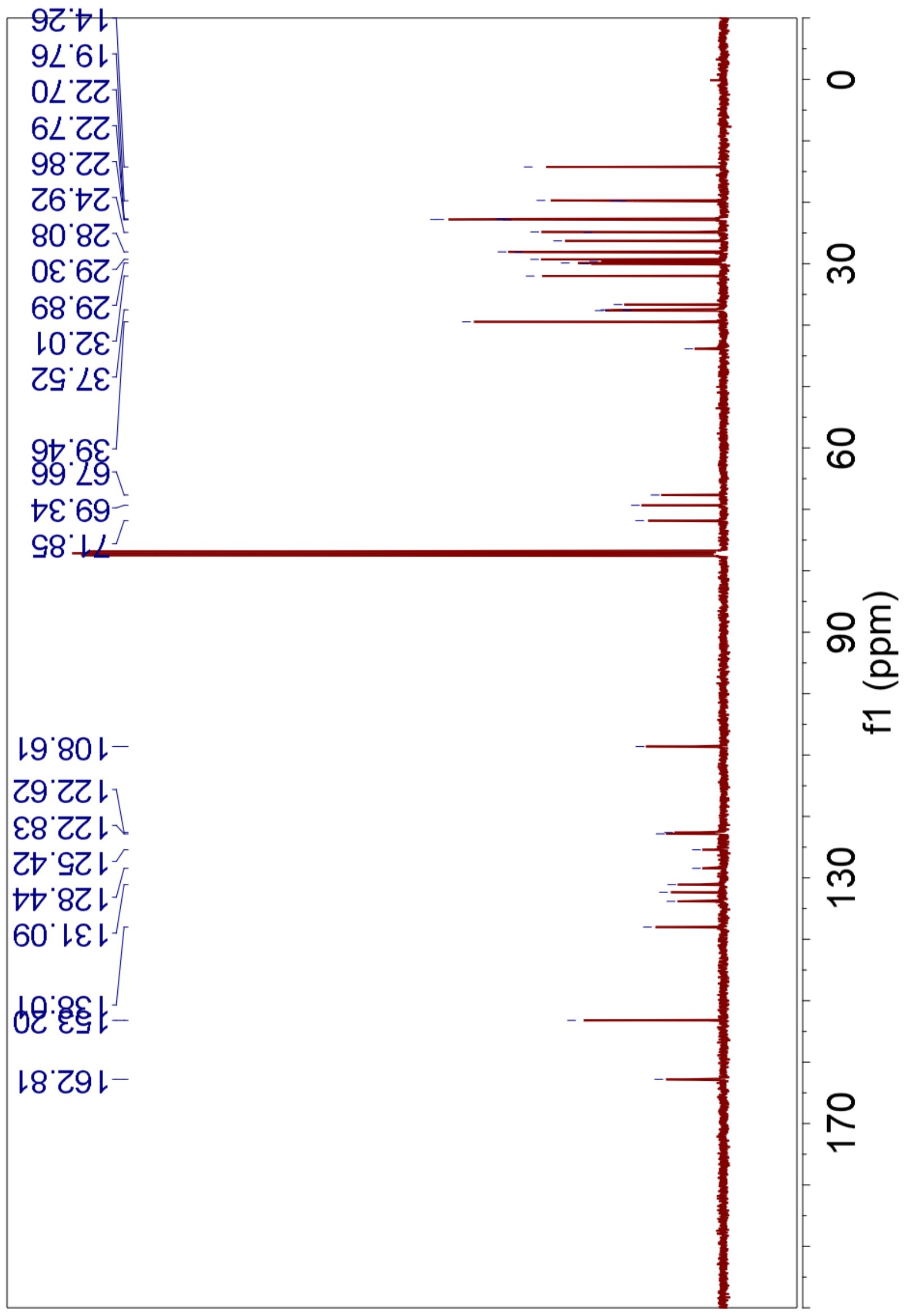




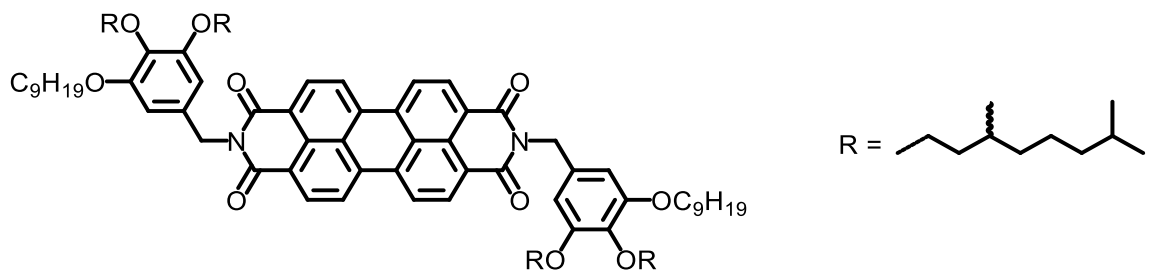

rr9-PBI. From 12-rr9 (0.81 g, $1.45 \mathrm{mmol})$, PTCDA $(0.26 \mathrm{~g}, 0.66 \mathrm{mmol})$ and $\mathrm{Zn}(\mathrm{OAc})_{2} \cdot 2 \mathrm{H}_{2} \mathrm{O}(0.145 \mathrm{~g}$, $0.66 \mathrm{mmol})$ in imidazole $(3 \mathrm{~g})$ at $180{ }^{\circ} \mathrm{C}$ for $6 \mathrm{~h}$, rr9-PBI was obtained as a red solid. Product: $0.63 \mathrm{~g}$. Yield: $65 \%$. Purity by HPLC: $99+\%$. mp: $204^{\circ} \mathrm{C}$.

${ }^{1} \mathrm{H}$ NMR (400 MHz, $\mathrm{CDCl}_{3}, \delta$, ppm): 8.60 (d, $J=7.9 \mathrm{~Hz}, 4 \mathrm{H}, \mathrm{PBI}, 1,6,7,12$-positions), 8.45 (d, $J=8.0$ $\mathrm{Hz}, 4 \mathrm{H}, \mathrm{PBI}, 2,5,8,11$-positions), 6.85 (d, $J=3.8 \mathrm{~Hz}, 4 \mathrm{H}, \mathrm{Ar} H$ of dendrons), 5.30 (s, 4H, $-\mathrm{NCH}_{2}-$ ), $4.11-$ $3.85\left(\mathrm{~m}, 12 \mathrm{H},-\mathrm{OCH}_{2}-\right), 1.90-1.73\left(\mathrm{~m}, 8 \mathrm{H},-\mathrm{OCH}_{2} \mathrm{CH}_{2} \mathrm{CH}_{2}-\right.$ and $\left.-\mathrm{CH}\left(\mathrm{CH}_{3}\right)_{2}\right), 1.73-1.63(\mathrm{~m}, 4 \mathrm{H},-$ $\left.\mathrm{CH}\left(\mathrm{CH}_{3}\right) \mathrm{CH}_{2}-\right), 1.63-1.41\left(\mathrm{~m}, 12 \mathrm{H},-\mathrm{O}\left(\mathrm{CH}_{2}\right)_{2} \mathrm{CH}_{2}-\right.$ and $\left.-\mathrm{OCH}_{2} \mathrm{CH}_{2} \mathrm{CH}\left(\mathrm{CH}_{3}\right)-\right), 1.39-1.05(\mathrm{~m}, 44 \mathrm{H},-$ $\mathrm{CH}\left(\mathrm{CH}_{3}\right)\left(\mathrm{CH}_{2}\right)_{3}-$ and $\left.-\left(\mathrm{CH}_{2}\right)_{3}\left(\mathrm{CH}_{2}\right)_{5}-\right), 0.93\left(\mathrm{~d}, J=6.5 \mathrm{~Hz}, 6 \mathrm{H},-\mathrm{CH}_{2} \mathrm{CH}\left(\mathrm{CH}_{3}\right)-\right), 0.91-0.79(\mathrm{~m}, 36 \mathrm{H},-$ $\mathrm{CH}_{2} \mathrm{CH}\left(\mathrm{CH}_{3}\right)-,-\mathrm{CH}\left(\mathrm{CH}_{3}\right)_{2}$ and $\left.-\mathrm{CH}_{2} \mathrm{CH}_{3}\right) .{ }^{13} \mathrm{C} \mathrm{NMR}\left(101 \mathrm{MHz}, \mathrm{CDCl}_{3}, \delta, \mathrm{ppm}\right): 162.75(\mathrm{C}=\mathrm{O}), 153.20$ (ArC-3,5), 138.00 (ArC-4), 133.74 (ArC-1), [132.34, 131.05, 128.35, 125.32, 122.78, 122.58] (perylene C), 108.60 ( $\mathrm{ArC}-2,6), 71.84\left(\mathrm{ArOCH}_{2}-5\right.$ position), $69.34\left(\mathrm{ArOCH}_{2}-4\right.$ position), $67.66\left(\mathrm{ArOCH}_{2}-3\right.$ position), $43.84\left(-\mathrm{CH}_{2} \mathrm{~N}\right),[39.47,37.64,37.61,37.58,37.52,36.66,32.06,30.02,29.89,29.79,29.66$, 29.47, 28.13, 28.08, 26.35, 24.92, 24.83] $\left(-\mathrm{OCH}_{2} \mathrm{CH}_{2} \mathrm{CH}\left(\mathrm{CH}_{3}\right)\left(\mathrm{CH}_{2}\right)_{3} \mathrm{CH}-\right.$ and $\left.-\mathrm{OCH}_{2}\left(\mathrm{CH}_{2}\right)_{6}-\right)$, [22.86, 22.82, 22.80, 22.75, 22.71, 19.79, 19.76, 19.70, 14.25] $\left(-\mathrm{CH}\left(\mathrm{CH}_{3}\right)_{2},-\mathrm{CH}\left(\mathrm{CH}_{3}\right) \mathrm{CH}_{2}-\right.$ and $\left.-\mathrm{CH}_{2} \mathrm{CH}_{3}\right)$. MALDI-TOF MS $m / z$ of $[\mathrm{M}+\mathrm{Na}]^{+}$calculated for $\mathrm{C}_{96} \mathrm{H}_{138} \mathrm{~N}_{2} \mathrm{O}_{10}$ : 1502.0; Found: 1502.7.
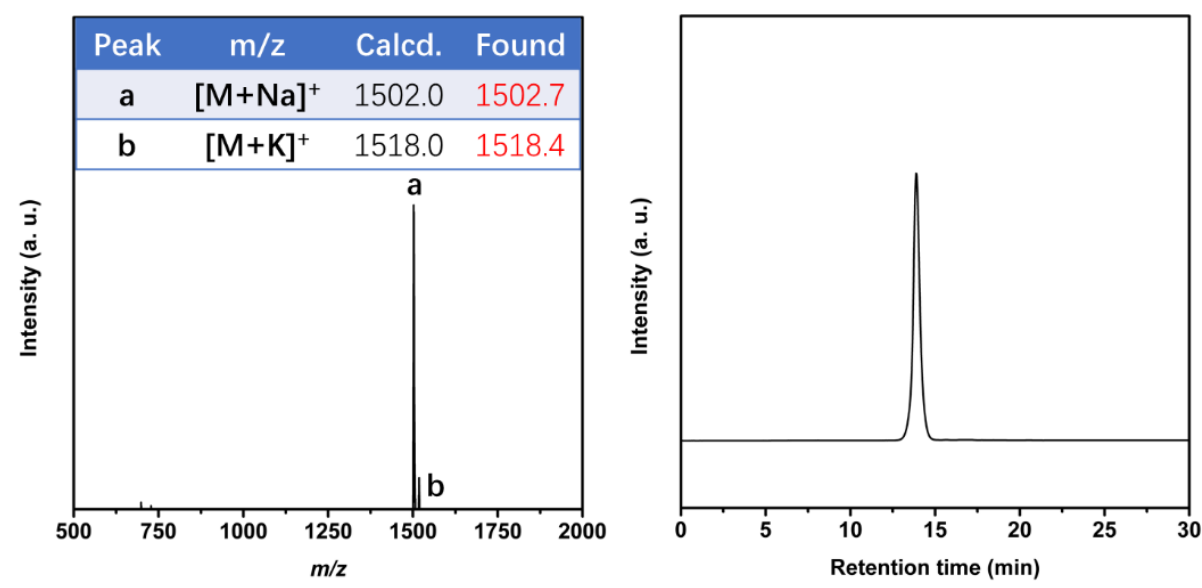

Supporting Figure S42. MALDI-TOF MS spectra (left) and HPLC trace (right) of rr9-PBI. Target molecule rr9-PBI has been successfully synthesized. ${ }^{1} \mathrm{H}$ and ${ }^{13} \mathrm{C}$ NMR spectra are presented on pages 129 and 130 , respectively. 
Supporting Figure $\mathbf{S 4 2}$ (continued). ${ }^{1} \mathrm{H}$ NMR of $\mathbf{r r 9}$-PBI.

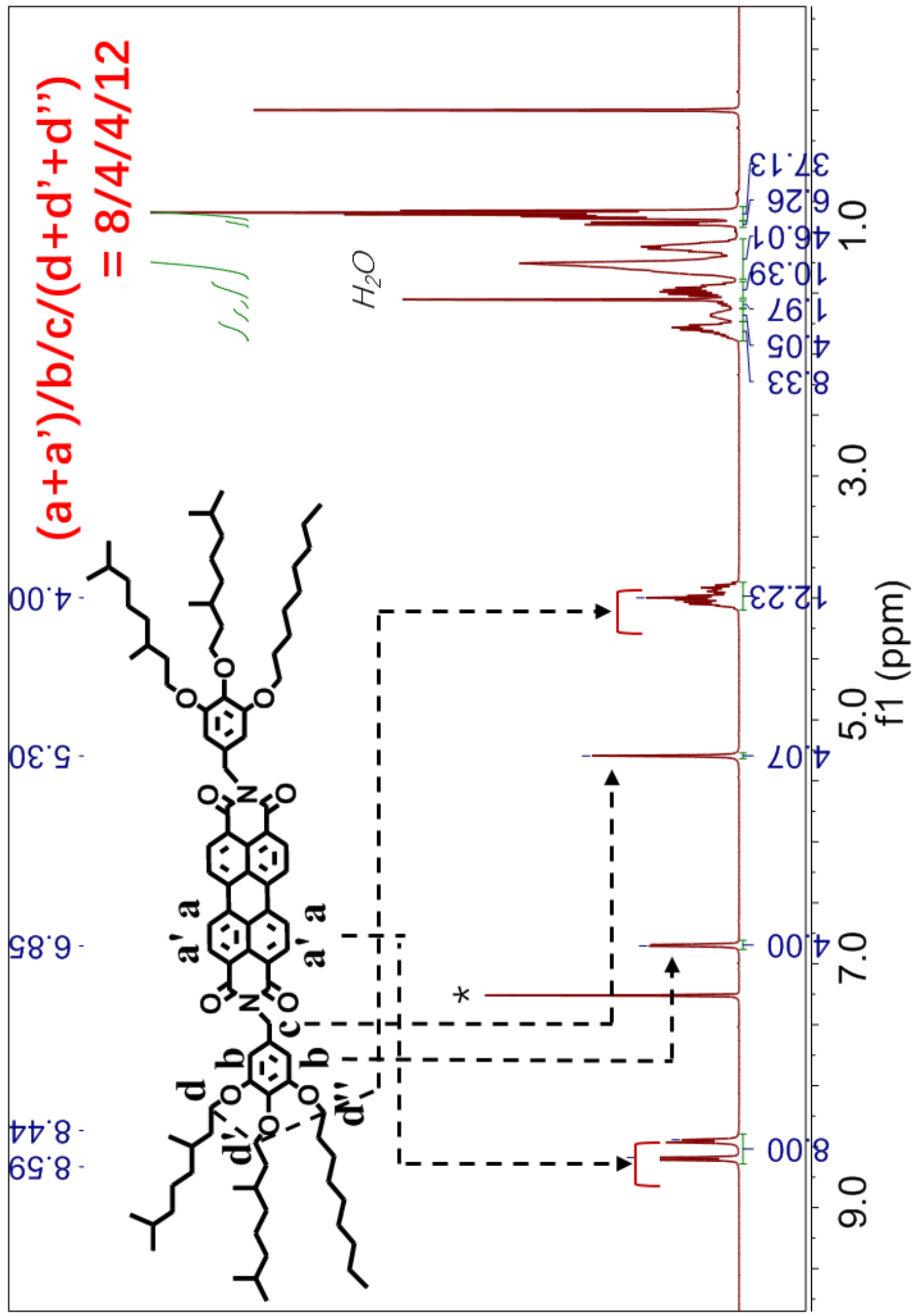


Supporting Figure $\mathbf{S 4 2}$ (continued). ${ }^{13} \mathrm{C}$ NMR of rr9-PBI.

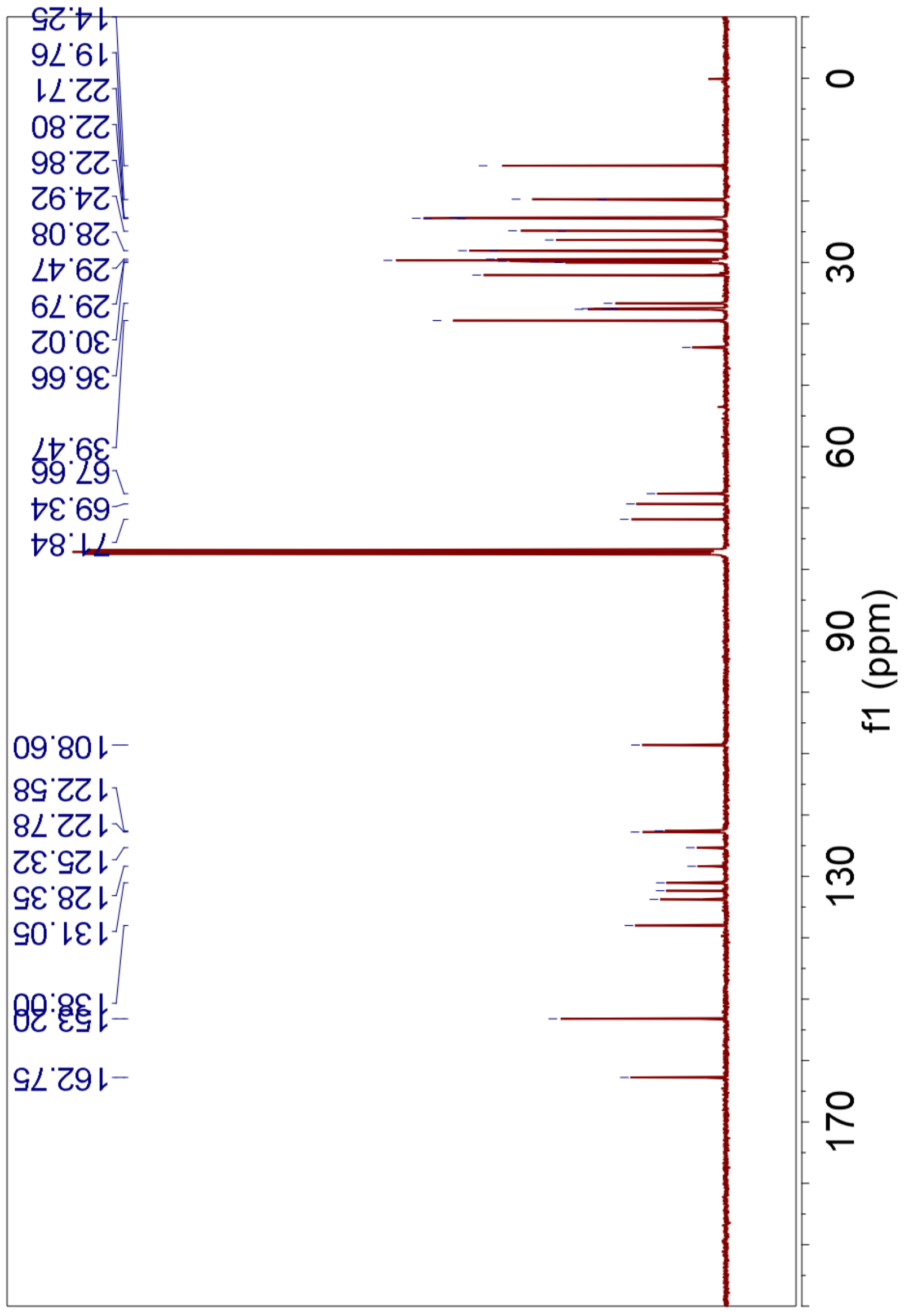




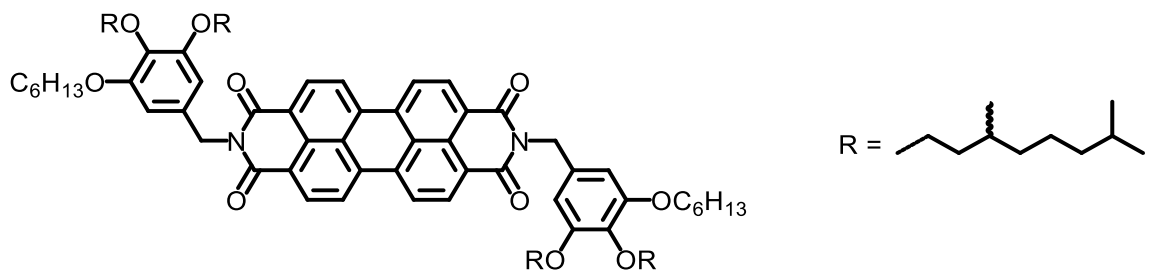

rr6-PBI. From 12-rr6 (1.07 g, $2.2 \mathrm{mmol})$, PTCDA $(0.39 \mathrm{~g}, 1.0 \mathrm{mmol})$ and $\mathrm{Zn}(\mathrm{OAc})_{2} \bullet 2 \mathrm{H}_{2} \mathrm{O}(0.22 \mathrm{~g}, 1.0$ $\mathrm{mmol})$ in imidazole $(3 \mathrm{~g})$ at $160^{\circ} \mathrm{C}$ for $16 \mathrm{~h}$, rr6-PBI was obtained as a red solid. Product: $0.77 \mathrm{~g}$. Yield: 59\%. Purity by HPLC: $99+\%$. mp: $207^{\circ} \mathrm{C}$.

${ }^{1} \mathrm{H}$ NMR (500 MHz, $\mathrm{CDCl}_{3}, \delta$, ppm): 8.53 (d, $J=8.0 \mathrm{~Hz}, 4 \mathrm{H}, \mathrm{PBI}, 1,6,7,12$-positions), 8.35 (d, $J=7.9$ $\mathrm{Hz}, 4 \mathrm{H}, \mathrm{PBI}, 2,5,8,11$-positions), 6.86 (d, $J=4.4 \mathrm{~Hz}, 4 \mathrm{H}, \mathrm{Ar} H$ of dendrons), 5.29 (s, 4H, $-\mathrm{NCH}_{2}-$ ), $4.11-$ $3.88\left(\mathrm{~m}, 12 \mathrm{H},-\mathrm{OCH}_{2}-\right), 1.91-1.75\left(\mathrm{~m}, 8 \mathrm{H},-\mathrm{OCH}_{2} \mathrm{CH}_{2} \mathrm{CH}_{2}-\right.$ and $\left.-\mathrm{CH}\left(\mathrm{CH}_{3}\right)_{2}\right), 1.75-1.62(\mathrm{~m}, 4 \mathrm{H},-$ $\left.\mathrm{CH}\left(\mathrm{CH}_{3}\right) \mathrm{CH}_{2}-\right), 1.62-1.42\left(\mathrm{~m}, 12 \mathrm{H},-\mathrm{O}\left(\mathrm{CH}_{2}\right)_{2} \mathrm{CH}_{2}-\right.$ and $\left.-\mathrm{OCH}_{2} \mathrm{CH}_{2} \mathrm{CH}\left(\mathrm{CH}_{3}\right)-\right), 1.39-1.05(\mathrm{~m}, 32 \mathrm{H},-$ $\mathrm{CH}\left(\mathrm{CH}_{3}\right)\left(\mathrm{CH}_{2}\right)_{3}-$ and $\left.-\left(\mathrm{CH}_{2}\right)_{3}\left(\mathrm{CH}_{2}\right)_{2}-\right), 0.94\left(\mathrm{~d}, J=6.6 \mathrm{~Hz}, 6 \mathrm{H},-\mathrm{CH}_{2} \mathrm{CH}\left(\mathrm{CH}_{3}\right)-\right), 0.91-0.80(\mathrm{~m}, 36 \mathrm{H},-$ $\mathrm{CH}_{2} \mathrm{CH}\left(\mathrm{CH}_{3}\right)-,-\mathrm{CH}\left(\mathrm{CH}_{3}\right)_{2}$ and $\left.-\mathrm{CH}_{2} \mathrm{CH}_{3}\right) .{ }^{13} \mathrm{C} \mathrm{NMR}\left(101 \mathrm{MHz}, \mathrm{CDCl}_{3}, \delta, \mathrm{ppm}\right): 162.73(\mathrm{C}=\mathrm{O}), 153.19$ (ArC-3,5), 137.99 (ArC-4), $133.72(\mathrm{ArC}-1)$, [132.35, 131.03, 128.33, 125.30, 122.76, 122.57] (perylene C), 108.62 ( $\mathrm{ArC}-2,6), 71.84$ ( $\mathrm{ArOCH}_{2}-5$ position), $69.33\left(\mathrm{ArOCH}_{2}-4\right.$ position), $67.65\left(\mathrm{ArOCH}_{2}-3\right.$ position), $43.82\left(-\mathrm{CH}_{2} \mathrm{~N}\right),[39.45,37.63,37.60,37.57,37.52,36.66,31.81,30.02,29.89,29.60,28.14$, 28.08, 26.00, 24.92, 24.83] $\left(-\mathrm{OCH}_{2} \mathrm{CH}_{2} \mathrm{CH}\left(\mathrm{CH}_{3}\right)\left(\mathrm{CH}_{2}\right)_{3} \mathrm{CH}-\right.$ and $\left.-\mathrm{OCH}_{2}\left(\mathrm{CH}_{2}\right)_{3}-\right)$, [22.86, 22.81, 22.80, $22.75,22.70,19.80,19.76,19.70,14.23]\left(-\mathrm{CH}\left(\mathrm{CH}_{3}\right)_{2},-\mathrm{CH}\left(\mathrm{CH}_{3}\right) \mathrm{CH}_{2}-\right.$ and $\left.-\mathrm{CH}_{2} \mathrm{CH}_{3}\right)$. MALDI-TOF MS $m / z$ of $[\mathrm{M}+\mathrm{Na}]^{+}$calculated for $\mathrm{C}_{90} \mathrm{H}_{126} \mathrm{~N}_{2} \mathrm{O}_{10}$ : 1417.9; Found: 1418.7.
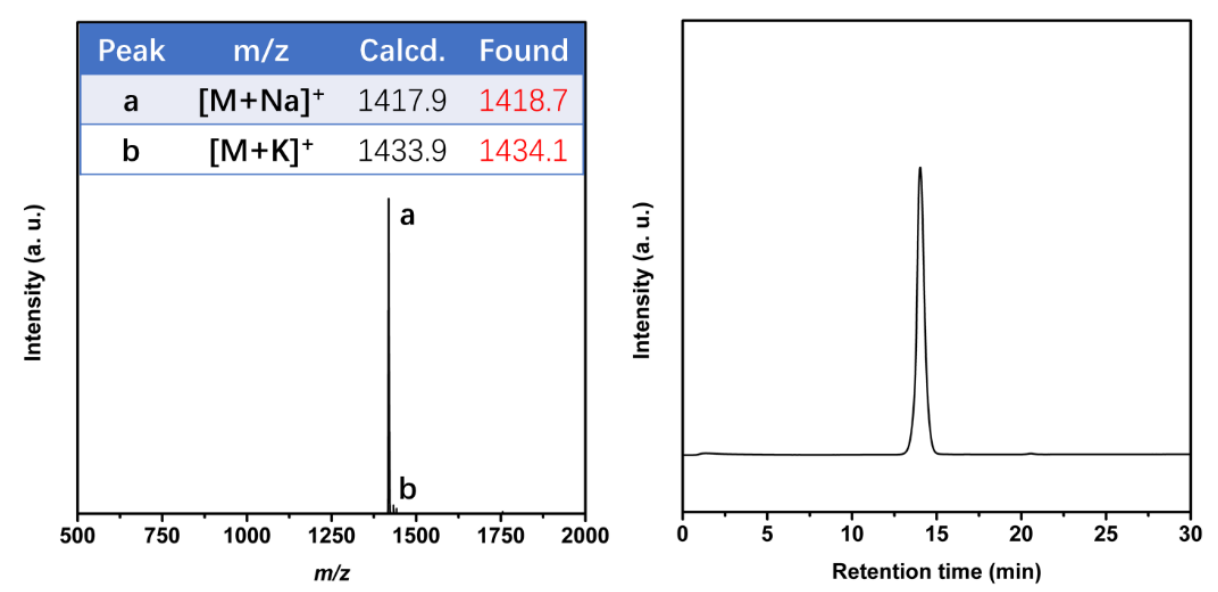

Supporting Figure S43. MALDI-TOF MS spectra (left) and HPLC trace (right) of rr6-PBI. Target molecule rr6-PBI has been successfully synthesized. ${ }^{1} \mathrm{H}$ and ${ }^{13} \mathrm{C}$ NMR spectra are presented on pages 132 and 133 , respectively. 
Supporting Figure $\mathbf{S 4 3}$ (continued). ${ }^{1} \mathrm{H}$ NMR of rr6-PBI.

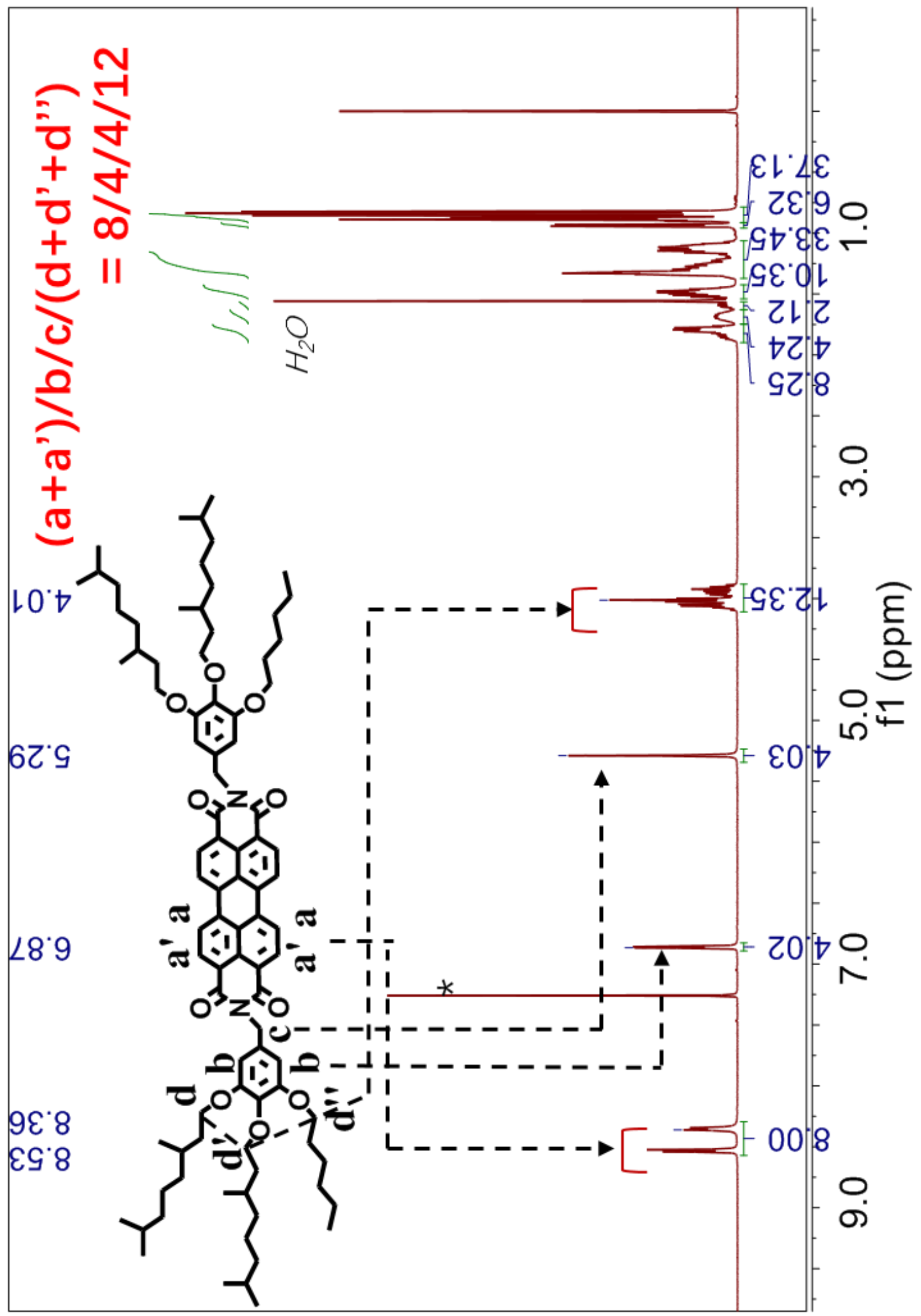


Supporting Figure $\mathbf{S 4 3}$ (continued). ${ }^{13} \mathrm{C}$ NMR of rr6-PBI.

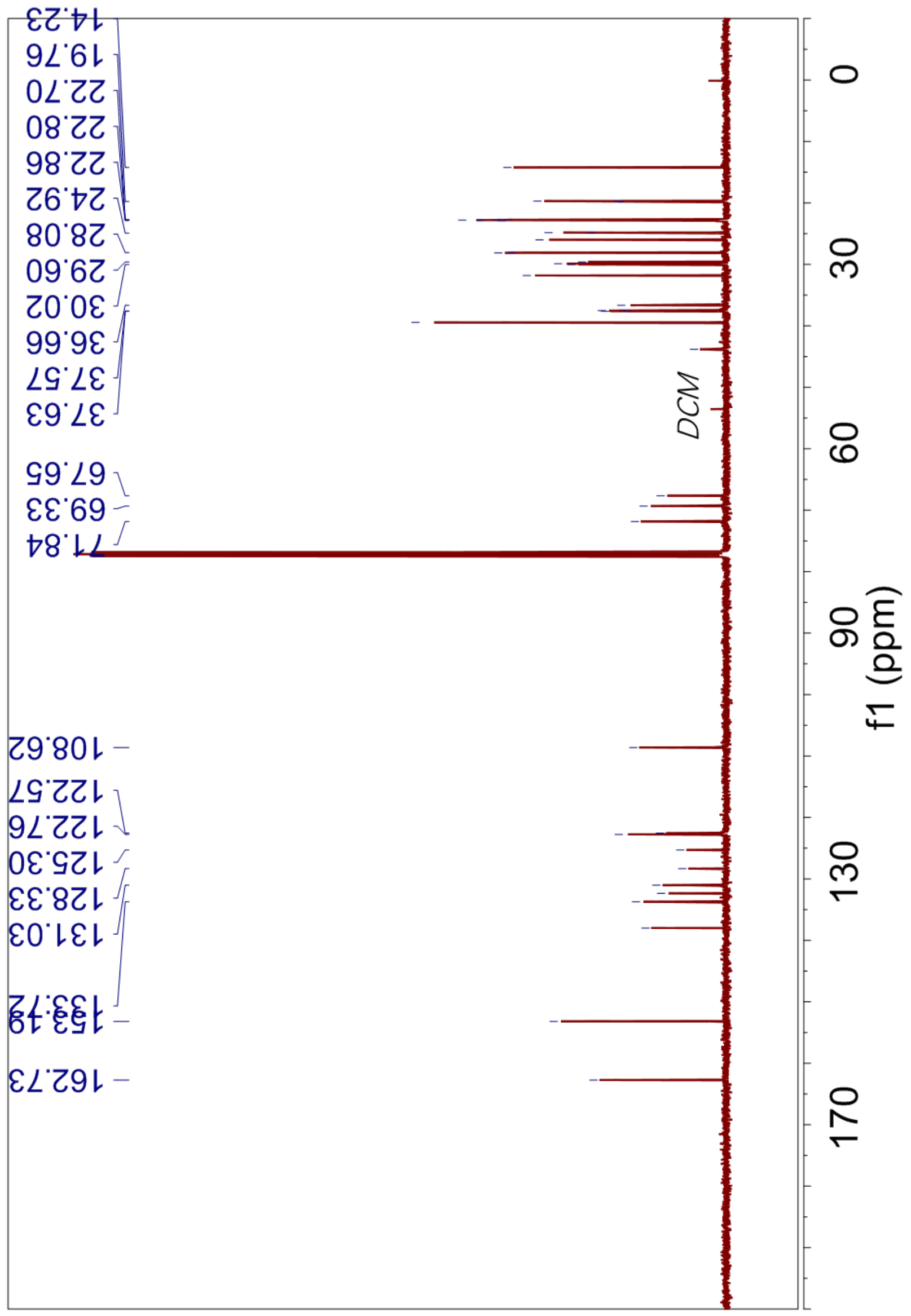




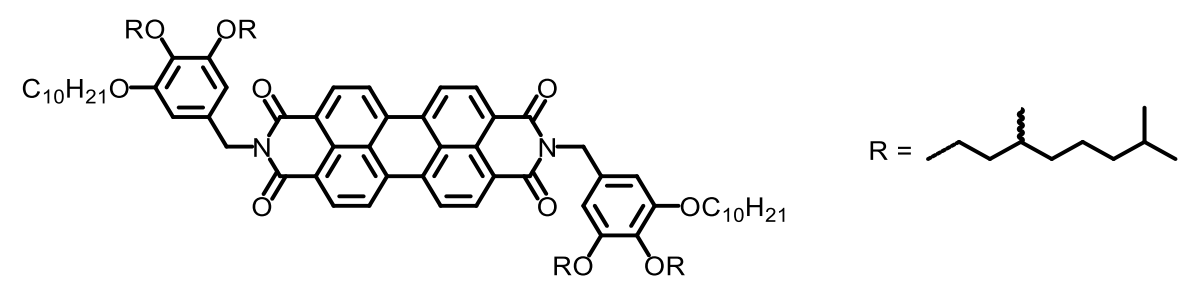

rr10-PBI. From 12-rr10 (0.39 g, $0.68 \mathrm{mmol})$, PTCDA ( $0.12 \mathrm{~g}, 0.31 \mathrm{mmol})$ and $\mathrm{Zn}(\mathrm{OAc})_{2} \cdot 2 \mathrm{H}_{2} \mathrm{O}(0.068$ $\mathrm{g}, 0.31 \mathrm{mmol})$ in imidazole $(3 \mathrm{~g})$ at $160{ }^{\circ} \mathrm{C}$ for $12 \mathrm{~h}$, rr10-PBI was obtained as a red solid. Product: 0.22 g. Yield: $48 \%$. Purity by HPLC: $99+\%$. mp: $204^{\circ} \mathrm{C}$.

${ }^{1} \mathrm{H}$ NMR (500 MHz, $\mathrm{CDCl}_{3}, \delta$, ppm): 8.44 (d, $J=7.8 \mathrm{~Hz}, 4 \mathrm{H}, \mathrm{PBI}, 1,6,7,12$-positions), 8.23 (d, $J=7.6$ $\mathrm{Hz}, 4 \mathrm{H}, \mathrm{PBI}, 2,5,8,11$-positions), 6.87 (d, $J=4.7 \mathrm{~Hz}, 4 \mathrm{H}, \mathrm{Ar} H$ of dendrons), 5.28 (s, 4H, $-\mathrm{NCH}_{2}-$ ), $4.12-$ $3.87\left(\mathrm{~m}, 12 \mathrm{H},-\mathrm{OCH}_{2}-\right), 1.91-1.75\left(\mathrm{~m}, 8 \mathrm{H},-\mathrm{OCH}_{2} \mathrm{CH}_{2} \mathrm{CH}_{2}-\right.$ and $\left.-\mathrm{CH}\left(\mathrm{CH}_{3}\right)_{2}\right), 1.75-1.64(\mathrm{~m}, 4 \mathrm{H},-$ $\left.\mathrm{CH}\left(\mathrm{CH}_{3}\right) \mathrm{CH}_{2}-\right), 1.64-1.42\left(\mathrm{~m}, 12 \mathrm{H},-\mathrm{O}\left(\mathrm{CH}_{2}\right)_{2} \mathrm{CH}_{2}-\right.$ and $\left.-\mathrm{OCH}_{2} \mathrm{CH}_{2} \mathrm{CH}\left(\mathrm{CH}_{3}\right)-\right), 1.40-1.05(\mathrm{~m}, 48 \mathrm{H},-$ $\mathrm{CH}\left(\mathrm{CH}_{3}\right)\left(\mathrm{CH}_{2}\right)_{3}-$ and $\left.-\left(\mathrm{CH}_{2}\right)_{3}\left(\mathrm{CH}_{2}\right)_{6}-\right), 0.95\left(\mathrm{~d}, J=6.4 \mathrm{~Hz}, 6 \mathrm{H},-\mathrm{CH}_{2} \mathrm{CH}\left(\mathrm{CH}_{3}\right)-\right), 0.92-0.79(\mathrm{~m}, 36 \mathrm{H},-$ $\mathrm{CH}_{2} \mathrm{CH}\left(\mathrm{CH}_{3}\right)-,-\mathrm{CH}\left(\mathrm{CH}_{3}\right)_{2}$ and $\left.-\mathrm{CH}_{2} \mathrm{CH}_{3}\right) .{ }^{13} \mathrm{C} \mathrm{NMR}\left(101 \mathrm{MHz}, \mathrm{CDCl}_{3}, \delta, \mathrm{ppm}\right): 162.45(\mathrm{C}=\mathrm{O}), 153.18$ (ArC-3,5), 137.92 (ArC-4), 133.35 (ArC-1), [132.38, 130.79, 127.90, 124.85, 122.47, 122.34] (perylene C), 108.64 ( $\mathrm{ArC}-2,6), 71.83$ ( $\mathrm{ArOCH}_{2}-3$ position), $69.30\left(\mathrm{ArOCH}_{2}-4\right.$ position), $67.64\left(\mathrm{ArOCH}_{2}-5\right.$ position), $43.69\left(-\mathrm{CH}_{2} \mathrm{~N}\right)$, [39.44, 37.63, 37.62, 37.58, 37.53, 36.66, 32.05, 30.03, 29.90, 29.85, 29.77, 29.66, 29.51, 28.13, 28.06, 26.36, 24.92, 24.83] $\left(-\mathrm{OCH}_{2} \mathrm{CH}_{2} \mathrm{CH}\left(\mathrm{CH}_{3}\right)\left(\mathrm{CH}_{2}\right)_{3} \mathrm{CH}-\right.$ and $\left.-\mathrm{OCH}_{2}\left(\mathrm{CH}_{2}\right)_{7}-\right)$, $[22.86,22.81,22.78,22.74,22.68,19.79,19.75,19.69,14.23]\left(-\mathrm{CH}\left(\mathrm{CH}_{3}\right)_{2},-\mathrm{CH}\left(\mathrm{CH}_{3}\right) \mathrm{CH}_{2}-\right.$ and $\mathrm{CH}_{2} \mathrm{CH}_{3}$ ). MALDI-TOF MS $m / z$ of $[\mathrm{M}+\mathrm{Na}]^{+}$calculated for $\mathrm{C}_{98} \mathrm{H}_{142} \mathrm{~N}_{2} \mathrm{O}_{10}: 1530.1$; Found: 1530.3 .
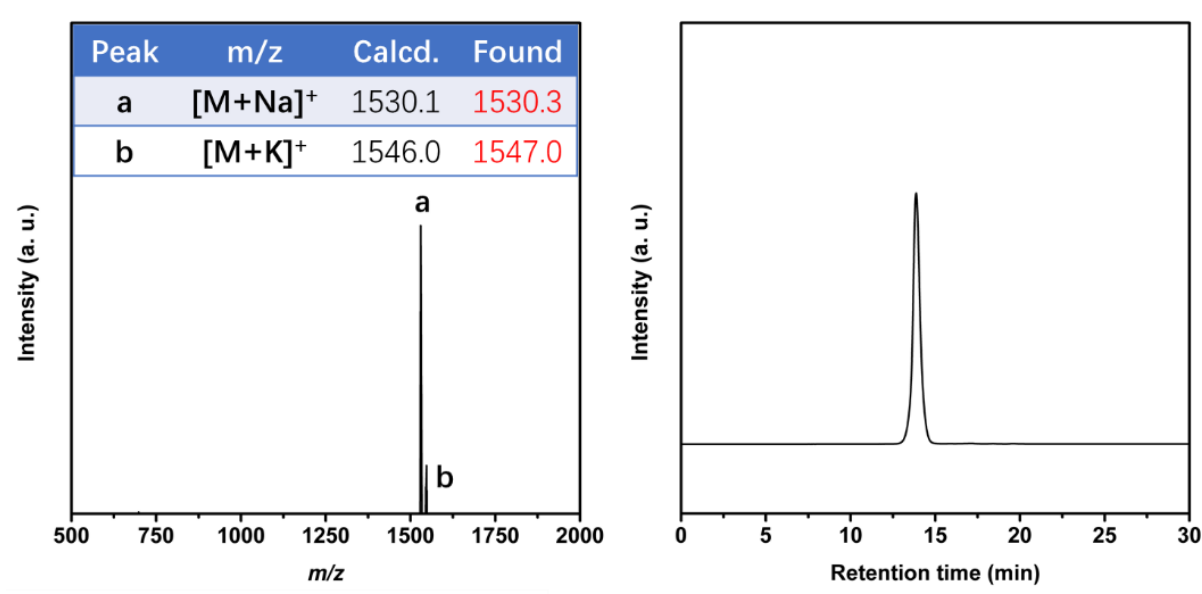

Supporting Figure S44. MALDI-TOF MS spectra (left) and HPLC trace (right) of rr10-PBI. Target molecule rr10-PBI has been successfully synthesized. ${ }^{1} \mathrm{H}$ and ${ }^{13} \mathrm{C}$ NMR spectra are presented on pages 135 and 136 , respectively. 
Supporting Figure S44 (continued). ${ }^{1} \mathrm{H}$ NMR of $\operatorname{rr10-PBI.~}$

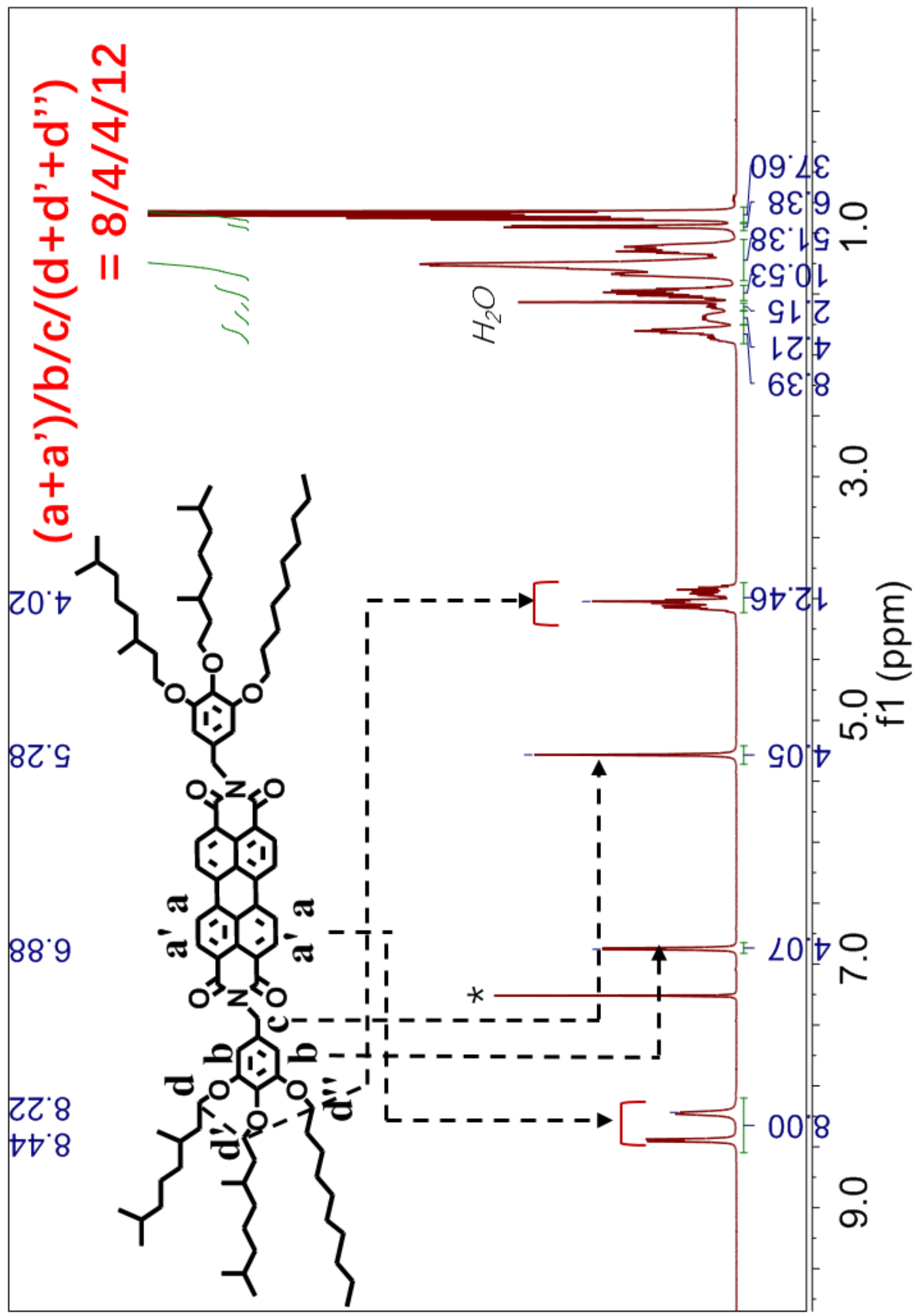


Supporting Figure S44 (continued). ${ }^{13} \mathrm{C}$ NMR of rr10-PBI.

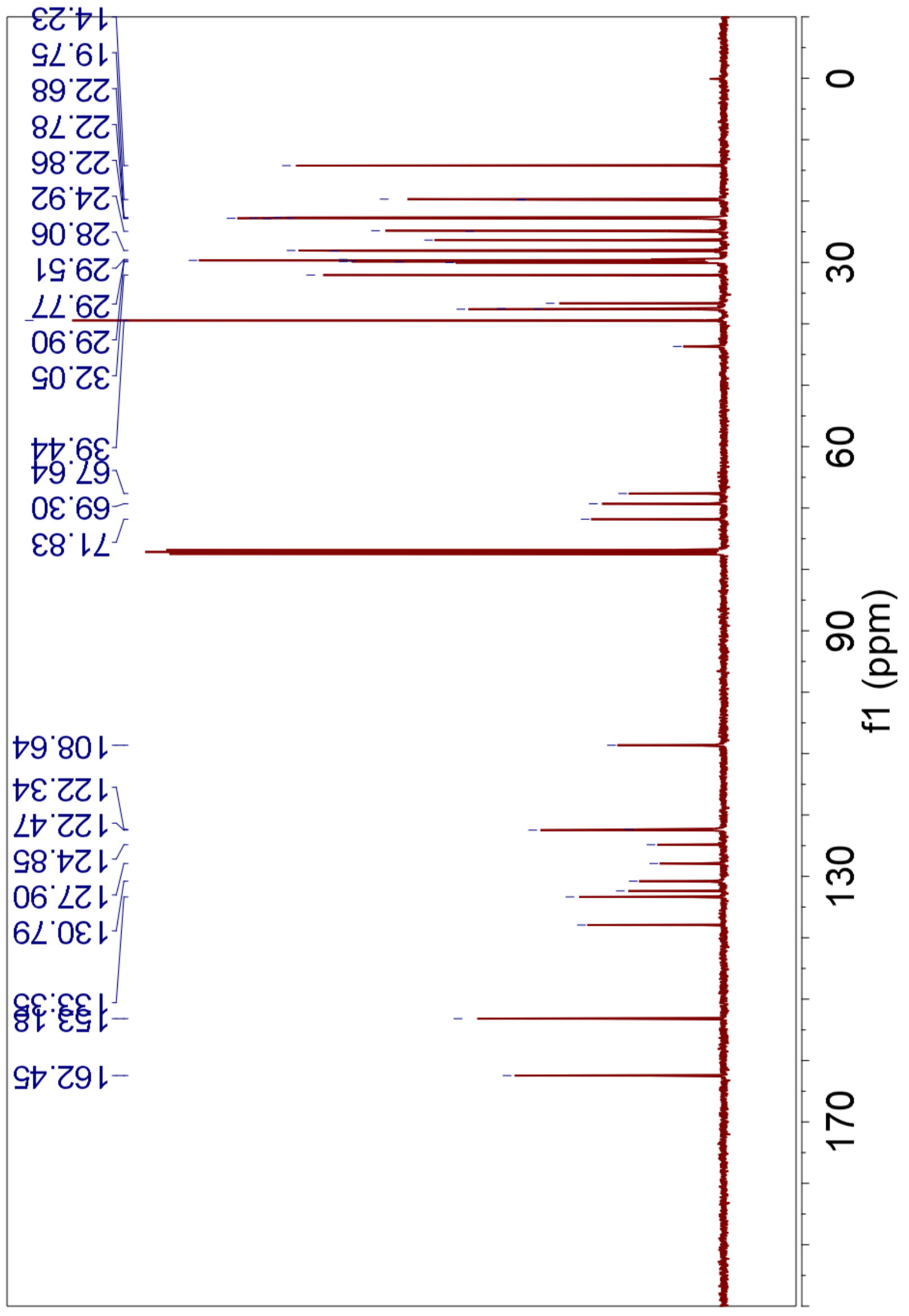




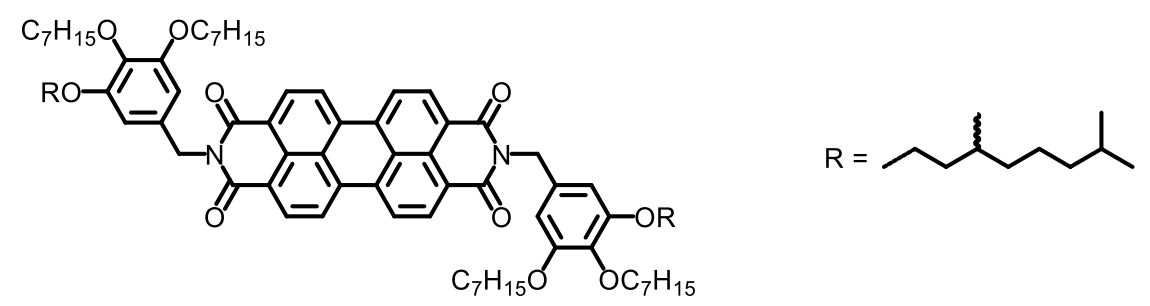

77r-PBI. From 12-77r $(0.71 \mathrm{~g}, 1.45 \mathrm{mmol})$, PTCDA $(0.26 \mathrm{~g}, 0.66 \mathrm{mmol})$ and $\mathrm{Zn}(\mathrm{OAc})_{2} \cdot 2 \mathrm{H}_{2} \mathrm{O}(0.145 \mathrm{~g}$, $0.66 \mathrm{mmol})$ in imidazole $(3 \mathrm{~g})$ at $180{ }^{\circ} \mathrm{C}$ for $6 \mathrm{~h}, 77 \mathrm{r}$-PBI was obtained as a red solid. Product: $0.74 \mathrm{~g}$. Yield: $84 \%$. Purity by HPLC: $99+\%$. mp: $229^{\circ} \mathrm{C}$.

${ }^{1} \mathrm{H}$ NMR (400 MHz, $\mathrm{CDCl}_{3}, \delta, \mathrm{ppm}$ ): 8.59 (d, $J=7.9 \mathrm{~Hz}, 4 \mathrm{H}, \mathrm{PBI}, 1,6,7,12$-positions), 8.45 (d, $J=8.1$ $\mathrm{Hz}, 4 \mathrm{H}, \mathrm{PBI}, 2,5,8,11$-positions), 6.85 (d, $J=3.7 \mathrm{~Hz} 4 \mathrm{H}, \mathrm{Ar} H$ of dendrons), 5.29 (s, 4H, $-\mathrm{NCH}_{2}-$ ), $4.10-$ $3.96\left(\mathrm{~m}, 8 \mathrm{H},-\mathrm{OCH}_{2} \mathrm{CH}_{2} \mathrm{CH}_{2}\right.$ and $\left.-\mathrm{OCH}_{2} \mathrm{CH}_{2} \mathrm{CH}(\mathrm{CH})_{3}-\right), 3.89\left(\mathrm{t}, J=6.6 \mathrm{~Hz}, 4 \mathrm{H},-\mathrm{OCH}_{2} \mathrm{CH}_{2} \mathrm{CH}_{2}-\right.$ ), 1.89-1.64 (m, 10H, $-\mathrm{CH}\left(\mathrm{CH}_{3}\right)_{2}$ and $\left.-\mathrm{OCH}_{2} \mathrm{CH}_{2} \mathrm{CH}_{2}-\right), 1.64-1.38\left(\mathrm{~m}, 14 \mathrm{H},-\mathrm{CH}\left(\mathrm{CH}_{3}\right) \mathrm{CH}_{2-},-\mathrm{O}\left(\mathrm{CH}_{2}\right)-\right.$ ${ }_{2} \mathrm{CH}_{2}-$ and $\left.-\mathrm{OCH}_{2} \mathrm{CH}_{2} \mathrm{CH}\left(\mathrm{CH}_{3}\right)-\right), 1.38-1.08\left(\mathrm{~m}, 36 \mathrm{H},-\mathrm{CH}\left(\mathrm{CH}_{3}\right)\left(\mathrm{CH}_{2}\right)_{3}-\right.$ and $\left.-\left(\mathrm{CH}_{2}\right)_{3}\left(\mathrm{CH}_{2}\right)_{3}-\right), 0.93(\mathrm{~d}$, $\left.J=6.5 \mathrm{~Hz}, 6 \mathrm{H},-\mathrm{CH}_{2} \mathrm{CH}\left(\mathrm{CH}_{3}\right)-\right), 0.90-0.80\left(\mathrm{~m}, 24 \mathrm{H},-\mathrm{CH}\left(\mathrm{CH}_{3}\right)_{2}\right.$ and $\left.-\mathrm{CH}_{2} \mathrm{CH}_{3}\right) .{ }^{13} \mathrm{C} \mathrm{NMR}(101 \mathrm{MHz}$, $\left.\mathrm{CDCl}_{3}, \delta, \mathrm{ppm}\right): 162.72(\mathrm{C}=\mathrm{O}), 153.17(\mathrm{ArC}-3,5), 137.97(\mathrm{ArC}-4), 133.71(\mathrm{ArC}-1)$, [132.34, 131.00, 128.31, 125.28, 122.74, 122.55] (perylene $C), 108.64(\mathrm{ArC}-2,6), 73.56\left(\mathrm{ArOCH}_{2}-4\right.$ position), 69.34 ( $\mathrm{ArOCH}_{2}-5$ position), 67.67 ( $\mathrm{ArOCH}_{2}-3$ position), $43.80\left(-\mathrm{CH}_{2} \mathrm{~N}\right)$, [39.44, 37.57, 36.67, 32.03, 32.01, $30.50,30.01,29.66,29.38,29.30,28.14,26.28,26.23,24.92]\left(-\mathrm{OCH}_{2} \mathrm{CH}_{2} \mathrm{CH}\left(\mathrm{CH}_{3}\right)\left(\mathrm{CH}_{2}\right)_{3} \mathrm{CH}-\right.$ and $\left.\mathrm{OCH}_{2}\left(\mathrm{CH}_{2}\right)_{4}-\right)$, [22.86, 22.79, 22.74, 19.78, 14.25, 14.21] $\left(-\mathrm{CH}\left(\mathrm{CH}_{3}\right)_{2},-\mathrm{CH}_{(} \mathrm{CH}_{3}\right) \mathrm{CH}_{2}-$ and $\left.-\mathrm{CH}_{2} \mathrm{CH}_{3}\right)$. MALDI-TOF MS $m / z$ of $[\mathrm{M}+\mathrm{Na}]^{+}$calculated for $\mathrm{C}_{86} \mathrm{H}_{118} \mathrm{~N}_{2} \mathrm{O}_{10}$ : 1361.9; Found: 1362.9 .
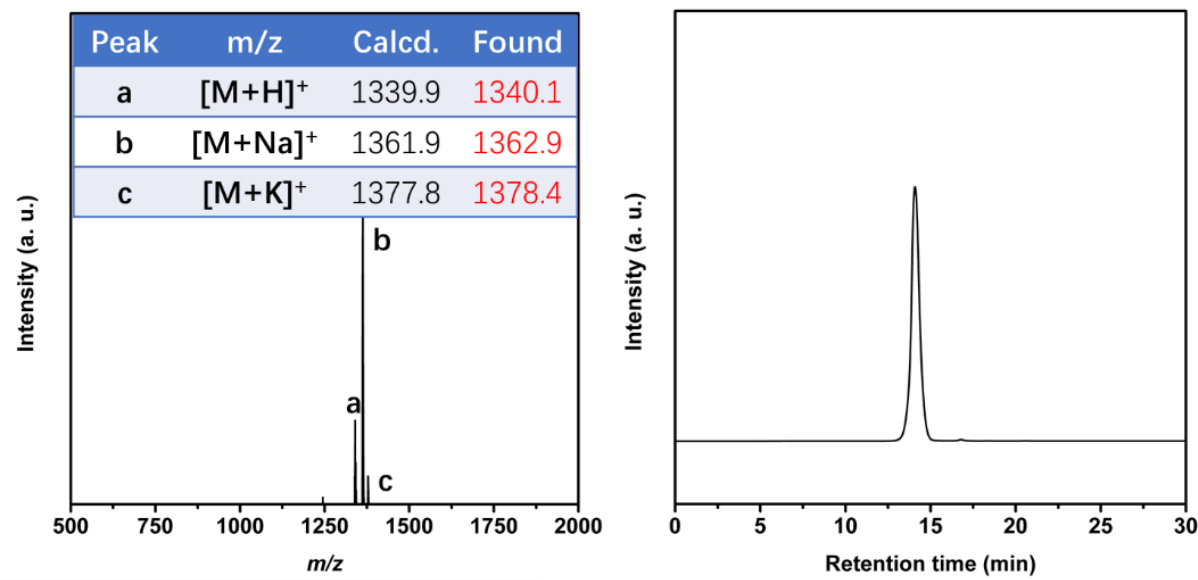

Supporting Figure S45. MALDI-TOF MS spectra (left) and HPLC trace (right) of 77r-PBI. Target molecule 77r-PBI has been successfully synthesized. ${ }^{1} \mathrm{H}$ and ${ }^{13} \mathrm{C}$ NMR spectra are presented on pages 138 and 139 , respectively. 
Supporting Figure S45 (continued). ${ }^{1} \mathrm{H}$ NMR of 77 r-PBI.

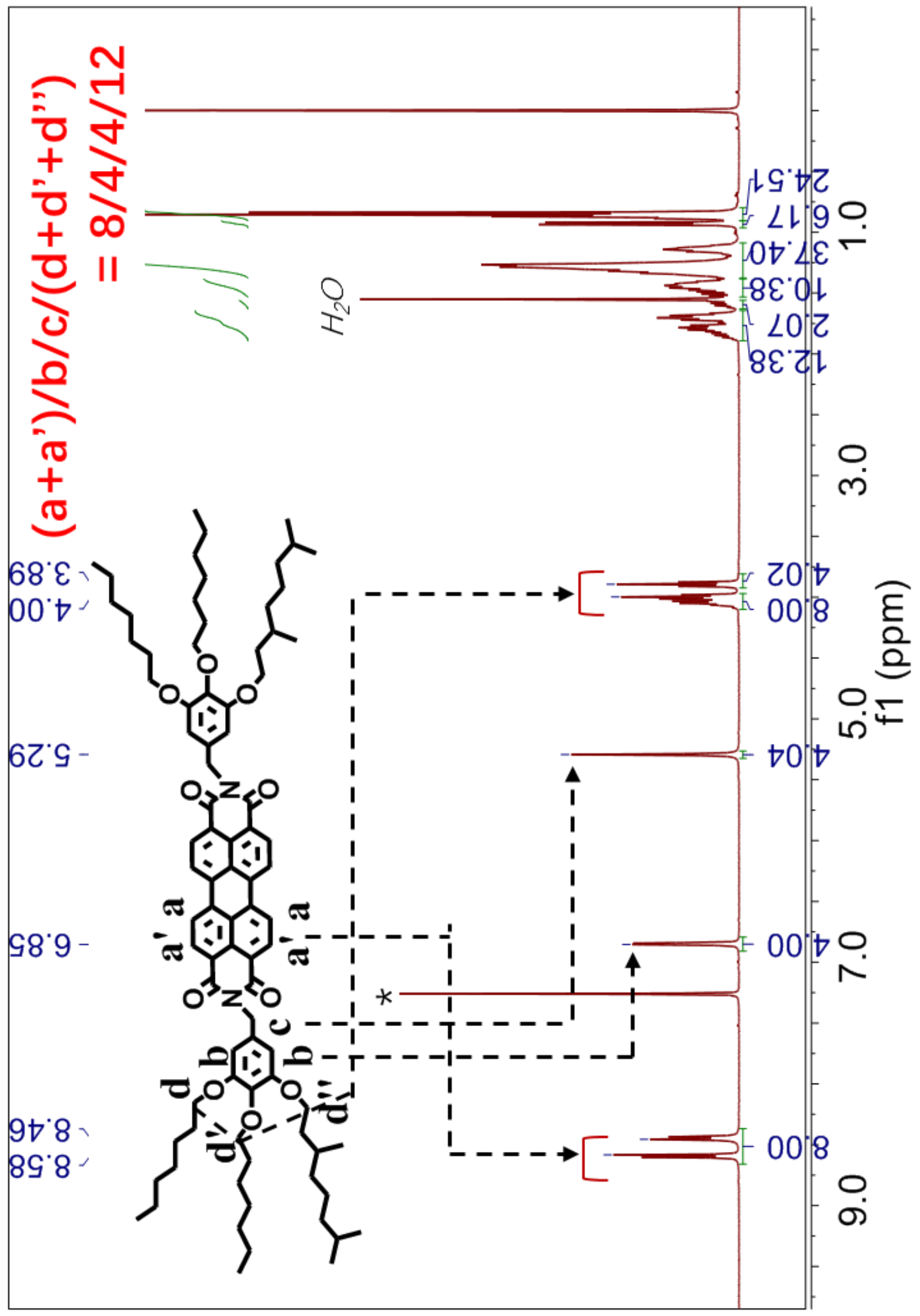


Supporting Figure $\mathbf{S 4 5}$ (continued). ${ }^{13} \mathrm{C}$ NMR of 77r-PBI.

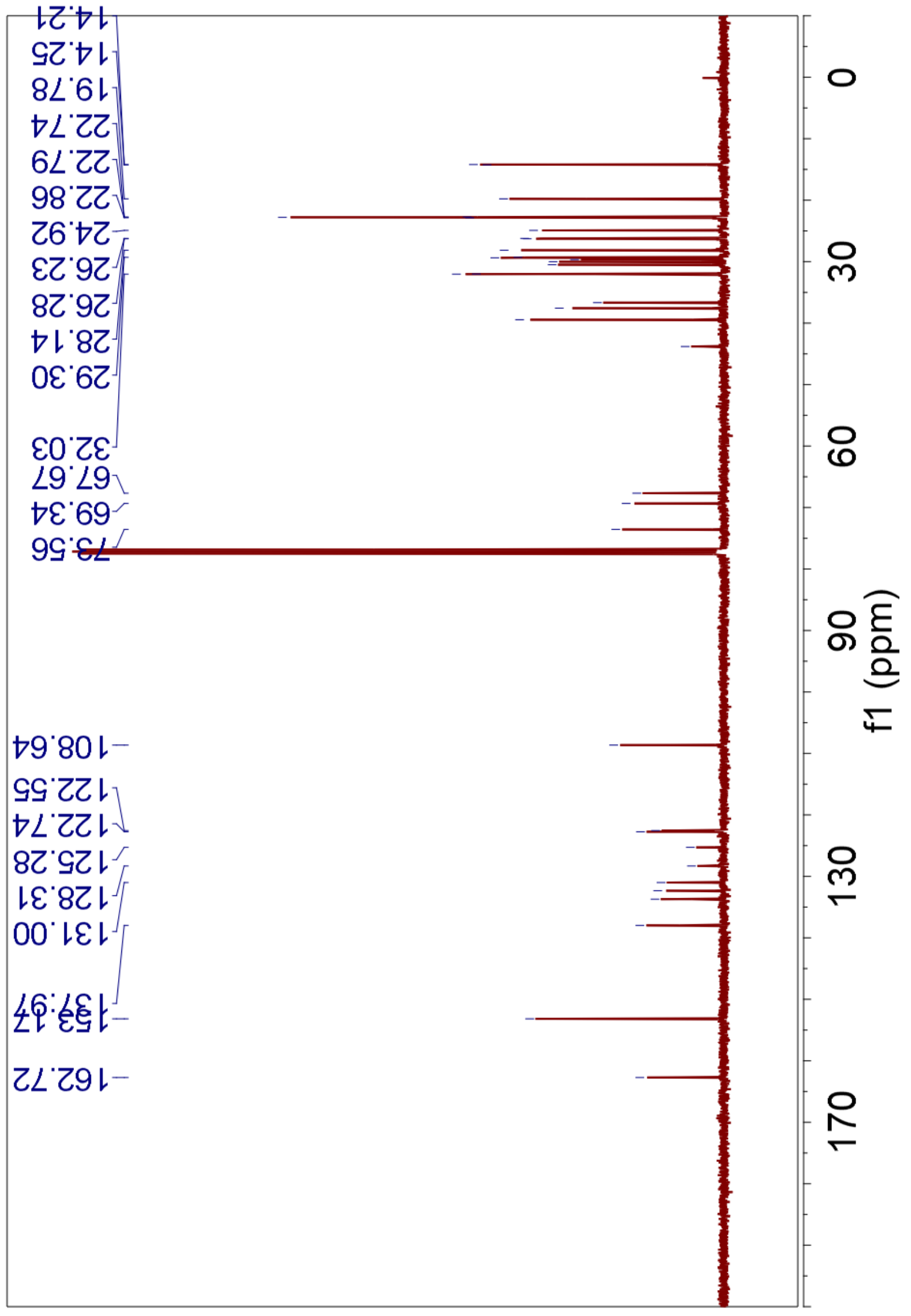




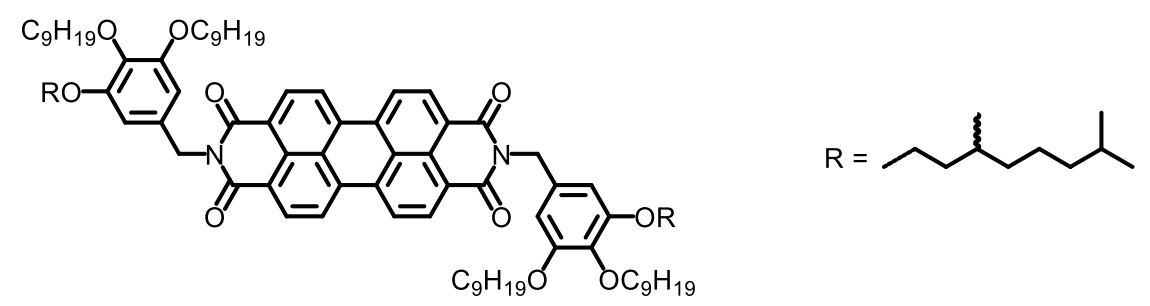

99r-PBI. From 12-99r (0.79 g, $1.45 \mathrm{mmol})$, PTCDA (0.26 g, $0.66 \mathrm{mmol})$ and $\mathrm{Zn}(\mathrm{OAc})_{2} \cdot 2 \mathrm{H}_{2} \mathrm{O}(0.145 \mathrm{~g}$, $0.66 \mathrm{mmol})$ in imidazole $(3 \mathrm{~g})$ at $180{ }^{\circ} \mathrm{C}$ for $6 \mathrm{~h}$, 99r-PBI was obtained as a red solid. Product: $0.73 \mathrm{~g}$. Yield: $76 \%$. Purity by HPLC: $99+\%$. mp: $225^{\circ} \mathrm{C}$.

${ }^{1} \mathrm{H}$ NMR (400 MHz, $\left.\mathrm{CDCl}_{3}, \delta, \mathrm{ppm}\right): 8.56$ (d, $J=7.9 \mathrm{~Hz}, 4 \mathrm{H}, \mathrm{PBI}, 1,6,7,12$-positions), 8.40 (d, $J=8.1$ $\mathrm{Hz}, 4 \mathrm{H}, \mathrm{PBI}, 2,5,8,11$-positions), 6.85 (d, $J=3.8 \mathrm{~Hz}, 4 \mathrm{H}, \mathrm{ArH}$ of dendrons), 5.29 (s, 4H, -NCH $\mathrm{N}_{2}$ ), 4.10$3.96\left(\mathrm{~m}, 8 \mathrm{H},-\mathrm{OCH}_{2} \mathrm{CH}_{2} \mathrm{CH}_{2}-\right.$ and $\left.-\mathrm{OCH}_{2} \mathrm{CH}_{2} \mathrm{CH}(\mathrm{CH})_{3}-\right), 3.89$ (t, $\left.J=6.6 \mathrm{~Hz}, 4 \mathrm{H},-\mathrm{OCH}_{2} \mathrm{CH}_{2} \mathrm{CH}_{2}-\right)$, 1.89-1.64 (m, $10 \mathrm{H},-\mathrm{CH}\left(\mathrm{CH}_{3}\right)_{2}$ and $\left.-\mathrm{OCH}_{2} \mathrm{CH}_{2} \mathrm{CH}_{2}-\right), 1.64-1.38\left(\mathrm{~m}, 14 \mathrm{H},-\mathrm{CH}\left(\mathrm{CH}_{3}\right) \mathrm{CH}_{2}-,-\mathrm{O}\left(\mathrm{CH}_{2}\right)-\right.$ ${ }_{2} \mathrm{CH}_{2}-$ and $\left.-\mathrm{OCH}_{2} \mathrm{CH}_{2} \mathrm{CH}\left(\mathrm{CH}_{3}\right)-\right), 1.38-1.09\left(\mathrm{~m}, 52 \mathrm{H},-\mathrm{CH}\left(\mathrm{CH}_{3}\right)\left(\mathrm{CH}_{2}\right)_{3}-\right.$ and $\left.-\left(\mathrm{CH}_{2}\right)_{3}\left(\mathrm{CH}_{2}\right)_{5}-\right), 0.93(\mathrm{~d}$, $\left.J=6.5 \mathrm{~Hz}, 6 \mathrm{H},-\mathrm{CH}_{2} \mathrm{CH}\left(\mathrm{CH}_{3}\right)-\right), 0.90-0.80\left(\mathrm{~m}, 24 \mathrm{H},-\mathrm{CH}\left(\mathrm{CH}_{3}\right)_{2}\right.$ and $\left.-\mathrm{CH}_{2} \mathrm{CH}_{3}\right) .{ }^{13} \mathrm{C} \mathrm{NMR}(101 \mathrm{MHz}$, $\left.\mathrm{CDCl}_{3}, \delta, \mathrm{ppm}\right): 162.66(C=\mathrm{O}), 153.17$ (ArC-3,5), 137.97 (ArC-4), 133.62 (ArC-1), [132.34, 130.95, 128.22, 125.18, 122.68, 122.50] (perylene C), 108.66 ( $\mathrm{ArC}-2,6), 73.57\left(\mathrm{ArOCH}_{2}-4\right.$ position), 69.33 ( $\mathrm{ArOCH} \mathrm{H}_{2}-5$ position), $67.67\left(\mathrm{ArOCH}_{2}-3\right.$ position), $43.78\left(-\mathrm{CH}_{2} \mathrm{~N}\right),[39.44,37.57,36.68,32.07,32.03$, $30.50, \quad 30.02, \quad 29.80, \quad 29.73, \quad 29.66, \quad 29.48, \quad 29.30, \quad 28.14, \quad 26.35, \quad 26.28, \quad 24.92] \quad(-$ $\mathrm{OCH}_{2} \mathrm{CH}_{2} \mathrm{CH}\left(\mathrm{CH}_{3}\right)\left(\mathrm{CH}_{2}\right)_{3} \mathrm{CH}-$ and $\left.-\mathrm{OCH}_{2}\left(\mathrm{CH}_{2}\right)_{6}-\right)$, [22.86, 22.83, 22.79, 22.74, 19.78, 14.24, 14.20] ($\mathrm{CH}\left(\mathrm{CH}_{3}\right)_{2},-\mathrm{CH}\left(\mathrm{CH}_{3}\right) \mathrm{CH}_{2}-$ and $\left.-\mathrm{CH}_{2} \mathrm{CH}_{3}\right)$. MALDI-TOF MS $m / z$ of $\left[\mathrm{M}+\mathrm{Na}^{+}\right.$calculated for $\mathrm{C}_{94} \mathrm{H}_{134} \mathrm{~N}_{2} \mathrm{O}_{10}$ : 1474.0; Found: 1474.9 .
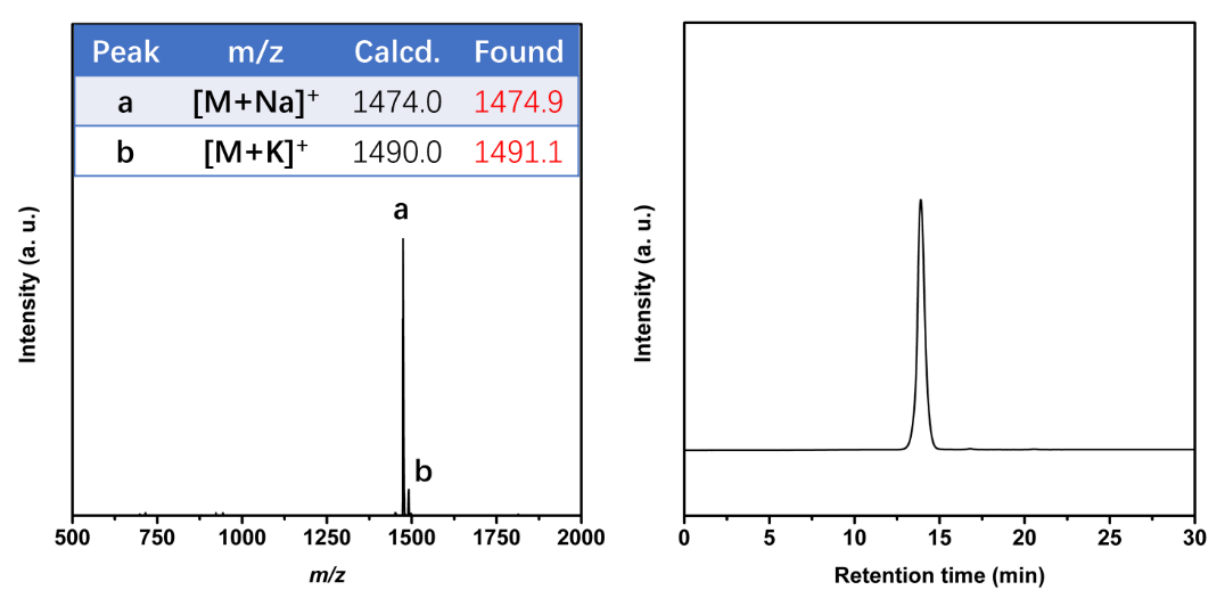

Supporting Figure S46. MALDI-TOF MS spectra (left) and HPLC trace (right) of 99r-PBI. Target molecule 99r-PBI has been successfully synthesized. ${ }^{1} \mathrm{H}$ and ${ }^{13} \mathrm{C}$ NMR spectra are presented on pages 141 and 142 , respectively. 
Supporting Figure S46 (continued). ${ }^{1} \mathrm{H}$ NMR of 99r-PBI.

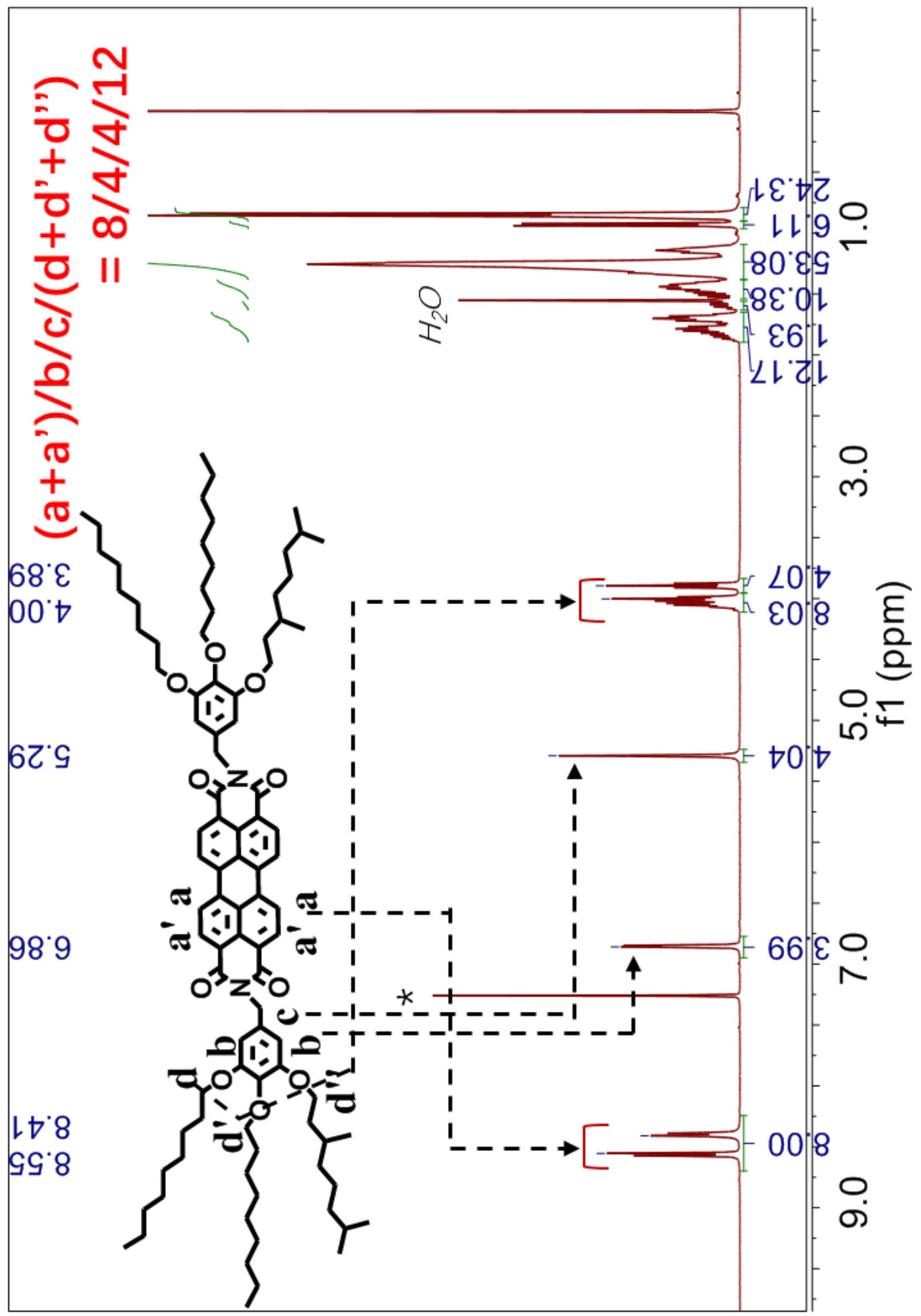


Supporting Figure S46 (continued). ${ }^{13} \mathrm{C}$ NMR of 99r-PBI.

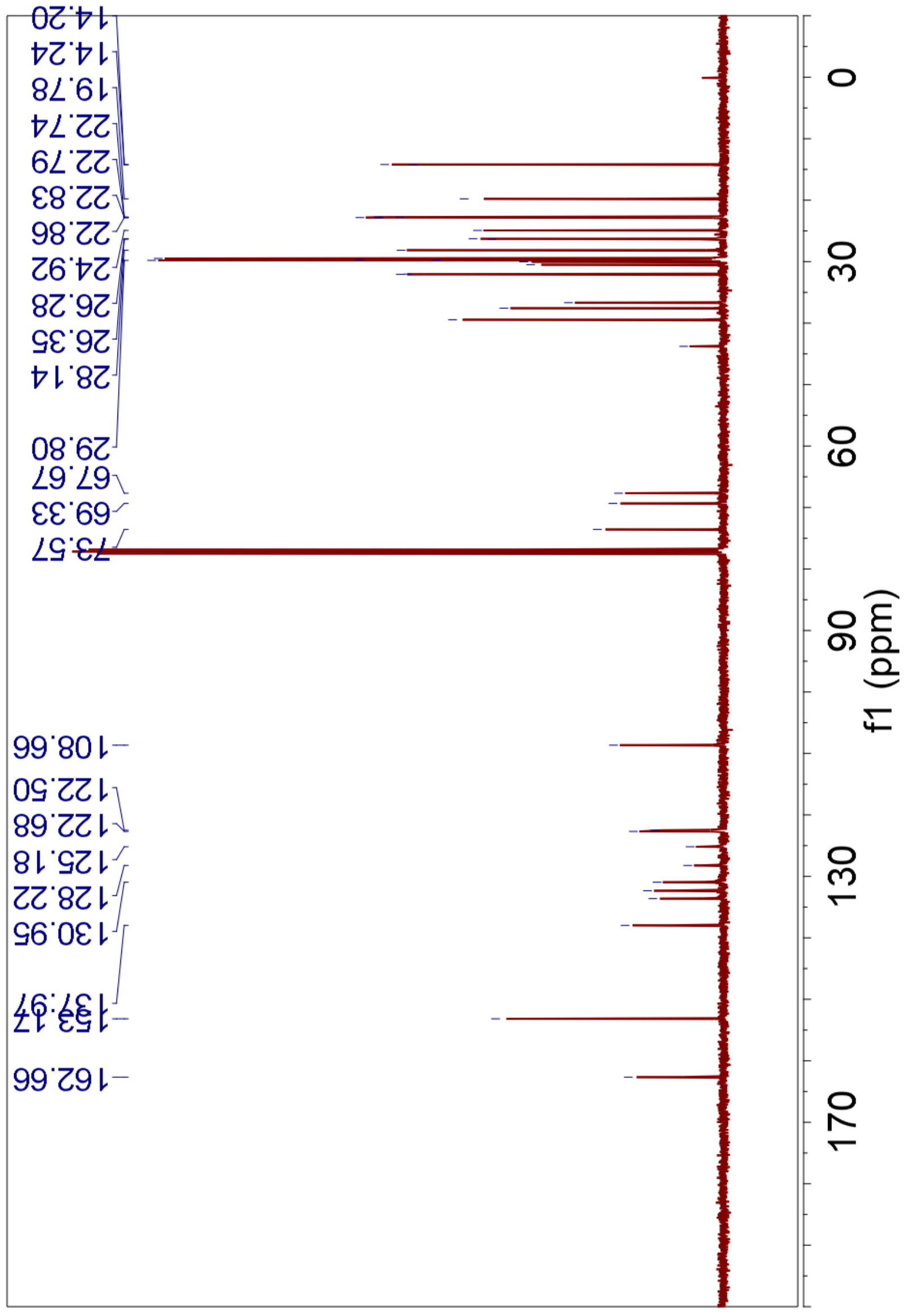



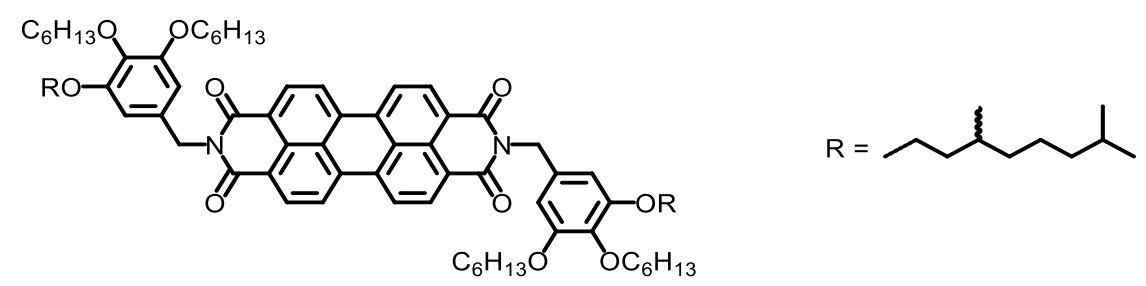

66r-PBI. From 12-66r (1.02 g, $2.2 \mathrm{mmol})$, PTCDA $(0.39 \mathrm{~g}, 1.0 \mathrm{mmol})$ and $\mathrm{Zn}(\mathrm{OAc})_{2} \cdot 2 \mathrm{H}_{2} \mathrm{O}(0.22 \mathrm{~g}, 1.0$ mmol) in imidazole $(3 \mathrm{~g})$ at $160^{\circ} \mathrm{C}$ for $14 \mathrm{~h}, \mathbf{6 6 r}-\mathbf{P B I}$ was obtained as a red solid. Product: $0.73 \mathrm{~g}$. Yield: 57\%. Purity by HPLC: $99+\%$. mp: $238^{\circ} \mathrm{C}$.

${ }^{1} \mathrm{H}$ NMR (500 MHz, $\mathrm{CDCl}_{3}, \delta$, ppm): 8.53 (d, $J=7.9 \mathrm{~Hz}, 4 \mathrm{H}, \mathrm{PBI}, 1,6,7,12$-positions), 8.35 (d, $J=8.0$ $\mathrm{Hz}, 4 \mathrm{H}, \mathrm{PBI}, 2,5,8,11$-positions), 6.86 (d, $J=4.5 \mathrm{~Hz} 4 \mathrm{H}, \mathrm{Ar} H$ of dendrons), 5.29 (s, 4H, $-\mathrm{NCH}_{2}-$ ), $4.13-$ $3.96\left(\mathrm{~m}, 8 \mathrm{H},-\mathrm{OCH}_{2} \mathrm{CH}_{2} \mathrm{CH}_{2}\right.$ and $\left.-\mathrm{OCH}_{2} \mathrm{CH}_{2} \mathrm{CH}(\mathrm{CH})_{3}-\right), 3.90\left(\mathrm{t}, J=6.7 \mathrm{~Hz}, 4 \mathrm{H},-\mathrm{OCH}_{2} \mathrm{CH}_{2} \mathrm{CH}_{2}-\right.$ ), 1.90-1.65 (m, $10 \mathrm{H},-\mathrm{CH}\left(\mathrm{CH}_{3}\right)_{2}$ and $\left.-\mathrm{OCH}_{2} \mathrm{CH}_{2} \mathrm{CH}_{2}-\right), 1.63-1.39\left(\mathrm{~m}, 14 \mathrm{H},-\mathrm{CH}\left(\mathrm{CH}_{3}\right) \mathrm{CH}_{2}-,-\mathrm{O}\left(\mathrm{CH}_{2}\right)-\right.$ ${ }_{2} \mathrm{CH}_{2}-$ and $\left.-\mathrm{OCH}_{2} \mathrm{CH}_{2} \mathrm{CH}\left(\mathrm{CH}_{3}\right)-\right), 1.38-1.09\left(\mathrm{~m}, 28 \mathrm{H},-\mathrm{CH}\left(\mathrm{CH}_{3}\right)\left(\mathrm{CH}_{2}\right)_{3}-\right.$ and $\left.-\left(\mathrm{CH}_{2}\right)_{3}\left(\mathrm{CH}_{2}\right)_{2}-\right), 0.94$ (d, $\left.J=6.6 \mathrm{~Hz}, 6 \mathrm{H},-\mathrm{CH}_{2} \mathrm{CH}\left(\mathrm{CH}_{3}\right)-\right), 0.92-0.83\left(\mathrm{~m}, 24 \mathrm{H},-\mathrm{CH}\left(\mathrm{CH}_{3}\right)_{2}\right.$ and $\left.-\mathrm{CH}_{2} \mathrm{CH}_{3}\right) .{ }^{13} \mathrm{C}$ NMR $(101 \mathrm{MHz}$, $\left.\mathrm{CDCl}_{3}, \delta, \mathrm{ppm}\right): 162.62(C=\mathrm{O}), 153.17(\mathrm{ArC}-3,5), 137.94(\mathrm{ArC}-4), 133.58(\mathrm{Ar} C-1),[132.35,130.92$, 128.15, 125.12, 122.63, 122.47] (perylene $C), 108.66$ ( $\mathrm{ArC}-2,6), 73.55\left(\mathrm{ArOCH}_{2}-4\right.$ position), 69.33 ( $\mathrm{ArOCH}_{2}-5$ position), 67.65 ( $\mathrm{ArOCH}_{2}-3$ position), $43.75\left(-\mathrm{CH}_{2} \mathrm{~N}\right)$, [39.44, 37.57, 36.67, 31.89, 31.79, $30.44,30.02,29.60,28.14,25.98,25.93,24.92]\left(-\mathrm{OCH}_{2} \mathrm{CH}_{2} \mathrm{CH}\left(\mathrm{CH}_{3}\right)\left(\mathrm{CH}_{2}\right)_{3} \mathrm{CH}-\right.$ and $\left.-\mathrm{OCH}_{2}\left(\mathrm{CH}_{2}\right)_{3}-\right)$, $[22.86,22.81,22.74,19.78,14.21,14.20]\left(-\mathrm{CH}\left(\mathrm{CH}_{3}\right)_{2},-\mathrm{CH}\left(\mathrm{CH}_{3}\right) \mathrm{CH}_{2}-\right.$ and $\left.-\mathrm{CH}_{2} \mathrm{CH}_{3}\right)$. MALDI-TOF MS $m / z$ of $[\mathrm{M}+\mathrm{Na}]^{+}$calculated for $\mathrm{C}_{82} \mathrm{H}_{110} \mathrm{~N}_{2} \mathrm{O}_{10}$ : 1305.8; Found: 1305.7 .
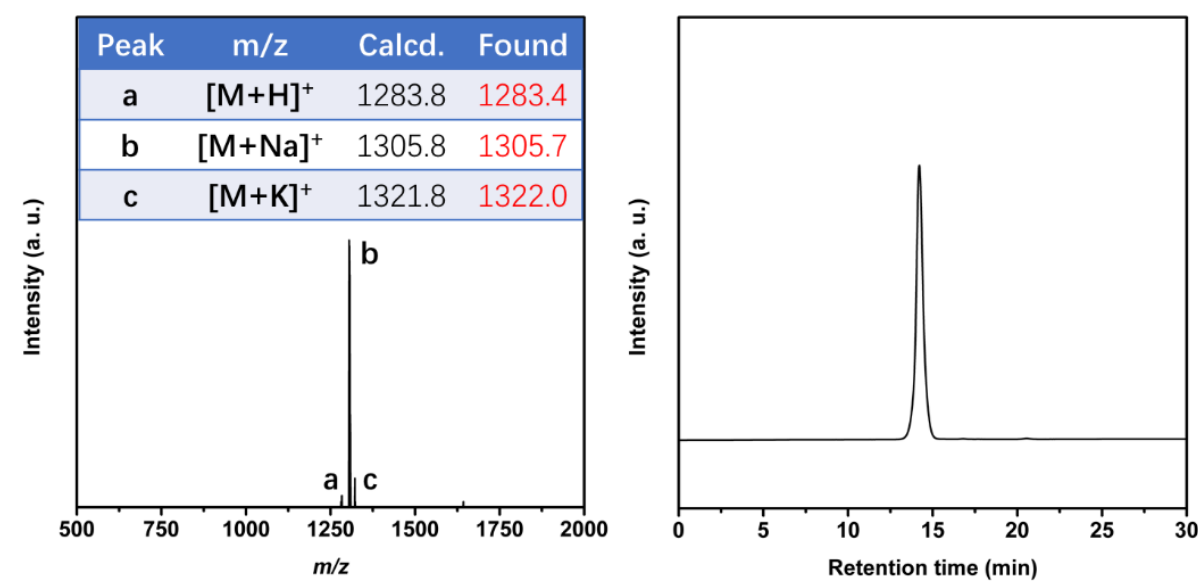

Supporting Figure S47. MALDI-TOF MS spectra (left) and HPLC trace (right) of 66r-PBI. Target molecule 66r-PBI has been successfully synthesized. ${ }^{1} \mathrm{H}$ and ${ }^{13} \mathrm{C}$ NMR spectra are presented on pages 144 and 145 , respectively. 
Supporting Figure S47 (continued). ${ }^{1} \mathrm{H}$ NMR of 66r-PBI.

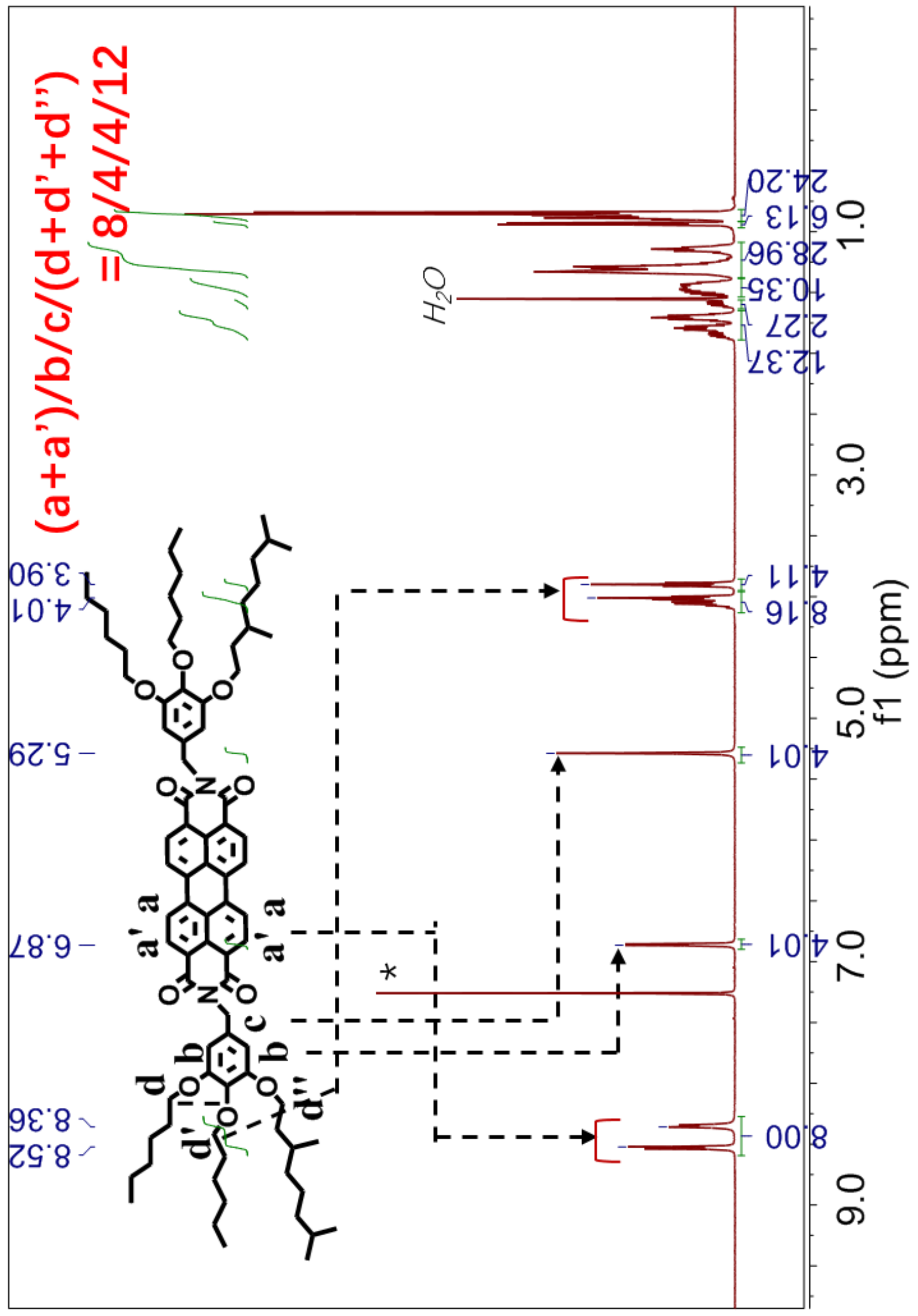


Supporting Figure S47 (continued). ${ }^{13} \mathrm{C}$ NMR of 66r-PBI.

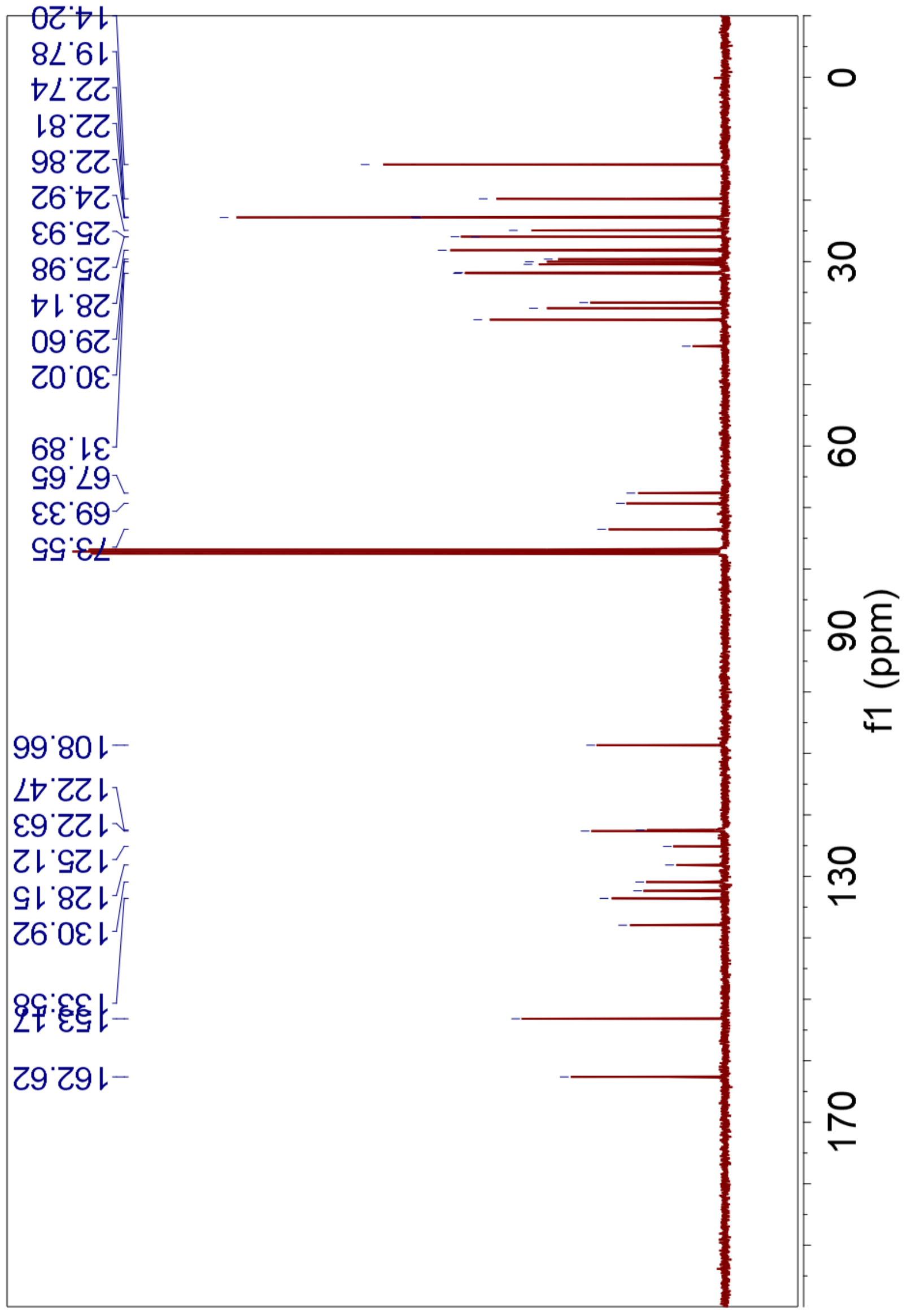




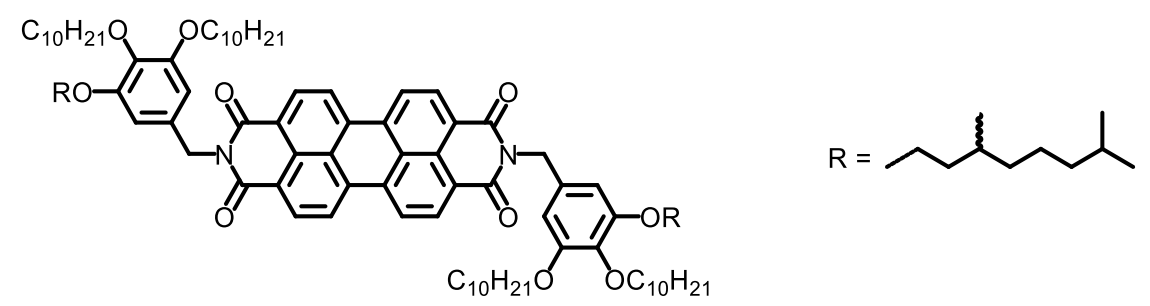

1010r-PBI. From 12-1010r (1.27 g, $2.2 \mathrm{mmol})$, PTCDA $(0.39 \mathrm{~g}, 1.0 \mathrm{mmol})$ and $\mathrm{Zn}(\mathrm{OAc})_{2} \cdot 2 \mathrm{H}_{2} \mathrm{O}(0.22 \mathrm{~g}$, $1.0 \mathrm{mmol})$ in imidazole $(3 \mathrm{~g})$ at $160{ }^{\circ} \mathrm{C}$ for $21 \mathrm{~h}, \mathbf{1 0 1 0 r}-\mathrm{PBI}$ was obtained as a red solid. Product: $1.08 \mathrm{~g}$. Yield: $72 \%$. Purity by HPLC: $99+\%$. mp: $225^{\circ} \mathrm{C}$.

${ }^{1} \mathrm{H}$ NMR (500 MHz, $\left.\mathrm{CDCl}_{3}, \delta, \mathrm{ppm}\right): 8.47$ (d, $J=7.9 \mathrm{~Hz}, 4 \mathrm{H}, \mathrm{PBI}, 1,6,7,12$-positions), 8.26 (d, $J=7.6$ $\mathrm{Hz}, 4 \mathrm{H}, \mathrm{PBI}, 2,5,8,11$-positions), 6.86 (d, $J=5.1 \mathrm{~Hz}, 4 \mathrm{H}$, ArH of dendrons), 5.28 (s, 4H, - $\mathrm{NCH}_{2}-$ ), 4.12$3.98\left(\mathrm{~m}, 8 \mathrm{H},-\mathrm{OCH}_{2} \mathrm{CH}_{2} \mathrm{CH}_{2}-\right.$ and $\left.-\mathrm{OCH}_{2} \mathrm{CH}_{2} \mathrm{CH}(\mathrm{CH})_{3}-\right), 3.90$ (t, $\left.J=6.4 \mathrm{~Hz}, 4 \mathrm{H},-\mathrm{OCH}_{2} \mathrm{CH}_{2} \mathrm{CH}_{2}-\right)$, 1.90-1.66 (m, $10 \mathrm{H},-\mathrm{CH}\left(\mathrm{CH}_{3}\right)_{2}$ and $\left.-\mathrm{OCH}_{2} \mathrm{CH}_{2} \mathrm{CH}_{2}-\right), 1.64-1.38\left(\mathrm{~m}, 14 \mathrm{H},-\mathrm{CH}\left(\mathrm{CH}_{3}\right) \mathrm{CH}_{2}-,-\mathrm{O}\left(\mathrm{CH}_{2}\right)-\right.$ ${ }_{2} \mathrm{CH}_{2}-$ and $\left.-\mathrm{OCH}_{2} \mathrm{CH}_{2} \mathrm{CH}\left(\mathrm{CH}_{3}\right)-\right), 1.38-1.09\left(\mathrm{~m}, 60 \mathrm{H},-\mathrm{CH}\left(\mathrm{CH}_{3}\right)\left(\mathrm{CH}_{2}\right)_{3}-\right.$ and $\left.-\left(\mathrm{CH}_{2}\right)_{3}\left(\mathrm{CH}_{2}\right)_{6}-\right), 0.94(\mathrm{~d}$, $\left.J=6.6 \mathrm{~Hz}, 6 \mathrm{H},-\mathrm{CH}_{2} \mathrm{CH}\left(\mathrm{CH}_{3}\right)-\right), 0.90-0.81\left(\mathrm{~m}, 24 \mathrm{H},-\mathrm{CH}\left(\mathrm{CH}_{3}\right)_{2}\right.$ and $\left.-\mathrm{CH}_{2} \mathrm{CH}_{3}\right) .{ }^{13} \mathrm{C} \mathrm{NMR}(101 \mathrm{MHz}$, $\left.\mathrm{CDCl}_{3}, \delta, \mathrm{ppm}\right): 162.66(C=\mathrm{O}), 153.17$ (ArC-3,5), 137.97 (ArC-4), 133.63 (ArC-1), [132.34, 130.96, 128.22, 125.19, 122.69, 122.50] (perylene $C), 108.65$ (ArC-2,6), $73.57\left(\mathrm{ArOCH}_{2}-4\right.$ position), 69.33 ( $\mathrm{ArOCH} \mathrm{H}_{2}-3$ position), 67.67 ( $\mathrm{ArOCH}_{2}-5$ position), $43.78\left(-\mathrm{CH}_{2} \mathrm{~N}\right),[39.45,37.57,36.68,32.07,32.04$, $30.51, \quad 30.02,29.85,29.78,29.74,29.66,29.53,29.49,28.14,26.35,26.28,24.93](-$ $\mathrm{OCH}_{2} \mathrm{CH}_{2} \mathrm{CH}\left(\mathrm{CH}_{3}\right)\left(\mathrm{CH}_{2}\right)_{3} \mathrm{CH}-$ and $\left.-\mathrm{OCH}_{2}\left(\mathrm{CH}_{2}\right)_{7}-\right)$, [22.86, 22.83, 22.79, 22.75, 19.78, 14.24, 14.21] ($\mathrm{CH}\left(\mathrm{CH}_{3}\right)_{2},-\mathrm{CH}\left(\mathrm{CH}_{3}\right) \mathrm{CH}_{2}-$ and $\left.-\mathrm{CH}_{2} \mathrm{CH}_{3}\right)$. MALDI-TOF MS $m / z$ of $\left[\mathrm{M}+\mathrm{Na}^{+}\right.$calculated for $\mathrm{C}_{98} \mathrm{H}_{142} \mathrm{~N}_{2} \mathrm{O}_{10}$ : 1530.1; Found: 1530.6 .
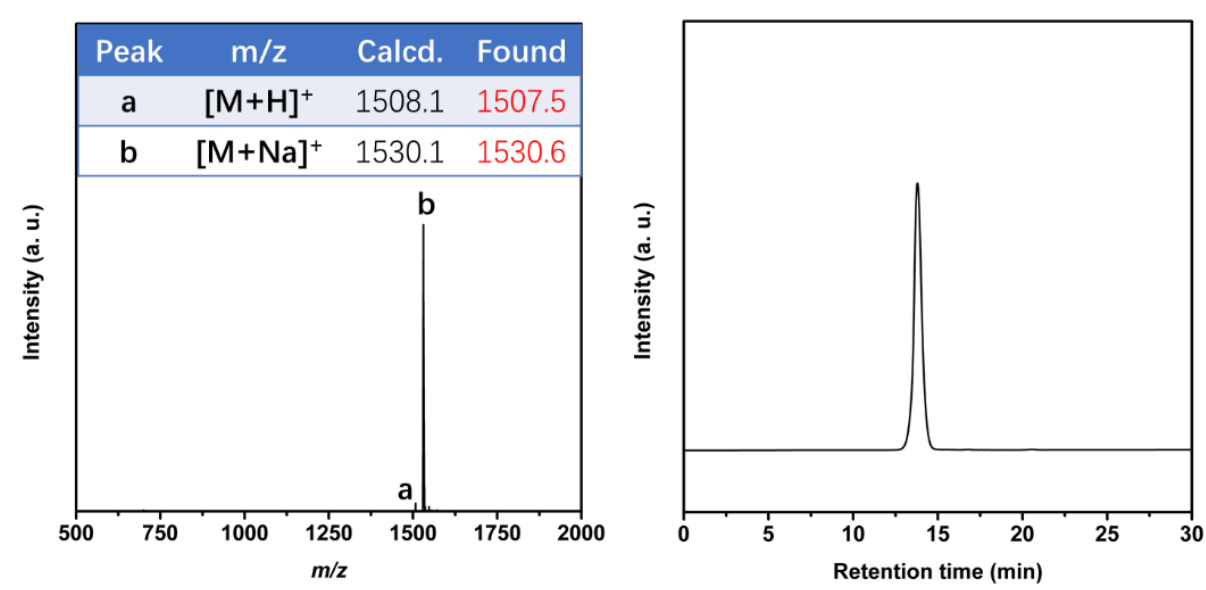

Supporting Figure S48. MALDI-TOF MS spectra (left) and HPLC trace (right) of 1010r-PBI. Target molecule 1010r-PBI has been successfully synthesized. ${ }^{1} \mathrm{H}$ and ${ }^{13} \mathrm{C}$ NMR spectra are presented on pages 147 and 148, respectively. 
Supporting Figure S48 (continued). ${ }^{1} \mathrm{H}$ NMR of 1010r-PBI.

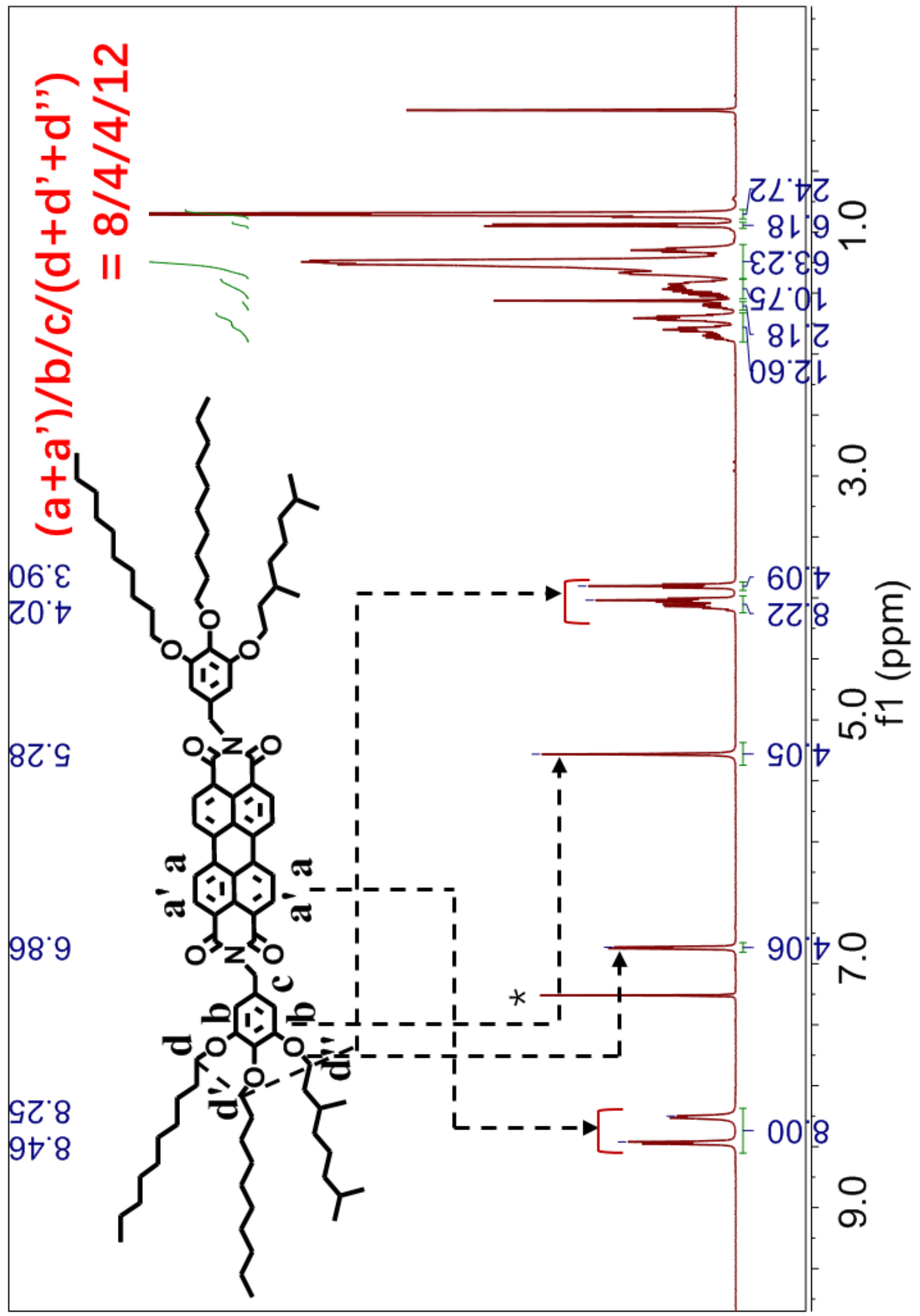


Supporting Figure S48 (continued). ${ }^{13} \mathrm{C}$ NMR of 1010r-PBI.

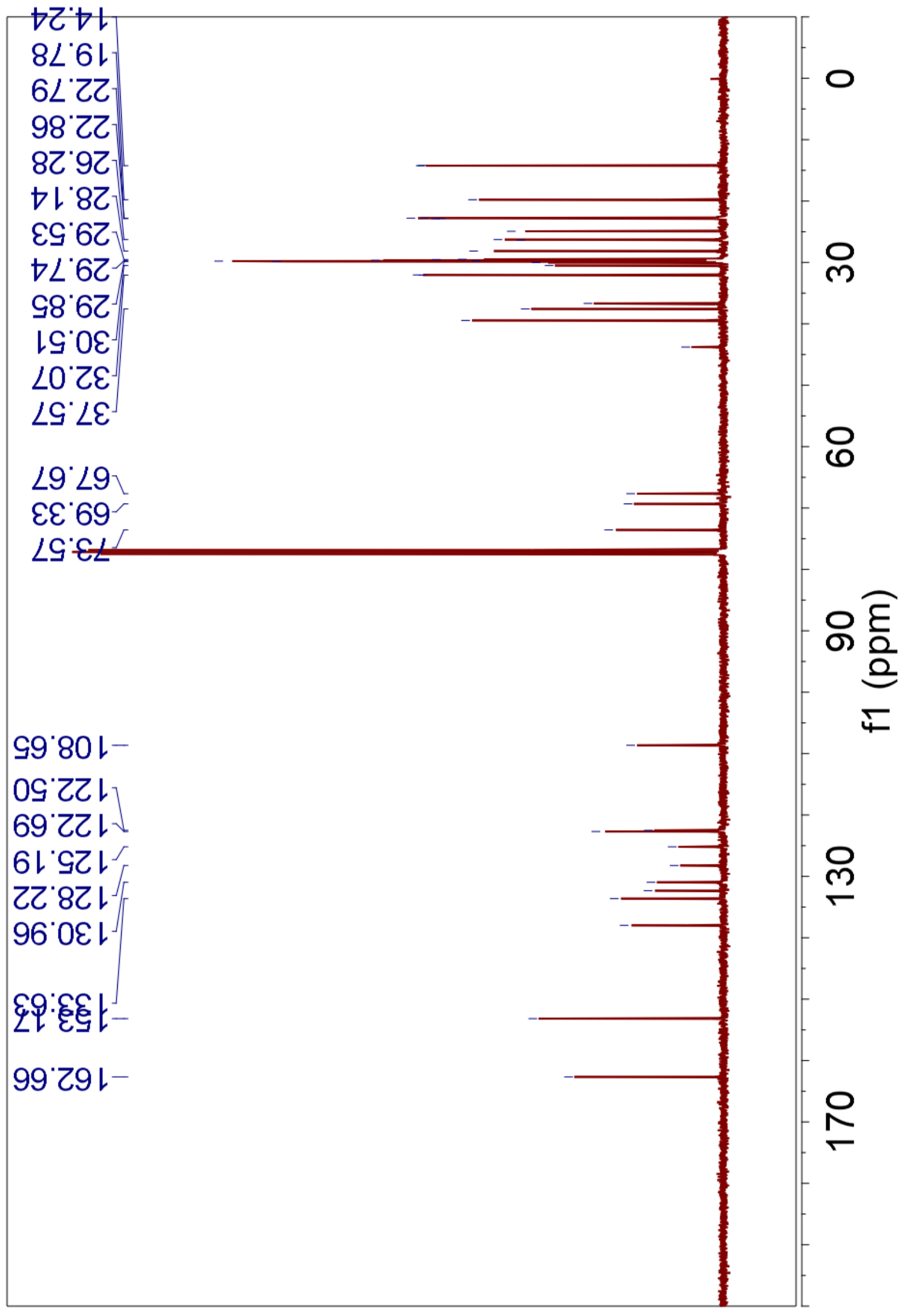




\section{Thermal Analysis of Sequence-Defined Hybrid PBIs by DSC}

\section{Supporting Table ST1. Transition Temperatures and Associated Enthalpy Changes of Sequence- Defined Hybrid Linear-Racemic PBIs Determined by DSC and Phases Determined by XRD}

\begin{tabular}{|c|c|c|}
\hline & \multicolumn{2}{|c|}{ Phase transition $\left({ }^{\circ} \mathrm{C}\right)$ and corresponding enthalpy changes $\left(\mathrm{kcal} \cdot \mathrm{mol}^{-1}\right)^{a}$} \\
\hline & Heating & Cooling \\
\hline rrr-PBI & $\begin{array}{l}\boldsymbol{\Phi}_{\mathbf{h}}{ }^{\mathbf{k} 1} 200(3.45) \boldsymbol{i} \\
\boldsymbol{\Phi}_{\mathbf{h}} \mathbf{k} 200(3.70) \boldsymbol{i}\end{array}$ & $i 198(-3.17) \boldsymbol{\Phi}_{\mathbf{h}}{ }^{\mathbf{k} 1}$ \\
\hline rr7-PBI & $\begin{array}{l}\boldsymbol{\Phi}_{\mathbf{h}}^{\mathbf{k} 2} 144(1.53) \boldsymbol{\Phi}_{\mathbf{h}}^{\mathbf{k} 1} 205(3.03) \boldsymbol{i} \\
\boldsymbol{\Phi}_{\mathbf{h}} \mathbf{k} 16(-0.66) \boldsymbol{\Phi}_{\mathbf{h}}{ }^{\mathbf{k} 2} 145(1.77) \boldsymbol{\Phi}_{\mathbf{h}} \mathbf{k} 1205(2.82) \boldsymbol{i}\end{array}$ & $\boldsymbol{i} 202(-2.94) \boldsymbol{\Phi}_{\mathbf{h}}{ }^{\mathbf{k} 1}$ \\
\hline r7r-PBI & $\begin{array}{l}\boldsymbol{\Phi}_{\mathbf{c}-\mathbf{0}}^{\mathbf{k}} 164(0.61) \boldsymbol{\Phi}_{\mathbf{h}}^{\mathbf{k} 1} 225(4.22) \boldsymbol{i} \\
\boldsymbol{\Phi}_{\mathbf{h}}^{\mathbf{k} 3} 70^{a} \boldsymbol{\Phi}_{\mathbf{c}-\mathbf{0}} \mathbf{k} 165(1.09) \boldsymbol{\Phi}_{\mathbf{h}}^{\mathbf{k} 1} 225(4.64) \boldsymbol{i}\end{array}$ & $\boldsymbol{i} 202(-2.94) \boldsymbol{\Phi}_{\mathbf{h}}{ }^{\mathbf{k} 3}$ \\
\hline 7r7-PBI & $\begin{array}{l}\boldsymbol{\Phi}_{\mathbf{c - 0}}{ }^{\mathbf{k}} 185 \boldsymbol{\Phi}_{\mathbf{h}}^{\text {io }} 198(2.82) \boldsymbol{\Phi}_{\mathbf{h}} 209(2.40) \boldsymbol{i} \\
54(0.60) \boldsymbol{\Phi}_{\mathbf{c}-\mathbf{0}}{ }^{\mathbf{k}} 185^{a} \boldsymbol{\Phi}_{\mathbf{h}}^{\text {io }} 193(2.84) \boldsymbol{\Phi}_{\mathbf{h}} 209(2.34) \boldsymbol{i}\end{array}$ & $i 207(-2.27) \boldsymbol{\Phi}_{\mathbf{h}} 183(-2.34) \boldsymbol{\Phi}_{\mathbf{c}-\mathbf{0}}^{\mathbf{k}}$ \\
\hline 77r-PBI & $\begin{array}{l}\boldsymbol{\Phi}_{\mathbf{c - 0}} \mathbf{k} 220(2.52) \boldsymbol{\Phi}_{\mathbf{h}} 229(3.14) \boldsymbol{i} \\
\boldsymbol{\Phi}_{\mathbf{c}-\mathbf{0}} \mathbf{k} 220(2.53) \boldsymbol{\Phi}_{\mathbf{h}} 229(3.43) \boldsymbol{i}\end{array}$ & $i 226(-3.30) \boldsymbol{\Phi}_{\mathbf{h}} 207(-2.53) \boldsymbol{\Phi}_{\mathbf{c - 0}} \mathbf{k}$ \\
\hline 777-PBI & $\begin{array}{l}\boldsymbol{\Phi}_{\mathrm{c}-\mathbf{0}}^{\mathrm{k}} 250(8.17) \boldsymbol{i} \\
\boldsymbol{\Phi}_{\mathrm{m}}^{\mathrm{k}-\mathrm{SL}} 9(4.08) \boldsymbol{\Phi}_{\mathrm{m}}^{\mathrm{k}-\mathrm{SL}} 22(1.28) \boldsymbol{\Phi}_{\mathrm{c}-0}{ }^{\mathrm{k}} 250(8.18) \boldsymbol{i}\end{array}$ & $\begin{array}{l}\boldsymbol{i} 241(-8.15) \boldsymbol{\Phi}_{\mathbf{c - 0}}{ }^{\mathrm{k}} 16(-1.68) \boldsymbol{\Phi}_{\mathbf{m}}^{\mathrm{k}-\mathrm{SL}} 16(-1.68) \boldsymbol{\Phi}_{\mathbf{m}}^{\mathrm{k}-\mathrm{SL}} \\
-6(-3.30)\end{array}$ \\
\hline rr9-PBI & $\begin{array}{l}\boldsymbol{\Phi}_{\mathbf{h}}^{\mathbf{k} 2} 135(1.09) \boldsymbol{\Phi}_{\mathbf{h}}^{\mathbf{k} 1} 204(3.85) \boldsymbol{i} \\
66(-0.64) \boldsymbol{\Phi}_{\mathbf{h}}^{\mathbf{k} 2} 136(1.82) \boldsymbol{\Phi}_{\mathbf{h}} \mathbf{k} 1204(3.33) \boldsymbol{i}\end{array}$ & $i 201(-3.66) \Phi_{\mathbf{h}}{ }^{\mathbf{k} 1} 50^{a} \boldsymbol{\Phi}_{\mathbf{h}}{ }^{\mathrm{k} 2}$ \\
\hline r9r-PBI & $\begin{array}{l}\boldsymbol{\Phi}_{\mathbf{c - 0}} \mathbf{k}^{\mathbf{k}} 145(1.20) \boldsymbol{\Phi}_{\mathbf{h}}^{\mathbf{k} 1} 222(4.85) \boldsymbol{i} \\
\boldsymbol{\Phi}_{\mathbf{h}}^{\mathbf{k} 1} 30^{a} \boldsymbol{\Phi}_{\mathbf{c}-\mathbf{0}} \mathbf{k}^{\mathbf{k}} 146(1.20) \boldsymbol{\Phi}_{\mathbf{h}}^{\mathbf{k} 1} 222(4.86) \boldsymbol{i}\end{array}$ & $\boldsymbol{i} 219(-4.85) \boldsymbol{\Phi}_{\mathbf{h}}^{\mathbf{k} 1}$ \\
\hline 9r9-PBI & $\begin{array}{l}\boldsymbol{\Phi}_{\mathbf{h}}^{\mathbf{k} 2} 136(1.15) \boldsymbol{\Phi}_{\mathbf{h}}{ }^{\mathbf{k} 1} 207(2.75) \boldsymbol{i} \\
\boldsymbol{\Phi}_{\mathbf{h}}{ }^{\mathbf{k} 2} 137(1.42) \boldsymbol{\Phi}_{\mathbf{h}} \mathbf{k} 1207(2.88) \boldsymbol{i}\end{array}$ & $\boldsymbol{i} 204(-2.78) \boldsymbol{\Phi}_{\mathbf{h}}{ }^{\mathbf{k} 1} 108(-1.17) \boldsymbol{\Phi}_{\mathbf{h}}^{\mathbf{k} 2}$ \\
\hline 99r-PBI & $\begin{array}{l}\boldsymbol{\Phi}_{\mathbf{c}-\mathbf{k}}^{\mathbf{k}} 157(1.18) \boldsymbol{\Phi}_{\mathbf{h}}^{\mathbf{k} 1} 225(3.97) \boldsymbol{i} \\
\boldsymbol{\Phi}_{\mathbf{c}-\mathbf{0}}^{\mathbf{k}} 155(0.93) \boldsymbol{\Phi}_{\mathbf{h}}^{\mathbf{k} \mathbf{1}} 225(4.08) \boldsymbol{i}\end{array}$ & $\boldsymbol{i} 222(-3.71) \boldsymbol{\Phi}_{\mathbf{h}} \mathbf{k 1} 122(-1.04) \boldsymbol{\Phi}_{\mathbf{c - 0}} \mathbf{k}$ \\
\hline 999-PBI & 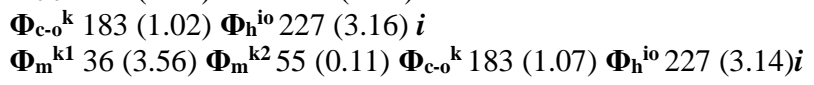 & $\boldsymbol{i} 225(-3.10) \boldsymbol{\Phi}_{\mathbf{h}}{ }^{\mathrm{io}} 175(-1.16) \boldsymbol{\Phi}_{\mathrm{c}-\mathrm{o}}^{\mathrm{k}} 15(-1.05)$ \\
\hline
\end{tabular}

${ }^{a}$ No first order phase transition observed by DSC. Phase notation: $\boldsymbol{\Phi}_{\mathbf{h}}{ }^{\mathbf{k} 1}-$ columnar hexagonal crystalline phase with offset dimers ${ }^{10} ; \boldsymbol{\Phi}_{\mathbf{h}}{ }^{\mathbf{k} 2}-$ columnar hexagonal crystalline phase with cogwheel assembly ${ }^{10} ; \boldsymbol{\Phi}_{\mathbf{h}}{ }^{\mathbf{k} 3}-$ unknown columnar hexagonal crystalline phase; $\boldsymbol{\Phi}_{\mathbf{c}-\mathbf{0}} \mathbf{k}-$ columnar centered orthorhombic crystalline phase; $\boldsymbol{\Phi}_{\mathbf{m}}{ }^{\mathbf{k}}$ - columnar monoclinic crystalline phase; $\boldsymbol{\Phi}_{\mathbf{h}}{ }^{\mathbf{i o}}-2 \mathrm{D}$ columnar hexagonal phase with short range intracolumnar order; $\boldsymbol{\Phi}_{\mathbf{h}}-2 \mathrm{D}$ columnar hexagonal phase; $\boldsymbol{i}$ - isotropic. Note: quantitative uncertainties are $\pm 1^{\circ} \mathrm{C}$ for thermal transition temperatures and $\sim 2 \%$ for the associated enthalpy changes reported in $\mathrm{kcal} / \mathrm{mol}$. 


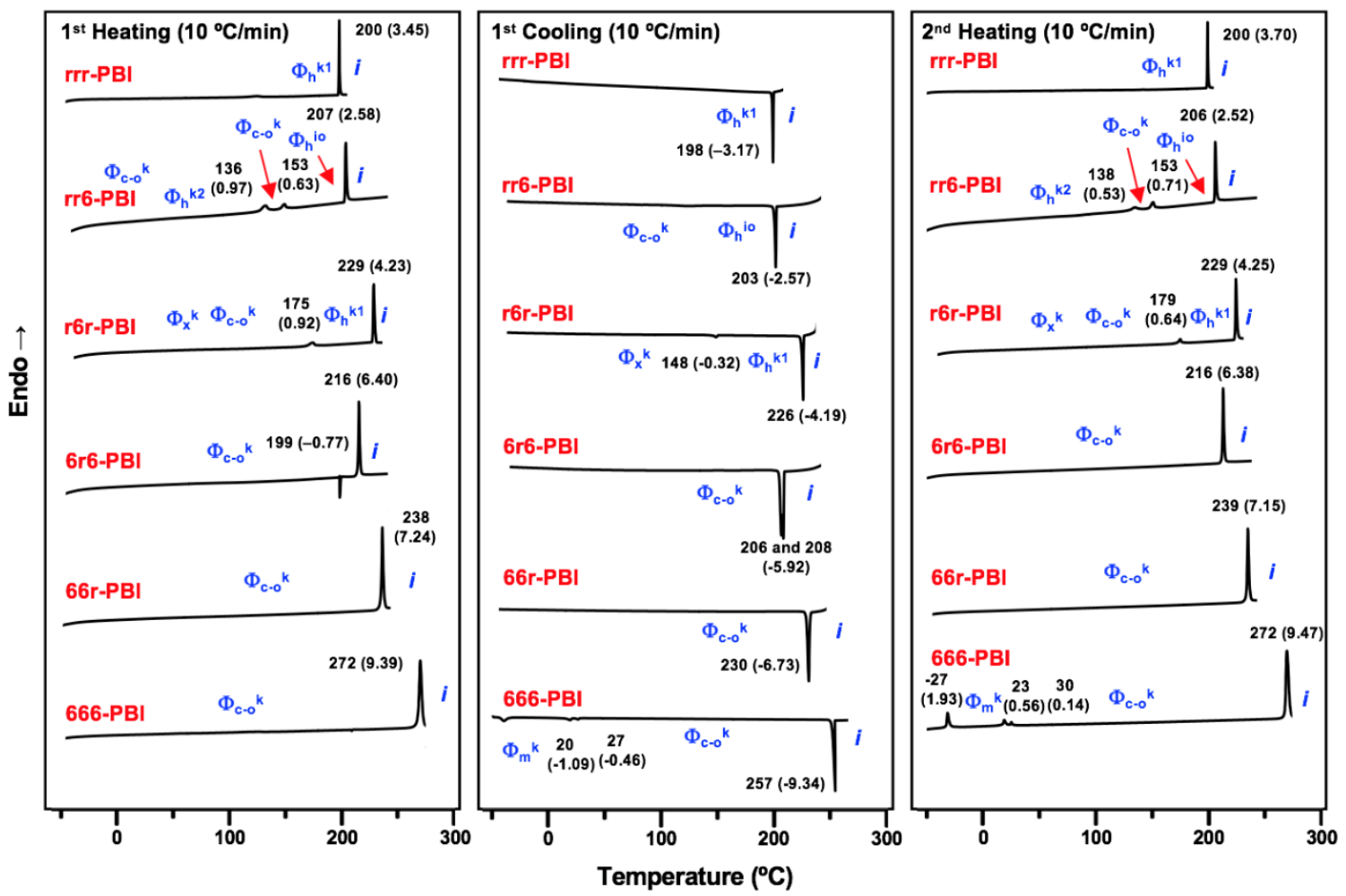

Figure S49. DSC traces of PBIs with sequence-defined hybrid r6 dendrons at heating and cooling rates of $10{ }^{\circ} \mathrm{C} / \mathrm{min}$. Phases determined by fiber XRD, transition temperatures (in ${ }^{\circ} \mathrm{C}$ ), and associated enthalpy changes (in parentheses, in $\mathrm{kcal} / \mathrm{mol}$ ) are indicated.
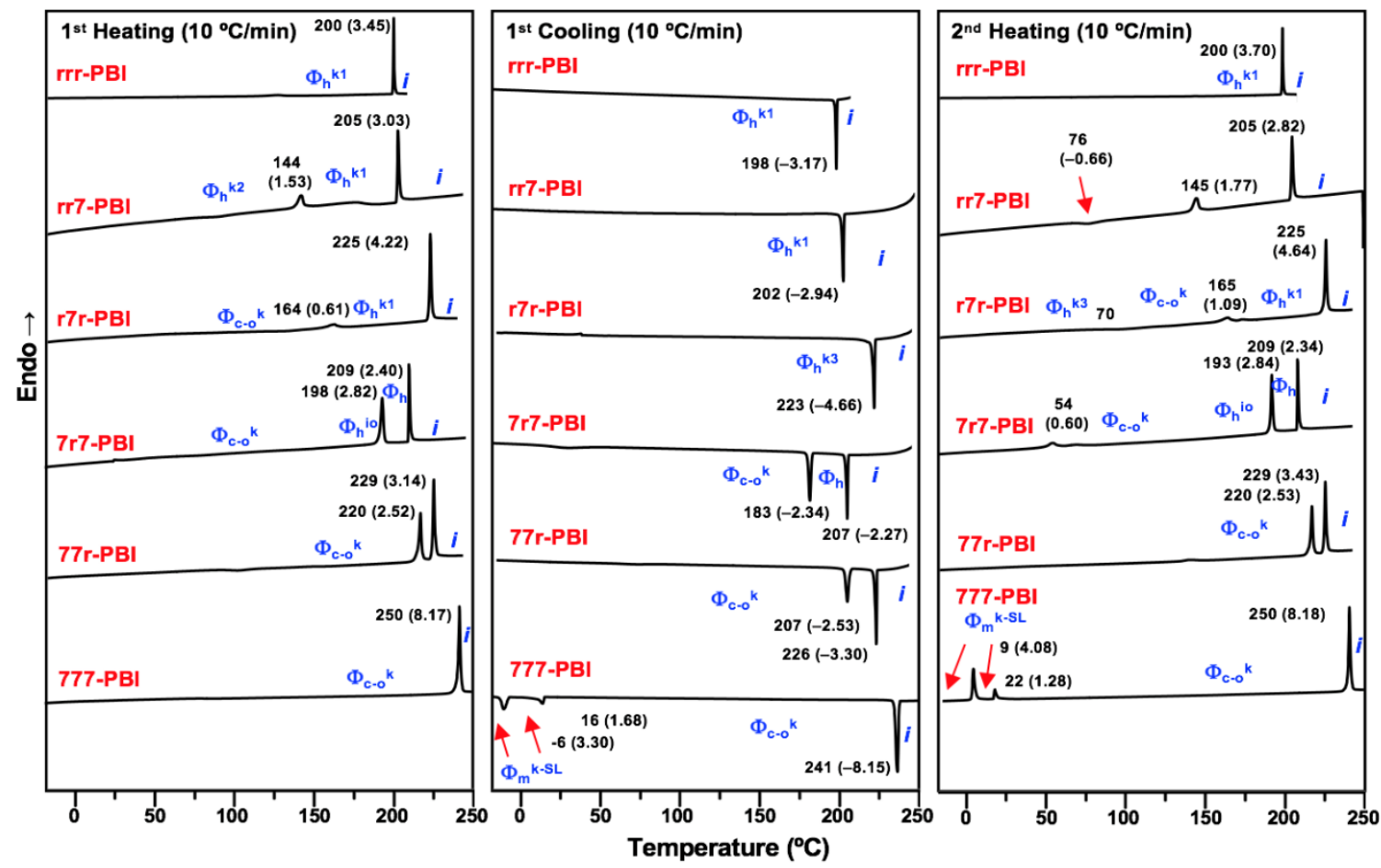

Figure S50. DSC traces of PBIs with sequence-defined hybrid $r 7$ dendrons at heating and cooling rates of $10{ }^{\circ} \mathrm{C} / \mathrm{min}$. Phases determined by fiber XRD, transition temperatures (in ${ }^{\circ} \mathrm{C}$ ), and associated enthalpy changes (in parentheses, in $\mathrm{kcal} / \mathrm{mol}$ ) are indicated. 

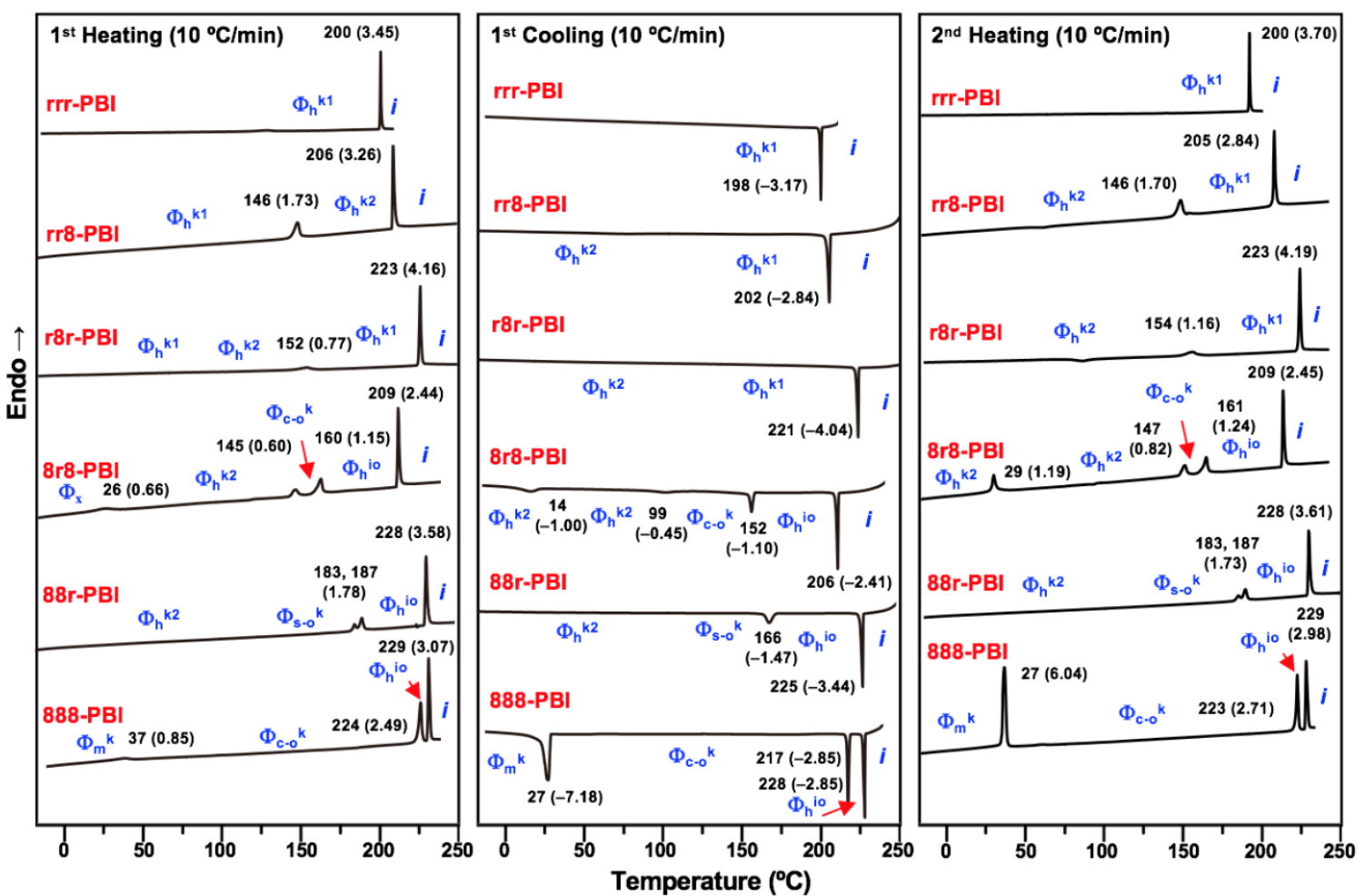

Figure S51. DSC traces of PBIs with sequence-defined hybrid $\mathrm{r} 8$ dendrons at heating and cooling rates of $10{ }^{\circ} \mathrm{C} / \mathrm{min}$. Phases determined by fiber XRD, transition temperatures (in ${ }^{\circ} \mathrm{C}$ ), and associated enthalpy changes (in parentheses, in $\mathrm{kcal} / \mathrm{mol}$ ) are indicated.
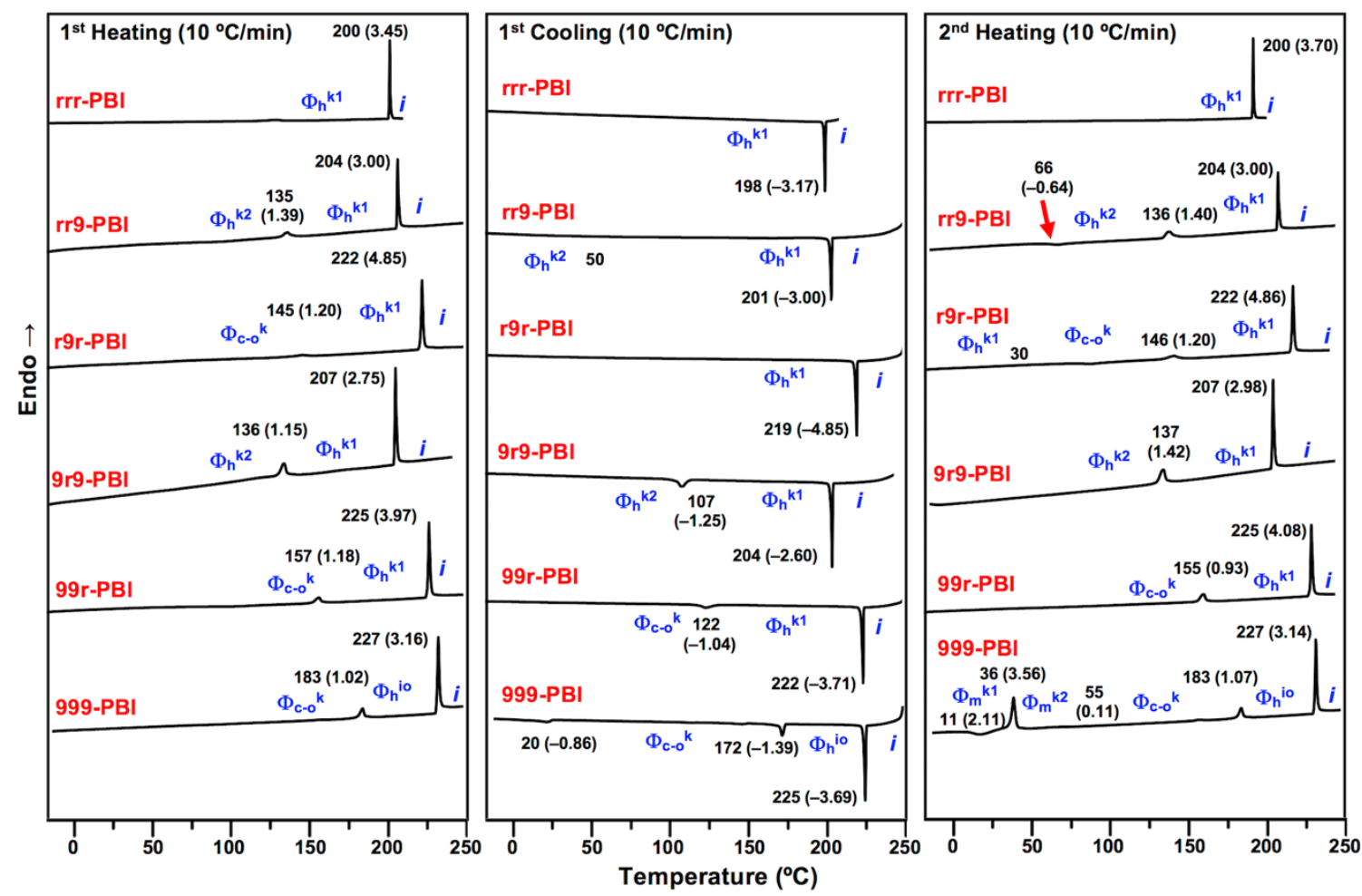

Figure S52. DSC traces of PBIs with sequence-defined hybrid $\mathrm{r} 9$ dendrons at heating and cooling rates of $10{ }^{\circ} \mathrm{C} / \mathrm{min}$. Phases determined by fiber XRD, transition temperatures (in ${ }^{\circ} \mathrm{C}$ ), and associated enthalpy changes (in parentheses, in $\mathrm{kcal} / \mathrm{mol}$ ) are indicated. 


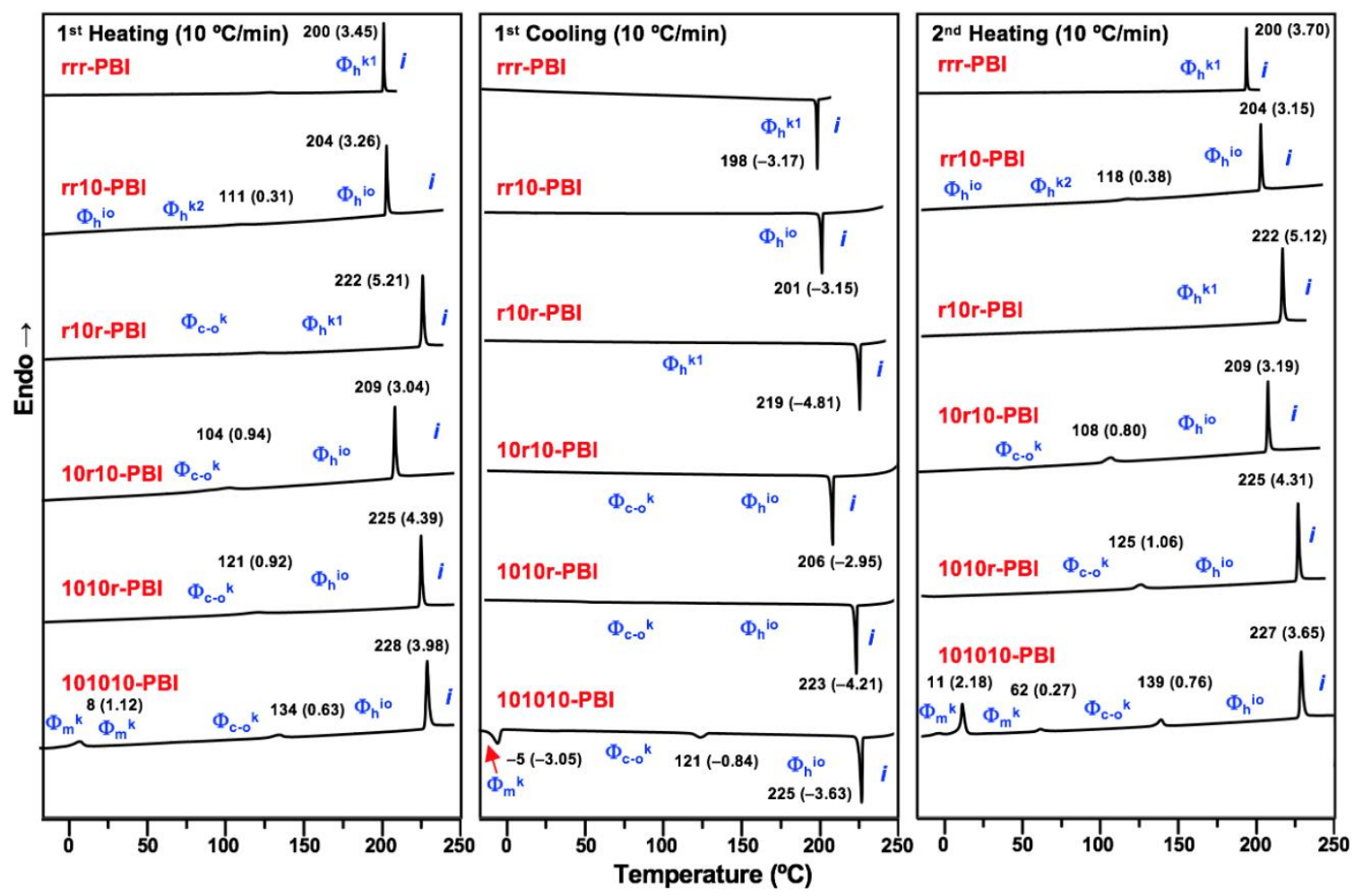

Figure S53. DSC traces of PBIs with sequence-defined hybrid r10 dendrons at heating and cooling rates of $10{ }^{\circ} \mathrm{C} / \mathrm{min}$. Phases determined by fiber XRD, transition temperatures (in ${ }^{\circ} \mathrm{C}$ ), and associated enthalpy changes (in parentheses, in $\mathrm{kcal} / \mathrm{mol}$ ) are indicated. 


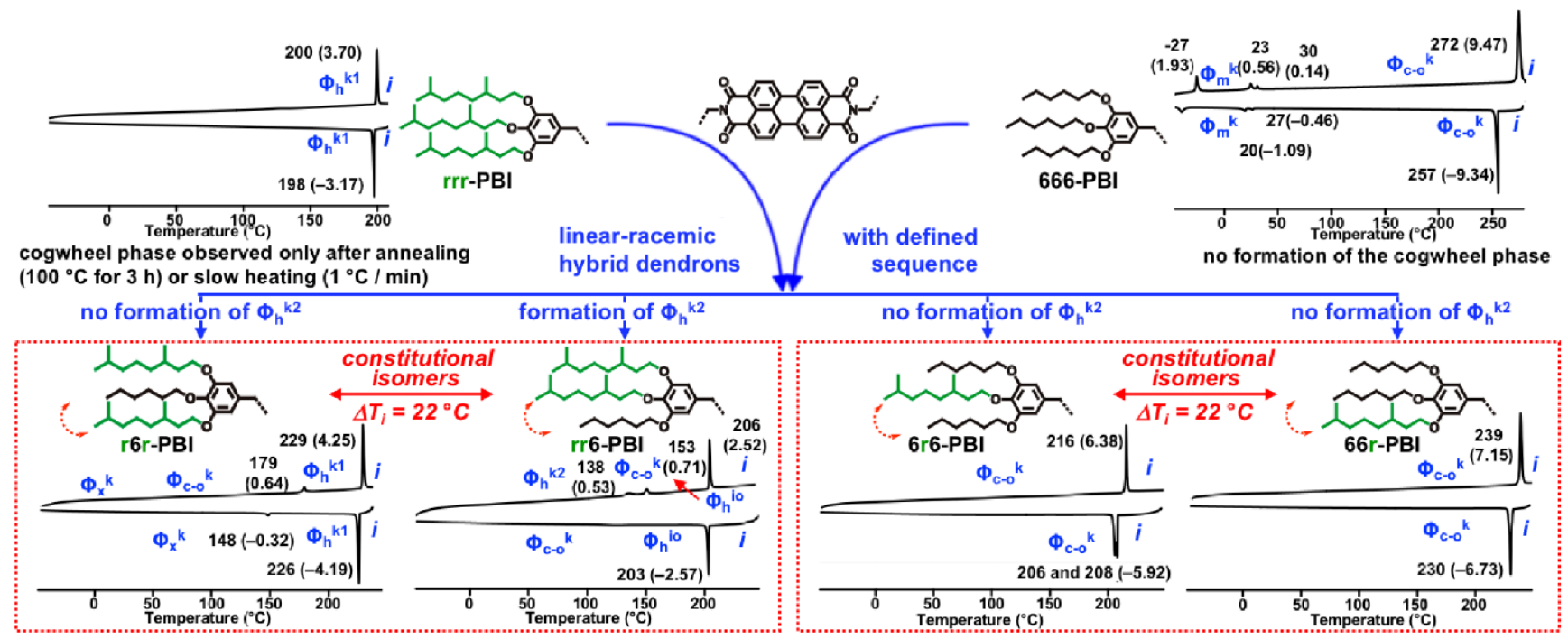

Figure S54. DSC traces of PBIs with sequence-defined hybrid r6 dendrons measured upon second heating and first cooling at $10{ }^{\circ} \mathrm{C} / \mathrm{min}$. Phases determined by fiber $\mathrm{XRD}$, transition temperatures (in ${ }^{\circ} \mathrm{C}$ ), and associated enthalpy changes (in parentheses, in $\mathrm{kcal} / \mathrm{mol}$ ) are indicated. Phase notation: $\Phi_{\mathrm{x}}{ }^{\mathrm{k}}$ unknown columnar crystal phase, $\Phi_{\mathrm{h}}{ }^{\mathrm{k} 1}$ columnar hexagonal crystal with offset dimers, $\Phi_{\mathrm{h}}{ }^{\mathrm{k} 2}$ columnar hexagonal crystal with cogwheel assembly, $\Phi_{\mathrm{c}-\mathrm{o}}{ }^{\mathrm{k}}$ columnar centered orthorhombic crystal, $\Phi_{\mathrm{h}}{ }^{\text {io }} 2 \mathrm{D}$ columnar hexagonal phase with short-range intracolumnar order, $i$ isotropic liquid.

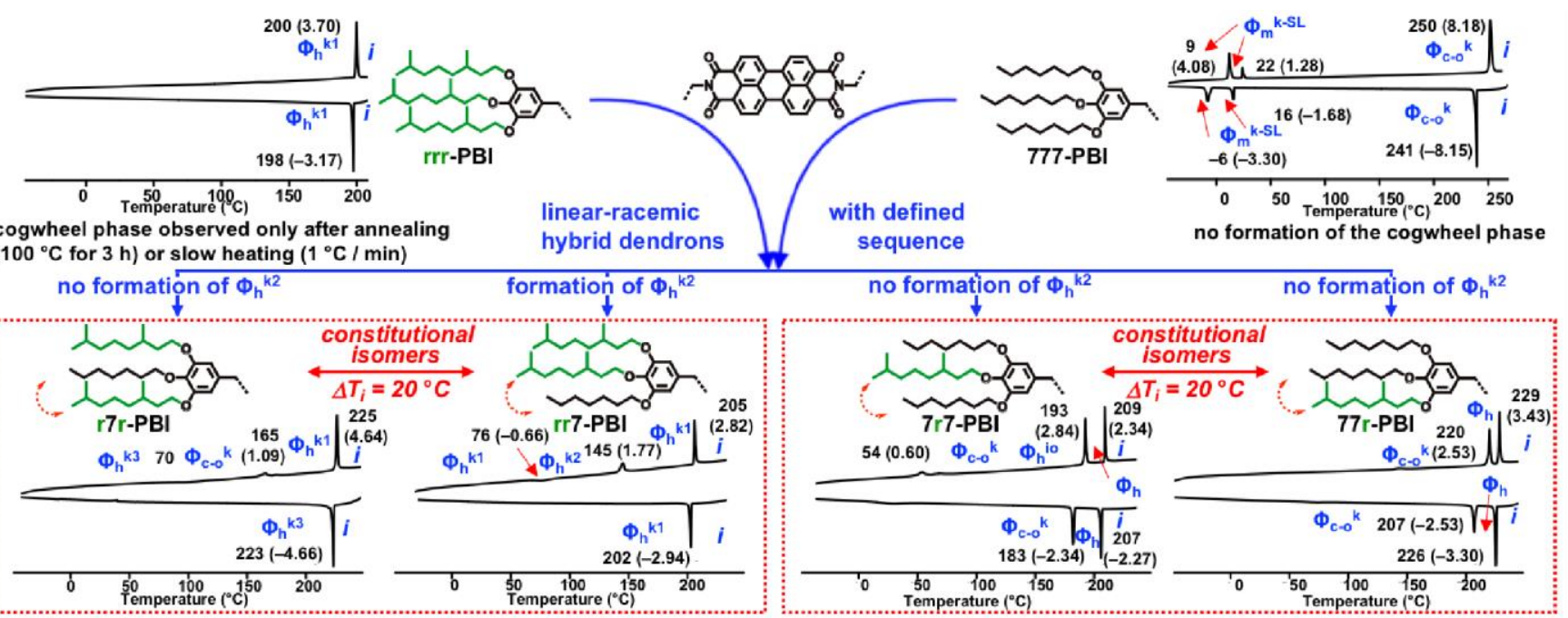

Figure S55. DSC traces of PBIs with sequence-defined hybrid $\mathrm{r} 7$ dendrons measured upon second heating and first cooling at $10^{\circ} \mathrm{C} / \mathrm{min}$. Phases determined by fiber $\mathrm{XRD}$, transition temperatures (in ${ }^{\circ} \mathrm{C}$ ), and associated enthalpy changes (in parentheses, in $\mathrm{kcal} / \mathrm{mol}$ ) are indicated. Phase notation: $\Phi_{\mathrm{h}}{ }^{\mathrm{k} 3}$ unknown columnar hexagonal crystal, $\Phi_{\mathrm{h}}{ }^{\mathrm{k} 1}$ columnar hexagonal crystal with offset dimers, $\Phi_{\mathrm{h}}{ }^{\mathrm{k} 2}$ columnar hexagonal crystal with cogwheel assembly, $\Phi_{\mathrm{c}-\mathrm{o}}{ }^{\mathrm{k}}$ columnar centered orthorhombic crystal, $\Phi_{\mathrm{h}}{ }^{\text {io }} 2 \mathrm{D}$ columnar hexagonal phase with short range intracolumnar order, $\Phi_{\mathrm{h}} 2 \mathrm{D}$ columnar hexagonal phase liquid crystal phase, $i$ isotropic liquid. 


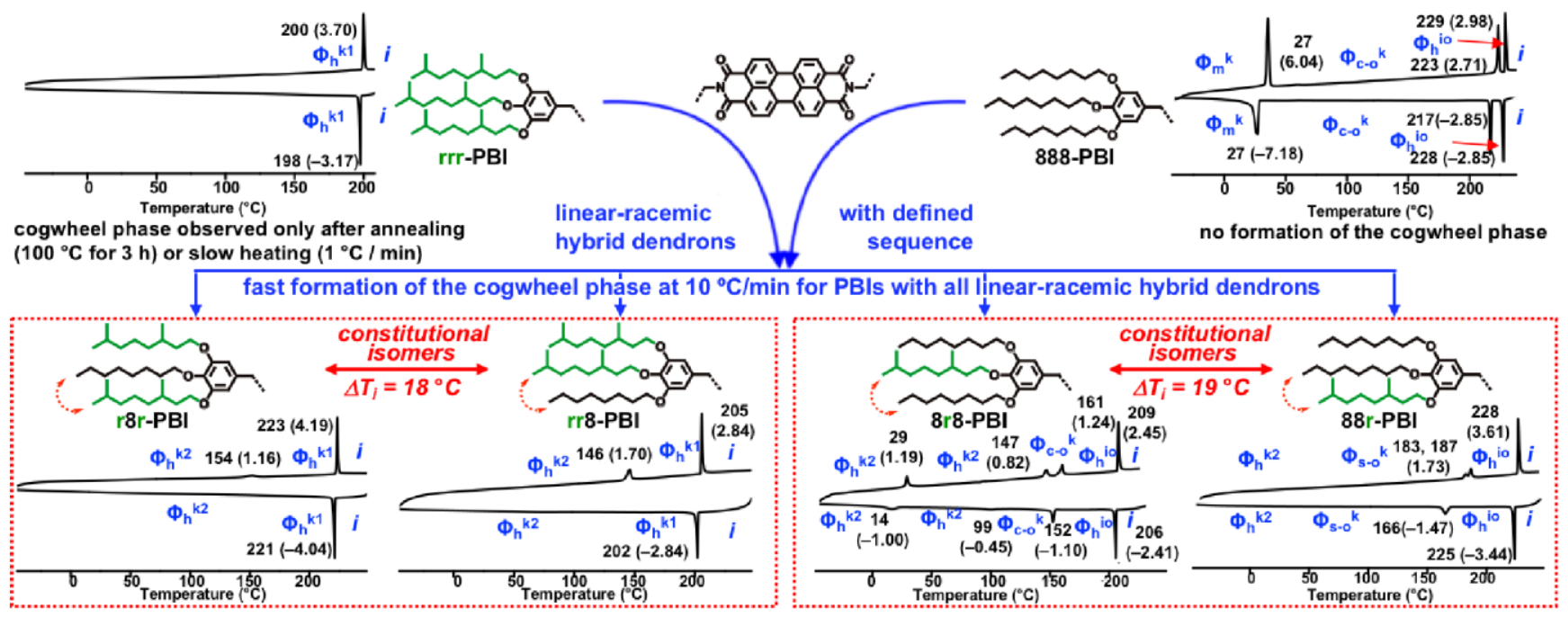

Figure S56. DSC traces of PBIs with sequence-defined hybrid r8 dendrons measured upon second heating and first cooling at $10{ }^{\circ} \mathrm{C} / \mathrm{min}$. Phases determined by fiber $\mathrm{XRD}$, transition temperatures (in ${ }^{\circ} \mathrm{C}$ ), and associated enthalpy changes (in parentheses, in $\mathrm{kcal} / \mathrm{mol}$ ) are indicated. Phase notation: $\Phi_{\mathrm{h}}{ }^{\mathrm{k} 1}$ columnar hexagonal crystal with offset dimers, $\Phi_{\mathrm{h}}{ }^{\mathrm{k} 2}$ columnar hexagonal crystal with cogwheel assembly, $\Phi_{\mathrm{c}-\mathrm{o}}{ }^{\mathrm{k}}$ columnar centered orthorhombic crystal, $\Phi_{\mathrm{h}}{ }^{\text {io }} 2 \mathrm{D}$ columnar hexagonal phase with short-range intracolumnar order, $i$ isotropic liquid.

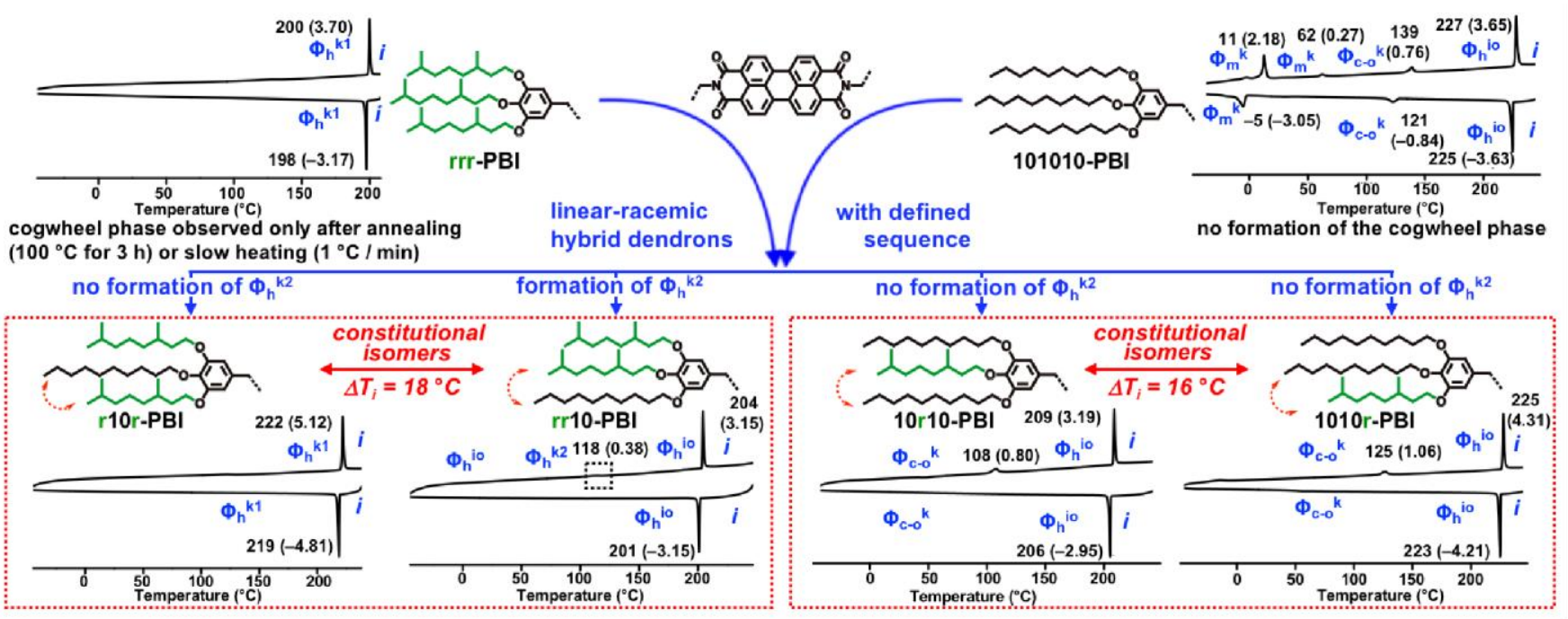

Figure S57. DSC traces of PBIs with sequence-defined hybrid r10 dendrons measured upon second heating and first cooling at $10^{\circ} \mathrm{C} / \mathrm{min}$. Phases determined by fiber $\mathrm{XRD}$, transition temperatures (in ${ }^{\circ} \mathrm{C}$ ), and associated enthalpy changes (in parentheses, in $\mathrm{kcal} / \mathrm{mol}$ ) are indicated. Phase notation: $\Phi_{\mathrm{h}}{ }^{\mathrm{k} 1}$ columnar hexagonal crystal with offset dimers, $\Phi_{\mathrm{h}}{ }^{\mathrm{k} 2}$ columnar hexagonal crystal with cogwheel assembly, $\Phi_{\mathrm{c}-\mathrm{o}}{ }^{\mathrm{k}}$ columnar centered orthorhombic crystal, $\Phi_{\mathrm{h}}{ }^{\text {io }} 2 \mathrm{D}$ columnar hexagonal phase with short-range intracolumnar order, $i$ isotropic liquid. 


\section{Structural Analysis of Sequence-Defined Hybrid PBIs by XRD}

\section{Supporting Table ST2. Structural Analysis of Sequence-Defined Hybrid PBIs by XRD}

\section{$\mathbf{T}$ \\ Phase $^{a} \quad a, b, c(\AA)^{b}$ \\ $d_{100}, d_{110}, d_{200}, d_{210}, d_{300}, d_{220}, d_{310}, d_{400}, d_{320}, d_{410} ; d_{101}, d_{111}, d_{201}(\AA)^{c}$}

$\left({ }^{\circ} \mathbf{C}\right)$

$d_{110}, d_{200}, d_{020}, d_{310}, d_{220}, d_{400}, d_{130} d_{420} ; d_{011}, d_{121}, d_{411}, d_{231} ; d_{002}, d_{112}(\AA)^{d}$

$d_{100}, d_{110}, d_{200}, d_{210}, d_{310}, d_{220}, d_{400}, d_{420}, d_{330}, d_{040} ; d_{011}, d_{201}, d_{411}, d_{002} ; d_{202}, d_{022}(\AA){ }^{e}$

$d_{110}, d_{200}, d_{020}, d_{310}, d_{220}, d_{400}, d_{420}, d_{330} ; d_{001}, d_{01-1}, d_{31-1}, d_{321} ; d_{113}, d_{023}, d_{203}, d_{016}, d_{036}$, $d_{006}(\AA)^{f}$

$d_{10}, d_{11}, d_{20}, d_{21}(\AA)^{g}$

$d_{010}, d_{110}, d_{1-10}, d_{020}, d_{220}, d_{2-20}, d_{240}, d_{4-10}, d_{340}$; do01, $d_{101}, d_{1-11}, d_{121}, d_{2-11}, d_{1-31}, d_{231}$, $\operatorname{do02}(\AA)^{h}$

$d_{100}, d_{020}, d_{120}, d_{200}, d_{220}, d_{2-20}, d_{140}, d_{2-40}, d_{260} ; d_{001}, d_{011}, d_{101}, d_{031}, d_{002}, d_{112}, d_{122}(\AA)^{i}$

\begin{tabular}{|c|c|c|c|}
\hline rrr-PBI & 70 & $\overline{\Phi_{h}}{ }^{k 1}$ & $28.5,28.5,26$ \\
\hline \multirow[t]{2}{*}{ rr7-PBI } & 100 & $\Phi_{h}^{k 2}$ & $26.8,26.8,14$ \\
\hline & 150 & $\Phi_{h}^{k 1}$ & $28.5,28.5$, \\
\hline \multirow[t]{3}{*}{ r7r-PBI } & 100 & $\Phi_{c-0} k$ & $43.4,27.7,1$ \\
\hline & 180 & $\Phi_{h}{ }^{k 1}$ & $28.5,28.5,27$ \\
\hline & 100 & $\Phi_{h}{ }^{k 3}$ & $28.3,28.3,47$ \\
\hline \multirow[t]{3}{*}{ 7r7-PBI } & 100 & $\Phi_{c-0} k$ & $38.8,28.8,14$ \\
\hline & 190 & $\Phi_{h}{ }^{\text {io }}$ & $27.7,27.7,3$. \\
\hline & 205 & $\Phi_{\mathrm{h}}$ & $27.7,27.7,-$ \\
\hline \multirow[t]{2}{*}{ 77r-PBI } & 100 & $\Phi_{c-0} k$ & $40.3,27.7,14$ \\
\hline & 224 & $\Phi_{h}$ & $29.0,29.0,-$ \\
\hline \multirow[t]{2}{*}{ 777-PBI } & 35 & $\Phi_{\mathrm{c}-\mathbf{0}} \mathbf{k}^{-}$ & $36.5,28.8,14$ \\
\hline & 0 & $\Phi_{m}^{k-S L}$ & $35.8,30.4,44$ \\
\hline
\end{tabular}

rr9-PBI $\quad 100 \quad \boldsymbol{\Phi}_{\mathbf{h}}{ }^{\mathbf{k} 2} \quad 27.0,27.0,14.5$

$150 \quad \boldsymbol{\Phi}_{\mathbf{h}}{ }^{\mathbf{k} 1} \quad 28.5,28.5,26.6$

r9r-PBI $100 \quad \boldsymbol{\Phi}_{\mathbf{c - 0}}{ }^{k} \quad 45.4,28.3,14.6$

$160 \quad \Phi_{\mathbf{h}} \mathbf{k} 1 \quad 29.4,29.4,27.0$

9r9-PBI $100 \quad \boldsymbol{\Phi}_{\mathbf{h}}{ }^{\mathbf{k} 2} \quad 26.7,26.7,14.6$

$150 \quad \boldsymbol{\Phi}_{\mathbf{h}}{ }^{\mathbf{k} 1} \quad 28.3,28.3,27.3$

99r-PBI $100 \quad \boldsymbol{\Phi}_{\mathbf{c - 0}} \mathbf{k} \quad 44.6,27.7,14.8$

$170 \quad \boldsymbol{\Phi}_{\mathbf{h}}{ }^{\mathbf{k} 1} \quad 28.8,28.8,26.9$

999-PBI $215 \quad \boldsymbol{\Phi}_{\mathbf{h}}{ }^{\text {io }} \quad 29.3,29.3$, -

$125 \quad \boldsymbol{\Phi}_{\mathrm{c}-0} \mathbf{k} \quad 40.5,30.8,14.3$

$45 \quad \boldsymbol{\Phi}_{\mathbf{m}}{ }^{\mathbf{k} 2} \quad 32.0,37.9,14.3$

$25 \quad \boldsymbol{\Phi}_{\mathbf{m}}{ }^{\mathbf{k} 1} \quad 25.8,47.3,14.2$ $24.7,14.5,12.3,9.4,-,-,-,-,-,-;-,-,-^{c}$

$23.2,13.4,11.6,8.8,7.7,6.7,6.4,5.8,5.3,5.1 ; 12.3,9.8,9.1^{c}$

$24.7,14.3,12.3,9.3,-,-,-,-,-,-;-,-,-^{c}$

$23.4,21.7,13.8,12.8,11.7,10.8,9.0,8.5 ; 12.6,9.8^{d}$

$24.4,14.1,12.2,9.2,-,-,-,-,-,-;-,-,-^{c}$

$24.3,14.0,12.2,9.2,-,-,-,-,-,-; 21.7,13.6,11.9^{c}$

$23.0,19.3,14.4,11.7,11.5,9.7,9.3,8.0 ; 12.9,9.8,7.7,7.4^{d}$

23.6, 13.6, $11.8,8.9^{g}$

$23.7,13.7,11.9,-,-,-,-,-,-,-;-,-,-^{c}$

$22.8,20.2,13.9,12.1,11.4,10.1,9.0,8.2 ; 12.7,9.7,7.9,7.2^{d}$

$25.0,14.1,12.2,-,-,-,-,-,-,-;-,-,-^{c}$

$22.6,18.3,14.4,11.2,11.3,9.1,7.7,7.5 ; 13.1,10.6,9.9,8.0^{d}$

$22.2,17.9,14.2,11.0,11.1,9.0,7.6,7.4 ; 40.1,19.5,10.3,9.4 ; 13.5,12.3,10.9,7.3,6.9$, $6.8^{f}$

$23.4,13.5,11.7,8.8,7.8,6.7,6.5,5.8 ; 12.3,9.9,9.1^{c}$

$24.7,14.3,12.4,9.3,-,-,-,-,-,-;-,-,-^{c}$

$24.0,22.7,14.2,13.3,12.0,11.3,9.3,8.9 ; 12.9,9.8,8.3,7.3^{d}$

25.3, 14.6, 12.6, 9.6,-,-,-,-,-,-; -, -, $-^{c}$

$23.1,13.3,11.6,8.7,7.7,6.7,6.4,5.9 ; 12.3,9.9,9.1^{c}$

$24.5,14.1,12.3,9.3,-,-,-,-,-,-;-,-,-^{c}$

$23.5,22.2,13.9,13.0,11.8,11.1,9.0,8.7 ; 13.1,9.9,8.5,7.4^{d}$

24.7, 14.3, 12.3, 9.3,-,-,-,-,-, -; -, -, $-^{c}$

$25.9,14.6,12.7,9.6^{g}$

$40.5,24.5,20.3,16.9,15.4,-, 12.3,-, 8.5,8.2,7.7 ; 13.0,11.7,10.5,8.0,7.2,6.7,6.5^{e}$

$37.8,25.2,23.7,18.9,12.6,11.8,8.4,7.7,7.3 ; 14.3,13.1,12.2,10.9,10.1,9.0,8.3,-{ }^{h}$ $25.6,23.5,18.4,12.8,11.9,10.7,11.2,-, 7.1,-; 13.6,12.4,-, 7.1,6.8,-, 6.6^{i}$

\footnotetext{
${ }^{a}$ Phase notation: $\Phi_{\mathrm{h}}{ }^{\mathrm{k} 1}-$ columnar hexagonal crystalline phase with offset dimers; $\Phi_{\mathrm{h}}{ }^{\mathrm{k} 2}-$ columnar hexagonal crystalline phase with cogwheel assembly; $\Phi_{\mathrm{h}}{ }^{\mathrm{k} 3}-$ unknown columnar hexagonal crystalline phase; $\Phi_{\mathrm{h}}{ }^{\mathrm{io}}-2 \mathrm{D}$ columnar hexagonal phase with short range intracolumnar order; $\Phi_{\mathrm{c}-\mathrm{o}}{ }^{\mathrm{k}}$ - columnar centered orthorhombic crystalline phase; $\Phi_{\mathrm{m}}{ }^{\mathrm{k}}-$ columnar monoclinic crystalline phase. ${ }^{b}$ Lattice parameters determined from fiber X-ray diffraction as follows: for hexagonal phases, $d_{h k l}=\left[4\left(h^{2}+k^{2}+h k\right) /\left(3 a^{2}\right)+(1 / c)^{2}\right]^{-1 / 2}$; for orthorhombic phases, $d_{h k l}=\left[(h / a)^{2}\right.$ $\left.+(\mathrm{k} / \mathrm{b})^{2}+(\mathrm{l} / \mathrm{c})^{2}\right]^{-1 / 2}$; for monoclinic phases: $\mathrm{dhkl}_{\mathrm{k}}=\left[(\mathrm{h} / \mathrm{a} \sin \gamma)^{2}+(\mathrm{k} / \mathrm{b} \sin \gamma)^{2}+(\mathrm{l} / \mathrm{c})^{2}-\left\{(2 \mathrm{hk} \cos \gamma) /\left(\mathrm{ab} \sin ^{2} \gamma\right)\right\}\right]^{-1 / 2}$. $^{c}$ Experimental diffraction peak d-spacings for the $\Phi_{\mathrm{h}}{ }^{\mathrm{k} 1}$ and $\Phi_{\mathrm{h}}{ }^{\mathrm{k} 2}$ phases. ${ }^{d}$ Experimental diffraction peak d-spacings for the $\Phi_{\mathrm{c}-\mathrm{o}}{ }^{\mathrm{k}}$ phase. ${ }^{e}$ Experimental diffraction peak d-spacings for the $\Phi_{\mathrm{h}}{ }^{\text {io }}$ phase. ${ }^{f}$ Experimental diffraction peak d-spacings for the $\Phi_{\mathrm{m}}{ }^{\mathrm{k}}$ phase. ${ }^{h}$ Data taken from ref. ${ }^{l} . *$ indicates overlapping features in XRD pattern.
} 
Supporting Table ST3. Retrostructural Analysis of Sequence-Defined Hybrid Linear-Racemic PBIs

\begin{tabular}{|c|c|c|c|c|c|c|c|c|}
\hline & $\begin{array}{c}\mathbf{T} \\
\left({ }^{\circ} \mathbf{C}\right)\end{array}$ & Phase $^{a}$ & $a, b, c(\AA)^{b}$ & $\mathrm{D}_{\text {col }}(\AA)^{c}$ & $t(\AA)^{d}$ & $\rho^{e}\left(\mathrm{~g} / \mathrm{cm}^{3}\right)$ & $\mathbf{M}_{\mathrm{wt}}{ }^{f}(\mathrm{~g} / \mathrm{mol})$ & $\mu^{g}$ \\
\hline rrr-PBI & 70 & $\Phi_{h}^{k 1}$ & $28.5,28.5,26.3$ & 28.5 & 3.3 & 1.05 & 1508.22 & 1 \\
\hline \multirow[t]{2}{*}{ rr7-PBI } & 100 & $\Phi_{h}{ }^{k 2}$ & $26.8,26.8,14.5$ & 26.8 & 3.6 & 1.05 & 1422.97 & 1 \\
\hline & 150 & $\Phi_{h}^{k 1}$ & $28.5,28.5,27.2$ & 28.5 & 3.4 & & & 1 \\
\hline \multirow[t]{3}{*}{ r7r-PBI } & 100 & $\Phi_{c-0}^{k}$ & $43.4,27.7,14.5$ & 25.7 & 3.6 & 1.04 & 1422.97 & 1 \\
\hline & 180 & $\Phi_{h}{ }^{k 1}$ & $28.5,28.5,27.3$ & 28.5 & 3.4 & & & 1 \\
\hline & 100 & $\Phi_{h}{ }^{k 3}$ & $28.3,28.3,47.1$ & 28.3 & 3.4 & & & 1 \\
\hline \multirow[t]{3}{*}{ 7r7-PBI } & 100 & $\Phi_{c-0} k$ & $38.8,28.8,14.4$ & 24.0 & 3.6 & 1.06 & 1338.88 & 1 \\
\hline & 190 & $\Phi_{h}{ }^{\text {io }}$ & $27.7,27.7,3.5$ & 27.7 & 3.5 & & & 1 \\
\hline & 205 & $\Phi_{h}$ & $27.7,27.7,-$ & 27.7 & - & & & 1 \\
\hline \multirow[t]{2}{*}{ 77r-PBI } & 100 & $\Phi_{c-0} k$ & $40.3,27.7,14.3$ & 24.4 & 3.6 & 1.07 & 1338.88 & 1 \\
\hline & 224 & $\Phi_{\mathrm{h}}$ & $29.0,29.0,-$ & 29.0 & & & & 1 \\
\hline \multirow[t]{2}{*}{ 777-PBI } & 35 & $\Phi_{c-0} k$ & $36.5,28.8,14.7$ & 23.2 & 3.7 & 1.09 & 1255.71 & 1 \\
\hline & 0 & $\Phi_{m}^{k-S L}$ & $35.8,30.4,44.1$ & 23.5 & 3.5 & & & 1 \\
\hline \multirow{2}{*}{ rr9-PBI } & 100 & $\Phi_{h}{ }^{k 2}$ & $27.0,27.0,14.5$ & 27.0 & 3.6 & 1.07 & 1479.04 & 1 \\
\hline & 150 & $\Phi_{h}^{k 1}$ & $28.5,28.5,26.6$ & 28.5 & 3.3 & & & 1 \\
\hline \multirow[t]{2}{*}{ r9r-PBI } & 100 & $\Phi_{c-0} k$ & $45.4,28.3,14.6$ & 26.7 & 3.6 & 1.06 & 1479.04 & 1 \\
\hline & 160 & $\Phi_{h}^{k 1}$ & $29.4,29.4,27.0$ & 29.4 & 3.4 & & & 1 \\
\hline \multirow[t]{2}{*}{ 9r9-PBI } & 100 & $\Phi_{h}{ }^{k 2}$ & $26.7,26.7,14.6$ & 26.7 & 3.6 & 1.05 & 1451.00 & 1 \\
\hline & 150 & $\Phi_{h}{ }^{k 1}$ & $28.3,28.3,27.3$ & 28.3 & 3.4 & & & 1 \\
\hline \multirow[t]{2}{*}{ 99r-PBI } & 100 & $\Phi_{c-0} k$ & $44.6,27.7,14.8$ & 26.6 & 3.7 & 1.05 & 1451.00 & 1 \\
\hline & 170 & $\Phi_{h}{ }^{k 1}$ & $28.8,28.8,26.9$ & 28.8 & 3.4 & & & 1 \\
\hline \multirow[t]{4}{*}{ 999-PBI } & 215 & $\Phi_{h}{ }^{\text {io }}$ & $29.3,29.3,-$ & 29.3 & - & 1.06 & 1424.02 & 1 \\
\hline & 125 & $\Phi_{c-0} k$ & $40.5,30.8,14.3$ & 25.4 & 3.6 & & & 1 \\
\hline & 45 & $\Phi_{m}^{k 2}$ & $32.0,37.9,14.3$ & 24.8 & 3.6 & & & 1 \\
\hline & 25 & $\Phi_{m}^{k 1}$ & $25.8,47.3,14.2$ & 25.8 & 3.6 & & & 1 \\
\hline
\end{tabular}

${ }^{a}$ Phase notation: $\boldsymbol{\Phi}_{\mathbf{h}}{ }^{\mathbf{k} 1}$ - columnar hexagonal crystalline phase with offset dimers; $\boldsymbol{\Phi}_{\mathbf{h}}{ }^{\mathbf{k} 2}$ - columnar hexagonal crystalline phase with cogwheel assembly; $\boldsymbol{\Phi}_{\mathbf{c}-\mathbf{0}} \mathbf{k}-$ columnar centered orthorhombic crystalline phase; $\boldsymbol{\Phi}_{\mathbf{h}}{ }^{\mathbf{i}}-2 \mathrm{D}$ columnar hexagonal phase with short range intracolumnar order; $\boldsymbol{\Phi}_{\mathbf{m}} \mathbf{k}$ - columnar monoclinic crystalline phase. ${ }^{b}$ Lattice parameters determined from fiber and powder X-ray diffraction as follows: for hexagonal phases, $d_{\mathrm{hkl}}=\left[4\left(h^{2}+k^{2}+h k\right) /\left(3 a^{2}\right)+(l / c)^{2}\right]^{-1 / 2}$; for orthorhombic phases, $d_{\mathrm{hkl}}=\left[(h / a)^{2}+(k / b)^{2}+(l / c)^{2}\right]^{-1 / 2}$; for monoclinic phases: $d_{\mathrm{hkl}}=\left[(h / a \sin \gamma)^{2}+(k / b \sin \gamma)^{2}+(l / c)^{2}-\left\{(2 h k \cos \gamma) /\left(a b \sin ^{2} \gamma\right)\right\}\right]^{-1 / 2} \cdot{ }^{c}$ Column diameter calculated using: $\mathrm{D}_{\mathrm{col}}=a$ for $\boldsymbol{\Phi}_{\mathbf{h}}{ }^{\mathbf{i o}}$ and $\boldsymbol{\Phi}_{\mathbf{h}}{ }^{\mathbf{k}}$, and $\mathrm{D}_{\mathrm{col}}=a /\left[2 \cos \left(\tan ^{-1} b / a\right)\right]$ for $\boldsymbol{\Phi}_{\mathbf{c}-\mathbf{o}}{ }^{\mathbf{k}} .{ }^{d}$ Stratum thickness calculated from the meridional pattern. ${ }^{e}$ Experimental density measured at 23 ${ }^{\circ} \mathrm{C} .{ }^{f}$ Molecular weight of the compound. ${ }^{g}$ Average number of dendrimers forming the supramolecular column stratum, calculated using: $\mu$ $=\left(\mathrm{N}_{\mathrm{A}} \cdot \rho \cdot \mathrm{A} \cdot t\right) \cdot\left(2 \mathrm{M}_{\mathrm{wt}}\right)^{-1}$, where $\mathrm{N}_{\mathrm{A}}=6.022 \times 10^{23} \mathrm{~mol}^{-1}$, A is the unit cell area of the $a b$-plane, and $t$ is the average stratum thickness calculated from the meridional pattern.

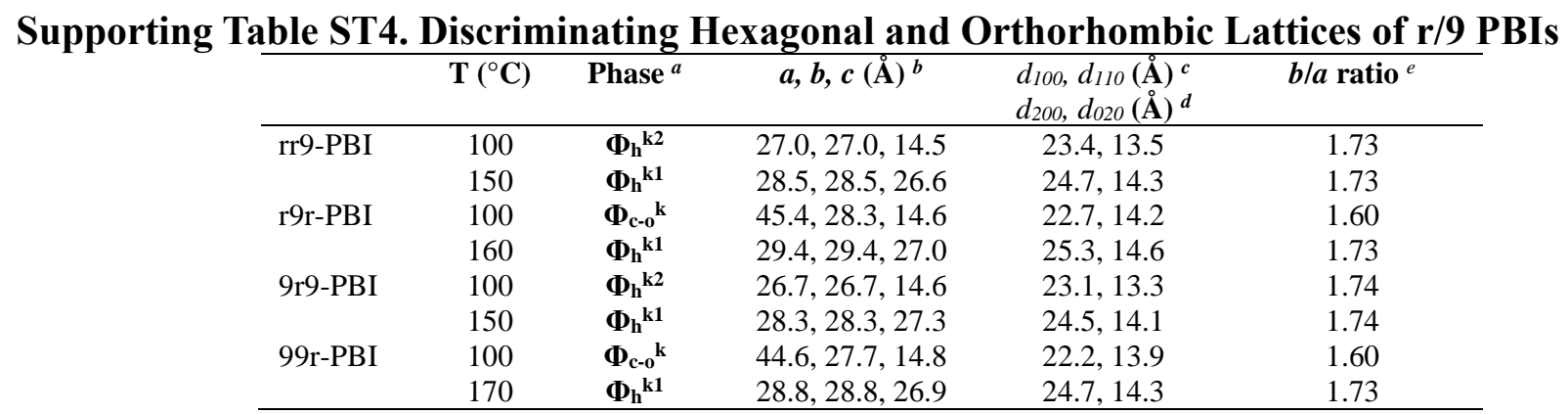

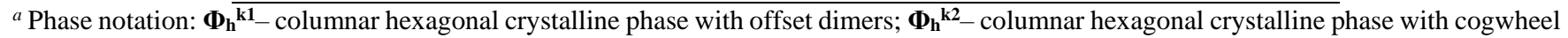
assembly; $\boldsymbol{\Phi}_{\mathbf{c}-\mathbf{0}} \mathbf{k}_{-}$columnar centered orthorhombic crystalline phase. ${ }^{b}$ Lattice parameters determined from fiber and powder X-ray diffraction as follows: for hexagonal phases, $d_{\mathrm{hkl}}=\left[4\left(h^{2}+k^{2}+h k\right) /\left(3 a^{2}\right)+(l / c)^{2}\right]^{-1 / 2}$; for orthorhombic phases, $d_{\mathrm{hkl}}=\left[(h / a)^{2}+(k / b)^{2}+(l / c)^{2}\right]^{-1 / 2}{ }^{c}$ Experimental diffraction peak d-spacings for the $\Phi_{\mathrm{h}}{ }^{\mathrm{k} 1}$ and $\Phi_{\mathrm{h}}{ }^{\mathrm{k} 2}$ phases. ${ }^{d}$ Experimental diffraction peak d-spacings for the $\Phi_{\mathrm{c}-\mathrm{o}}{ }^{\mathrm{k}}$ phase. ${ }^{e} b / a$ ratio of a theoretical orthorhombic cell determined as follows: for hexagonal phases, $d_{100} / d_{110}$; for orthorhombic phases, $d_{200} / d_{020}$. In the case where $b / a=\sqrt{3}(\approx 1.73)$, the orthorhombic cell is equivalent to a hexagonal cell. 


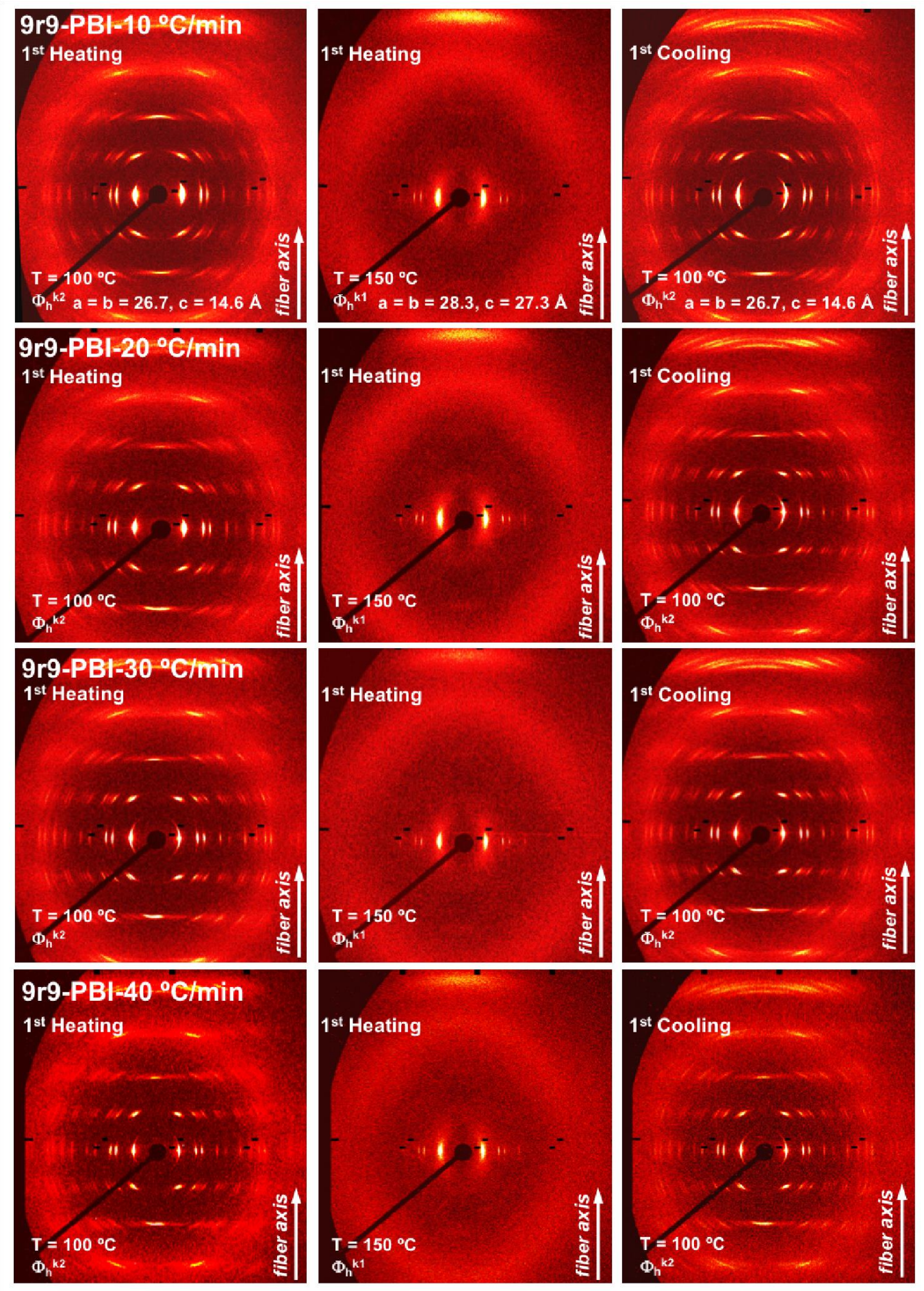

Figure S58. Fiber XRD of the cogwheel 9r9-PBI measured upon heating and cooling at 10 to $50{ }^{\circ} \mathrm{C} / \mathrm{min}$ in the $\Phi_{\mathrm{h}}{ }^{\mathrm{k} 2}$ and $\Phi_{\mathrm{h}}{ }^{\mathrm{k} 1}$ phases. Transition temperatures (in ${ }^{\circ} \mathrm{C}$ ) and heating and cooling rates indicated. 

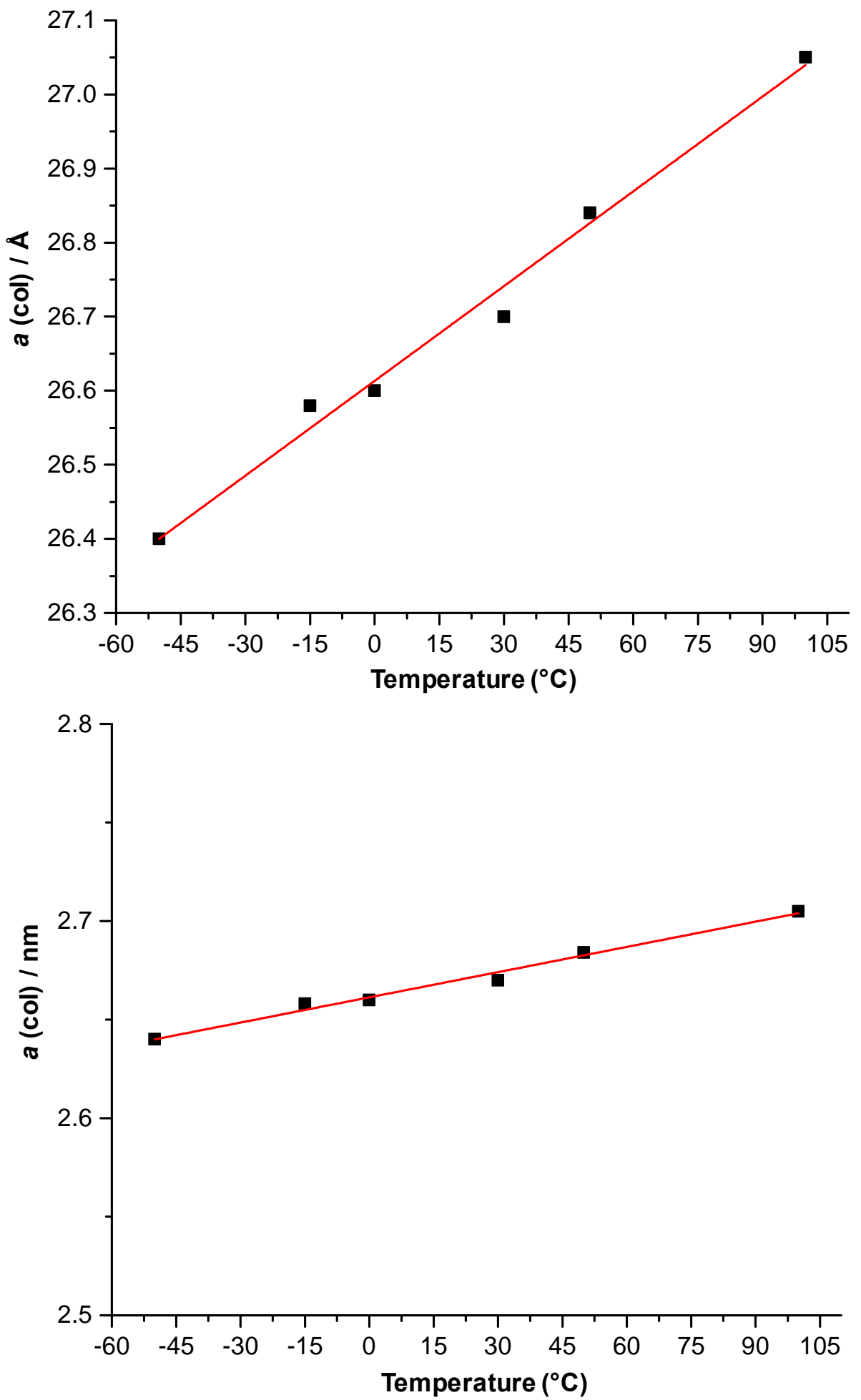

Figure S59. Temperature dependence of the unit cell parameter a in the $\Phi_{\mathrm{h}}{ }^{\mathrm{k} 2}$ of $\operatorname{rr} 9-\mathrm{PBI}$. Top in Angstroms, bottom in $\mathrm{nm}$. 


\section{Further Discussion of Solid State NMR Studies}

As the cogwheel phases have only been observed for PBI derivatives with $(3,7)$ dimethyl octane $(\operatorname{dm} 8)$ side chains independent of the enantiomeric form of $\mathrm{dm} 8$, the conformation behavior of the branching point closest to the phenyl ring should be particularly informative for understanding the local side chain organization in the columnar packing of the cogwheel phases. Unfortunately, the signal of this $\mathrm{CH}$ branching site overlaps with signals from the central part of linear chains and therefore cannot be observed unambiguously. However, the signals of $\mathrm{CH}_{2}$ sites next to the branching sites in the $\mathrm{dm} 8$ main chain as well as the signal of the methyl branch closer to the phenyl ring are spectrally resolved and can be monitored selectively. In Figure 6, colored labels show the assignment of dm8 sites to spectral features in the ${ }^{13} \mathrm{C}$ CP-MAS NMR spectra acquired at $60^{\circ} \mathrm{C}$ sample temperature. Figure $6 \mathrm{a}$ ) shows spectra of PDI derivatives with $\mathrm{dm} 8$ side chains in meta position. The ${ }^{13} \mathrm{C} \mathrm{NMR}$ signal of the $\mathrm{CH}_{2}$ group next to the branching site of the two terminal methyl groups forms a relatively sharp peak at 40 ppm with a shoulder at $40.4 \mathrm{ppm}$, indicating that the conformational averaging is quite efficient towards the chain ends of the dm8 side chains. In contrast, the signals of the $\mathrm{CH}_{2}$ groups next to the other branching site as well as the methyl branch spreads out over 3-4 ppm (18 - $22 \mathrm{ppm})$ with several spectral features. The signal of the methyl branch splits into 3 peaks, a most intense central peak at $20.2 \mathrm{ppm}$ and two weaker side peaks at 20.7 and 19.4 ppm of similar intensity. The signals of the $\mathrm{CH}_{2}$ groups next to the central branching site are overlapping and show a much more complex line shape, where an analysis in terms of separated broadened lines is not appropriate. Here it is important to note, that this line shape originates from a distribution of isotropic chemical shifts for these two sites resulting from the conformational distribution of the dm8 side chain attached in meta-position at the outer phenyl ring of the PBI derivative. The difference spectrum of the 99r-PBI and the r9r-PBI, weighting the spectra with the number of dm8 per molecule, given Figure 6a) in red color. demonstrates that the conformational distribution of the $\operatorname{dm} 8$ side chains attached to the outer phenyl ring in meta-position does not change when there are one or two dm 8 side chains attached to the phenyl ring in meta-position. Only the methyl-signal of the C9 side chains remain, because the weighting is different.

For $\mathrm{dm} 8$ side chains attached in para position, the observed ${ }^{13} \mathrm{C}$ MAS NMR signals are relatively narrow peaks as shown in the middle row of Figure 6b) for 9r9-PBI. The signals of the $\mathrm{CH}_{2}$ group next to the terminal branching point at $40.3 \mathrm{ppm}$ as well as the signal of the central methyl branch at $20.2 \mathrm{ppm}$ have both weak high field shoulder, whereas the signal of the two $\mathrm{CH}_{2}$ groups next to the central branching point are observed as single narrow peak at $38.1 \mathrm{ppm}$. The narrow peak could indicate a rapid conformational averaging coupled with pronounced local fluctuations. However, studies of site selective residual hetero-nuclear dipolar couplings do not confirm the locally increased molecular mobility in this region of the $\mathrm{dm} 8$ side chain, but provide the expected picture with the highest molecular mobility at the 
chain ends of the side chains. Therefore, the narrow signal of the $\mathrm{CH}_{2}$ groups next to the central branching site indicates a remarkably high conformational order of the $\mathrm{dm} 8$ side chains in the 9r9-PBI. This high conformational order of the dm8 chains attached in para position is present not only in 9r9-PBI. In 9rrPBI similar sharp features are observed for the signals assigned to the spectrally isolated main chain $\mathrm{CH}_{2}$ groups. The dashed red line in the top spectrum of Figure $6 \mathrm{~b}$ ) shows the sum spectrum of the ${ }^{13} \mathrm{C} \mathrm{CP}-\mathrm{MAS}$ NMR spectra of 9r9- and 99r-PBI, which matches almost perfectly for the main chain $\mathrm{CH}_{2}$ sites in the spectral range between $36-41.5 \mathrm{ppm}$. In the case of the central methyl branch (18 - 22ppm), the sum spectrum of 9r9- and 99r-PBI (dashed red line) is significantly narrower compared to the spectrum of 9rrPBI (solid black line), even though the overall line shape is similar. This suggests a higher local mobility of the methyl groups in 9r9- and 99r-PBI averaging more efficient different packing arrangements of the methyl branch as confirmed by residual ${ }^{1} \mathrm{H}^{13} \mathrm{C}$ hetero-nuclear dipolar coupling measurements. Please note, that these robust conformational distributions are not only observed for sequence defined PBIs with linear C9 alkyl chains.

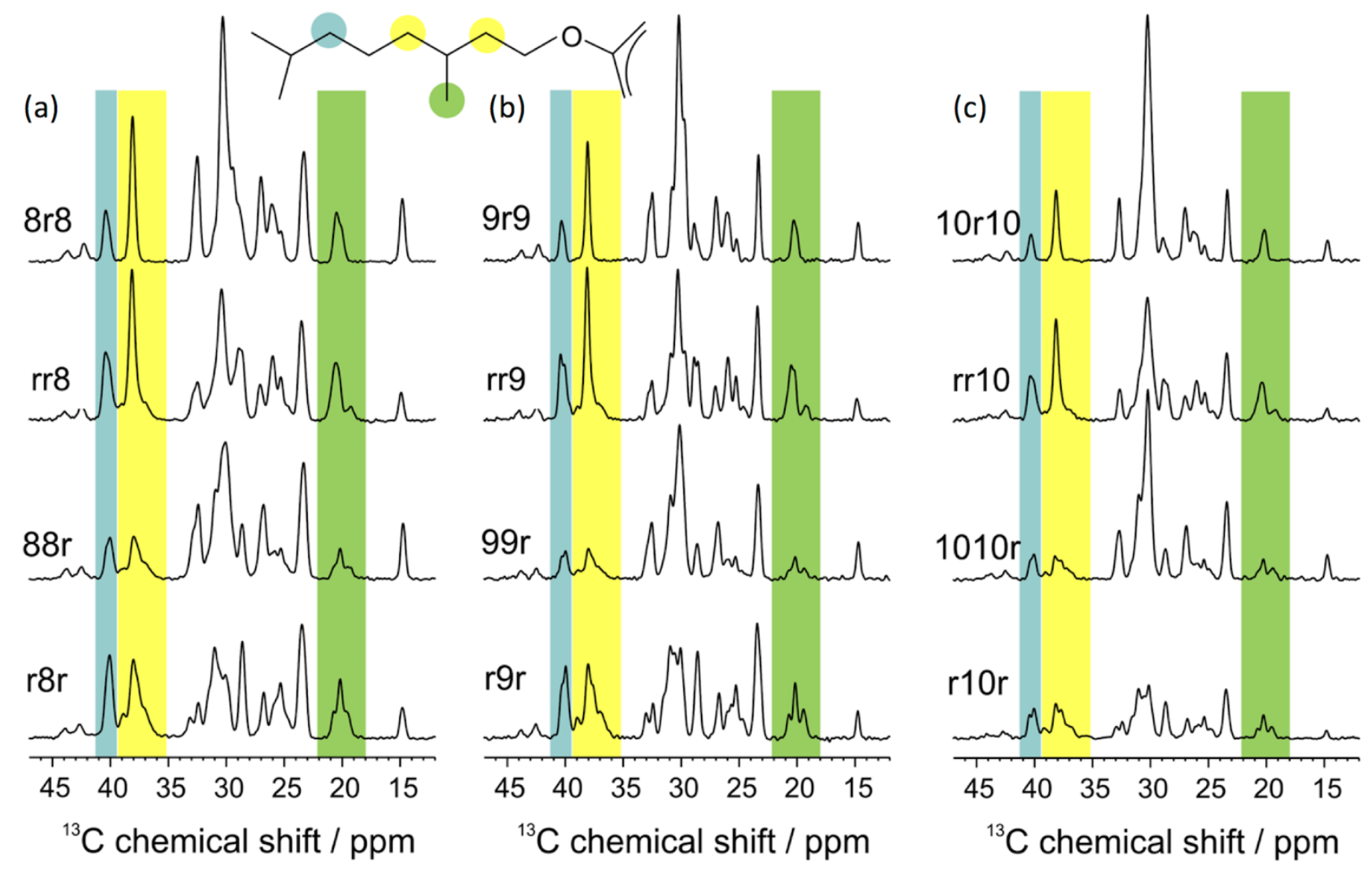

Figure S60: ${ }^{13} \mathrm{C}$ CP-MAS spectra of sequence defined C8 - C10 PBI derivatives recorded at $60^{\circ} \mathrm{C}, 25$ $\mathrm{kHz}$ MAS and $700 \mathrm{MHz}{ }^{1} \mathrm{H}$ Larmor frequency. The NMR spectra of PBI-nrn samples show remarkable narrow signals for the $\mathrm{CH}_{2}$ groups next to the upper branching site (yellow background) and the upper methyl branch (green background), indicating a highly restricted conformational distribution for the $\mathrm{dm} 8$ main chain and a well-defined (or nicely averaged) packing arrangement for the methyl branch.

Minor variations of the linear alkyl chain length leave the conformational distributions of the $\mathrm{dm} 8$ side chains almost unchanged (see Figure S60). Remarkably, temperature changes in the range from $60-$ 
$100{ }^{\circ} \mathrm{C}$ do not change NMR line shapes reflecting the conformational distributions and conformational averaging, whereas the CP-MAS signal intensities decay in the same temperature range in average by more than a factor of two due to increased molecular fluctuations and thus reduced residual hetero-nuclear dipolar couplings (see Figure S61).
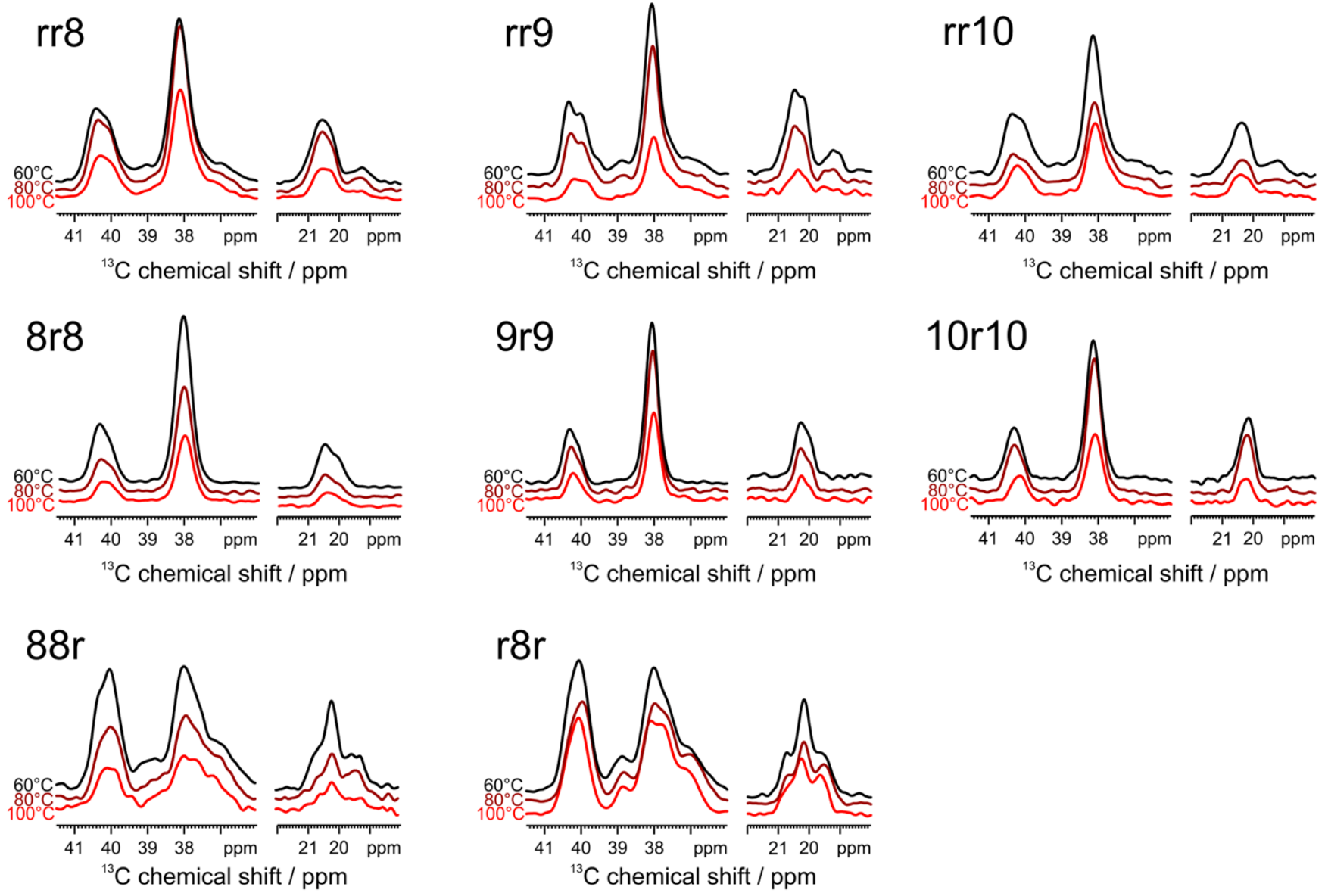

Figure S61: Temperature dependent 13C CP-MAS NMR spectra of sequence defined PBI derivatives. Temperature variations between 60 and $100^{\circ} \mathrm{C}$ cause a reduction of the signal intensity due to growing molecular fluctuations with increasing temperature reducing the hetero-nuclear dipolar couplings required

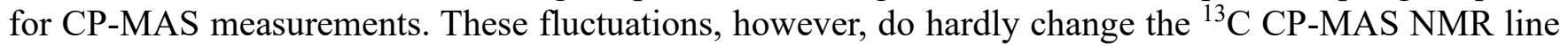
shape and thus do not affect the conformational distribution and averaging of the $\operatorname{dm} 8$ side chains at these temperatures. 


\section{Procedure of XRD Measurements}

The aligned sample fibers are exposed to XRD under vacuumn for 150 seconds for the first exposure. Then the position of the detector will move slightly (programed by Xeuss 2.0) for another 150 seconds exposure. The two XRD measurements will be integrated and the blocking lines of the detectors will be removed. In the final XRD pattern, $300 \mathrm{~s}$ exposure in total is presented. Figure S14 shows the second cooling of 9r9-PBI with the rate $40{ }^{\circ} \mathrm{C} / \mathrm{min}$ at $100{ }^{\circ} \mathrm{C}$.
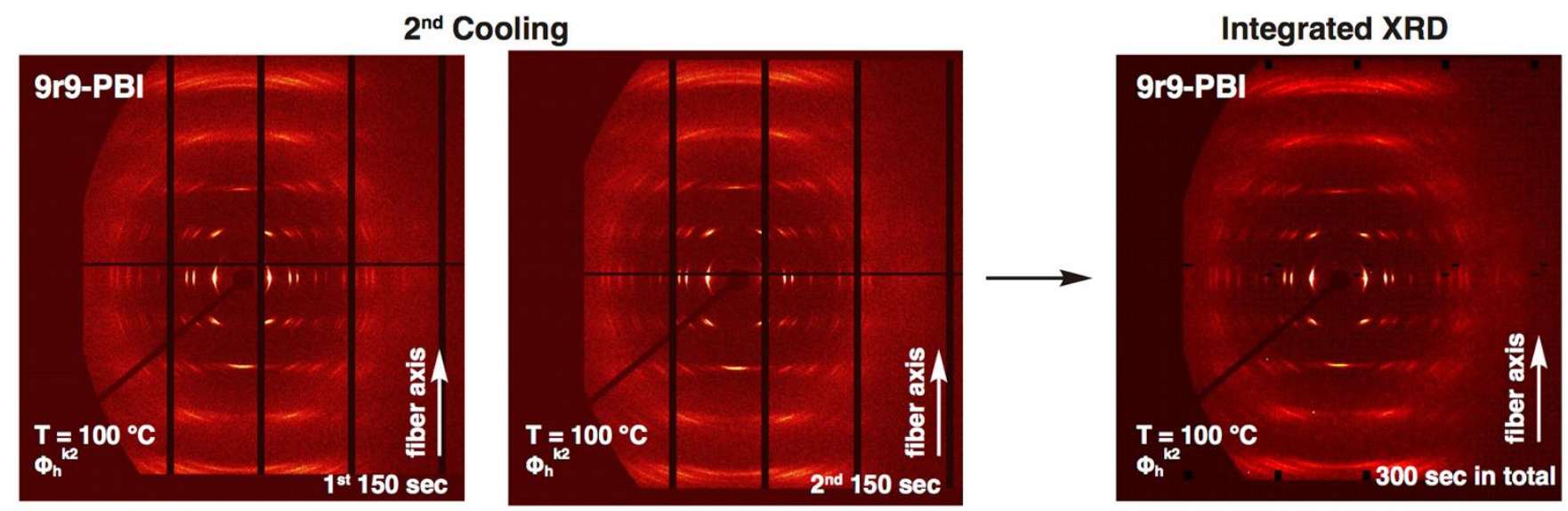

Figure S62. Fiber XRD of the cogwheel 9r9-PBI measured upon heating and cooling at $40{ }^{\circ} \mathrm{C} / \mathrm{min}$ in the $\Phi_{\mathrm{h}}{ }^{\mathrm{k} 2}$ phase. XRD patterns of the first and second 150 seconds exposures are presented as well as the final integrated XRD pattern. The combination of eight diffraction images from eight detectors resulted in the black stripe lines in the diffractograms obtained after only $2.5 \mathrm{~min}$ exposure, which were subsequently removed in the 5 min images by combination with images recorded in the next 2.5 min where the eight detectors were moved to another position. 


\section{References for the Supporting Information}

(1) Percec, V.; Sun, H.-J.; Leowanawat, P.; Peterca, M.; Graf, R.; Spiess, H. W.; Zeng, X.; Ungar, G.; Heiney, P. A. Transformation from Kinetically into Thermodynamically Controlled SelfOrganization of Complex Helical Columns with 3D Periodicity Assembled from Dendronized Perylene Bisimides. J. Am. Chem. Soc. 2013, 135, 4129-4148.

(2) Heiney, P. A. Datasqueeze: A Software Tool for Powder and Small-Angle X-Ray Diffraction Analysis. Comm. Powder Diffr. Newsl. 2005, 32, 9-11.

(3) Partridge, B. E., Wang, L., Sahoo, D., Olsen, J. T., Leowanawat, P., Roche, C., Ferreira, H., Reilly, K. J., Zeng, X., Ungar, G.; Heiney, P. A. Sequence-Defined Dendrons Dictate Supramolecular Cogwheel Assembly of Dendronized Perylene Bisimides. J. Am. Chem. Soc. 2019, 141, 1576115766.

(4) Pearson, A. J.; Bruhn, P. R. Studies on the Synthesis of Aryl Ethers Using Arene-Manganese Chemistry. J. Org. Chem. 1991, 56, 7092-7097.

(5) Buzzacchera, I.; Xiao, Q.; Han, H.; Rahimi, K.; Li, S.; Kostina, N. Y.; Toebes, B. J.; Wilner, S. E.; Möller, M.; Rodriguez-Emmenegger, C.; Baumgart, T.; Wilson, D. A.; Wilson, C. J.; Klein, M. L.; Percec, V. Screening Libraries of Amphiphilic Janus Dendrimers Based on Natural Phenolic Acids to Discover Monodisperse Unilamellar Dendrimersomes. Biomacromolecules 2019, 20, 712-727.

(6) Zhang, S.; Xiao, Q.; Sherman, S. E.; Muncan, A.; Ramos Vicente, A. D. M.; Wang, Z.; Hammer, D. A.; Williams, D.; Chen, Y.; Pochan, D. J.; Vértesy, S.; André, S.; Klein, M. L.; Gabius, H.-J.; Percec, V. Glycodendrimersomes from Sequence-Defined Janus Glycodendrimers Reveal High Activity and Sensor Capacity for the Agglutination by Natural Variants of Human Lectins. J. Am. Chem. Soc. 2015, 137, 13334-13344.

(7) Percec, V.; Imam, M. R.; Peterca, M.; Leowanawat, P. Self-Organizable Vesicular Columns Assembled from Polymers Dendronized with Semifluorinated Janus Dendrimers Act As Reverse Thermal Actuators. J. Am. Chem. Soc. 2012, 134, 4408-4420.

(8) Percec, V.; Imam, M. R.; Peterca, M.; Wilson, D. A.; Heiney, P. A. Self-Assembly of Dendritic Crowns into Chiral Supramolecular Spheres. J. Am. Chem. Soc. 2009, 131, 1294-1304.

(9) Percec, V.; Peterca, M.; Tsuda, Y.; Rosen, B. M.; Uchida, S.; Imam, M. R.; Ungar, G.; Heiney, P. A. Elucidating the Structure of the Pm3n Cubic Phase of Supramolecular Dendrimers through the Modification of Their Aliphatic to Aromatic Volume Ratio. Chem. Eur. J. 2009, 15, 8994-9004.

(10) Percec, V.; Peterca, M.; Tadjiev, T.; Zeng, X.; Ungar, G.; Leowanawat, P.; Aqad, E.; Imam, M. R.; Rosen, B. M.; Akbey, U.; Graf, R.; Sekharan, S.; Sebastiani, D.; Spiess, H. W.; Heineu, P. A.; Hudson, S. D. Self-Assembly of Dendronized Perylene Bisimides into Complex Helical Columns. J. Am. Chem. Soc. 2011, 133, 12197-12219. 